\title{
DNA barcoding a nightmare taxon: Assessing barcode index numbers and barcode gaps for sweat bees
}

\begin{tabular}{|r|l|}
\hline Journal: & Genome \\
\hline Manuscript ID & gen-2017-0096.R2 \\
\hline Manuscript Type: & Article \\
\hline Date Submitted by the Author: & 18 -Aug-2017 \\
\hline Complete List of Authors: & Gibbs, Jason; University of Manitoba, Entomology \\
\hline $\begin{array}{r}\text { Is the invited manuscript for } \\
\text { consideration in a Special } \\
\text { Issue? : }\end{array}$ & This submission is not invited \\
\hline Keyword: & Apoidea, species delimitation, identification, taxonomy, Hymenoptera \\
\hline & \\
\hline
\end{tabular}

SCHOLARONE ${ }^{m}$

Manuscripts 
1 DNA barcoding a nightmare taxon: Assessing barcode index numbers and barcode gaps

2 for sweat bees

3 Jason GIBBS

4 University of Manitoba,

5 Department of Entomology,

612 Dafoe Rd.

7 Winnipeg, Manitoba, R3T 2N2

8

$9 \quad$ Email: jason.gibbs@umanitoba.ca

10

11

12

13

14

15

16

17

18 


\section{Abstract}

There is an ongoing campaign to DNA barcode the world's $>20,000$ bee species. Recent

21 revisions of Lasioglossum (Dialictus) (Hymenoptera: Halictidae) for Canada and the eastern

22 United States were completed using integrative taxonomy. DNA barcode data from 110 species

23 of $L$. (Dialictus) are examined for their value in identification and discovering additional

24 taxonomic diversity. Specimen identification success was estimated using the best close match

25 method. Error rates were 20\% relative to current taxonomic understanding. Barcode Index

26 Numbers (BINs) assigned using Refined Single Linkage Analysis (RESL) and 'barcode gaps'

27 using the Automatic Barcode Gap Discovery (ABGD) method were also assessed. RESL is

28 incongruent for $44.5 \%$ species, although some cryptic diversity may exist. Forty-three of 110

29 species were part of merged BINs with multiple species. The barcode gap is non-existent for the

30 data set as a whole and ABGD showed levels of discordance similar to the RESL. The

31 'viridatum species-group' is particularly problematic, so that DNA barcodes alone would be

32 misleading for species delimitation and specimen identification. Character-based methods using

33 fixed nucleotide substitutions could improve specimen identification success in some cases. The

34 use of DNA barcoding for species discovery for standard taxonomic practice in the absence of a

35 well-defined 'barcode gap' is discussed.

36 Keywords. Apoidea, Hymenoptera, identification, species delimitation, taxonomy 
40

41

42

43

44

45

46

47

48

49

50

\section{Introduction}

DNA barcoding is a method for identifying biological samples to species using the sequence of a standardized gene fragment (Hebert et al. 2003a, Hebert and Gregory 2005). DNA barcoding standards for animals require sequencing a fragment of mitochondrial cytochrome $c$ oxidase subunit 1 not less than 500 bp in length (Hebert et al. 2003a, Hubert et al. 2008), although shorter fragments also can be used for identification (Hajibabaei et al. 2006b) and individual nucleotide substitutions can be diagnostic (Burns et al. 2007, Gibbs 2009b). DNA barcodes are also commonly used to aid in the delimitation of species boundaries (Gibbs 2009a, Rehan and Sheffield 2011, González-Vaquero et al. 2016b, Packer and Ruz 2017). I prefer to use the terms `specimen identification` and 'species discovery' (Collins and Cruickshank 2012) to prevent confusion over the more ambiguous term 'species identification'.

DNA barcoding for specimen identification works by comparing unknown samples to a database of sequences generated from identified material available on GenBank and the Barcode of Life Data System (BOLD; Ratnasingham \& Hebert 2007). BOLD is the product of collaboration between computer programmers, molecular biologists and taxonomists (Smith et al. 2005, Hajibabaei et al. 2005, Ratnasingham and Hebert 2007). Taxonomists provide the scientific context for sequence data used for specimen identification (Goldstein and DeSalle 2011). The advantage of this enterprise from the taxonomists' perspective is a potential wealth of molecular data that can be used to test hypotheses of species limits (DeSalle et al. 2005). The combination of traditional taxonomic approaches with molecular methods can create a 'taxonomic feedback loop', which can lead to species discovery and more well resolved taxonomies (Page et al. 2005). Inclusion of molecular data in taxonomic studies is one part of a 
62 broader integrative approach to the science sometimes referred to as 'integrative taxonomy'

63 (Dayrat 2005).

DNA barcoding success is often related to the presence of a barcoding 'gap' (Meyer and

65

66

67

68

69

70

71

72

73

74

75

76

77

78

79

80

81

82

83

84

Paulay 2005). If genetic divergence within species does not overlap with divergence between congeners then DNA barcodes can identify specimens effectively. DNA barcoding loses efficacy when the barcoding 'gap' becomes small or absent (Meyer and Paulay 2005). Sequence divergence thresholds such as 2 or $3 \%$ have been suggested for grouping specimens into provisional species (Hebert et al. 2004b, Smith et al. 2005). High success rates using similar thresholds have been reported for some taxa (Hebert et al. 2003b, 2004b, Barrett and Hebert 2005), but would not be sufficient for some species-complexes, including groups of butterflies (Hebert et al. 2004a), flies (Meier et al. 2006, Virgilio et al. 2012), and bees (Gibbs 2009a, 2009b, Almeida et al. 2009). DNA barcoding thresholds work best if matched to the specific taxon library (Meier et al. 2006). A regression method for determining ad hoc thresholds for DNA barcoding libraries has been proposed (Virgilio et al. 2012). An alternative to sequence divergence thresholds is to use diagnostic nucleotide substitutions or unique patterns of nucleotide polymorphism for identifying closely related species (DeSalle et al. 2005, Goldstein and DeSalle 2011, Gibbs et al. 2013).

Ratnasingham and Hebert (2013) recently developed the Barcode Index Number (BIN) system for categorizing DNA barcodes into operational taxonomic units (OTUs) in the absence of taxonomic information. BINs are assigned using Refined Single Linkage Analysis (RESL), an algorithm that doesn't use prior taxonomic knowledge (Ratnasingham and Hebert 2013). RESL uses a 2.2\% threshold of sequence divergence to delimit preliminary OTUs and then refines them using a graphical Markov clustering analysis (Ratnasingham and Hebert 2013). A BIN can have 
85 four possible relationships for any species pair, which Ratnasingham and Hebert (2013) refer to 86 as 'match', 'split', 'merge' and 'mixture' (their figure 1). When traditional taxonomy and BINs 87 are concordant, they are said to 'match'. Splits occur when single species are assigned multiple 88 BINs. Merging occurs when a single BIN number is assigned to two or more species, what has long been referred to informally as 'lumping'. In the 'mixture' scenario, two BINs are assigned to two species, but sequences of at least one species fall into both BINs. Merging and mixture of BINs may occur in situations of introgression, incomplete lineage sorting, or if species are inappropriately assigned too many names (Rheindt et al. 2009). Other methods have been developed for assigning OTUs using sequence data (Pons et al. 2006), including the Automatic recursively allowing for different barcode gaps across the dataset. ABGD has been used in several barcoding studies, typically using default or slightly modified settings (Hendrixson et al. 2013, Kekkonen and Hebert 2014). flowering plants (Klein et al. 2007, Ollerton et al. 2011). Pollination services provided by bees are crucial for most terrestrial ecosystem functioning and much of agricultural production (Klein et al. 2007). Bees are also potentially valuable as indicator taxa of ecosystem health (Zayed et al. 2004), which stems from their increased extinction risk due to a haplodiploid sex determination mechanism (Zayed and Packer 2005, Zayed 2009). Haplodiploidy also has potential impacts on

105 the rates of mitochondrial introgression (Patten et al. 2015), which may reduce the utility of 106 DNA barcodes in species that hybridize (Nicholls et al. 2012, Patten et al. 2015). Nevertheless, 107 DNA barcodes have been used successfully in bees for faunal studies (Sheffield et al. 2009, 
108 Magnacca and Brown 2012, Schmidt et al. 2015, Packer and Ruz 2017), species and subspecies

109 discovery and delimitation (Gibbs 2009a, Rehan and Sheffield 2011, Pauly et al. 2015,

110 González-Vaquero et al. 2016a, Sheffield et al. 2016), and specimen identification (Sheffield et

111 al. 2011).

112

I examine DNA barcode data generated for a group of taxonomically challenging bees in

113 North America using BINs and barcode gaps. Sweat bees (Hymenoptera: Halictidae) have been

114 called 'morphologically monotonous' (Michener 1974) and 'the despair of taxonomists'

115 (Wheeler 1928), and the large subgenus Lasioglossum (Dialictus) is notoriously the most

116 difficult to identify to species. Lasioglossum (Dialictus) are extremely abundant in surveys of

117 North American bees (MacKay and Knerer 1979, Grixti and Packer 2006, Campbell et al. 2007,

118 Droege et al. 2010, Ngo et al. 2013), making identification tools crucial for studies of bee

119 diversity. Thousands of L. (Dialictus) are collected each year in North America, but taxonomic

120 keys have only become available recently for a subset of species based on geographic regions

121 (Gibbs 2009b, 2010a, 2011). Consequently, many studies have been published for which $L$.

122 (Dialictus) specimens are unidentified or misidentified (Kalhorn et al. 2003, Giles and Ascher

123 2006, Grixti and Packer 2006, Kearns and Oliveras 2009, Smith et al. 2012a, Wheelock and

124 O’Neal 2016). A very small pool of taxonomists is available to provide reliable identifications to

125 this group, limited primarily to species included in recent revisions (Gibbs 2010a, 2011, Gibbs et

126 al. 2013). DNA barcodes have proven useful for facilitating species discovery within small

127 species groups of L. (Dialictus) in the past (Gibbs 2009a, 2009b), and they have the potential to

128 aid in the identification of difficult taxa for which taxonomic expertise is limited (Hebert et al.

129 2003a, Packer et al. 2009). 

Lasioglossum (Dialictus) has a nearly cosmopolitan distribution including nearly the

131

132

133

134

135

136

137

138

139

140

141

142

143

144

145

146

147

148

149

150

151

152

entire Nearctic, Neotropical, Palaearctic and Afrotropical regions. The genus as a whole has a relatively recent origin (31 Mya, 95CI 24-48 Mya) (Gibbs et al. 2012), which has diversified rapidly into more than 1800 described species (Gibbs et al. 2012, Ascher and Pickering 2016).

The genus has not been revised for many parts of the world (Michener 2007), so the total species richness may be much higher. The recent and rapid diversification of Lasioglossum is likely to blame for taxonomic challenges associated with the genus (Gibbs et al. 2012, Groom et al. 2013). In addition, sexual dimorphism and caste variation within Lasioglossum has led to taxonomic errors in the past (Knerer and Atwood 1964). It is unclear why Lasioglossum has diversified so rapidly, but their generalist nature seems to allow them to thrive in varied conditions. The small body size of $L$. (Dialictus) may also allow for geographic isolation of populations and subsequent speciation.

I restricted the study of $L$. (Dialictus) DNA barcoding to the species occurring in Canada and the United States for two reasons. There have been recent revisions of L. (Dialictus) in these two areas (Gibbs 2010a, 2011) and DNA barcode data for these species are most complete. The species in these regions as currently defined were based on an integrative approach using an evolutionary species concept. Data used in delimiting species included morphology, DNA barcodes, ecology, and geography (Gibbs 2009a, 2010a, 2011). These data were used in combination to corroborate taxonomic hypotheses (DeSalle et al. 2005). In cases where corroboration failed, individuals or populations were considered conspecific. Species were not defined by a single character where polymorphism could not be distinguished from species level differences. Some highly variable species were considered tentative species complexes in need of additional study (Gibbs 2010a). 
Given that taxonomic information is lacking globally, including western North America,

154 I explore the effectiveness of molecular methods for identifying specimens and inferring species

155 boundaries in this group. Both RESL and ABGD have been reported to be relatively successful

156 for delimiting species boundaries using public data sets (Puillandre et al. 2012, Ratnasingham

157 and Hebert 2013, Kekkonen and Hebert 2014), but these have often been across broader taxon

158 groups where the taxonomy overall is less problematic. I focus attention on whether current

159 DNA barcode data are sufficient for identification and delimitation of L. (Dialictus) specimens

160 based upon the best close match method (Virgilio et al. 2012) and the BIN system. I also address

161 remaining taxonomic issues in the group and the use of DNA barcodes for species delimitation in

162 a difficult taxon.

163 Materials and methods

164 Data set

Compliant DNA barcodes for 110 species were generated concurrently with traditional

166 taxonomic study that culminated in taxonomic monographs on L. (Dialictus) for Canada (Gibbs

167 2010a) and the eastern United States (Gibbs 2011). Species limits used herein were based on

168 morphological study of many specimens, including name-bearing types, and through integrative

169 approaches that incorporated barcode data with morphology and geography (Gibbs 2009a).

170 Specimens were sequenced from the greatest possible geographic range of each species so that

171 some sequences originate from specimens collected outside the geographic boundaries of Canada

172 and the eastern United States. Whenever possible, samples were included from the type locality

173 or as close as was possible. In delimiting species, sequence divergence from DNA barcodes in

174 the absence of other supporting evidence was not considered sufficient to justify recognition of 
175 species (Gibbs 2010a, 2011). Instead, such situations were considered candidate species

176 complexes in need of additional study, such as examination of nuclear loci (Carman and Packer

177 1996, Danforth et al. 1998).

The DNA barcoding methods were described in previous studies of Lasioglossum (Gibbs

179

180

181

182

183

184

185

186

187

188

189

190

191

192

193

194

195

196

197 2009a, 2009b, Gibbs et al. 2013). Briefly, sequencing was performed at the Canadian Centre for DNA Barcoding at the University of Guelph (Guelph, Ontario). DNA extractions were performed using standard methods for 96-well plates (Ivanova et al. 2006). Either a single hind leg or both the middle and hind leg (for small specimens) from the right side were used for extractions. "Universal" primer pairs were used to amplify the DNA barcode region; either LCO1490 and HCO2198 (Folmer et al. 1994) or the variants LepF1 and LepR1 (Hebert et al. 2004a). LepF1 and LepR1 are variations of the Folmer primers designed for Lepidoptera and often used for DNA barcoding Insecta. The LepR1 primer has since been shown to be a poor fit for halictid bees (Smith et al. 2012b). Samples that failed to amplify were then reattempted using internal primer pairs LepF1 and C_AntMr1D-RonIIdeg_R and LepR1 with MLepF1. (Hajibabaei et al. 2006b, Smith et al. 2007). PCR and sequencing reactions followed standard protocols (Hajibabaei et al. 2005). Sequences were uploaded to the Barcode of Life Data Systems (BOLD) (Ratnasingham and Hebert 2007) and GenBank (accession numbers in prep.).

A full-length barcode sequence for these bees is $658 \mathrm{bp}$. Since internal primers were often necessary, many sequences did not approach this length. To limit spurious results from small sequence overlap I limited most data analyses to barcode-compliant sequences greater than $500 \mathrm{bp}$, with two or more trace files, meeting minimum recommended standards for DNA barcoding (Hubert et al. 2008). Samples were also excluded from analyses if they had more than $1 \%$ ambiguous base calls. Any sequences with stop codons or showing evidence of 
198 contamination (e.g., best match sequence identity to non-bee taxa or other bee genera than to

199 Lasioglossum) were also removed from the dataset. A larger number of non-compliant sequences

200 and extra-limital species are also available on BOLD. These are not analyzed fully, but are

201 referenced with respect to their inclusion in BINs and their potential effects on successful

202 identification using DNA barcodes.

204 with default settings (gap penalty of -400 and extension penalty of -0.01 ) as implemented by

205 MEGA7 (Kumar et al. 2016). Re-alignment was necessary to remove seven inappropriate 206 insertion/deletions in the sequences generated in the BOLD alignment. The final alignment had

207 no indels or stop codons. Aligned sequences were renamed in MESQUITE (Maddison and 208 Maddison 2017) for compatibility with downstream analyses.

210 Statistical analyses were conducted using R version 2.3.3 (R Core Team 2015), unless otherwise

211 specified. The minimum interspecific and maximum intraspecific genetic distances for each

212 species were calculated using the nonConDist and maxInDist functions in the spider package

213 (Brown et al. 2012) using a Kimura two-parameter model of substitution (Kimura 1980). To

214 assess the potential barcode gap, we examined the proportion of cases where minimum

215 interspecific distance was greater than intraspecific distance for each sequence. The

216 identification success rate was estimated using the best close match method (Meier et al. 2006)

217 implemented by the threshOpt function. The ad hoc package (Sonet et al. 2013) was also used to 218 assess the identification success rate and optimal threshold values for the DNA barcoding library 219 based on the methods described by Virgilio et al. (2012). The checkDNAbcd function was used 
220 with a Kimura two-parameter model to calculate pairwise distances. Identification error rates

221 using the best close match method were determined using the adhocTHR function. Diagnostic

222 nucleotide substitutions were determined for species complexes using the function nucDiag in

223 the spider package for species with merged BINs.

224 Species discovery

225 BOLD automatically implements RESL to group sequences into BINs (Ratnasingham

226 and Hebert 2013). BIN contents were compared to currently accepted species limits to determine

227 the congruence of RESL to integrative taxonomic assignment of species limits. In addition, the

228 Automatic Barcode Gap Discovery method (Puillandre et al. 2012) was used to estimate species

229 limits using the online web application (http://wwwabi.snv.jussieu.fr/public/abgd/abgdweb.html,

230 accessed $18 \mathrm{Jul} 2017$ ). The results of our optimal threshold analyses were used to improve $a$

231 priori input values for intraspecific diversity limits $(P)$ and minimum gap width $(X)$. Default

232 values of $P$ between 0.001 and 0.1 were used. The number of steps was set to 20 . Several values

233 of $X$ from 0.1 to 1.5 were evaluated. Simple distances are reported as these seemed to perform

234 better than the Jukes-Cantor or Kimura 2-parameter method (Srivathsan and Meier 2011).

235 ABGD output includes several options for partitioning the data over values of $P$. The preferred

236 partition was selected using an intermediate value of $P$ after an initial steep decline in number of

237 species estimated (Puillandre et al. 2012).

\section{Results}

239 Data set

240 In total, 1279 DNA barcode-compliant sequences were generated for L. (Dialictus)

241 species revised in Canada and the eastern United States (supplementary table). Sequences had a 
242 strong AT bias in the $3^{\text {rd }}$ codon position (GC content: $7.1 \pm 0.07 \mathrm{SE}$ ). One hundred and ten

243 species identified using integrative approaches were included (11.6 \pm 1.3 sequences per species,

244 range $=1-97)$. The mean number of haplotypes per species was $5.2 \pm 0.46$ (range $1-24)$. Mean

245 maximum intraspecific distance was $1.69 \pm 0.05 \%$ (range $0-7.5 \%$ ), excluding singletons, while

246 the mean minimum interspecific distance was $2.60 \pm 0.21 \%$ (range $0-11.5 \%$ ).

247 Specimen identification

248

249

250

251

252

253

254

255

256

257

258

259

260 261 identification using these characters (Table 3).

262

Species discovery

The DNA barcoding threshold with the lowest relative error $(20.3 \%)$ in comparison to current taxonomy was $1.4 \%$ based on the methods of Virgilio et al. (2012). The best match method implemented in spider resulted in an optimal threshold of $0.2-0.3 \%$, but an overall error rate of $33.3 \%$. Eleven species were represented by a single DNA barcode sequence, necessitating a false positive identification using the best match method. However, these species only made up a small proportion of the false positives and removing them from analyses did not improve results. For nine of these species the distance to their best match was greater than the ad hoc threshold of $1.4 \%$ (mean $3.2 \pm 0.5 \%$ ) suggesting that with additional sampling these species would be correctly identified. The singleton L. reticulatum (Robertson) differed from its closest match L. bruneri (Crawford) by $1.3 \%$. Of the singletons, only L. alachuense (Mitchell) had no local gap with its closest match L. oblongum (Lovell). An analysis of species monophyly using neighbour joining revealed that 30 of the 110 species were non-monophyletic. Diagnostic nucleotide substitutions were found for a subset of problematic species that could allow 
Ninety-four BINs corresponded to these 110 species in the data set, comprising 61

264

265

266

267

268

270

271

272

273

274

275

276

277

278

279

280

281

282

283

284

285 'matches' (Table 1), 6 'splits' (Table 2), 43 'merged' (Table 3) and 6 'mixed' BINs (Tables 2 and 3). Species with minimum interspecific distance greater than $1.4 \%$ usually ( $85 \%)$ showed concordance between traditional taxonomy and BINs (Fig. 1). Four species above this cut-off had multiple BINs suggestive of cryptic species and two species were morphologically distinct singletons grouped with the viridatum species complex.

The ABGD performed poorly with the Jukes-Cantor and Kimura 2-parameter when the relative gap width value $(X)$ was greater than one. The results from simple p-distances were less impacted by values of $X$ and more often estimated species richness similar to integrative taxonomy. The simple distance ABGD results with relative gap width set to 1.0 are summarized in Tables 1-3. The performance of ABGD was comparable to RESL. Examining pairwise distances across DNA barcode-compliant sequences reveals no definitive DNA barcode gap (Fig. 2).

Species with 'merged' BINs were sometimes identifiable using fixed nucleotide substitutions, although they did not show clear barcode gaps (Table 3). For example, Lasioglossum knereri Gibbs and L. subversans (Mitchell) were grouped together in both the ABGD and RESL analyses, but they are distinguished by four fixed nucleotide substitutions. In the case of the L. viridatum (Lovell) species complex, 19 morphospecies with barcode-compliant sequences were combined in a single BIN. Three of these could be distinguished from all others by fixed substitutions and four were treated as distinct groups in the ABGD analysis (Table 3). The remaining species lacked fixed differences with one or more species in the complex. One putative species in this complex, L. subviridatum (Cockerell), shows two distinct DNA barcode clusters, with 15 fixed nucleotide substitutions between them. Treated as a single species, as is 
286

287

288

289

290

291

292

293

294

295

296

297

298

299

300

301

302

303

304

305

306

307

currently the situation, the consensus sequence shows substantial overlap with other L. viridatum group species, with 0 fixed substitutions with 8 other species in the group (mean 2.1). If $L$. subviridatum were ultimately treated as two species, which seems plausible, only two other species in the viridatum complex would show zero fixed substitutions with either of the candidate species (mean 8.1). These results imply that even if L. subviridatum is maintained as a single species other taxa in the species group could be distinguished from it based on unique patterns of polymorphism.

In other species with merged BINs, many are distinguishable by one or more fixed nucleotide substitutions. The exceptions are L. callidum (Sandhouse) and L. versatum (Robertson), which show identical barcode haplotypes; L. leucocomum (Lovell) and $L$. succinipenne (Ellis); L. puteulanum Gibbs and L. tegulare (Robertson), and the group of L. hitchensi Gibbs, L. leviense (Mitchell) and L. weemsi (Mitchell). In combination with the $L$. viridatum group above, 21 of 110 species are not readily identifiable using DNA barcodes even when considering single base pair substitutions. In the case of L. puteulanum and L. tegulare, the majority of individuals are readily distinguishable, but four individuals that seem to match $L$. tegulare morphologically group with L. puteulanum based on barcodes. The range of these individuals might indicate that there may be unresolved aspects of the taxonomy of this complex. Similarly, $L$. weemsi is distinguishable from all but one individual of each of $L$. leviense and $L$. hitchensi. The morphological identifications have been carefully checked multiple times, so the discrepancy is either due to unreliability of the distinguishing anatomical traits or the barcode sequences. Cross-contamination of samples within a plate is a possibility, although attempts were made to keep closely related species and putative conspecifics well separated within a plate. 
Some species have BINs and ABGD groups congruent with taxonomic species

309

310

311

312

313

314

315

316

317

318

319

320

321

322

323

324

325

326

327

328

329

330

delimitation, but may have incongruence with extra-limital species. The eastern North American species $L$. trigeminum Gibbs has distinct barcodes from the morphologically similar species $L$. versatum and L. callidum. However, L. connexum (Cresson), a species found in Texas and therefore not treated in previous revisions, has only a single nucleotide difference from $L$. trigeminum, but due to insufficient amplification success in L. connexum, it remains unclear if that difference is fixed.

\section{Discussion}

The value of DNA barcodes in an integrative taxonomic approach is difficult to quantify retroactively. Species delimitation is a process that occurs over time as taxonomists familiarize themselves with a taxon and gather evidence from multiple sources. In the context of $L$. (Dialictus), the availability of an independent data set was invaluable even in cases were RESL and ABGD were deemed unsuccessful. As an illustration, I describe the situation of L. ephialtum Gibbs, which has a specific epithet that literally means 'nightmare' in Greek. Lasioglossum ephialtum was not described until 2010, but it is a relatively common species, particularly in urban areas (Gibbs 2010a). The species was often misidentified as L. versatum (or the junior synonym rohweri Ellis) by earlier workers leading to confusion over the diagnostic characters for L. versatum. Although DNA barcodes of L. ephialtum are not easily distinguished from those of members of the L. viridatum species group, they are clearly distinct from L. versatum, which helped to clarify the latter species' limits. Other advantages of incorporating DNA barcodes into these revisions was the ability to associate dimorphic sexes and female castes (Gibbs 2010a).

Many Lasioglossum are social with queens and workers, which can be so different that they have been described as distinct species (Knerer and Atwood 1964), and nearly two thirds of North 
331 American L. (Dialictus) prior to recent revisionary studies were described from a single sex

332 (Moure and Hurd 1987).

DNA barcodes may also benefit taxonomy by showing clear variation in morphologically distinct species that were often not recognized due to insufficient study. Species with distinct characters in otherwise problematic taxa may not be scrutinized as closely and incorrectly lumped (Packer and Taylor 1997). DNA barcodes sequence variation can highlight the variation

337 in a species complex leading to more careful taxonomic study. Examples in L. (Dialictus) include the L. tegulare and L. petrellum (Cockerell) species-groups (Gibbs 2009a, 2009b). When DNA barcodes suggest splitting species it could be evidence of cryptic species, but it might also represent highly divergent barcodes within a single species. Heteroplasmy (i.e. the presence of multiple haplotypes within an individual) has been shown to occur in the bee genus Hylaeus

342 (Magnacca and Brown 2010). Heteroplasmic individuals may still be identified using DNA 343 barcodes, but the reference database must include multiple divergent haplotypes for each species.

344 The best example of cryptic species among bees is in the Halictus ligatus Say/Halictus poeyi 345 Lepeletier species pair (Packer et al. 2016). Originally separated based upon extensive allozyme 346 data (Carman and Packer 1996), these two species were subsequently distinguished using both 347 nuclear and mitochondrial DNA sequences including DNA barcodes (Danforth et al. 1998, 348 1999). The two are sympatric in a narrow area with almost no heterozygosity at the allozyme loci 349 (never more than one of 7 loci out of hundreds of individuals from sympatric populations sampled) (Packer 1999). No morphological differences have been discovered despite intensive 351 investigation, although there are slight phenological differences in sympatry (Dunn et al. 1998). 352 DNA barcodes have recently recovered a third Neotropical species in this complex, presumably H. townsendi Cockerell based on geography (Packer et al. 2016). 

Lasioglossum (Dialictus) is taxonomically challenging and it is likely that species-level 355 diversity has not been completely described even within areas that have received detailed study 356 (Gibbs 2010b, 2011). Lasioglossum is the most species rich of all bee genera, but few revisionary 357 studies exist. This group of small bees has diversified rapidly to occupy virtually every terrestrial 358 habitat on the planet (Michener 1979, 2007). Although broadly generalist and highly adaptable, 359 L. (Dialictus) are small-bodied species that are likely limited in their dispersal ability. As a 360 result, this subgenus seems able to successfully establish and persist in novel and often marginal 361 habitats, but is also susceptible to physical barriers to gene flow. Species rich taxa with recent 362 diversification are not expected to perform well with single locus automated species delimitation 363 methods (Puillandre et al. 2012).

The highest level of intra-specific divergence in this study, $6.9 \%$, is seen in L. ruidosense, 365 putatively a single species with an enormous north-south distribution, ranging from southern 366 New Mexico to Alaska (Gibbs 2010a). Southern populations of L. ruidosense are primarily 367 limited to high elevations, which have the potential to isolate populations leading to allopatric 368 speciation (Coyne and Orr 2004) as seems to be the case with another species, L. boreale 369 Svensson, Ebmer and Sakagami, with a similar distribution in North America (Packer and Taylor 370 2002). Allozyme data from L. boreale showed little genetic variation from individuals spanning

371 large geographical distances, but high elevation populations in the southwestern USA were

372 found to have fixed differences. Additional study is required to determine and describe the extent 373 of diversity in this apparent complex. In the ruidosense case, the incongruence between current 374 taxonomy and DNA barcoding is likely caused by insufficient taxonomic study. High intra375 specific genetic divergence is also present in L. cressonii (Robertson) and L. sagax (Sandhouse). 376 Neither of these two species has a distinct geographical or morphological pattern correlated with 
377 sequence divergence. In fact, highly divergent sequences have been found from a single locality

378 during the same collecting event for both species (Gibbs 2010a). Substitutions between

379 haplotypes are strongly biased in the $3^{\text {rd }}$ codon position, which does not suggest amplification of

380 a nuclear pseudogene, although a recently derived pseudogene is possible. A more detailed

381 examination of these species that includes morphology and nuclear DNA is required. In the

382 meantime, they can at most be considered "unconfirmed candidate species" (Padial et al. 2010).

383 Lasioglossum cressonii is a common, distinctive species and similar cases in the past have turned

384 out to be cryptic species (Danforth et al. 1998, Gibbs 2009a). Lasioglossum sagax is a less well-

385 known species that until recently was only known from the holotype (Wolf and Ascher 2009,

386 Gibbs 2010a). Future work that includes multiple loci will be productive for delimiting cryptic

387 species where morphological characters are lacking. It should be noted that DNA barcoding may

388 overestimate the number of species (Dasmahapatra et al. 2010), so the deep divergences within

389 species like L. cressonii may not be true evidence of speciation.

Mitochondrial DNA has proven capable of identifying specimens and clarifying species

boundaries in other taxonomically challenging bees (Danforth et al. 1998, Murray et al. 2007,

Bertsch 2009, Sheffield et al. 2009, Gibbs 2009b, Magnacca and Brown 2010, Rehan and

393 Sheffield 2011). However, DNA barcoding is only moderately successful for specimen

394 identification in L. (Dialictus) using either DNA barcode gaps or BINs. The mean maximum

395 intra-specific value of $1.69 \%$ reported here is higher than mean intra-specific values reported for

396 some taxa, including bats (Clare et al. 2007), birds (Hebert et al. 2004b), marine fish (Ward et al.

397 2005), Lepidoptera (Hajibabaei et al. 2006a), bumble bees (Bertsch 2009), and a general bee

398 fauna (Sheffield et al. 2009) but does correspond to some previous studies of insect taxa, such as

399 mayflies (Alexander et al. 2009) and black flies (Rivera and Currie 2009). Direct comparisons 
400 are not always reliable due to differences in sampling effort and the use of mean intra-specific 401 divergences in some studies (e.g., Barrett \& Hebert 2005) and the more relevant value, the mean 402 maximum intra-specific divergence used here (Collins and Cruickshank 2012). Minimum 403 distances between species pairs was on average four times the mean intra-specific genetic 404 divergence but less than double the mean maximum intra-specific divergence. This gap between 405 inter- and intra-specific genetic divergences is less than those of most barcoding studies and falls 406 well below the 10-fold difference suggested in an earlier study of birds (Hebert et al. 2004b).

407 Importantly, the intra-specific and inter-specific divergence varies among taxa and in recently 408 diverged groups any simple criterion for delimiting species will have flaws. importance of traditional taxonomic practices. In another group of bees, DNA barcodes do not

411 distinguish morphologically similar but ecologically distinct species in the Colletes succinctus

412 (L.) group, although differences in nuclear sequences were present (Kuhlmann et al. 2007). The

413 L. viridatum species-group defined by Gibbs (2010a) is comprised of many of the most

414 taxonomically challenging L. (Dialictus) species in Canada and the eastern USA. Members of 415 this complex are often distinguishable based on multiple lines of evidence including ecological 416 differences (e.g., L. georgeickworti Gibbs is the only member restricted to coastal areas of the 417 northeastern United States and L. subviridatum nests in logs unlike the ground nests of most 418 other species; Gibbs 2011). Unfortunately, few members of the viridatum group have had their 419 nests discovered, and none has had their nesting biology described in detail. The nesting biology 420 of L. viridatum described by Atwood (1933) is unreliable (Zarrillo et al. 2016), and may pertain 421 to L. laevissimum. DNA barcodes of L. viridatum group species are among the most difficult to 422 interpret, including a surprising 19 species lumped into a single BIN. It is tempting to consider 
423 this as a case of traditional taxonomy over-splitting species. However, this BIN includes

424 morphologically distinct, geographically separated populations that occupy distinct ecological

425 niches (Gibbs 2010a, 2011). It's possible that the species complex in its entirety has not been

426 accurately delimited, but multiple lines of evidence suggest that it is not a single species (Gibbs

427 2010a, 2011). The absence of clear DNA barcode clusters is presumably due to incomplete

428 lineage sorting or introgression in a species complex that has undergone recent and rapid

429 diversification. A neighbour-joining analysis suggested that $27 \%$ of species in this study had

430 non-monophyletic DNA barcode clusters. Neighbour-joining is not a preferred method of

431 phylogenetic analysis, being generally outperformed by other methods of phylogenetic analysis

432 that are better able to account for evolutionary rate variation (Huelsenbeck 1995; Felsenstein

433 2004). However, neighbour-joining was assessed here because it is commonly used to examine

434 DNA barcode sequences and is the standard tree building algorithm used in BOLD, due to its

435 speed and relatively good performance when sequences have recently diverged (Holder and

436 Lewis 2003). The high level of paraphyly found here for Lasioglossum should be taken into

437 account when interpreting results from neighbour-joining trees as part of future DNA barcoding

438 efforts.

Although DNA barcodes may be insufficient for species identification using RESL or

ABGD in some cases, fixed substitutions can be informative in an integrative taxonomic

441 approach. Lasioglossum (Dialictus) is a recently derived subgenus that has diversified into many

442 hundreds of species (Gibbs et al. 2012, Ascher and Pickering 2016). Closely related species that

443 have recently separated will have evolved fewer neutral mutations than other species.

444 Morphological characters, if under selection, can evolve rapidly (Owen and Harder 1995,

445 Thompson 1998) and could result in clearly defined species with little genetic divergence in 
446 mitochondrial haplotypes. In addition, the possibility of introgression is presumably higher in

447 closely related species and could confound specimen identification and species discovery using

448 DNA barcodes by reducing intraspecific variation among species (Pentinsaari et al. 2014).

449 Lasioglossum hitchensi and L. weemsi are closely related, but distinguishable based on hair 450 patterns of the metasoma; however, a single individual of L. hitchensi was found to have a DNA 451 barcode consistent with $L$. weemsi. This example is a candidate for introgression between closely 452 related species.

In some cases, character-based methods of identification may still allow correct

454 determination of species where distance-based methods are misleading (Burns et al. 2007).

455 Previous results from Lasioglossum species suggest that unique fixed substitutions or unique 456 polymorphism patterns may be sufficient to distinguish species in the absence of a clear DNA 457 barcode gap (Gibbs 2009a, 2009b, Gibbs et al. 2013). Similar methods have also been used in the 458 Bombus lucorum (L.) species complex of bumble bees (Murray et al. 2007, Bertsch 2009) and 459 Hawaiian Hylaeus (Magnacca and Brown 2010). All of the species recognized in the taxonomic 460 revisions are diagnosable using morphological characters (Gibbs 2010b, 2011). If voucher 461 specimens are retained, then additional morphological or geographic characters could be used in 462 cases where DNA barcodes fail entirely. For example, a DNA barcode sequence matching that of 463 L. versatum and L. callidum, which share an identical DNA barcode haplotype, can be 464 differentiated by examining morphological characters, including the shape of the female 465 mandible and protrochanter and male facial pubescence (Gibbs 2010a, 2011). Although DNA 466 barcodes do not distinguish, L. tenax (Sandhouse) and L. cattellae (Ellis), these two species can 467 be distinguished by geographical distribution alone, in addition to aspects of their 468 microsculpture, colour, and pubescence (Gibbs 2010a). Lasioglossum tenax is an alpine/boreal 
469 species restricted to Canada and the Rocky Mountains, while l. cattellae is largely an open

470 grassland species in the mid-Atlantic and Midwestern states of the USA. The BIN system on

471 BOLD allows for expert opinion to annotate BINs believed to include multiple species

472 (Ratnasingham and Hebert 2013). Until BINs are fully annotated and taxonomic revisions are

473 complete, users would be wise to treat BINs with care and use alternative data, including

474 morphology and geographical distribution, to make species identifications using DNA barcodes.

In conclusion, DNA barcodes in L. (Dialictus) lack a well-defined 'barcode gap' that can

476 be used for delimiting species, and RESL and ABGD worked unambiguously in only about half

477 the cases. Nevertheless, DNA barcodes are sufficient for identifying specimens of many species

478 in this taxonomically challenging group even with simple distance-based methods. Character-

479 based identifications have the potential for a greater level of success in identifying specimens

480 with low inter-specific genetic divergence. Unique combinations of nucleotide substitutions may

481 allow specimen identification even when inter-specific divergences are low and fixed nucleotide

482 substitutions are absent (Gibbs et al. 2013). Although some species pairs cannot be identified

483 using DNA barcodes, the sequences are nevertheless informative for reducing the list of possible

484 species names. Simple annotations to the BOLD system (e.g., 'viridatum complex') could flag

485 such species for additional study or sequencing of additional loci. For this reason, I reiterate the

486 need of morphology-based taxonomy and integrative approaches using multiple loci as further

487 tests of species identity. DNA barcodes, even with a weak 'barcode gap', are nevertheless a

488 useful taxonomic tool for bees when used in conjunction with morphology, behaviour, and other

489 data.

490 Acknowledgements 
491 Laurence Packer (York University) was instrumental to the success of this project, providing full 492 support for the research at all stages and providing valuable comments and additions to the 493 manuscript. The editor and two anonymous reviewers also provided useful comments that helped 494 to improve the manuscript greatly. This research was supported through funding to the Canadian 495 Barcode of Life Network from Genome Canada, NSERC, and other sponsors listed at 496 www.BOLNET.ca. Ontario Graduate Scholarships in Science and Technology awarded during 497 the time of data collection were greatly appreciated.

498

499 References

500 Alexander, L.C., Delion, M., Hawthorne, D.J., Lamp, W.O., and Funk, D.H. 2009.

501 Mitochondrial lineages and DNA barcoding of closely related species in the mayfly genus 502 Ephemerella (Ephemeroptera: Ephemerellidae). J. North Am. Benthol. Soc. 28(3): 584-595. 503 doi:10.1899/08-150.1.

504 Almeida, E., Laurenne, N., Kuhlmann, M., and Quicke, D. 2009. Molecular phylogeny and 505 historical biogeography of the bee genus Colletes Latreille, 1802 (Hymenoptera: Apiformes:

506 Colletidae), based on mitochondrial COI and nuclear 28S sequence data. Insect Syst. Evol. 507 40(3): 291-318. doi:10.1163/139956009X12475840653733.

508 Ascher, J.S., and Pickering, J. 2016. Discover Life bee species guide and world checklist 509 (Hymenoptera: Apoidea: Anthophila). Draft-45. Available from 510 http://www.discoverlife.org/mp/20q?guide=Apoidea_species.

511 Atwood, C.E. 1933. Studies on the Apoidea of western Nova Scotia with special reference to 512 visitors of apple bloom. Can. J. Res. 9(5): 443-457. doi:10.1139/cjr33-101. 
513 Barrett, R.D.., and Hebert, P.D.. 2005. Identifying spiders through DNA barcodes. Can. J. Zool.

514 83(3): 481-491. doi:10.1139/z05-024.

515 Bertsch, A. 2009. Barcoding cryptic bumblebee taxa: B. lucorum, B. crytarum and B. magnus, a 516 case study. Beiträge zur Entomol. 59(2): 287-310.

517 Brown, S.D.J., Collins, R.A., Boyer, S., Lefort, M.-C., Malumbres-Olarte, J., Vink, C.J., and 518 Cruickshank, R.H. 2012. Spider: An R package for the analysis of species identity and evolution, 519 with particular reference to DNA barcoding. Mol. Ecol. Resour. 12(3): 562-565. 520 doi:10.1111/j.1755-0998.2011.03108.x.

521 Burns, J.M., Janzen, D.., Hajibabaei, M., Hallwachs, W., and Hebert, P.D.N. 2007. DNA 522 barcodes of closely related (but morphologically and ecologically distinct) species of skipper 523 butterflies (Hesperiidae) can differ by only one to three nucleotides. J. Lepid. Soc. 61: 138-153.

524 Campbell, J.W., Hanula, J.L., and Waldrop, T.A. 2007. Effects of prescribed fire and fire 525 surrogates on floral visiting insects of the blue ridge province in North Carolina. Biol. Conserv. 526 (134): 393-404.

527 Carman, G.M., and Packer, L. 1996. A cryptic species allied to Halictus ligatus Say

528 (Hymenoptera: Halictidae) detected by allozyme electrophoresis. J. Kansas Entomol. Soc. 69: $529 \quad 168-176$.

530 Clare, E.L., Lim, B.K., Engstrom, M.D., Eger, J.L., and Hebert, P.D.N. 2007. DNA barcoding of 531 Neotropical bats: species identification and discovery within Guyana. Mol. Ecol. Notes 7(2): 532 184-190. doi:10.1111/j.1471-8286.2006.01657.x. 
533 Collins, R.A., and Cruickshank, R.H. 2012. The seven deadly sins of DNA barcoding. Mol. Ecol.

534 Resour. 13(6): 969-975. doi:10.1111/1755-0998.12046.

535 Coyne, J.A., and Orr, H.A. 2004. Speciation. Sinauer Associates, Inc., Sunderland, M.A..

536 Danforth, B.N., Mitchell, P.L., and Packer, L. 1998. Mitochondrial DNA differentiation between

537 two cryptic Halictus (Hymenoptera: Halictidae) species. Ann. Entomol. Soc. Am. 91(4): 387-

$538 \quad 391$.

539 Danforth, B.N., Sauquet, H., and Packer, L. 1999. Phylogeny of the bee genus Halictus

540 (Hymenoptera: Halictidae) based on parsimony and likelihood analyses of nuclear EF-1a

541 sequence data. Mol. Phylogenet. Evol. 13(3): 605-618. doi:mpev.1999.0670.

542 Dasmahapatra, K.K., Elias, M., Hill, R.I., Hoffman, J.I., and Mallet, J. 2010. Mitochondrial

543 DNA barcoding detects some species that are real, and some that are not. Mol. Ecol. Resour.

544 10(2): 264-273. doi:10.1111/j.1755-0998.2009.02763.x.

545 Dayrat, B. 2005. Towards integrative taxonomy. Biol. J. Linn. Soc. 85(3): 407-415.

546 doi:10.1111/j.1095-8312.2005.00503.x.

547 DeSalle, R., Egan, M.G., and Siddall, M. 2005. The unholy trinity: taxonomy, species

548 delimitation and DNA barcoding. Philos. Trans. R. Soc. B Biol. Sci. 360(1462): 1905-1916.

549 doi:10.1098/rstb.2005.1722.

550 Droege, S., Tepedino, V.J., LeBuhn, G., Link, W., Minckley, R.L., Chen, Q., and Conrad, C.

551 2010. Spatial patterns of bee captures in North American bowl trapping surveys. Insect Conserv.

552 Divers. 3: 15-23. doi:10.1111/j.1752-4598.2009.00074.x. 
553 Dunn, M., Mitchell, P.L., and Packer, L. 1998. Phenology and social biology of two sibling

554 species of Halictus in an area of sympatry. Can. J. Zool. 76(12): 2207-2213. doi:10.1139/cjz-76-

$555 \quad$ 12-2207.

556 Edgar, R.C. 2004. MUSCLE: multiple sequence alignment with high accuracy and high

557 throughput. Nucleic Acids Res. 32(5): 1792-1797. doi:10.1093/nar/gkh340.

558 Felsenstein, J. (2004) Inferring phylogenies. Sinauer Associates, Inc., Sunderland, M.A.

559 Folmer, O., Black, M., Hoeh, W., Lutz, R., and Vrijenhoek, R. 1994. DNA primers for 560 amplification of mitochondrial cytochrome c oxidase subunit I from diverse metazoan 561 invertebrates. Mol. Mar. Biol. Biotechnol. 3(5): 294-9.

562 Gibbs, J. 2009a. Integrative taxonomy identifies new (and old) species in the Lasioglossum

563 (Dialictus) tegulare (Robertson) species group (Hymenoptera, Halictidae). Zootaxa 2032: 1-38.

564 Gibbs, J. 2010a. Revision of the metallic species of Lasioglossum (Dialictus) in Canada 565 (Hymenoptera, Halictidae, Halictini). Zootaxa 3073: 1-382.

566 Gibbs, J. 2010b. Atypical wing venation in Dialictus and Hemihalictus and its implications for 567 subgeneric classification of Lasioglossum. doi:10.1155/2010/605390.

568 Gibbs, J. 2011. Revision of the metallic Lasioglossum (Dialictus) of eastern North America 569 (Hymenoptera: Halictidae: Halictini). Zootaxa (3073): 1-216.

570 Gibbs, J., Brady, S.G., Kanda, K., and Danforth, B.N. 2012. Phylogeny of halictine bees supports 571 a shared origin of eusociality for Halictus and Lasioglossum (Apoidea: Anthophila: Halictidae).

572 Mol. Phylogenet. Evol. 65(3): 926-939. doi:10.1016/j.ympev.2012.08.013. 
573 Gibbs, J., Packer, L., Dumesh, S., and Danforth, B.N. 2013. Revision and reclassification of

574 Lasioglossum (Evylaeus), L. (Hemihalictus) and L. (Sphecodogastra) in eastern North America

575 (Hymenoptera: Apoidea: Halictidae). Zootaxa 3672: 1-117.

576 Gibbs, J.J. 2009b. New species in the Lasioglossum petrellum species group identified through

577 an integrative taxonomic approach. Can. Entomol. 141: 371-396. doi:10.4039/n09-020.

578 Giles, V., and Ascher, J.S. 2006. A survey of the bees of the Black Rock Forest Preserve, New 579 York (Hymenoptera: Apoidea). J. Hymenopt. Res. 15(2): 208-231.

580 Goldstein, P.Z., and DeSalle, R. 2011. Integrating DNA barcode data and taxonomic practice:

581 determination, discovery, and description. Bioessays 33(2): 135-47.

582 doi:10.1002/bies.201000036.

583 González-Vaquero, R.A., Roig-Alsina, A., Packer, L., and Wilson, J.-J. 2016a. DNA barcoding 584 as a useful tool in the systematic study of wild bees of the tribe Augochlorini (Hymenoptera:

585 Halictidae). Genome 59(10): 889-898. doi:10.1139/gen-2016-0006.

586 González-Vaquero, R.A., Roig-Alsina, A., Packer, L., and Wilson, J.-J. 2016b. DNA barcoding

587 as a useful tool in the systematic study of wild bees of the tribe Augochlorini (Hymenoptera:

588 Halictidae). Genome 59(10): 889-898. doi:10.1139/gen-2016-0006.

589 Grixti, J.C., and Packer, L. 2006. Changes in the bee fauna (Hymenoptera: Apoidea) of an old 590 field site in southern Ontario, revisited after 34 years. Can. Entomol. 138(2): 147-164.

591 doi:10.4039/n05-034. 
592 Groom, S.V.C., Stevens, M.I., and Schwarz, M.P. 2013. Diversification of Fijian halictine bees:

593 Insights into a recent island radiation. Mol. Phylogenet. Evol. 68(3): 582-594.

594 doi:10.1016/j.ympev.2013.04.015.

595 Hajibabaei, M., deWaard, J.R., Ivanova, N. V, Ratnasingham, S., Dooh, R.T., Kirk, S.L.,

596 Mackie, P.M., and Hebert, P.D.N. 2005. Critical factors for assembling a high volume of DNA

597 barcodes. Philos. Trans. R. Soc. Lond. B. Biol. Sci. 360(1462): 1959-67.

598 doi:10.1098/rstb.2005.1727.

599 Hajibabaei, M., Janzen, D.H., Burns, J.M., Hallwachs, W., and Hebert, P.D.N. 2006a. DNA 600 barcodes distinguish species of tropical Lepidoptera. Proc. Natl. Acad. Sci. 103(4): 968-71. 601 doi:10.1073/pnas.0510466103.

602 Hajibabaei, M., Smith, M.A., Janzen, D.H., Rodriguez, J.J., Whitfield, J.B., and Hebert, P.D.N. 603 2006b. A minimalist barcode can identify a specimen whose DNA is degraded. Mol. Ecol. Notes 604 6(4): 959-964. doi:10.1111/j.1471-8286.2006.01470.x.

605 Hebert, P.D.N., Cywinska, A., Ball, S.L., deWaard, J.R., and Jeremy, R. 2003a. Biological 606 identifications through DNA barcodes. Proc. Biol. Sci. 270(1512): 313-21. 607 doi:10.1098/rspb.2002.2218.

608 Hebert, P.D.N., and Gregory, T.R. 2005. The promise of DNA barcoding for taxonomy. Syst. 609 Biol. 54(5): 852-9. doi:10.1080/10635150500354886.

610 Hebert, P.D.N., Penton, E.H., Burns, J.M., Janzen, D.H., and Hallwachs, W. 2004a. Ten species 611 in one: DNA barcoding reveals cryptic species in the neotropical skipper butterfly Astraptes 612 fulgerator. Proc. Natl. Acad. Sci. 101(41): 14812-7. doi:10.1073/pnas.0406166101. 
613 Hebert, P.D.N., Ratnasingham, S., and DeWaard, J.R. 2003b. Barcoding animal life: cytochrome 614 c oxidase subunit 1 divergences among closely related species. Proc. Biol. Sci.

615 doi:10.1098/rsbl.2003.0025.

616 Hebert, P.D.N., Stoeckle, M.Y., Zemlak, T.S., and Francis, C.M. 2004b. Identification of birds 617 through DNA barcodes. PLoS Biol. 2(10). doi:10.1371/journal.pbio.0020312.

618 Hendrixson, B.E., DeRussy, B.M., Hamilton, C.A., and Bond, J.E. 2013. An exploration of 619 species boundaries in turret-building tarantulas of the Mojave Desert (Araneae, Mygalomorphae, 620 Theraphosidae, Aphonopelma). Mol. Phylogenet. Evol. 66(1): 327-340.

621 doi:10.1016/j.ympev.2012.10.004.

622 Holder, M., and Lewis, P.O. 2003. Phylogeny estimation: traditional and Bayesian approaches.

623 Nat. Rev. Genet. 4: 275-284. doi:10.1038/nrg1044

624 Hubert, N., Hanner, R., Holm, E., Mandrak, N.E., Taylor, E., Burridge, M., Watkinson, D., 625 Dumont, P., Curry, A., Bentzen, P., Zhang, J., April, J., and Bernatchez, L. 2008. Identifying 626 Canadian freshwater fishes through DNA barcodes. PLoS One 3(6): e2490. Public Library of 627 Science. doi:10.1371/journal.pone.0002490.

628 Huelsenbeck, J.P. 1995. The robustness of two phylogenetic methos: four-taxon simulations 629 reveal a slight superiority of maximum likelihood over neighbor joining. Mol. Biol. Evol. 12(5): 630 843-849. doi: 10.1093/oxfordjournals.molbev.a040261

631 Ivanova, N. V., DeWaard, J.R., and Hebert, P.D.N. 2006. An inexpensive, automation-friendly 632 protocol for recovering high-quality DNA. Mol. Ecol. Notes 6(4): 998-1002.

633 doi:10.1111/j.1471-8286.2006.01428.x. 
634 Kalhorn, K.D., Barrows, E.M., and LaBerge, W.E. 2003. Bee (Hymenoptera: Apoidea:

635 Apiformes) Diversity in an Appalachian Shale Barrens. J. Kansas Entomol. Soc. 76(3): 455-468.

636 Kearns, C.A., and Oliveras, D.M. 2009. Boulder County Bees revisited: a resampling of Boulder

637 Colorado Bees a century later. J. Insect Conserv. 13(6): 603-613. doi:10.1007/s 10841-009-9211-

6388.

639 Kekkonen, M., and Hebert, P.D.N. 2014. DNA barcode-based delineation of putative species:

640 efficient start for taxonomic workflows. Mol. Ecol. Resour. 14(4): 706-15. Wiley-Blackwell.

641 doi:10.1111/1755-0998.12233.

642 Kimura, M. 1980. A simple method for estimating evolutionary rates of base substitutions

643 through comparative studies of nucleotide sequences. J. Mol. Evol. 16(2): 111-120. Springer-

644 Verlag. doi:10.1007/BF01731581.

645 Klein, A.-M., Vaissière, B.E., Cane, J.H., Steffan-Dewenter, I., Cunningham, S.A., Kremen, C., 646 and Tscharntke, T. 2007. Importance of pollinators in changing landscapes for world crops. Proc.

647 R. Soc. London B 274(1608): 303-313.

648 Knerer, G., and Atwood, C.E. 1964. Further notes on the genus Evylaeus Robertson

649 (Hymenoptera: Halictidae). Can. Entomol. 96(7): 957-962. doi:10.4039/Ent96957-7.

650 Kuhlmann, M., Else, G., Dawson, A., and Quicke, D. 2007. Molecular, biogeographical and

651 phenological evidence for the existence of three western European sibling species in the Colletes

652 succinctus group (Hymenoptera: Apidae). Org. Divers. Evol. 7(2): 155-165.

653 doi:10.1016/j.ode.2006.04.001. 
654 Kumar, S., Stecher, G., and Tamura, K. 2016. MEGA7: Molecular Evolutionary Genetics

655 Analysis Version 7.0 for Bigger Datasets. Mol. Biol. Evol. 33(7): 1870-1874.

656 doi:10.1093/molbev/msw054.

657 MacKay, P.A., and Knerer, G. 1979. Seasonal occurrence and abundance in a community of wild 658 bees from an old field habitat in southern Ontario. Can. Entomol. 111(3): 367-376. Cambridge 659 University Press. doi:10.4039/Ent111367-3.

660 Maddison, W.P., and Maddison, D.R. 2017. Mesquite: A modular system for evolutionary 661 analysis. Version 3.2. Available from http://mesquiteproject.org.

662 Magnacca, K.N., and Brown, M.J.F. 2010. Mitochondrial heteroplasmy and DNA barcoding in 663 Hawaiian Hylaeus (Nesoprosopis) bees (Hymenoptera: Colletidae). BMC Evol. Biol. 10: 174. 664 doi:10.1186/1471-2148-10-174.

665 Magnacca, K.N., and Brown, M.J.F. 2012. DNA barcoding a regional fauna: Irish solitary bees. 666 Mol. Ecol. Resour. 12(6): 990-8. doi:10.1111/1755-0998.12001.

667 Meier, R., Shiyang, K., Vaidya, G., Ng, P.K.L., and Hedin, M. 2006. DNA Barcoding and 668 Taxonomy in Diptera: A Tale of High Intraspecific Variability and Low Identification Success. 669 Syst. Biol. 55(5): 715-728. doi:10.1080/10635150600969864.

670 Meyer, C.P., and Paulay, G. 2005. DNA barcoding: error rates based on comprehensive 671 sampling. PLoS Biol. 3(12): e422. Public Library of Science. doi:10.1371/journal.pbio.0030422.

672 Michener, C.D. 1974. The social behavior of the bees. Belknap Press, Cambridge, M.A..

673 Michener, C.D. 1979. Biogeography of the bees. Ann. Missouri Bot. Gard. 66(3): 277. 674 doi:10.2307/2398833. 
675 Michener, C.D. 2007. The bees of the world. In 2nd edition. The Johns Hopkins University 676 Press, Baltimore, M.D..

677 Moure, J.S., and Hurd, P.D. 1987. An annotated catalog of the halictid bees of the Western

678 Hemisphere (Hymenoptera: Halictidae). Smithsonian Institution Press, Washington, D.C.

679 Murray, T.E., Fitzpatrick, Ú., Brown, M.J.F., and Paxton, R.J. 2007. Cryptic species diversity in 680 a widespread bumble bee complex revealed using mitochondrial DNA RFLPs. Conserv. Genet. 681 9(3): 653-666. doi:10.1007/s10592-007-9394-Z.

682 Ngo, H.T., Gibbs, J., Griswold, T., and Packer, L. 2013. Evaluating bee (Hymenoptera: Apoidea) 683 diversity using Malaise traps in coffee landscapes of Costa Rica. Can. Entomol. 145(4): 435684 453. doi:10.4039/tce.2013.16.

685 Nicholls, J.A., Challis, R.J., Mutun, S., and Stone, G.N. 2012. Mitochondrial barcodes are 686 diagnostic of shared refugia but not species in hybridizing oak gallwasps. Mol. Ecol. 21(16): 687 4051-62. doi:10.1111/j.1365-294X.2012.05683.x.

688 Ollerton, J., Winfree, R., and Tarrant, S. 2011. How many flowering plants are pollinated by 689 animals? Oikos 120(3): 321-326. doi:10.1111/j.1600-0706.2010.18644.x.

690 Owen, R.E., and Harder, L.D. 1995. Heritable allometric variation in bumble bees: opportunities 691 for colony-level selection of foraging ability. J. Evol. Biol. 8(6): 725-738. doi:10.1046/j.1420$6929101.1995 .8060725 . x$.

693 Packer, L. 1999. The distribution of Halictus ligatus Say and H. poeyi Lep. (Hymenoptera:

694 Halictidae) in North America. Univ. Kansas Nat. Hist. Museum Spec. Publ. 24: 81-84. 
695 Packer, L., Ali, E., Dumesh, S., and Walker, K. 2016. The identification of pollinators: Where 696 are we and where should we go? In Pollination services to agriculture: Sustaining and enhancing 697 a key ecosystem service. Edited by B. Gemmill-Herren. Routledge, New York City. pp. 57-73.

698 Packer, L., Gibbs, J., Sheffield, C., and Hanner, R. 2009. DNA barcoding and the mediocrity of 699 morphology. Mol. Ecol. Resour. 9 Suppl s1: 42-50. doi:10.1111/j.1755-0998.2009.02631.x.

700 Packer, L., and Ruz, L. 2017. DNA barcoding the bees (Hymenoptera: Apoidea) of Chile:

701 species discovery in a reasonably well known bee fauna with the description of a new species of 702 Lonchopria (Colletidae). Genome 60(5): 414-430. doi:10.1139/gen-2016-0071.

703 Packer, L., and Taylor, J.S. 1997. How many cryptic species are there? An application of the 704 phylogenetic species concept to genetic data for some comparatively well known bee "species." 705 Can. Entomol. 129(4): 587-594. doi:10.4039/Ent129587-4.

706 Packer, L., and Taylor, J.S. 2002. Genetic variation within and among populations of an 707 arctic/alpine sweat bee (Hymenoptera: Halictidae). Can. Entomol. 134(5): 619-631. 708 doi:10.4039/Ent134619-5.

709 Padial, J.M., Miralles, A., De la Riva, I., and Vences, M. 2010. The integrative future of 710 taxonomy. Front. Zool. 7: 16. doi:10.1186/1742-9994-7-16.

711 Patten, M.M., Carioscia, S.A., and Linnen, C.R. 2015. Biased introgression of mitochondrial and 712 nuclear genes: a comparison of diploid and haplodiploid systems. Mol. Ecol. 24(20): 5200-10. 713 doi:10.1111/mec.13318.

714 Pauly, A., Devalez, J., Sonet, G., Nagy, Z.T., and Boevé, J.-L. 2015. DNA barcoding and male 715 genital morphology reveal five new cryptic species in the West Palearctic bee Seladonia 
716 smaragdula (Vachal, 1895) (Hymenoptera: Apoidea: Halictidae). Zootaxa 4034(2): 257.

717 doi:10.11646/zootaxa.4034.2.2.

718 Pentinsaari, M., Mutanen, M., and Kaila, L. 2014. Cryptic diversity and signs of mitochondrial 719 introgression in the Agrilus viridis species complex (Coleoptera: Buprestidae). Eur. J. Entomol. 720 111(4): 475-486. doi:10.14411/eje.2014.072.

721 Pons, J., Barraclough, T.G., Gomez-Zurita, J., Cardoso, A., Duran, D.P., Hazell, S., Kamoun, S., 722 Sumlin, W.D., and Vogler, A.P. 2006. Sequence-based species delimitation for the DNA 723 taxonomy of undescribed insects. Syst. Biol. 55(4): 595-609.

724 Puillandre, N., Lamber, A., Brouillet, S., and Achaz, G. 2012. ABGD, Automatic Barcode Gap 725 Discovery for primary species delimitation. Mol. Ecol. 21(8): 1864-1877. doi:10.1111/j.1365726 294X.2011.05239.x.

727 R Core Team. 2015. R: A language and environment for statistical computing. R version 3.2.2. R 728 Foundation for Statistical Computing. doi:10.1007/978-3-540-74686-7.

729 Ratnasingham, S., and Hebert, P.D.N. 2007. BOLD: The Barcode of Life Data System 730 (www.barcodinglife.org). Mol. Ecol. Notes 7(3): 355-364. doi:10.1111/j.1471$731 \quad 8286.2007 .01678 . x$.

732 Ratnasingham, S., and Hebert, P.D.N. 2013. A DNA-based registry for all animal species: the 733 barcode index number (BIN) system. PLoS One 8(7): e66213.

734 doi:10.1371/journal.pone.0066213. 
735 Rehan, S.M., and Sheffield, C.S. 2011. Morphological and molecular delineation of a new 736 species in the Ceratina dupla species-group (Hymenoptera: Apidae: Xylocopinae) of eastern 737 North America. Zootaxa 2873: 35-50.

738 Rheindt, F.E., Christidis, L., and Norman, J.A. 2009. Genetic introgression, incomplete lineage 739 sorting and faulty taxonomy create multiple cases of polyphyly in a montane clade of tyrant740 flycatchers (Elaenia, Tyrannidae). Zool. Scr. 38(2): 143-153. Blackwell Publishing Ltd. 741 doi:10.1111/j.1463-6409.2008.00369.x.

742 Rivera, J., and Currie, D.C. 2009. Identification of Nearctic black flies using DNA barcodes

743 (Diptera: Simuliidae). Mol. Ecol. Resour. 9 Suppl s1: 224-36. doi:10.1111/j.1755$744 \quad$ 0998.2009.02648.x.

745 Schmidt, S., Schmid-Egger, C., Morinière, J., Haszprunar, G., and Hebert, P.D.N. 2015. DNA 746 barcoding largely supports 250 years of classical taxonomy: identifications for Central European 747 bees (Hymenoptera, Apoidea partim). Mol. Ecol. Resour. 15(4): 985-1000. doi:10.1111/17557480998.12363.

749 Sheffield, C.S., Dumesh, S., and Cheryomina, M. 2011. Hylaeus punctatus (Hymenoptera: 750 Colletidae), a bee species new to Canada, with notes on other non-native species. J. Entomol. 751 Soc. Ontario 142: 29-43.

752 Sheffield, C.S., Hebert, P.D.N., Kevan, P.G., and Packer, L. 2009. DNA barcoding a regional 753 bee (Hymenoptera: Apoidea) fauna and its potential for ecological studies. Mol. Ecol. Resour. 9 754 Suppl s1: 196-207. doi:10.1111/j.1755-0998.2009.02645.x. 
755 Sheffield, C.S., Richardson, L., Cannings, S., Ngo, H., Heron, J., and Williams, P.H. 2016.

756 Biogeography and designatable units of Bombus occidentalis Greene and B. terricola Kirby

757 (Hymenoptera: Apidae) with implications for conservation status assessments. J. Insect Conserv.

758 20(2): 189-199. doi:10.1007/s10841-016-9853-2.

759 Smith, B.A., Brown, R.L., LaBerge, W., and Griswold, T.L. 2012a. A faunistic survey of bees

760 (Hymenoptera: Apoidea) in the Black Belt Prairie of Mississippi. J. Kansas Entomol. Soc. 85(1):

$76132-47$.

762 Smith, M.A., Bertrand, C., Crosby, K., Eveleigh, E.S., Fernandez-Triana, J., Fisher, B.L., Gibbs,

763 J., Hajibabaei, M., Hallwachs, W., Hind, K., Hrcek, J., Huang, D.-W., Janda, M., Janzen, D.H.,

764 Li, Y., Miller, S.E., Packer, L., Quicke, D., Ratnasingham, S., Rodriguez, J., Rougerie, R., Shaw,

765 M.R., Sheffield, C., Stahlhut, J.K., Steinke, D., Whitfield, J., Wood, M., and Zhou, X. 2012 b.

766 Wolbachia and DNA barcoding insects: patterns, potential, and problems. PLoS One 7(5):

767 e36514. doi:10.1371/journal.pone.0036514.

768 Smith, M.A., Fisher, B.L., and Hebert, P.D.N. 2005. DNA barcoding for effective biodiversity

769 assessment of a hyperdiverse arthropod group: the ants of Madagascar. Philos. Trans. R. Soc.

770 Lond. B. Biol. Sci. 360(1462): 1825-1834. doi:10.1098/rstb.2005.1714.

771 Smith, M.A., Wood, D.M., Janzen, D.H., Hallwachs, W., and Hebert, P.D.N. 2007. DNA

772 barcodes affirm that 16 species of apparently generalist tropical parasitoid flies (Diptera,

773 Tachinidae) are not all generalists. Proc. Natl. Acad. Sci. 104(12): 4967-72.

774 doi:10.1073/pnas.0700050104. 
775 Sonet, G., Jordaens, K., Nagy, Z.T., Breman, F., de Meyer, M., Backeljau, T., and Virgilio, M.

776 2013. Adhoc: an R package to calculate ad hoc distance thresholds for DNA barcoding

777 identification. Zookeys 365: 329-336. doi:10.3897/zookeys.365.6034.

778 Srivathsan, A., and Meier, R. 2011. Cladistics On the inappropriate use of Kimura-2-parameter 779 (K2P ) divergences in the DNA-barcoding literature. 27: 1-5.

780 Thompson, J.N. 1998. Rapid evolution as an ecological process. Trends Ecol. Evol. 13(8): 329781 332. doi:10.1016/S0169-5347(98)01378-0.

782 Virgilio, M., Jordaens, K., Breman, F.C., Backeljau, T., and De Meyer, M. 2012. Identifying 783 Insects with Incomplete DNA Barcode Libraries, African Fruit Flies (Diptera: Tephritidae) as a 784 Test Case. PLoS One 7(2): e31581. doi:10.1371/journal.pone.0031581.

785 Ward, R.D., Zemlak, T.S., Innes, B.H., Last, P.R., and Hebert, P.D.N. 2005. DNA barcoding 786 Australia's fish species. Philos. Trans. R. Soc. Lond. B. Biol. Sci. 360(1462): 1847-57. 787 doi:10.1098/rstb.2005.1716.

788 Wheelock, M.J., and O’Neal, M.E. 2016. Insect pollinators in Iowa cornfields: Community 789 identification and trapping method analysis. PLoS One 11(7): e0143479.

790 doi:10.1371/journal.pone.0143479.

791 Wolf, A.T., and Ascher, J.S. 2009. Bees of Wisconsin (Hymenoptera: Apoidea: Anthophila). Gt.

792 Lakes Entomol. 41: 129-168.

793 Zarrillo, T.A., Ascher, J.S., Gibbs, J., and Stoner, K.A. 2016. New and noteworthy records of 794 bees (Hymenoptera: Apoidea: Anthophila) for Connecticut. J. Kansas Entomol. Soc. 89(2): 138795 157. doi:10.2317/0022-8567-89.2.138. 
796 Zayed, A. 2009. Bee genetics and conservation. Apidologie 40(3): 237-262.

797 doi:10.1051/apido/2009026.

798 Zayed, A., and Packer, L. 2005. Complementary sex determination substantially increases

799 extinction proneness of haplodiploid populations. Proc. Natl. Acad. Sci. 102(30): 10742-6.

800 doi:10.1073/pnas.0502271102.

801 Zayed, A., Roubik, D.W., and Packer, L. 2004. Use of diploid male frequency data as an

802 indicator of pollinator decline. Proc. R. Soc. B 271 Suppl: S9-12. doi:10.1098/rsbl.2003.0109.

803

804

805

806

807

808

809

810

811

812

813 
814 Table 1. Taxonomically concordant Barcode Index Numbers (BINs) based on analysis of 1279

815 barcode-compliant sequences and morphology of voucher specimens. BIN members include

816 non-compliant DNA barcodes. BIN numbers only represented by non-compliant barcodes are

817 indicated with NC. ABGD groups assigned to compliant sequences are included. Species are

818 categorized by regions based on the taxonomic revisions used to delimit species boundaries:

819 Canada (CAN) or eastern United States (EUS) (see Gibbs 2010a, 2011).

\begin{tabular}{|c|c|c|c|c|}
\hline Identification & BIN & $\begin{array}{l}\text { ABGD } \\
\text { group }\end{array}$ & Region & TotalNo \\
\hline $\begin{array}{l}\text { Lasioglossum } \\
\text { abundipunctum Gibbs }\end{array}$ & BOLD:AAE1318 & 1 & CAN & 5 \\
\hline $\begin{array}{l}\text { Lasioglossum achilleae } \\
\text { (Mitchell) }\end{array}$ & BOLD:AAW9967 & ( & CAN/EUS & 1 \\
\hline $\begin{array}{l}\text { Lasioglossum albipenne } \\
\text { (Robertson) }\end{array}$ & BOLD:AAB 6738 & 5 & CAN/EUS & 11 \\
\hline $\begin{array}{l}\text { Lasioglossum albohirtum } \\
\text { (Crawford)* }^{*}\end{array}$ & BOLD:ABZ7019 & 6 & CAN & 8 \\
\hline $\begin{array}{l}\text { Lasioglossum anomalum } \\
\text { (Robertson) }\end{array}$ & BOLD:AAA7867 & 7 & CAN/EUS & 6 \\
\hline $\begin{array}{l}\text { Lasioglossum apopkense } \\
\text { (Robertson) }\end{array}$ & BOLD:AAE1289 & 8 & EUS & 5 \\
\hline
\end{tabular}




\begin{tabular}{|c|c|c|c|c|}
\hline Lasioglossum batya Gibbs & BOLD:AAE1190 & 10 & EUS & 2 \\
\hline $\begin{array}{l}\text { Lasioglossum } \\
\text { brunneiventre (Crawford) }\end{array}$ & BOLD:AAB7689 & 12 & $\mathrm{CAN}$ & 14 \\
\hline $\begin{array}{l}\text { Lasioglossum coreopsis } \\
\text { (Robertson) }\end{array}$ & BOLD:AAC1424 & 17 & EUS & 16 \\
\hline $\begin{array}{l}\text { Lasioglossum creberrimum } \\
\text { (Robertson) }\end{array}$ & BOLD:AAC2514 & 18 & EUS & 12 \\
\hline $\begin{array}{l}\text { Lasioglossum dashwoodi } \\
\text { Gibbs }\end{array}$ & BOLD:AAJ1718 & 25 & CAN & 2 \\
\hline $\begin{array}{l}\text { Lasioglossum disparile } \\
\text { (Cresson) }\end{array}$ & BOLD:AAC8559 & 26 & EUS & 9 \\
\hline $\begin{array}{l}\text { Lasioglossum ebmerellum } \\
\text { Gibbs }\end{array}$ & BOLD:AAD7578 & 27 & CAN & 6 \\
\hline $\begin{array}{l}\text { Lasioglossum gotham } \\
\text { Gibbs }\end{array}$ & $\begin{array}{l}\text { BOLD:AAK8338 } \\
\text { (NC) }\end{array}$ & NA & EUS & 1 \\
\hline $\begin{array}{l}\text { Lasioglossum halophitum } \\
\text { (Graenicher) }\end{array}$ & BOLD:AAC5496 & 33,34 & EUS & 9,2 \\
\hline $\begin{array}{l}\text { Lasioglossum hartii } \\
\text { (Robertson) }\end{array}$ & BOLD:AAL9893 & 35 & EUS & 2 \\
\hline
\end{tabular}




\begin{tabular}{|c|c|c|c|c|}
\hline $\begin{array}{l}\text { Lasioglossum hemimelas } \\
\text { (Cockerell) }\end{array}$ & BOLD:ABZ6179 & 36 & EUS & 1 \\
\hline $\begin{array}{l}\text { Lasioglossum } \\
\text { heterognathum (Mitchell) }\end{array}$ & BOLD:ACE5057 & 37 & CAN/EUS & 3 \\
\hline $\begin{array}{l}\text { Lasioglossum hyalinum } \\
\text { (Crawford) }\end{array}$ & BOLD:AAE1411 & 44 & CAN & 4 \\
\hline $\begin{array}{l}\text { Lasioglossum illinoense } \\
\text { (Robertson) }\end{array}$ & BOLD:AAD2971 & 45 & CAN/EUS & 5 \\
\hline $\begin{array}{l}\text { Lasioglossum imitatum } \\
\text { (Smith) }\end{array}$ & BOLD:AAA7868 & 47 & CAN/EUS & 26 \\
\hline $\begin{array}{l}\text { Lasioglossum incompletum } \\
\text { (Crawford) }\end{array}$ & BOLD:AAB8298 & 48 & CAN & 13 \\
\hline $\begin{array}{l}\text { Lasioglossum izawsum } \\
\text { Gibbs }\end{array}$ & BOLD:ABZ7461 & 49 & CAN/EUS & 2 \\
\hline $\begin{array}{l}\text { Lasioglossum laevissimum } \\
\text { (Smith) }\end{array}$ & BOLD:AAA7513 & 52 & CAN/EUS & 42 \\
\hline $\begin{array}{l}\text { Lasioglossum lepidii } \\
\text { (Graenicher) }\end{array}$ & BOLD:ABY5206 & 53 & EUS & 3 \\
\hline Lasioglossum lineatulum & BOLD:AAA2141 & 57,58 & CAN/EUS & 13,1 \\
\hline
\end{tabular}




\begin{tabular}{|c|c|c|c|c|}
\hline (Crawford) & & & & \\
\hline $\begin{array}{l}\text { Lasioglossum lionotum } \\
\text { (Sandhouse) }\end{array}$ & BOLD:AAE5826 & 59 & CAN/EUS & 3 \\
\hline $\begin{array}{l}\text { Lasioglossum longifrons } \\
\text { (Baker) }\end{array}$ & BOLD:AAC8294 & 60 & EUS & 9 \\
\hline $\begin{array}{l}\text { Lasioglossum } \\
\text { macroprosopum Gibbs }\end{array}$ & BOLD:AAE1153 & 61 & $\mathrm{CAN}$ & 2 \\
\hline $\begin{array}{l}\text { Lasioglossum marinum } \\
\text { (Crawford) }\end{array}$ & BOLD:AAD0611 & 62 & EUS & 8 \\
\hline $\begin{array}{l}\text { Lasioglossum } \\
\text { michiganense (Mitchell) }\end{array}$ & BOLD:ACE8520 & 63 & CAN/EUS & 3 \\
\hline $\begin{array}{l}\text { Lasioglossum nevadense } \\
\text { (Crawford) }\end{array}$ & BOLD:AAB0372 & 64,65 & $\mathrm{CAN}$ & 21,1 \\
\hline $\begin{array}{l}\text { Lasioglossum nigroviride } \\
\text { (Graenicher) }\end{array}$ & BOLD:AAD3968 & 66 & CAN/EUS & 1 \\
\hline $\begin{array}{l}\text { Lasioglossum nymphale } \\
\text { (Smith) }\end{array}$ & BOLD:AAB6637 & 68 & EUS & 17 \\
\hline $\begin{array}{l}\text { Lasioglossum occidentale } \\
\text { (Crawford) }\end{array}$ & BOLD:AAC7155 & 69,70 & $\mathrm{CAN}$ & 10,1 \\
\hline
\end{tabular}




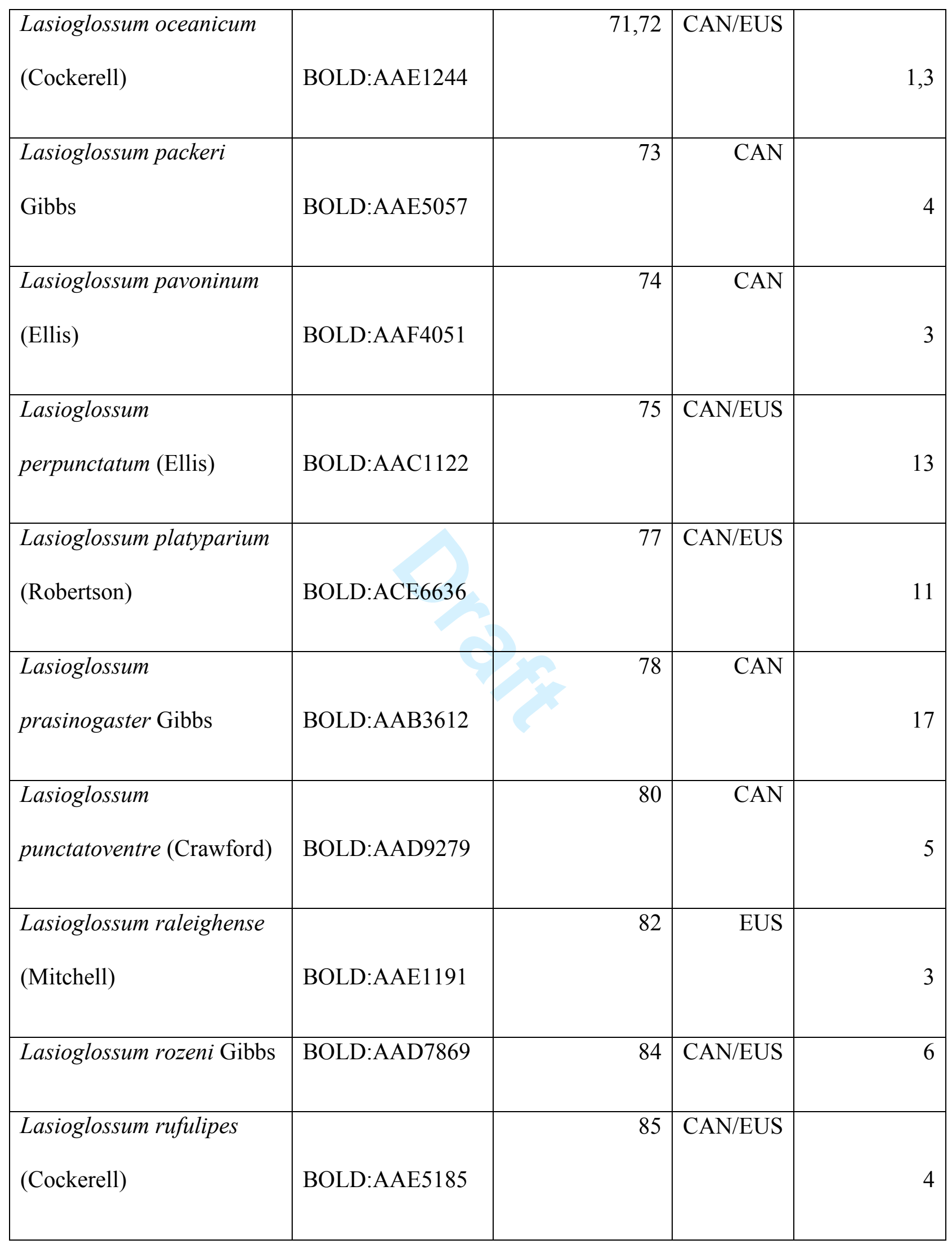




\begin{tabular}{|c|c|c|c|c|}
\hline $\begin{array}{l}\text { Lasioglossum sedi } \\
\text { (Sandhouse) }\end{array}$ & BOLD:ABZ3764 & 94 & CAN & 16 \\
\hline $\begin{array}{l}\text { Lasioglossum } \\
\text { semicaeruleum (Cockerell) }\end{array}$ & BOLD:AAB1546 & 95 & $\mathrm{CAN}$ & 28 \\
\hline $\begin{array}{l}\text { Lasioglossum simplex } \\
\text { (Robertson) }\end{array}$ & BOLD:ACE5372 & 97 & EUS & 2 \\
\hline $\begin{array}{l}\text { Lasioglossum smilacinae } \\
\text { (Robertson) }\end{array}$ & BOLD:AAE1871 & 98 & CAN/EUS & 1 \\
\hline $\begin{array}{l}\text { Lasioglossum } \mathrm{cf} . \\
\text { stuartense (Mitchell) }\end{array}$ & BOLD:AAW9918 & 16 & EUS & 1 \\
\hline $\begin{array}{l}\text { Lasioglossum tamiamense } \\
\text { (Mitchell) }\end{array}$ & BOLD:AAX0169 & 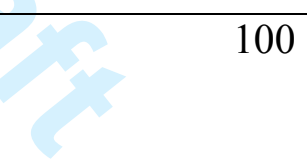 & EUS & 1 \\
\hline $\begin{array}{l}\text { Lasioglossum tarponense } \\
\text { (Mitchell) }\end{array}$ & BOLD:AAE5196 & 101 & EUS & 4 \\
\hline $\begin{array}{l}\text { Lasioglossum tenax } \\
\text { (Sandhouse) }\end{array}$ & BOLD:AAB6998 & 102 & CAN/EUS & 19 \\
\hline $\begin{array}{l}\text { Lasioglossum timothyi } \\
\text { Gibbs }\end{array}$ & BOLD:AAE1693 & 103 & CAN/EUS & 3 \\
\hline Lasioglossum versans & BOLD:ABZ6180 & $105,106,107,108$ & CAN/EUS & $6,1,1,1$ \\
\hline
\end{tabular}




\begin{tabular}{|l|r|r|r|r|}
\hline (Lovell) & & 109 & CAN/EUS & \\
\hline Lasioglossum vierecki & BOLD:AAB4651 & 111 & CAN/EUS & \\
\hline $\begin{array}{l}\text { Lasioglossum zephyrum } \\
\text { (Smith) }\end{array}$ & BOLD:AAD0272 & & & 6 \\
\hline Lasioglossum n. sp. (ex. & & 99 & CAN & \\
Alberta) & BOLD:AAX015 & & & 1 \\
\hline
\end{tabular}

820

One BIN but geographic and morphological variation consistent with two species.

821

822

823

824

825

826

827

828

829

830 
831 Table 2. Morphologically identifiable species represented by multiple BINs based on analysis of 8321279 barcode-compliant sequences. Total BIN members includes non-compliant DNA barcodes 833 with less than 500 bp or greater than 1\% ambiguous nucleotides. Additional BINs based on non834 compliant sequences are indicated by NC. ABGD group numbers are provided.

\begin{tabular}{|c|c|c|c|c|}
\hline Species & Included BINS & ABGD group & Region & $\begin{array}{l}\text { Total BIN } \\
\text { members }\end{array}$ \\
\hline $\begin{array}{l}\text { Lasioglossum } \\
\text { cressonii (Robertson) }\end{array}$ & $\begin{array}{l}\text { BOLD:AAA5974 } \\
\text { BOLD:AAP0913 } \\
\text { BOLD:ABY7290 } \\
\text { BOLD:ACE7675 } \\
\text { BOLD:ABY8200(NC) } \\
\text { BOLD:AAA5973 }\end{array}$ & $19,20,21,22,23,24$ & CAN/EUS & $5,1,18,1,1,1$ \\
\hline $\begin{array}{l}\text { Lasioglossum } \\
\text { hitchensi Gibbs }\end{array}$ & $\begin{array}{l}\text { BOLD:AAE5827 } \\
\text { BOLD:ABY8629 } \\
\text { BOLD:AAA3782* }\end{array}$ & $38,39,40$ & $\begin{array}{l}\text { CAN, } \\
\text { EUS }\end{array}$ & $73,1,1$ \\
\hline $\begin{array}{l}\text { Lasioglossum } \\
\text { hudsoniellum } \\
\text { (Cockerell) }\end{array}$ & $\begin{array}{l}\text { BOLD:AAB5530 } \\
\text { BOLD:ACE7697 } \\
\text { BOLD:ABZ0512 }\end{array}$ & $41,43,43$ & CAN & $16,2,1$ \\
\hline
\end{tabular}




\begin{tabular}{|c|c|c|c|c|}
\hline & [singleton] & & & \\
\hline $\begin{array}{l}\text { Lasioglossum } \\
\text { leucocomum (Lovell) }\end{array}$ & $\begin{array}{l}\text { BOLD:ACE3786 } \\
\text { BOLD:ACE9642 } \\
\text { BOLD:ACF2785* }\end{array}$ & $54,55,56$ & CAN/EUS & $12,3,2$ \\
\hline $\begin{array}{l}\text { Lasioglossum } \\
\text { pruinosum } \\
\text { (Robertson) }\end{array}$ & $\begin{array}{l}\text { BOLD:AAC1101 } \\
\text { BOLD:ABY7039 }\end{array}$ & 79 & CAN/EUS & 8 \\
\hline $\begin{array}{l}\text { Lasioglossum } \\
\text { ruidosense } \\
\text { (Cockerell) }\end{array}$ & $\begin{array}{l}\text { BOLD:AAA5893 } \\
\text { BOLD:ACE3475 } \\
\text { BOLD:ACE3476 } \\
\text { BOLD:AAA5895 } \\
\text { BOLD:AAA5894 } \\
\text { [singleton] }\end{array}$ & $86,87,88,89,90,91$ & $\mathrm{CAN}$ & $3,9,3,9,2,1$ \\
\hline
\end{tabular}

* see table 3

836

837

838 
839 Table 3. Taxonomically discordant Barcode Index Numbers (BINs) that merge multiple

840 morphospecies into a single BIN. The status of ABGD groups for these taxa are provided. The

841 pattern of discordance between taxonomy and either BINs or ABGD groups is not identical. The

842 number of diagnostic substitutions calculated with the nucDiag function in the R package spider

843 are provided and where available the base pair positions relative to the standard 658 bp DNA

844 barcode fragment for bees are provided. In BINs with more than two species, there may be 845 additional pairwise diagnostic characters.

\begin{tabular}{|c|c|c|c|c|}
\hline $\mathrm{BIN}$ & Included taxa & ABGD & $\begin{array}{l}\text { Diagnostic } \\
\text { substitutions }\end{array}$ & Sample size \\
\hline BOLD:AAA3781 & $\begin{array}{l}\text { L. callidum (Sandhouse), } \\
\text { Lasioglossum versatum } \\
\text { (Robertson) }\end{array}$ & 13 & 0 & $33+97$ \\
\hline BOLD:AAA3782 & $\begin{array}{l}\text { L. hitchensi Gibbs, L. } \\
\text { leviense (Mitchell), L. } \\
\text { weemsi (Mitchell) }\end{array}$ & 102 & 0 & $73+4+25$ \\
\hline BOLD:AAA $8348^{*}$ & $\begin{array}{l}\text { L. knereri Gibbs, L. } \\
\text { subversans (Mitchell) }\end{array}$ & 51 & $\begin{array}{l}4(270,384, \\
504,631)\end{array}$ & $36+3$ \\
\hline BOLD:AAB0760* & $\begin{array}{l}\text { L. ellisae (Sandhouse), L. } \\
\text { imbrex Gibbs }\end{array}$ & 28,46 & $\begin{array}{l}7(81,282,312, \\
315,573,603, \\
640)\end{array}$ & 21,9 \\
\hline
\end{tabular}




\begin{tabular}{|c|c|c|c|c|}
\hline BOLD:AAB2245* & L. trigeminum Gibbs & 104 & NA & 29 \\
\hline BOLD:AAB6361 & $\begin{array}{l}\text { L. floridanum (Robertson), L. } \\
\text { pilosum } \dagger \text { (Smith) }\end{array}$ & $31(76)$ & 0 & $4+18,(1)$ \\
\hline BOLD:AAC1104* & $\begin{array}{l}\text { L. ceanothi (Mitchell)†, } L . \\
\text { foveolatum (Robertson), } L \text {. } \\
\text { pictum (Crawford) }\end{array}$ & $\begin{array}{l}15, \\
112,32\end{array}$ & $\begin{array}{l}6,1,0(219, \\
315,327,360, \\
402,462,594, \\
618,625,634)\end{array}$ & $2,1,9+9$ \\
\hline BOLD:AAB6998 & $\begin{array}{l}\text { L. cattellae (Ellis) (NC), L. } \\
\text { tenax (Sandhouse) }\end{array}$ & 102 & $0(\mathrm{NC})$ & 19 \\
\hline BOLD:AAC7048 & $\begin{array}{l}\text { L. bruneri (Crawford), L. } \\
\text { reticulatum (Robertson) }\end{array}$ & 11,83 & $\begin{array}{l}8(159,189, \\
201,234,360, \\
363,432,634)\end{array}$ & 11,1 \\
\hline BOLD:AAD7708* & $\begin{array}{l}\text { L. puteulanum Gibbs, } L \text {. } \\
\text { tegulare (Robertson) }\end{array}$ & 46 & 0 & $20+26$ \\
\hline BOLD:AAD9325 & $\begin{array}{l}\text { L. arantium Gibbs, } L \text {. } \\
\text { miniatulum (Mitchell) }\end{array}$ & 9 & $3(66,177,585)$ & 5,6 \\
\hline BOLD:AAW9735* & L. flaveriae (Robertson) & 30 & $\mathrm{NA}$ & 1 \\
\hline BOLD:ABY5205* & L. carlinvillense Gibbs & 14 & NA & 3 \\
\hline BOLD:ABZ0652* & $\begin{array}{l}\text { L. abanci (Crawford) } \dagger, L . \\
\text { admirandum (Sandhouse), } L .\end{array}$ & $\begin{array}{l}(3), 3 \\
(29),\end{array}$ & $\begin{array}{l}0 \text { except abanci } \\
3(200,201 \text {, }\end{array}$ & $\begin{array}{l}1,8+16+6+4 \\
+(44,1)+4+8\end{array}$ \\
\hline
\end{tabular}




\begin{tabular}{|c|c|c|c|c|}
\hline & $\begin{array}{l}\text { apocyni (Mitchell), L. } \\
\text { atwoodi Gibbs, L. dreisbachi } \\
\text { (Mitchell), L. ephialtum } \\
\text { (Gibbs) ††, L. fattigi } \\
\text { (Mitchell), L. georgeickworti } \\
\text { Gibbs, L. katherineae } \\
\text { Gibbs } \dagger, \text { L. marinense } \\
\text { (Michener), L. novascotiae } \\
\text { (Mitchell) } \text {, L. obscurum } \\
\text { (Robertson), L. pacatum } \\
\text { (Sandhouse), L. } \\
\text { taylorae Gibbs, L. viridatum } \\
\text { (Lovell) } \dagger \\
\text { (Saradmirandum (Knerer \& }\end{array}$ & $\begin{array}{l}(50), \\
(67), \\
(110)\end{array}$ & $\begin{array}{l}212), \\
\text { admirandum } 2 \\
(310,561) \text { and } \\
\text { novascotiae } 6 \\
(25,141,201, \\
384,387,390)\end{array}$ & $\begin{array}{l}+(6)+9+ \\
(1)+10+20 \\
+8+8+ \\
(18,4)+38 \\
+3,(6)\end{array}$ \\
\hline BOLD:ABZ0968 & $\begin{array}{l}\text { L. alachuense (Mitchell), L. } \\
\text { oblongum (Lovell) }\end{array}$ & 4 & $1(180)$ & $1+10$ \\
\hline BOLD:ACF2785 & $\begin{array}{l}\text { L. leucocomum (Lovell) } \dagger \dagger, \\
\text { L. succinipenne (Ellis) }\end{array}$ & $\begin{array}{l}54,55, \\
56\end{array}$ & 0 & $7+5,(3,2)$ \\
\hline
\end{tabular}




\begin{tabular}{|l|l|l|l|l|}
\hline BOLD:ACF5350* & L. sandhousiellum Gibbs & 93 & NA & 7 \\
\hline BOLD:ACF5692 & L. sheffieldi Gibbs, L. & 96 & $2(267,561)$ & $7+3$ \\
& & & & \\
\hline
\end{tabular}

846

847

848

849

850

851

852

853

854

855

856

857

858

859

860 $\uparrow$ ABGD groups discordant with BINs

$\dagger \dagger$ ABGD groups partially discordant with BINs 
861 Figure 1. Summary of minimum interspecific distance and maximum intraspecific distance for

862110 Lasioglossum species with DNA barcode-compliant sequences. Horizontal dashed line

863 indicates 1.4\% interspecific distance. Diagonal solid line indicates the expected barcode gap

864 where interspecific divergence is greater than intraspecific divergence. Shape and colour indicate 865 relationship of BIN to traditional taxonomic identification.

867 Figure 2. Summary of DNA barcode gap from all pairwise comparison of 110 Lasioglossum 868 species with DNA barcode-compliant sequences. Most pairwise comparisons are between 869 different species with large genetic distances (light gray bars). Intraspecific distances (dark gray 870 bars) overlap extensively with interspecific distances, preventing the use of DNA barcode gap 871 for correctly reliably diagnosing some species. Vertical dashed line indicates $1.4 \%$ genetic 872 distance.

873 Supplementary Figure. Neighbour-joining tree of Lasioglossum DNA barcodes used in the 874 analyses generated in the Barcode of Life Data Systems (http://www.boldsystems.org/) with a 875 Kimura 2-parameter substitution model and aligned using an amino acid based hidden Markov 876 model. Individual sequences are indicated with the species name and DNA barcode process ID. 


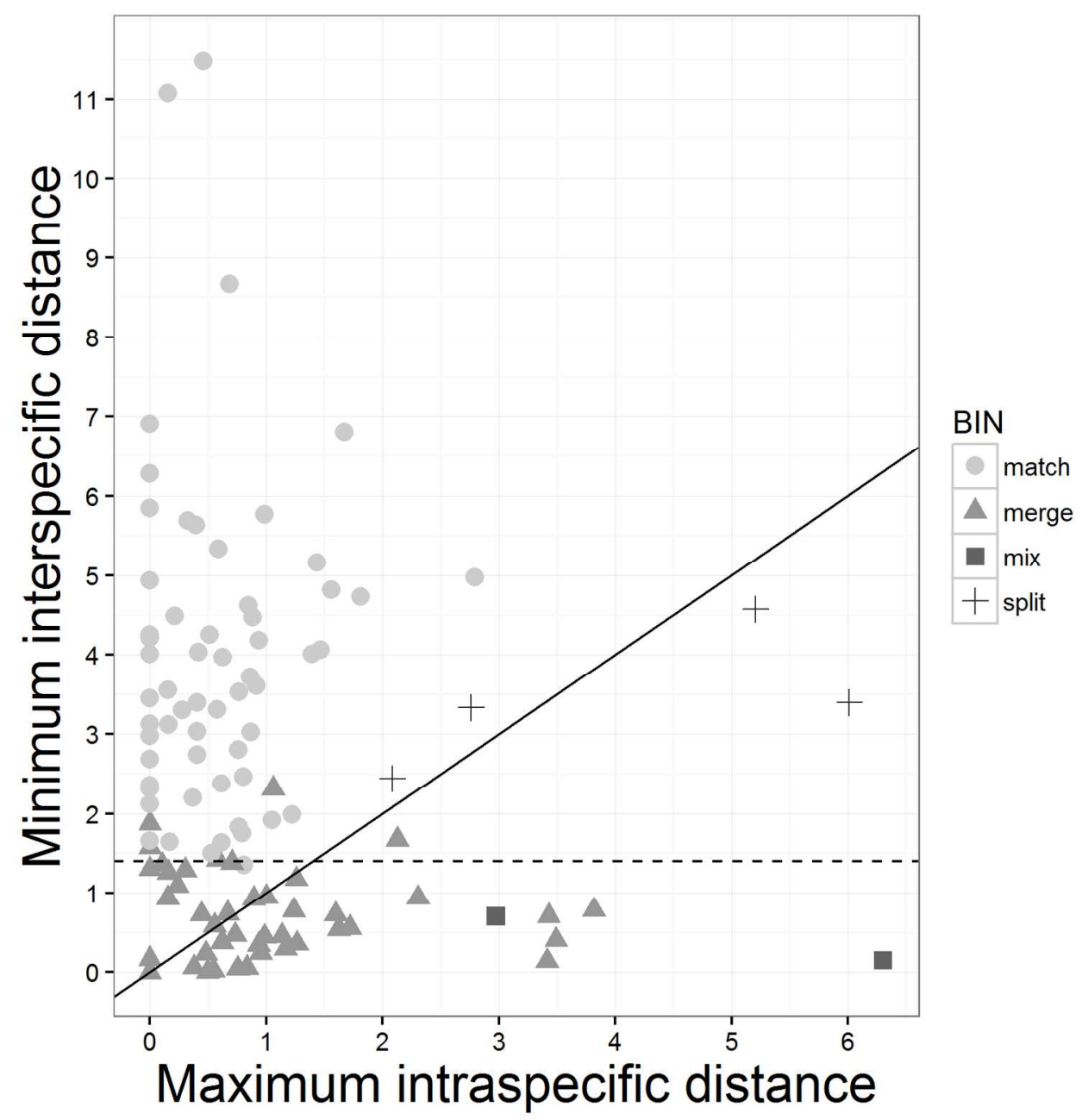

Figure 1. Summary of minimum interspecific distance and maximum intraspecific distance for 110 Lasioglossum species with DNA barcode-compliant sequences. Horizontal dashed line indicates $1.4 \%$ interspecific distance. Diagonal solid line indicates the expected barcode gap where interspecific divergence is greater than intraspecific divergence. Shape and colour indicate relationship of BIN to traditional taxonomic identification.

$152 \times 152 \mathrm{~mm}(300 \times 300 \mathrm{DPI})$ 


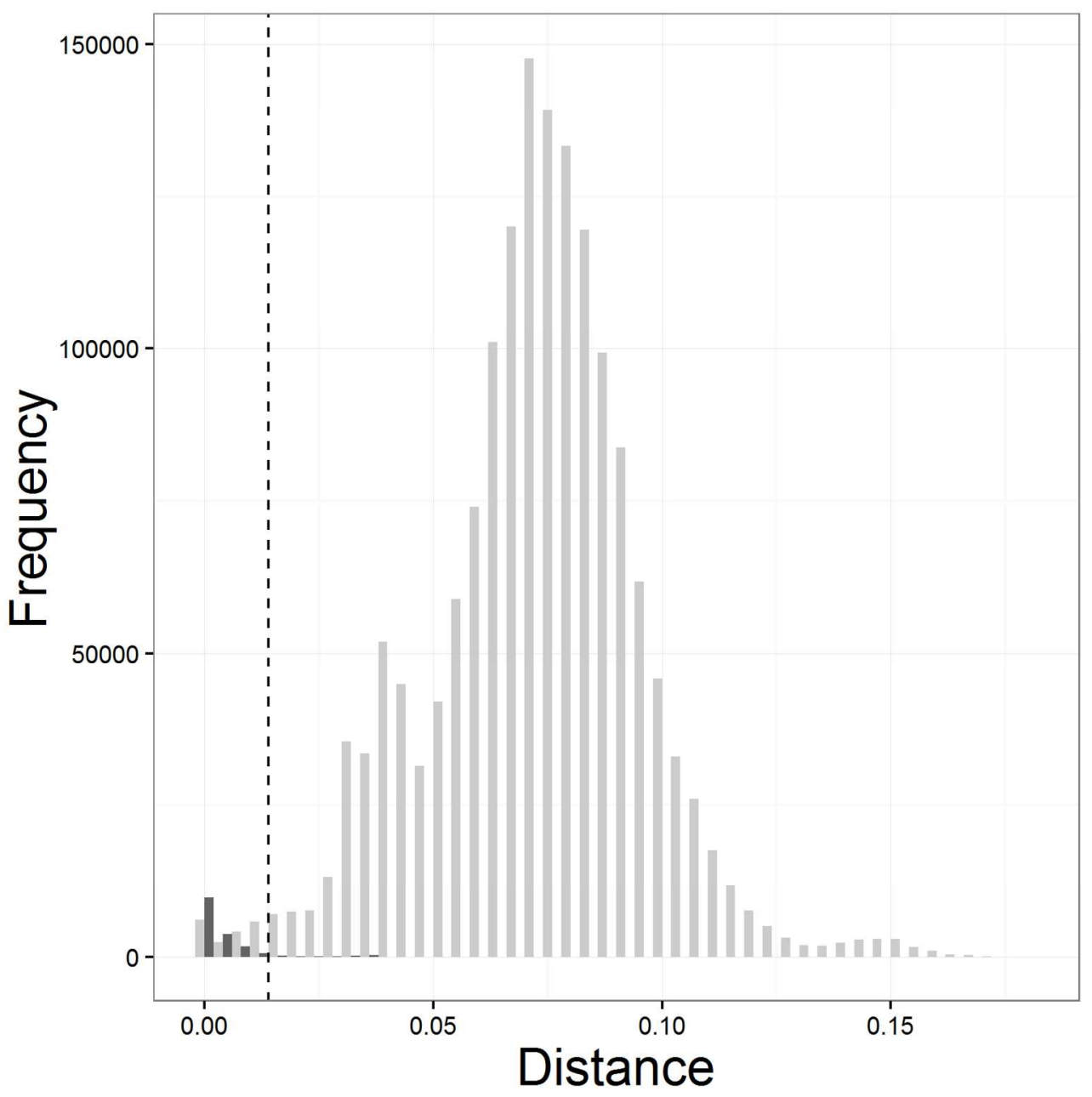

Figure 2. Summary of DNA barcode gap from all pairwise comparison of 110 Lasioglossum species with DNA barcode-compliant sequences. Most pairwise comparisons are between different species with large genetic distances (light gray bars). Intraspecific distances (dark gray bars) overlap extensively with interspecific distances, preventing the use of DNA barcode gap for correctly reliably diagnosing some species. Vertical dashed line indicates $1.4 \%$ genetic distance.

$152 \times 152 \mathrm{~mm}(300 \times 300 \mathrm{DPI})$ 


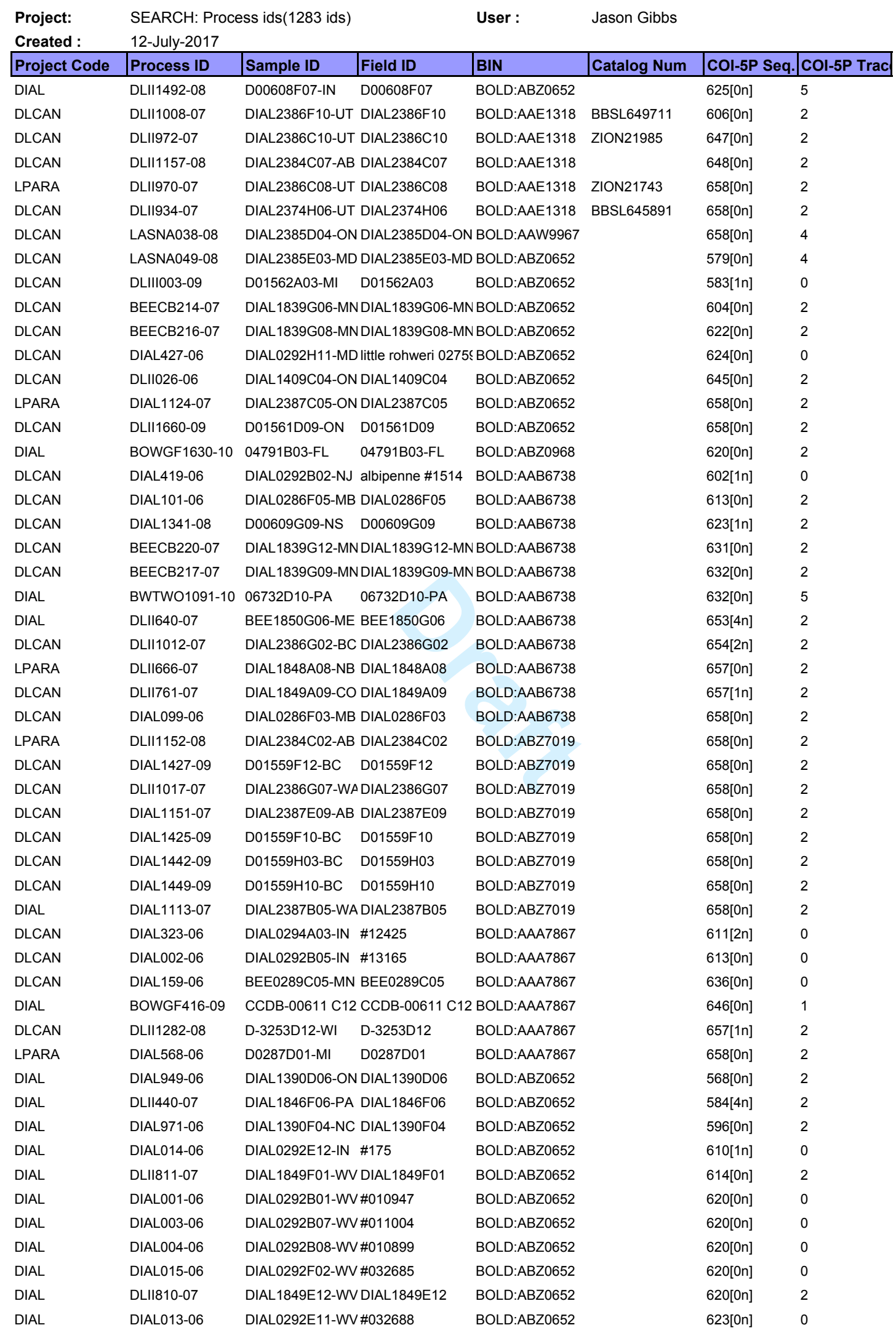




\begin{tabular}{|c|c|c|c|c|c|c|}
\hline DIAL & DIAL973-06 & DIAL1390F06-NC & DIAL1390F06 & BOLD:ABZ0652 & & $633[0 n]$ \\
\hline LPARA & DIAL983-06 & DIAL1390G04-NC & DIAL1390G04 & BOLD:ABZ0652 & & $653[1 \mathrm{n}]$ \\
\hline DIAL & DIAL985-06 & DIAL1390G06-NC & DIAL1390G06 & BOLD:ABZ0652 & & $654[0 \mathrm{n}]$ \\
\hline DIAL & DIAL981-06 & DIAL1390G02-WI & DIAL1390G02 & BOLD:ABZ0652 & & $654[0 n]$ \\
\hline DIAL & DLII701-07 & DIAL1848D09-MD & DIAL1848D09 & BOLD:ABZ0652 & & $657[0 \mathrm{n}]$ \\
\hline DIAL & DLII422-07 & DIAL1846D12-MS & DIAL1846D12 & BOLD:AAE1289 & & $645[0 \mathrm{n}]$ \\
\hline DIAL & DIAL805-06 & D1414H02-VA & $\mathrm{D} 1414 \mathrm{H} 02$ & BOLD:AAE1289 & & $658[0 \mathrm{n}]$ \\
\hline DIAL & DLII1219-08 & DIAL2384H09-SC & DIAL2384H09-SC & BOLD:AAE1289 & & $658[0 \mathrm{n}]$ \\
\hline DIAL & DIAL1388-09 & D01559C09-GA & D01559C09 & BOLD:AAE1289 & & $658[0 \mathrm{n}]$ \\
\hline DIAL & DIAL769-06 & D1414E02-VA & D1414E02 & BOLD:AAE1289 & & $658[0 \mathrm{n}]$ \\
\hline DIAL & DIAL018-06 & DIAL0293C03-NJ & \#2488 & BOLD:AAD9325 & & $618[1 \mathrm{n}]$ \\
\hline DIAL & DIAL019-06 & DIAL0293C04-NJ & $\# 1247$ & BOLD:AAD9325 & & $618[1 \mathrm{n}]$ \\
\hline LPARA & DLIII018-09 & D01562B06-MD & D01562B06 & BOLD:AAD9325 & & $658[0 \mathrm{n}]$ \\
\hline DIAL & DLIII014-09 & D01562B02-MD & D01562B02 & BOLD:AAD9325 & & $658[0 n]$ \\
\hline DIAL & DLIII036-09 & D01562C12-MD & D01562C12 & BOLD:AAD9325 & & $658[0 \mathrm{n}]$ \\
\hline DLCAN & DLIII022-09 & D01562B10-ON & D01562B10 & BOLD:ABZ0652 & & $594[1 \mathrm{n}]$ \\
\hline DLCAN & DLIII032-09 & D01562C08-ON & D01562C08 & BOLD:ABZ0652 & & $601[0 n]$ \\
\hline DLII & DIAL392-06 & DIAL0294G02-ML & Z020228 & BOLD:ABZ0652 & & $613[0 n]$ \\
\hline DLCAN & DLII672-07 & DIAL1848B03-ON & J DIAL1848B03 & BOLD:ABZ0652 & & $646[1 \mathrm{n}]$ \\
\hline DLCAN & DIAL1277-08 & D00609B05-WI & D00609B05 & BOLD:ABZ0652 & & $658[0 n]$ \\
\hline DLCAN & DIAL1335-08 & D00609G03-NS & D00609G03 & BOLD:ABZ0652 & & $658[0 \mathrm{n}]$ \\
\hline DIAL & DIAL504-06 & D0288F07-SC & D0288F07 & BOLD:AAE1190 & & $647[0 n]$ \\
\hline DIAL & DIAL506-06 & D0288F09-SC & D0288F09 & BOLD:AAE1190 & & $658[0 \mathrm{n}]$ \\
\hline DLCAN & DIAL325-06 & DIAL0294A05-in & $\# 12565$ & BOLD:AAC7048 & & $610[1 \mathrm{n}]$ \\
\hline DLCAN & DIAL012-06 & DIAL0292E08-MD & \#028623 & BOLD:AAC7048 & & $617[0 n]$ \\
\hline DIAL & BWTWO1090-10 & 06732D09-VA & 06732D09-VA & BOLD:AAC7048 & & $620[0 n]$ \\
\hline DLCAN & DIAL1291-08 & D00609C07-KS & D00609C07 & BOLD:AAC7048 & & $623[1 \mathrm{n}]$ \\
\hline DLCAN & DLII397-07 & DIAL1846B10-WV & DIAL1846B10 & BOLD:AAC7048 & & $632[0 n]$ \\
\hline DLCAN & DIAL939-06 & DIAL1390C08-ON & N DIAL1390C08 & BOLD:AAC7048 & & $643[0 \mathrm{n}]$ \\
\hline DLCAN & DLII009-06 & DIAL1409A09-NC & DIAL1409A09 & BOLD:AAC7048 & & $645[0 n]$ \\
\hline DLCAN & DLII450-07 & DIAL1846G04-VA & DIAL1846G04 & BOLD:AAC7048 & & $645[0 n]$ \\
\hline DLCAN & DIAL922-06 & DIAL1390B02-ON & JIAL1390B02 & BOLD:AAC7048 & & $654[0 n]$ \\
\hline LPARA & DLII716-07 & DIAL1848E12-MD & DIAL1848E12 & BOLD:AAC7048 & & $657[0 \mathrm{n}]$ \\
\hline DLCAN & DIAL849-06 & DIAL1413C12-TN & DIAL1413C12 & BOLD:AAC7048 & & $658[0 n]$ \\
\hline DLCAN & DIAL1438-09 & D01559G11-BC & D01559G11 & BOLD:AAB7689 & & $526[0 \mathrm{n}]$ \\
\hline DLCAN & DLII325-07 & DIAL1845D09-CA & DIAL1845D09 & BOLD:AAB7689 & & $614[0 n]$ \\
\hline DLCAN & DLII928-07 & DIAL2374G12-CA & A DIAL2374G12 & BOLD:AAB7689 & BBSL612783 & $618[0 \mathrm{n}]$ \\
\hline DLCAN & DLII309-07 & DIAL1845C05-CA & DIAL1845C05 & BOLD:AAB7689 & & $619[0 \mathrm{n}]$ \\
\hline DLCAN & DLII319-07 & DIAL1845D03-CA & DIAL1845D03 & BOLD:AAB7689 & & $645[0 n]$ \\
\hline DLCAN & DLII315-07 & DIAL1845C11-CA & DIAL1845C11 & BOLD:AAB7689 & & $645[0 n]$ \\
\hline DLCAN & DLII1068-07 & DIAL2388C12-UT & DIAL2388C12-UT & BOLD:AAB7689 & & $655[0 \mathrm{n}]$ \\
\hline DLCAN & DLII927-07 & DIAL2374G11-CA & AIAL2374G11 & BOLD:AAB7689 & BBSL612913 & $655[0 \mathrm{n}]$ \\
\hline LPARA & DLII1382-08 & D00607E04-CA & D00607E04 & BOLD:AAB7689 & & $658[0 \mathrm{n}]$ \\
\hline DLCAN & DIAL1413-09 & D01559E10-BC & D01559E10 & BOLD:AAB7689 & & $658[0 \mathrm{n}]$ \\
\hline DLCAN & DIAL1431-09 & D01559G04-BC & D01559G04 & BOLD:AAB7689 & & $658[0 \mathrm{n}]$ \\
\hline DLCAN & DLII1051-07 & DIAL2388B06-UT & DIAL2388B06-UT & BOLD:AAB7689 & & $658[0 \mathrm{n}]$ \\
\hline DLCAN & DLII1371-08 & D00607D05-CA & D00607D05 & BOLD:AAB7689 & & $658[0 \mathrm{n}]$ \\
\hline DIAL & DLII937-07 & DIAL2374H09-UT & DIAL2374H09 & BOLD:AAB7689 & BBSL648068 & $658[0 \mathrm{n}]$ \\
\hline DLCAN & LASNA025-08 & DIAL2385C03-MD & DIAL2385C03-MD & DOLD:AAA3781 & & $582[0 \mathrm{n}]$ \\
\hline DLCAN & DIAL872-06 & DIAL1413E11-SC & DIAL1413E11 & BOLD:AAA3781 & & $606[3 n]$ \\
\hline DLCAN & DIAL424-06 & DIAL0292D03-NC & coeruleum 02390 & BOLD:AAA3781 & & $610[1 \mathrm{n}]$ \\
\hline DLCAN & DIAL369-06 & DIAL0294E03-VA & $\# 046377$ & BOLD:AAA3781 & & $612[1 \mathrm{n}]$ \\
\hline DLCAN & DIAL368-06 & DIAL0294E02-TN & \#055668 & BOLD:AAA3781 & & $612[1 \mathrm{n}]$ \\
\hline DLCAN & DIAL436-06 & DIAL0293E11-NC & versatum 023948 & BOLD:AAA3781 & & $618[1 \mathrm{n}]$ \\
\hline DLCAN & DIAL030-06 & DIAL0293E10-MD & \#023822 & BOLD:AAA3781 & & $619[0 n]$ \\
\hline
\end{tabular}




\begin{tabular}{|c|c|c|c|c|c|}
\hline DLCAN & DIAL465-06 & D0288C04-DC & D0288C04 & BOLD:AAA3781 & $622[0 \mathrm{n}]$ \\
\hline DLCAN & DIAL437-06 & DIAL0293E12-DC & versatum 026920 & BOLD:AAA3781 & $623[1 \mathrm{n}]$ \\
\hline DLCAN & DIAL810-06 & D1414H07-VA & D1414H07 & BOLD:AAA3781 & $625[0 \mathrm{n}]$ \\
\hline DLCAN & DIAL078-06 & BEE0286D06-VA & BEE0286D06 & BOLD:AAA3781 & $627[3 \mathrm{n}]$ \\
\hline DLCAN & DIAL853-06 & DIAL1413D04-NC & DIAL1413D04 & BOLD:AAA3781 & $629[0 \mathrm{n}]$ \\
\hline DLCAN & DIAL1047-06 & DIAL1391D10-NC & DIAL1391D10 & BOLD:AAA3781 & $632[0 \mathrm{n}]$ \\
\hline DLCAN & DIAL775-06 & D1414E08-VA & D1414E08 & BOLD:AAA3781 & $636[0 \mathrm{n}]$ \\
\hline DLCAN & DLII017-06 & DIAL1409B06-NC & DIAL1409B06 & BOLD:AAA3781 & $645[0 \mathrm{n}]$ \\
\hline DLCAN & DIAL869-06 & DIAL1413E08-NC & DIAL1413E08 & BOLD:AAA3781 & $650[0 \mathrm{n}]$ \\
\hline DLCAN & DIAL771-06 & D1414E04-VA & D1414E04 & BOLD:AAA3781 & $653[0 \mathrm{n}]$ \\
\hline DLCAN & DIAL1012-06 & DIAL1391A09-SC & DIAL1391A09 & BOLD:AAA3781 & $653[0 \mathrm{n}]$ \\
\hline DLCAN & DIAL880-06 & DIAL1413F07-TN & DIAL1413F07 & BOLD:AAA3781 & $655[0 \mathrm{n}]$ \\
\hline DLCAN & DIAL1046-06 & DIAL1391D09-NC & DIAL1391D09 & BOLD:AAA3781 & $655[0 \mathrm{n}]$ \\
\hline DLCAN & DIAL1044-06 & DIAL1391D07-NC & DIAL1391D07 & BOLD:AAA3781 & $657[0 \mathrm{n}]$ \\
\hline DLCAN & DIAL850-06 & DIAL1413D01-TN & DIAL1413D01 & BOLD:AAA3781 & $658[0 \mathrm{n}]$ \\
\hline DLCAN & DIAL774-06 & D1414E07-VA & D1414E07 & BOLD:AAA3781 & $658[0 \mathrm{n}]$ \\
\hline DLCAN & DIAL462-06 & D0288C01-DC & D0288C01 & BOLD:AAA3781 & $658[0 \mathrm{n}]$ \\
\hline DLCAN & DIAL467-06 & D0288C06-SC & D0288C06 & BOLD:AAA3781 & $658[0 \mathrm{n}]$ \\
\hline DLCAN & DIAL482-06 & D0288D09-SC & D0288D09 & BOLD:AAA3781 & $658[0 \mathrm{n}]$ \\
\hline DLCAN & DLII1384-08 & D00607E06-MO & D00607E06 & BOLD:AAA3781 & $658[0 \mathrm{n}]$ \\
\hline DLCAN & DLII1339-08 & D00607A09-MO & D00607A09 & BOLD:AAA3781 & $658[0 \mathrm{n}]$ \\
\hline DLCAN & DIAL512-06 & D0288G03-SC & D0288G03 & BOLD:AAA3781 & $658[0 \mathrm{n}]$ \\
\hline DLCAN & DIAL499-06 & D0288F02-SC & D0288F02 & BOLD:AAA3781 & $658[0 \mathrm{n}]$ \\
\hline DLCAN & DIAL1015-06 & DIAL1391B01-SC & DIAL1391B01 & BOLD:AAA3781 & $658[0 \mathrm{n}]$ \\
\hline DLCAN & DIAL1053-06 & DIAL1391E04-SC & DIAL1391E04 & BOLD:AAA3781 & $658[0 \mathrm{n}]$ \\
\hline DLCAN & DIAL1048-06 & DIAL1391D11-SC & DIAL1391D11 & BOLD:AAA3781 & $658[0 \mathrm{n}]$ \\
\hline LTEG & DIAL832-06 & DIAL1413B06-IL & DIAL1413B06 & BOLD:ABY5205 & $636[0 \mathrm{n}]$ \\
\hline LTEG & DIAL728-06 & D1414A07-IL & D1414A07 & BOLD:ABY5205 & $658[0 \mathrm{n}]$ \\
\hline LTEG & DIAL837-06 & DIAL1413B11-IL & DIAL1413B11 & BOLD:ABY5205 & $658[0 \mathrm{n}]$ \\
\hline DIAL & BOWGF1452-10 & 04485C03-MD & 04485C03-MD & BOLD:AAC1104 & $658[0 \mathrm{n}]$ \\
\hline DIAL & DLII1396-08 & D00607F06-MD & D00607F06 & BOLD:AAC1104 & $658[0 \mathrm{n}]$ \\
\hline DLII & DLII1281-08 & D-3253D11-FL & D-3253D11 & BOLD:AAW9918 & $658[0 \mathrm{n}]$ \\
\hline DIAL & DLII1344-08 & D00607B02-KS & D00607B02 & BOLD:AAC1424 & $588[0 \mathrm{n}]$ \\
\hline DIAL & DIAL006-06 & DIAL0292D09-MD & \#012960 & BOLD:AAC1424 & $605[0 \mathrm{n}]$ \\
\hline DIAL & DIAL007-06 & DIAL0292D10-MD & D \#002309 & BOLD:AAC1424 & $606[0 \mathrm{n}]$ \\
\hline DIAL & DIAL005-06 & DIAL0292D08-NC & \#023927 & BOLD:AAC1424 & $610[2 n]$ \\
\hline DIAL & DIAL009-06 & DIAL0292D12-MD & \#002291 & BOLD:AAC1424 & $612[0 n]$ \\
\hline DIAL & DIAL008-06 & DIAL0292D11-MD & \#029860 & BOLD:AAC1424 & $614[0 n]$ \\
\hline DIAL & DLII464-07 & DIAL1846H06-VA & DIAL1846H06 & BOLD:AAC1424 & $634[0 n]$ \\
\hline DIAL & DLII399-07 & DIAL1846C01-MS & DIAL1846C01 & BOLD:AAC1424 & $645[0 \mathrm{n}]$ \\
\hline DIAL & DLII433-07 & DIAL1846E11-MS & DIAL1846E11 & BOLD:AAC1424 & $645[0 n]$ \\
\hline DIAL & DLII049-06 & DIAL1409E03-TX & DIAL1409E03 & BOLD:AAC1424 & $645[0 \mathrm{n}]$ \\
\hline DIAL & DLII453-07 & DIAL1846G07-MS & SIAL1846G07 & BOLD:AAC1424 & $645[0 n]$ \\
\hline DIAL & DLIII821-07 & DIAL1849F11-DE & DIAL1849F11 & BOLD:AAC1424 & $657[1 \mathrm{n}]$ \\
\hline LPARA & DLII825-07 & DIAL1849G03-DE & DIAL1849G03 & BOLD:AAC1424 & $658[0 \mathrm{n}]$ \\
\hline DIAL & DIAL1041-06 & DIAL1391D04-NC & DIAL1391D04 & BOLD:AAC1424 & $658[0 \mathrm{n}]$ \\
\hline DIAL & BWTW01077-10 & 06732C08-MA & 06732C08-MA & BOLD:AAC1424 & $658[0 \mathrm{n}]$ \\
\hline DIAL & BWTW01079-10 & 06732C10-VA & 06732C10-VA & BOLD:AAC1424 & $658[0 \mathrm{n}]$ \\
\hline DIAL & DLII1277-08 & D-3253D07-FL & D-3253D07 & BOLD:AAC2514 & $503[1 \mathrm{n}]$ \\
\hline DIAL & BOWGF944-09 & B01566H05-TX & B01566H05 & BOLD:AAC2514 & $594[0 \mathrm{n}]$ \\
\hline DIAL & DIAL010-06 & DIAL0292E02-MD & \#017872 & BOLD:AAC2514 & $603[0 \mathrm{n}]$ \\
\hline DIAL & DIAL380-06 & DIAL0294F02-NC & \#046248 & BOLD:AAC2514 & $605[1 n]$ \\
\hline DIAL & DIAL381-06 & DIAL0294F03-NC & ;046252 & BOLD:AAC2514 & $605[1 \mathrm{n}]$ \\
\hline DIAL & DIAL371-06 & DIAL0294E05-NC & ; \#056272 & BOLD:AAC2514 & $612[0 \mathrm{n}]$ \\
\hline DIAL & DIAL011-06 & DIAL0292E04-MD & \#017878 & BOLD:AAC2514 & $612[0 n]$ \\
\hline
\end{tabular}




\begin{tabular}{|c|c|c|c|c|c|c|}
\hline DIAL & DIAL861-06 & DIAL1413D12-FL & DIAL1413D12 & BOLD:AAC2514 & & $617[0 \mathrm{n}]$ \\
\hline DIAL & DLII404-07 & DIAL1846C06-MS & DIAL1846C06 & BOLD:AAC2514 & & $637[0 n]$ \\
\hline LPARA & DLII460-07 & DIAL1846H02-MS & DIAL1846H02 & BOLD:AAC2514 & & $645[0 \mathrm{n}]$ \\
\hline DIAL & DLII409-07 & DIAL1846C11-MS & DIAL1846C11 & BOLD:AAC2514 & & $645[0 n]$ \\
\hline DIAL & BOWGF433-09 & CCDB-00611 E05 & CCDB-00611 E05 & BOLD:AAC2514 & & $658[0 \mathrm{n}]$ \\
\hline DIAL & DIAL068-06 & BEE0286C08-NC & BEE0286C08 & BOLD:AAA5974 & & $608[5 n]$ \\
\hline DLCAN & DIAL331-06 & DIAL0294A11-IN & \#1791 & BOLD:ABY8200 & & $611[1 n]$ \\
\hline DLCAN & DIAL330-06 & DIAL0294A10-IN & $\# 12521$ & BOLD:AAA5973 & & $611[1 \mathrm{n}]$ \\
\hline DLCAN & DIAL933-06 & DIAL1390C02-ON & DIAL1390C02 & BOLD:ACE7675 & & $611[1 n]$ \\
\hline DLCAN & DIAL043-06 & BEE0286A05-GA & BEE0286A05 & BOLD:AAA5974 & & $612[1 \mathrm{n}]$ \\
\hline DLCAN & DIAL329-06 & DIAL0294A09-in & \#14033 & BOLD:ACE7675 & & $612[1 \mathrm{n}]$ \\
\hline DIAL & DIAL426-06 & DIAL0292E09-MD & cressonii 035210 & BOLD:AAA5973 & & $612[3 n]$ \\
\hline DLCAN & DLII373-07 & DIAL1845H09-BC & DIAL1845H09 & BOLD:AAA5973 & & $618[1 \mathrm{n}]$ \\
\hline DLCAN & DLII371-07 & DIAL1845H07-BC & DIAL1845H07 & BOLD:AAA5973 & & $619[0 n]$ \\
\hline DLCAN & DIAL425-06 & DIAL0292E07-ME & cressonii 010595 & BOLD:AAA5973 & & $622[2 n]$ \\
\hline DLCAN & DLII351-07 & DIAL1845F11-BC & DIAL1845F11 & BOLD:AAA5973 & & $624[0 n]$ \\
\hline DLCAN & DIAL1006-06 & DIAL1391A03-NC & DIAL1391A03 & BOLD:AAA5973 & & $630[0 \mathrm{n}]$ \\
\hline DLCAN & DIAL171-06 & BEE0289D05-MN & BEE0289D05 & BOLD:AAA5973 & & $636[0 n]$ \\
\hline DIAL & DIAL173-06 & BEE0289D07-MN & BEE0289D07 & BOLD:AAA5973 & & $648[1 \mathrm{n}]$ \\
\hline LPARA & DIAL1008-06 & DIAL1391A05-NC & DIAL1391A05 & BOLD:AAA5973 & & $655[0 n]$ \\
\hline DLCAN & DIAL1339-08 & D00609G07-NS & D00609G07 & BOLD:AAA5973 & & $657[0 \mathrm{n}]$ \\
\hline DLCAN & DLII661-07 & DIAL1848A03-NB & DIAL1848A03 & BOLD:AAA5973 & & $657[0 \mathrm{n}]$ \\
\hline DLCAN & DLII639-07 & BEE1850G05-ME & BEE1850G05 & BOLD:AAA5973 & & $657[0 \mathrm{n}]$ \\
\hline DLCAN & DIAL172-06 & BEE0289D06-MN & BEE0289D06 & BOLD:AAA5973 & & $658[0 n]$ \\
\hline DLCAN & DLII1688-09 & D01561G01-MD & D01561G01 & BOLD:ACE7675 & & $658[0 \mathrm{n}]$ \\
\hline DLCAN & DLII1025-07 & DIAL2386H03-WA & DIAL2386H03 & BOLD:AAA5973 & & $658[0 \mathrm{n}]$ \\
\hline DLCAN & DIAL1009-06 & DIAL1391A06-NC & DIAL1391A06 & BOLD:AAA5973 & & $658[0 \mathrm{n}]$ \\
\hline DLCAN & DIAL609-06 & D0287G06-IL & D0287G06 & BOLD:ABY7290 & & $658[0 \mathrm{n}]$ \\
\hline DLCAN & DIAL519-06 & D0288G10-ON & D0288G10 & BOLD:AAA5973 & & $658[0 \mathrm{n}]$ \\
\hline DLCAN & DLII1016-07 & DIAL2386G06-BC & DIAL2386G06 & BOLD:AAA5973 & & $658[0 \mathrm{n}]$ \\
\hline DIAL & BOWGF1886-10 & 03767G09-BC & 03767G09-BC & BOLD:AAA5973 & & $658[0 \mathrm{n}]$ \\
\hline DIAL & BWTWO1069-10 & 06732B12-NY & 06732B12-NY & BOLD:AAP0913 & & $658[0 \mathrm{n}]$ \\
\hline LPARA & DLII1005-07 & DIAL2386F07-WA & DIAL2386F07 & BOLD:AAJ1718 & BBSL696686 & $658[0 \mathrm{n}]$ \\
\hline DLCAN & DLII1027-07 & DIAL2386H05-WA & DIAL2386H05 & BOLD:AAJ1718 & & $658[0 n]$ \\
\hline DIAL & DLII1292-08 & D-3253E10-OK & D-3253E10 & BOLD:AAC8559 & & $557[1 \mathrm{n}]$ \\
\hline DIAL & DLII1351-08 & D00607B09-KS & D00607B09 & BOLD:AAC8559 & & $606[0 n]$ \\
\hline DIAL & DLII041-06 & DIAL1409D07-TX & DIAL1409D07 & BOLD:AAC8559 & & $644[1 \mathrm{n}]$ \\
\hline DIAL & DLII038-06 & DIAL1409D04-TX & DIAL1409D04 & BOLD:AAC8559 & & $645[0 \mathrm{n}]$ \\
\hline DIAL & DLII054-06 & DIAL1409E08-TX & DIAL1409E08 & BOLD:AAC8559 & & $645[0 \mathrm{n}]$ \\
\hline DIAL & DLII056-06 & DIAL1409E10-TX & DIAL1409E10 & BOLD:AAC8559 & & $645[0 n]$ \\
\hline DIAL & DLII059-06 & DIAL1409F01-TX & DIAL1409F01 & BOLD:AAC8559 & & $645[0 \mathrm{n}]$ \\
\hline DIAL & DLII1343-08 & D00607B01-KS & D00607B01 & BOLD:AAC8559 & & $651[0 n]$ \\
\hline LPARA & DLII728-07 & DIAL1848F12-NC & DIAL1848F12 & BOLD:AAC8559 & & $657[0 \mathrm{n}]$ \\
\hline DLCAN & DLII1639-09 & D01561B12-ON & D01561B12 & BOLD:ABZ0652 & & $656[2 n]$ \\
\hline DLCAN & DLII726-07 & DIAL1848F10-ON & IIAL1848F10 & BOLD:ABZ0652 & & $656[1 \mathrm{n}]$ \\
\hline LPARA & DLII729-07 & DIAL1848G01-ON & I DIAL1848G01 & BOLD:ABZ0652 & & $657[0 \mathrm{n}]$ \\
\hline DLCAN & DLII1666-09 & D01561E03-ON & D01561E03 & BOLD:ABZ0652 & & $657[1 \mathrm{n}]$ \\
\hline DLCAN & DLII920-07 & DIAL2374G04-UT & DIAL2374G04 & BOLD:AAD7578 & ZION23251 & $612[0 n]$ \\
\hline LPARA & DLII1049-07 & DIAL2388B04-UT & DIAL2388B04-UT & BOLD:AAD7578 & & $658[0 \mathrm{n}]$ \\
\hline DLCAN & DLII1059-07 & DIAL2388C03-UT & DIAL2388C03-UT & BOLD:AAD7578 & & $658[0 \mathrm{n}]$ \\
\hline DLCAN & DLII956-07 & DIAL2386B05-NV & DIAL2386B05 & BOLD:AAD7578 & BBSL536805 & $658[0 \mathrm{n}]$ \\
\hline DLCAN & DLII1158-08 & DIAL2384C08-AB & DIAL2384C08 & BOLD:AAD7578 & & $658[0 \mathrm{n}]$ \\
\hline DLCAN & DLII921-07 & DIAL2374G05-UT & DIAL2374G05 & BOLD:AAD7578 & ZION22357 & $658[0 \mathrm{n}]$ \\
\hline LTEG & DIAL356-06 & DIAL0294D02-IN & $\# 14702$ & BOLD:AAB0760 & & $586[0 \mathrm{n}]$ \\
\hline DLCAN & DLII1559-09 & D01560D03-IA & D01560D03 & BOLD:AAB0760 & & $599[0 n]$ \\
\hline
\end{tabular}




\begin{tabular}{|c|c|c|c|c|c|}
\hline DLCAN & DLII1527-09 & D01560A07-WI & D01560A07 & BOLD:AAB0760 & $628[0 \mathrm{n}]$ \\
\hline LTEG & DLII031-06 & DIAL1409C09-ON & DIAL1409C09 & BOLD:AAB0760 & $645[0 \mathrm{n}]$ \\
\hline LTEG & DIAL918-06 & DIAL1390A09-ON & DIAL1390A09 & BOLD:AAB0760 & $654[0 n]$ \\
\hline LTEG & BEECB213-07 & DIAL1839G05-MN & DIAL1839G05-MN & BOLD:AAB0760 & $658[0 \mathrm{n}]$ \\
\hline LTEG & DIAL476-06 & D0288D03-IN & D0288D03 & BOLD:AAB0760 & $658[0 \mathrm{n}]$ \\
\hline LTEG & DIAL888-06 & DIAL1413G03-NC & DIAL1413G03 & BOLD:AAB0760 & $658[0 \mathrm{n}]$ \\
\hline LTEG & DIAL653-06 & D0295C04-ON & D0295C04 & BOLD:AAB0760 & $658[0 \mathrm{n}]$ \\
\hline LTEG & DIAL1089-06 & DIAL1391H04-NC & DIAL1391H04 & BOLD:AAB0760 & $658[0 \mathrm{n}]$ \\
\hline LTEG & DLII833-07 & DIAL1849G11-MA & DIAL1849G11 & BOLD:AAB0760 & $658[0 \mathrm{n}]$ \\
\hline LTEG & BEECB221-07 & DIAL1839H01-MN & DIAL1839H01-MN & BOLD:AAB0760 & $658[0 \mathrm{n}]$ \\
\hline LTEG & DIAL654-06 & D0295C05-ON & D0295C05 & BOLD:AAB0760 & $658[0 \mathrm{n}]$ \\
\hline DLCAN & DLIII026-09 & D01562C02-MB & D01562C02 & BOLD:AAB0760 & $658[0 \mathrm{n}]$ \\
\hline DLCAN & DLIII019-09 & D01562B07-MI & D01562B07 & BOLD:AAB0760 & $658[0 \mathrm{n}]$ \\
\hline DIAL & BWTWO899-10 & B03765D08-IA & B03765D08-IA & BOLD:AAB0760 & $658[0 \mathrm{n}]$ \\
\hline DIAL & BOWGF1482-10 & 04485E09-MI & 04485E09-MI & BOLD:AAB0760 & $658[0 \mathrm{n}]$ \\
\hline DIAL & BWTWO897-10 & B03765D06-IA & B03765D06-IA & BOLD:AAB0760 & $658[0 \mathrm{n}]$ \\
\hline DIAL & BOWGF1475-10 & 04485E02-MI & 04485E02-MI & BOLD:AAB0760 & $658[0 \mathrm{n}]$ \\
\hline DIAL & BWTWO902-10 & B03765D11-IA & B03765D11-IA & BOLD:AAB0760 & $658[0 \mathrm{n}]$ \\
\hline DIAL & BWTWO904-10 & B03765E01-IA & B03765E01-IA & BOLD:AAB0760 & $658[0 \mathrm{n}]$ \\
\hline DIAL & DIAL797-06 & D1414G06-DC & D1414G06 & BOLD:ABZ0652 & $552[3 n]$ \\
\hline DLCAN & DLII469-07 & DIAL1846H11-DC & DIAL1846H11 & BOLD:ABZ0652 & $559[0 \mathrm{n}]$ \\
\hline DLCAN & DIAL135-06 & BEE0289A03-MN & BEE0289A03 & BOLD:ABZ0652 & $565[0 \mathrm{n}]$ \\
\hline DLII & DLII425-07 & DIAL1846E03-PA & DIAL1846E03 & BOLD:ABZ0652 & $581[3 n]$ \\
\hline DIAL & DIAL676-06 & D0295E03-VA & D0295E03 & BOLD:ABZ0652 & $596[4 n]$ \\
\hline DLCAN & DIAL139-06 & BEE0289A07-MN & BEE0289A07 & BOLD:ABZ0652 & $598[0 n]$ \\
\hline DLCAN & DIAL410-06 & DIAL0294H08-ON & | DIAL0294H08 & BOLD:ABZ0652 & $606[2 n]$ \\
\hline DLCAN & DIAL979-06 & DIAL1390F12-NC & DIAL1390F12 & BOLD:ABZ0652 & $607[0 \mathrm{n}]$ \\
\hline DLCAN & DIAL656-06 & D0295C07-ON & D0295C07 & BOLD:ABZ0652 & $607[0 \mathrm{n}]$ \\
\hline DLCAN & DIAL1121-07 & DIAL2387C02-ON & | DIAL2387C02 & BOLD:ABZ0652 & $610[2 n]$ \\
\hline DLCAN & DLII837-07 & DIAL1849H03-DC & DIAL1849H03 & BOLD:ABZ0652 & $620[0 n]$ \\
\hline DLCAN & DLII381-07 & DIAL1846A05-ON & DIAL1846A05 & BOLD:ABZ0652 & $624[0 n]$ \\
\hline DLCAN & DLII383-07 & DIAL1846A07-ON & DIAL1846A07 & BOLD:ABZ0652 & $628[0 \mathrm{n}]$ \\
\hline DLCAN & DLII378-07 & DIAL1846A02-ON & DIAL1846A02 & BOLD:ABZ0652 & $629[0 n]$ \\
\hline DIAL & DIAL975-06 & DIAL1390F08-WV & DIAL1390F08 & BOLD:ABZ0652 & $630[0 \mathrm{n}]$ \\
\hline DLII & DIAL1067-06 & DIAL1391F06-ON & DIAL1391F06 & BOLD:ABZ0652 & $632[0 \mathrm{n}]$ \\
\hline DLCAN & DIAL1080-06 & DIAL1391G07-ON & I DIAL1391G07 & BOLD:ABZ0652 & $639[1 \mathrm{n}]$ \\
\hline DLCAN & DIAL970-06 & DIAL1390F03-ON & DIAL1390F03 & BOLD:ABZ0652 & $640[0 n]$ \\
\hline DLCAN & DLII389-07 & DIAL1846B02-ON & DIAL1846B02 & BOLD:ABZ0652 & $642[0 n]$ \\
\hline DLCAN & DLII379-07 & DIAL1846A03-ON & DIAL1846A03 & BOLD:ABZ0652 & $645[0 n]$ \\
\hline DLCAN & DLIII024-06 & DIAL1409C02-ON & | DIAL1409C02 & BOLD:ABZ0652 & $645[0 \mathrm{n}]$ \\
\hline DLCAN & DLII025-06 & DIAL1409C03-ON & DIAL1409C03 & BOLD:ABZ0652 & $645[0 \mathrm{n}]$ \\
\hline DLCAN & DLII029-06 & DIAL1409C07-ON & DIAL1409C07 & BOLD:ABZ0652 & $645[0 \mathrm{n}]$ \\
\hline DLCAN & DLII030-06 & DIAL1409C08-ON & DIAL1409C08 & BOLD:ABZ0652 & $645[0 n]$ \\
\hline DIAL & DLIII034-09 & D01562C10-MB & D01562C10 & BOLD:ABZ0652 & $652[6 n]$ \\
\hline DLCAN & DIAL956-06 & DIAL1390E01-ON & DIAL1390E01 & BOLD:ABZ0652 & $653[1 \mathrm{n}]$ \\
\hline DLCAN & DIAL968-06 & DIAL1390F01-ON & DIAL1390F01 & BOLD:ABZ0652 & $654[0 \mathrm{n}]$ \\
\hline DLCAN & DIAL980-06 & DIAL1390G01-NC & DIAL1390G01 & BOLD:ABZ0652 & $654[0 \mathrm{n}]$ \\
\hline DLCAN & DIAL976-06 & DIAL1390F09-ON & DIAL1390F09 & BOLD:ABZ0652 & $654[0 n]$ \\
\hline DLCAN & DIAL972-06 & DIAL1390F05-ON & DIAL1390F05 & BOLD:ABZ0652 & $654[0 \mathrm{n}]$ \\
\hline DLCAN & DIAL1076-06 & DIAL1391G03-ON & I DIAL1391G03 & BOLD:ABZ0652 & $654[0 \mathrm{n}]$ \\
\hline DLCAN & DIAL958-06 & DIAL1390E03-ON & DIAL1390E03 & BOLD:ABZ0652 & $654[0 \mathrm{n}]$ \\
\hline DLCAN & DIAL961-06 & DIAL1390E06-ON & DIAL1390E06 & BOLD:ABZ0652 & $654[0 n]$ \\
\hline DLCAN & DIAL962-06 & DIAL1390E07-ON & DIAL1390E07 & BOLD:ABZ0652 & $654[0 \mathrm{n}]$ \\
\hline DLII & DIAL1078-06 & DIAL1391G05-ON & IDIAL1391G05 & BOLD:ABZ0652 & $654[0 \mathrm{n}]$ \\
\hline DLCAN & DIAL1119-07 & DIAL2387B11-ON & DIAL2387B11 & BOLD:ABZ0652 & $657[1 \mathrm{n}]$ \\
\hline
\end{tabular}




\begin{tabular}{|c|c|c|c|c|c|}
\hline DLCAN & DLII618-07 & BEE1850E08-MN & BEE1850E08 & BOLD:ABZ0652 & $657[0 n]$ \\
\hline DIAL & DLII1618-09 & D01561A03-IN & D01561A03 & BOLD:ABZ0652 & $657[1 \mathrm{n}]$ \\
\hline LPARA & DIAL1075-06 & DIAL1391G02-ON & V DIAL1391G02 & BOLD:ABZ0652 & $658[0 \mathrm{n}]$ \\
\hline DLCAN & DLII1524-09 & D01560A04-WI & D01560A04 & BOLD:ABZ0652 & $658[0 n]$ \\
\hline DLCAN & DLII1542-09 & D01560B10-ON & D01560B10 & BOLD:ABZ0652 & $658[0 n]$ \\
\hline DLCAN & DLII830-07 & DIAL1849G08-WI & DIAL1849G08 & BOLD:ABZ0652 & $658[0 n]$ \\
\hline DLCAN & DIAL1114-07 & DIAL2387B06-ON & NIAL2387B06 & BOLD:ABZ0652 & $658[0 n]$ \\
\hline DLCAN & DIAL795-06 & D1414G04-DC & D1414G04 & BOLD:ABZ0652 & $658[0 \mathrm{n}]$ \\
\hline DLII & BOWGF1487-10 & 04485F02-MI & 04485F02-MI & BOLD:ABZ0652 & $658[0 n]$ \\
\hline DLCAN & DIAL947-06 & DIAL1390D04-ON & V DIAL1390D04 & BOLD:ABZ0652 & $626[0 n]$ \\
\hline DLCAN & DLII002-06 & DIAL1409A02-ON & JIAL1409A02 & BOLD:ABZ0652 & $645[0 n]$ \\
\hline LPARA & DIAL1126-07 & DIAL2387C07-ON & V DIAL2387C07 & BOLD:ABZ0652 & $658[0 n]$ \\
\hline DLCAN & DIAL1091-06 & DIAL1391H06-NC & DIAL1391H06 & BOLD:ABZ0652 & $658[0 \mathrm{n}]$ \\
\hline DIAL & DLII1276-08 & D-3253D06-FL & D-3253D06 & BOLD:AAW9735 & $561[2 n]$ \\
\hline DIAL & DIAL049-06 & BEE0286A11-GA & BEE0286A11 & BOLD:AAB6361 & $574[0 \mathrm{n}]$ \\
\hline DIAL & DLII444-07 & DIAL1846F10-SC & DIAL1846F10 & BOLD:AAB6361 & $583[0 \mathrm{n}]$ \\
\hline DIAL & DLII446-07 & DIAL1846F12-SC & DIAL1846F12 & BOLD:AAB6361 & $589[0 \mathrm{n}]$ \\
\hline DIAL & DIAL057-06 & BEE0286B08-GA & BEE0286B08 & BOLD:AAB6361 & $613[0 n]$ \\
\hline DLCAN & DIAL353-06 & DIAL0294C11-IN & \#3182 & BOLD:AAC1104 & $582[3 n]$ \\
\hline DLCAN & DIAL355-06 & DIAL0294D01-IN & $\# 8126$ & BOLD:AAC1104 & $584[2 n]$ \\
\hline DLCAN & LASNA050-08 & DIAL2385E04-ON & N DIAL2385E04-ON & BOLD:AAC1104 & $586[3 n]$ \\
\hline DIAL & DIAL354-06 & DIAL0294C12-IL & \#8348 & BOLD:AAC1104 & $589[4 n]$ \\
\hline DLCAN & DIAL025-06 & DIAL0293E02-MD & \#11D & BOLD:AAC1104 & $610[2 n]$ \\
\hline LPARA & DIAL024-06 & DIAL0293E01-IN & $\# 11182$ & BOLD:AAC1104 & $621[2 n]$ \\
\hline DLCAN & DLII1502-08 & D00608G05-IN & D00608G05 & BOLD:AAC1104 & $643[0 n]$ \\
\hline DLCAN & DLII1498-08 & D00608G01-IN & D00608G01 & BOLD:AAC1104 & $658[0 \mathrm{n}]$ \\
\hline DLCAN & DLII1624-09 & D01561A09-MD & D01561A09 & BOLD:AAC1104 & $658[0 n]$ \\
\hline DLCAN & DLII1646-09 & D01561C07-MD & D01561C07 & BOLD:AAC1104 & $658[0 \mathrm{n}]$ \\
\hline DIAL & LASNA029-08 & DIAL2385C07-DE & DIAL2385C07-DE & BOLD:ABZ0652 & $538[0 n]$ \\
\hline DIAL & DLII1398-08 & D00607F08-MA & D00607F08 & BOLD:ABZ0652 & $591[3 n]$ \\
\hline DIAL & DLII627-07 & BEE1850F05-NY & BEE1850F05 & BOLD:ABZ0652 & $656[1 \mathrm{n}]$ \\
\hline DIAL & LASNA015-08 & DIAL2385B04-NY & DIAL2385B04-NY & BOLD:ABZ0652 & $656[0 \mathrm{n}]$ \\
\hline LPARA & DIAL806-06 & D1414H03-VA & D1414H03 & BOLD:ABZ0652 & $657[0 \mathrm{n}]$ \\
\hline DIAL & DLII1222-08 & DIAL2384H12-NY & DIAL2384H12-NY & BOLD:ABZ0652 & $657[0 \mathrm{n}]$ \\
\hline DIAL & DIAL1394-09 & D01559D03-MA & D01559D03 & BOLD:ABZ0652 & $657[0 \mathrm{n}]$ \\
\hline DIAL & BOWGF1122-10 & B04743G05-MA & B04743G05-MA & BOLD:ABZ0652 & $658[0 \mathrm{n}]$ \\
\hline DIAL & DLII449-07 & DIAL1846G03-VA & DIAL1846G03 & BOLD:AAC5496 & $582[1 \mathrm{n}]$ \\
\hline DIAL & DIAL815-06 & D1414H12-VA & D1414H12 & BOLD:AAC5496 & $594[3 n]$ \\
\hline DIAL & DIAL813-06 & D1414H10-VA & D1414H10 & BOLD:AAC5496 & $601[0 \mathrm{n}]$ \\
\hline DIAL & DIAL363-06 & DIAL0294D09-NC & \#046341 & BOLD:AAC5496 & $601[5 n]$ \\
\hline DIAL & DIAL755-06 & D1414C12-VA & D1414C12 & BOLD:AAC5496 & $603[0 \mathrm{n}]$ \\
\hline DIAL & DIAL362-06 & DIAL0294D08-NC & \#046295 & BOLD:AAC5496 & $604[2 n]$ \\
\hline DIAL & DIAL858-06 & DIAL1413D09-FL & DIAL1413D09 & BOLD:AAC5496 & $611[0 \mathrm{n}]$ \\
\hline DIAL & DLII447-07 & DIAL1846G01-VA & DIAL1846G01 & BOLD:AAC5496 & $645[0 \mathrm{n}]$ \\
\hline DIAL & DLII714-07 & DIAL1848E10-MD & DIAL1848E10 & BOLD:AAC5496 & $657[0 \mathrm{n}]$ \\
\hline LPARA & DIAL825-06 & DIAL1413A10-VA & DIAL1413A10 & BOLD:AAC5496 & $658[0 n]$ \\
\hline DIAL & DIAL826-06 & DIAL1413A11-VA & DIAL1413A11 & BOLD:AAC5496 & $658[0 \mathrm{n}]$ \\
\hline DIAL & BOWGF418-09 & CCDB-00611 D02 & 2 CCDB-00611 D02 & BOLD:AAL9893 & $521[0 \mathrm{n}]$ \\
\hline DIAL & BOWGF1499-10 & 04485G02-MI & 04485G02-MI & BOLD:AAL9893 & $658[0 \mathrm{n}]$ \\
\hline DIAL & DLII693-07 & DIAL1848D01-WI & DIAL1848D01 & BOLD:ABZ6179 & $657[0 \mathrm{n}]$ \\
\hline DLCAN & DLII430-07 & DIAL1846E08-WV & DIAL1846E08 & BOLD:ACE5057 & $576[0 n]$ \\
\hline DLCAN & DIAL1040-06 & DIAL1391D03-NC & DIAL1391D03 & BOLD:ACE5057 & $618[1 \mathrm{n}]$ \\
\hline LPARA & DIAL1036-06 & DIAL1391C11-NC & DIAL1391C11 & BOLD:ACE5057 & $658[0 \mathrm{n}]$ \\
\hline DLCAN & DIAL811-06 & D1414H08-VA & D1414H08 & BOLD:AAA3782 & $520[0 n]$ \\
\hline DIAL & DIAL908-06 & DIAL1413H11-NC & DIAL1413H11 & BOLD:ABY8629 & $531[2 n]$ \\
\hline
\end{tabular}




\begin{tabular}{|c|c|c|c|c|c|}
\hline DLCAN & DIAL1013-06 & DIAL1391A10-SC & DIAL1391A10 & BOLD:AAA3782 & $534[0 \mathrm{n}]$ \\
\hline DLCAN & DIAL803-06 & D1414G12-VA & D1414G12 & BOLD:AAA3782 & $545[1 n]$ \\
\hline DLCAN & DIAL870-06 & DIAL1413E09-NC & DIAL1413E09 & BOLD:AAA3782 & $557[0 \mathrm{n}]$ \\
\hline DLCAN & DIAL827-06 & DIAL1413B01-VA & DIAL1413B01 & BOLD:AAA3782 & $557[0 \mathrm{n}]$ \\
\hline DLCAN & DIAL892-06 & DIAL1413G07-NC & DIAL1413G07 & BOLD:AAA3782 & $583[0 n]$ \\
\hline DLCAN & DLII1424-08 & D00607H10-KS & $\mathrm{D} 00607 \mathrm{H} 10$ & BOLD:AAA3782 & $592[0 n]$ \\
\hline DLCAN & DIAL450-06 & D0288A11-MD & D0288A11 & BOLD:AAA3782 & $596[0 \mathrm{n}]$ \\
\hline DLCAN & DIAL448-06 & D0288A09-WV & D0288A09 & BOLD:AAA3782 & $598[0 \mathrm{n}]$ \\
\hline DIAL & DIAL570-06 & D0287D03-IL & D0287D03 & BOLD:AAE5827 & $598[0 \mathrm{n}]$ \\
\hline DIAL & DIAL463-06 & D0288C02-DC & D0288C02 & BOLD:AAA3782 & $599[1 n]$ \\
\hline DLCAN & DIAL582-06 & D0287E03-IL & D0287E03 & BOLD:AAA3782 & $600[1 n]$ \\
\hline DIAL & DIAL321-06 & DIAL0294A01-IN & \#3770 & & $600[6 n]$ \\
\hline DLCAN & DIAL598-06 & D0287F07-IL & D0287F07 & BOLD:AAA3782 & $601[0 \mathrm{n}]$ \\
\hline DLCAN & DIAL583-06 & D0287E04-IL & D0287E04 & BOLD:AAA3782 & $601[1 n]$ \\
\hline DLCAN & DLII439-07 & DIAL1846F05-WV & DIAL1846F05 & BOLD:AAA3782 & $606[0 \mathrm{n}]$ \\
\hline DLCAN & DIAL322-06 & DIAL0294A02-il & $\# 8619$ & BOLD:AAA3782 & $607[0 \mathrm{n}]$ \\
\hline DLCAN & DIAL374-06 & DIAL0294E08-MD & \#029472 & BOLD:AAA3782 & $607[0 \mathrm{n}]$ \\
\hline DLCAN & DIAL384-06 & DIAL0294F06-VA & $\# 046373$ & BOLD:AAA3782 & $608[0 \mathrm{n}]$ \\
\hline DLCAN & DIAL376-06 & DIAL0294E10-VA & $\# 046372$ & BOLD:AAA3782 & $608[0 \mathrm{n}]$ \\
\hline DLCAN & DIAL375-06 & DIAL0294E09-MD & $\# 046450$ & BOLD:AAA3782 & $610[1 n]$ \\
\hline DLCAN & DIAL386-06 & DIAL0294F08-MD & \#039978 & BOLD:AAA3782 & $611[0 n]$ \\
\hline DLCAN & DIAL036-06 & DIAL0292A09-VA & $\# 017382$ & BOLD:AAA3782 & $611[0 \mathrm{n}]$ \\
\hline DLCAN & DIAL816-06 & DIAL1413A01-IL & DIAL1413A01 & BOLD:AAA3782 & $612[1 \mathrm{n}]$ \\
\hline DLCAN & DIAL989-06 & DIAL1390G10-NC & DIAL1390G10 & BOLD:AAA3782 & $612[0 \mathrm{n}]$ \\
\hline DLCAN & DIAL382-06 & DIAL0294F04-VA & $\# 046370$ & BOLD:AAA3782 & $613[0 n]$ \\
\hline DLCAN & DIAL1043-06 & DIAL1391D06-NC & DIAL1391D06 & BOLD:AAA3782 & $614[0 n]$ \\
\hline DIAL & BWTWO879-10 & B03765B12-MD & B03765B12-MD & BOLD:AAA3782 & $614[0 n]$ \\
\hline DLII & DLII560-07 & DIAL1847H08-MD & DIAL1847H08 & BOLD:AAA3782 & $614[0 n]$ \\
\hline DIAL & DIAL1082-06 & DIAL1391G09-MI & DIAL1391G09 & BOLD:AAA3782 & $618[0 \mathrm{n}]$ \\
\hline DLCAN & DIAL905-06 & DIAL1413H08-NC & DIAL1413H08 & BOLD:AAA3782 & $620[0 \mathrm{n}]$ \\
\hline DLCAN & DIAL433-06 & DIAL0293C02-NC & perpunctatum 023 & BOLD:AAA3782 & $620[0 \mathrm{n}]$ \\
\hline DLCAN & DIAL417-06 & DIAL0292A08-VA & admirandum 0223 & BOLD:AAA3782 & $620[0 \mathrm{n}]$ \\
\hline DLCAN & DIAL819-06 & DIAL1413A04-IL & DIAL1413A04 & BOLD:AAA3782 & $624[0 n]$ \\
\hline DLCAN & DIAL821-06 & DIAL1413A06-IL & DIAL1413A06 & BOLD:AAA3782 & $624[0 \mathrm{n}]$ \\
\hline DLCAN & DIAL890-06 & DIAL1413G05-NC & DIAL1413G05 & BOLD:AAA3782 & $632[0 \mathrm{n}]$ \\
\hline DLCAN & DIAL1052-06 & DIAL1391E03-SC & DIAL1391E03 & BOLD:AAA3782 & $633[0 \mathrm{n}]$ \\
\hline DLCAN & DIAL834-06 & DIAL1413B08-IL & DIAL1413B08 & BOLD:AAA3782 & $637[0 \mathrm{n}]$ \\
\hline DLCAN & DLII434-07 & DIAL1846E12-SC & DIAL1846E12 & BOLD:AAA3782 & $637[0 \mathrm{n}]$ \\
\hline DLCAN & DLII388-07 & DIAL1846B01-TN & DIAL1846B01 & BOLD:AAA3782 & $645[0 n]$ \\
\hline DLCAN & DLII387-07 & DIAL1846A11-NC & DIAL1846A11 & BOLD:AAA3782 & $645[0 \mathrm{n}]$ \\
\hline DLCAN & DLII427-07 & DIAL1846E05-MS & DIAL1846E05 & BOLD:AAA3782 & $645[0 n]$ \\
\hline DLCAN & DIAL900-06 & DIAL1413H03-NC & DIAL1413H03 & BOLD:AAA3782 & $648[0 \mathrm{n}]$ \\
\hline DLCAN & DIAL841-06 & DIAL1413C04-TN & DIAL1413C04 & BOLD:AAA3782 & $649[0 \mathrm{n}]$ \\
\hline DLCAN & DIAL593-06 & D0287F02-IL & D0287F02 & BOLD:AAA3782 & $651[0 \mathrm{n}]$ \\
\hline DLCAN & DIAL875-06 & DIAL1413F02-TN & DIAL1413F02 & BOLD:AAA3782 & $651[0 \mathrm{n}]$ \\
\hline DLCAN & DIAL469-06 & D0288C08-DC & D0288C08 & BOLD:AAA3782 & $653[0 \mathrm{n}]$ \\
\hline DLCAN & DIAL845-06 & DIAL1413C08-TN & DIAL1413C08 & BOLD:AAA3782 & $655[0 \mathrm{n}]$ \\
\hline DLCAN & DIAL842-06 & DIAL1413C05-TN & DIAL1413C05 & BOLD:AAA3782 & $655[0 \mathrm{n}]$ \\
\hline DLCAN & DIAL828-06 & DIAL1413B02-VA & DIAL1413B02 & BOLD:AAA3782 & $655[0 \mathrm{n}]$ \\
\hline DLCAN & DIAL843-06 & DIAL1413C06-TN & DIAL1413C06 & BOLD:AAA3782 & $655[0 \mathrm{n}]$ \\
\hline DLCAN & DIAL1081-06 & DIAL1391G08-MI & DIAL1391G08 & BOLD:AAA3782 & $656[0 \mathrm{n}]$ \\
\hline DLCAN & DIAL848-06 & DIAL1413C11-NC & DIAL1413C11 & BOLD:AAA3782 & $657[0 \mathrm{n}]$ \\
\hline DLCAN & DIAL604-06 & D0287G01-IL & D0287G01 & BOLD:AAA3782 & $657[1 \mathrm{n}]$ \\
\hline DLCAN & DIAL835-06 & DIAL1413B09-IL & DIAL1413B09 & BOLD:AAA3782 & $657[0 \mathrm{n}]$ \\
\hline LPARA & DIAL838-06 & DIAL1413C01-IL & DIAL1413C01 & BOLD:AAA3782 & $658[0 \mathrm{n}]$ \\
\hline
\end{tabular}




\begin{tabular}{|c|c|c|c|c|c|c|}
\hline DLCAN & DIAL885-06 & DIAL1413F12-TN & DIAL1413F12 & BOLD:AAA3782 & & $658[0 n]$ \\
\hline DLCAN & DIAL854-06 & DIAL1413D05-SC & DIAL1413D05 & BOLD:AAA3782 & & $658[0 n]$ \\
\hline DLCAN & DIAL846-06 & DIAL1413C09-TN & DIAL1413C09 & BOLD:AAA3782 & & $658[0 n]$ \\
\hline DLCAN & DIAL607-06 & D0287G04-IL & D0287G04 & BOLD:AAA3782 & & $658[0 n]$ \\
\hline DLCAN & DIAL605-06 & D0287G02-IL & D0287G02 & BOLD:AAA3782 & & $658[0 n]$ \\
\hline DLCAN & DIAL611-06 & D0287G08-IL & D0287G08 & BOLD:AAA3782 & & $658[0 n]$ \\
\hline DLCAN & LASNA039-08 & DIAL2385D05-NC & DIAL2385D05-NC & BOLD:AAA3782 & & $658[0 n]$ \\
\hline DLCAN & DIAL839-06 & DIAL1413C02-TN & DIAL1413C02 & BOLD:AAA3782 & & $658[0 \mathrm{n}]$ \\
\hline DLCAN & DIAL840-06 & DIAL1413C03-TN & DIAL1413C03 & BOLD:AAA3782 & & $658[0 \mathrm{n}]$ \\
\hline DLCAN & DIAL610-06 & D0287G07-IL & D0287G07 & BOLD:AAA3782 & & $658[0 n]$ \\
\hline DLCAN & DIAL615-06 & D0287G12-IL & D0287G12 & BOLD:AAA3782 & & $658[0 n]$ \\
\hline DLCAN & DIAL624-06 & D0287H09-IL & D0287H09 & BOLD:AAA3782 & & $658[0 \mathrm{n}]$ \\
\hline DLCAN & DIAL818-06 & DIAL1413A03-IL & DIAL1413A03 & BOLD:AAA3782 & & $658[0 n]$ \\
\hline DLCAN & DIAL566-06 & D0287C11-IL & D0287C11 & BOLD:AAA3782 & & $658[0 n]$ \\
\hline DLCAN & DIAL564-06 & D0287C09-IL & D0287C09 & BOLD:AAA3782 & & $658[0 n]$ \\
\hline DLCAN & DIAL569-06 & D0287D02-MI & D0287D02 & BOLD:AAA3782 & & $658[0 \mathrm{n}]$ \\
\hline DLCAN & DIAL573-06 & D0287D06-IL & D0287D06 & BOLD:AAA3782 & & $658[0 n]$ \\
\hline DLII & BWTWO875-10 & B03765B08-MD & B03765B08-MD & BOLD:AAA3782 & & $658[0 \mathrm{n}]$ \\
\hline DIAL & DLII1266-08 & D-3253C08-NM & D-3253C08 & BOLD:AAB5530 & & $565[0 n]$ \\
\hline DIAL & DLII851-07 & DIAL2374A05-UT & DIAL2374A05 & BOLD:ACE7697 & BBSL418326 & $568[0 n]$ \\
\hline DIAL & DIAL1130-07 & DIAL2387C12-TX & DIAL2387C12 & BOLD:AAB5530 & & $599[3 n]$ \\
\hline DIAL & DLII874-07 & DIAL2374C06-UT & DIAL2374C06 & BOLD:AAB5530 & BBSL459644 & $614[5 n]$ \\
\hline DLCAN & DLII852-07 & DIAL2374A06-UT & DIAL2374A06 & BOLD:AAB5530 & BBSL364307 & $631[0 n]$ \\
\hline DLCAN & DLII785-07 & DIAL1849C11-CO & DIAL1849C11 & BOLD:AAB5530 & & $635[1 \mathrm{n}]$ \\
\hline DLCAN & DLII021-06 & DIAL1409B10-TX & DIAL1409B10 & BOLD:AAB5530 & & $645[0 n]$ \\
\hline DLCAN & DLII052-06 & DIAL1409E06-TX & DIAL1409E06 & BOLD:AAB5530 & & $645[0 n]$ \\
\hline DLCAN & DIAL1111-07 & DIAL2387B03-NM & IDIAL2387B03 & BOLD:AAB5530 & & $654[1 n]$ \\
\hline DLCAN & DLII1032-07 & DIAL2386H10-UT & DIAL2386H10 & BOLD:AAB5530 & ZION19521 & $655[0 n]$ \\
\hline DIAL & DLII1273-08 & D-3253D03-NM & D-3253D03 & BOLD:AAB5530 & & $656[2 n]$ \\
\hline LPARA & DLII912-07 & DIAL2374F08-UT & DIAL2374F08 & BOLD:AAB5530 & ZION23744 & $657[1 \mathrm{n}]$ \\
\hline DLCAN & DLII1079-07 & DIAL2388D11-NM & I DIAL2388D11-NM & 1 BOLD:AAB5530 & & $657[1 \mathrm{n}]$ \\
\hline DLCAN & DLII999-07 & DIAL2386F01-NV & DIAL2386F01 & BOLD:AAB5530 & BBSL671665 & $658[0 n]$ \\
\hline DLCAN & DLII1472-08 & D00608D11-NM & D00608D11 & BOLD:AAB5530 & & $658[0 n]$ \\
\hline DLCAN & DLII913-07 & DIAL2374F09-UT & DIAL2374F09 & BOLD:AAB5530 & ZION22507 & $658[0 n]$ \\
\hline DLCAN & DLII983-07 & DIAL2386D09-UT & DIAL2386D09 & BOLD:AAB5530 & ZION20631 & $658[0 \mathrm{n}]$ \\
\hline DIAL & DLII801-07 & DIAL1849E03-AZ & DIAL1849E03 & BOLD:ACE7697 & & $658[0 n]$ \\
\hline DIAL & DLII1083-07 & DIAL2388E03-NM & DIAL2388E03-NM & 1 BOLD:ABZ0512 & & $658[0 \mathrm{n}]$ \\
\hline DLCAN & DLII1056-07 & DIAL2388B11-UT & DIAL2388B11-UT & BOLD:AAE1411 & & $584[1 \mathrm{n}]$ \\
\hline DLCAN & LASNA844-08 & B-3252H08-NV & B-3252H08 & BOLD:AAE1411 & & $646[0 n]$ \\
\hline LPARA & DLII853-07 & DIAL2374A07-UT & DIAL2374A07 & BOLD:AAE1411 & BBSL460140 & $658[0 n]$ \\
\hline DLCAN & DLII1515-08 & D00608H06-NV & D00608H06 & BOLD:AAE1411 & & $658[0 n]$ \\
\hline DLCAN & DIAL877-06 & DIAL1413F04-TN & DIAL1413F04 & BOLD:AAD2971 & & $619[1 n]$ \\
\hline DLCAN & DIAL995-06 & DIAL1390H04-SC & DIAL1390H04 & BOLD:AAD2971 & & $636[0 n]$ \\
\hline DLCAN & DLII563-07 & DIAL1847H11-MD & DIAL1847H11 & BOLD:AAD2971 & & $645[0 n]$ \\
\hline DLCAN & DIAL734-06 & D1414B02-NC & D1414B02 & BOLD:AAD2971 & & $651[1 \mathrm{n}]$ \\
\hline LPARA & DIAL891-06 & DIAL1413G06-NC & DIAL1413G06 & BOLD:AAD2971 & & $657[0 n]$ \\
\hline DLCAN & DLII300-07 & DIAL1845B07-CA & DIAL1845B07 & BOLD:AAB0760 & & $637[0 \mathrm{n}]$ \\
\hline DLCAN & DLII288-07 & DIAL1845A06-CA & DIAL1845A06 & BOLD:AAB0760 & & $645[0 n]$ \\
\hline DLCAN & DLII294-07 & DIAL1845B01-CA & DIAL1845B01 & BOLD:AAB0760 & & $645[0 n]$ \\
\hline DLCAN & DLII306-07 & DIAL1845C02-CA & DIAL1845C02 & BOLD:AAB0760 & & $645[0 n]$ \\
\hline DLCAN & DLII1518-08 & D00608H09-OR & D00608H09 & BOLD:AAB0760 & & $649[0 n]$ \\
\hline LPARA & DLII1416-08 & D00607H02-CA & $\mathrm{D} 00607 \mathrm{H} 02$ & BOLD:AAB0760 & & $658[0 n]$ \\
\hline DLCAN & DLII1375-08 & D00607D09-CA & D00607D09 & BOLD:AAB0760 & & $658[0 n]$ \\
\hline DLCAN & DLII1400-08 & D00607F10-CA & D00607F10 & BOLD:AAB0760 & & $658[0 n]$ \\
\hline DLCAN & DIAL1403-09 & D01559D12-BC & D01559D12 & BOLD:AAB0760 & & $658[0 \mathrm{n}]$ \\
\hline
\end{tabular}




\begin{tabular}{|c|c|c|c|c|c|c|}
\hline DLCAN & DLII1303-08 & D-3253F09-KY & D-3253F09 & BOLD:AAA7868 & & $506[3 n]$ \\
\hline DLCAN & DIAL502-06 & D0288F05-SC & D0288F05 & BOLD:AAA7868 & & $581[1 \mathrm{n}]$ \\
\hline DLCAN & DIAL579-06 & D0287D12-IL & D0287D12 & BOLD:AAA7868 & & $591[1 n]$ \\
\hline DLCAN & DIAL599-06 & D0287F08-IL & D0287F08 & BOLD:AAA7868 & & $601[0 n]$ \\
\hline DLCAN & DLII458-07 & DIAL1846G12-SC & DIAL1846G12 & BOLD:AAA7868 & & $601[0 n]$ \\
\hline DLCAN & DIAL596-06 & D0287F05-IL & D0287F05 & BOLD:AAA7868 & & $601[0 n]$ \\
\hline DLCAN & DIAL898-06 & DIAL1413H01-NC & DIAL1413H01 & BOLD:AAA7868 & & $609[0 n]$ \\
\hline DLCAN & DIAL617-06 & D0287H02-IL & D0287H02 & BOLD:AAA7868 & & $614[0 n]$ \\
\hline DLCAN & DIAL1042-06 & DIAL1391D05-NC & DIAL1391D05 & BOLD:AAA7868 & & $616[1 \mathrm{n}]$ \\
\hline DLCAN & DLII019-06 & DIAL1409B08-VA & DIAL1409B08 & BOLD:AAA7868 & & $619[0 n]$ \\
\hline DIAL & DLII020-06 & DIAL1409B09-VA & DIAL1409B09 & BOLD:AAA7868 & & $619[0 n]$ \\
\hline DLCAN & DIAL847-06 & DIAL1413C10-NC & DIAL1413C10 & BOLD:AAA7868 & & $621[0 n]$ \\
\hline DLCAN & DIAL833-06 & DIAL1413B07-IL & DIAL1413B07 & BOLD:AAA7868 & & $623[0 n]$ \\
\hline DLCAN & DIAL851-06 & DIAL1413D02-TN & DIAL1413D02 & BOLD:AAA7868 & & $628[0 n]$ \\
\hline DLCAN & DIAL1055-06 & DIAL1391E06-SC & DIAL1391E06 & BOLD:AAA7868 & & $649[0 n]$ \\
\hline DLCAN & DIAL516-06 & D0288G07-NC & D0288G07 & BOLD:AAA7868 & & $649[0 n]$ \\
\hline DLCAN & DIAL887-06 & DIAL1413G02-TN & DIAL1413G02 & BOLD:AAA7868 & & $651[0 n]$ \\
\hline DLCAN & DIAL594-06 & D0287F03-IL & D0287F03 & BOLD:AAA7868 & & $653[0 n]$ \\
\hline DLCAN & DIAL987-06 & DIAL1390G08-NC & DIAL1390G08 & BOLD:AAA7868 & & $654[0 n]$ \\
\hline DLCAN & DIAL886-06 & DIAL1413G01-TN & DIAL1413G01 & BOLD:AAA7868 & & $655[0 n]$ \\
\hline DLCAN & DIAL561-06 & D0287C06-IL & D0287C06 & BOLD:AAA7868 & & $657[1 \mathrm{n}]$ \\
\hline LPARA & DIAL1069-06 & DIAL1391F08-ON & JIAL1391F08 & BOLD:AAA7868 & & $658[0 \mathrm{n}]$ \\
\hline DLCAN & DIAL903-06 & DIAL1413H06-NC & DIAL1413H06 & BOLD:AAA7868 & & $658[0 n]$ \\
\hline DLCAN & DIAL1063-06 & DIAL1391F02-NC & DIAL1391F02 & BOLD:AAA7868 & & $658[0 n]$ \\
\hline DLCAN & DIAL1016-06 & DIAL1391B02-SC & DIAL1391B02 & BOLD:AAA7868 & & $658[0 n]$ \\
\hline DLCAN & DLII832-07 & DIAL1849G10-MA & A DIAL1849G10 & BOLD:AAA7868 & & $658[0 n]$ \\
\hline DLCAN & DLII1246-08 & D-3253A10-BC & D-3253A10 & BOLD:AAB8298 & & $515[0 n]$ \\
\hline DLCAN & DLII1240-08 & D-3253A04-BC & D-3253A04 & BOLD:AAB8298 & & $576[0 n]$ \\
\hline DLCAN & DLII1048-07 & DIAL2388B03-UT & DIAL2388B03-UT & BOLD:AAB8298 & & $604[1 \mathrm{n}]$ \\
\hline DLCAN & DLII548-07 & DIAL1847G08-OR & RIAL1847G08 & BOLD:AAB8298 & & $643[0 n]$ \\
\hline DLCAN & DLII042-06 & DIAL1409D08-OR & २ DIAL1409D08 & BOLD:AAB8298 & & $645[0 \mathrm{n}]$ \\
\hline DLCAN & DLII549-07 & DIAL1847G09-OR & RIAL1847G09 & BOLD:AAB8298 & & $645[0 n]$ \\
\hline DLCAN & DLII551-07 & DIAL1847G11-OR & DIAL1847G11 & BOLD:AAB8298 & & $645[0 n]$ \\
\hline DLCAN & DLII1435-08 & D00608A10-CA & D00608A10 & BOLD:AAB8298 & & $645[0 n]$ \\
\hline DLCAN & DLII1034-07 & DIAL2386H12-UT & DIAL2386H12 & BOLD:AAB8298 & ZION20195 & $655[0 \mathrm{n}]$ \\
\hline LPARA & DLII1024-07 & DIAL2386H02-WA & $\triangle \mathrm{DIAL} 2386 \mathrm{H} 02$ & BOLD:AAB8298 & & $658[0 n]$ \\
\hline DLCAN & DLII1376-08 & D00607D10-CA & D00607D10 & BOLD:AAB8298 & & $658[0 \mathrm{n}]$ \\
\hline DLCAN & DLII1373-08 & D00607D07-CA & D00607D07 & BOLD:AAB8298 & & $658[0 n]$ \\
\hline DLCAN & DLII977-07 & DIAL2386D03-UT & DIAL2386D03 & BOLD:AAB8298 & ZION20813 & $658[0 n]$ \\
\hline DLCAN & DLII979-07 & DIAL2386D05-UT & DIAL2386D05 & BOLD:AAB8298 & ZION22265 & $658[0 n]$ \\
\hline DIAL & BWTWO1231-10 & 06717H07-MA & CCDB-06717 H07 & BOLD:ABZ7461 & & $525[1 \mathrm{n}]$ \\
\hline DIAL & LASNA043-08 & DIAL2385D09-WV & VIAL2385D09-WV & BOLD:ABZ7461 & & $600[0 n]$ \\
\hline DIAL & DLII1565-09 & D01560D09-IA & D01560D09 & BOLD:ABZ0652 & & $568[1 n]$ \\
\hline DIAL & BHTT190-09 & B03751H11-MA & B03751H11-MA & BOLD:ABZ0652 & & $575[0 n]$ \\
\hline DIAL & DLII1573-09 & D01560E05-IA & D01560E05 & BOLD:ABZ0652 & & $635[0 n]$ \\
\hline LPARA & BHTT016-09 & B03750B04-MA & В03750B04-MA & BOLD:ABZ0652 & & $658[0 n]$ \\
\hline DIAL & BOWGF1133-10 & B04743H04-MA & B04743H04-MA & BOLD:ABZ0652 & & $658[0 \mathrm{n}]$ \\
\hline DIAL & BWTWO1310-10 & 06735G03-MA & CCDB-06735G03 & BOLD:ABZ0652 & & $658[0 \mathrm{n}]$ \\
\hline DLCAN & DLII1254-08 & D-3253B07-BC & D-3253B07 & BOLD:AAA8348 & BBSL704328 & $526[0 n]$ \\
\hline DLCAN & DLII1243-08 & D-3253A07-BC & D-3253A07 & BOLD:AAA8348 & BBSL705735 & $560[1 \mathrm{n}]$ \\
\hline DIAL & DLII1252-08 & D-3253B05-BC & D-3253B05 & BOLD:AAA8348 & BBSL701926 & $569[5 n]$ \\
\hline DLCAN & DLII1260-08 & D-3253C02-BC & D-3253C02 & BOLD:AAA8348 & BBSL704151 & $574[0 n]$ \\
\hline DLCAN & DLII312-07 & DIAL1845C08-CA & DIAL1845C08 & BOLD:AAA8348 & & $576[0 n]$ \\
\hline DLCAN & DLII1256-08 & D-3253B09-BC & D-3253B09 & BOLD:AAA8348 & BBSL701194 & $577[0 \mathrm{n}]$ \\
\hline DLCAN & DLII311-07 & DIAL1845C07-CA & DIAL1845C07 & BOLD:AAA8348 & & $596[0 n]$ \\
\hline
\end{tabular}




\begin{tabular}{|c|c|c|c|c|c|c|}
\hline DLCAN & DIAL688-06 & D0295F03-CO & D0295F03 & BOLD:AAA8348 & & $596[0 n]$ \\
\hline DLCAN & DLII1239-08 & D-3253A03-BC & D-3253A03 & BOLD:AAA8348 & BBSL705988 & $617[2 n]$ \\
\hline DLCAN & DLII1467-08 & D00608D06-BC & D00608D06 & BOLD:AAA8348 & & $621[0 \mathrm{n}]$ \\
\hline DLCAN & DLII1481-08 & D00608E08-BC & D00608E08 & BOLD:AAA8348 & & $626[1 n]$ \\
\hline DLCAN & DLII1469-08 & D00608D08-BC & D00608D08 & BOLD:AAA8348 & & $632[0 \mathrm{n}]$ \\
\hline DLCAN & DLII1355-08 & D00607C01-CO & D00607C01 & BOLD:AAA8348 & & $632[0 n]$ \\
\hline DLCAN & DLII360-07 & DIAL1845G08-BC & DIAL1845G08 & BOLD:AAA8348 & & $641[0 n]$ \\
\hline DIAL & DLII898-07 & DIAL2374E06-WA & A DIAL2374E06 & BOLD:AAA8348 & BBSL696000 & $643[0 n]$ \\
\hline DLCAN & DLII032-06 & DIAL1409C10-OR & RIAL1409C10 & BOLD:AAA8348 & & $644[1 \mathrm{n}]$ \\
\hline DLCAN & DLII349-07 & DIAL1845F09-BC & DIAL1845F09 & BOLD:AAA8348 & & $645[0 n]$ \\
\hline DLCAN & DLII323-07 & DIAL1845D07-CA & DIAL1845D07 & BOLD:AAA8348 & & $645[0 n]$ \\
\hline DLCAN & DLII322-07 & DIAL1845D06-CA & DIAL1845D06 & BOLD:AAA8348 & & $645[0 n]$ \\
\hline DLCAN & DLII327-07 & DIAL1845D11-CA & DIAL1845D11 & BOLD:AAA8348 & & $645[0 n]$ \\
\hline DLCAN & DLII332-07 & DIAL1845E04-CA & DIAL1845E04 & BOLD:AAA8348 & & $645[0 n]$ \\
\hline DLCAN & DLII343-07 & DIAL1845F03-BC & DIAL1845F03 & BOLD:AAA8348 & & $645[0 \mathrm{n}]$ \\
\hline DLCAN & DLIII57-07 & DIAL1845G05-BC & DIAL1845G05 & BOLD:AAA8348 & & $645[0 \mathrm{n}]$ \\
\hline DIAL & BOWGF1888-10 & 03767G11-BC & 03767G11-BC & BOLD:AAA8348 & & $646[0 \mathrm{n}]$ \\
\hline DLCAN & DIAL1153-07 & DIAL2387E11-BC & DIAL2387E11 & BOLD:AAA8348 & & $652[0 n]$ \\
\hline DLCAN & DIAL1157-07 & DIAL2387F03-BC & DIAL2387F03 & BOLD:AAA8348 & & $653[0 \mathrm{n}]$ \\
\hline DLCAN & DIAL1102-07 & DIAL2387A05-ID & DIAL2387A05 & BOLD:AAA8348 & & $653[0 n]$ \\
\hline DLCAN & DLII945-07 & DIAL2386A05-CA & DIAL2386A05 & BOLD:AAA8348 & BBSL563254 & $657[0 \mathrm{n}]$ \\
\hline LPARA & DLII1471-08 & D00608D10-BC & D00608D10 & BOLD:AAA8348 & & $658[0 \mathrm{n}]$ \\
\hline DLCAN & DLII1022-07 & DIAL2386G12-ID & DIAL2386G12 & BOLD:AAA8348 & & $658[0 \mathrm{n}]$ \\
\hline DLCAN & DLII1458-08 & D00608C09-CO & D00608C09 & BOLD:AAA8348 & & $658[0 \mathrm{n}]$ \\
\hline DLCAN & DLII1451-08 & D00608C02-CO & D00608C02 & BOLD:AAA8348 & & $658[0 \mathrm{n}]$ \\
\hline DLCAN & DLII1489-08 & D00608F04-BC & D00608F04 & BOLD:AAA8348 & & $658[0 \mathrm{n}]$ \\
\hline DLCAN & DLIII004-09 & D01562A04-MT & D01562A04 & BOLD:AAA8348 & & $658[0 \mathrm{n}]$ \\
\hline DLCAN & DIAL715-06 & D0295H06-CO & D0295H06 & BOLD:AAA8348 & & $658[0 \mathrm{n}]$ \\
\hline DIAL & BWTWO913-10 & B03765E10-BC & B03765E10-BC & BOLD:AAA8348 & & $658[0 \mathrm{n}]$ \\
\hline DLCAN & DLII1259-08 & D-3253C01-BC & D-3253C01 & BOLD:AAA7513 & & $576[0 \mathrm{n}]$ \\
\hline DLCAN & LASNA054-08 & DIAL2385E08-MD & DIAL2385E08-MD & BOLD:AAA7513 & & $586[1 n]$ \\
\hline DLCAN & DLII752-07 & DIAL1848H12-PE & DIAL1848H12 & BOLD:AAA7513 & & $594[0 \mathrm{n}]$ \\
\hline DLCAN & DLII369-07 & DIAL1845H05-BC & DIAL1845H05 & BOLD:AAA7513 & & $595[0 \mathrm{n}]$ \\
\hline DLCAN & DLII1238-08 & D-3253A02-BC & D-3253A02 & BOLD:AAA7513 & & $599[1 \mathrm{n}]$ \\
\hline DLCAN & DIAL1338-08 & D00609G06-NS & D00609G06 & BOLD:AAA7513 & & $608[0 \mathrm{n}]$ \\
\hline DLCAN & DIAL383-06 & DIAL0294F05-MD & \#035032 & BOLD:AAA7513 & & $610[3 n]$ \\
\hline DLCAN & DIAL413-06 & DIAL0294H11-NB & 3 DIAL0294H11 & BOLD:AAA7513 & & $610[2 n]$ \\
\hline DLCAN & DLII1410-08 & D00607G08-BC & D00607G08 & BOLD:AAA7513 & & $613[0 \mathrm{n}]$ \\
\hline DLCAN & DLII384-07 & DIAL1846A08-NC & DIAL1846A08 & BOLD:AAA7513 & & $614[0 n]$ \\
\hline DLCAN & DLIII372-07 & DIAL1845H08-BC & DIAL1845H08 & BOLD:AAA7513 & & $619[0 \mathrm{n}]$ \\
\hline DLCAN & DLII348-07 & DIAL1845F08-BC & DIAL1845F08 & BOLD:AAA7513 & & $619[0 \mathrm{n}]$ \\
\hline DLCAN & DIAL1025-06 & DIAL1391B11-NC & DIAL1391B11 & BOLD:AAA7513 & & $621[0 n]$ \\
\hline DLCAN & DIAL1336-08 & D00609G04-NS & D00609G04 & BOLD:AAA7513 & & $630[0 \mathrm{n}]$ \\
\hline DLCAN & DLII697-07 & DIAL1848D05-NS & DIAL1848D05 & BOLD:AAA7513 & & $636[0 \mathrm{n}]$ \\
\hline DLCAN & DLII094-06 & DIAL1409H12-ON & N DIAL1409H12 & BOLD:AAA7513 & & $645[0 \mathrm{n}]$ \\
\hline DLCAN & DLII366-07 & DIAL1845H02-BC & DIAL1845H02 & BOLD:AAA7513 & & $645[0 \mathrm{n}]$ \\
\hline DLCAN & DLII353-07 & DIAL1845G01-BC & DIAL1845G01 & BOLD:AAA7513 & & $645[0 \mathrm{n}]$ \\
\hline DLCAN & DLII354-07 & DIAL1845G02-BC & DIAL1845G02 & BOLD:AAA7513 & & $645[0 \mathrm{n}]$ \\
\hline DLCAN & DLII344-07 & DIAL1845F04-BC & DIAL1845F04 & BOLD:AAA7513 & & $645[0 \mathrm{n}]$ \\
\hline DLCAN & DLII016-06 & DIAL1409B05-NC & DIAL1409B05 & BOLD:AAA7513 & & $645[0 \mathrm{n}]$ \\
\hline DLCAN & DLII346-07 & DIAL1845F06-BC & DIAL1845F06 & BOLD:AAA7513 & & $645[0 \mathrm{n}]$ \\
\hline DLCAN & DLII347-07 & DIAL1845F07-BC & DIAL1845F07 & BOLD:AAA7513 & & $645[0 \mathrm{n}]$ \\
\hline DLCAN & DIAL868-06 & DIAL1413E07-NC & DIAL1413E07 & BOLD:AAA7513 & & $655[0 \mathrm{n}]$ \\
\hline DLCAN & DLII725-07 & DIAL1848F09-PE & DIAL1848F09 & BOLD:AAA7513 & & $656[1 \mathrm{n}]$ \\
\hline DLCAN & DLII663-07 & DIAL1848A05-NB & DIAL1848A05 & BOLD:AAA7513 & & $657[0 \mathrm{n}]$ \\
\hline
\end{tabular}




\begin{tabular}{|c|c|c|c|c|c|}
\hline DLCAN & DLII664-07 & DIAL1848A06-NB & DIAL1848A06 & BOLD:AAA7513 & $657[0 \mathrm{n}]$ \\
\hline DLCAN & DLII734-07 & DIAL1848G06-PE & DIAL1848G06 & BOLD:AAA7513 & $657[0 \mathrm{n}]$ \\
\hline LPARA & DLII840-07 & DIAL1849H06-ME & DIAL1849H06 & BOLD:AAA7513 & $658[0 \mathrm{n}]$ \\
\hline DLCAN & DLII1073-07 & DIAL2388D05-CO & DIAL2388D05-CO & BOLD:AAA7513 & $658[0 \mathrm{n}]$ \\
\hline DLCAN & DLIII038-09 & D01562D02-ON & D01562D02 & BOLD:AAA7513 & $658[0 \mathrm{n}]$ \\
\hline DLCAN & DLII1335-08 & D00607A05-AB & D00607A05 & BOLD:AAA7513 & $658[0 \mathrm{n}]$ \\
\hline DLCAN & DLII1364-08 & D00607C10-AB & D00607C10 & BOLD:AAA7513 & $658[0 \mathrm{n}]$ \\
\hline DLCAN & DLII1363-08 & D00607C09-AB & D00607C09 & BOLD:AAA7513 & $658[0 \mathrm{n}]$ \\
\hline DLCAN & DIAL1010-06 & DIAL1391A07-NC & DIAL1391A07 & BOLD:AAA7513 & $658[0 \mathrm{n}]$ \\
\hline DLCAN & DIAL648-06 & D0295B10-ON & D0295B10 & BOLD:AAA7513 & $658[0 \mathrm{n}]$ \\
\hline DLCAN & DIAL650-06 & D0295C01-ON & D0295C01 & BOLD:AAA7513 & $658[0 \mathrm{n}]$ \\
\hline DLCAN & DIAL1027-06 & DIAL1391C02-NC & DIAL1391C02 & BOLD:AAA7513 & $658[0 \mathrm{n}]$ \\
\hline DLCAN & DIAL1017-06 & DIAL1391B03-OH & DIAL1391B03 & BOLD:AAA7513 & $658[0 \mathrm{n}]$ \\
\hline DLCAN & DIAL1026-06 & DIAL1391C01-NC & DIAL1391C01 & BOLD:AAA7513 & $658[0 \mathrm{n}]$ \\
\hline DLCAN & DLII1072-07 & DIAL2388D04-CO & DIAL2388D04-CO & BOLD:AAA7513 & $658[0 \mathrm{n}]$ \\
\hline DIAL & BOWGF1958-10 & 06745E10-ON & 06745E10-ON & BOLD:AAA7513 & $658[0 \mathrm{n}]$ \\
\hline LTEG & DIAL863-06 & DIAL1413E02-FL & DIAL1413E02 & BOLD:ABY5206 & $655[0 \mathrm{n}]$ \\
\hline LTEG & DIAL865-06 & DIAL1413E04-FL & DIAL1413E04 & BOLD:ABY5206 & $658[0 \mathrm{n}]$ \\
\hline LTEG & DIAL867-06 & DIAL1413E06-FL & DIAL1413E06 & BOLD:ABY5206 & $658[0 \mathrm{n}]$ \\
\hline DIAL & BOWGF1489-10 & 04485F04-MI & 04485F04-MI & BOLD:ACF2785 & $566[0 \mathrm{n}]$ \\
\hline DIAL & BOWGF1493-10 & 04485F08-MI & 04485F08-MI & BOLD:ACF2785 & $595[3 n]$ \\
\hline DIAL & BOWGF1481-10 & 04485E08-MI & 04485E08-MI & BOLD:ACF2785 & $619[0 \mathrm{n}]$ \\
\hline DLCAN & DLII681-07 & DIAL1848C01-NB & DIAL1848C01 & BOLD:ACE3786 & $620[0 \mathrm{n}]$ \\
\hline DIAL & BOWGF1494-10 & 04485F09-MI & 04485F09-MI & BOLD:ACF2785 & $620[0 \mathrm{n}]$ \\
\hline DIAL & BOWGF1496-10 & 04485F11-MI & 04485F11-MI & BOLD:ACF2785 & $620[0 n]$ \\
\hline DIAL & BOWGF1515-10 & 04485H06-MI & 04485H06-MI & BOLD:ACF2785 & $620[0 n]$ \\
\hline DLCAN & DLII380-07 & DIAL1846A04-ON & DIAL1846A04 & BOLD:ACE 9642 & $621[0 n]$ \\
\hline DIAL & BOWGF1513-10 & 04485H04-MI & 04485H04-MI & BOLD:ACF2785 & $630[0 \mathrm{n}]$ \\
\hline DLCAN & DLII680-07 & DIAL1848B11-NB & DIAL1848B11 & BOLD:ACE3786 & $657[0 \mathrm{n}]$ \\
\hline LPARA & DIAL1334-08 & D00609G02-NS & D00609G02 & BOLD:ACE9642 & $658[0 \mathrm{n}]$ \\
\hline DLCAN & DIAL1353-08 & D00609H09-NS & D00609H09 & BOLD:ACE3786 & $658[0 \mathrm{n}]$ \\
\hline DIAL & DLII331-07 & DIAL1845E03-TX & DIAL1845E03 & BOLD:AAA3782 & $595[1 \mathrm{n}]$ \\
\hline DIAL & DLII417-07 & DIAL1846D07-MS & DIAL1846D07 & BOLD:AAA3782 & $601[0 n]$ \\
\hline DLII & DLII445-07 & DIAL1846F11-MS & DIAL1846F11 & BOLD:AAA3782 & $619[1 \mathrm{n}]$ \\
\hline DIAL & DIAL1014-06 & DIAL1391A11-SC & DIAL1391A11 & BOLD:AAA3782 & $621[0 \mathrm{n}]$ \\
\hline DLCAN & LASNA053-08 & DIAL2385E07-WV & DIAL2385E07-WV & BOLD:AAA2141 & $549[3 n]$ \\
\hline DLCAN & DIAL148-06 & BEE0289B05-MN & BEE0289B05 & BOLD:AAA2141 & $598[0 \mathrm{n}]$ \\
\hline DLCAN & DIAL129-06 & DIAL0286H09-MB & DIAL0286H09 & BOLD:AAA2141 & $612[1 n]$ \\
\hline DLCAN & DIAL124-06 & DIAL0286H04-MB & DIAL0286H04 & BOLD:AAA2141 & $613[0 n]$ \\
\hline DLCAN & DIAL646-06 & D0295B08-ON & D0295B08 & BOLD:AAA2141 & $620[0 \mathrm{n}]$ \\
\hline DLCAN & DIAL943-06 & DIAL1390C12-ON & DIAL1390C12 & BOLD:AAA2141 & $625[0 n]$ \\
\hline DLCAN & DIAL016-06 & DIAL0292H06-WV & \#018478 & BOLD:AAA2141 & $625[0 \mathrm{n}]$ \\
\hline DLCAN & BEECB215-07 & DIAL1839G07-MN & DIAL1839G07-MN & BOLD:AAA2141 & $632[0 \mathrm{n}]$ \\
\hline DIAL & BWTW01073-10 & 06732C04-WV & 06732C04-WV & BOLD:AAA2141 & $634[0 \mathrm{n}]$ \\
\hline DIAL & DLII1349-08 & D00607B07-KS & D00607B07 & BOLD:AAA2141 & $641[0 \mathrm{n}]$ \\
\hline DLCAN & DLII012-06 & DIAL1409B01-NC & DIAL1409B01 & BOLD:AAA2141 & $645[0 \mathrm{n}]$ \\
\hline DLCAN & DIAL938-06 & DIAL1390C07-ON & DIAL1390C07 & BOLD:AAA2141 & $654[0 \mathrm{n}]$ \\
\hline DLCAN & DIAL935-06 & DIAL1390C04-ON & DIAL1390C04 & BOLD:AAA2141 & $654[0 n]$ \\
\hline DIAL & DIAL126-06 & DIAL0286H06-MB & DIAL0286H06 & BOLD:AAA2141 & $657[0 \mathrm{n}]$ \\
\hline DLCAN & DLII392-07 & DIAL1846B05-MD & DIAL1846B05 & BOLD:AAE5826 & $599[0 \mathrm{n}]$ \\
\hline LPARA & DLII564-07 & DIAL1847H12-MD & DIAL1847H12 & BOLD:AAE5826 & $635[0 \mathrm{n}]$ \\
\hline DLCAN & DLII1084-07 & DIAL2388E04-AL & DIAL2388E04-AL & BOLD:AAE5826 & $658[0 \mathrm{n}]$ \\
\hline DIAL & DLII462-07 & DIAL1846H04-MS & DIAL1846H04 & BOLD:AAC8294 & $613[0 n]$ \\
\hline DIAL & DLII424-07 & DIAL1846E02-MS & DIAL1846E02 & BOLD:AAC8294 & $623[0 \mathrm{n}]$ \\
\hline DIAL & DLII431-07 & DIAL1846E09-MS & DIAL1846E09 & BOLD:AAC8294 & $631[0 \mathrm{n}]$ \\
\hline
\end{tabular}




\begin{tabular}{|c|c|c|c|c|c|c|}
\hline DIAL & DLII428-07 & DIAL1846E06-MS & DIAL1846E06 & BOLD:AAC8294 & & $635[0 n]$ \\
\hline DIAL & DLII415-07 & DIAL1846D05-MS & DIAL1846D05 & BOLD:AAC8294 & & $645[0 n]$ \\
\hline DIAL & DIAL1160-07 & DIAL2387F06-TX & DIAL2387F06 & BOLD:AAC8294 & & $657[0 n]$ \\
\hline LPARA & DIAL505-06 & D0288F08-NC & D0288F08 & BOLD:AAC8294 & & $658[0 n]$ \\
\hline DIAL & DIAL058-06 & BEE0286B09-GA & BEE0286B09 & BOLD:AAC8294 & & $658[0 n]$ \\
\hline DIAL & DIAL507-06 & D0288F10-SC & D0288F10 & BOLD:AAC8294 & & $658[0 \mathrm{n}]$ \\
\hline LPARA & DIAL1406-09 & D01559E03-BC & D01559E03 & BOLD:AAE1153 & & $658[0 n]$ \\
\hline DLCAN & DIAL1421-09 & D01559F06-BC & D01559F06 & BOLD:AAE1153 & & $658[0 \mathrm{n}]$ \\
\hline DLCAN & DLII1447-08 & D00608B10-BC & D00608B10 & BOLD:ABZ0652 & & $621[0 n]$ \\
\hline DLCAN & DLII1465-08 & D00608D04-BC & D00608D04 & BOLD:ABZ0652 & & $633[0 n]$ \\
\hline DLCAN & DIAL1351-08 & D00609H07-BC & D00609H07 & BOLD:ABZ0652 & & $644[0 n]$ \\
\hline LPARA & DIAL1347-08 & D00609H03-BC & $\mathrm{D} 00609 \mathrm{H} 03$ & BOLD:ABZ0652 & & $658[0 \mathrm{n}]$ \\
\hline DLCAN & DIAL1349-08 & D00609H05-BC & D00609H05 & BOLD:ABZ0652 & & $658[0 n]$ \\
\hline DIAL & DLII1050-07 & DIAL2388B05-WA & A DIAL2388B05-WA & BOLD:ABZ0652 & & $658[0 n]$ \\
\hline DIAL & DLII1668-09 & D01561E05-CA & D01561E05 & BOLD:ABZ0652 & & $658[0 n]$ \\
\hline DIAL & BOWGF847-09 & CCDB - $01565 \mathrm{H} 3$ & 3 CCDB - 01565 H3 & 3 BOLD:ABZ0652 & & $658[0 \mathrm{n}]$ \\
\hline DIAL & BOWGF852-09 & CCDB - $01565 \mathrm{H} 8$ & 3 CCDB - $01565 \mathrm{H} 8$ & 3 BOLD:ABZ0652 & & $658[0 n]$ \\
\hline LTEG & DIAL746-06 & D1414C03-VA & D1414C03 & BOLD:AAD0611 & & $614[0 n]$ \\
\hline LTEG & DLII418-07 & DIAL1846D08-VA & DIAL1846D08 & BOLD:AAD0611 & & $645[0 n]$ \\
\hline LTEG & DLII685-07 & DIAL1848C05-MD & DIAL1848C05 & BOLD:AAD0611 & & $657[0 n]$ \\
\hline LTEG & DIAL763-06 & D1414D08-VA & D1414D08 & BOLD:AAD0611 & & $658[0 n]$ \\
\hline LTEG & DIAL749-06 & D1414C06-VA & D1414C06 & BOLD:AAD0611 & & $658[0 n]$ \\
\hline LTEG & DIAL748-06 & D1414C05-VA & D1414C05 & BOLD:AAD0611 & & $658[0 n]$ \\
\hline LTEG & DIAL747-06 & D1414C04-VA & D1414C04 & BOLD:AAD0611 & & $658[0 n]$ \\
\hline DIAL & DLII1671-09 & D01561E08-NY & D01561E08 & BOLD:AAD0611 & & $658[0 n]$ \\
\hline DLCAN & DLII815-07 & DIAL1849F05-DC & DIAL1849F05 & BOLD:ACE8520 & & $572[0 n]$ \\
\hline LPARA & DLII013-06 & DIAL1409B02-NC & DIAL1409B02 & BOLD:ACE8520 & & $645[0 n]$ \\
\hline DLCAN & DLII001-06 & DIAL1409A01-ON & NIAL1409A01 & BOLD:ACE8520 & & $645[0 n]$ \\
\hline DIAL & DLII1294-08 & D-3253E12-FL & D-3253E12 & BOLD:AAD9325 & & $574[1 \mathrm{n}]$ \\
\hline DIAL & DLII1284-08 & D-3253E02-FL & D-3253E02 & BOLD:AAD9325 & & $575[1 n]$ \\
\hline DIAL & DIAL1358-09 & D01559A03-FL & D01559A03 & BOLD:AAD9325 & & $655[3 n]$ \\
\hline DIAL & DLII1707-09 & D01561H08-FL & D01561H08 & BOLD:AAD9325 & & $657[1 \mathrm{n}]$ \\
\hline DIAL & DIAL1400-09 & D01559D09-FL & D01559D09 & BOLD:AAD9325 & & $657[1 \mathrm{n}]$ \\
\hline LPARA & DLIII039-09 & D01562D03-FL & D01562D03 & BOLD:AAD9325 & & $658[0 \mathrm{n}]$ \\
\hline DLCAN & DLII1248-08 & D-3253B01-BC & D-3253B01 & BOLD:AAB0372 & & $558[3 n]$ \\
\hline DLCAN & DLII1241-08 & D-3253A05-BC & D-3253A05 & BOLD:AAB0372 & & $567[1 n]$ \\
\hline DLCAN & DLII1257-08 & D-3253B10-BC & D-3253B10 & BOLD:AAB0372 & & $596[3 n]$ \\
\hline DLCAN & DLII1258-08 & D-3253B11-BC & D-3253B11 & BOLD:AAB0372 & & $597[1 \mathrm{n}]$ \\
\hline DLCAN & DLII1255-08 & D-3253B08-BC & D-3253B08 & BOLD:AAB0372 & & $598[0 n]$ \\
\hline DLCAN & ASBEE072-08 & D-3262G02-UT & D-3262G02-UT & BOLD:AAB0372 & & $601[0 n]$ \\
\hline DLCAN & DLII1244-08 & D-3253A08-BC & D-3253A08 & BOLD:AAB0372 & & $617[0 \mathrm{n}]$ \\
\hline DLCAN & DLII316-07 & DIAL1845C12-CA & DIAL1845C12 & BOLD:AAB0372 & & $628[1 n]$ \\
\hline DLCAN & DLII326-07 & DIAL1845D10-CA & DIAL1845D10 & BOLD:AAB0372 & & $636[0 n]$ \\
\hline DLCAN & DIAL1154-07 & DIAL2387E12-BC & DIAL2387E12 & BOLD:AAB0372 & & $654[1 \mathrm{n}]$ \\
\hline DLCAN & DLII1397-08 & D00607F07-CA & D00607F07 & BOLD:AAB0372 & & $656[1 \mathrm{n}]$ \\
\hline DLCAN & DLII626-07 & BEE1850F04-OR & BEE1850F04 & BOLD:AAB0372 & & $656[1 \mathrm{n}]$ \\
\hline DLCAN & DLII1412-08 & D00607G10-BC & D00607G10 & BOLD:AAB0372 & & $657[0 \mathrm{n}]$ \\
\hline DLCAN & DLII965-07 & DIAL2386C03-CA & DIAL2386C03 & BOLD:AAB0372 & BBSL566129 & $657[1 \mathrm{n}]$ \\
\hline LPARA & DLII857-07 & DIAL2374A11-UT & DIAL2374A11 & BOLD:AAB0372 & BBSL370394 & $658[0 n]$ \\
\hline DLCAN & DLII982-07 & DIAL2386D08-UT & DIAL2386D08 & BOLD:AAB0372 & ZION19814 & $658[0 n]$ \\
\hline DLCAN & DLII980-07 & DIAL2386D06-UT & DIAL2386D06 & BOLD:AAB0372 & ZION121776 & $658[0 n]$ \\
\hline DLCAN & DLII966-07 & DIAL2386C04-CA & DIAL2386C04 & BOLD:AAB0372 & BBSL564884 & $658[0 n]$ \\
\hline DLCAN & DIAL1415-09 & D01559E12-BC & D01559E12 & BOLD:AAB0372 & & $658[0 n]$ \\
\hline DLCAN & DLII889-07 & DIAL2374D09-UT & DIAL2374D09 & BOLD:AAB0372 & BBSL437336 & $658[0 n]$ \\
\hline DLCAN & DLII903-07 & DIAL2374E11-WA & A DIAL2374E11 & BOLD:AAB0372 & BBSL696899 & $658[0 \mathrm{n}]$ \\
\hline
\end{tabular}




\begin{tabular}{|c|c|c|c|c|c|}
\hline DIAL & BWTWO1106-10 & 06732F01-WY & 06732F01-WY & BOLD:AAB0372 & $658[0 \mathrm{n}]$ \\
\hline DLCAN & LASNA023-08 & DIAL2385C01-MD & DIAL2385C01-MD & BOLD:AAD3968 & $575[1 n]$ \\
\hline DLCAN & DLII823-07 & DIAL1849G01-ME & DIAL1849G01 & BOLD:AAD3968 & $598[0 n]$ \\
\hline DIAL & BOWGF1433-10 & 04485A08-MD & 04485A08-MD & BOLD:AAD3968 & $623[0 \mathrm{n}]$ \\
\hline DLCAN & DIAL1340-08 & D00609G08-NS & D00609G08 & BOLD:AAD3968 & $628[0 \mathrm{n}]$ \\
\hline DLCAN & DLII822-07 & DIAL1849F12-MA & DIAL1849F12 & BOLD:AAD3968 & $630[0 \mathrm{n}]$ \\
\hline LPARA & DLII719-07 & DIAL1848F03-ON & JIAL1848F03 & BOLD:AAD3968 & $657[0 \mathrm{n}]$ \\
\hline DLCAN & DLII1543-09 & D01560B11-VT & D01560B11 & BOLD:AAD3968 & $658[0 \mathrm{n}]$ \\
\hline DLCAN & DLII721-07 & DIAL1848F05-PE & DIAL1848F05 & BOLD:ABZ0652 & $609[1 \mathrm{n}]$ \\
\hline DIAL & DIAL460-06 & D0288B10-NC & D0288B10 & BOLD:AAB6637 & $584[0 n]$ \\
\hline DIAL & DIAL753-06 & D1414C10-VA & D1414C10 & BOLD:AAB6637 & $603[0 \mathrm{n}]$ \\
\hline DIAL & DIAL752-06 & D1414C09-VA & D1414C09 & BOLD:AAB6637 & $603[0 \mathrm{n}]$ \\
\hline DIAL & DLII1305-08 & D-3253F11-FL & D-3253F11 & BOLD:AAB6637 & $611[0 n]$ \\
\hline DIAL & DIAL781-06 & D1414F02-NC & D1414F02 & BOLD:AAB6637 & $616[0 \mathrm{n}]$ \\
\hline DIAL & DIAL779-06 & D1414E12-NC & D1414E12 & BOLD:AAB6637 & $618[0 n]$ \\
\hline DIAL & DIAL778-06 & D1414E11-NC & D1414E11 & BOLD:AAB6637 & $618[0 n]$ \\
\hline DIAL & DIAL464-06 & D0288C03-SC & D0288C03 & BOLD:AAB6637 & $620[0 n]$ \\
\hline DIAL & DIAL751-06 & D1414C08-VA & D1414C08 & BOLD:AAB6637 & $627[0 \mathrm{n}]$ \\
\hline DIAL & DIAL461-06 & D0288B11-NC & D0288B11 & BOLD:AAB6637 & $637[0 \mathrm{n}]$ \\
\hline DIAL & DLII421-07 & DIAL1846D11-MS & DIAL1846D11 & BOLD:AAB6637 & $645[0 \mathrm{n}]$ \\
\hline LPARA & DLII813-07 & DIAL1849F03-NC & DIAL1849F03 & BOLD:AAB6637 & $658[0 \mathrm{n}]$ \\
\hline DIAL & DIAL764-06 & D1414D09-VA & D1414D09 & BOLD:AAB6637 & $658[0 \mathrm{n}]$ \\
\hline DIAL & BWTWO870-10 & B03765B03-VA & B03765B03-VA & BOLD:AAB6637 & $658[0 \mathrm{n}]$ \\
\hline DIAL & DIAL472-06 & D0288C11-SC & D0288C11 & BOLD:AAB6637 & $658[0 \mathrm{n}]$ \\
\hline DIAL & DIAL784-06 & D1414F05-NC & D1414F05 & BOLD:AAB6637 & $658[0 \mathrm{n}]$ \\
\hline DIAL & DIAL750-06 & D1414C07-VA & D1414C07 & BOLD:AAB6637 & $658[0 \mathrm{n}]$ \\
\hline DIAL & DIAL673-06 & D0295D12-MD & D0295D12 & BOLD:ABZ0968 & $595[5 n]$ \\
\hline DLCAN & DIAL738-06 & D1414B06-VA & D1414B06 & BOLD:ABZ0968 & $602[0 n]$ \\
\hline DLCAN & DIAL017-06 & DIAL0293B05-MD & \#011917 & BOLD:ABZ0968 & $610[1 \mathrm{n}]$ \\
\hline DLCAN & DIAL739-06 & D1414B07-VA & D1414B07 & BOLD:ABZ0968 & $611[0 \mathrm{n}]$ \\
\hline DLCAN & DLII1378-08 & D00607D12-MA & D00607D12 & BOLD:ABZ0968 & $612[3 n]$ \\
\hline DLCAN & DLII1497-08 & D00608F12-IN & D00608F12 & BOLD:ABZ0968 & $622[2 n]$ \\
\hline DLCAN & DIAL740-06 & D1414B08-VA & D1414B08 & BOLD:ABZ0968 & $626[0 \mathrm{n}]$ \\
\hline LPARA & DIAL768-06 & D1414E01-VA & D1414E01 & BOLD:ABZ0968 & $658[0 \mathrm{n}]$ \\
\hline DLCAN & DIAL741-06 & D1414B09-VA & D1414B09 & BOLD:ABZ0968 & $658[0 \mathrm{n}]$ \\
\hline DLCAN & DLII824-07 & DIAL1849G02-MA & A DIAL1849G02 & BOLD:ABZ0968 & $658[0 \mathrm{n}]$ \\
\hline DLCAN & DIAL873-06 & DIAL1413E12-TN & I DIAL1413E12 & BOLD:ABZ0652 & $527[0 n]$ \\
\hline DIAL & DIAL389-06 & DIAL0294F11-VA & $\# 048070$ & BOLD:ABZ0652 & $547[4 n]$ \\
\hline DLCAN & DLII484-07 & DIAL1847B03-WV & DIAL1847B03 & BOLD:ABZ0652 & $577[0 \mathrm{n}]$ \\
\hline DLCAN & DLII691-07 & DIAL1848C11-NY & DIAL1848C11 & BOLD:ABZ0652 & $591[1 n]$ \\
\hline DIAL & DIAL679-06 & D0295E06-MD & D0295E06 & BOLD:ABZ0652 & $595[5 n]$ \\
\hline DLCAN & DLII395-07 & DIAL1846B08-WV & DIAL1846B08 & BOLD:ABZ0652 & $599[0 \mathrm{n}]$ \\
\hline DLCAN & DIAL081-06 & BEE0286D09-VA & BEE0286D09 & BOLD:ABZ0652 & $606[0 \mathrm{n}]$ \\
\hline DLCAN & DIAL1007-06 & DIAL1391A04-NC & DIAL1391A04 & BOLD:ABZ0652 & $613[0 \mathrm{n}]$ \\
\hline LPARA & DIAL990-06 & DIAL1390G11-NC & DIAL1390G11 & BOLD:ABZ0652 & $654[0 \mathrm{n}]$ \\
\hline DLCAN & DLII842-07 & DIAL1849H08-VT & DIAL1849H08 & BOLD:ABZ0652 & $658[0 \mathrm{n}]$ \\
\hline DLCAN & LASNA014-08 & DIAL2385B03-MB & 3 DIAL2385B03-MB & BOLD:AAC7155 & $628[1 \mathrm{n}]$ \\
\hline DLCAN & DIAL1163-07 & DIAL2387F09-NM & 1 DIAL2387F09 & BOLD:AAC7155 & $654[1 \mathrm{n}]$ \\
\hline DLCAN & DIAL1175-07 & DIAL2387H11-NM & I DIAL2387H11 & BOLD:AAC7155 & $657[1 \mathrm{n}]$ \\
\hline DLCAN & DIAL544-06 & D0287A11-CO & D0287A11 & BOLD:AAC7155 & $657[0 \mathrm{n}]$ \\
\hline LPARA & LASNA010-08 & DIAL2385A10-MB & 3 DIAL2385A10-MB & BOLD:AAC7155 & $658[0 \mathrm{n}]$ \\
\hline DLCAN & DIAL702-06 & D0295G05-CO & D0295G05 & BOLD:AAC7155 & $658[0 \mathrm{n}]$ \\
\hline DLCAN & LASNA004-08 & DIAL2385A04-CO & DIAL2385A04-CO & BOLD:AAC7155 & $658[0 \mathrm{n}]$ \\
\hline DLCAN & DIAL555-06 & D0287B11-CO & D0287B11 & BOLD:AAC7155 & $658[0 \mathrm{n}]$ \\
\hline DIAL & DLII1628-09 & D01561B01-MT & D01561B01 & BOLD:AAC7155 & $658[0 \mathrm{n}]$ \\
\hline
\end{tabular}




\begin{tabular}{|c|c|c|c|c|c|}
\hline DIAL & BWTWO1114-10 & 06732F09-WY & 06732F09-WY & BOLD:AAC7155 & $658[0 \mathrm{n}]$ \\
\hline DIAL & DIAL707-06 & D0295G10-CO & D0295G10 & BOLD:AAC7155 & $658[0 \mathrm{n}]$ \\
\hline DLCAN & DIAL940-06 & DIAL1390C09-ON & N DIAL1390C09 & BOLD:AAE1244 & $617[0 \mathrm{n}]$ \\
\hline DLCAN & DLII831-07 & DIAL1849G09-MA & A DIAL1849G09 & BOLD:AAE1244 & $636[0 \mathrm{n}]$ \\
\hline LPARA & DLII006-06 & DIAL1409A06-NC & DIAL1409A06 & BOLD:AAE1244 & $645[0 n]$ \\
\hline DLCAN & DLII011-06 & DIAL1409A11-NC & DIAL1409A11 & BOLD:AAE1244 & $645[0 \mathrm{n}]$ \\
\hline DLCAN & DLII358-07 & DIAL1845G06-BC & DIAL1845G06 & BOLD:ABZ0652 & $602[0 \mathrm{n}]$ \\
\hline DLCAN & DLII374-07 & DIAL1845H10-BC & DIAL $1845 \mathrm{H} 10$ & BOLD:ABZ0652 & $619[0 \mathrm{n}]$ \\
\hline DLCAN & DLIII356-07 & DIAL1845G04-BC & DIAL1845G04 & BOLD:ABZ0652 & $621[0 \mathrm{n}]$ \\
\hline DLCAN & DLIII345-07 & DIAL1845F05-BC & DIAL1845F05 & BOLD:ABZ0652 & $632[0 n]$ \\
\hline DLCAN & DLII367-07 & DIAL1845H03-BC & DIAL1845H03 & BOLD:ABZ0652 & $632[0 \mathrm{n}]$ \\
\hline DLCAN & DLII375-07 & DIAL1845H11-BC & DIAL1845H11 & BOLD:ABZ0652 & $637[0 \mathrm{n}]$ \\
\hline DLCAN & DLII1503-08 & D00608G06-BC & D00608G06 & BOLD:ABZ0652 & $638[0 \mathrm{n}]$ \\
\hline DLCAN & DLII376-07 & DIAL1845H12-BC & DIAL1845H12 & BOLD:ABZ0652 & $643[0 n]$ \\
\hline DLCAN & DLII355-07 & DIAL1845G03-BC & DIAL1845G03 & BOLD:ABZ0652 & $644[0 \mathrm{n}]$ \\
\hline DLCAN & DLIII59-07 & DIAL1845G07-BC & DIAL1845G07 & BOLD:ABZ0652 & $645[0 \mathrm{n}]$ \\
\hline DLCAN & DLII364-07 & DIAL1845G12-BC & DIAL1845G12 & BOLD:ABZ0652 & $645[0 \mathrm{n}]$ \\
\hline DLCAN & DLII352-07 & DIAL1845F12-BC & DIAL1845F12 & BOLD:ABZ0652 & $645[0 \mathrm{n}]$ \\
\hline DLCAN & DLII350-07 & DIAL1845F10-BC & DIAL1845F10 & BOLD:ABZ0652 & $645[0 \mathrm{n}]$ \\
\hline DLCAN & DLII342-07 & DIAL1845F02-BC & DIAL1845F02 & BOLD:ABZ0652 & $645[0 \mathrm{n}]$ \\
\hline DLCAN & DLII361-07 & DIAL1845G09-BC & DIAL1845G09 & BOLD:ABZ0652 & $645[0 n]$ \\
\hline DLCAN & DLII362-07 & DIAL1845G10-BC & DIAL1845G10 & BOLD:ABZ0652 & $645[0 n]$ \\
\hline DLCAN & DLII363-07 & DIAL1845G11-BC & DIAL1845G11 & BOLD:ABZ0652 & $645[0 \mathrm{n}]$ \\
\hline DLCAN & DIAL1169-07 & DIAL2387H05-CO & DIAL2387H05 & BOLD:ABZ0652 & $658[0 \mathrm{n}]$ \\
\hline DIAL & BWTWO908-10 & B03765E05-BC & B03765E05-BC & BOLD:ABZ0652 & $658[0 \mathrm{n}]$ \\
\hline DIAL & BOWGF473-09 & CCDB-00611 H09 & 9 CCDB-00611 H09 & BOLD:ABZ0652 & $658[0 \mathrm{n}]$ \\
\hline DLCAN & DIAL1395-09 & D01559D04-AB & D01559D04 & BOLD:AAE5057 & $634[0 \mathrm{n}]$ \\
\hline LPARA & LASNA008-08 & DIAL2385A08-SK & DIAL2385A08-SK & BOLD:AAE5057 & $658[0 \mathrm{n}]$ \\
\hline DLCAN & DIAL1428-09 & D01559G01-AB & D01559G01 & BOLD:AAE5057 & $658[0 \mathrm{n}]$ \\
\hline DLCAN & LASNA013-08 & DIAL2385B02-SK & DIAL2385B02-SK & BOLD:AAE5057 & $658[0 \mathrm{n}]$ \\
\hline DLCAN & DIAL037-06 & DIAL0293B11-WV & N032687 & BOLD:ABZ0652 & $620[0 n]$ \\
\hline DLCAN & DIAL817-06 & DIAL1413A02-IL & DIAL1413A02 & BOLD:ABZ0652 & $625[0 \mathrm{n}]$ \\
\hline DLCAN & DLII008-06 & DIAL1409A08-NC & DIAL1409A08 & BOLD:ABZ0652 & $645[0 n]$ \\
\hline DLCAN & DIAL964-06 & DIAL1390E09-ON & J DIAL1390E09 & BOLD:ABZ0652 & $654[0 \mathrm{n}]$ \\
\hline DLCAN & DIAL831-06 & DIAL1413B05-IL & DIAL1413B05 & BOLD:ABZ0652 & $655[0 \mathrm{n}]$ \\
\hline LPARA & DIAL1287-08 & D00609C03-WI & D00609C03 & BOLD:ABZ0652 & $657[0 \mathrm{n}]$ \\
\hline DLCAN & DLII1553-09 & D01560C09-IA & D01560C09 & BOLD:ABZ0652 & $658[0 \mathrm{n}]$ \\
\hline DIAL & BOWGF583-09 & CCDB-01557 B01 & I CCDB-01557 B01 & BOLD:ABZ0652 & $658[0 \mathrm{n}]$ \\
\hline DLCAN & DIAL944-06 & DIAL1390D01-NT & DIAL1390D01 & BOLD:AAF4051 & $653[1 n]$ \\
\hline DLCAN & DIAL941-06 & DIAL1390C10-NT & DIAL1390C10 & BOLD:AAF4051 & $654[0 \mathrm{n}]$ \\
\hline DLCAN & DLII1148-08 & DIAL2384B09-YT & DIAL2384B09 & BOLD:AAF4051 & $658[0 \mathrm{n}]$ \\
\hline DLCAN & DIAL695-06 & D0295F10-CO & D0295F10 & BOLD:AAC1122 & $575[1 \mathrm{n}]$ \\
\hline DLCAN & DIAL113-06 & DIAL0286G05-MB & 3 DIAL0286G05 & BOLD:AAC1122 & $613[0 \mathrm{n}]$ \\
\hline DLCAN & DIAL114-06 & DIAL0286G06-MB & 3 DIAL0286G06 & BOLD:AAC1122 & $613[0 \mathrm{n}]$ \\
\hline DLCAN & DIAL431-06 & DIAL0293B09-IN & perpunctatum \#12 & BOLD:AAC1122 & $624[1 \mathrm{n}]$ \\
\hline DLCAN & DIAL1354-08 & D00609H10-NS & D00609H10 & BOLD:AAC1122 & $632[0 \mathrm{n}]$ \\
\hline LPARA & DIAL115-06 & DIAL0286G07-MB & 3 DIAL0286G07 & BOLD:AAC1122 & $658[0 \mathrm{n}]$ \\
\hline DLCAN & DIAL111-06 & DIAL0286G03-MB & 3 DIAL0286G03 & BOLD:AAC1122 & $658[0 \mathrm{n}]$ \\
\hline DLCAN & DLII1629-09 & D01561B02-MT & D01561B02 & BOLD:AAC1122 & $658[0 \mathrm{n}]$ \\
\hline DLCAN & DIAL536-06 & D0287A03-CO & D0287A03 & BOLD:AAC1122 & $658[0 \mathrm{n}]$ \\
\hline DLCAN & DIAL118-06 & DIAL0286G10-MB & 3 DIAL0286G10 & BOLD:AAC1122 & $658[0 \mathrm{n}]$ \\
\hline DLCAN & DIAL117-06 & DIAL0286G09-MB & 3 DIAL0286G09 & BOLD:AAC1122 & $658[0 \mathrm{n}]$ \\
\hline DLCAN & DIAL112-06 & DIAL0286G04-MB & 3 DIAL0286G04 & BOLD:AAC1122 & $658[0 \mathrm{n}]$ \\
\hline DLCAN & DIAL123-06 & DIAL0286H03-MB & 3 DIAL0286H03 & BOLD:AAC1122 & $658[0 \mathrm{n}]$ \\
\hline DLCAN & DIAL434-06 & DIAL0293C07-MN & $\checkmark$ pictum \#03 & BOLD:AAC1104 & $553[0 \mathrm{n}]$ \\
\hline
\end{tabular}




\begin{tabular}{|c|c|c|c|c|c|c|}
\hline DLCAN & DIAL435-06 & DIAL0293C08-MN & pictum \#04 & BOLD:AAC1104 & $553[0 \mathrm{n}]$ & 0 \\
\hline DLCAN & DIAL021-06 & DIAL0293C06-MN & |\#02 & BOLD:AAC1104 & $562[0 \mathrm{n}]$ & 0 \\
\hline DLCAN & DIAL343-06 & DIAL0294C01-IN & \#15765 & BOLD:AAC1104 & $613[0 \mathrm{n}]$ & 0 \\
\hline DLCAN & DIAL020-06 & DIAL0293C05-MN & \#01 & BOLD:AAC1104 & $616[1 \mathrm{n}]$ & 0 \\
\hline LPARA & DIAL103-06 & DIAL0286F07-MB & DIAL0286F07 & BOLD:AAC1104 & $658[0 n]$ & 2 \\
\hline DLCAN & DIAL104-06 & DIAL0286F08-MB & DIAL0286F08 & BOLD:AAC1104 & $658[0 \mathrm{n}]$ & 2 \\
\hline DLCAN & DIAL102-06 & DIAL0286F06-MB & DIAL0286F06 & BOLD:AAC1104 & $658[0 \mathrm{n}]$ & 2 \\
\hline DLCAN & LASNA022-08 & DIAL2385B11-MI & DIAL2385B11-MI & BOLD:AAC1104 & $658[0 \mathrm{n}]$ & 4 \\
\hline DLCAN & DIAL1281-08 & D00609B09-WI & D00609B09 & BOLD:AAB6361 & $577[0 n]$ & 2 \\
\hline DLCAN & DIAL767-06 & D1414D12-VA & D1414D12 & BOLD:AAB6361 & $579[0 n]$ & 2 \\
\hline DLCAN & DIAL1039-06 & DIAL1391D02-NC & DIAL1391D02 & BOLD:AAB6361 & $608[0 \mathrm{n}]$ & 1 \\
\hline DIAL & DIAL390-06 & DIAL0294F12-NY & \#046092 & BOLD:AAB6361 & $609[3 n]$ & 0 \\
\hline DLCAN & DIAL925-06 & DIAL1390B05-ON & DIAL1390B05 & BOLD:AAB6361 & $612[0 \mathrm{n}]$ & 1 \\
\hline DIAL & BOWGF1509-10 & 04485G12-MI & 04485G12-MI & BOLD:AAB6361 & $612[2 n]$ & 2 \\
\hline DLCAN & DLIII382-07 & DIAL1846A06-ON & DIAL1846A06 & BOLD:AAB6361 & $616[0 \mathrm{n}]$ & 2 \\
\hline DLCAN & DLII558-07 & DIAL1847H06-MD & DIAL1847H06 & BOLD:AAB6361 & $623[0 \mathrm{n}]$ & 2 \\
\hline DLCAN & DIAL1004-06 & DIAL1391A01-VA & DIAL1391A01 & BOLD:AAB6361 & $624[0 \mathrm{n}]$ & 2 \\
\hline DLCAN & DIAL844-06 & DIAL1413C07-TN & DIAL1413C07 & BOLD:AAB6361 & $624[0 \mathrm{n}]$ & 2 \\
\hline DIAL & BOWGF1486-10 & 04485F01-MI & 04485F01-MI & BOLD:AAB6361 & $628[3 n]$ & 2 \\
\hline DLCAN & DLII005-06 & DIAL1409A05-NC & DIAL1409A05 & BOLD:AAB6361 & $633[0 n]$ & 2 \\
\hline DLCAN & DIAL823-06 & DIAL1413A08-VA & DIAL1413A08 & BOLD:AAB6361 & $640[0 \mathrm{n}]$ & 2 \\
\hline DLCAN & DIAL996-06 & DIAL1390H05-NC & DIAL1390H05 & BOLD:AAB6361 & $643[0 n]$ & 2 \\
\hline DLCAN & DIAL878-06 & DIAL1413F05-TN & DIAL1413F05 & BOLD:AAB6361 & $645[0 \mathrm{n}]$ & 2 \\
\hline DLCAN & DIAL852-06 & DIAL1413D03-NC & DIAL1413D03 & BOLD:AAB6361 & $649[1 \mathrm{n}]$ & 2 \\
\hline DLCAN & DIAL830-06 & DIAL1413B04-ON & DIAL1413B04 & BOLD:AAB6361 & $653[0 n]$ & 2 \\
\hline DIAL & DLII628-07 & BEE1850F06-MN & BEE1850F06 & BOLD:AAB6361 & $655[2 n]$ & 2 \\
\hline LPARA & DIAL1037-06 & DIAL1391C12-NC & DIAL1391C12 & BOLD:AAB6361 & $657[1 \mathrm{n}]$ & 2 \\
\hline DLCAN & DLII1408-08 & D00607G06-BC & D00607G06 & BOLD:ABZ0652 & $600[0 n]$ & 2 \\
\hline DLCAN & DIAL412-06 & DIAL0294H10-NB & DIAL0294H10 & BOLD:ABZ0652 & $613[0 \mathrm{n}]$ & 0 \\
\hline DLCAN & DLII660-07 & DIAL1848A02-NB & DIAL1848A02 & BOLD:ABZ0652 & $655[2 n]$ & 2 \\
\hline DLCAN & DLII730-07 & DIAL1848G02-NB & DIAL1848G02 & BOLD:ABZ0652 & $657[0 \mathrm{n}]$ & 2 \\
\hline DLCAN & DLII659-07 & DIAL1848A01-NB & DIAL1848A01 & BOLD:ABZ0652 & $657[0 \mathrm{n}]$ & 2 \\
\hline DLCAN & DLII1087-07 & DIAL2388E07-NB & DIAL2388E07-NB & BOLD:ABZ0652 & $658[0 \mathrm{n}]$ & 2 \\
\hline DLCAN & DIAL1066-06 & DIAL1391F05-NT & DIAL1391F05 & BOLD:ABZ0652 & $658[0 \mathrm{n}]$ & 2 \\
\hline DIAL & BOWGF450-09 & CCDB-00611 F10. & CCDB-00611 F10 & BOLD:ABZ0652 & $658[0 \mathrm{n}]$ & 1 \\
\hline DLCAN & DLII1567-09 & D01560D11-DC & D01560D11 & BOLD:ACE6636 & $522[0 n]$ & 2 \\
\hline DLCAN & LASNA033-08 & DIAL2385C11-DC & DIAL2385C11-DC & BOLD:ACE6636 & $582[2 \mathrm{n}]$ & 4 \\
\hline DLCAN & DIAL857-06 & DIAL1413D08-NC & DIAL1413D08 & BOLD:ACE6636 & $614[0 \mathrm{n}]$ & 2 \\
\hline DLCAN & DIAL023-06 & DIAL0293D07-WV & BBSL429788 & BOLD:ACE6636 & $620[0 n]$ & 0 \\
\hline DLCAN & DIAL022-06 & DIAL0293D03-MD & \#028159 & BOLD:ACE6636 & $620[0 n]$ & 0 \\
\hline DLCAN & DLII836-07 & DIAL1849H02-DC & DIAL1849H02 & BOLD:ACE6636 & $641[0 \mathrm{n}]$ & 2 \\
\hline DLCAN & DLII561-07 & DIAL1847H09-MD & IDIAL1847H09 & BOLD:ACE6636 & $645[0 \mathrm{n}]$ & 2 \\
\hline LPARA & DLII713-07 & DIAL1848E09-MD & DIAL1848E09 & BOLD:ACE6636 & $657[0 \mathrm{n}]$ & 2 \\
\hline DLCAN & DLII1534-09 & D01560B02-DC & D01560B02 & BOLD:ACE6636 & $658[0 \mathrm{n}]$ & 2 \\
\hline DLCAN & DLII1537-09 & D01560B05-DC & D01560B05 & BOLD:ACE6636 & $658[0 \mathrm{n}]$ & 3 \\
\hline DLCAN & DLII818-07 & DIAL1849F08-MD & DIAL1849F08 & BOLD:ACE6636 & $658[0 n]$ & 2 \\
\hline DLCAN & DIAL1311-08 & D00609E03-MT & D00609E03 & BOLD:AAB3612 & $550[0 n]$ & 2 \\
\hline DLCAN & DIAL710-06 & D0295H01-CO & D0295H01 & BOLD:AAB3612 & $600[0 \mathrm{n}]$ & 2 \\
\hline DLCAN & DIAL1416-09 & D01559F01-AB & D01559F01 & BOLD:AAB3612 & $606[0 \mathrm{n}]$ & 2 \\
\hline DLCAN & DLII1019-07 & DIAL2386G09-WA & DIAL2386G09 & BOLD:AAB3612 & $641[0 n]$ & 2 \\
\hline DLCAN & DIAL700-06 & D0295G03-CO & D0295G03 & BOLD:AAB3612 & $654[0 \mathrm{n}]$ & 2 \\
\hline DLCAN & DLII1155-08 & DIAL2384C05-AB & DIAL2384C05 & BOLD:AAB3612 & $657[1 \mathrm{n}]$ & 2 \\
\hline DLCAN & DLII717-07 & DIAL1848F01-CO & DIAL1848F01 & BOLD:AAB3612 & $657[0 \mathrm{n}]$ & 2 \\
\hline LPARA & DIAL1389-09 & D01559C10-AB & D01559C10 & BOLD:AAB3612 & $658[0 \mathrm{n}]$ & 2 \\
\hline DLCAN & DLII1026-07 & DIAL2386H04-WA & DIAL2386H04 & BOLD:AAB3612 & $658[0 \mathrm{n}]$ & 2 \\
\hline
\end{tabular}




\begin{tabular}{|c|c|c|c|c|c|}
\hline DLCAN & DIAL698-06 & D0295G01-CO & D0295G01 & BOLD:AAB3612 & $658[0 n]$ \\
\hline DLCAN & DIAL1445-09 & D01559H06-BC & D01559H06 & BOLD:AAB3612 & $658[0 n]$ \\
\hline DLCAN & DIAL557-06 & D0287C02-CO & D0287C02 & BOLD:AAB3612 & $658[0 n]$ \\
\hline DLCAN & DIAL1433-09 & D01559G06-BC & D01559G06 & BOLD:AAB3612 & $658[0 n]$ \\
\hline DLCAN & DIAL1448-09 & D01559H09-BC & D01559H09 & BOLD:AAB3612 & $658[0 n]$ \\
\hline DLCAN & DIAL1366-09 & D01559A11-MT & D01559A11 & BOLD:AAB3612 & $658[0 n]$ \\
\hline DLCAN & DLII1156-08 & DIAL2384C06-AB & DIAL2384C06 & BOLD:AAB3612 & $658[0 n]$ \\
\hline DLCAN & DLII1101-07 & DIAL2388F09-UT & DIAL2388F09-UT & BOLD:AAB3612 & $658[0 \mathrm{n}]$ \\
\hline DLCAN & DIAL223-06 & BEE0289H09-MB & BEE0289H09 & BOLD:AAC1101 & $596[2 n]$ \\
\hline DLCAN & DIAL221-06 & BEE0289H07-MB & BEE0289H07 & BOLD:AAC1101 & $598[1 n]$ \\
\hline DLCAN & DLII085-06 & DIAL1409H03-MB & 3 DIAL1409H03 & BOLD:AAC1101 & $604[1 n]$ \\
\hline DLCAN & DIAL220-06 & BEE0289H06-MB & BEE0289H06 & BOLD:AAC1101 & $609[0 \mathrm{n}]$ \\
\hline DLCAN & DIAL224-06 & BEE0289H10-MB & BEE0289H10 & BOLD:AAC1101 & $613[0 n]$ \\
\hline DLCAN & DLII1116-07 & DIAL2388G12-AZ & DIAL2388G12-AZ & BOLD:AAC1101 & $623[1 n]$ \\
\hline LPARA & DLII918-07 & DIAL2374G02-UT & DIAL2374G02 & BOLD:AAC1101 ZION21740 & $658[0 n]$ \\
\hline DLCAN & DLII862-07 & DIAL2374B05-UT & DIAL2374B05 & BOLD:AAC1101 BBSL428648 & $658[0 \mathrm{n}]$ \\
\hline DLCAN & DLII301-07 & DIAL1845B08-CA & DIAL1845B08 & BOLD:AAD9279 & $624[0 n]$ \\
\hline DLCAN & DLII1456-08 & D00608C07-CA & D00608C07 & BOLD:AAD9279 & $636[0 n]$ \\
\hline DLCAN & DLII295-07 & DIAL1845B02-CA & DIAL1845B02 & BOLD:AAD9279 & $637[0 n]$ \\
\hline DLCAN & DIAL1104-07 & DIAL2387A07-ID & DIAL2387A07 & BOLD:AAD9279 & $654[1 \mathrm{n}]$ \\
\hline DIAL & BWTWO930-10 & B03765G03-BC & B03765G03-BC & BOLD:AAD9279 & $658[0 n]$ \\
\hline LTEG & DLII696-07 & DIAL1848D04-FL & DIAL1848D04 & BOLD:AAD7708 & $611[0 n]$ \\
\hline LTEG & DIAL027-06 & DIAL0293E07-FL & \#032210 & BOLD:AAD7708 & $614[0 n]$ \\
\hline LTEG & DIAL029-06 & DIAL0293E09-FL & $\# 032211$ & BOLD:AAD7708 & $615[0 n]$ \\
\hline LTEG & DIAL028-06 & DIAL0293E08-FL & $\# 032175$ & BOLD:AAD7708 & $615[0 n]$ \\
\hline LTEG & DLII465-07 & DIAL1846H07-SC & DIAL1846H07 & BOLD:AAD7708 & $619[0 n]$ \\
\hline LTEG & DLII474-07 & DIAL1847A04-FL & DIAL1847A04 & BOLD:AAD7708 & $619[0 n]$ \\
\hline LTEG & DIAL866-06 & DIAL1413E05-FL & DIAL1413E05 & BOLD:AAD7708 & $622[0 n]$ \\
\hline DIAL & DLII398-07 & DIAL1846B11-MS & DIAL1846B11 & BOLD:AAD7708 & $634[0 \mathrm{n}]$ \\
\hline LTEG & DLII472-07 & DIAL1847A02-FL & DIAL1847A02 & BOLD:AAD7708 & $635[0 n]$ \\
\hline LTEG & DIAL496-06 & D0288E11-SC & D0288E11 & BOLD:AAD7708 & $644[0 \mathrm{n}]$ \\
\hline LTEG & DLII435-07 & DIAL1846F01-SC & DIAL1846F01 & BOLD:AAD7708 & $645[0 n]$ \\
\hline DIAL & DLII396-07 & DIAL1846B09-MS & DIAL1846B09 & BOLD:AAD7708 & $645[0 n]$ \\
\hline DIAL & DLII411-07 & DIAL1846D01-MS & DIAL1846D01 & BOLD:AAD7708 & $645[0 n]$ \\
\hline LTEG & DIAL514-06 & D0288G05-FL & D0288G05 & BOLD:AAD7708 & $657[1 \mathrm{n}]$ \\
\hline LTEG & DIAL513-06 & D0288G04-FL & D0288G04 & BOLD:AAD7708 & $657[1 \mathrm{n}]$ \\
\hline LTEG & DIAL501-06 & D0288F04-SC & D0288F04 & BOLD:AAD7708 & $657[1 \mathrm{n}]$ \\
\hline LTEG & DIAL487-06 & D0288E02-NC & D0288E02 & BOLD:AAD7708 & $657[1 \mathrm{n}]$ \\
\hline LTEG & DIAL473-06 & D0288C12-FL & D0288C12 & BOLD:AAD7708 & $657[1 \mathrm{n}]$ \\
\hline LTEG & DIAL498-06 & D0288F01-SC & D0288F01 & BOLD:AAD7708 & $658[0 n]$ \\
\hline DIAL & BWTWO856-10 & B03765A01-GA & B03765A01-GA & BOLD:AAD7708 & $658[0 n]$ \\
\hline LPARA & DIAL1375-09 & D01559B08-GA & D01559B08 & BOLD:AAE1191 & $658[0 n]$ \\
\hline DIAL & DIAL1390-09 & D01559C11-GA & D01559C11 & BOLD:AAE1191 & $658[0 n]$ \\
\hline DIAL & DIAL1404-09 & D01559E01-GA & D01559E01 & BOLD:AAE1191 & $658[0 n]$ \\
\hline LPARA & DLII413-07 & DIAL1846D03-MS & DIAL1846D03 & BOLD:AAC7048 & $645[0 n]$ \\
\hline DLCAN & DLII401-07 & DIAL1846C03-MS & DIAL1846C03 & BOLD:AAD7869 & $645[0 n]$ \\
\hline DIAL & DLII1664-09 & D01561E01-MA & D01561E01 & BOLD:AAD7869 & $653[5 n]$ \\
\hline LPARA & DLII700-07 & DIAL1848D08-MD & DIAL1848D08 & BOLD:AAD7869 & $657[0 \mathrm{n}]$ \\
\hline DLCAN & DLII698-07 & DIAL1848D06-WV & DIAL1848D06 & BOLD:AAD7869 & $657[0 n]$ \\
\hline DLCAN & LASNA027-08 & DIAL2385C05-MD & DIAL2385C05-MD & BOLD:AAD7869 & $658[0 n]$ \\
\hline DLCAN & DLII820-07 & DIAL1849F10-MD & DIAL1849F10 & BOLD:AAD7869 & $658[0 n]$ \\
\hline DIAL & DLII1140-08 & DIAL2384B01-YT & DIAL2384B01 & BOLD:AAE5185 & $642[1 \mathrm{n}]$ \\
\hline DIAL & DLII1138-08 & DIAL2384A10-YT & DIAL2384A10 & BOLD:AAE5185 & $658[0 n]$ \\
\hline DIAL & DLII1142-08 & DIAL2384B03-YT & DIAL2384B03 & BOLD:AAE5185 & $658[0 n]$ \\
\hline CNBEE & DLII1136-08 & DIAL2384A08-YT & DIAL2384A08 & BOLD:AAE5185 & $658[0 n]$ \\
\hline
\end{tabular}




\begin{tabular}{|c|c|c|c|c|c|c|}
\hline DIAL & BWTWO126-09 & B01558C07-AB & CCDB-01558C7 & BOLD:ACE3475 & & $558[4 n]$ \\
\hline DLCAN & DIAL1123-07 & DIAL2387C04-NM & A DIAL2387C04 & BOLD:ACE3475 & & $580[0 n]$ \\
\hline DLCAN & DIAL1274-08 & D00609B02-MT & D00609B02 & BOLD:AAA5895 & & $603[2 \mathrm{n}]$ \\
\hline DLCAN & DLIII001-09 & D01562A01-MT & D01562A01 & BOLD:AAA5895 & & $626[0 \mathrm{n}]$ \\
\hline DLCAN & DLII919-07 & DIAL2374G03-NV & / DIAL2374G03 & BOLD:ACE3476 & BBSL577864 & $636[0 \mathrm{n}]$ \\
\hline DIAL & BOWGF1881-10 & 03767G04-BC & 03767G04-BC & BOLD:AAA5895 & & $636[0 \mathrm{n}]$ \\
\hline DIAL & BOWGF466-09 & CCDB-00611 H02 & 2 CCDB-00611 H02 & BOLD:AAA5895 & & $645[0 \mathrm{n}]$ \\
\hline DIAL & DIAL716-06 & D0295H07-CO & D0295H07 & BOLD:AAA5893 & & $648[6 n]$ \\
\hline DIAL & BOWGF571-09 & CCDB-01557 A01 & CCDB-01557 A01 & BOLD:AAA5893 & & $655[2 \mathrm{n}]$ \\
\hline DLCAN & DLII1153-08 & DIAL2384C03-AB & 3 DIAL2384C03 & BOLD:ACE3475 & & $657[1 \mathrm{n}]$ \\
\hline DLCAN & DIAL712-06 & D0295H03-CO & D0295H03 & BOLD:AAA5893 & & $657[1 \mathrm{n}]$ \\
\hline DLCAN & DIAL1439-09 & D01559G12-BC & D01559G12 & BOLD:AAA5895 & & $658[0 \mathrm{n}]$ \\
\hline DLCAN & DLII1417-08 & D00607H03-SK & D00607H03 & BOLD:AAA5893 & & $658[0 \mathrm{n}]$ \\
\hline DLCAN & DLII1013-07 & DIAL2386G03-BC & DIAL2386G03 & BOLD:AAA5895 & & $658[0 \mathrm{n}]$ \\
\hline DLCAN & DLII864-07 & DIAL2374B07-UT & DIAL2374B07 & BOLD:AAA5894 & BBSL475705 & $658[0 \mathrm{n}]$ \\
\hline DLCAN & DLII1063-07 & DIAL2388C07-NV & / DIAL2388C07-NV & BOLD:ACE3476 & & $658[0 \mathrm{n}]$ \\
\hline DLCAN & DLII1670-09 & D01561E07-SK & D01561E07 & BOLD:AAA5893 & & $658[0 \mathrm{n}]$ \\
\hline DLCAN & DLII1665-09 & D01561E02-SK & D01561E02 & BOLD:AAA5893 & & $658[0 \mathrm{n}]$ \\
\hline DLCAN & DLIII048-09 & D01562D12-BC & D01562D12 & BOLD:AAA5895 & & $658[0 \mathrm{n}]$ \\
\hline DLCAN & DIAL1363-09 & D01559A08-AB & D01559A08 & BOLD:AAA5893 & & $658[0 \mathrm{n}]$ \\
\hline DIAL & BOWGF1573-10 & 04486E05-WA & 04486E05-WA & BOLD:AAA5895 & & $658[0 \mathrm{n}]$ \\
\hline DIAL & BOWGF475-09 & CCDB-00611 H11 & 1 CCDB-00611 H11 & BOLD:AAA5893 & & $658[0 \mathrm{n}]$ \\
\hline DIAL & BOWGF1883-10 & 03767G06-BC & 03767G06-BC & BOLD:AAA5895 & & $658[0 \mathrm{n}]$ \\
\hline DIAL & BWTWO909-10 & B03765E06-BC & B03765E06-BC & BOLD:AAA5895 & & $658[0 \mathrm{n}]$ \\
\hline CNBEE & BOWGF574-09 & CCDB-01557 A04 & 4 CCDB-01557 A04 & BOLD:AAA5893 & & $658[0 \mathrm{n}]$ \\
\hline DIAL & BWTWO1105-10 & 06732E12-WY & 06732E12-WY & BOLD:AAA5895 & & $658[0 \mathrm{n}]$ \\
\hline DIAL & DLII948-07 & DIAL2386A08-NV & DIAL2386A08 & BOLD:ACE3476 & BBSL530045 & $658[0 \mathrm{n}]$ \\
\hline DLCAN & DLIII046-09 & D01562D10-ON & D01562D10 & BOLD:ABZ0652 & & $530[1 n]$ \\
\hline DIAL & DIAL130-06 & DIAL0286H10-MB & 3 DIAL0286H10 & BOLD:ABZ0652 & & $597[4 n]$ \\
\hline DLCAN & DIAL315-06 & DIAL0291H07-MB & 3 DIAL0291H07 & BOLD:ABZ0652 & & $598[0 \mathrm{n}]$ \\
\hline DLCAN & DIAL313-06 & DIAL0291H05-MB & 3 DIAL0291H05 & BOLD:ABZ0652 & & $598[0 n]$ \\
\hline DLCAN & DIAL314-06 & DIAL0291H06-MB & 3 DIAL0291H06 & BOLD:ABZ0652 & & $601[0 n]$ \\
\hline DLCAN & DIAL131-06 & DIAL0286H11-MB & 3 DIAL0286H11 & BOLD:ABZ0652 & & $603[1 n]$ \\
\hline DLCAN & DIAL127-06 & DIAL0286H07-MB & 3 DIAL0286H07 & BOLD:ABZ0652 & & $604[0 n]$ \\
\hline DLCAN & DIAL128-06 & DIAL0286H08-MB & 3 DIAL0286H08 & BOLD:ABZ0652 & & $611[0 \mathrm{n}]$ \\
\hline DIAL & BWTWO927-10 & B03765F12-BC & B03765F12-BC & BOLD:ABZ0652 & & $614[0 \mathrm{n}]$ \\
\hline DLCAN & DLII086-06 & DIAL1409H04-MB & 3 DIAL1409H04 & BOLD:ABZ0652 & & $617[0 \mathrm{n}]$ \\
\hline DLCAN & DLII084-06 & DIAL1409H02-MB & 3 DIAL1409H02 & BOLD:ABZ0652 & & $645[0 n]$ \\
\hline DIAL & BOWGF579-09 & CCDB-01557 A09 & CCDB-01557 A09 & BOLD:ABZ0652 & & $654[3 n]$ \\
\hline DIAL & DLIII028-09 & D01562C04-MB & D01562C04 & BOLD:ABZ0652 & & $654[4 n]$ \\
\hline DLCAN & DLII1530-09 & D01560A10-WI & D01560A10 & BOLD:ABZ0652 & & $658[0 \mathrm{n}]$ \\
\hline DLCAN & DIAL316-06 & DIAL0291H08-MB & 3 DIAL0291H08 & BOLD:ABZ0652 & & $658[0 \mathrm{n}]$ \\
\hline DLCAN & DLIII050-09 & D01562E02-MB & D01562E02 & BOLD:ABZ0652 & & $658[0 \mathrm{n}]$ \\
\hline DLCAN & DLIII033-09 & D01562C09-ON & D01562C09 & BOLD:ABZ0652 & & $658[0 \mathrm{n}]$ \\
\hline DIAL & BOWGF577-09 & CCDB-01557 A07. & CCDB-01557 A07 & BOLD:ABZ0652 & & $658[0 \mathrm{n}]$ \\
\hline DIAL & BWTWO924-10 & B03765F09-BC & B03765F09-BC & BOLD:ABZ0652 & & $658[0 \mathrm{n}]$ \\
\hline DIAL & BOWGF580-09 & CCDB-01557 A10 & CCDB-01557 A10 & BOLD:ABZ0652 & & $658[0 \mathrm{n}]$ \\
\hline DIAL & BOWGF581-09 & CCDB-01557 A11 & CCDB-01557 A11 & BOLD:ABZ0652 & & $658[0 \mathrm{n}]$ \\
\hline DIAL & BWTWO921-10 & B03765F06-BC & B03765F06-BC & BOLD:ABZ0652 & & $658[0 \mathrm{n}]$ \\
\hline DLII & DLII1491-08 & D00608F06-BC & D00608F06 & BOLD:ACF5350 & & $634[1 \mathrm{n}]$ \\
\hline LPARA & BHTT184-09 & B03751H05-BC & B03751H05-BC & BOLD:ACF5350 & & $658[0 \mathrm{n}]$ \\
\hline DLCAN & DIAL1411-09 & D01559E08-BC & D01559E08 & BOLD:ACF5350 & & $658[0 \mathrm{n}]$ \\
\hline DLCAN & DLII1390-08 & D00607E12-UT & D00607E12 & BOLD:ACF5350 & & $658[0 \mathrm{n}]$ \\
\hline DLCAN & DLII1474-08 & D00608E01-BC & D00608E01 & BOLD:ACF5350 & & $658[0 \mathrm{n}]$ \\
\hline DLCAN & DLII1681-09 & D01561F06-BC & D01561F06 & BOLD:ACF5350 & & $658[0 \mathrm{n}]$ \\
\hline
\end{tabular}




\begin{tabular}{|c|c|c|c|c|c|c|}
\hline DIAL & BWTWO915-10 & B03765E12-BC & B03765E12-BC & BOLD:ACF5350 & & $658[0 \mathrm{n}]$ \\
\hline DLCAN & DIAL686-06 & D0295F01-CO & D0295F01 & BOLD:ABZ3764 & & $574[0 n]$ \\
\hline DLCAN & DLII897-07 & DIAL2374E05-WA & A DIAL2374E05 & BOLD:ABZ3764 & BBSL696917 & $599[0 n]$ \\
\hline DIAL & BOWGF468-09 & CCDB-00611 H04 & 4 CCDB-00611 H04 & BOLD:ABZ3764 & & $611[0 \mathrm{n}]$ \\
\hline DLCAN & DIAL1436-09 & D01559G09-BC & D01559G09 & BOLD:ABZ3764 & & $632[0 \mathrm{n}]$ \\
\hline DLCAN & DIAL708-06 & D0295G11-CO & D0295G11 & BOLD:ABZ3764 & & $651[0 n]$ \\
\hline DLCAN & DIAL1122-07 & DIAL2387C03-ID & DIAL2387C03 & BOLD:ABZ3764 & & $653[2 n]$ \\
\hline LPARA & DIAL689-06 & D0295F04-CO & D0295F04 & BOLD:ABZ3764 & & $658[0 \mathrm{n}]$ \\
\hline DLCAN & DLII896-07 & DIAL2374E04-WA & A DIAL2374E04 & BOLD:ABZ3764 & BBSL696048 & $658[0 \mathrm{n}]$ \\
\hline DLCAN & DIAL1378-09 & D01559B11-BC & D01559B11 & BOLD:ABZ3764 & & $658[0 \mathrm{n}]$ \\
\hline DLCAN & DIAL1382-09 & D01559C03-BC & D01559C03 & BOLD:ABZ3764 & & $658[0 \mathrm{n}]$ \\
\hline DLCAN & DIAL720-06 & D0295H11-CO & D0295H11 & BOLD:ABZ3764 & & $658[0 \mathrm{n}]$ \\
\hline DLCAN & DIAL556-06 & D0287C01-CO & D0287C01 & BOLD:ABZ3764 & & $658[0 \mathrm{n}]$ \\
\hline DLCAN & DIAL545-06 & D0287B01-CO & D0287B01 & BOLD:ABZ3764 & & $658[0 \mathrm{n}]$ \\
\hline DLCAN & DLIII010-09 & D01562A10-MT & D01562A10 & BOLD:ABZ3764 & & $658[0 \mathrm{n}]$ \\
\hline DLCAN & DIAL703-06 & D0295G06-CO & D0295G06 & BOLD:ABZ3764 & & $658[0 \mathrm{n}]$ \\
\hline DIAL & BOWGF467-09 & CCDB-00611 H03 & 3 CCDB-00611 H03 & BOLD:ABZ3764 & & $658[0 \mathrm{n}]$ \\
\hline DIAL & DLII1080-07 & DIAL2388D12-NM & A DIAL2388D12-NM & 1 BOLD:AAB1546 & & $555[3 n]$ \\
\hline DIAL & DIAL1260-08 & DIAL0293H09-AZ & DIAL0293H09 & BOLD:AAB1546 & & $582[1 \mathrm{n}]$ \\
\hline DLCAN & ASBEE065-08 & D-3262F07-NM & D-3262F07-NM & BOLD:AAB1546 & & $601[0 n]$ \\
\hline DLCAN & DIAL704-06 & D0295G07-CO & D0295G07 & BOLD:AAB1546 & & $607[2 n]$ \\
\hline DLCAN & DLII684-07 & DIAL1848C04-MN & V DIAL1848C04 & BOLD:AAB1546 & & $619[0 n]$ \\
\hline DLCAN & DLII718-07 & DIAL1848F02-MN & J DIAL1848F02 & BOLD:AAB1546 & & $619[1 \mathrm{n}]$ \\
\hline DLCAN & DLII674-07 & DIAL1848B05-AZ & DIAL1848B05 & BOLD:AAB1546 & & $633[0 n]$ \\
\hline DIAL & DLII768-07 & DIAL1849B05-AZ & DIAL1849B05 & BOLD:AAB1546 & & $644[4 n]$ \\
\hline DLCAN & DLII531-07 & DIAL1847F03-TX & DIAL1847F03 & BOLD:AAB1546 & & $645[0 \mathrm{n}]$ \\
\hline DLCAN & DLII808-07 & DIAL1849E10-AZ & DIAL1849E10 & BOLD:AAB1546 & & $655[0 \mathrm{n}]$ \\
\hline DLCAN & DIAL539-06 & D0287A06-CO & D0287A06 & BOLD:AAB1546 & & $656[2 n]$ \\
\hline LPARA & DLII916-07 & DIAL2374F12-UT & DIAL2374F12 & BOLD:AAB1546 & ZION21139 & $657[0 \mathrm{n}]$ \\
\hline DLCAN & DIAL699-06 & D0295G02-CO & D0295G02 & BOLD:AAB1546 & & $657[1 \mathrm{n}]$ \\
\hline DLCAN & DLII800-07 & DIAL1849E02-CO & DIAL1849E02 & BOLD:AAB1546 & & $657[1 \mathrm{n}]$ \\
\hline DLCAN & DLII670-07 & DIAL1848B01-AZ & DIAL1848B01 & BOLD:AAB1546 & & $657[0 \mathrm{n}]$ \\
\hline DLCAN & DLII683-07 & DIAL1848C03-MN & N DIAL1848C03 & BOLD:AAB1546 & & $657[0 \mathrm{n}]$ \\
\hline DLCAN & DLII1630-09 & D01561B03-CA & D01561B03 & BOLD:AAB1546 & & $658[0 \mathrm{n}]$ \\
\hline DLCAN & DLII953-07 & DIAL2386B02-NV & / DIAL2386B02 & BOLD:AAB1546 & BBSL575216 & $658[0 \mathrm{n}]$ \\
\hline DLCAN & DLII917-07 & DIAL2374G01-UT & DIAL2374G01 & BOLD:AAB1546 & ZION22020 & $658[0 \mathrm{n}]$ \\
\hline DLCAN & DLII1082-07 & DIAL2388E02-NM & A DIAL2388E02-NM & 1 BOLD:AAB1546 & & $658[0 \mathrm{n}]$ \\
\hline DLCAN & DLII780-07 & DIAL1849C06-CO & DIAL1849C06 & BOLD:AAB1546 & & $658[0 \mathrm{n}]$ \\
\hline DLCAN & DIAL119-06 & DIAL0286G11-MB & 3 DIAL0286G11 & BOLD:AAB1546 & & $658[0 \mathrm{n}]$ \\
\hline DLCAN & DIAL537-06 & D0287A04-CO & D0287A04 & BOLD:AAB1546 & & $658[0 \mathrm{n}]$ \\
\hline DLCAN & DIAL713-06 & $\mathrm{D} 0295 \mathrm{H} 04-\mathrm{CO}$ & $\mathrm{D} 0295 \mathrm{H} 04$ & BOLD:AAB1546 & & $658[0 \mathrm{n}]$ \\
\hline DLCAN & DIAL701-06 & D0295G04-CO & D0295G04 & BOLD:AAB1546 & & $658[0 \mathrm{n}]$ \\
\hline DLCAN & DIAL120-06 & DIAL0286G12-MB & 3 DIAL0286G12 & BOLD:AAB1546 & & $658[0 \mathrm{n}]$ \\
\hline DLCAN & DIAL116-06 & DIAL0286G08-MB & 3 DIAL0286G08 & BOLD:AAB1546 & & $658[0 \mathrm{n}]$ \\
\hline DIAL & BWTW01132-10 & 06732H03-CO & 06732H03-CO & BOLD:AAB1546 & & $658[0 \mathrm{n}]$ \\
\hline DLCAN & LASNA057-08 & DIAL2385E11-NS & DIAL2385E11-NS & BOLD:ACF5692 & & $574[0 \mathrm{n}]$ \\
\hline DLCAN & LASNA002-08 & DIAL2385A02-NS & DIAL2385A02-NS & BOLD:ACF5692 & & $597[1 \mathrm{n}]$ \\
\hline DLCAN & LASNA059-08 & DIAL2385F01-NS & DIAL2385F01-NS & BOLD:ACF5692 & & $599[0 \mathrm{n}]$ \\
\hline DLCAN & LASNA062-08 & DIAL2385F04-NS & DIAL2385F04-NS & BOLD:ACF5692 & & $612[0 \mathrm{n}]$ \\
\hline LPARA & DLII695-07 & DIAL1848D03-NS & DIAL1848D03 & BOLD:ACF5692 & & $657[0 \mathrm{n}]$ \\
\hline DLCAN & DLII694-07 & DIAL1848D02-NS & DIAL1848D02 & BOLD:ACF5692 & & $657[0 \mathrm{n}]$ \\
\hline DLCAN & DLII1525-09 & D01560A05-WI & D01560A05 & BOLD:ACF5692 & & $658[0 \mathrm{n}]$ \\
\hline LPARA & DLII1218-08 & DIAL2384H08-MD & DIAL2384H08-MD & BOLD:ACE5372 & & $658[0 \mathrm{n}]$ \\
\hline DIAL & DLII839-07 & DIAL1849H05-MD & DIAL1849H05 & BOLD:ACE5372 & & $658[0 \mathrm{n}]$ \\
\hline DIAL & DIAL337-06 & DIAL0294B06-IN & \#6716 & BOLD:AAE1871 & & $609[4 n]$ \\
\hline
\end{tabular}




\begin{tabular}{|c|c|c|c|c|c|}
\hline DLII & DLIII37-07 & DIAL1848G09-AB & DIAL1848G09 & BOLD:AAX0151 & $610[1 \mathrm{n}]$ \\
\hline LPARA & DLIII051-09 & D01562E03-NS & D01562E03 & BOLD:AAA8348 & $658[0 n]$ \\
\hline DLCAN & LASNA001-08 & DIAL2385A01-SK & DIAL2385A01-SK & BOLD:AAA8348 & $658[0 \mathrm{n}]$ \\
\hline DLCAN & LASNA005-08 & DIAL2385A05-SK & DIAL2385A05-SK & BOLD:AAA8348 & $658[0 n]$ \\
\hline DLII & DLII1551-09 & D01560C07-IA & D01560C07 & BOLD:ABZ0652 & $547[0 \mathrm{n}]$ \\
\hline DLII & DLII1297-08 & D-3253F03-MO & D-3253F03 & BOLD:ABZ0652 & $553[2 n]$ \\
\hline DLCAN & DIAL486-06 & D0288E01-VA & D0288E01 & BOLD:ABZ0652 & $576[0 n]$ \\
\hline DLII & LASNA055-08 & DIAL2385E09-NS & DIAL2385E09-NS & BOLD:ABZ0652 & $583[1 \mathrm{n}]$ \\
\hline DLII & DIAL471-06 & D0288C10-VA & D0288C10 & BOLD:ABZ0652 & $600[0 \mathrm{n}]$ \\
\hline DLII & DIAL385-06 & DIAL0294F07-MD & \#045294 & BOLD:ABZ0652 & $606[2 n]$ \\
\hline DLCAN & DIAL430-06 & DIAL0293B04-MD & obongum 020194 & BOLD:ABZ0652 & $609[2 n]$ \\
\hline DLCAN & DIAL393-06 & DIAL0294G03-ML & \#012348 & BOLD:ABZ0652 & $610[1 \mathrm{n}]$ \\
\hline DLII & DIAL429-06 & DIAL0293B03-MD & oblongum 020202 & BOLD:ABZ0652 & $610[1 \mathrm{n}]$ \\
\hline DLCAN & DIAL394-06 & DIAL0294G04-ML & \#012349 & BOLD:ABZ0652 & $611[2 n]$ \\
\hline DLII & DIAL350-06 & DIAL0294C08-IN & /lineat\#15367 & BOLD:ABZ0652 & $612[1 \mathrm{n}]$ \\
\hline DLCAN & DIAL415-06 & DIAL0292A03-MD & abanci 019384 & BOLD:ABZ0652 & $618[2 n]$ \\
\hline DLCAN & DIAL416-06 & DIAL0292A04-MD & abanci 009068 & BOLD:ABZ0652 & $619[1 \mathrm{n}]$ \\
\hline DIAL & BOWGF1124-10 & B04743G07-MA & B04743G07-MA & BOLD:ABZ0652 & $621[1 \mathrm{n}]$ \\
\hline DIAL & BOWGF1118-10 & B04743G01-MA & B04743G01-MA & BOLD:ABZ0652 & $625[0 \mathrm{n}]$ \\
\hline DLCAN & DLII442-07 & DIAL1846F08-WV & DIAL1846F08 & BOLD:ABZ0652 & $628[0 \mathrm{n}]$ \\
\hline DLII & DLII692-07 & DIAL1848C12-WV & DIAL1848C12 & BOLD:ABZ0652 PCYU & $631[0 n]$ \\
\hline DIAL & BOWGF1086-10 & B04743D05-NS & B04743D05-NS & BOLD:ABZ0652 & $632[0 \mathrm{n}]$ \\
\hline DLCAN & DLII1483-08 & D00608E10-IN & D00608E10 & BOLD:ABZ0652 & $637[0 \mathrm{n}]$ \\
\hline DLCAN & DLIII017-09 & D01562B05-ON & D01562B05 & BOLD:ABZ0652 & $652[1 \mathrm{n}]$ \\
\hline DIAL & BOWGF1135-10 & B04743H06-MA & B04743H06-MA & BOLD:ABZ0652 & $654[0 n]$ \\
\hline DLII & DIAL1054-06 & DIAL1391E05-TN & DIAL1391E05 & BOLD:ABZ0652 & $654[0 \mathrm{n}]$ \\
\hline DLCAN & DLII1385-08 & D00607E07-CT & D00607E07 & BOLD:ABZ0652 & $658[0 n]$ \\
\hline DLCAN & DIAL452-06 & D0288B02-MD & D0288B02 & BOLD:ABZ0652 & $658[0 \mathrm{n}]$ \\
\hline DLCAN & DIAL1409-09 & D01559E06-ON & D01559E06 & BOLD:ABZ0652 & $658[0 \mathrm{n}]$ \\
\hline DLCAN & DLII1340-08 & D00607A10-CT & D00607A10 & BOLD:ABZ0652 & $658[0 \mathrm{n}]$ \\
\hline DLCAN & DLII828-07 & DIAL1849G06-NH & DIAL1849G06 & BOLD:ABZ0652 & $658[0 \mathrm{n}]$ \\
\hline DLCAN & DLII1521-09 & D01560A01-WI & D01560A01 & BOLD:ABZ0652 & $658[0 n]$ \\
\hline DIAL & ВНTT014-09 & B03750B02-MA & B03750B02-MA & BOLD:ABZ0652 & $658[0 n]$ \\
\hline DIAL & BOWGF460-09 & CCDB-00611 G08 & CCDB-00611 G08 & B BOLD:ABZ0652 & $658[0 \mathrm{n}]$ \\
\hline DIAL & BOWGF443-09 & CCDB-00611 F03. & CCDB-00611 F03 & BOLD:ABZ0652 & $658[0 \mathrm{n}]$ \\
\hline DIAL & BOWGF1120-10 & B04743G03-MA & B04743G03-MA & BOLD:ABZ0652 & $658[0 \mathrm{n}]$ \\
\hline DIAL & BOWGF1131-10 & B04743H02-MA & B04743H02-MA & BOLD:ABZ0652 & $658[0 n]$ \\
\hline DIAL & BOWGF455-09 & CCDB-00611 G03 & CCDB-00611 G03 & BOLD:ABZ0652 & $658[0 n]$ \\
\hline DIAL & BOWGF456-09 & CCDB-00611 G04 & CCDB-00611 G04 & BOLD:ABZ0652 & $658[0 n]$ \\
\hline DLII & BOWGF1084-10 & B04743D03-NS & B04743D03-NS & BOLD:ABZ0652 & $658[0 \mathrm{n}]$ \\
\hline DLII & DLII1485-08 & D00608E12-IN & D00608E12 & BOLD:ABZ0652 & $658[0 n]$ \\
\hline DLII & DLIII055-09 & D01562E07-NS & D01562E07 & BOLD:ABZ0652 & $658[0 n]$ \\
\hline DLCAN & DLII1556-09 & D01560C12-IA & D01560C12 & BOLD:ACF2785 & $600[0 n]$ \\
\hline DLCAN & DIAL108-06 & DIAL0286F12-MB & DIAL0286F12 & BOLD:ACF2785 & $604[0 \mathrm{n}]$ \\
\hline DLCAN & DIAL106-06 & DIAL0286F10-MB & DIAL0286F10 & BOLD:ACF2785 & $609[0 n]$ \\
\hline LPARA & DLII743-07 & DIAL1848H03-AB & DIAL1848H03 & BOLD:ACF2785 & $620[0 \mathrm{n}]$ \\
\hline DLCAN & DIAL921-06 & DIAL1390B01-ON & DIAL1390B01 & BOLD:ACF2785 & $654[0 n]$ \\
\hline DIAL & DIAL026-06 & DIAL0293E04-FL & $\# 031875$ & BOLD:AAX0169 & $603[0 \mathrm{n}]$ \\
\hline DIAL & DLII1279-08 & D-3253D09-FL & D-3253D09 & BOLD:AAE5196 & $574[1 \mathrm{n}]$ \\
\hline DIAL & DLII1286-08 & D-3253E04-FL & D-3253E04 & BOLD:AAE5196 & $632[1 \mathrm{n}]$ \\
\hline DIAL & DLII1661-09 & D01561D10-FL & D01561D10 & BOLD:AAE5196 & $658[0 \mathrm{n}]$ \\
\hline DIAL & DIAL1359-09 & D01559A04-FL & D01559A04 & BOLD:AAE5196 & $658[0 n]$ \\
\hline DIAL & DIAL663-06 & D0295D02-DC & D0295D02 & BOLD:ABZ0652 & $593[4 n]$ \\
\hline DLCAN & DLII1442-08 & D00608B05-ON & D00608B05 & BOLD:ABZ0652 & $607[0 \mathrm{n}]$ \\
\hline DLCAN & DLII1440-08 & D00608B03-ON & D00608B03 & BOLD:ABZ0652 & $630[0 n]$ \\
\hline
\end{tabular}




\begin{tabular}{|c|c|c|c|c|c|}
\hline LTEG & DIAL745-06 & D1414C02-VA & D1414C02 & BOLD:AAD7708 & $604[0 n]$ \\
\hline LTEG & DIAL367-06 & DIAL0294E01-VA & \#032884 & BOLD:AAD7708 & $614[0 n]$ \\
\hline LTEG & DIAL366-06 & DIAL0294D12-NY & \#046015 & BOLD:AAD7708 & $614[0 n]$ \\
\hline LTEG & DIAL742-06 & D1414B10-VA & D1414B10 & BOLD:AAD7708 & $614[0 n]$ \\
\hline LTEG & DIAL744-06 & D1414C01-VA & D1414C01 & BOLD:AAD7708 & $618[0 n]$ \\
\hline DLCAN & DLII1300-08 & D-3253F06-MO & D-3253F06 & BOLD:AAD7708 & $622[0 n]$ \\
\hline DIAL & DLII393-07 & DIAL1846B06-MS & DIAL1846B06 & BOLD:AAD7708 & $641[0 n]$ \\
\hline DLCAN & DLII562-07 & DIAL1847H10-MD & DIAL1847H10 & BOLD:AAD7708 & $643[2 n]$ \\
\hline LTEG & DLII468-07 & DIAL1846H10-MD & DIAL1846H10 & BOLD:AAD7708 & $645[0 \mathrm{n}]$ \\
\hline DIAL & DLII391-07 & DIAL1846B04-MS & DIAL1846B04 & BOLD:AAD7708 & $645[0 \mathrm{n}]$ \\
\hline LTEG & DIAL824-06 & DIAL1413A09-VA & DIAL1413A09 & BOLD:AAD7708 & $653[0 \mathrm{n}]$ \\
\hline DIAL & BOWGF895-09 & B01566D04-TX & B01566D04 & BOLD:AAD7708 & $656[2 n]$ \\
\hline LTEG & DIAL493-06 & D0288E08-WV & D0288E08 & BOLD:AAD7708 & $657[1 \mathrm{n}]$ \\
\hline LTEG & DIAL743-06 & D1414B11-VA & D1414B11 & BOLD:AAD7708 & $658[0 n]$ \\
\hline LTEG & DIAL1051-06 & DIAL1391E02-SC & DIAL1391E02 & BOLD:AAD7708 & $658[0 \mathrm{n}]$ \\
\hline LTEG & DIAL1050-06 & DIAL1391E01-SC & DIAL1391E01 & BOLD:AAD7708 & $658[0 n]$ \\
\hline LTEG & DIAL518-06 & D0288G09-SC & D0288G09 & BOLD:AAD7708 & $658[0 \mathrm{n}]$ \\
\hline LTEG & DIAL495-06 & D0288E10-NC & D0288E10 & BOLD:AAD7708 & $658[0 n]$ \\
\hline LTEG & DIAL490-06 & D0288E05-WV & D0288E05 & BOLD:AAD7708 & $658[0 \mathrm{n}]$ \\
\hline LTEG & DIAL485-06 & D0288D12-WV & D0288D12 & BOLD:AAD7708 & $658[0 n]$ \\
\hline LTEG & DIAL684-06 & D0295E11-DC & D0295E11 & BOLD:AAD7708 & $658[0 \mathrm{n}]$ \\
\hline LTEG & DIAL683-06 & D0295E10-DC & D0295E10 & BOLD:AAD7708 & $658[0 n]$ \\
\hline LTEG & DIAL091-06 & BEE0286E07-VA & BEE0286E07 & BOLD:AAD7708 & $658[0 \mathrm{n}]$ \\
\hline DLCAN & DLII1356-08 & D00607C02-MO & D00607C02 & BOLD:AAD7708 & $658[0 n]$ \\
\hline DLCAN & DLII1331-08 & D00607A01-NE & D00607A01 & BOLD:AAD7708 & $658[0 \mathrm{n}]$ \\
\hline DIAL & BWTW01071-10 & 06732C02-DC & 06732C02-DC & BOLD:AAD7708 & $658[0 \mathrm{n}]$ \\
\hline DLCAN & DIAL1125-07 & DIAL2387C06-YT & DIAL2387C06 & BOLD:AAB6998 & $550[3 n]$ \\
\hline DLCAN & DLII337-07 & DIAL1845E09-NL & DIAL1845E09 & BOLD:AAB6998 & $576[0 n]$ \\
\hline DLCAN & DLII334-07 & DIAL1845E06-NL & DIAL1845E06 & BOLD:AAB6998 & $610[1 \mathrm{n}]$ \\
\hline DLCAN & DLII1139-08 & DIAL2384A11-YT & DIAL2384A11 & BOLD:AAB6998 & $617[1 \mathrm{n}]$ \\
\hline DLCAN & DIAL1028-06 & DIAL1391C03-NC & DIAL1391C03 & BOLD:AAB6998 & $619[0 \mathrm{n}]$ \\
\hline DLCAN & DLII1131-08 & DIAL2384A03-YT & DIAL2384A03 & BOLD:AAB6998 & $634[0 n]$ \\
\hline DLCAN & DLII1130-08 & DIAL2384A02-YT & DIAL2384A02 & BOLD:AAB6998 & $637[0 \mathrm{n}]$ \\
\hline DLCAN & DLII340-07 & DIAL1845E12-NL & DIAL1845E12 & BOLD:AAB6998 & $645[0 n]$ \\
\hline DLCAN & DLII341-07 & DIAL1845F01-NL & DIAL1845F01 & BOLD:AAB6998 & $645[0 \mathrm{n}]$ \\
\hline DIAL & DIAL1118-07 & DIAL2387B10-YT & DIAL2387B10 & BOLD:AAB6998 & $650[5 n]$ \\
\hline DLCAN & DIAL1029-06 & DIAL1391C04-NC & DIAL1391C04 & BOLD:AAB6998 & $652[0 \mathrm{n}]$ \\
\hline DLCAN & DIAL1112-07 & DIAL2387B04-YT & DIAL2387B04 & BOLD:AAB6998 & $655[0 \mathrm{n}]$ \\
\hline DLCAN & DLII1134-08 & DIAL2384A06-YT & DIAL2384A06 & BOLD:AAB6998 & $657[0 \mathrm{n}]$ \\
\hline DLCAN & DLII668-07 & DIAL1848A10-NB & DIAL1848A10 & BOLD:AAB6998 & $657[0 \mathrm{n}]$ \\
\hline DLCAN & DLII1137-08 & DIAL2384A09-YT & DIAL2384A09 & BOLD:AAB6998 & $657[0 \mathrm{n}]$ \\
\hline DLCAN & DLII1147-08 & DIAL2384B08-YT & DIAL2384B08 & BOLD:AAB6998 & $658[0 \mathrm{n}]$ \\
\hline DLCAN & DLII1129-08 & DIAL2384A01-YT & DIAL2384A01 & BOLD:AAB6998 & $658[0 \mathrm{n}]$ \\
\hline DLCAN & DLII1141-08 & DIAL2384B02-YT & DIAL2384B02 & BOLD:AAB6998 & $658[0 \mathrm{n}]$ \\
\hline DLCAN & DLII1149-08 & DIAL2384B10-YT & DIAL2384B10 & BOLD:AAB6998 & $658[0 \mathrm{n}]$ \\
\hline DLCAN & DIAL932-06 & DIAL1390C01-ON & N DIAL1390C01 & BOLD:AAE1693 & $614[3 n]$ \\
\hline DLCAN & DIAL934-06 & DIAL1390C03-ON & N DIAL1390C03 & BOLD:AAE1693 & $654[0 \mathrm{n}]$ \\
\hline DLCAN & DLII645-07 & BEE1850G11-ME & BEE1850G11 & BOLD:AAE1693 & $657[0 \mathrm{n}]$ \\
\hline DIAL & DIAL804-06 & D1414H01-VA & D1414H01 & BOLD:AAB2245 & $600[0 \mathrm{n}]$ \\
\hline DIAL & DIAL418-06 & DIAL0292A11-WV & V admirandum 0109 & BOLD:AAB2245 & $611[0 n]$ \\
\hline DIAL & DIAL372-06 & DIAL0294E06-MD & \#041908 & BOLD:AAB2245 & $611[1 \mathrm{n}]$ \\
\hline DIAL & LASNA060-08 & DIAL2385F02-MD & DIAL2385F02-MD & BOLD:AAB2245 & $612[0 n]$ \\
\hline DIAL & DIAL378-06 & DIAL0294E12-MD & \#023397 & BOLD:AAB2245 & $612[1 \mathrm{n}]$ \\
\hline DIAL & DIAL373-06 & DIAL0294E07-IN & \#021006 & BOLD:AAB2245 & $613[0 n]$ \\
\hline DIAL & DIAL432-06 & DIAL0293B10-WV & perpunctatum 032 & BOLD:AAB2245 & $619[0 n]$ \\
\hline
\end{tabular}




\begin{tabular}{|c|c|c|c|c|c|}
\hline DIAL & DIAL031-06 & DIAL0293F02-NC & $\# 023965$ & BOLD:AAB2245 & $619[0 n]$ \\
\hline DIAL & DLII1425-08 & D00607H11-KS & D00607H11 & BOLD:AAB2245 & $622[0 n]$ \\
\hline DIAL & BWTW0138-09 & B01558D07-NC & CCDB-01558D7 & BOLD:AAB2245 & $623[0 n]$ \\
\hline DIAL & DLII1432-08 & D00608A07-KS & D00608A07 & BOLD:AAB2245 & $624[0 n]$ \\
\hline DIAL & DIAL466-06 & D0288C05-DC & D0288C05 & BOLD:AAB2245 & $643[0 n]$ \\
\hline DIAL & DLII018-06 & DIAL1409B07-NC & DIAL1409B07 & BOLD:AAB2245 & $645[0 n]$ \\
\hline DIAL & DLII414-07 & DIAL1846D04-MS & DIAL1846D04 & BOLD:AAB2245 & $645[0 \mathrm{n}]$ \\
\hline DIAL & DIAL899-06 & DIAL1413H02-NC & DIAL1413H02 & BOLD:AAB2245 & $655[0 \mathrm{n}]$ \\
\hline LPARA & DIAL481-06 & D0288D08-NC & D0288D08 & BOLD:AAB2245 & $658[0 \mathrm{n}]$ \\
\hline DIAL & LASNA034-08 & DIAL2385C12-SC & DIAL2385C12-SC & BOLD:AAB2245 & $658[0 n]$ \\
\hline DIAL & DLII784-07 & DIAL1849C10-CO & DIAL1849C10 & BOLD:AAB2245 & $658[0 n]$ \\
\hline DIAL & DLII1345-08 & D00607B03-KS & D00607B03 & BOLD:AAB2245 & $658[0 n]$ \\
\hline DIAL & DLII1423-08 & D00607H09-KS & D00607H09 & BOLD:AAB2245 & $658[0 \mathrm{n}]$ \\
\hline DIAL & BWTWO1094-10 & 06732E01-MD & 06732E01-MD & BOLD:AAB2245 & $658[0 n]$ \\
\hline DIAL & DIAL727-06 & D1414A06-IL & D1414A06 & BOLD:AAB2245 & $658[0 n]$ \\
\hline DIAL & BWTWO876-10 & B03765B09-GA & B03765B09-GA & BOLD:AAB2245 & $658[0 \mathrm{n}]$ \\
\hline DIAL & BWTWO139-09 & B01558D08-NC & CCDB-01558D8 & BOLD:AAB2245 & $658[0 \mathrm{n}]$ \\
\hline DIAL & BWTW0134-09 & B01558D03-NC & CCDB-01558D3 & BOLD:AAB2245 & $658[0 \mathrm{n}]$ \\
\hline DIAL & BWTW0131-09 & B01558C12-NC & CCDB-01558C12 & BOLD:AAB2245 & $658[0 \mathrm{n}]$ \\
\hline DIAL & DIAL458-06 & D0288B08-SC & D0288B08 & BOLD:AAB2245 & $658[0 n]$ \\
\hline DIAL & DIAL456-06 & D0288B06-NC & D0288B06 & BOLD:AAB2245 & $658[0 n]$ \\
\hline DIAL & DIAL1045-06 & DIAL1391D08-NC & DIAL1391D08 & BOLD:AAB2245 & $658[0 n]$ \\
\hline DLCAN & DIAL1059-06 & DIAL1391E10-NC & DIAL1391E10 & & $501[3 n]$ \\
\hline DLCAN & DIAL438-06 & DIAL0293F04-TN & versans 000503 & BOLD:ABZ6180 & $606[1 n]$ \\
\hline DLCAN & DIAL032-06 & DIAL0293F05-TN & $\# 000504$ & BOLD:ACE3474 & $614[0 n]$ \\
\hline DLCAN & DIAL1030-06 & DIAL1391C05-NC & DIAL1391C05 & BOLD:ABZ6180 & $617[0 n]$ \\
\hline DLCAN & DIAL1011-06 & DIAL1391A08-NC & DIAL1391A08 & BOLD:ABZ6180 & $619[0 n]$ \\
\hline DLCAN & DLII841-07 & DIAL1849H07-ME & DIAL1849H07 & BOLD:ABZ6180 & $642[0 n]$ \\
\hline DIAL & DLII723-07 & DIAL1848F07-PE & DIAL1848F07 & BOLD:ABZ6180 & $653[0 \mathrm{n}]$ \\
\hline DLCAN & DLII712-07 & DIAL1848E08-WV & DIAL1848E08 & BOLD:ABZ6180 & $657[0 n]$ \\
\hline DLCAN & DLII1402-08 & D00607F12-NS & D00607F12 & BOLD:ABZ6180 & $658[0 n]$ \\
\hline DLCAN & LASNA028-08 & DIAL2385C06-MD & DIAL2385C06-MD & BOLD:AAA3781 & $512[0 \mathrm{n}]$ \\
\hline DLCAN & DIAL758-06 & D1414D03-VA & D1414D03 & BOLD:AAA3781 & $575[0 n]$ \\
\hline DLCAN & DLII405-07 & DIAL1846C07-WV & DIAL1846C07 & BOLD:AAA3781 & $576[0 \mathrm{n}]$ \\
\hline DIAL & DIAL575-06 & D0287D08-IL & D0287D08 & BOLD:AAA3781 & $576[3 n]$ \\
\hline DIAL & DIAL1230-08 & DIAL0293B02-DC & DIAL0293B02 & BOLD:AAA3781 & $584[2 n]$ \\
\hline DLCAN & DIAL1088-06 & DIAL1391H03-NC & DIAL1391H03 & BOLD:AAA3781 & $599[0 \mathrm{n}]$ \\
\hline DLCAN & DLII557-07 & DIAL1847H05-MD & DIAL1847H05 & BOLD:AAA3781 & $608[0 \mathrm{n}]$ \\
\hline DLCAN & DIAL391-06 & DIAL0294G01-ML & \#035111 & BOLD:AAA3781 & $612[1 \mathrm{n}]$ \\
\hline DLCAN & DIAL348-06 & DIAL0294C06-IL & \#9074 & BOLD:AAA3781 & $612[1 \mathrm{n}]$ \\
\hline DLCAN & DIAL349-06 & DIAL0294C07-IN & $\# 15$ & BOLD:AAA3781 & $612[1 \mathrm{n}]$ \\
\hline DLCAN & DIAL365-06 & DIAL0294D11-NY & \#046076 & BOLD:AAA3781 & $613[0 \mathrm{n}]$ \\
\hline DLCAN & DLII706-07 & DIAL1848E02-MD & DIAL1848E02 & BOLD:AAA3781 & $615[0 n]$ \\
\hline DLCAN & DIAL644-06 & D0295B06-ON & D0295B06 & BOLD:AAA3781 & $617[3 n]$ \\
\hline DLCAN & DIAL1087-06 & DIAL1391H02-NC & DIAL1391H02 & BOLD:AAA3781 & $618[0 n]$ \\
\hline DLCAN & DIAL1038-06 & DIAL1391D01-NC & DIAL1391D01 & BOLD:AAA3781 & $618[0 n]$ \\
\hline DLCAN & DIAL1093-06 & DIAL1391H08-NC & DIAL1391H08 & BOLD:AAA3781 & $619[0 n]$ \\
\hline DLCAN & DLIII556-07 & DIAL1847H04-MD & DIAL1847H04 & BOLD:AAA3781 & $619[0 \mathrm{n}]$ \\
\hline DLCAN & DIAL1085-06 & DIAL1391G12-NC & DIAL1391G12 & BOLD:AAA3781 & $620[0 \mathrm{n}]$ \\
\hline DLCAN & DIAL420-06 & DIAL0292B09-MD & big rohweri 01605 & BOLD:AAA3781 & $622[1 n]$ \\
\hline DLCAN & DIAL422-06 & DIAL0292C01-WV & big rohweri 01105 & ، BOLD:AAA3781 & $622[2 n]$ \\
\hline DLCAN & DIAL421-06 & DIAL0292B11-MD & big rohweri 01395 ! & BOLD:AAA3781 & $623[1 \mathrm{n}]$ \\
\hline DLCAN & DIAL423-06 & DIAL0292C02-MD & big rohweri 01602 & (BOLD:AAA3781 & $623[1 \mathrm{n}]$ \\
\hline DLCAN & DIAL895-06 & DIAL1413G10-NC & DIAL1413G10 & BOLD:AAA3781 & $626[0 n]$ \\
\hline DLCAN & DIAL1035-06 & DIAL1391C10-NC & DIAL1391C10 & BOLD:AAA3781 & $632[0 \mathrm{n}]$ \\
\hline
\end{tabular}




\begin{tabular}{|c|c|c|c|c|c|}
\hline DLCAN & DIAL1024-06 & DIAL1391B10-NC & DIAL1391B10 & BOLD:AAA3781 & $632[0 \mathrm{n}]$ \\
\hline DIAL & BWTWO1099-10 & 06732E06-WV & 06732E06-WV & BOLD:AAA3781 & $635[0 \mathrm{n}]$ \\
\hline DLCAN & DIAL621-06 & D0287H06-IL & D0287H06 & BOLD:AAA3781 & $636[0 \mathrm{n}]$ \\
\hline DLCAN & DIAL592-06 & D0287F01-IL & D0287F01 & BOLD:AAA3781 & $636[0 \mathrm{n}]$ \\
\hline DLCAN & DIAL623-06 & D0287H08-IL & D0287H08 & BOLD:AAA3781 & $643[0 n]$ \\
\hline DLCAN & DIAL894-06 & DIAL1413G09-NC & DIAL1413G09 & BOLD:AAA3781 & $643[0 \mathrm{n}]$ \\
\hline DLCAN & DIAL627-06 & D0287H12-IL & D0287H12 & BOLD:AAA3781 & $645[0 n]$ \\
\hline DLCAN & DLII023-06 & DIAL1409C01-PQ & DIAL1409C01 & BOLD:AAA3781 & $645[0 \mathrm{n}]$ \\
\hline DLCAN & DLII022-06 & DIAL1409B11-PQ & DIAL1409B11 & BOLD:AAA3781 & $645[0 \mathrm{n}]$ \\
\hline DLCAN & DLII437-07 & DIAL1846F03-MD & DIAL1846F03 & BOLD:AAA3781 & $645[0 \mathrm{n}]$ \\
\hline DLCAN & DLII553-07 & DIAL1847H01-MD & DIAL1847H01 & BOLD:AAA3781 & $645[0 n]$ \\
\hline DLCAN & DLII554-07 & DIAL1847H02-MD & DIAL1847H02 & BOLD:AAA3781 & $645[0 \mathrm{n}]$ \\
\hline DLCAN & DLII390-07 & DIAL1846B03-MD & DIAL1846B03 & BOLD:AAA3781 & $645[0 \mathrm{n}]$ \\
\hline DLCAN & DLII1421-08 & D00607H07-KS & D00607H07 & BOLD:AAA3781 & $647[0 \mathrm{n}]$ \\
\hline DLCAN & DIAL1090-06 & DIAL1391H05-NC & DIAL1391H05 & BOLD:AAA3781 & $648[0 \mathrm{n}]$ \\
\hline DLCAN & DIAL1021-06 & DIAL1391B07-NC & DIAL1391B07 & BOLD:AAA3781 & $648[0 \mathrm{n}]$ \\
\hline DLCAN & DIAL1019-06 & DIAL1391B05-NC & DIAL1391B05 & BOLD:AAA3781 & $649[1 n]$ \\
\hline DLCAN & DIAL591-06 & D0287E12-IL & D0287E12 & BOLD:AAA3781 & $651[0 n]$ \\
\hline DLCAN & DIAL585-06 & D0287E06-IL & D0287E06 & BOLD:AAA3781 & $653[0 \mathrm{n}]$ \\
\hline DLCAN & DIAL829-06 & DIAL1413B03-IL & DIAL1413B03 & BOLD:AAA3781 & $653[0 \mathrm{n}]$ \\
\hline DLCAN & DIAL911-06 & DIAL1390A02-NC & DIAL1390A02 & BOLD:AAA3781 & $654[0 \mathrm{n}]$ \\
\hline DLCAN & DIAL910-06 & DIAL1390A01-NC & DIAL1390A01 & BOLD:AAA3781 & $654[0 \mathrm{n}]$ \\
\hline DLCAN & DIAL969-06 & DIAL1390F02-NC & DIAL1390F02 & BOLD:AAA3781 & $654[0 \mathrm{n}]$ \\
\hline DLCAN & DIAL893-06 & DIAL1413G08-NC & DIAL1413G08 & BOLD:AAA3781 & $654[0 \mathrm{n}]$ \\
\hline DLCAN & DIAL1097-06 & DIAL1391H12-NC & DIAL1391H12 & BOLD:AAA3781 & $655[0 \mathrm{n}]$ \\
\hline DLCAN & DIAL904-06 & DIAL1413H07-NC & DIAL1413H07 & BOLD:AAA3781 & $655[0 \mathrm{n}]$ \\
\hline DLCAN & DIAL901-06 & DIAL1413H04-NC & DIAL1413H04 & BOLD:AAA3781 & $655[0 \mathrm{n}]$ \\
\hline DLCAN & DIAL1023-06 & DIAL1391B09-NC & DIAL1391B09 & BOLD:AAA3781 & $655[0 \mathrm{n}]$ \\
\hline DLCAN & DIAL1022-06 & DIAL1391B08-NC & DIAL1391B08 & BOLD:AAA3781 & $655[0 \mathrm{n}]$ \\
\hline DLCAN & DIAL626-06 & D0287H11-IL & D0287H11 & BOLD:AAA3781 & $656[0 \mathrm{n}]$ \\
\hline DLCAN & DIAL1018-06 & DIAL1391B04-NC & DIAL1391B04 & BOLD:AAA3781 & $656[0 \mathrm{n}]$ \\
\hline DLCAN & DIAL1092-06 & DIAL1391H07-NC & DIAL1391H07 & BOLD:AAA3781 & $657[0 \mathrm{n}]$ \\
\hline DLCAN & DIAL581-06 & D0287E02-IL & D0287E02 & BOLD:AAA3781 & $657[0 \mathrm{n}]$ \\
\hline DLCAN & DIAL1020-06 & DIAL1391B06-NC & DIAL1391B06 & BOLD:AAA3781 & $657[1 \mathrm{n}]$ \\
\hline DLCAN & DLII720-07 & DIAL1848F04-MD & DIAL1848F04 & BOLD:AAA3781 & $657[0 \mathrm{n}]$ \\
\hline LPARA & DIAL906-06 & DIAL1413H09-NC & DIAL1413H09 & BOLD:AAA3781 & $658[0 \mathrm{n}]$ \\
\hline DLCAN & DIAL587-06 & D0287E08-IL & D0287E08 & BOLD:AAA3781 & $658[0 \mathrm{n}]$ \\
\hline DLCAN & DIAL645-06 & D0295B07-ON & D0295B07 & BOLD:AAA3781 & $658[0 \mathrm{n}]$ \\
\hline DLCAN & DIAL639-06 & D0295B01-ON & D0295B01 & BOLD:AAA3781 & $658[0 \mathrm{n}]$ \\
\hline DLCAN & DIAL640-06 & D0295B02-ON & D0295B02 & BOLD:AAA3781 & $658[0 \mathrm{n}]$ \\
\hline DLCAN & DIAL622-06 & D0287H07-IL & D0287H 07 & BOLD:AAA3781 & $658[0 \mathrm{n}]$ \\
\hline DLCAN & DIAL1096-06 & DIAL1391H11-NC & DIAL1391H11 & BOLD:AAA3781 & $658[0 \mathrm{n}]$ \\
\hline DLCAN & DIAL614-06 & D0287G11-IL & D0287G11 & BOLD:AAA3781 & $658[0 \mathrm{n}]$ \\
\hline DLCAN & DIAL618-06 & D0287H03-IL & $\mathrm{D} 0287 \mathrm{H} 03$ & BOLD:AAA3781 & $658[0 \mathrm{n}]$ \\
\hline DLCAN & DIAL619-06 & D0287H04-IL & D0287H04 & BOLD:AAA3781 & $658[0 \mathrm{n}]$ \\
\hline DLCAN & DIAL620-06 & D0287H05-IL & D0287H05 & BOLD:AAA3781 & $658[0 \mathrm{n}]$ \\
\hline DLCAN & DIAL608-06 & D0287G05-IL & D0287G05 & BOLD:AAA3781 & $658[0 \mathrm{n}]$ \\
\hline DLCAN & DIAL590-06 & D0287E11-IL & D0287E11 & BOLD:AAA3781 & $658[0 \mathrm{n}]$ \\
\hline DLCAN & DIAL586-06 & D0287E07-IL & D0287E07 & BOLD:AAA3781 & $658[0 \mathrm{n}]$ \\
\hline DLCAN & DIAL574-06 & D0287D07-IL & D0287D07 & BOLD:AAA3781 & $658[0 \mathrm{n}]$ \\
\hline DLCAN & DIAL560-06 & D0287C05-IL & D0287C05 & BOLD:AAA3781 & $658[0 \mathrm{n}]$ \\
\hline DLCAN & DIAL773-06 & D1414E06-VA & D1414E06 & BOLD:AAA3781 & $658[0 \mathrm{n}]$ \\
\hline DLCAN & DIAL558-06 & D0287C03-IL & D0287C03 & BOLD:AAA3781 & $658[0 \mathrm{n}]$ \\
\hline DLCAN & DIAL559-06 & D0287C04-IL & D0287C04 & BOLD:AAA3781 & $658[0 \mathrm{n}]$ \\
\hline DLCAN & DIAL588-06 & D0287E09-IL & D0287E09 & BOLD:AAA3781 & $658[0 \mathrm{n}]$ \\
\hline
\end{tabular}




\begin{tabular}{|c|c|c|c|c|c|}
\hline DLCAN & DIAL770-06 & D1414E03-VA & D1414E03 & BOLD:AAA3781 & $658[0 \mathrm{n}]$ \\
\hline DLCAN & DIAL523-06 & D0288H02-MD & $\mathrm{D} 0288 \mathrm{H} 02$ & BOLD:AAA3781 & $658[0 \mathrm{n}]$ \\
\hline DLCAN & DIAL521-06 & D0288G12-MD & D0288G12 & BOLD:AAA3781 & $658[0 \mathrm{n}]$ \\
\hline DLCAN & DIAL483-06 & D0288D10-MD & D0288D10 & BOLD:AAA3781 & $658[0 \mathrm{n}]$ \\
\hline DLCAN & DIAL457-06 & D0288B07-MD & D0288B07 & BOLD:AAA3781 & $658[0 \mathrm{n}]$ \\
\hline DLCAN & DIAL809-06 & D1414H06-VA & D1414H06 & BOLD:AAA3781 & $658[0 \mathrm{n}]$ \\
\hline DLCAN & DIAL808-06 & D1414H05-VA & D1414H05 & BOLD:AAA3781 & $658[0 \mathrm{n}]$ \\
\hline DLCAN & DIAL772-06 & D1414E05-VA & D1414E05 & BOLD:AAA3781 & $658[0 \mathrm{n}]$ \\
\hline DLCAN & DIAL642-06 & D0295B04-ON & D0295B04 & BOLD:AAA3781 & $658[0 \mathrm{n}]$ \\
\hline DLCAN & DLII1393-08 & D00607F03-KS & D00607F03 & BOLD:AAA3781 & $658[0 \mathrm{n}]$ \\
\hline DLCAN & DLII1358-08 & D00607C04-MO & D00607C04 & BOLD:AAA3781 & $658[0 \mathrm{n}]$ \\
\hline DLCAN & DLII1480-08 & D00608E07-IN & D00608E07 & BOLD:AAA3781 & $658[0 \mathrm{n}]$ \\
\hline DLCAN & LASNA030-08 & DIAL2385C08-MD & DIAL2385C08-MD & BOLD:AAA3781 & $658[0 \mathrm{n}]$ \\
\hline DLCAN & LASNA040-08 & DIAL2385D06-MD & DIAL2385D06-MD & BOLD:AAA3781 & $658[0 \mathrm{n}]$ \\
\hline DIAL & BWTW01087-10 & 06732D06-WV & 06732D06-WV & BOLD:AAA3781 & $658[0 \mathrm{n}]$ \\
\hline DIAL & BOWGF425-09 & CCDB-00611 D09 & CCDB-00611 D09 & BOLD:AAA3781 & $658[0 \mathrm{n}]$ \\
\hline DIAL & BOWGF419-09 & CCDB-00611 D03 & 3 CCDB-00611 D03 & BOLD:AAA3781 & $658[0 \mathrm{n}]$ \\
\hline DIAL & BWTWO1135-10 & 06732H06-RI & 06732H06-RI & BOLD:AAA3781 & $658[0 \mathrm{n}]$ \\
\hline DLCAN & DLII314-07 & DIAL1845C10-TX & DIAL1845C10 & BOLD:AAB4651 & $599[0 \mathrm{n}]$ \\
\hline DLCAN & DLII471-07 & DIAL1847A01-SC & DIAL1847A01 & BOLD:AAB4651 & $602[1 \mathrm{n}]$ \\
\hline LTEG & DIAL358-06 & DIAL0294D04-IN & \#9696 & BOLD:AAB4651 & $614[0 \mathrm{n}]$ \\
\hline LTEG & DIAL370-06 & DIAL0294E04-NY & $\# 046048$ & BOLD:AAB4651 & $614[0 n]$ \\
\hline LTEG & DIAL035-06 & DIAL0293F11-MN & J \#000000 & BOLD:AAB4651 & $619[0 \mathrm{n}]$ \\
\hline LTEG & DIAL033-06 & DIAL0293F08-MD & \#028663 & BOLD:AAB4651 & $619[0 \mathrm{n}]$ \\
\hline LTEG & DIAL479-06 & D0288D06-NC & D0288D06 & BOLD:AAB4651 & $622[0 \mathrm{n}]$ \\
\hline LTEG & DIAL034-06 & DIAL0293F09-MD & \#028012 & BOLD:AAB4651 & $624[0 \mathrm{n}]$ \\
\hline DIAL & BWTWO1088-10 & 06732D07-MA & 06732D07-MA & BOLD:AAB4651 & $632[0 \mathrm{n}]$ \\
\hline LTEG & DIAL915-06 & DIAL1390A06-ON & DIAL1390A06 & BOLD:AAB4651 & $654[0 \mathrm{n}]$ \\
\hline LTEG & DIAL121-06 & DIAL0286H01-MB & 3 DIAL0286H01 & BOLD:AAB4651 & $658[0 \mathrm{n}]$ \\
\hline LTEG & DIAL480-06 & D0288D07-SC & D0288D07 & BOLD:AAB4651 & $658[0 \mathrm{n}]$ \\
\hline LTEG & DIAL484-06 & D0288D11-SC & D0288D11 & BOLD:AAB4651 & $658[0 \mathrm{n}]$ \\
\hline DLCAN & DIAL1337-08 & D00609G05-NS & D00609G05 & BOLD:ABZ0652 & $545[0 n]$ \\
\hline DLCAN & LASNA056-08 & DIAL2385E10-MB & 3 DIAL2385E10-MB & BOLD:ABZ0652 & $575[2 n]$ \\
\hline DLCAN & DLII1656-09 & D01561D05-ON & D01561D05 & BOLD:ABZ0652 & $590[2 n]$ \\
\hline DLCAN & DLII1643-09 & D01561C04-IN & D01561C04 & BOLD:ABZ0652 & $617[0 \mathrm{n}]$ \\
\hline DLCAN & DIAL988-06 & DIAL1390G09-NC & DIAL1390G09 & BOLD:ABZ0652 & $654[0 n]$ \\
\hline DLCAN & DLII662-07 & DIAL1848A04-NB & DIAL1848A04 & BOLD:ABZ0652 & $657[0 \mathrm{n}]$ \\
\hline DLCAN & DIAL664-06 & D0295D03-MD & D0295D03 & BOLD:AAA3782 & $550[0 \mathrm{n}]$ \\
\hline DLII & DLII403-07 & DIAL1846C05-MS & DIAL1846C05 & BOLD:AAA3782 & $576[0 n]$ \\
\hline DLCAN & DIAL855-06 & DIAL1413D06-NC & DIAL1413D06 & BOLD:AAA3782 & $605[3 n]$ \\
\hline DIAL & DIAL379-06 & DIAL0294F01-PA & \#039353 & BOLD:AAA3782 & $605[2 n]$ \\
\hline DLCAN & DIAL820-06 & DIAL1413A05-IL & DIAL1413A05 & BOLD:AAA3782 & $609[2 n]$ \\
\hline DLCAN & DIAL038-06 & DIAL0293A03-MD & \#027019 & BOLD:AAA3782 & $609[2 n]$ \\
\hline DLCAN & DIAL439-06 & DIAL0293G02-ML & viridatum 035222 & BOLD:AAA3782 & $611[1 n]$ \\
\hline DLCAN & DIAL1057-06 & DIAL1391E08-NC & DIAL1391E08 & BOLD:AAA3782 & $611[3 n]$ \\
\hline DLCAN & DIAL377-06 & DIAL0294E11-MD & \#036466 & BOLD:AAA3782 & $611[2 n]$ \\
\hline DLCAN & DIAL428-06 & DIAL0292H12-MD & little rohweri 0270 & (BOLD:AAA3782 & $622[2 n]$ \\
\hline DLCAN & DIAL957-06 & DIAL1390E02-ON & NIAL1390E02 & BOLD:AAA3782 & $624[0 \mathrm{n}]$ \\
\hline DLCAN & DIAL874-06 & DIAL1413F01-TN & DIAL1413F01 & BOLD:AAA3782 & $624[0 n]$ \\
\hline DLCAN & DIAL822-06 & DIAL1413A07-VA & DIAL1413A07 & BOLD:AAA3782 & $628[1 n]$ \\
\hline DLCAN & DIAL902-06 & DIAL1413H05-NC & DIAL1413H05 & BOLD:AAA3782 & $629[3 n]$ \\
\hline DLCAN & DIAL836-06 & DIAL1413B10-IL & DIAL1413B10 & BOLD:AAA3782 & $643[1 n]$ \\
\hline DLCAN & DLII423-07 & DIAL1846E01-WV & VIAL1846E01 & BOLD:AAA3782 & $643[2 n]$ \\
\hline DLCAN & DLII010-06 & DIAL1409A10-NC & DIAL1409A10 & BOLD:AAA3782 & $643[2 n]$ \\
\hline DLCAN & DLII093-06 & DIAL1409H11-ON & NIAL1409H11 & BOLD:AAA3782 & $645[0 \mathrm{n}]$ \\
\hline
\end{tabular}




\begin{tabular}{|c|c|c|c|c|}
\hline DLCAN & DIAL994-06 & DIAL1390H03-SC DIAL1390H03 & BOLD:AAA3782 & $651[3 n]$ \\
\hline DLCAN & DIAL937-06 & DIAL1390C06-ON DIAL1390C06 & BOLD:AAA3782 & $653[1 \mathrm{n}]$ \\
\hline DLCAN & DIAL993-06 & DIAL1390H02-SC DIAL1390H02 & BOLD:AAA3782 & $654[0 \mathrm{n}]$ \\
\hline DLCAN & DIAL984-06 & DIAL1390G05-NC DIAL1390G05 & BOLD:AAA3782 & $654[0 \mathrm{n}]$ \\
\hline DIAL & DIAL1005-06 & DIAL1391A02-VA DIAL1391A02 & BOLD:AAA3782 & $654[4 n]$ \\
\hline DLCAN & DIAL856-06 & DIAL1413D07-NC DIAL1413D07 & BOLD:AAA3782 & $657[1 \mathrm{n}]$ \\
\hline DLCAN & DLII722-07 & DIAL1848F06-MD DIAL1848F06 & BOLD:AAA3782 & $657[0 \mathrm{n}]$ \\
\hline DLCAN & DIAL722-06 & D1414A01-YT & BOLD:ACF5692 & $593[0 n]$ \\
\hline DLCAN & DIAL1162-07 & DIAL2387F08-BC DIAL2387F08 & BOLD:ACF5692 & $655[0 \mathrm{n}]$ \\
\hline LPARA & DLII682-07 & DIAL1848C02-YT DIAL1848C02 & BOLD:ACF5692 & $657[0 \mathrm{n}]$ \\
\hline DLCAN & DIAL071-06 & BEE0286C11-ON BEE0286C11 & BOLD:AAD0272 & $607[2 n]$ \\
\hline DLCAN & DLII1519-08 & D00608H10-OR D00608H10 & BOLD:AAD0272 & $618[0 \mathrm{n}]$ \\
\hline DIAL & BOWGF1435-10 & 04485A10-VA & BOLD:AAD0272 & $621[0 \mathrm{n}]$ \\
\hline DLCAN & DLII702-07 & DIAL1848D10-MD DIAL1848D10 & BOLD:AAD0272 & $642[0 \mathrm{n}]$ \\
\hline DLCAN & DIAL952-06 & DIAL1390D09-ON DIAL1390D09 & BOLD:AAD0272 & $654[0 \mathrm{n}]$ \\
\hline LPARA & DLII675-07 & DIAL1848B06-WV DIAL1848B06 & BOLD:AAD0272 & $657[0 \mathrm{n}]$ \\
\hline DLCAN & DLII688-07 & DIAL1848C08-WV DIAL1848C08 & BOLD:AAD0272 & $657[0 \mathrm{n}]$ \\
\hline
\end{tabular}




\begin{tabular}{|c|c|c|c|c|c|}
\hline COI-5P Acc & Image Coun & Barcode Col Contamination & Stop Codon & Flagged Record & Collection Date \\
\hline \multirow[t]{4}{*}{ HQ945439 } & 0 & Yes & & & 05-Aug-2004 \\
\hline & 1 & Yes & & & 16-Jun-2005 \\
\hline & 1 & Yes & & & \\
\hline & 1 & Yes & & & 15-Jun-2007 \\
\hline \multirow[t]{9}{*}{ JF903493 } & 1 & Yes & & & \\
\hline & 1 & Yes & & & 28-May-1998 \\
\hline & 1 & Yes & & & 03-Aug-2001 \\
\hline & 2 & Yes & & & 22-Sep-2004 \\
\hline & 2 & No & & & \\
\hline & 2 & Yes & & & 21-Jul-2006 \\
\hline & 2 & Yes & & & 21-Jul-2006 \\
\hline & 2 & No & & & 22-Jul-2004 \\
\hline & 2 & Yes & & & 07-Oct-2006 \\
\hline \multirow[t]{2}{*}{ JF903494 } & 2 & Yes & & & \\
\hline & 2 & Yes & & & 26-Sep-2003 \\
\hline \multirow[t]{6}{*}{ HM905839 } & 1 & Yes & & & 04-Jun-2005 \\
\hline & 3 & No & & & 21-Jul-2004 \\
\hline & 3 & Yes & & & 23-Aug-2005 \\
\hline & 2 & Yes & & & 07-Jun-1999 \\
\hline & 3 & Yes & & & 02-Jun-2006 \\
\hline & 3 & Yes & & & 21-Jul-2006 \\
\hline \multirow[t]{3}{*}{ HQ937904 } & 1 & Yes & & & 20-Aug-2008 \\
\hline & 1 & Yes & & & \\
\hline & 3 & Yes & & & \\
\hline \multirow[t]{3}{*}{ JF903495 } & 1 & Yes & & & 31-Jul-2005 \\
\hline & 3 & Yes & & & 02-Jun-2005 \\
\hline & 3 & Yes & & & 23-Aug-2005 \\
\hline \multirow[t]{11}{*}{ JF903496 } & 1 & Yes & & & 20-Jun-2007 \\
\hline & 2 & Yes & & & 12-Jul-2008 \\
\hline & 3 & Yes & & & \\
\hline & 3 & Yes & & & 20-Jun-2007 \\
\hline & 2 & Yes & & & 27-Jul-2008 \\
\hline & 2 & Yes & & & 27-Jul-2008 \\
\hline & 2 & Yes & & & 18-Jul-2008 \\
\hline & 1 & Yes & & & 29-Мау-2007 \\
\hline & 3 & No & & & 01-Jul-2003 \\
\hline & 4 & No & & & 16-Jul-2003 \\
\hline & 3 & No & & & 01-Jun-2005 \\
\hline \multirow[t]{2}{*}{ GU708187 } & 1 & No & & & \\
\hline & 2 & Yes & & & 05-Sep-2007 \\
\hline \multirow[t]{12}{*}{ JF903497 } & 1 & Yes & & & 27-Jun-2006 \\
\hline & 1 & Yes & & & 20-Jun-2005 \\
\hline & 1 & Yes & & & 23-Aug-2005 \\
\hline & 1 & No & & & 15-Aug-2006 \\
\hline & 1 & No & & & 11-Мay-1999 \\
\hline & 0 & Yes & & & 11-Jul-2005 \\
\hline & 4 & No & & & \\
\hline & 1 & No & & & \\
\hline & 1 & No & & & \\
\hline & 1 & No & & & 30-May-2004 \\
\hline & 0 & Yes & & & 11-Jul-2005 \\
\hline & 1 & No & & & 30-May-2004 \\
\hline
\end{tabular}




\begin{tabular}{|c|c|c|c|}
\hline & 1 & No & 15-Aug-2006 \\
\hline \multirow[t]{11}{*}{ JF903498 } & 1 & Yes & 15-Aug-2006 \\
\hline & 1 & No & 15-Aug-2006 \\
\hline & 1 & Yes & 15-Aug-2006 \\
\hline & 1 & Yes & \\
\hline & 1 & Yes & 04-Jun-2006 \\
\hline & 1 & Yes & 01-Jul-2006 \\
\hline & 1 & Yes & 11-Jul-2007 \\
\hline & 0 & Yes & 18-Jul-2008 \\
\hline & 1 & Yes & \\
\hline & 0 & No & 17-Jun-2003 \\
\hline & 3 & No & 06-Jun-2003 \\
\hline GU666313 & 0 & Yes & 22-Jul-2008 \\
\hline GU666312 & 0 & Yes & 22-Jul-2008 \\
\hline \multirow[t]{11}{*}{ GU666310 } & 0 & Yes & 22-Jul-2008 \\
\hline & 1 & No & 19-Jun-2007 \\
\hline & 1 & No & 19-Jun-2007 \\
\hline & 1 & No & \\
\hline & 1 & Yes & \\
\hline & 1 & Yes & 25-May-2005 \\
\hline & 1 & Yes & 10-Jul-2003 \\
\hline & 1 & Yes & \\
\hline & 1 & Yes & 18-May-2006 \\
\hline & 6 & No & 01-Jul-2003 \\
\hline & 5 & No & 27-Jul-2004 \\
\hline \multirow[t]{7}{*}{ HQ937903 } & 1 & Yes & 14-Aug-2008 \\
\hline & 2 & Yes & 09-Aug-2004 \\
\hline & 3 & Yes & 30-Мay-2005 \\
\hline & 3 & Yes & \\
\hline & 3 & Yes & 13-Aug-2006 \\
\hline & 3 & Yes & 30-Jun-2006 \\
\hline & 3 & Yes & \\
\hline \multirow[t]{10}{*}{ JF903499 } & 1 & Yes & 21-Aug-2005 \\
\hline & 3 & Yes & 05-Aug-2006 \\
\hline & 2 & Yes & 29-Jul-2008 \\
\hline & 3 & Yes & \\
\hline & 3 & Yes & \\
\hline & 3 & Yes & \\
\hline & 3 & Yes & \\
\hline & 3 & Yes & \\
\hline & 3 & Yes & 26-May-2006 \\
\hline & 3 & Yes & \\
\hline \multirow[t]{13}{*}{ JF903500 } & 2 & Yes & 30-May-2008 \\
\hline & 2 & Yes & 15-Aug-2008 \\
\hline & 2 & Yes & 28-Jul-2008 \\
\hline & 3 & Yes & 05-Jul-2006 \\
\hline & 2 & Yes & 30-May-2008 \\
\hline & 1 & Yes & 11-May-1998 \\
\hline & 2 & Yes & 30-Sep-2003 \\
\hline & 3 & Yes & 08-Aug-2006 \\
\hline & 3 & No & 01-Oct-2003 \\
\hline & 3 & No & 08-Jun-2005 \\
\hline & 3 & No & 08-Mar-2006 \\
\hline & 3 & No & 01-Oct-2003 \\
\hline & 3 & No & 03-Sep-2003 \\
\hline
\end{tabular}




\begin{tabular}{|c|c|c|c|}
\hline & 3 & \multicolumn{2}{|l|}{ No } \\
\hline & 3 & No & 30-Jul-2004 \\
\hline & 3 & Yes & \\
\hline & 3 & No & 06-Jun-2005 \\
\hline & 3 & Yes & 07-Aug-2006 \\
\hline & 3 & Yes & 10-Aug-2006 \\
\hline & 3 & No & \\
\hline & 3 & Yes & 10-Aug-2006 \\
\hline & 3 & Yes & 08-Aug-2006 \\
\hline & 3 & Yes & \\
\hline & 3 & Yes & 09-Aug-2006 \\
\hline & 3 & Yes & 08-Aug-2006 \\
\hline & 3 & Yes & 10-Aug-2006 \\
\hline & 3 & Yes & 10-Aug-2006 \\
\hline & 3 & Yes & 05-Aug-2006 \\
\hline & 3 & Yes & \\
\hline & 3 & Yes & \\
\hline & 3 & Yes & \\
\hline & 3 & Yes & \\
\hline & 2 & Yes & 20-Jun-2005 \\
\hline & 2 & Yes & 02-Jun-2005 \\
\hline & 3 & Yes & \\
\hline & 3 & Yes & \\
\hline & 3 & Yes & 09-Aug-2006 \\
\hline & 3 & Yes & 09-Aug-2006 \\
\hline & 3 & Yes & 09-Aug-2006 \\
\hline FJ663059 & 2 & Yes & 25-Jun-2006 \\
\hline FJ663058 & 2 & Yes & 24-Jun-2006 \\
\hline FJ663060 & 2 & Yes & 25-Jun-2006 \\
\hline HM905722 & 0 & Yes & 09-Jul-2007 \\
\hline & 0 & Yes & 13-May-2005 \\
\hline & 0 & Yes & \\
\hline & 0 & Yes & 26-Aug-2004 \\
\hline & 1 & No & 13-Aug-2003 \\
\hline & 1 & No & 17-Jul-2002 \\
\hline & 3 & No & \\
\hline & 1 & No & 17-Jul-2002 \\
\hline & 1 & No & 25-Sep-2004 \\
\hline & 1 & Yes & 01-Jul-2006 \\
\hline & 1 & Yes & 04-Jun-2006 \\
\hline & 1 & Yes & 04-Jun-2006 \\
\hline & 1 & Yes & 27-Apr-2002 \\
\hline & 1 & Yes & 04-Jun-2006 \\
\hline & 1 & Yes & 26-Aug-2006 \\
\hline JF903503 & 1 & Yes & 26-Aug-2005 \\
\hline & 1 & Yes & 10-Aug-2006 \\
\hline HQ558108 & 0 & Yes & 06-Aug-2008 \\
\hline HQ558109 & 0 & Yes & 20-Aug-2008 \\
\hline & 0 & Yes & 03-Jun-2007 \\
\hline GU708160 & 0 & Yes & 19-Apr-2008 \\
\hline & 3 & No & 21-Aug-2003 \\
\hline & 1 & No & \\
\hline & 2 & No & \\
\hline & 2 & No & 07-Jun-2005 \\
\hline & 2 & No & 21-Aug-2003 \\
\hline
\end{tabular}




\begin{tabular}{|c|c|c|c|}
\hline & 2 & Yes & 30-Aug-2006 \\
\hline & 2 & Yes & 04-Jun-2006 \\
\hline \multirow[t]{2}{*}{ JF903505 } & 2 & Yes & 04-Jun-2006 \\
\hline & 2 & Yes & 04-Jun-2006 \\
\hline \multirow[t]{15}{*}{ GU708189 } & 1 & No & 16-May-2005 \\
\hline & 1 & No & 04-Jun-2005 \\
\hline & 3 & No & 13-Apr-2001 \\
\hline & 3 & No & 01-Jul-2003 \\
\hline & 3 & Yes & \\
\hline & 3 & Yes & 01-Jun-2005 \\
\hline & 3 & No & 02-Jul-2003 \\
\hline & 1 & No & 01-Jan-2004 \\
\hline & 3 & Yes & 19-Jun-2003 \\
\hline & 3 & Yes & 01-Jun-2003 \\
\hline & 3 & No & 20-Jun-2002 \\
\hline & 3 & Yes & 14-May-2003 \\
\hline & 3 & No & 13-Aug-2006 \\
\hline & 3 & No & 01-Jun-2005 \\
\hline & 1 & No & 01-Jun-2005 \\
\hline \multirow[t]{11}{*}{ JF903506 } & 1 & Yes & 13-Aug-2006 \\
\hline & 2 & Yes & 24-Jun-2003 \\
\hline & 3 & Yes & 31-Jul-2005 \\
\hline & 3 & Yes & \\
\hline & 3 & Yes & 01-Jun-2005 \\
\hline & 2 & Yes & 03-Sep-2003 \\
\hline & 3 & Yes & \\
\hline & 3 & Yes & 13-Aug-2006 \\
\hline & 3 & Yes & \\
\hline & 3 & Yes & 17-Jun-2006 \\
\hline & 3 & Yes & \\
\hline HQ557881 & 0 & Yes & 15-Мау-2009 \\
\hline HQ558103 & 1 & Yes & 18-Aug-2008 \\
\hline \multirow[t]{10}{*}{ JF903508 } & 1 & Yes & 09-Aug-2004 \\
\hline & 1 & Yes & \\
\hline & 0 & Yes & \\
\hline & 0 & Yes & 02-Aug-2004 \\
\hline & 1 & Yes & 27-Apr-2002 \\
\hline & 1 & Yes & 27-Apr-2002 \\
\hline & 1 & Yes & 27-Apr-2002 \\
\hline & 1 & Yes & 27-Apr-2002 \\
\hline & 1 & Yes & 27-Apr-2002 \\
\hline & 0 & Yes & 09-Aug-2004 \\
\hline \multirow[t]{3}{*}{ JF903509 } & 1 & Yes & \\
\hline & 2 & Yes & 09-Sep-2003 \\
\hline & 4 & Yes & 07-Jun-2006 \\
\hline \multirow[t]{3}{*}{ JF903510 } & 4 & Yes & 07-Jun-2006 \\
\hline & 2 & Yes & 07-Jun-2006 \\
\hline & 2 & Yes & \\
\hline \multirow[t]{5}{*}{ JF903511 } & 2 & Yes & 02-Jun-2006 \\
\hline & 2 & Yes & 24-May-2006 \\
\hline & 2 & Yes & \\
\hline & 2 & Yes & 13-Jun-2007 \\
\hline & 2 & Yes & \\
\hline \multirow[t]{2}{*}{ FJ663063 } & 2 & No & 16-Jul-2003 \\
\hline & 2 & No & 14-Aug-2005 \\
\hline
\end{tabular}




\begin{tabular}{|c|c|c|c|}
\hline & 2 & No & 23-Jul-2006 \\
\hline FJ663070 & 1 & Yes & 01-Oct-2006 \\
\hline FJ663067 & 1 & Yes & \\
\hline FJ663069 & 1 & Yes & 21-Jul-2006 \\
\hline FJ663064 & 1 & Yes & 26-Jul-2003 \\
\hline FJ663065 & 1 & Yes & 08-Aug-2006 \\
\hline FJ663061 & 1 & Yes & 24-May-2006 \\
\hline FJ663066 & 1 & Yes & 06-Aug-2006 \\
\hline FJ663071 & 2 & Yes & 19-Jul-2006 \\
\hline FJ663068 & 1 & Yes & 21-Jul-2006 \\
\hline FJ663062 & 1 & Yes & 24-May-2006 \\
\hline GU666303 & 2 & Yes & 05-Aug-2007 \\
\hline GU666306 & 2 & Yes & \\
\hline HM405336 & 1 & Yes & 19-Jun-2007 \\
\hline HM905750 & 0 & Yes & 30-Jul-2009 \\
\hline HM405335 & 1 & Yes & 01-Aug-2007 \\
\hline HM905744 & 0 & Yes & 23-Sep-2009 \\
\hline HM405338 & 1 & Yes & 31-Jul-2007 \\
\hline \multirow[t]{25}{*}{ HM405339 } & 1 & Yes & 20-Jun-2007 \\
\hline & 0 & Yes & \\
\hline & 2 & Yes & 08-Jul-2005 \\
\hline & 2 & No & 01-Jun-2005 \\
\hline & 0 & Yes & 23-Aug-2005 \\
\hline & 0 & Yes & \\
\hline & 2 & Yes & 01-Jun-2005 \\
\hline & 3 & No & \\
\hline & 2 & No & 15-Aug-2006 \\
\hline & 2 & Yes & \\
\hline & 2 & Yes & \\
\hline & 2 & Yes & 16-Jul-2004 \\
\hline & 2 & Yes & 17-Jul-2006 \\
\hline & 2 & Yes & 17-Jul-2006 \\
\hline & 2 & Yes & 17-Jul-2006 \\
\hline & 0 & No & 15-Aug-2006 \\
\hline & 0 & Yes & 23-Sep-2006 \\
\hline & 2 & Yes & 18-Aug-2006 \\
\hline & 2 & Yes & \\
\hline & 2 & Yes & 17-Jul-2006 \\
\hline & 2 & Yes & 17-Jul-2006 \\
\hline & 2 & Yes & 06-Oct-2006 \\
\hline & 2 & Yes & 06-Oct-2006 \\
\hline & 2 & Yes & \\
\hline & 2 & Yes & \\
\hline \multirow[t]{12}{*}{ GU666301 } & 0 & Yes & 09-Jun-2007 \\
\hline & 2 & Yes & \\
\hline & 2 & Yes & \\
\hline & 2 & No & 15-Aug-2006 \\
\hline & 2 & Yes & \\
\hline & 2 & Yes & \\
\hline & 2 & No & 23-Sep-2006 \\
\hline & 2 & Yes & \\
\hline & 2 & Yes & \\
\hline & 2 & Yes & \\
\hline & 0 & Yes & 23-Sep-2006 \\
\hline & 2 & Yes & \\
\hline
\end{tabular}




\begin{tabular}{|c|c|c|c|}
\hline & 2 & Yes & 17-Aug-2004 \\
\hline & 0 & Yes & 04-May-2004 \\
\hline \multirow[t]{6}{*}{ JF903512 } & 2 & No & 23-Sep-2006 \\
\hline & 2 & Yes & 23-Jul-2006 \\
\hline & 2 & Yes & 04-Aug-2006 \\
\hline & 2 & Yes & 11-Jul-2005 \\
\hline & 2 & Yes & 17-Aug-2006 \\
\hline & 2 & Yes & \\
\hline \multirow[t]{3}{*}{ HM905754 } & 0 & Yes & 12-Aug-2009 \\
\hline & 3 & Yes & 20-Jun-2005 \\
\hline & 3 & Yes & 03-Oct-2006 \\
\hline \multirow[t]{12}{*}{ JF903513 } & 3 & Yes & 17-Aug-2006 \\
\hline & 3 & Yes & 06-Aug-2006 \\
\hline & 0 & Yes & \\
\hline & 1 & Yes & 02-Jun-2005 \\
\hline & 1 & Yes & 07-Sep-2006 \\
\hline & 1 & Yes & 07-Sep-2006 \\
\hline & 1 & Yes & 02-Jun-2005 \\
\hline & 4 & No & 01-Aug-2001 \\
\hline & 4 & No & 22-Jun-2003 \\
\hline & 2 & Yes & \\
\hline & 2 & No & 02-Jul-2003 \\
\hline & 3 & No & 06-Jun-2001 \\
\hline \multirow[t]{9}{*}{ JF903514 } & 5 & No & 22-Мay-2003 \\
\hline & 2 & Yes & 09-Aug-2004 \\
\hline & 2 & Yes & \\
\hline & 2 & Yes & \\
\hline & 2 & Yes & \\
\hline & 0 & Yes & \\
\hline & 0 & Yes & 14-Jul-2006 \\
\hline & 0 & Yes & 08-Sep-2005 \\
\hline & 0 & Yes & 07-Sep-2005 \\
\hline \multirow[t]{3}{*}{ JF903523 } & 0 & Yes & 30-Jun-2006 \\
\hline & 0 & Yes & 06-Sep-2005 \\
\hline & 0 & Yes & 21-Aug-2008 \\
\hline \multirow[t]{10}{*}{ HM404563 } & 0 & Yes & 30-Jul-2008 \\
\hline & 1 & Yes & 30-Jun-2006 \\
\hline & 1 & Yes & \\
\hline & 1 & Yes & \\
\hline & 1 & No & 07-Jun-2005 \\
\hline & 1 & Yes & \\
\hline & 1 & No & 07-Jun-2005 \\
\hline & 1 & Yes & 30-Aug-2006 \\
\hline & 1 & Yes & 01-Jul-2006 \\
\hline & 1 & Yes & 21-Aug-2003 \\
\hline \multirow[t]{2}{*}{ JF903515 } & 1 & Yes & 01-Jul-2006 \\
\hline & 1 & Yes & 01-Jul-2006 \\
\hline HM905893 & 1 & No & \\
\hline \multirow[t]{4}{*}{ HM905760 } & 0 & Yes & 25-Jun-2009 \\
\hline & 1 & Yes & 24-May-2005 \\
\hline & 3 & Yes & 30-Mar-2006 \\
\hline & 3 & No & 06-Aug-2006 \\
\hline \multirow[t]{3}{*}{ JF903516 } & 1 & Yes & 06-Aug-2006 \\
\hline & 3 & Yes & \\
\hline & 1 & Yes & 08-Aug-2006 \\
\hline
\end{tabular}




\begin{tabular}{|c|c|c|c|}
\hline & 3 & Yes & 09-Aug-2006 \\
\hline & 3 & Yes & \\
\hline & 3 & Yes & 08-Aug-2006 \\
\hline & 3 & Yes & 01-Jul-2006 \\
\hline & 3 & Yes & 08-Aug-2006 \\
\hline & 2 & No & 26-Jul-2004 \\
\hline & 3 & Yes & 05-Sep-2005 \\
\hline & 3 & Yes & \\
\hline & 1 & Yes & \\
\hline & 1 & Yes & \\
\hline & 3 & Yes & \\
\hline & 2 & No & 24-Apr-2002 \\
\hline & 3 & Yes & \\
\hline & 3 & Yes & \\
\hline & 3 & Yes & 17-Sep-2005 \\
\hline & 4 & No & 11-Apr-2003 \\
\hline & 4 & No & 21-Sep-2004 \\
\hline & 4 & No & \\
\hline & 4 & No & 08-Jun-2005 \\
\hline & 4 & No & 13-Apr-2005 \\
\hline & 4 & No & \\
\hline & 5 & No & 14-Apr-2003 \\
\hline & 2 & Yes & 25-Jun-2006 \\
\hline & 3 & Yes & 06-Aug-2006 \\
\hline & 4 & No & \\
\hline & 3 & Yes & 10-Aug-2006 \\
\hline HM405324 & 1 & Yes & 05-Oct-2009 \\
\hline & 0 & Yes & 30-Sep-2003 \\
\hline & 1 & Yes & 21-Sep-2006 \\
\hline & 3 & Yes & 08-Aug-2006 \\
\hline & 3 & No & 01-Oct-2003 \\
\hline & 3 & No & 24-Apr-2003 \\
\hline & 3 & Yes & 25-Jun-2006 \\
\hline & 3 & Yes & 25-Jun-2006 \\
\hline & 3 & Yes & 08-Aug-2006 \\
\hline & 3 & No & 09-Aug-2006 \\
\hline & 3 & Yes & 25-Jun-2006 \\
\hline & 3 & Yes & 07-Sep-2006 \\
\hline & 3 & Yes & 05-Aug-2006 \\
\hline & 3 & Yes & 07-Aug-2006 \\
\hline & 3 & Yes & 04-Jun-2006 \\
\hline & 3 & Yes & 08-Aug-2006 \\
\hline & 3 & Yes & 05-Aug-2006 \\
\hline & 3 & Yes & \\
\hline & 3 & Yes & 08-Aug-2006 \\
\hline & 3 & Yes & \\
\hline & 3 & Yes & 05-Aug-2006 \\
\hline & 3 & Yes & 05-Aug-2006 \\
\hline & 3 & Yes & 01-Jul-2006 \\
\hline & 3 & Yes & 05-Aug-2006 \\
\hline & 3 & Yes & 21-Sep-2006 \\
\hline & 3 & Yes & 10-Aug-2006 \\
\hline & 3 & Yes & \\
\hline & 3 & Yes & 25-Jun-2006 \\
\hline JF903536 & 1 & Yes & 25-Jun-2006 \\
\hline
\end{tabular}




\begin{tabular}{|c|c|c|c|}
\hline & 3 & Yes & 08-Aug-2006 \\
\hline & 3 & Yes & 09-Aug-2006 \\
\hline & 3 & Yes & 05-Aug-2006 \\
\hline & 3 & Yes & \\
\hline & 3 & Yes & \\
\hline & 3 & Yes & \\
\hline & 2 & Yes & 08-Jul-2006 \\
\hline & 2 & Yes & 05-Aug-2006 \\
\hline & 3 & Yes & 05-Aug-2006 \\
\hline & 3 & Yes & \\
\hline & 3 & Yes & \\
\hline & 3 & Yes & \\
\hline & 3 & Yes & 25-Jun-2006 \\
\hline & 3 & Yes & \\
\hline & 3 & Yes & \\
\hline & 3 & Yes & 27-Jun-2006 \\
\hline & 3 & Yes & \\
\hline \multirow[t]{12}{*}{ HM405322 } & 1 & Yes & 05-Oct-2009 \\
\hline & 0 & Yes & \\
\hline & 0 & Yes & \\
\hline & 1 & Yes & 27-Apr-2002 \\
\hline & 1 & Yes & \\
\hline & 3 & Yes & \\
\hline & 2 & Yes & 07-Jul-2004 \\
\hline & 3 & Yes & 15-May-2003 \\
\hline & 3 & Yes & 27-Apr-2002 \\
\hline & 3 & Yes & 10-Aug-2007 \\
\hline & 2 & Yes & \\
\hline & 0 & Yes & \\
\hline \multirow[t]{10}{*}{ JF903517 } & 1 & Yes & 02-May-2006 \\
\hline & 2 & Yes & 23-Aug-2007 \\
\hline & 3 & Yes & \\
\hline & 2 & Yes & 19-Oct-2004 \\
\hline & 3 & Yes & \\
\hline & 3 & Yes & \\
\hline & 0 & Yes & 05-Jul-2001 \\
\hline & 0 & Yes & 23-Aug-2007 \\
\hline & 3 & No & 06-May-2002 \\
\hline & 2 & Yes & 05-Feb-2004 \\
\hline \multirow[t]{6}{*}{ JF903518 } & 1 & Yes & 17-May-2002 \\
\hline & 2 & Yes & 24-Aug-2004 \\
\hline & 4 & Yes & 08-Aug-2006 \\
\hline & 4 & Yes & 09-Aug-2006 \\
\hline & 3 & Yes & 09-Sep-2005 \\
\hline & 4 & Yes & \\
\hline \multirow[t]{6}{*}{ JF903519 } & 2 & Yes & 08-Aug-2006 \\
\hline & 1 & Yes & \\
\hline & 1 & Yes & \\
\hline & 1 & Yes & \\
\hline & 1 & Yes & \\
\hline & 1 & Yes & 06-Jul-2007 \\
\hline \multirow[t]{4}{*}{ JF903520 } & 1 & Yes & 01-Jun-2008 \\
\hline & 1 & Yes & 31-May-2008 \\
\hline & 1 & Yes & 30-May-2008 \\
\hline & 1 & Yes & 16-Jul-2008 \\
\hline
\end{tabular}




\begin{tabular}{|c|c|c|c|}
\hline & & & \multirow{3}{*}{ 26-Jul-2007 } \\
\hline & 2 & Yes & \\
\hline & 4 & Yes & \\
\hline & 4 & Yes & \\
\hline & 4 & Yes & \\
\hline & 4 & Yes & 06-Sep-2006 \\
\hline & 4 & Yes & \\
\hline & 4 & No & 08-Aug-2006 \\
\hline & 4 & Yes & \\
\hline & 4 & Yes & 10-Aug-2006 \\
\hline & 4 & Yes & 14-Aug-2006 \\
\hline & 2 & Yes & 14-Aug-2006 \\
\hline & 4 & Yes & 10-Aug-2006 \\
\hline & 4 & Yes & 25-Jun-2006 \\
\hline & 4 & Yes & 05-Aug-2006 \\
\hline & 4 & Yes & 09-Aug-2006 \\
\hline & 4 & Yes & \\
\hline & 4 & Yes & 08-Aug-2006 \\
\hline & 4 & Yes & \\
\hline & 4 & Yes & 06-Aug-2006 \\
\hline & 4 & Yes & 08-Aug-2006 \\
\hline & 4 & Yes & \\
\hline \multirow[t]{14}{*}{ JF903521 } & 2 & Yes & 23-Sep-2006 \\
\hline & 4 & Yes & 08-Aug-2006 \\
\hline & 4 & No & 10-Aug-2006 \\
\hline & 4 & No & 09-Aug-2006 \\
\hline & 4 & Yes & 19-Jul-2006 \\
\hline & 2 & Yes & \\
\hline & 2 & Yes & \\
\hline & 3 & Yes & 14-May-1997 \\
\hline & 3 & Yes & 13-Aug-2005 \\
\hline & 3 & Yes & 13-Aug-2005 \\
\hline & 3 & Yes & 13-Aug-2005 \\
\hline & 3 & Yes & 13-Aug-2005 \\
\hline & 2 & Yes & 31-May-2008 \\
\hline & 3 & Yes & \\
\hline \multirow[t]{8}{*}{ JF903522 } & 1 & Yes & 29-May-2007 \\
\hline & 2 & Yes & 31-May-2008 \\
\hline & 2 & Yes & 30-May-2008 \\
\hline & 3 & Yes & \\
\hline & 3 & Yes & \\
\hline & 0 & Yes & 08-Oct-2008 \\
\hline & 0 & Yes & 11-Jul-2002 \\
\hline & 0 & Yes & 18-Jul-2005 \\
\hline \multirow[t]{2}{*}{ HM884228 } & 1 & Yes & 08-Oct-2008 \\
\hline & 0 & Yes & 20-May-2004 \\
\hline HM407358 & 1 & Yes & 02-May-2008 \\
\hline HM404570 & 0 & Yes & 08-Oct-2008 \\
\hline \multirow[t]{8}{*}{ HQ938022 } & 1 & Yes & 20-Oct-2009 \\
\hline & 2 & Yes & \\
\hline & 2 & Yes & \\
\hline & 0 & Yes & \\
\hline & 2 & Yes & \\
\hline & 2 & Yes & \\
\hline & 2 & Yes & 01-May-2005 \\
\hline & 2 & Yes & 16-Jun-2005 \\
\hline
\end{tabular}




\begin{tabular}{|c|c|c|c|}
\hline & 2 & \multicolumn{2}{|l|}{ Yes } \\
\hline & 2 & Yes & \\
\hline & 2 & Yes & 01-May-2005 \\
\hline & 2 & Yes & 28-Jun-2005 \\
\hline & 2 & Yes & 30-May-2005 \\
\hline & 2 & No & 11-May-2004 \\
\hline & 2 & Yes & 26-May-2003 \\
\hline & 0 & Yes & \\
\hline & 2 & Yes & 13-Aug-2005 \\
\hline & 2 & Yes & 14-May-2003 \\
\hline & 2 & Yes & \\
\hline & 2 & Yes & \\
\hline & 2 & Yes & \\
\hline & 2 & Yes & \\
\hline & 2 & Yes & 14-Маy-2003 \\
\hline & 2 & Yes & 14-May-2003 \\
\hline \multirow[t]{5}{*}{ HQ557882 } & 0 & Yes & 14-May-2009 \\
\hline & 2 & Yes & 23-Apr-2004 \\
\hline & 2 & Yes & 07-May-2004 \\
\hline & 2 & Yes & 23-Jun-2007 \\
\hline & 2 & Yes & 13-Aug-2004 \\
\hline \multirow[t]{5}{*}{ JF903526 } & 2 & Yes & 20-Jun-2005 \\
\hline & 2 & Yes & 28-Мay-2007 \\
\hline & 2 & Yes & 12-May-2004 \\
\hline & 2 & Yes & 11-May-2004 \\
\hline & 2 & Yes & 27-Apr-2005 \\
\hline \multirow[t]{2}{*}{ GU666307 } & 2 & Yes & 22-Jun-2008 \\
\hline & 2 & Yes & \\
\hline \multirow[t]{27}{*}{ HM405345 } & 1 & Yes & 02-Aug-2009 \\
\hline & 2 & Yes & \\
\hline & 2 & Yes & \\
\hline & 4 & Yes & 07-Jul-2006 \\
\hline & 4 & Yes & 04-Jul-2003 \\
\hline & 2 & Yes & \\
\hline & 2 & No & 08-Jul-2003 \\
\hline & 4 & No & \\
\hline & 5 & No & \\
\hline & 2 & Yes & 07-Aug-2001 \\
\hline & 4 & Yes & 07-Aug-2006 \\
\hline & 4 & Yes & 03-Jul-2003 \\
\hline & 4 & Yes & 13-May-2003 \\
\hline & 4 & Yes & 06-Aug-2006 \\
\hline & 2 & No & 29-May-2002 \\
\hline & 4 & Yes & 29-May-2002 \\
\hline & 4 & Yes & 06-Jun-2006 \\
\hline & 4 & Yes & 23-Apr-2004 \\
\hline & 4 & Yes & 12-Jul-2003 \\
\hline & 4 & Yes & 13-May-2003 \\
\hline & 4 & Yes & 31-May-2003 \\
\hline & 4 & Yes & 13-Aug-2006 \\
\hline & 4 & Yes & 13-May-2003 \\
\hline & 4 & Yes & 08-Jul-2003 \\
\hline & 4 & Yes & 07-Aug-2006 \\
\hline & 4 & Yes & 06-Jul-2006 \\
\hline & 4 & Yes & 31-Jul-2005 \\
\hline
\end{tabular}




\begin{tabular}{|c|c|c|c|}
\hline & 4 & Yes & 31-Jul-2005 \\
\hline & 4 & Yes & 08-Jul-2006 \\
\hline \multirow[t]{2}{*}{ JF903527 } & 2 & Yes & 16-Aug-2006 \\
\hline & 4 & Yes & \\
\hline \multirow[t]{11}{*}{ GU666299 } & 2 & Yes & 11-Jul-2007 \\
\hline & 2 & Yes & 02-Sep-2007 \\
\hline & 2 & Yes & 02-Sep-2007 \\
\hline & 2 & Yes & 02-Sep-2007 \\
\hline & 4 & Yes & 13-Aug-2006 \\
\hline & 4 & Yes & \\
\hline & 4 & Yes & \\
\hline & 4 & No & 04-Aug-2006 \\
\hline & 4 & No & 16-Aug-2006 \\
\hline & 4 & Yes & 04-Aug-2006 \\
\hline & 4 & Yes & \\
\hline HQ557920 & 1 & Yes & 30-May-2010 \\
\hline FJ663072 & 2 & Yes & 31-Aug-2006 \\
\hline FJ663073 & 2 & Yes & 31-Aug-2006 \\
\hline \multirow[t]{11}{*}{ FJ663074 } & 2 & Yes & 31-Aug-2006 \\
\hline & 0 & Yes & 09-Jul-2009 \\
\hline & 0 & Yes & 06-Jun-2009 \\
\hline & 0 & Yes & 22-Jul-2009 \\
\hline & 2 & Yes & 31-Jul-2005 \\
\hline & 0 & Yes & 27-Jun-2009 \\
\hline & 0 & Yes & 28-Jul-2009 \\
\hline & 0 & Yes & 24-Jun-2009 \\
\hline & 2 & Yes & 17-Jul-2006 \\
\hline & 0 & Yes & 19-Aug-2009 \\
\hline & 2 & Yes & 31-Jul-2005 \\
\hline \multirow[t]{14}{*}{ JF903528 } & 1 & Yes & 21-Jul-2003 \\
\hline & 1 & Yes & 17-May-2003 \\
\hline & 1 & Yes & 12-May-2000 \\
\hline & 1 & Yes & 04-Jun-2006 \\
\hline & 1 & Yes & 04-Jun-2006 \\
\hline & 1 & Yes & 09-Aug-2006 \\
\hline & 2 & Yes & 14-Jul-2003 \\
\hline & 3 & Yes & \\
\hline & 5 & No & 06-Jun-2005 \\
\hline & 3 & Yes & 25-Jul-2005 \\
\hline & 3 & Yes & \\
\hline & 3 & Yes & \\
\hline & 4 & No & 24-May-2003 \\
\hline & 3 & Yes & 10-Jun-2005 \\
\hline \multirow[t]{7}{*}{ HQ937902 } & 1 & Yes & 11-Маy-2009 \\
\hline & 0 & Yes & 14-Jun-2005 \\
\hline & 3 & Yes & 13-Aug-2006 \\
\hline & 3 & Yes & \\
\hline & 3 & Yes & \\
\hline & 1 & Yes & 06-Jun-2005 \\
\hline & 3 & Yes & 22-Sep-2006 \\
\hline \multirow[t]{5}{*}{ JF903529 } & 1 & Yes & 10-Sep-2005 \\
\hline & 3 & Yes & 31-Мay-2007 \\
\hline & 1 & Yes & 04-Jun-2006 \\
\hline & 1 & Yes & 04-Jun-2006 \\
\hline & 1 & Yes & 04-Jun-2006 \\
\hline
\end{tabular}




\begin{tabular}{|c|c|c|c|}
\hline & 1 & Yes & 04-Jun-2006 \\
\hline & 1 & Yes & 04-Jun-2006 \\
\hline & 1 & Yes & \\
\hline \multirow[t]{3}{*}{ JF903530 } & 1 & Yes & 19-May-2006 \\
\hline & 2 & Yes & 02-Jun-2005 \\
\hline & 1 & Yes & \\
\hline \multirow[t]{5}{*}{ JF903532 } & 2 & Yes & 25-Jul-2008 \\
\hline & 2 & Yes & 27-Jul-2008 \\
\hline & 1 & Yes & 28-Jun-2005 \\
\hline & 1 & Yes & 26-Jul-2005 \\
\hline & 1 & Yes & 12-Jun-2005 \\
\hline \multirow[t]{4}{*}{ JF903533 } & 1 & Yes & 25-Jun-2005 \\
\hline & 1 & Yes & 13-Jul-2005 \\
\hline & 0 & Yes & 09-Aug-2004 \\
\hline & 0 & Yes & 03-Aug-2005 \\
\hline GU708012 & 0 & Yes & 16-Jul-2008 \\
\hline GU708010 & 0 & Yes & 13-Jul-2008 \\
\hline FJ663076 & 1 & Yes & 01-Jul-2006 \\
\hline FJ663081 & 1 & Yes & 01-Jul-2006 \\
\hline FJ663082 & 1 & Yes & 18-Jul-2006 \\
\hline FJ663080 & 1 & Yes & 01-Jul-2006 \\
\hline FJ663079 & 1 & Yes & 01-Jul-2006 \\
\hline FJ663078 & 1 & Yes & 01-Jul-2006 \\
\hline \multirow[t]{3}{*}{ FJ663077 } & 1 & Yes & 01-Jul-2006 \\
\hline & 0 & Yes & 08-Sep-2005 \\
\hline & 4 & Yes & 18-Oct-2004 \\
\hline \multirow[t]{7}{*}{ JF903534 } & 2 & Yes & 13-Aug-2006 \\
\hline & 4 & Yes & \\
\hline & 0 & Yes & \\
\hline & 0 & Yes & \\
\hline & 0 & Yes & 04-Jun-2007 \\
\hline & 0 & Yes & 04-Jun-2007 \\
\hline & 0 & Yes & 04-Jun-2007 \\
\hline \multirow[t]{15}{*}{ GU666311 } & 0 & Yes & 04-Jun-2007 \\
\hline & 2 & Yes & \\
\hline & 2 & Yes & \\
\hline & 2 & Yes & \\
\hline & 2 & Yes & \\
\hline & 2 & Yes & \\
\hline & 2 & Yes & 23-Jun-1999 \\
\hline & 2 & Yes & \\
\hline & 3 & Yes & \\
\hline & 3 & Yes & \\
\hline & 3 & Yes & 05-Jul-2003 \\
\hline & 2 & Yes & 31-May-2008 \\
\hline & 3 & Yes & 27-Jul-2001 \\
\hline & 2 & Yes & 22-May-2001 \\
\hline & 3 & Yes & \\
\hline \multirow[t]{7}{*}{ JF903537 } & 1 & Yes & 07-Sep-2000 \\
\hline & 3 & Yes & \\
\hline & 3 & Yes & \\
\hline & 3 & Yes & \\
\hline & 2 & Yes & 16-Aug-2008 \\
\hline & 3 & Yes & \\
\hline & 3 & Yes & \\
\hline
\end{tabular}




\begin{tabular}{|c|c|c|c|}
\hline \multirow[t]{6}{*}{ HQ558129 } & 1 & Yes & 28-Aug-2009 \\
\hline & 2 & Yes & \\
\hline & 3 & Yes & 18-Aug-2006 \\
\hline & 0 & Yes & 09-Jul-2007 \\
\hline & 2 & Yes & 25-Jun-2003 \\
\hline & 3 & Yes & 28-May-2006 \\
\hline \multirow[t]{14}{*}{ JF903538 } & 1 & Yes & 07-Jun-2006 \\
\hline & 2 & Yes & 28-Jul-2006 \\
\hline & 3 & Yes & 07-Jul-2006 \\
\hline & 2 & Yes & \\
\hline & 2 & Yes & \\
\hline & 2 & Yes & \\
\hline & 0 & Yes & \\
\hline & 2 & Yes & \\
\hline & 2 & Yes & \\
\hline & 2 & Yes & \\
\hline & 2 & No & \\
\hline & 2 & No & \\
\hline & 2 & Yes & \\
\hline & 2 & Yes & 04-Jun-2006 \\
\hline \multirow[t]{2}{*}{ JF903539 } & 2 & Yes & 13-Jul-2006 \\
\hline & 2 & Yes & \\
\hline \multirow[t]{9}{*}{ HM405321 } & 1 & Yes & 09-Aug-2008 \\
\hline & 2 & Yes & \\
\hline & 2 & Yes & \\
\hline & 2 & Yes & \\
\hline & 0 & Yes & \\
\hline & 2 & Yes & \\
\hline & 6 & No & 31-Jul-2002 \\
\hline & 2 & Yes & \\
\hline & 2 & Yes & 21-Jul-2006 \\
\hline \multirow[t]{2}{*}{ HQ561111 } & 2 & Yes & 31-Aug-2004 \\
\hline & 2 & No & \\
\hline \multirow[t]{11}{*}{ JF903540 } & 2 & Yes & \\
\hline & 2 & Yes & \\
\hline & 2 & Yes & 05-Aug-2006 \\
\hline & 3 & No & 08-Aug-2006 \\
\hline & 2 & No & \\
\hline & 2 & Yes & 30-Jul-2005 \\
\hline & 2 & Yes & 21-Jul-2005 \\
\hline & 0 & Yes & \\
\hline & 2 & Yes & 07-Aug-2006 \\
\hline & 3 & Yes & 06-Jun-2005 \\
\hline & 3 & Yes & 13-Aug-2006 \\
\hline \multirow[t]{6}{*}{ JF903541 } & 1 & Yes & 06-Aug-2006 \\
\hline & 2 & Yes & 28-Jul-2006 \\
\hline & 2 & Yes & 12-Jun-2007 \\
\hline & 3 & Yes & \\
\hline & 3 & Yes & 06-Aug-2007 \\
\hline & 3 & Yes & \\
\hline \multirow[t]{5}{*}{ JF903542 } & 2 & Yes & 12-Jun-2007 \\
\hline & 3 & Yes & \\
\hline & 2 & Yes & 11-May-2004 \\
\hline & 3 & Yes & \\
\hline & 0 & Yes & \\
\hline
\end{tabular}




\begin{tabular}{|c|c|c|c|}
\hline \multirow[t]{4}{*}{ HQ558137 } & 0 & Yes & 28-Aug-2009 \\
\hline & 1 & Yes & 12-May-2004 \\
\hline & 3 & Yes & \\
\hline & 3 & Yes & 05-Aug-2006 \\
\hline \multirow[t]{20}{*}{ JF903543 } & 1 & Yes & 13-Aug-2006 \\
\hline & 3 & Yes & 13-Aug-2006 \\
\hline & 1 & Yes & 04-Jun-2003 \\
\hline & 1 & Yes & 18-Jun-2004 \\
\hline & 1 & Yes & 13-May-2003 \\
\hline & 1 & Yes & 14-May-2003 \\
\hline & 1 & Yes & 12-May-2003 \\
\hline & 1 & Yes & 01-Jul-2003 \\
\hline & 1 & Yes & 11-May-2005 \\
\hline & 1 & Yes & 09-Apr-2004 \\
\hline & 1 & Yes & 16-Маy-2003 \\
\hline & 1 & Yes & 22-May-2003 \\
\hline & 1 & Yes & 25-May-2003 \\
\hline & 1 & Yes & 19-Мау-2003 \\
\hline & 1 & Yes & 14-Мау-2003 \\
\hline & 1 & Yes & 20-May-2003 \\
\hline & 1 & Yes & 22-Мay-2003 \\
\hline & 1 & Yes & 31-May-2003 \\
\hline & 1 & Yes & 14-Маy-2003 \\
\hline & 1 & Yes & 15-Aug-2007 \\
\hline HM405342 & 1 & Yes & 03-Мау-2009 \\
\hline \multirow[t]{2}{*}{ GU708184 } & 1 & No & 01-Jul-2007 \\
\hline & 2 & No & 13-Jun-2007 \\
\hline \multirow[t]{8}{*}{ JF903544 } & 2 & Yes & 04-Jun-2007 \\
\hline & 2 & Yes & 13-Jun-2007 \\
\hline & 2 & Yes & 04-Jun-2007 \\
\hline & 2 & No & 30-Мау-2004 \\
\hline & 2 & Yes & 25-Jun-2006 \\
\hline & 2 & Yes & 13-Aug-2006 \\
\hline & 2 & Yes & 28-Jun-2005 \\
\hline & 2 & Yes & 25-Jun-2006 \\
\hline \multirow[t]{2}{*}{ JF903545 } & 2 & Yes & 23-Jul-2006 \\
\hline & 2 & Yes & 18-Jun-2005 \\
\hline \multirow[t]{9}{*}{ GU707990 } & 0 & Yes & 20-Jul-2007 \\
\hline & 3 & Yes & \\
\hline & 3 & Yes & 30-Jun-2005 \\
\hline & 4 & Yes & 22-Jul-1992 \\
\hline & 3 & Yes & \\
\hline & 3 & Yes & 06-Jun-2005 \\
\hline & 3 & Yes & 01-Jun-2005 \\
\hline & 3 & No & 01-Jul-2003 \\
\hline & 2 & Yes & 03-Jul-2002 \\
\hline \multirow[t]{9}{*}{ JF903547 } & 1 & Yes & 15-Jun-2005 \\
\hline & 3 & Yes & 20-Jun-2005 \\
\hline & 2 & Yes & \\
\hline & 3 & Yes & \\
\hline & 3 & Yes & 20-Jul-2005 \\
\hline & 3 & Yes & 22-Aug-2005 \\
\hline & 3 & Yes & 20-Jul-2005 \\
\hline & 3 & Yes & 11-Sep-2005 \\
\hline & 3 & No & 10-Jul-2001 \\
\hline
\end{tabular}




\begin{tabular}{|c|c|c|c|}
\hline & 3 & No & 10-Jul-2001 \\
\hline & 3 & No & 10-Jul-2001 \\
\hline & 4 & No & 30-Jul-2003 \\
\hline & 3 & No & 10-Jul-2001 \\
\hline \multirow[t]{22}{*}{ JF903548 } & 1 & Yes & 16-Aug-2005 \\
\hline & 3 & Yes & 15-Jun-2005 \\
\hline & 3 & Yes & 20-Jun-2005 \\
\hline & 2 & Yes & 18-Aug-2003 \\
\hline & 2 & Yes & 23-Jul-2006 \\
\hline & 3 & Yes & \\
\hline & 3 & No & 06-Aug-2006 \\
\hline & 1 & No & \\
\hline & 3 & No & \\
\hline & 0 & Yes & 30-Jul-2009 \\
\hline & 3 & Yes & 17-Jul-2006 \\
\hline & 3 & Yes & 20-Aug-2004 \\
\hline & 3 & Yes & 14-Aug-2006 \\
\hline & 3 & Yes & 05-Aug-2006 \\
\hline & 0 & Yes & 22-Jul-2009 \\
\hline & 3 & Yes & 14-Aug-2006 \\
\hline & 3 & Yes & 01-Jul-2006 \\
\hline & 3 & Yes & 14-Aug-2006 \\
\hline & 3 & Yes & 08-Aug-2006 \\
\hline & 3 & Yes & 07-Aug-2006 \\
\hline & 3 & Yes & 14-Jul-2001 \\
\hline & 1 & Yes & 16-Jul-2004 \\
\hline \multirow[t]{8}{*}{ JF903549 } & 1 & Yes & 06-Aug-2006 \\
\hline & 1 & Yes & 11-Jul-2002 \\
\hline & 2 & No & \\
\hline & 1 & Yes & 31-Jul-2005 \\
\hline & 1 & Yes & 31-Jul-2005 \\
\hline & 1 & Yes & 31-Jul-2005 \\
\hline & 1 & Yes & 31-Jul-2005 \\
\hline & 1 & Yes & 21-Jun-2005 \\
\hline \multirow[t]{8}{*}{ GU708181 } & 1 & No & 23-Jun-2007 \\
\hline & 2 & Yes & 07-Oct-2004 \\
\hline & 2 & Yes & 18-Oct-2004 \\
\hline & 3 & Yes & 07-Aug-2006 \\
\hline & 3 & No & 07-Apr-1999 \\
\hline & 3 & No & 22-Aug-2004 \\
\hline & 3 & Yes & 18-Oct-2004 \\
\hline & 3 & Yes & 03-Sep-2003 \\
\hline \multirow[t]{11}{*}{ JF903550 } & 1 & Yes & 02-Apr-2002 \\
\hline & 2 & Yes & 07-Oct-2004 \\
\hline & 2 & Yes & 07-Oct-2004 \\
\hline & 3 & Yes & 19-Apr-2006 \\
\hline & 2 & Yes & 21-Jun-2007 \\
\hline & 3 & Yes & \\
\hline & 2 & Yes & 07-Aug-2008 \\
\hline & 3 & Yes & \\
\hline & 3 & Yes & \\
\hline & 2 & Yes & 11-Jun-2007 \\
\hline & 3 & Yes & 12-Мау-2005 \\
\hline \multirow[t]{2}{*}{ JF903551 } & 2 & Yes & 13-Jun-2007 \\
\hline & 3 & Yes & \\
\hline
\end{tabular}




\begin{tabular}{|c|c|c|c|}
\hline & 3 & Yes & \multirow[b]{2}{*}{ 18-Jul-2008 } \\
\hline & 2 & Yes & \\
\hline & 3 & Yes & \\
\hline & 2 & Yes & 11-Jul-2008 \\
\hline & 2 & Yes & 18-Jul-2008 \\
\hline & 2 & Yes & 21-Jun-2007 \\
\hline & 2 & Yes & \\
\hline & 2 & Yes & 12-Jul-2002 \\
\hline & 3 & No & 20-Sep-2005 \\
\hline & 3 & No & 05-Sep-2005 \\
\hline & 3 & Yes & 30-Aug-2005 \\
\hline & 3 & Yes & 20-Sep-2005 \\
\hline & 3 & Yes & 16-Aug-2005 \\
\hline & 3 & Yes & 14-Jun-1999 \\
\hline \multirow[t]{6}{*}{ JF903552 } & 1 & Yes & 05-Jul-2006 \\
\hline & 3 & Yes & \\
\hline & 2 & Yes & \\
\hline & 1 & Yes & 03-Jul-2007 \\
\hline & 2 & Yes & \\
\hline & 2 & Yes & 24-Jun-2007 \\
\hline HM405351 & 1 & Yes & 04-May-2009 \\
\hline FJ663098 & 2 & Yes & 25-Jan-2005 \\
\hline FJ663083 & 2 & No & 27-Jan-2005 \\
\hline FJ663085 & 2 & No & 27-Jan-2005 \\
\hline FJ663084 & 2 & No & 27-Jan-2005 \\
\hline FJ663095 & 2 & Yes & 07-Sep-2005 \\
\hline FJ663097 & 2 & Yes & 27-Jan-2005 \\
\hline \multirow[t]{2}{*}{ FJ663093 } & 2 & Yes & 31-Aug-2006 \\
\hline & 0 & Yes & 04-Jun-2006 \\
\hline FJ663096 & 2 & Yes & 27-Jan-2005 \\
\hline FJ663088 & 2 & Yes & 19-May-2006 \\
\hline \multirow[t]{3}{*}{ FJ663094 } & 2 & Yes & 06-Sep-2006 \\
\hline & 0 & Yes & 04-Jun-2006 \\
\hline & 0 & Yes & 04-Jun-2006 \\
\hline FJ663092 & 2 & Yes & 27-Jan-2005 \\
\hline FJ663091 & 2 & Yes & 25-Jan-2005 \\
\hline FJ663090 & 2 & Yes & 19-May-2006 \\
\hline FJ663087 & 2 & Yes & 19-May-2006 \\
\hline FJ663086 & 2 & Yes & 25-Jan-2005 \\
\hline FJ663089 & 2 & Yes & 19-May-2006 \\
\hline HM405312 & 1 & Yes & 23-Oct-2009 \\
\hline \multirow[t]{3}{*}{ JF903553 } & 0 & Yes & 18-Jul-2008 \\
\hline & 0 & Yes & 18-Jul-2008 \\
\hline & 0 & Yes & 18-Jul-2008 \\
\hline \multirow[t]{3}{*}{ JF903554 } & 1 & Yes & 04-Jun-2006 \\
\hline & 3 & Yes & 08-May-2005 \\
\hline & 0 & Yes & 01-Sep-2006 \\
\hline \multirow[t]{8}{*}{ JF903524 } & 1 & Yes & 02-Oct-2002 \\
\hline & 3 & Yes & 20-Jul-2001 \\
\hline & 2 & Yes & 21-Jul-2004 \\
\hline & 3 & Yes & 08-Mar-2005 \\
\hline & 1 & Yes & 04-Aug-1992 \\
\hline & 1 & Yes & 04-Aug-1992 \\
\hline & 1 & Yes & 04-Aug-1992 \\
\hline & 1 & Yes & 04-Aug-1992 \\
\hline
\end{tabular}




\begin{tabular}{|c|c|c|c|}
\hline \multirow[t]{3}{*}{ HQ937997 } & 1 & Yes & 12-Jun-2007 \\
\hline & 3 & Yes & \\
\hline & 2 & Yes & 23-Jun-2007 \\
\hline \multirow[t]{2}{*}{ GU666309 } & 2 & Yes & 24-Jun-2008 \\
\hline & 3 & Yes & \\
\hline HQ557879 & 0 & Yes & 09-May-2007 \\
\hline \multirow[t]{2}{*}{ GU708193 } & 1 & No & 27-Jun-2007 \\
\hline & 1 & Yes & \\
\hline \multirow[t]{10}{*}{ GU707996 } & 0 & Yes & 15-Jun-2007 \\
\hline & 4 & Yes & 12-Jun-2007 \\
\hline & 3 & Yes & \\
\hline & 2 & Yes & 29-Jul-2008 \\
\hline & 2 & Yes & 04-Jun-2007 \\
\hline & 3 & Yes & \\
\hline & 3 & Yes & \\
\hline & 4 & Yes & 15-Jul-2005 \\
\hline & 2 & Yes & 04-Jun-2007 \\
\hline & 2 & Yes & 04-Jun-2007 \\
\hline \multirow[t]{2}{*}{ GU666295 } & 2 & Yes & 23-Aug-2008 \\
\hline & 2 & Yes & 12-Jun-2007 \\
\hline HM905811 & 1 & Yes & 23-Jun-2009 \\
\hline GU708190 & 1 & No & 30-Jul-2007 \\
\hline HQ557880 & 0 & Yes & 22-May-2007 \\
\hline HM405343 & 1 & Yes & 02-May-2009 \\
\hline GU707997 & 0 & Yes & 04-Jun-2007 \\
\hline \multirow[t]{10}{*}{ HQ558128 } & 1 & Yes & 28-Aug-2009 \\
\hline & 1 & Yes & 27-Aug-2004 \\
\hline & 2 & No & 27-May-2007 \\
\hline & 0 & Yes & 16-May-2005 \\
\hline & 2 & Yes & \\
\hline & 2 & Yes & \\
\hline & 2 & Yes & \\
\hline & 2 & Yes & 16-May-2005 \\
\hline & 2 & Yes & 06-Jun-2005 \\
\hline & 2 & Yes & 15-Jun-2005 \\
\hline \multirow[t]{3}{*}{ HM405350 } & 1 & No & 22-Apr-2009 \\
\hline & 2 & Yes & 24-May-2005 \\
\hline & 2 & Yes & 16-May-2005 \\
\hline GU707995 & 0 & Yes & 16-Jun-2007 \\
\hline \multirow[t]{3}{*}{ GU666304 } & 0 & Yes & 09-Jun-2007 \\
\hline & 2 & Yes & 30-Jul-2005 \\
\hline & 2 & Yes & 08-Aug-2005 \\
\hline GU666296 & 2 & Yes & 03-Jun-2007 \\
\hline GU666300 & 2 & Yes & 27-May-2007 \\
\hline GU707994 & 0 & Yes & 16-Jun-2007 \\
\hline HM405348 & 1 & Yes & 22-Apr-2009 \\
\hline GU707992 & 0 & Yes & 11-Jun-2007 \\
\hline GU707993 & 0 & Yes & 16-Jun-2007 \\
\hline HM405347 & 1 & Yes & 22-Apr-2009 \\
\hline HQ561110 & 0 & Yes & 12-Мау-2005 \\
\hline \multirow[t]{5}{*}{ HM407455 } & 1 & Yes & 03-May-2009 \\
\hline & 2 & Yes & 26-Jul-2008 \\
\hline & 2 & Yes & 21-Jun-1999 \\
\hline & 2 & Yes & 25-Jun-2005 \\
\hline & 2 & Yes & 26-May-2005 \\
\hline
\end{tabular}




\begin{tabular}{|c|c|c|c|}
\hline \multirow[t]{3}{*}{ HM405346 } & 1 & Yes & 03-May-2009 \\
\hline & 3 & Yes & \\
\hline & 3 & Yes & \\
\hline \multirow[t]{4}{*}{ HM905895 } & 1 & Yes & 27-Jun-2007 \\
\hline & 2 & No & 18-Jul-2008 \\
\hline & 3 & Yes & \\
\hline & 2 & Yes & 23-Jun-2007 \\
\hline \multirow[t]{7}{*}{ JF903555 } & 3 & Yes & \\
\hline & 3 & Yes & \\
\hline & 2 & Yes & 13-Jul-2008 \\
\hline & 2 & Yes & 13-Jul-2008 \\
\hline & 3 & Yes & \\
\hline & 3 & Yes & \\
\hline & 3 & Yes & \\
\hline \multirow[t]{2}{*}{ GU666308 } & 2 & Yes & 22-Jun-2008 \\
\hline & 3 & Yes & \\
\hline \multirow[t]{12}{*}{ GU708186 } & 1 & No & 27-Jun-2007 \\
\hline & 1 & Yes & 23-Aug-2007 \\
\hline & 0 & No & \\
\hline & 2 & Yes & \\
\hline & 3 & Yes & \\
\hline & 3 & No & 13-Jul-2004 \\
\hline & 3 & Yes & 21-Jul-2004 \\
\hline & 3 & Yes & 01-Aug-2005 \\
\hline & 1 & Yes & 03-Mar-2001 \\
\hline & 3 & Yes & 27-Apr-2007 \\
\hline & 3 & Yes & 01-Sep-2004 \\
\hline & 3 & Yes & 11-May-2004 \\
\hline \multirow[t]{16}{*}{ JF903557 } & 1 & Yes & 29-May-2006 \\
\hline & 3 & Yes & \\
\hline & 3 & Yes & 08-Jun-2004 \\
\hline & 3 & Yes & 01-Aug-2005 \\
\hline & 3 & Yes & 26-Jul-2004 \\
\hline & 2 & Yes & \\
\hline & 3 & Yes & \\
\hline & 3 & Yes & \\
\hline & 3 & Yes & 23-Aug-2007 \\
\hline & 3 & Yes & 01-Jun-2005 \\
\hline & 4 & Yes & 20-Sep-2005 \\
\hline & 3 & Yes & \\
\hline & 3 & Yes & \\
\hline & 3 & Yes & \\
\hline & 4 & Yes & 28-Jun-2005 \\
\hline & 4 & Yes & 05-Sep-2005 \\
\hline \multirow[t]{5}{*}{ HQ558150 } & 1 & Yes & 31-Aug-2007 \\
\hline & 2 & Yes & 01-Aug-2001 \\
\hline & 2 & Yes & 18-Jul-2002 \\
\hline & 2 & Yes & 20-Sep-2001 \\
\hline & 2 & Yes & 09-Oct-2002 \\
\hline \multirow[t]{3}{*}{ JF903558 } & 2 & Yes & 29-May-2002 \\
\hline & 2 & Yes & 29-May-2002 \\
\hline & 2 & Yes & 30-Jul-2005 \\
\hline \multirow[t]{3}{*}{ JF903559 } & 2 & Yes & 13-Apr-2005 \\
\hline & 2 & Yes & 20-Sep-2006 \\
\hline & 1 & No & 03-Apr-2003 \\
\hline
\end{tabular}




\begin{tabular}{|c|c|c|c|}
\hline & 0 & Yes & 28-Jul-1994 \\
\hline \multirow[t]{16}{*}{ GU666297 } & 2 & Yes & 31-Jul-2002 \\
\hline & 2 & Yes & 04-Jun-2007 \\
\hline & 2 & Yes & 04-Jun-2007 \\
\hline & 0 & Yes & 20-May-2004 \\
\hline & 0 & Yes & 29-Mar-1998 \\
\hline & 2 & Yes & 11-Apr-2005 \\
\hline & 0 & Yes & 20-Jun-2002 \\
\hline & 0 & Yes & \\
\hline & 1 & No & \\
\hline & 2 & No & 07-Jul-2001 \\
\hline & 5 & No & 16-Jul-2003 \\
\hline & 0 & No & 07-Jul-2001 \\
\hline & 3 & No & 15-Jul-2003 \\
\hline & 2 & No & 17-Jul-2003 \\
\hline & 2 & No & 13-Apr-2003 \\
\hline & 2 & No & \\
\hline HM404564 & 0 & Yes & 15-May-2008 \\
\hline \multirow[t]{5}{*}{ HM404559 } & 0 & Yes & 08-Oct-2008 \\
\hline & 2 & Yes & 07-Aug-2006 \\
\hline & 0 & Yes & 30-May-2004 \\
\hline & 0 & Yes & 20-Jun-2002 \\
\hline & 2 & Yes & 28-Apr-2004 \\
\hline GU666305 & 2 & No & 11-Jul-2007 \\
\hline \multirow[t]{8}{*}{ HM404572 } & 0 & Yes & 08-Aug-2009 \\
\hline & 0 & Yes & 05-Aug-2006 \\
\hline & 2 & Yes & 01-Jun-2006 \\
\hline & 2 & Yes & \\
\hline & 2 & Yes & 15-Jul-2008 \\
\hline & 2 & Yes & 19-Jul-2006 \\
\hline & 2 & Yes & 23-Jun-2006 \\
\hline & 2 & Yes & 01-Jul-2005 \\
\hline HM407357 & 1 & Yes & 08-Oct-2008 \\
\hline GU708182 & 1 & No & 14-Jul-2008 \\
\hline GU708179 & 1 & No & 07-Jul-2007 \\
\hline HM404561 & 0 & Yes & 15-May-2008 \\
\hline HM404569 & 0 & Yes & 03-Jul-2008 \\
\hline GU708180 & 1 & No & 14-Jul-2008 \\
\hline GU708178 & 1 & No & 12-Aug-2008 \\
\hline \multirow[t]{2}{*}{ HM404532 } & 0 & Yes & 06-Jun-2002 \\
\hline & 0 & Yes & 25-Jun-2003 \\
\hline \multirow[t]{4}{*}{ GU666294 } & 0 & Yes & 20-Jun-2002 \\
\hline & 2 & Yes & 17-Aug-2004 \\
\hline & 3 & Yes & 01-Jun-2005 \\
\hline & 3 & Yes & 29-Aug-2005 \\
\hline \multirow[t]{10}{*}{ JF903560 } & 1 & Yes & 29-Jun-1994 \\
\hline & 3 & Yes & \\
\hline & 3 & No & 27-Jan-2005 \\
\hline & 0 & Yes & \\
\hline & 0 & Yes & \\
\hline & 0 & Yes & 04-Jun-2007 \\
\hline & 0 & Yes & 04-Jun-2007 \\
\hline & 0 & Yes & 24-Jun-2006 \\
\hline & 1 & Yes & 07-Jul-2007 \\
\hline & 1 & Yes & 22-Jun-2007 \\
\hline
\end{tabular}




\begin{tabular}{|c|c|c|c|}
\hline FJ663108 & 1 & Yes & 01-Jul-2006 \\
\hline FJ663110 & 2 & No & 02-Oct-2004 \\
\hline FJ663109 & 2 & No & 06-Sep-2005 \\
\hline FJ663105 & 1 & Yes & 01-Jul-2006 \\
\hline \multirow[t]{4}{*}{ FJ663107 } & 1 & Yes & \\
\hline & 2 & Yes & \\
\hline & 0 & Yes & 04-Jun-2006 \\
\hline & 2 & Yes & 12-Aug-2003 \\
\hline \multirow[t]{2}{*}{ FJ663101 } & 2 & Yes & 07-Jul-2006 \\
\hline & 0 & Yes & 04-Jun-2006 \\
\hline FJ663116 & 2 & Yes & 01-Jul-2006 \\
\hline GU708168 & 0 & Yes & 22-May-2003 \\
\hline FJ663113 & 1 & Yes & 30-May-2004 \\
\hline FJ663106 & 1 & Yes & 01-Jul-2006 \\
\hline FJ663118 & 2 & No & 09-Aug-2006 \\
\hline FJ663117 & 2 & Yes & 09-Aug-2006 \\
\hline FJ663115 & 2 & Yes & 19-May-2006 \\
\hline FJ663114 & 2 & Yes & 01-Oct-2003 \\
\hline FJ663112 & 1 & Yes & 30-May-2004 \\
\hline FJ663111 & 1 & Yes & 30-May-2004 \\
\hline FJ663104 & 1 & Yes & 24-Jun-2006 \\
\hline FJ663103 & 1 & Yes & 24-Jun-2006 \\
\hline \multirow[t]{3}{*}{ FJ663102 } & 1 & Yes & 07-Jun-2005 \\
\hline & 2 & Yes & 16-Jul-2006 \\
\hline & 2 & Yes & 10-May-2007 \\
\hline \multirow[t]{30}{*}{ HQ558104 } & 0 & Yes & 08-Mar-2009 \\
\hline & 2 & Yes & 08-Jul-2008 \\
\hline & 2 & Yes & 05-Jul-2006 \\
\hline & 2 & Yes & 21-Jul-2006 \\
\hline & 2 & Yes & 04-Aug-1992 \\
\hline & 2 & No & 04-Aug-2006 \\
\hline & 2 & Yes & 04-Aug-1992 \\
\hline & 2 & Yes & 04-Aug-1992 \\
\hline & 2 & Yes & 07-Jul-2006 \\
\hline & 2 & Yes & 05-Jul-2006 \\
\hline & 0 & Yes & 08-Jul-2008 \\
\hline & 2 & Yes & 04-Aug-2006 \\
\hline & 2 & Yes & 08-Jul-2008 \\
\hline & 2 & Yes & 04-Aug-1992 \\
\hline & 2 & Yes & 31-Jul-2005 \\
\hline & 2 & Yes & 04-Aug-1992 \\
\hline & 2 & Yes & 22-Jul-1992 \\
\hline & 2 & Yes & 04-Aug-1992 \\
\hline & 2 & Yes & 04-Aug-1992 \\
\hline & 2 & Yes & 22-Jul-1992 \\
\hline & 3 & No & \\
\hline & 3 & Yes & \\
\hline & 3 & Yes & 17-Jun-2002 \\
\hline & 1 & Yes & \\
\hline & 1 & No & \\
\hline & 1 & No & 08-Sep-2005 \\
\hline & 0 & Yes & 17-Sep-2006 \\
\hline & 1 & No & 14-Jun-2005 \\
\hline & 1 & No & 26-Jul-2003 \\
\hline & 1 & No & 30-May-2004 \\
\hline
\end{tabular}




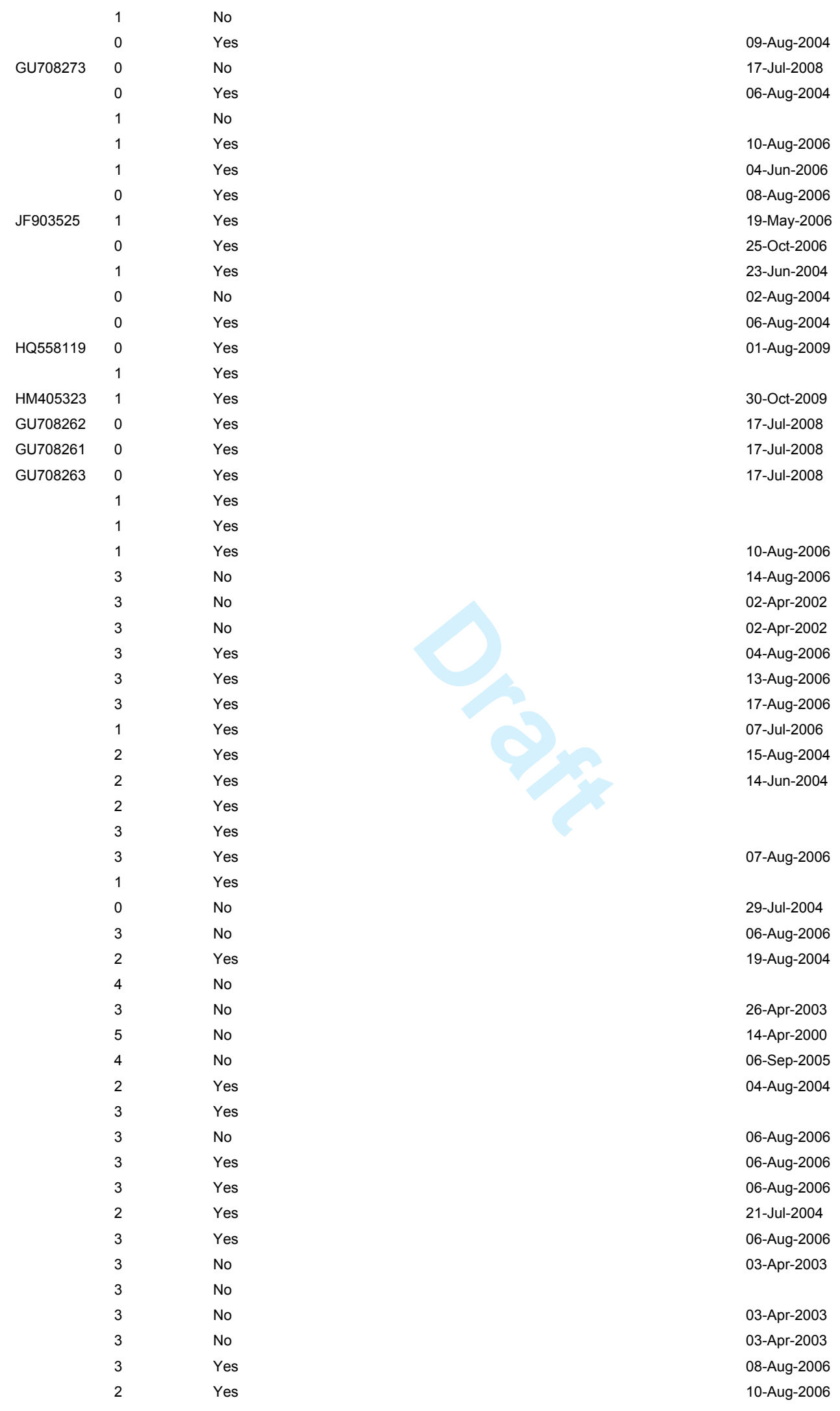




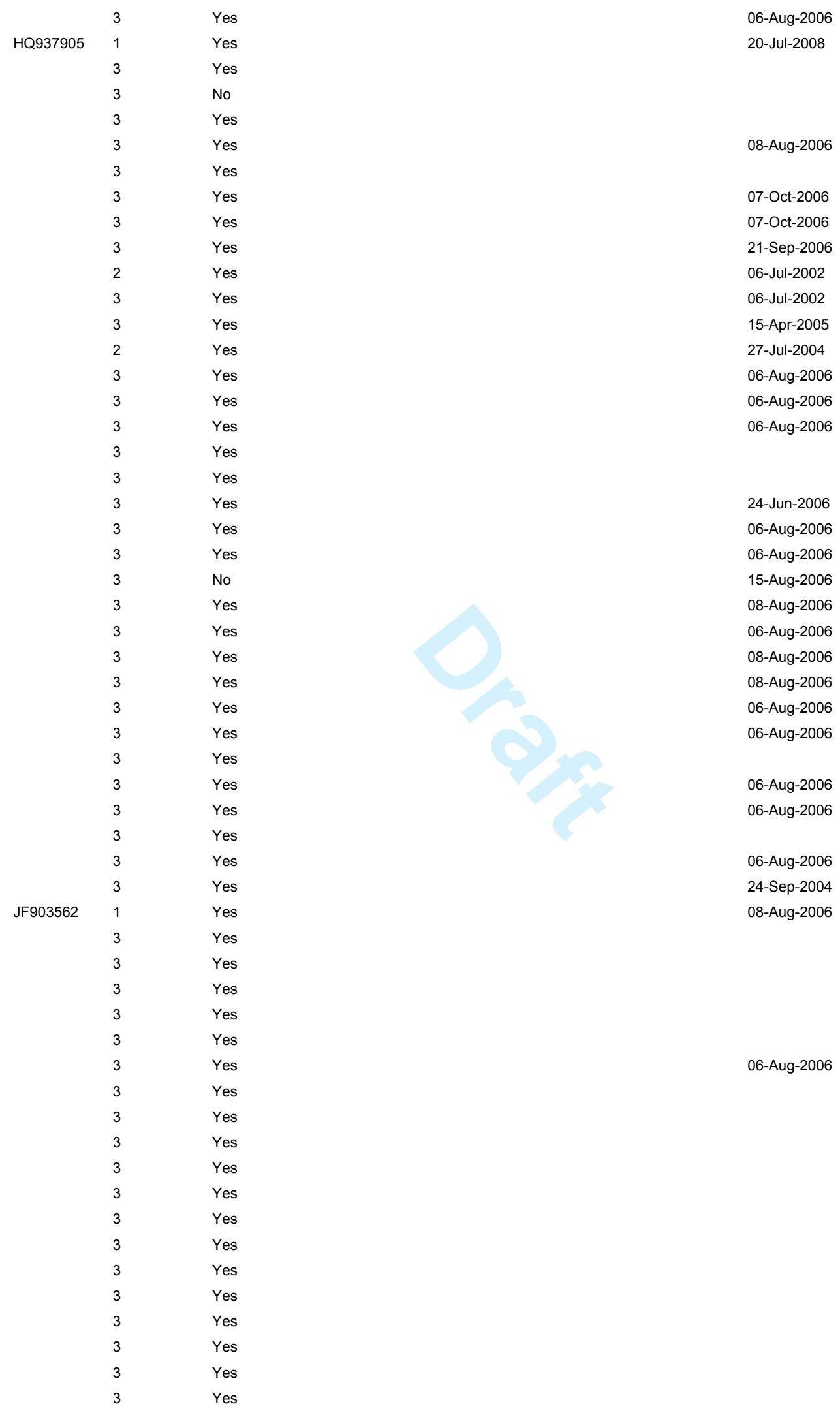




\begin{tabular}{|c|c|c|c|}
\hline & 3 & \multicolumn{2}{|l|}{ Yes } \\
\hline & 3 & \multicolumn{2}{|l|}{ Yes } \\
\hline & 3 & \multicolumn{2}{|l|}{ Yes } \\
\hline & 3 & \multicolumn{2}{|l|}{ Yes } \\
\hline & 3 & \multicolumn{2}{|l|}{ Yes } \\
\hline & 3 & \multicolumn{2}{|l|}{ Yes } \\
\hline & 3 & \multicolumn{2}{|l|}{ Yes } \\
\hline & 3 & \multicolumn{2}{|l|}{ Yes } \\
\hline & 3 & \multicolumn{2}{|l|}{ Yes } \\
\hline & 2 & No & 01-Aug-2004 \\
\hline & 2 & Yes & 24-Jun-2001 \\
\hline & 2 & Yes & 23-Jun-2004 \\
\hline & 2 & Yes & 21-Jul-2004 \\
\hline & 2 & Yes & 01-Aug-2005 \\
\hline HQ558114 & 1 & Yes & 20-Jul-2008 \\
\hline GU708183 & 1 & No & \\
\hline GU708185 & 1 & \multicolumn{2}{|l|}{ No } \\
\hline \multirow[t]{3}{*}{ HQ558153 } & 1 & Yes & 28-Aug-2008 \\
\hline & 3 & Yes & 12-May-2000 \\
\hline & 3 & Yes & 18-May-2006 \\
\hline FJ663121 & 2 & No & 27-Apr-2003 \\
\hline FJ663122 & 2 & No & 06-Sep-2005 \\
\hline FJ663119 & 1 & No & 10-Jul-2001 \\
\hline FJ663128 & 1 & No & 27-Jul-2004 \\
\hline FJ663123 & 1 & Yes & 19-May-2006 \\
\hline FJ663127 & 1 & No & 20-Aug-2004 \\
\hline HQ558115 & 0 & Yes & 22-Aug-2008 \\
\hline FJ663126 & 1 & \multicolumn{2}{|l|}{ Yes } \\
\hline FJ663120 & 1 & Yes & 22-Aug-2005 \\
\hline FJ663124 & 1 & Yes & 19-Мау-2006 \\
\hline \multirow[t]{25}{*}{ FJ663125 } & 1 & Yes & 19-Мау-2006 \\
\hline & 2 & Yes & 10-Jul-2003 \\
\hline & 2 & Yes & 11-Jun-2007 \\
\hline & 2 & Yes & 30-Jul-2005 \\
\hline & 2 & Yes & 05-Jun-2003 \\
\hline & 2 & Yes & 06-Aug-2006 \\
\hline & 2 & Yes & 31-Jul-2005 \\
\hline & 2 & Yes & 24-Jun-2006 \\
\hline & 1 & Yes & 04-Jun-2006 \\
\hline & 2 & No & 07-Aug-2006 \\
\hline & 2 & \multicolumn{2}{|l|}{ No } \\
\hline & 2 & Yes & 25-Jun-2006 \\
\hline & 2 & No & 19-Jul-2004 \\
\hline & 2 & No & 01-Jan-2004 \\
\hline & 2 & Yes & 14-Aug-2006 \\
\hline & 3 & No & 10-May-2005 \\
\hline & 2 & No & 19-Jul-2004 \\
\hline & 2 & \multicolumn{2}{|l|}{ Yes } \\
\hline & 2 & Yes & 08-Aug-2006 \\
\hline & 1 & Yes & 01-Jul-2006 \\
\hline & 2 & Yes & 08-Aug-2006 \\
\hline & 1 & Yes & 25-Jun-2006 \\
\hline & 2 & Yes & 08-Aug-2006 \\
\hline & 2 & Yes & 13-Aug-2006 \\
\hline & 2 & Yes & 06-Jun-2006 \\
\hline
\end{tabular}




\begin{tabular}{|c|c|}
\hline & 2 \\
\hline & 2 \\
\hline & 2 \\
\hline & 2 \\
\hline & 1 \\
\hline & 2 \\
\hline & 2 \\
\hline & 2 \\
\hline & 2 \\
\hline JF903564 & 2 \\
\hline & 4 \\
\hline & 2 \\
\hline & 0 \\
\hline & 4 \\
\hline & 4 \\
\hline JF903565 & 2 \\
\hline & 4 \\
\hline
\end{tabular}
09-Aug-2006
09-Aug-2006
15-Aug-2006
14-Aug-2006
07-Aug-2006
03-Sep-2003
25-Jun-2004
30-Apr-2006
06-Jul-2007
18-Jul-2009
20-Aug-2004
15-Aug-2006
15-Aug-2006 


\begin{tabular}{|c|c|c|c|c|}
\hline Identification & Life Stage & Extra Info & Voucher Type & Institution \\
\hline Lasioglossum_abanci & A & & & York University, Packer Colle \\
\hline Lasioglossum_abundipunctu & A & & & York University, Packer Colle \\
\hline Lasioglossum_abundipunctu & & & & York University, Packer Colle \\
\hline Lasioglossum_abundipunctu & & & & York University, Packer Colle \\
\hline Lasioglossum_abundipunctu & & & & York University, Packer Colle \\
\hline Lasioglossum_abundipunctu & A & & & York University, Packer Collesp. C \\
\hline Lasioglossum_achilleae & A & & & York University, Packer Colle \\
\hline Lasioglossum_admirandum & A & & & York University, Packer Colle \\
\hline Lasioglossum_admirandum & A & & & York University, Packer Colle \\
\hline Lasioglossum_admirandum & A & & & York University, Packer Colle \\
\hline Lasioglossum_admirandum & A & & & York University, Packer Colle \\
\hline Lasioglossum_admirandum & A & & & York University, Packer Colle \\
\hline Lasioglossum_admirandum & A & & & York University, Packer Colle \\
\hline Lasioglossum_admirandum & A & & & York University, Packer Colle \\
\hline Lasioglossum_admirandum & A & & & York University, Packer Colle \\
\hline Lasioglossum_alachuense & A & & & York University, Packer Colle \\
\hline Lasioglossum_albipenne & A & Col on: tomato & & York University, Packer Coll€ \\
\hline Lasioglossum_albipenne & A & cress7 MB & & York University, Packer Colle \\
\hline Lasioglossum_albipenne & A & & & York University, Packer Colle \\
\hline Lasioglossum_albipenne & A & & & York University, Packer Colle \\
\hline Lasioglossum_albipenne & A & & & York University, Packer Colle \\
\hline Lasioglossum_albipenne & A & & & York University, Packer Coll€ \\
\hline Lasioglossum_albipenne & A & & & York University, Packer Colle \\
\hline Lasioglossum_albipenne & A & & & York University, Packer Colle \\
\hline Lasioglossum_albipenne & A & DIAL1412A08 & & York University, Packer Colle \\
\hline Lasioglossum_albipenne & A & carina weak & & York University, Packer Coll€ \\
\hline Lasioglossum_albipenne & A & cress5 MB & & York University, Packer Colle \\
\hline Lasioglossum_albohirtum & A & & & York University, Packer Coll€ \\
\hline Lasioglossum_albohirtum & A & & & York University, Packer Colle \\
\hline Lasioglossum_albohirtum & A & & & York University, Packer Coll€ \\
\hline Lasioglossum_albohirtum & A & & & York University, Packer Coll€ \\
\hline Lasioglossum_albohirtum & A & & & York University, Packer Coll€ \\
\hline Lasioglossum_albohirtum & A & & & York University, Packer Colle \\
\hline Lasioglossum_albohirtum & A & & & York University, Packer Coll€ \\
\hline Lasioglossum_albohirtum & A & & & York University, Packer Colle \\
\hline Lasioglossum_anomalum & A & & & York University, Packer Colle White pan trap \\
\hline Lasioglossum_anomalum & A & anomalum1 & & York University, Packer Coll€ Col on: Monarda fistulosa \\
\hline Lasioglossum_anomalum & A & Lanoma-MN1 & & York University, Packer Colle \\
\hline Lasioglossum_anomalum & A & & & York University, Packer Colle \\
\hline Lasioglossum_anomalum & A & & & York University, Packer Coll $\epsilon$ \\
\hline Lasioglossum_anomalum & A & & & York University, Packer Colle \\
\hline Lasioglossum_apocyni & A & & & York University, Packer Colle \\
\hline Lasioglossum_apocyni & A & & & York University, Packer Colle \\
\hline Lasioglossum_apocyni & A & & & York University, Packer Coll \\
\hline Lasioglossum_apocyni & A & apocyniE12 & & York University, Packer Colle original ID by S.D. as fattigi \\
\hline Lasioglossum_apocyni & A & & & York University, Packer Coll \\
\hline Lasioglossum_apocyni & A & apocyniB01 & & York University, Packer Coll€ 20 -Sep-year? Original ID by S. [ \\
\hline Lasioglossum_apocyni & A & apocyni1 & & York University, Packer Coll€ 20-Sep-year? \\
\hline Lasioglossum_apocyni & A & apocyni2 & & York University, Packer Coll€ 20-Sep-year? \\
\hline Lasioglossum_apocyni & A & apocyniF02 & & York University, Packer Colle original ID by S.D. as fattigi \\
\hline Lasioglossum_apocyni & A & & & York University, Packer Colle \\
\hline Lasioglossum_apocyni & A & apocyniE11 & & York University, Packer Colle original ID by S.D. as fattigi \\
\hline
\end{tabular}




\begin{tabular}{|c|c|c|}
\hline Lasioglossum_apocyni & A & York University, Packer Colle \\
\hline Lasioglossum_apocyni & A & York University, Packer Colle \\
\hline Lasioglossum_apocyni & A & York University, Packer Colle \\
\hline Lasioglossum_apocyni & A & York University, Packer Colle \\
\hline Lasioglossum_apocyni & 293G09 & York University, Packer Colle \\
\hline Lasioglossum_apopkense & A & York University, Packer Colle \\
\hline Lasioglossum_apopkense & A & York University, Packer Colle \\
\hline Lasioglossum_apopkense & A & York University, Packer Colle \\
\hline Lasioglossum_apopkense & A & York University, Packer Colle \\
\hline Lasioglossum_apopkense & A & York University, Packer Colle \\
\hline Lasioglossum_arantium & pictum1 & York University, Packer Colle \\
\hline Lasioglossum_arantium & pictum2 & York University, Packer Colle \\
\hline Lasioglossum_arantium & A & York University, Packer Colle \\
\hline Lasioglossum_arantium & A & York University, Packer Colle \\
\hline Lasioglossum_arantium & A & York University, Packer Colle \\
\hline Lasioglossum_atwoodi & A & York University, Packer Colle \\
\hline Lasioglossum_atwoodi & A & York University, Packer Colle \\
\hline Lasioglossum_atwoodi & DIAL6MD & York University, Packer Colle \\
\hline Lasioglossum_atwoodi & A & York University, Packer Colle \\
\hline Lasioglossum_atwoodi & A & York University, Packer Colle \\
\hline Lasioglossum_atwoodi & A & York University, Packer Colle \\
\hline Lasioglossum_batya & A & York University, Packer Colle \\
\hline Lasioglossum_batya & A & York University, Packer Colle \\
\hline Lasioglossum_bruneri & brune IN2 & York University, Packer Colle White pan trap \\
\hline Lasioglossum_bruneri & bruneriE08 & York University, Packer Colle original ID by S.D. as cressonii \\
\hline Lasioglossum_bruneri & A & York University, Packer Colle \\
\hline Lasioglossum_bruneri & A & York University, Packer Colle \\
\hline Lasioglossum_bruneri & A & York University, Packer Colle \\
\hline Lasioglossum_bruneri & A & York University, Packer Colle \\
\hline Lasioglossum_bruneri & A & York University, Packer Colle \\
\hline Lasioglossum_bruneri & A & York University, Packer Colle \\
\hline Lasioglossum_bruneri & A & York University, Packer Colle \\
\hline Lasioglossum_bruneri & DIAL1412E12 & York University, Packer Colle \\
\hline Lasioglossum_bruneri & A & York University, Packer Colle \\
\hline Lasioglossum_brunneiventre $A$ & & York University, Packer Colle \\
\hline Lasioglossum_brunneiventre $A$ & & York University, Packer Colle \\
\hline Lasioglossum_brunneiventre $A$ & long face & York University, Packer Colle \\
\hline Lasioglossum_brunneiventre $A$ & & York University, Packer Colle \\
\hline Lasioglossum_brunneiventre $A$ & & York University, Packer Colle \\
\hline Lasioglossum_brunneiventre $A$ & & York University, Packer Colle \\
\hline Lasioglossum_brunneiventre $A$ & & York University, Packer Colle \\
\hline Lasioglossum_brunneiventre $A$ & long face & York University, Packer Colle \\
\hline Lasioglossum_brunneiventre $A$ & & York University, Packer Colle \\
\hline Lasioglossum_brunneiventre $A$ & & York University, Packer Colle \\
\hline Lasioglossum_brunneiventre $A$ & & York University, Packer Colle \\
\hline Lasioglossum_brunneiventre $A$ & & York University, Packer Colle \\
\hline Lasioglossum_brunneiventre $A$ & & York University, Packer Colle \\
\hline Lasioglossum_brunneiventre $A$ & & York University, Packer Colle \\
\hline Lasioglossum_callidum & A & York University, Packer Colle \\
\hline Lasioglossum_callidum & A & York University, Packer Colle \\
\hline Lasioglossum_callidum & A & York University, Packer Coll€ \\
\hline Lasioglossum_callidum & versa2 VA & York University, Packer Colle \\
\hline Lasioglossum_callidum & versa1 TN & York University, Packer Colle \\
\hline Lasioglossum_callidum & A & York University, Packer Colle \\
\hline Lasioglossum_callidum & versatum1 & York University, Packer Colle \\
\hline
\end{tabular}




\begin{tabular}{|c|c|c|}
\hline Lasioglossum_callidum & A & \\
\hline Lasioglossum_callidum & A & \\
\hline Lasioglossum_callidum & A & \\
\hline Lasioglossum_callidum & A & versa1 VA \\
\hline Lasioglossum_callidum & A & \\
\hline Lasioglossum_callidum & A & \\
\hline Lasioglossum_callidum & A & \\
\hline Lasioglossum_callidum & A & \\
\hline Lasioglossum_callidum & A & \\
\hline Lasioglossum_callidum & A & \\
\hline Lasioglossum_callidum & A & \\
\hline Lasioglossum_callidum & A & \\
\hline Lasioglossum_callidum & A & \\
\hline Lasioglossum_callidum & A & \\
\hline Lasioglossum_callidum & A & \\
\hline Lasioglossum_callidum & A & \\
\hline Lasioglossum_callidum & A & \\
\hline Lasioglossum_callidum & A & \\
\hline Lasioglossum_callidum & A & \\
\hline Lasioglossum_callidum & A & \\
\hline Lasioglossum_callidum & A & \\
\hline Lasioglossum_callidum & A & \\
\hline Lasioglossum_callidum & A & \\
\hline Lasioglossum_callidum & A & \\
\hline Lasioglossum_callidum & A & \\
\hline Lasioglossum_callidum & A & \\
\hline Lasioglossum_carlinvillense & A & CARLINVILLEI \\
\hline Lasioglossum_carlinvillense & A & CARLINVILLEI \\
\hline Lasioglossum_carlinvillense & A & CARLINVILLEI \\
\hline Lasioglossum_ceanothi & A & \\
\hline Lasioglossum_ceanothi & A & \\
\hline \multicolumn{3}{|l|}{ Lasioglossum_cf._stuartens $€ \mathrm{~A}$} \\
\hline Lasioglossum_coreopsis & A & \\
\hline Lasioglossum_coreopsis & A & coreopsis2 \\
\hline Lasioglossum_coreopsis & A & coreopsis3 \\
\hline Lasioglossum_coreopsis & A & coreopsis1 \\
\hline Lasioglossum_coreopsis & A & coreopsis 5 \\
\hline Lasioglossum_coreopsis & A & coreopsis 4 \\
\hline Lasioglossum_coreopsis & A & \\
\hline Lasioglossum_coreopsis & A & \\
\hline Lasioglossum_coreopsis & A & \\
\hline Lasioglossum_coreopsis & A & \\
\hline Lasioglossum_coreopsis & A & \\
\hline Lasioglossum_coreopsis & A & \\
\hline Lasioglossum_coreopsis & A & \\
\hline Lasioglossum_coreopsis & A & \\
\hline Lasioglossum_coreopsis & A & \\
\hline Lasioglossum_coreopsis & A & \\
\hline Lasioglossum_creberrimum & A & \\
\hline Lasioglossum_creberrimum & A & \\
\hline Lasioglossum_creberrimum & A & crebberimum2 \\
\hline Lasioglossum_creberrimum & A & crebe2 NC \\
\hline Lasioglossum_creberrimum & A & crebe3 NC \\
\hline Lasioglossum_creberrimum & A & crebe1 NC \\
\hline Lasioglossum_creberrimum & A & crebberimum4 \\
\hline
\end{tabular}

York University, Packer Colle York University, Packer Colle York University, Packer Colle York University, Packer Colle York University, Packer Colle York University, Packer Colle York University, Packer Colle York University, Packer Colle York University, Packer Colle York University, Packer Colle York University, Packer Colle York University, Packer Colle York University, Packer Colle York University, Packer Colle York University, Packer Colle York University, Packer Colle York University, Packer Colle York University, Packer Colle York University, Packer Colle York University, Packer Colle York University, Packer Colle York University, Packer Colle York University, Packer Colle York University, Packer Colle York University, Packer Colle York University, Packer Colle mix up? York University, Packer Colle York University, Packer Colle York University, Packer Colle York University, Packer Colle York University, Packer Colle York University, Packer Colle York University, Packer Colle York University, Packer Colle York University, Packer Colle York University, Packer Colle Sep or Oct-03 York University, Packer Colle York University, Packer Colle York University, Packer Colle York University, Packer Colle York University, Packer Colle York University, Packer Colle York University, Packer Colle York University, Packer Colle York University, Packer Colle York University, Packer Colle York University, Packer Colle York University, Packer Colle York University, Packer Colle York University, Packer Colle York University, Packer Colle York University, Packer Colle York University, Packer Colle York University, Packer Colle York University, Packer Colle 


\begin{tabular}{|c|c|c|c|}
\hline Lasioglossum_creberrimum & A & & York University, Packer Colle \\
\hline Lasioglossum_creberrimum & A & & York University, Packer Colle \\
\hline Lasioglossum_creberrimum & A & & York University, Packer Colle \\
\hline Lasioglossum_creberrimum & A & & York University, Packer Colle \\
\hline Lasioglossum_creberrimum & A & & York University, Packer Colle \\
\hline Lasioglossum_cressonii & A & cress2 $\sim \mathrm{NC}$ & York University, Packer Colle \\
\hline Lasioglossum_cressonii & A & cress $\sim$ IN2 & York University, Packer Colle Col on: Claytonia virginica \\
\hline Lasioglossum_cressonii & A & cress $\sim \mathrm{IN} 1$ & York University, Packer Colle Blue pan trap \\
\hline Lasioglossum_cressonii & A & & York University, Packer Colle \\
\hline Lasioglossum_cressonii & A & cress1 GA & York University, Packer Colle \\
\hline Lasioglossum_cressonii & A & cress $\sim$ IL1 & York University, Packer Colle Blue pan trap \\
\hline Lasioglossum_cressonii & A & & York University, Packer Colle \\
\hline Lasioglossum_cressonii & A & & York University, Packer Colle \\
\hline Lasioglossum_cressonii & A & & York University, Packer Colle \\
\hline Lasioglossum_cressonii & A & & York University, Packer Colle \\
\hline Lasioglossum_cressonii & A & & York University, Packer Colle \\
\hline Lasioglossum_cressonii & A & & York University, Packer Colle \\
\hline Lasioglossum_cressonii & A & Lcress-MN1 & York University, Packer Colle \\
\hline Lasioglossum_cressonii & A & Lcress-MN3 & York University, Packer Colle \\
\hline Lasioglossum_cressonii & A & & York University, Packer Colle \\
\hline Lasioglossum_cressonii & A & & York University, Packer Colle \\
\hline Lasioglossum_cressonii & A & DIAL1412A03 & York University, Packer Colle \\
\hline Lasioglossum_cressonii & A & & York University, Packer Colle \\
\hline Lasioglossum_cressonii & A & Lcress-MN2 & York University, Packer Colle \\
\hline Lasioglossum_cressonii & A & & York University, Packer Colle \\
\hline Lasioglossum_cressonii & A & & York University, Packer Colle \\
\hline Lasioglossum_cressonii & A & & York University, Packer Colle \\
\hline Lasioglossum_cressonii & A & & York University, Packer Colle \\
\hline Lasioglossum_cressonii & A & & York University, Packer Colle \\
\hline Lasioglossum_cressonii & A & & York University, Packer Colle \\
\hline Lasioglossum_cressonii & A & & York University, Packer Colle \\
\hline Lasioglossum_cressonii & A & & York University, Packer Colle \\
\hline Lasioglossum_dashwoodi & A & & York University, Packer Colle \\
\hline Lasioglossum_dashwoodi & A & WA-3 & York University, Packer Coll $\epsilon$ \\
\hline Lasioglossum_disparile & A & & York University, Packer Colle \\
\hline Lasioglossum_disparile & A & & York University, Packer Colle \\
\hline Lasioglossum_disparile & A & & York University, Packer Colle \\
\hline Lasioglossum_disparile & A & & York University, Packer Colle \\
\hline Lasioglossum_disparile & A & & York University, Packer Colle \\
\hline Lasioglossum_disparile & A & & York University, Packer Colle \\
\hline Lasioglossum_disparile & A & & York University, Packer Colle \\
\hline Lasioglossum_disparile & A & & York University, Packer Colle \\
\hline Lasioglossum_disparile & A & & York University, Packer Colle \\
\hline Lasioglossum_dreisbachi & A & & York University, Packer Colle \\
\hline Lasioglossum_dreisbachi & A & & York University, Packer Colle \\
\hline Lasioglossum_dreisbachi & A & & York University, Packer Colle \\
\hline Lasioglossum_dreisbachi & A & & York University, Packer Colle \\
\hline Lasioglossum_ebmerellum & A & & York University, Packer Colle \\
\hline Lasioglossum_ebmerellum & A & & York University, Packer Colle \\
\hline Lasioglossum_ebmerellum & A & & York University, Packer Colle \\
\hline Lasioglossum_ebmerellum & A & & York University, Packer Coll€ \\
\hline Lasioglossum_ebmerellum & A & & York University, Packer Colle \\
\hline Lasioglossum_ebmerellum & A & & York University, Packer Colle \\
\hline Lasioglossum_ellisiae & A & tegul IN1 & York University, Packer Colle Blue pan trap \\
\hline Lasioglossum_ellisiae & A & & York University, Packer Colle \\
\hline
\end{tabular}




\begin{tabular}{|c|c|}
\hline Lasioglossum_ellisiae & $A$ \\
\hline Lasioglossum_ellisiae & \\
\hline Lasioglossum_ellisiae & \\
\hline Lasioglossum_ellisiae & \\
\hline Lasioglossum_ellisiae & \\
\hline Lasioglossum_ellisiae & \\
\hline Lasioglossum_ellisiae & \\
\hline Lasioglossum_ellisiae & \\
\hline Lasioglossum_ellisiae & A \\
\hline Lasioglossum_ellisiae & \\
\hline Lasioglossum_ellisiae & \\
\hline Lasioglossum_ellisiae & \\
\hline Lasioglossum_ellisiae & \\
\hline Lasioglossum_ellisiae & \\
\hline Lasioglossum_ellisiae & A \\
\hline Lasioglossum_ellisiae & \\
\hline Lasioglossum_ellisiae & $A$ \\
\hline Lasioglossum_ellisiae & \\
\hline Lasioglossum_ellisiae & \\
\hline Lasioglossum_ephialtum & $A$ \\
\hline Lasioglossum_ephialtum & A \\
\hline Lasioglossum_ephialtum & \\
\hline Lasioglossum_ephialtum & A \\
\hline Lasioglossum_ephialtum & \\
\hline Lasioglossum_ephialtum & \\
\hline Lasioglossum_ephialtum & A \\
\hline Lasioglossum_ephialtum & $A$ \\
\hline Lasioglossum_ephialtum & \\
\hline Lasioglossum_ephialtum & A \\
\hline Lasioglossum_ephialtum & \\
\hline Lasioglossum_ephialtum & A \\
\hline Lasioglossum_ephialtum & A \\
\hline Lasioglossum_ephialtum & \\
\hline Lasioglossum_ephialtum & $A$ \\
\hline Lasioglossum_ephialtum & A \\
\hline Lasioglossum_ephialtum & \\
\hline Lasioglossum_ephialtum & \\
\hline Lasioglossum_ephialtum & A \\
\hline Lasioglossum_ephialtum & \\
\hline Lasioglossum_ephialtum & $A$ \\
\hline Lasioglossum_ephialtum & A \\
\hline Lasioglossum_ephialtum & \\
\hline Lasioglossum_ephialtum & \\
\hline Lasioglossum_ephialtum & A \\
\hline Lasioglossum_ephialtum & A \\
\hline Lasioglossum_ephialtum & \\
\hline Lasioglossum_ephialtum & A \\
\hline Lasioglossum_ephialtum & \\
\hline Lasioglossum_ephialtum & \\
\hline Lasioglossum_ephialtum & $A$ \\
\hline Lasioglossum_ephialtum & \\
\hline Lasioglossum_ephialtum & \\
\hline Lasioglossum_ephialtum & \\
\hline Lasioglossum_ephialtum & \\
\hline Lasioglossum_ephialtum & \\
\hline
\end{tabular}

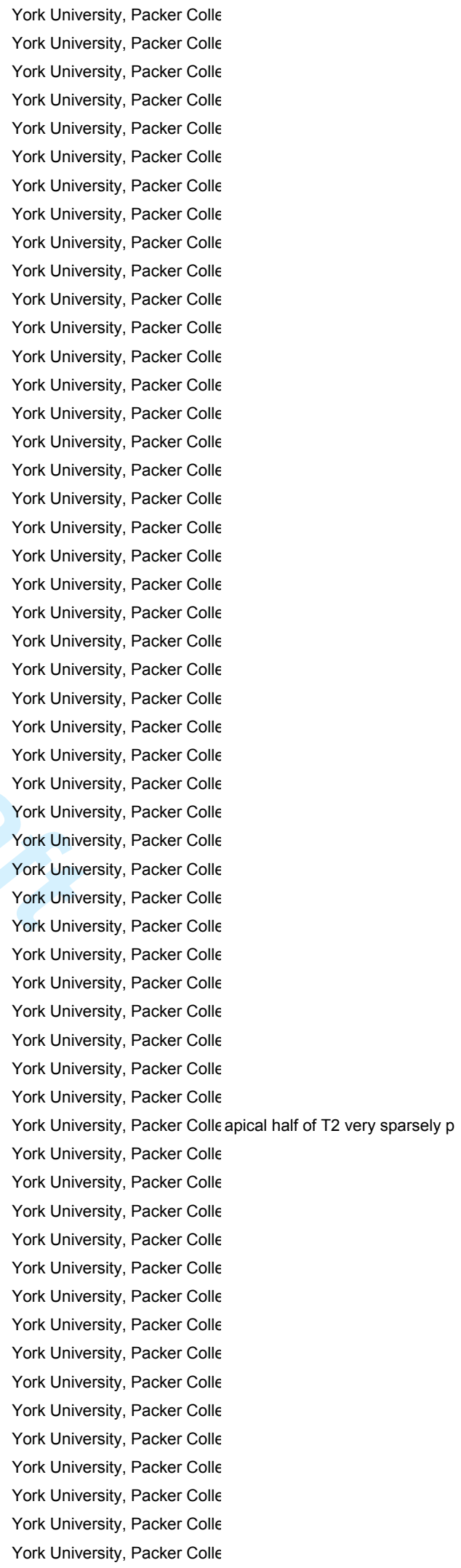




\begin{tabular}{|c|c|c|}
\hline Lasioglossum_ephialtum & A & \\
\hline Lasioglossum_ephialtum & A & \\
\hline Lasioglossum_ephialtum & A & \\
\hline Lasioglossum_ephialtum & A & \\
\hline Lasioglossum_ephialtum & A & \\
\hline Lasioglossum_ephialtum & A & \\
\hline Lasioglossum_ephialtum & A & \\
\hline Lasioglossum_ephialtum & A & \\
\hline Lasioglossum_ephialtum & A & \\
\hline Lasioglossum_fattigi & A & \\
\hline Lasioglossum_fattigi & A & \\
\hline Lasioglossum_fattigi & A & \\
\hline Lasioglossum_fattigi & A & \\
\hline Lasioglossum_flaveriae & A & \\
\hline Lasioglossum_floridanum & A & Lpilos1 GA \\
\hline Lasioglossum_floridanum & A & \\
\hline Lasioglossum_floridanum & A & \\
\hline Lasioglossum_floridanum & A & Lpilos3 GA \\
\hline Lasioglossum_foveolatum & A & supra IN1 \\
\hline Lasioglossum_foveolatum & A & supra IN2 \\
\hline Lasioglossum_foveolatum & A & \\
\hline Lasioglossum_foveolatum & A & supra IL1 \\
\hline Lasioglossum_foveolatum & A & supraclypeatur \\
\hline Lasioglossum_foveolatum & A & supraclypeatur \\
\hline Lasioglossum_foveolatum & A & \\
\hline Lasioglossum_foveolatum & A & \\
\hline Lasioglossum_foveolatum & A & \\
\hline Lasioglossum_foveolatum & A & \\
\hline Lasioglossum_georgeickwo & & \\
\hline Lasioglossum_georgeickwo & & \\
\hline Lasioglossum_georgeickwo & & \\
\hline Lasioglossum_georgeickwo & & \\
\hline Lasioglossum_georgeickwo & & \\
\hline Lasioglossum_georgeickwo & & \\
\hline Lasioglossum_georgeickwo & & \\
\hline Lasioglossum_georgeickwo & & \\
\hline Lasioglossum_halophitum & A & \\
\hline Lasioglossum_halophitum & A & \\
\hline Lasioglossum_halophitum & A & \\
\hline Lasioglossum_halophitum & A & halop2 NC \\
\hline Lasioglossum_halophitum & A & \\
\hline Lasioglossum_halophitum & A & halop1 NC \\
\hline Lasioglossum_halophitum & A & \\
\hline Lasioglossum_halophitum & A & \\
\hline Lasioglossum_halophitum & A & \\
\hline Lasioglossum_halophitum & A & \\
\hline Lasioglossum_halophitum & A & \\
\hline Lasioglossum_hartii & A & \\
\hline Lasioglossum_hartii & A & \\
\hline Lasioglossum_hemimelas & A & DIAL1412D01 \\
\hline \multicolumn{3}{|c|}{ Lasioglossum_heterognathuı A } \\
\hline \multicolumn{3}{|c|}{ Lasioglossum_heterognathuı A } \\
\hline \multicolumn{3}{|l|}{ Lasioglossum_heterognathuı A } \\
\hline Lasioglossum_hitchensi & A & \\
\hline Lasioglossum hitchensi & A & \\
\hline
\end{tabular}

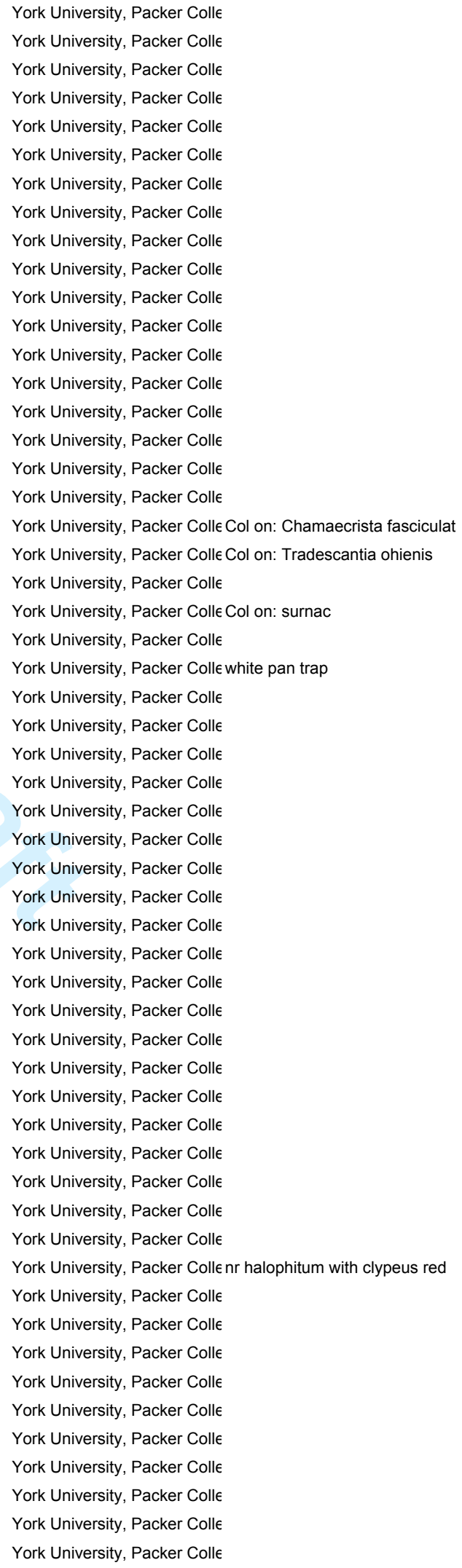


York University, Packer Colle York University, Packer Colle York University, Packer Colle York University, Packer Colle York University, Packer Colle York University, Packer Colle York University, Packer Colle York University, Packer Colle York University, Packer Colle York University, Packer Colle York University, Packer Colle

admirlN1 York University, Packer Coll $\epsilon$ Col on: Ranunculus abortivus York University, Packer Colle York University, Packer Colle York University, Packer Colle

admir IL1

DIAL1MD

laevi3 VA

DIAL1VA

DIAL2MD

oblon MD2

admirandum4

York University, Packer Colle Yellow pan trap

York University, Packer Colle

York University, Packer Colle

York University, Packer Colle

York University, Packer Colle

York University, Packer Colle

York University, Packer Colle

York University, Packer Colle

York University, Packer Colle

York University, Packer Colle

York University, Packer Colle

York University, Packer Colle

York University, Packer Colle

York University, Packer Colle

York University, Packer Colle

York University, Packer Colle near admirandum with relatively

York University, Packer Colle close to flaveriae

York University, Packer Colle

York University, Packer Colle

York University, Packer Colle

York University, Packer Colle

York University, Packer Colle

York University, Packer Colle

York University, Packer Colle

York University, Packer Colle

York University, Packer Colle

York University, Packer Colle

York University, Packer Colle

York University, Packer Colle

York University, Packer Colle

York University, Packer Colle

York University, Packer Colle

York University, Packer Colle

York University, Packer Colle

York University, Packer Colle

York University, Packer Colle

York University, Packer Colle

York University, Packer Colle

York University, Packer Colle

York University, Packer Colle 


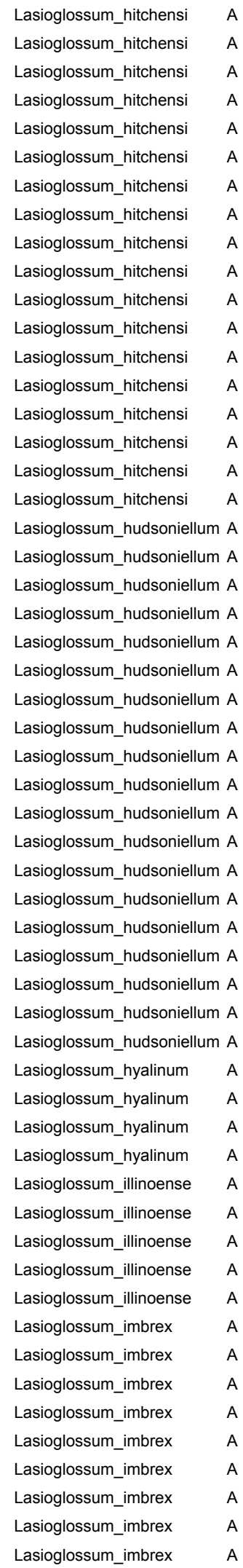

M10

brown metasor

Z16 or 27

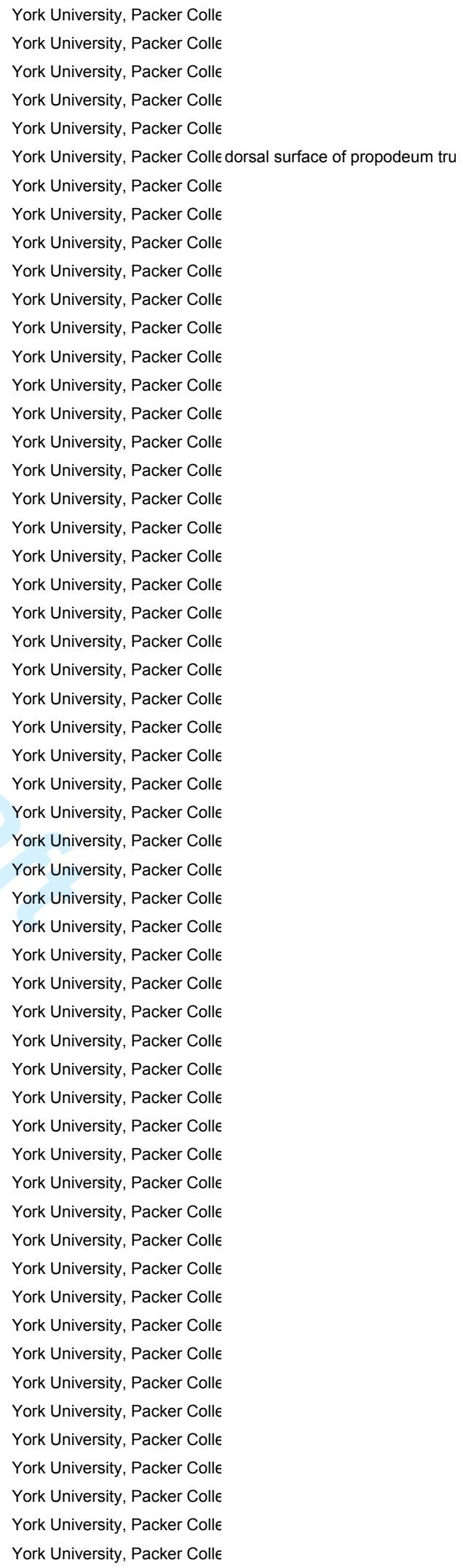




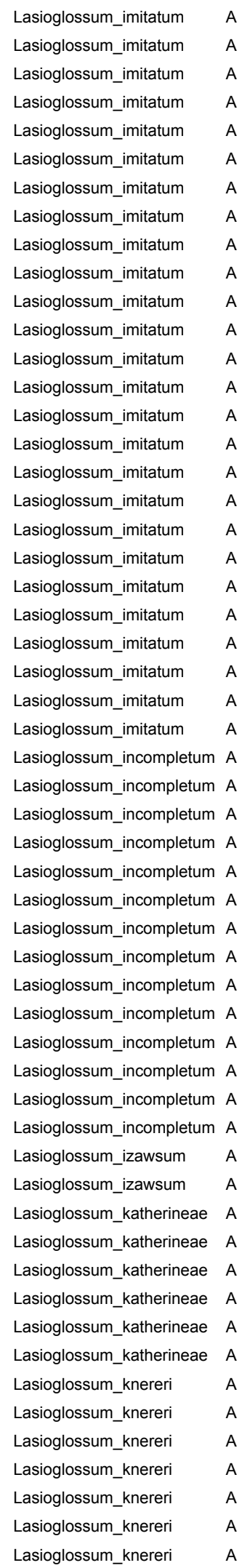

York University, Packer Colle York University, Packer Colle York University, Packer Colle York University, Packer Colle York University, Packer Colle York University, Packer Colle York University, Packer Colle York University, Packer Colle York University, Packer Colle York University, Packer Colle York University, Packer Colle York University, Packer Colle York University, Packer Colle York University, Packer Colle York University, Packer Colle York University, Packer Colle York University, Packer Colle York University, Packer Colle York University, Packer Colle York University, Packer Colle weird propodeum York University, Packer Colle York University, Packer Colle York University, Packer Colle York University, Packer Colle York University, Packer Colle York University, Packer Colle York University, Packer Colle York University, Packer Colle York University, Packer Colle York University, Packer Colle York University, Packer Colle York University, Packer Colle York University, Packer Colle York University, Packer Colle York University, Packer Colle York University, Packer Colle York University, Packer Colle York University, Packer Colle York University, Packer Colle York University, Packer Colle York University, Packer Colle York University, Packer Colle York University, Packer Colle York University, Packer Colle York University, Packer Colle York University, Packer Colle York University, Packer Colle York University, Packer Colle York University, Packer Colle York University, Packer Colle York University, Packer Colle York University, Packer Colle York University, Packer Colle York University, Packer Colle York University, Packer Colle 


\begin{tabular}{|c|c|c|}
\hline Lasioglossum_knereri & A & York University, Packer Colle \\
\hline Lasioglossum_knereri & A & York University, Packer Colle \\
\hline Lasioglossum_knereri & A & York University, Packer Colle \\
\hline Lasioglossum_knereri & A & York University, Packer Colle \\
\hline Lasioglossum_knereri & A & York University, Packer Colle \\
\hline Lasioglossum_knereri & A & York University, Packer Colle \\
\hline Lasioglossum_knereri & A & York University, Packer Colle \\
\hline Lasioglossum_knereri & A & York University, Packer Colle \\
\hline Lasioglossum_knereri & A & York University, Packer Colle \\
\hline Lasioglossum_knereri & A & York University, Packer Colle \\
\hline Lasioglossum_knereri & A & York University, Packer Colle \\
\hline Lasioglossum_knereri & A & York University, Packer Colle \\
\hline Lasioglossum_knereri & A & York University, Packer Colle \\
\hline Lasioglossum_knereri & A & York University, Packer Colle \\
\hline Lasioglossum_knereri & A & York University, Packer Colle \\
\hline Lasioglossum_knereri & A & York University, Packer Colle \\
\hline Lasioglossum_knereri & A & York University, Packer Colle \\
\hline Lasioglossum_knereri & A & York University, Packer Colle \\
\hline Lasioglossum_knereri & A & York University, Packer Colle \\
\hline Lasioglossum_knereri & A & York University, Packer Colle \\
\hline Lasioglossum_knereri & A & York University, Packer Colle \\
\hline Lasioglossum_knereri & A & York University, Packer Colle \\
\hline Lasioglossum_knereri & A & York University, Packer Colle ID-11 \\
\hline Lasioglossum_knereri & A & York University, Packer Colle \\
\hline Lasioglossum_knereri & A & York University, Packer Colle \\
\hline Lasioglossum_knereri & A & York University, Packer Colle \\
\hline Lasioglossum_knereri & A & York University, Packer Colle \\
\hline Lasioglossum_knereri & A & York University, Packer Colle \\
\hline Lasioglossum_knereri & A & York University, Packer Colle \\
\hline Lasioglossum_laevissimum & A & York University, Packer Colle \\
\hline Lasioglossum_laevissimum & A & York University, Packer Colle \\
\hline Lasioglossum_laevissimum & DIAL1412H12 & York University, Packer Colle \\
\hline Lasioglossum_laevissimum & A & York University, Packer Colle \\
\hline Lasioglossum_laevissimum & A & York University, Packer Colle \\
\hline Lasioglossum_laevissimum & A & York University, Packer Colle \\
\hline Lasioglossum_laevissimum & laev2 MD & York University, Packer Colle \\
\hline Lasioglossum_laevissimum & DIAL2NB & York University, Packer Colle \\
\hline Lasioglossum_laevissimum & A & York University, Packer Colle \\
\hline Lasioglossum_laevissimum & A & York University, Packer Colle \\
\hline Lasioglossum_laevissimum & A & York University, Packer Colle \\
\hline Lasioglossum_laevissimum & A & York University, Packer Colle \\
\hline Lasioglossum_laevissimum & A & York University, Packer Colle \\
\hline Lasioglossum_laevissimum & A & York University, Packer Colle \\
\hline Lasioglossum_laevissimum & DIAL1412D05 & York University, Packer Colle \\
\hline Lasioglossum_laevissimum & A & York University, Packer Colle \\
\hline Lasioglossum_laevissimum & A & York University, Packer Colle \\
\hline Lasioglossum_laevissimum & A & York University, Packer Colle \\
\hline Lasioglossum_laevissimum & A & York University, Packer Colle \\
\hline Lasioglossum_laevissimum & A & York University, Packer Colle \\
\hline Lasioglossum_laevissimum & A & York University, Packer Colle \\
\hline Lasioglossum_laevissimum & A & York University, Packer Colle \\
\hline Lasioglossum_laevissimum & A & York University, Packer Colle \\
\hline Lasioglossum_laevissimum & A & York University, Packer Colle \\
\hline Lasioglossum_laevissimum & DIAL1412F09 & York University, Packer Colle \\
\hline Lasioglossum_laevissimum & DIAL1412A05 & York University, Packer Colle \\
\hline
\end{tabular}




\begin{tabular}{|c|c|c|}
\hline Lasioglossum_laevissimum & A & DIAL1412A06 \\
\hline Lasioglossum_laevissimum & A & DIAL1412G06 \\
\hline Lasioglossum_laevissimum & A & \\
\hline Lasioglossum_laevissimum & A & \\
\hline Lasioglossum_laevissimum & A & \\
\hline Lasioglossum_laevissimum & A & \\
\hline Lasioglossum_laevissimum & A & \\
\hline Lasioglossum_laevissimum & A & \\
\hline Lasioglossum_laevissimum & A & \\
\hline Lasioglossum_laevissimum & A & \\
\hline Lasioglossum_laevissimum & A & \\
\hline Lasioglossum_laevissimum & A & \\
\hline Lasioglossum_laevissimum & A & \\
\hline Lasioglossum_laevissimum & A & \\
\hline Lasioglossum_laevissimum & A & \\
\hline Lasioglossum_laevissimum & A & \\
\hline Lasioglossum_lepidii & A & \\
\hline Lasioglossum_lepidii & A & \\
\hline Lasioglossum_lepidii & A & \\
\hline Lasioglossum_leucocomum & A & \\
\hline Lasioglossum_leucocomum & A & \\
\hline Lasioglossum_leucocomum & A & \\
\hline Lasioglossum_leucocomum & A & DIAL1412C01 \\
\hline Lasioglossum_leucocomum & A & \\
\hline Lasioglossum_leucocomum & A & \\
\hline Lasioglossum_leucocomum & A & \\
\hline Lasioglossum_leucocomum & A & \\
\hline Lasioglossum_leucocomum & A & \\
\hline Lasioglossum_leucocomum & A & DIAL1412B11 \\
\hline Lasioglossum_leucocomum & A & \\
\hline Lasioglossum_leucocomum & A & \\
\hline Lasioglossum_leviense & A & yellow legs \\
\hline Lasioglossum_leviense & A & yellowish legs \\
\hline Lasioglossum_leviense & A & \\
\hline Lasioglossum_leviense & A & \\
\hline Lasioglossum_lineatulum & A & \\
\hline Lasioglossum_lineatulum & A & Ladmir-MN1 \\
\hline Lasioglossum_lineatulum & A & DIAL8-MB \\
\hline Lasioglossum_lineatulum & A & DIAL3-MB \\
\hline Lasioglossum_lineatulum & A & \\
\hline Lasioglossum_lineatulum & A & \\
\hline Lasioglossum_lineatulum & A & lineatulum1 \\
\hline Lasioglossum_lineatulum & A & \\
\hline Lasioglossum_lineatulum & A & \\
\hline Lasioglossum_lineatulum & A & \\
\hline Lasioglossum_lineatulum & A & \\
\hline Lasioglossum_lineatulum & A & \\
\hline Lasioglossum_lineatulum & A & \\
\hline Lasioglossum_lineatulum & A & DIAL5-MB \\
\hline Lasioglossum_lionotum & A & \\
\hline Lasioglossum_lionotum & A & \\
\hline Lasioglossum_lionotum & A & \\
\hline Lasioglossum_longifrons & A & \\
\hline Lasioglossum_longifrons & A & \\
\hline Lasioglossum_longifrons & A & \\
\hline
\end{tabular}

York University, Packer Colle York University, Packer Colle York University, Packer Colle York University, Packer Colle York University, Packer Colle York University, Packer Colle York University, Packer Colle York University, Packer Colle York University, Packer Colle York University, Packer Colle York University, Packer Colle York University, Packer Colle York University, Packer Colle York University, Packer Colle York University, Packer Colle York University, Packer Colle York University, Packer Colle York University, Packer Colle York University, Packer Colle York University, Packer Colle York University, Packer Colle York University, Packer Colle York University, Packer Colle York University, Packer Colle York University, Packer Colle York University, Packer Colle York University, Packer Colle York University, Packer Colle York University, Packer Colle York University, Packer Colle York University, Packer Colle York University, Packer Colle York University, Packer Colle York University, Packer Colle York University, Packer Colle York University, Packer Colle York University, Packer Colle York University, Packer Colle York University, Packer Colle York University, Packer Colle York University, Packer Colle York University, Packer Colle York University, Packer Colle York University, Packer Colle York University, Packer Colle York University, Packer Colle York University, Packer Colle York University, Packer Colle York University, Packer Colle York University, Packer Colle York University, Packer Colle York University, Packer Colle York University, Packer Colle York University, Packer Colle York University, Packer Colle 


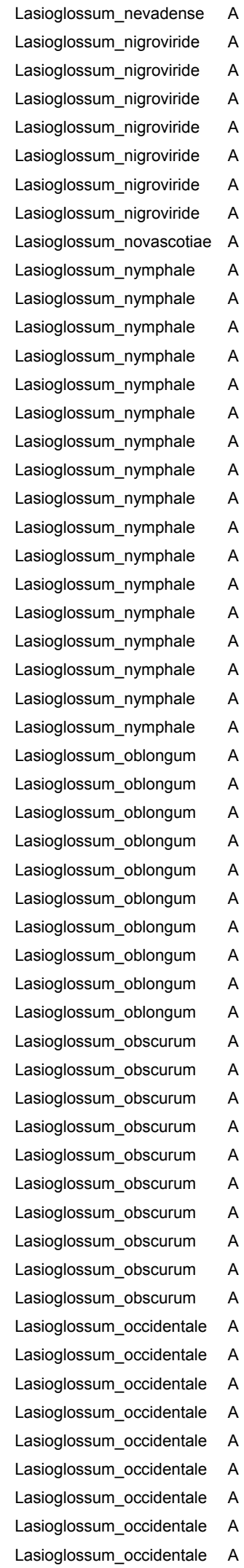

York University, Packer Colle York University, Packer Colle York University, Packer Colle York University, Packer Colle York University, Packer Colle York University, Packer Colle York University, Packer Colle York University, Packer Colle York University, Packer Colle York University, Packer Colle York University, Packer Colle York University, Packer Colle York University, Packer Colle York University, Packer Colle York University, Packer Colle York University, Packer Colle York University, Packer Colle York University, Packer Colle York University, Packer Colle York University, Packer Colle York University, Packer Colle York University, Packer Colle York University, Packer Colle York University, Packer Colle York University, Packer Colle York University, Packer Colle York University, Packer Colle York University, Packer Colle York University, Packer Colle York University, Packer Colle York University, Packer Colle York University, Packer Colle York University, Packer Colle York University, Packer Colle York University, Packer Colle York University, Packer Colle York University, Packer Colle York University, Packer Colle York University, Packer Colle York University, Packer Colle York University, Packer Colle York University, Packer Colle York University, Packer Colle York University, Packer Colle York University, Packer Colle weird York University, Packer Colle York University, Packer Colle York University, Packer Colle York University, Packer Colle York University, Packer Colle York University, Packer Colle York University, Packer Colle York University, Packer Colle York University, Packer Colle York University, Packer Colle 


\begin{tabular}{|c|c|c|}
\hline Lasioglossum_occidentale & A & York University, Packer Colle \\
\hline Lasioglossum_occidentale & A & York University, Packer Colle \\
\hline Lasioglossum_oceanicum & A & York University, Packer Colle \\
\hline Lasioglossum_oceanicum & A & York University, Packer Colle \\
\hline Lasioglossum_oceanicum & A & York University, Packer Colle \\
\hline Lasioglossum_oceanicum & A & York University, Packer Colle \\
\hline Lasioglossum_pacatum & A & York University, Packer Colle \\
\hline Lasioglossum_pacatum & A & York University, Packer Colle \\
\hline Lasioglossum_pacatum & A & York University, Packer Colle \\
\hline Lasioglossum_pacatum & A & York University, Packer Colle \\
\hline Lasioglossum_pacatum & A & York University, Packer Colle \\
\hline Lasioglossum_pacatum & A & York University, Packer Colle \\
\hline Lasioglossum_pacatum & A & York University, Packer Colle \\
\hline Lasioglossum_pacatum & A & York University, Packer Colle \\
\hline Lasioglossum_pacatum & A & York University, Packer Colle \\
\hline Lasioglossum_pacatum & A & York University, Packer Colle \\
\hline Lasioglossum_pacatum & A & York University, Packer Colle \\
\hline Lasioglossum_pacatum & A & York University, Packer Colle \\
\hline Lasioglossum_pacatum & A & York University, Packer Colle \\
\hline Lasioglossum_pacatum & A & York University, Packer Colle \\
\hline Lasioglossum_pacatum & A & York University, Packer Colle \\
\hline Lasioglossum_pacatum & A & York University, Packer Colle \\
\hline Lasioglossum_pacatum & A & York University, Packer Colle \\
\hline Lasioglossum_pacatum & A & York University, Packer Colle \\
\hline Lasioglossum_pacatum & A & York University, Packer Colle \\
\hline Lasioglossum_pacatum & A & York University, Packer Colle \\
\hline Lasioglossum_packeri & A & York University, Packer Colle \\
\hline Lasioglossum_packeri & A & York University, Packer Colle \\
\hline Lasioglossum_packeri & A & York University, Packer Colle \\
\hline Lasioglossum_packeri & A & York University, Packer Colle \\
\hline Lasioglossum_paradmirandu A & laevissimumB1 & York University, Packer Colle original ID by S. D. as perpuncte \\
\hline Lasioglossum_paradmirandu A & & York University, Packer Colle \\
\hline Lasioglossum_paradmirandu A & & York University, Packer Colle \\
\hline Lasioglossum_paradmirandu A & & York University, Packer Colle \\
\hline Lasioglossum_paradmirandu A & & York University, Packer Colle \\
\hline Lasioglossum_paradmirandu A & & York University, Packer Colle \\
\hline Lasioglossum_paradmirandu A & & York University, Packer Colle \\
\hline Lasioglossum_paradmirandu A & & York University, Packer Colle \\
\hline Lasioglossum_pavoninum & A & York University, Packer Colle \\
\hline Lasioglossum_pavoninum & A & York University, Packer Colle \\
\hline Lasioglossum_pavoninum & A & York University, Packer Colle \\
\hline Lasioglossum_perpunctatum A & & York University, Packer Colle \\
\hline Lasioglossum_perpunctatum A & perpu3 MB & York University, Packer Colle \\
\hline Lasioglossum_perpunctatum A & perpu4 MB & York University, Packer Colle \\
\hline Lasioglossum_perpunctatum A & & York University, Packer Colle \\
\hline Lasioglossum_perpunctatum A & & York University, Packer Colle \\
\hline Lasioglossum_perpunctatum A & perpu5 MB & York University, Packer Colle \\
\hline Lasioglossum_perpunctatum A & perpu1 MB & York University, Packer Colle \\
\hline Lasioglossum_perpunctatum A & & York University, Packer Colle \\
\hline Lasioglossum_perpunctatum A & & York University, Packer Colle \\
\hline Lasioglossum_perpunctatum A & pruin3 MB & York University, Packer Colle \\
\hline Lasioglossum_perpunctatum A & pruin2 MB & York University, Packer Colle \\
\hline Lasioglossum_perpunctatum A & perpu2 MB & York University, Packer Colle \\
\hline Lasioglossum_perpunctatum A & DIAL2-MB & York University, Packer Coll $\epsilon$ \\
\hline Lasioglossum_pictum & A & York University, Packer Colle \\
\hline
\end{tabular}




\begin{tabular}{|c|c|c|}
\hline Lasioglossum_pictum & A & York University, Packer Colle \\
\hline Lasioglossum_pictum & pictum4 & York University, Packer Colle bowl survey \\
\hline Lasioglossum_pictum & pictu $\sim \mathrm{IN} 1$ & York University, Packer Colle Yellow pan trap \\
\hline Lasioglossum_pictum & pictum3 & York University, Packer Colle bowl survey \\
\hline Lasioglossum_pictum & pictu2 MB & York University, Packer Coll€ \\
\hline Lasioglossum_pictum & pictu3 MB & York University, Packer Coll€ \\
\hline Lasioglossum_pictum & pictu1 MB & York University, Packer Colle \\
\hline Lasioglossum_pictum & $A$ & York University, Packer Colle \\
\hline Lasioglossum_pilosum & $A$ & York University, Packer Colle \\
\hline Lasioglossum_pilosum & A & York University, Packer Coll€ \\
\hline Lasioglossum_pilosum & $A$ & York University, Packer Colle \\
\hline Lasioglossum_pilosum & pilos1 NY & York University, Packer Colle \\
\hline Lasioglossum_pilosum & $A$ & York University, Packer Colle \\
\hline Lasioglossum_pilosum & A & York University, Packer Colle \\
\hline Lasioglossum_pilosum & A & York University, Packer Colle \\
\hline Lasioglossum_pilosum & A & York University, Packer Coll€ \\
\hline Lasioglossum_pilosum & $A$ & York University, Packer Colle \\
\hline Lasioglossum_pilosum & A & York University, Packer Colle \\
\hline Lasioglossum_pilosum & A & York University, Packer Colle \\
\hline Lasioglossum_pilosum & A & York University, Packer Colle \\
\hline Lasioglossum_pilosum & A & York University, Packer Colle \\
\hline Lasioglossum_pilosum & A & York University, Packer Colle \\
\hline Lasioglossum_pilosum & A & York University, Packer Colle \\
\hline Lasioglossum_pilosum & A & York University, Packer Colle \\
\hline Lasioglossum_pilosum & A & York University, Packer Colle \\
\hline Lasioglossum_pilosum & A & York University, Packer Coll€ \\
\hline Lasioglossum_pilosum & A & York University, Packer Coll€ \\
\hline Lasioglossum_planatum & A & York University, Packer Colle \\
\hline Lasioglossum_planatum & DIAL1NB & York University, Packer Colle \\
\hline Lasioglossum_planatum & DIAL1412A02 & York University, Packer Colle \\
\hline Lasioglossum_planatum & $293 \mathrm{H} 06$ & York University, Packer Colle \\
\hline Lasioglossum_planatum & DIAL1412A01 & York University, Packer Coll€ \\
\hline Lasioglossum_planatum & A & York University, Packer Coll€ \\
\hline Lasioglossum_planatum & A & York University, Packer Coll€ \\
\hline Lasioglossum_planatum & A & York University, Packer Colle \\
\hline Lasioglossum_platyparium & A & York University, Packer Coll€ \\
\hline Lasioglossum_platyparium & A & York University, Packer Coll€ \\
\hline Lasioglossum_platyparium & A & York University, Packer Coll€ \\
\hline Lasioglossum_platyparium & P. platyparium! & York University, Packer Colle Paralictus; pan trap \\
\hline Lasioglossum_platyparium & P. platyparium & York University, Packer Colle Paralictus \\
\hline Lasioglossum_platyparium & A & York University, Packer Colle \\
\hline Lasioglossum_platyparium & A & York University, Packer Coll€ \\
\hline Lasioglossum_platyparium A & A & York University, Packer Coll€ \\
\hline Lasioglossum_platyparium & A & York University, Packer Coll€ \\
\hline Lasioglossum_platyparium A & A & York University, Packer Coll€ \\
\hline Lasioglossum_platyparium & A & York University, Packer Coll€ \\
\hline Lasioglossum_prasinogaster A & & York University, Packer Coll€ \\
\hline Lasioglossum_prasinogaster A & & York University, Packer Coll€ \\
\hline Lasioglossum_prasinogaster A & & York University, Packer Coll€ \\
\hline Lasioglossum_prasinogaster A & WA-4 & York University, Packer Colle \\
\hline Lasioglossum_prasinogaster A & & York University, Packer Coll€ \\
\hline Lasioglossum_prasinogaster A & & York University, Packer Coll€ \\
\hline Lasioglossum_prasinogaster A & DIAL1412F01 & York University, Packer Coll€ \\
\hline Lasioglossum_prasinogaster A & & York University, Packer Colle \\
\hline Lasioglossum_prasinogaster $\mathrm{A}$ & WA-4 & York University, Packer Colle \\
\hline
\end{tabular}


Lasioglossum_prasinogaster A Lasioglossum_prasinogaster A Lasioglossum_prasinogaster A Lasioglossum_prasinogaster A Lasioglossum_prasinogaster A Lasioglossum_prasinogaster A Lasioglossum_prasinogaster A Lasioglossum_prasinogaster A Lasioglossum_pruinosum A Lasioglossum_pruinosum A Lasioglossum_pruinosum A Lasioglossum_pruinosum A Lasioglossum_pruinosum A Lasioglossum_pruinosum A Lasioglossum_pruinosum A Lasioglossum_pruinosum A Lasioglossum_punctatoventr A Lasioglossum_punctatoventr A Lasioglossum_punctatoventr A Lasioglossum_punctatoventr A Lasioglossum_punctatoventr A Lasioglossum_puteulanum A Lasioglossum_puteulanum A Lasioglossum_puteulanum A Lasioglossum_puteulanum A Lasioglossum_puteulanum A Lasioglossum_puteulanum A Lasioglossum_puteulanum A Lasioglossum_puteulanum A Lasioglossum_puteulanum A Lasioglossum_puteulanum A Lasioglossum_puteulanum A Lasioglossum_puteulanum A Lasioglossum_puteulanum A Lasioglossum_puteulanum A Lasioglossum_puteulanum A Lasioglossum_puteulanum A Lasioglossum_puteulanum A Lasioglossum_puteulanum A Lasioglossum_puteulanum A Lasioglossum_puteulanum A Lasioglossum_raleighense A Lasioglossum_raleighense A Lasioglossum_raleighense A Lasioglossum_reticulatum A Lasioglossum_rozeni A Lasioglossum_rozeni A Lasioglossum_rozeni A Lasioglossum_rozeni A Lasioglossum_rozeni A Lasioglossum_rozeni A Lasioglossum_rufulipes A Lasioglossum_rufulipes A Lasioglossum_rufulipes A Lasioglossum_rufulipes A

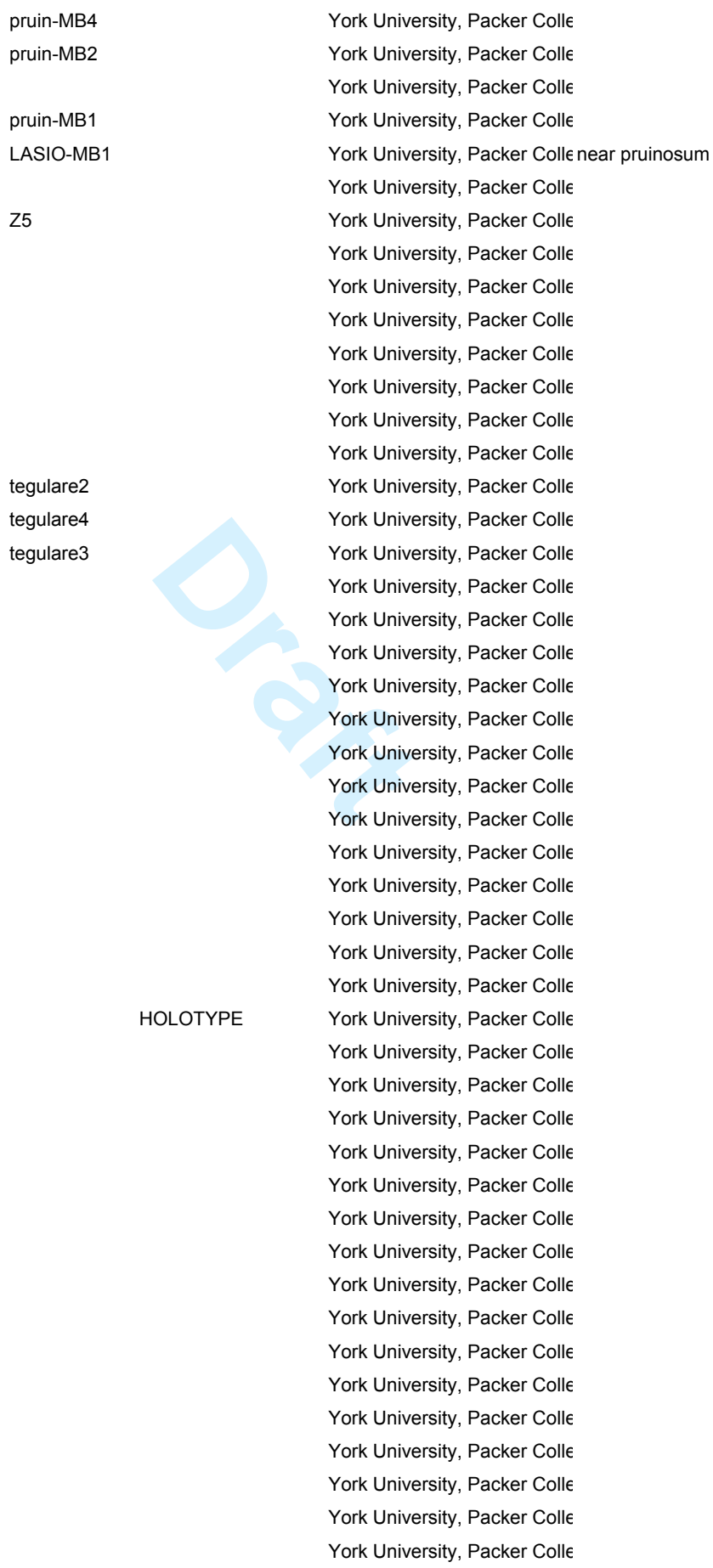




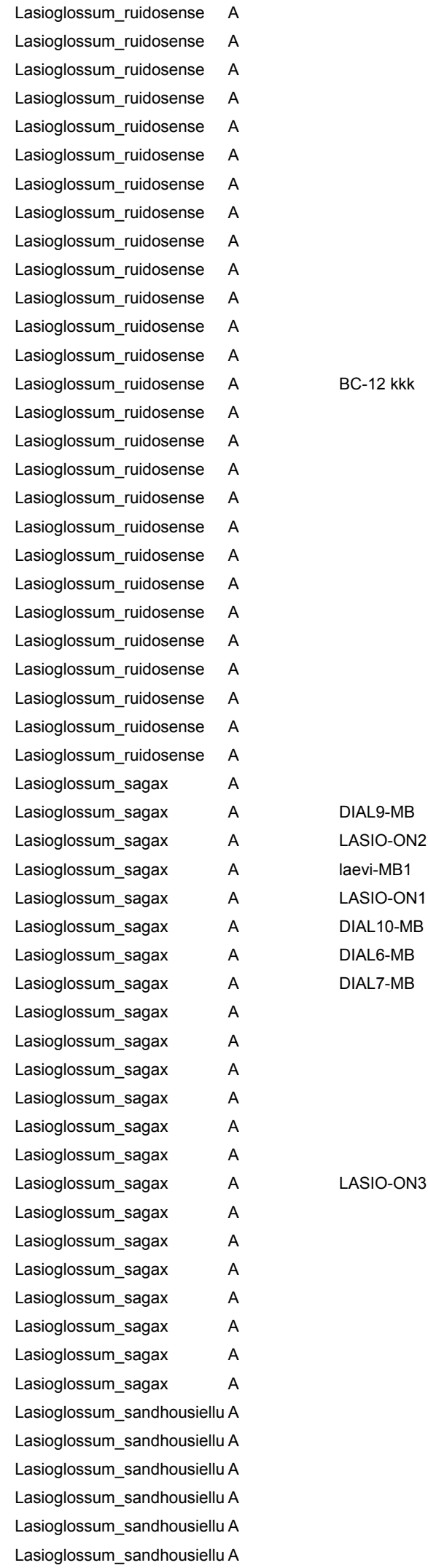


Lasioglossum_sandhousiellu A

Lasioglossum_sedi A

Lasioglossum_sedi A

Lasioglossum_sedi A

Lasioglossum_sedi A

Lasioglossum_sedi A

Lasioglossum_sedi A

Lasioglossum_sedi A

Lasioglossum_sedi A

Lasioglossum_sedi A

Lasioglossum_sedi A

Lasioglossum_sedi A

Lasioglossum_sedi A

Lasioglossum_sedi A

Lasioglossum_sedi A

Lasioglossum_sedi A

Lasioglossum_sedi A

Lasioglossum_semicaeruleu A

Lasioglossum_semicaeruleu A

Lasioglossum_semicaeruleu A

Lasioglossum_semicaeruleuı A

Lasioglossum_semicaeruleu A

Lasioglossum_semicaeruleu A

Lasioglossum_semicaeruleu A

Lasioglossum_semicaeruleu A

Lasioglossum_semicaeruleuı A

Lasioglossum_semicaeruleu A

Lasioglossum_semicaeruleu A

Lasioglossum_semicaeruleu A

Lasioglossum_semicaeruleuı A

Lasioglossum_semicaeruleuı A

Lasioglossum_semicaeruleu A

Lasioglossum_semicaeruleu A

Lasioglossum_semicaeruleuı A

Lasioglossum_semicaeruleu A

Lasioglossum_semicaeruleu A

Lasioglossum_semicaeruleu A

Lasioglossum_semicaeruleu A

Lasioglossum_semicaeruleu A

Lasioglossum_semicaeruleuı A

Lasioglossum_semicaeruleuı A

Lasioglossum_semicaeruleu A

Lasioglossum_semicaeruleu A

Lasioglossum_semicaeruleuı A

Lasioglossum_semicaeruleuı A

Lasioglossum_sheffieldi A

Lasioglossum_sheffieldi A

Lasioglossum_sheffieldi A

Lasioglossum_sheffieldi A

Lasioglossum_sheffieldi A

Lasioglossum_sheffieldi A

Lasioglossum_sheffieldi A

Lasioglossum_simplex A

Lasioglossum_simplex A

Lasioglossum_smilacinae A ok 1

York University, Packer Colle

York University, Packer Colle

York University, Packer Colle

York University, Packer Colle

York University, Packer Colle

York University, Packer Colle

York University, Packer Colle

York University, Packer Colle

York University, Packer Colle

York University, Packer Colle

York University, Packer Colle

York University, Packer Colle

York University, Packer Colle

York University, Packer Colle

York University, Packer Colle

York University, Packer Colle

York University, Packer Colle

York University, Packer Colle

York University, Packer Colle

York University, Packer Colle

York University, Packer Colle

York University, Packer Colle

DIAL1412F02 York University, Packer Colle

York University, Packer Colle

York University, Packer Colle

York University, Packer Colle

York University, Packer Colle

York University, Packer Colle

York University, Packer Colle

York University, Packer Colle

York University, Packer Colle

York University, Packer Colle

York University, Packer Colle

York University, Packer Colle

York University, Packer Colle

York University, Packer Colle

York University, Packer Colle

York University, Packer Colle

pruin4 MB

York University, Packer Colle

York University, Packer Colle

York University, Packer Colle

York University, Packer Colle

pruin5 MB

pruin1 MB

York University, Packer Colle

York University, Packer Colle

York University, Packer Colle

York University, Packer Colle

York University, Packer Colle

York University, Packer Colle

York University, Packer Colle

York University, Packer Colle

York University, Packer Colle

York University, Packer Colle

York University, Packer Colle

York University, Packer Colle

laevi IN1

York University, Packer Colle Col on: Selix humilis 
Lasioglossum_sp. nov_A A Lasioglossum_subversans A Lasioglossum_subversans A Lasioglossum_subversans A Lasioglossum_subviridatum A Lasioglossum_subviridatum A Lasioglossum_subviridatum A Lasioglossum_subviridatum A Lasioglossum_subviridatum A Lasioglossum_subviridatum A Lasioglossum_subviridatum A Lasioglossum_subviridatum A Lasioglossum_subviridatum A Lasioglossum_subviridatum A Lasioglossum_subviridatum A Lasioglossum_subviridatum A Lasioglossum_subviridatum A Lasioglossum_subviridatum A Lasioglossum_subviridatum A Lasioglossum_subviridatum A Lasioglossum_subviridatum A Lasioglossum_subviridatum A Lasioglossum_subviridatum A Lasioglossum_subviridatum A Lasioglossum_subviridatum A Lasioglossum_subviridatum A Lasioglossum_subviridatum A Lasioglossum_subviridatum A Lasioglossum_subviridatum A Lasioglossum_subviridatum A Lasioglossum_subviridatum A Lasioglossum_subviridatum A Lasioglossum_subviridatum A Lasioglossum_subviridatum A Lasioglossum_subviridatum A Lasioglossum_subviridatum A Lasioglossum_subviridatum A Lasioglossum_subviridatum A Lasioglossum_subviridatum A Lasioglossum_subviridatum A Lasioglossum_subviridatum A Lasioglossum_subviridatum A Lasioglossum_succinipenne A Lasioglossum_succinipenne A Lasioglossum_succinipenne A Lasioglossum_succinipenne A Lasioglossum_succinipenne A Lasioglossum_tamiamense A Lasioglossum_tarponense A Lasioglossum_tarponense A Lasioglossum_tarponense A Lasioglossum_tarponense A Lasioglossum_taylorae A Lasioglossum_taylorae A Lasioglossum_taylorae A

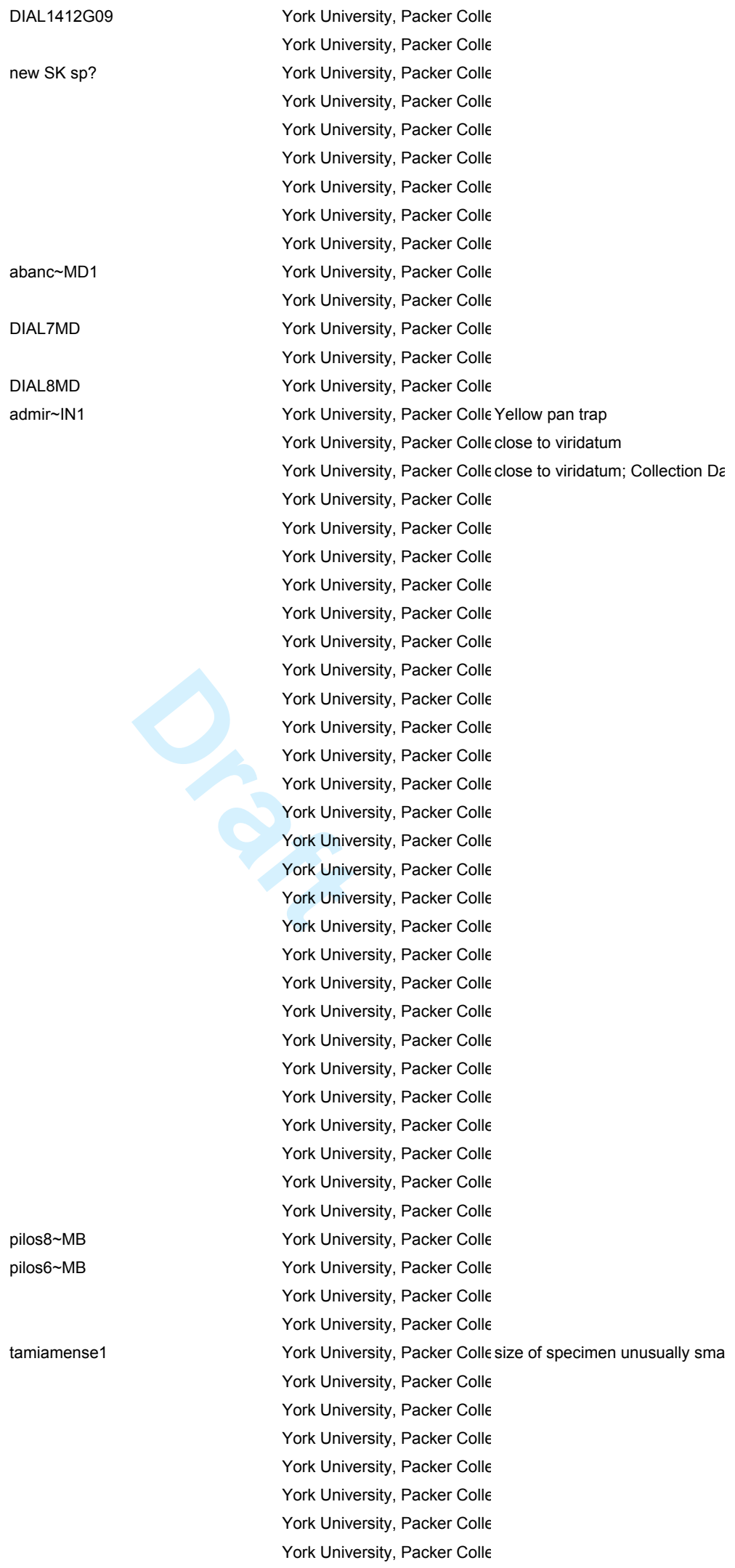




\begin{tabular}{|c|c|c|}
\hline Lasioglossum_tegulare & A & \\
\hline Lasioglossum_tegulare & A & tegul VA1 \\
\hline Lasioglossum_tegulare & A & tegul NY1 \\
\hline Lasioglossum_tegulare & A & \\
\hline Lasioglossum_tegulare & A & \\
\hline Lasioglossum_tegulare & A & \\
\hline Lasioglossum_tegulare & A & \\
\hline Lasioglossum_tegulare & A & \\
\hline Lasioglossum_tegulare & A & \\
\hline Lasioglossum_tegulare & A & \\
\hline Lasioglossum_tegulare & A & \\
\hline Lasioglossum_tegulare & A & \\
\hline Lasioglossum_tegulare & A & \\
\hline Lasioglossum_tegulare & A & \\
\hline Lasioglossum_tegulare & A & \\
\hline Lasioglossum_tegulare & A & \\
\hline Lasioglossum_tegulare & A & \\
\hline Lasioglossum_tegulare & A & \\
\hline Lasioglossum_tegulare & A & \\
\hline Lasioglossum_tegulare & A & \\
\hline Lasioglossum_tegulare & A & \\
\hline Lasioglossum_tegulare & A & \\
\hline Lasioglossum_tegulare & A & tegul1 VA \\
\hline Lasioglossum_tegulare & A & \\
\hline Lasioglossum_tegulare & A & \\
\hline Lasioglossum_tegulare & A & \\
\hline Lasioglossum_tenax & A & \\
\hline Lasioglossum_tenax & A & \\
\hline Lasioglossum_tenax & A & \\
\hline Lasioglossum_tenax & A & \\
\hline Lasioglossum_tenax & A & \\
\hline Lasioglossum_tenax & A & \\
\hline Lasioglossum_tenax & A & \\
\hline Lasioglossum_tenax & A & \\
\hline Lasioglossum_tenax & A & \\
\hline Lasioglossum_tenax & A & \\
\hline Lasioglossum_tenax & A & \\
\hline Lasioglossum_tenax & A & \\
\hline Lasioglossum_tenax & A & \\
\hline Lasioglossum_tenax & A & DIAL1412A10 \\
\hline Lasioglossum_tenax & A & \\
\hline Lasioglossum_tenax & A & \\
\hline Lasioglossum_tenax & A & \\
\hline Lasioglossum_tenax & A & \\
\hline Lasioglossum_tenax & A & \\
\hline Lasioglossum_timothyi & A & \\
\hline Lasioglossum_timothyi & A & \\
\hline Lasioglossum_timothyi & A & \\
\hline Lasioglossum_trigeminum & A & \\
\hline Lasioglossum_trigeminum & A & \\
\hline Lasioglossum_trigeminum & A & admir1 MD \\
\hline Lasioglossum_trigeminum & A & \\
\hline Lasioglossum_trigeminum & A & DIAL4MD \\
\hline Lasioglossum_trigeminum & A & admir2 IN \\
\hline Lasioglossum trigeminum & A & \\
\hline
\end{tabular}

York University, Packer Colle York University, Packer Colle York University, Packer Colle York University, Packer Colle York University, Packer Colle York University, Packer Colle York University, Packer Colle York University, Packer Colle York University, Packer Colle York University, Packer Colle York University, Packer Colle York University, Packer Colle York University, Packer Colle York University, Packer Colle York University, Packer Colle York University, Packer Colle York University, Packer Colle York University, Packer Colle York University, Packer Colle York University, Packer Colle York University, Packer Colle York University, Packer Colle York University, Packer Colle York University, Packer Colle York University, Packer Colle York University, Packer Colle York University, Packer Colle York University, Packer Colle York University, Packer Colle York University, Packer Colle York University, Packer Colle York University, Packer Colle York University, Packer Colle York University, Packer Colle York University, Packer Colle York University, Packer Colle York University, Packer Colle York University, Packer Colle York University, Packer Colle York University, Packer Colle York University, Packer Colle York University, Packer Colle York University, Packer Colle York University, Packer Colle York University, Packer Colle York University, Packer Colle York University, Packer Colle York University, Packer Colle York University, Packer Colle York University, Packer Coll€ Collection Date: 20-Sep-? York University, Packer Colle York University, Packer Colle York University, Packer Colle York University, Packer Colle York University, Packer Colle 


\begin{tabular}{|c|c|c|}
\hline Lasioglossum_trigeminum & A & versatum5 \\
\hline Lasioglossum_trigeminum & A & \\
\hline Lasioglossum_trigeminum & A & \\
\hline Lasioglossum_trigeminum & A & \\
\hline Lasioglossum_trigeminum & A & \\
\hline Lasioglossum_trigeminum & A & \\
\hline Lasioglossum_trigeminum & A & \\
\hline Lasioglossum_trigeminum & A & \\
\hline Lasioglossum_trigeminum & A & \\
\hline Lasioglossum_trigeminum & A & \\
\hline Lasioglossum_trigeminum & A & \\
\hline Lasioglossum_trigeminum & A & \\
\hline Lasioglossum_trigeminum & A & \\
\hline Lasioglossum_trigeminum & A & \\
\hline Lasioglossum_trigeminum & A & \\
\hline Lasioglossum_trigeminum & A & \\
\hline Lasioglossum_trigeminum & A & \\
\hline Lasioglossum_trigeminum & A & \\
\hline Lasioglossum_trigeminum & A & \\
\hline Lasioglossum_trigeminum & A & \\
\hline Lasioglossum_trigeminum & A & \\
\hline Lasioglossum_trigeminum & A & \\
\hline Lasioglossum_versans & A & \\
\hline Lasioglossum_versans & A & \\
\hline Lasioglossum_versans & A & versans3 \\
\hline Lasioglossum_versans & A & \\
\hline Lasioglossum_versans & A & \\
\hline Lasioglossum_versans & A & \\
\hline Lasioglossum_versans & A & \\
\hline Lasioglossum_versans & A & DIAL1412E08 \\
\hline Lasioglossum_versans & A & \\
\hline Lasioglossum_versatum & A & \\
\hline Lasioglossum_versatum & A & \\
\hline Lasioglossum_versatum & A & \\
\hline Lasioglossum_versatum & A & \\
\hline Lasioglossum_versatum & A & \\
\hline Lasioglossum_versatum & A & \\
\hline Lasioglossum_versatum & A & \\
\hline Lasioglossum_versatum & A & DIAL5MD \\
\hline Lasioglossum_versatum & A & rohwe IL1 \\
\hline Lasioglossum_versatum & A & rohwe IN2 \\
\hline Lasioglossum_versatum & A & rohwe $\sim \mathrm{NY} 1$ \\
\hline Lasioglossum_versatum & A & DIAL1412E02 \\
\hline Lasioglossum_versatum & A & \\
\hline Lasioglossum_versatum & A & \\
\hline Lasioglossum_versatum & A & \\
\hline Lasioglossum_versatum & A & \\
\hline Lasioglossum_versatum & A & \\
\hline Lasioglossum_versatum & A & gynandromorp \\
\hline Lasioglossum_versatum & A & \\
\hline Lasioglossum_versatum & A & \\
\hline Lasioglossum_versatum & A & \\
\hline Lasioglossum_versatum & A & \\
\hline Lasioglossum_versatum & A & \\
\hline Lasioglossum_versatum & A & \\
\hline
\end{tabular}

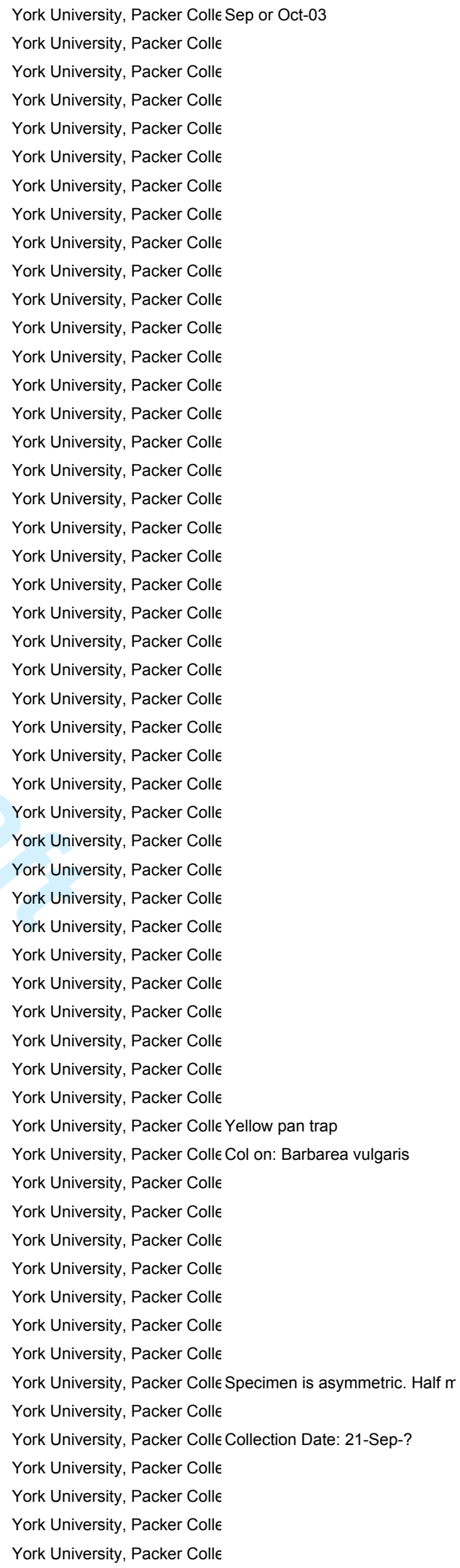




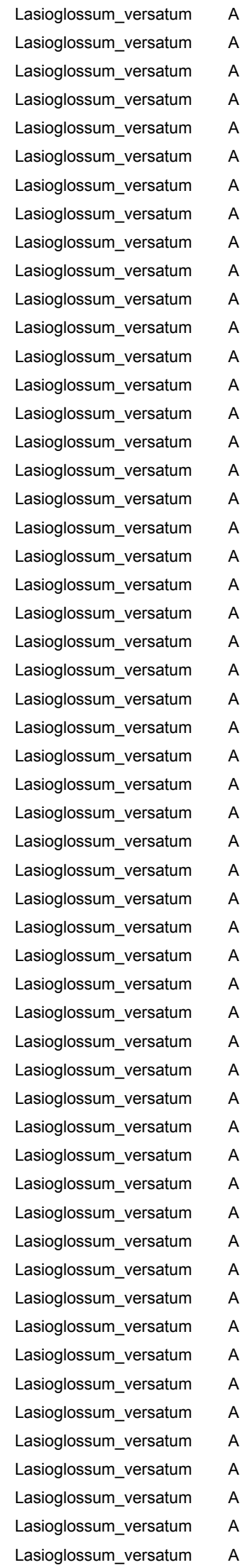
York University, Packer Colle York University, Packer Colle York University, Packer Colle York University, Packer Colle York University, Packer Colle York University, Packer Colle York University, Packer Colle York University, Packer Colle York University, Packer Colle York University, Packer Colle York University, Packer Colle York University, Packer Colle York University, Packer Colle York University, Packer Colle York University, Packer Colle York University, Packer Colle York University, Packer Colle York University, Packer Colle York University, Packer Colle York University, Packer Colle York University, Packer Colle York University, Packer Colle York University, Packer Colle York University, Packer Colle York University, Packer Colle York University, Packer Colle York University, Packer Colle York University, Packer Colle York University, Packer Colle York University, Packer Colle York University, Packer Colle York University, Packer Colle York University, Packer Colle York University, Packer Colle York University, Packer Colle York University, Packer Colle York University, Packer Colle York University, Packer Colle York University, Packer Colle York University, Packer Colle York University, Packer Colle York University, Packer Colle York University, Packer Colle York University, Packer Colle York University, Packer Colle York University, Packer Colle York University, Packer Colle York University, Packer Colle York University, Packer Colle York University, Packer Colle York University, Packer Colle York University, Packer Colle York University, Packer Colle York University, Packer Colle York University, Packer Colle 


\begin{tabular}{|c|c|c|}
\hline Lasioglossum_versatum & A & \\
\hline Lasioglossum_versatum & A & \\
\hline Lasioglossum_versatum & A & \\
\hline Lasioglossum_versatum & A & \\
\hline Lasioglossum_versatum & A & \\
\hline Lasioglossum_versatum & A & \\
\hline Lasioglossum_versatum & A & \\
\hline Lasioglossum_versatum & A & \\
\hline Lasioglossum_versatum & A & \\
\hline Lasioglossum_versatum & A & \\
\hline Lasioglossum_versatum & A & \\
\hline Lasioglossum_versatum & A & \\
\hline Lasioglossum_versatum & A & \\
\hline Lasioglossum_versatum & A & \\
\hline Lasioglossum_versatum & A & \\
\hline Lasioglossum_versatum & A & \\
\hline Lasioglossum_versatum & A & \\
\hline Lasioglossum_versatum & A & \\
\hline Lasioglossum_vierecki & A & \\
\hline Lasioglossum_vierecki & A & \\
\hline Lasioglossum_vierecki & A & viere IN1 \\
\hline Lasioglossum_vierecki & A & viere1 NY \\
\hline Lasioglossum_vierecki & A & vierecki4 \\
\hline Lasioglossum_vierecki & A & vierecki1 \\
\hline Lasioglossum_vierecki & A & \\
\hline Lasioglossum_vierecki & A & vierecki2 \\
\hline Lasioglossum_vierecki & A & \\
\hline Lasioglossum_vierecki & A & \\
\hline Lasioglossum_vierecki & A & viere1 MB \\
\hline Lasioglossum_vierecki & A & \\
\hline Lasioglossum_vierecki & A & \\
\hline Lasioglossum_viridatum & A & \\
\hline Lasioglossum_viridatum & A & \\
\hline Lasioglossum_viridatum & A & \\
\hline Lasioglossum_viridatum & A & \\
\hline Lasioglossum_viridatum & A & \\
\hline Lasioglossum_viridatum & A & DIAL1412A04 \\
\hline Lasioglossum_weemsi & A & \\
\hline Lasioglossum_weemsi & A & yellow legs \\
\hline Lasioglossum_weemsi & A & \\
\hline Lasioglossum_weemsi & A & admir1 PA \\
\hline Lasioglossum_weemsi & A & \\
\hline Lasioglossum_weemsi & A & atlanticumA03 \\
\hline Lasioglossum_weemsi & A & \\
\hline Lasioglossum_weemsi & A & \\
\hline Lasioglossum_weemsi & A & DIAL3MD \\
\hline Lasioglossum_weemsi & A & \\
\hline Lasioglossum_weemsi & A & \\
\hline Lasioglossum_weemsi & A & \\
\hline Lasioglossum_weemsi & A & \\
\hline Lasioglossum_weemsi & A & \\
\hline Lasioglossum_weemsi & A & \\
\hline Lasioglossum_weemsi & A & \\
\hline Lasioglossum_weemsi & A & \\
\hline Lasioglossum_weemsi & A & \\
\hline
\end{tabular}

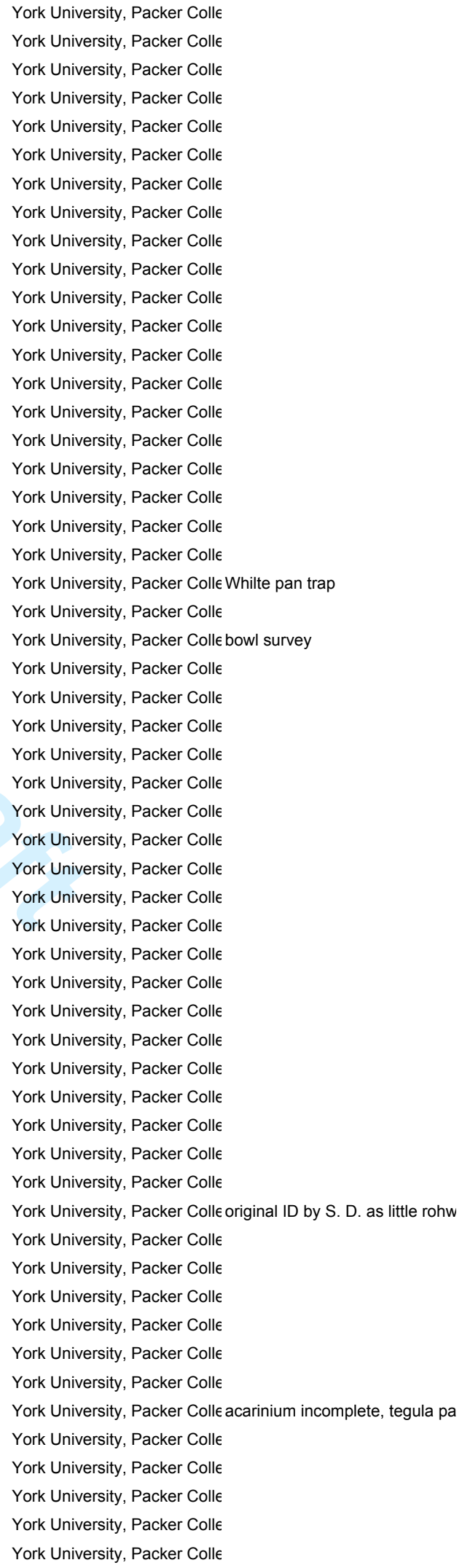




$\begin{array}{ll}\text { Lasioglossum_weemsi } & \text { A } \\ \text { Lasioglossum_weemsi } & \text { A } \\ \text { Lasioglossum_weemsi } & \text { A } \\ \text { Lasioglossum_weemsi } & \text { A } \\ \text { Lasioglossum_weemsi } & \text { A } \\ \text { Lasioglossum_weemsi } & \text { A } \\ \text { Lasioglossum_weemsi } & \text { A } \\ \text { Lasioglossum_yukonae } & \text { A } \\ \text { Lasioglossum_yukonae } & \text { A } \\ \text { Lasioglossum_yukonae } & \text { A } \\ \text { Lasioglossum_zephyrum } & \text { A } \\ \text { Lasioglossum_zephyrum } & \text { A } \\ \text { Lasioglossum_zephyrum } & \text { A } \\ \text { Lasioglossum_zephyrum } & \text { A } \\ \text { Lasioglossum_zephyrum } & \text { A } \\ \text { Lasioglossum_zephyrum } & \text { A } \\ \text { Lasioglossum_zephyrum } & \text { A }\end{array}$

\author{
York University, Packer Coll \\ York University, Packer Colle \\ York University, Packer Colle \\ York University, Packer Colle obscure punctures visible on pl€ \\ York University, Packer Colle \\ York University, Packer Colle \\ York University, Packer Colle \\ York University, Packer Colle \\ York University, Packer Colle \\ York University, Packer Colle \\ York University, Packer Colle \\ York University, Packer Colle \\ York University, Packer Colle \\ York University, Packer Colle sculpturing unusual 293G10 \\ York University, Packer Colle \\ York University, Packer Colle \\ York University, Packer Colle
}


). as admirandum 
dense punctures in central area of scutum 
Incate with complete rugae 
ite: 15-Jul-?

\section{॥}


ale/Half female 
le, dorsal surface of propodeum plicate

https://mc06.manuscriptcentral.com/genome-pubs 


\begin{tabular}{|c|c|c|c|c|}
\hline Project: & SEARCH: Process & ids(1283 ids) & & Jason Gibbs \\
\hline Created : & 12-July-2017 & & & \\
\hline Sample ID & \begin{tabular}{|l} 
Field ID \\
\end{tabular} & Museum ID & Collection Code & Institution Storing \\
\hline D00608F07-IN & D00608F07 & & & York University, Packer Collection \\
\hline DIAL2386F10-UT & DIAL2386F10 & BBSL649711 & & York University, Packer Collection \\
\hline DIAL2386C10-UT & DIAL2386C10 & ZION21985 & & York University, Packer Collection \\
\hline DIAL2384C07-AB & DIAL2384C07 & & & York University, Packer Collection \\
\hline DIAL2386C08-UT & DIAL2386C08 & ZION21743 & & York University, Packer Collection \\
\hline DIAL2374H06-UT & DIAL2374H06 & BBSL645891 & & York University, Packer Collection \\
\hline DIAL2385D04-ON & JIAL2385D04-ON & & & York University, Packer Collection \\
\hline DIAL2385E03-MD & DIAL2385E03-MD & & & York University, Packer Collection \\
\hline D01562A03-Ml & D01562A03 & & & York University, Packer Collection \\
\hline DIAL1839G06-MN & DIAL1839G06-MN & & & York University, Packer Collection \\
\hline DIAL1839G08-MN & DIAL1839G08-MN & & & York University, Packer Collection \\
\hline DIAL0292H11-MD & little rohweri 027590 & & & York University, Packer Collection \\
\hline DIAL1409C04-ON & DIAL1409C04 & & & York University, Packer Collection \\
\hline DIAL2387C05-ON & J DIAL2387C05 & & & York University, Packer Collection \\
\hline D01561D09-ON & D01561D09 & & & York University, Packer Collection \\
\hline 04791B03-FL & 04791B03-FL & & & York University, Packer Collection \\
\hline DIAL0292B02-NJ & albipenne \#1514 & & & York University, Packer Collection \\
\hline DIAL0286F05-MB & DIAL0286F05 & & & York University, Packer Collection \\
\hline D00609G09-NS & D00609G09 & & & York University, Packer Collection \\
\hline DIAL1839G12-MN & DIAL1839G12-MN & & & York University, Packer Collection \\
\hline DIAL1839G09-MN & DIAL1839G09-MN & & & York University, Packer Collection \\
\hline 06732D10-PA & 06732D10-PA & & & York University, Packer Collection \\
\hline BEE1850G06-ME & BEE1850G06 & & & York University, Packer Collection \\
\hline DIAL2386G02-BC & DIAL2386G02 & & & York University, Packer Collection \\
\hline DIAL1848A08-NB & DIAL1848A08 & & & York University, Packer Collection \\
\hline DIAL1849A09-CO & DIAL1849A09 & & & York University, Packer Collection \\
\hline DIAL0286F03-MB & DIAL0286F03 & & & York University, Packer Collection \\
\hline DIAL2384C02-AB & DIAL2384C02 & & & York University, Packer Collection \\
\hline D01559F12-BC & D01559F12 & & & York University, Packer Collection \\
\hline DIAL2386G07-WA & DIAL2386G07 & & & York University, Packer Collection \\
\hline DIAL2387E09-AB & DIAL2387E09 & & & York University, Packer Collection \\
\hline D01559F10-BC & D01559F10 & & & York University, Packer Collection \\
\hline D01559H03-BC & D01559H03 & & & York University, Packer Collection \\
\hline D01559H10-BC & $\mathrm{D} 01559 \mathrm{H} 10$ & & & York University, Packer Collection \\
\hline DIAL2387B05-WA & DIAL2387B05 & & & York University, Packer Collection \\
\hline DIAL0294A03-IN & $\# 12425$ & & & York University, Packer Collection \\
\hline DIAL0292B05-IN & $\# 13165$ & & & York University, Packer Collection \\
\hline BEE0289C05-MN & BEE0289C05 & & & York University, Packer Collection \\
\hline CCDB-00611 C12 & CCDB-00611 C12 & & & York University, Packer Collection \\
\hline D-3253D12-WI & D-3253D12 & & & York University, Packer Collection \\
\hline D0287D01-MI & D0287D01 & & & York University, Packer Collection \\
\hline DIAL1390D06-ON & JIAL1390D06 & & & York University, Packer Collection \\
\hline DIAL1846F06-PA & DIAL1846F06 & & & York University, Packer Collection \\
\hline DIAL1390F04-NC & DIAL1390F04 & & & York University, Packer Collection \\
\hline DIAL0292E12-IN & \#175 & & & York University, Packer Collection \\
\hline DIAL1849F01-WV & / DIAL1849F01 & & & York University, Packer Collection \\
\hline DIAL0292B01-WV & /\#010947 & & & York University, Packer Collection \\
\hline DIAL0292B07-WV & \#011004 & & & York University, Packer Collection \\
\hline DIAL0292B08-WV & /\#010899 & & & York University, Packer Collection \\
\hline DIAL0292F02-WV & / \#032685 & & & York University, Packer Collection \\
\hline DIAL1849E12-WV & / DIAL1849E12 & & & York University, Packer Collection \\
\hline DIAL0292E11-WV & \#032688 & & & York University, Packer Collection \\
\hline
\end{tabular}


DIAL1390F06-NC DIAL1390F06 DIAL1390G04-NC DIAL1390G04 DIAL1390G06-NC DIAL1390G06 DIAL1390G02-WV DIAL1390G02 DIAL1848D09-MD DIAL1848D09 DIAL1846D12-MS DIAL1846D12 D1414H02-VA D1414H02 DIAL2384H09-SC DIAL2384H09-SC D01559C09-GA D01559C09 D1414E02-VA D1414E02 DIAL0293C03-NJ \#2488 DIAL0293C04-NJ \#1247 D01562B06-MD D01562B06 D01562B02-MD D01562B02 D01562C12-MD D01562C12 D01562B10-ON D01562B10 D01562C08-ON D01562C08 DIAL0294G02-ML \#020228 DIAL1848B03-ON DIAL1848B03 D00609B05-WI D00609B05 D00609G03-NS D00609G03 D0288F07-SC D0288F07 D0288F09-SC D0288F09 DIAL0294A05-in \#12565 DIAL0292E08-MD \#028623 06732D09-VA 06732D09-VA D00609C07-KS D00609C07 DIAL1846B10-WV DIAL1846B10 DIAL1390C08-ON DIAL1390C08 DIAL1409A09-NC DIAL1409A09 DIAL1846G04-VA DIAL1846G04 DIAL1390B02-ON DIAL1390B02 DIAL1848E12-MD DIAL1848E12 DIAL1413C12-TN DIAL1413C12 D01559G11-BC D01559G11 DIAL1845D09-CA DIAL1845D09 DIAL2374G12-CA DIAL2374G12 DIAL1845C05-CA DIAL1845C05 DIAL1845D03-CA DIAL1845D03 DIAL1845C11-CA DIAL1845C11 DIAL2388C12-UT DIAL2388C12-UT DIAL2374G11-CA DIAL2374G11 D00607E04-CA D00607E04 D01559E10-BC D01559E10 D01559G04-BC D01559G04 DIAL2388B06-UT DIAL2388B06-UT D00607D05-CA D00607D05 DIAL2374H09-UT DIAL2374H09 DIAL2385C03-MD DIAL2385C03-MD DIAL1413E11-SC DIAL1413E11 DIAL0292D03-NC coeruleum 023909 DIAL0294E03-VA \#046377 DIAL0294E02-TN \#055668 DIAL0293E11-NC versatum 023948 DIAL0293E10-MD \#023822
York University, Packer Collection York University, Packer Collection York University, Packer Collection York University, Packer Collection York University, Packer Collection York University, Packer Collection York University, Packer Collection York University, Packer Collection York University, Packer Collection York University, Packer Collection York University, Packer Collection York University, Packer Collection York University, Packer Collection York University, Packer Collection York University, Packer Collection York University, Packer Collection York University, Packer Collection York University, Packer Collection 1390D05 York University, Packer Collection York University, Packer Collection York University, Packer Collection York University, Packer Collection York University, Packer Collection York University, Packer Collection York University, Packer Collection York University, Packer Collection York University, Packer Collection York University, Packer Collection York University, Packer Collection York University, Packer Collection York University, Packer Collection York University, Packer Collection York University, Packer Collection York University, Packer Collection York University, Packer Collection York University, Packer Collection York University, Packer Collection York University, Packer Collection York University, Packer Collection York University, Packer Collection York University, Packer Collection York University, Packer Collection York University, Packer Collection York University, Packer Collection York University, Packer Collection York University, Packer Collection York University, Packer Collection York University, Packer Collection York University, Packer Collection York University, Packer Collection York University, Packer Collection York University, Packer Collection York University, Packer Collection York University, Packer Collection York University, Packer Collection 
D0288C04-DC D0288C04

DIAL0293E12-DC versatum 026920

D1414H07-VA D1414H07

BEE0286D06-VA BEE0286D06

DIAL1413D04-NC DIAL1413D04

DIAL1391D10-NC DIAL1391D10

D1414E08-VA D1414E08

DIAL1409B06-NC DIAL1409B06

DIAL1413E08-NC DIAL1413E08

D1414E04-VA D1414E04

DIAL1391A09-SC DIAL1391A09

DIAL1413F07-TN DIAL1413F07

DIAL1391D09-NC DIAL1391D09

DIAL1391D07-NC DIAL1391D07

DIAL1413D01-TN DIAL1413D01

D1414E07-VA D1414E07

D0288C01-DC D0288C01

D0288C06-SC D0288C06

D0288D09-SC D0288D09

D00607E06-MO D00607E06

D00607A09-MO D00607A09

D0288G03-SC D0288G03

D0288F02-SC D0288F02

DIAL1391B01-SC DIAL1391B01

DIAL1391E04-SC DIAL1391E04

DIAL1391D11-SC DIAL1391D11

DIAL1413B06-IL DIAL1413B06

D1414A07-IL D1414A07

DIAL1413B11-IL DIAL1413B11

04485C03-MD 04485C03-MD

D00607F06-MD D00607F06

D-3253D11-FL D-3253D11

D00607B02-KS D00607B02

DIAL0292D09-MD\#012960

DIAL0292D10-MD \#002309

DIAL0292D08-NC \#023927

DIAL0292D12-MD \#002291

DIAL0292D11-MD \#029860

DIAL1846H06-VA DIAL1846H06

DIAL1846C01-MS DIAL1846C01

DIAL1846E11-MS DIAL1846E11

DIAL1409E03-TX DIAL1409E03

DIAL1846G07-MS DIAL1846G07

DIAL1849F11-DE DIAL1849F11

DIAL1849G03-DE DIAL1849G03

DIAL1391D04-NC DIAL1391D04

06732C08-MA 06732C08-MA

06732C10-VA 06732C10-VA

D-3253D07-FL D-3253D07

B01566H05-TX B01566H05

DIAL0292E02-MD \#017872

DIAL0294F02-NC \#046248

DIAL0294F03-NC \#046252

DIAL0294E05-NC \#056272

DIAL0292E04-MD \#017878
York University, Packer Collection

York University, Packer Collection

York University, Packer Collection

York University, Packer Collection

York University, Packer Collection

York University, Packer Collection

York University, Packer Collection

York University, Packer Collection

York University, Packer Collection

York University, Packer Collection

York University, Packer Collection

York University, Packer Collection

York University, Packer Collection

York University, Packer Collection

York University, Packer Collection

York University, Packer Collection

York University, Packer Collection

York University, Packer Collection

York University, Packer Collection

York University, Packer Collection

York University, Packer Collection

York University, Packer Collection

York University, Packer Collection

York University, Packer Collection

York University, Packer Collection

York University, Packer Collection

York University, Packer Collection

York University, Packer Collection

York University, Packer Collection

York University, Packer Collection

York University, Packer Collection

York University, Packer Collection

York University, Packer Collection

York University, Packer Collection

York University, Packer Collection

York University, Packer Collection

York University, Packer Collection

York University, Packer Collection

York University, Packer Collection

York University, Packer Collection

York University, Packer Collection

York University, Packer Collection

York University, Packer Collection

York University, Packer Collection

York University, Packer Collection

York University, Packer Collection

York University, Packer Collection

York University, Packer Collection

York University, Packer Collection

York University, Packer Collection

York University, Packer Collection

York University, Packer Collection

York University, Packer Collection

York University, Packer Collection

York University, Packer Collection 
DIAL1413D12-FL DIAL1413D12 DIAL1846C06-MS DIAL1846C06 DIAL1846H02-MS DIAL1846H02 DIAL1846C11-MS DIAL1846C11 CCDB-00611 E05 CCDB-00611 E05 BEE0286C08-NC BEE0286C08 DIAL0294A11-IN \#1791 DIAL0294A10-IN \#12521 DIAL1390C02-ON DIAL1390C02 BEE0286A05-GA BEE0286A05 DIAL0294A09-in \#14033 DIAL0292E09-MD cressonii 035210 DIAL1845H09-BC DIAL1845H09 DIAL1845H07-BC DIAL1845H07 DIAL0292E07-ME cressonii 010595 DIAL1845F11-BC DIAL1845F11 DIAL1391A03-NC DIAL1391A03 BEE0289D05-MN BEE0289D05 BEE0289D07-MN BEE0289D07 DIAL1391A05-NC DIAL1391A05 D00609G07-NS D00609G07 DIAL1848A03-NB DIAL1848A03 BEE1850G05-ME BEE1850G05 BEE0289D06-MN BEE0289D06 D01561G01-MD D01561G01 DIAL2386H03-WA DIAL2386H03 DIAL1391A06-NC DIAL1391A06 D0287G06-IL D0287G06 D0288G10-ON D0288G10 DIAL2386G06-BC DIAL2386G06 03767G09-BC 03767G09-BC 06732B12-NY 06732B12-NY DIAL2386F07-WA DIAL2386F07 DIAL2386H05-WA DIAL2386H05 D-3253E10-OK D-3253E10 D00607B09-KS D00607B09 DIAL1409D07-TX DIAL1409D07 DIAL1409D04-TX DIAL1409D04 DIAL1409E08-TX DIAL1409E08 DIAL1409E10-TX DIAL1409E10 DIAL1409F01-TX DIAL1409F01 D00607B01-KS D00607B01 DIAL1848F12-NC DIAL1848F12 D01561B12-ON D01561B12 DIAL1848F10-ON DIAL1848F10 DIAL1848G01-ON DIAL1848G01 D01561E03-ON D01561E03 DIAL2374G04-UT DIAL2374G04 DIAL2388B04-UT DIAL2388B04-UT DIAL2388C03-UT DIAL2388C03-UT DIAL2386B05-NV DIAL2386B05 DIAL2384C08-AB DIAL2384C08 DIAL2374G05-UT DIAL2374G05 DIAL0294D02-IN \#14702 D01560D03-IA D01560D03
York University, Packer Collection York University, Packer Collection York University, Packer Collection York University, Packer Collection York University, Packer Collection York University, Packer Collection York University, Packer Collection York University, Packer Collection York University, Packer Collection York University, Packer Collection York University, Packer Collection York University, Packer Collection York University, Packer Collection York University, Packer Collection York University, Packer Collection York University, Packer Collection York University, Packer Collection York University, Packer Collection York University, Packer Collection York University, Packer Collection York University, Packer Collection York University, Packer Collection York University, Packer Collection York University, Packer Collection York University, Packer Collection York University, Packer Collection York University, Packer Collection York University, Packer Collection York University, Packer Collection York University, Packer Collection York University, Packer Collection York University, Packer Collection York University, Packer Collection York University, Packer Collection York University, Packer Collection York University, Packer Collection York University, Packer Collection York University, Packer Collection York University, Packer Collection York University, Packer Collection York University, Packer Collection York University, Packer Collection York University, Packer Collection York University, Packer Collection DIAL1412F10 York University, Packer Collection DIAL1412G01 York University, Packer Collection York University, Packer Collection York University, Packer Collection York University, Packer Collection York University, Packer Collection York University, Packer Collection York University, Packer Collection York University, Packer Collection York University, Packer Collection York University, Packer Collection 
D01560A07-WI D01560A07 DIAL1409C09-ON DIAL1409C09 DIAL1390A09-ON DIAL1390A09 DIAL1839G05-MN DIAL1839G05-MN D0288D03-IN D0288D03 DIAL1413G03-NC DIAL1413G03 D0295C04-ON D0295C04 DIAL1391H04-NC DIAL1391H04 DIAL1849G11-MA DIAL1849G11 DIAL1839H01-MN DIAL1839H01-MN D0295C05-ON D0295C05 D01562C02-MB D01562C02 D01562B07-MI D01562B07 B03765D08-IA B03765D08-IA 04485E09-MI 04485E09-MI B03765D06-IA B03765D06-IA 04485E02-MI 04485E02-MI B03765D11-IA B03765D11-IA B03765E01-IA B03765E01-IA D1414G06-DC D1414G06 DIAL1846H11-DC DIAL1846H11 BEE0289A03-MN BEE0289A03 DIAL1846E03-PA DIAL1846E03 D0295E03-VA D0295E03 BEE0289A07-MN BEE0289A07 DIAL0294H08-ON DIAL0294H08 DIAL1390F12-NC DIAL1390F12 D0295C07-ON D0295C07 DIAL2387C02-ON DIAL2387C02 DIAL1849H03-DC DIAL1849H03 DIAL1846A05-ON DIAL1846A05 DIAL1846A07-ON DIAL1846A07 DIAL1846A02-ON DIAL1846A02 DIAL1390F08-WV DIAL1390F08 DIAL1391F06-ON DIAL1391F06 DIAL1391G07-ON DIAL1391G07 DIAL1390F03-ON DIAL1390F03 DIAL1846B02-ON DIAL1846B02 DIAL1846A03-ON DIAL1846A03 DIAL1409C02-ON DIAL1409C02 DIAL1409C03-ON DIAL1409C03 DIAL1409C07-ON DIAL1409C07 DIAL1409C08-ON DIAL1409C08 D01562C10-MB D01562C10 DIAL1390E01-ON DIAL1390E01 DIAL1390F01-ON DIAL1390F01 DIAL1390G01-NC DIAL1390G01 DIAL1390F09-ON DIAL1390F09 DIAL1390F05-ON DIAL1390F05 DIAL1391G03-ON DIAL1391G03 DIAL1390E03-ON DIAL1390E03 DIAL1390E06-ON DIAL1390E06 DIAL1390E07-ON DIAL1390E07 DIAL1391G05-ON DIAL1391G05 DIAL2387B11-ON DIAL2387B11
York University, Packer Collection York University, Packer Collection York University, Packer Collection York University, Packer Collection York University, Packer Collection York University, Packer Collection York University, Packer Collection York University, Packer Collection York University, Packer Collection York University, Packer Collection York University, Packer Collection York University, Packer Collection York University, Packer Collection York University, Packer Collection York University, Packer Collection York University, Packer Collection York University, Packer Collection York University, Packer Collection York University, Packer Collection York University, Packer Collection York University, Packer Collection York University, Packer Collection York University, Packer Collection York University, Packer Collection York University, Packer Collection York University, Packer Collection York University, Packer Collection York University, Packer Collection York University, Packer Collection York University, Packer Collection York University, Packer Collection York University, Packer Collection York University, Packer Collection York University, Packer Collection York University, Packer Collection York University, Packer Collection York University, Packer Collection York University, Packer Collection York University, Packer Collection York University, Packer Collection York University, Packer Collection York University, Packer Collection York University, Packer Collection York University, Packer Collection York University, Packer Collection York University, Packer Collection York University, Packer Collection York University, Packer Collection York University, Packer Collection York University, Packer Collection York University, Packer Collection York University, Packer Collection York University, Packer Collection York University, Packer Collection York University, Packer Collection 
BEE1850E08-MN BEE1850E08 D01561A03-IN D01561A03 DIAL1391G02-ON DIAL1391G02 D01560A04-WI D01560A04 D01560B10-ON D01560B10 DIAL1849G08-W DIAL1849G08 DIAL2387B06-ON DIAL2387B06 D1414G04-DC D1414G04 04485F02-MI 04485F02-MI DIAL1390D04-ON DIAL1390D04 DIAL1409A02-ON DIAL1409A02 DIAL2387C07-ON DIAL2387C07 DIAL1391H06-NC DIAL1391H06 D-3253D06-FL D-3253D06 BEE0286A11-GA BEE0286A11 DIAL1846F10-SC DIAL1846F10 DIAL1846F12-SC DIAL1846F12 BEE0286B08-GA BEE0286B08 DIAL0294C11-IN \#3182 DIAL0294D01-IN \#8126 DIAL2385E04-ON DIAL2385E04-ON DIAL0294C12-IL \#8348 DIAL0293E02-MD \#11D DIAL0293E01-IN \#11182 D00608G05-IN D00608G05 D00608G01-IN D00608G01 D01561A09-MD D01561A09 D01561C07-MD D01561C07 DIAL2385C07-DE DIAL2385C07-DE D00607F08-MA D00607F08 BEE1850F05-NY BEE1850F05 DIAL2385B04-NY DIAL2385B04-NY D1414H03-VA D1414H03 DIAL2384H12-NY DIAL2384H12-NY D01559D03-MA D01559D03 B04743G05-MA B04743G05-MA DIAL1846G03-VA DIAL1846G03 D1414H12-VA D1414H12 D1414H10-VA D1414H10 DIAL0294D09-NC \#046341 D1414C12-VA D1414C12 DIAL0294D08-NC \#046295 DIAL1413D09-FL DIAL1413D09 DIAL1846G01-VA DIAL1846G01 DIAL1848E10-MD DIAL1848E10 DIAL1413A10-VA DIAL1413A10 DIAL1413A11-VA DIAL1413A11 CCDB-00611 D02 CCDB-00611 D02 04485G02-MI 04485G02-MI DIAL1848D01-WI DIAL1848D01 DIAL1846E08-WV DIAL1846E08 DIAL1391D03-NC DIAL1391D03 DIAL1391C11-NC DIAL1391C11 D1414H08-VA D1414H08 DIAL1413H11-NC DIAL1413H11
York University, Packer Collection York University, Packer Collection York University, Packer Collection York University, Packer Collection York University, Packer Collection York University, Packer Collection York University, Packer Collection York University, Packer Collection York University, Packer Collection York University, Packer Collection York University, Packer Collection York University, Packer Collection York University, Packer Collection York University, Packer Collection York University, Packer Collection York University, Packer Collection York University, Packer Collection York University, Packer Collection York University, Packer Collection York University, Packer Collection York University, Packer Collection York University, Packer Collection York University, Packer Collection York University, Packer Collection York University, Packer Collection York University, Packer Collection York University, Packer Collection York University, Packer Collection York University, Packer Collection York University, Packer Collection York University, Packer Collection York University, Packer Collection York University, Packer Collection York University, Packer Collection York University, Packer Collection York University, Packer Collection York University, Packer Collection York University, Packer Collection York University, Packer Collection York University, Packer Collection York University, Packer Collection York University, Packer Collection York University, Packer Collection York University, Packer Collection York University, Packer Collection York University, Packer Collection York University, Packer Collection York University, Packer Collection York University, Packer Collection York University, Packer Collection York University, Packer Collection York University, Packer Collection York University, Packer Collection York University, Packer Collection York University, Packer Collection 
DIAL1391A10-SC DIAL1391A10 D1414G12-VA D1414G12 DIAL1413E09-NC DIAL1413E09 DIAL1413B01-VA DIAL1413B01 DIAL1413G07-NC DIAL1413G07 D00607H10-KS D00607H10 D0288A11-MD D0288A11 D0288A09-WV D0288A09 D0287D03-IL D0287D03 D0288C02-DC D0288C02 D0287E03-IL D0287E03 DIAL0294A01-IN \#3770 D0287F07-IL D0287F07 D0287E04-IL D0287E04 DIAL1846F05-WV DIAL1846F05 DIAL0294A02-il \#8619 DIAL0294E08-MD \#029472 DIAL0294F06-VA \#046373 DIAL0294E10-VA \#046372 DIAL0294E09-MD \#046450 DIAL0294F08-MD \#039978 DIAL0292A09-VA \#017382 DIAL1413A01-IL DIAL1413A01 DIAL1390G10-NC DIAL1390G10 DIAL0294F04-VA \#046370 DIAL1391D06-NC DIAL1391D06 B03765B12-MD B03765B12-MD DIAL1847H08-MD DIAL1847H08 DIAL1391G09-MI DIAL1391G09 DIAL1413H08-NC DIAL1413H08 DIAL0293C02-NC perpunctatum 023922 DIAL0292A08-VA admirandum 022345 DIAL1413A04-IL DIAL1413A04 DIAL1413A06-IL DIAL1413A06 DIAL1413G05-NC DIAL1413G05 DIAL1391E03-SC DIAL1391E03 DIAL1413B08-IL DIAL1413B08 DIAL1846E12-SC DIAL1846E12 DIAL1846B01-TN DIAL1846B01 DIAL1846A11-NC DIAL1846A11 DIAL1846E05-MS DIAL1846E05 DIAL1413H03-NC DIAL1413H03 DIAL1413C04-TN DIAL1413C04 D0287F02-IL D0287F02 DIAL1413F02-TN DIAL1413F02 D0288C08-DC D0288C08 DIAL1413C08-TN DIAL1413C08 DIAL1413C05-TN DIAL1413C05 DIAL1413B02-VA DIAL1413B02 DIAL1413C06-TN DIAL1413C06 DIAL1391G08-MI DIAL1391G08 DIAL1413C11-NC DIAL1413C11 D0287G01-IL D0287G01 DIAL1413B09-IL DIAL1413B09 DIAL1413C01-IL DIAL1413C01
York University, Packer Collection York University, Packer Collection York University, Packer Collection York University, Packer Collection York University, Packer Collection York University, Packer Collection York University, Packer Collection York University, Packer Collection York University, Packer Collection York University, Packer Collection York University, Packer Collection York University, Packer Collection York University, Packer Collection York University, Packer Collection York University, Packer Collection York University, Packer Collection York University, Packer Collection York University, Packer Collection York University, Packer Collection York University, Packer Collection York University, Packer Collection York University, Packer Collection York University, Packer Collection York University, Packer Collection York University, Packer Collection York University, Packer Collection York University, Packer Collection York University, Packer Collection York University, Packer Collection York University, Packer Collection York University, Packer Collection York University, Packer Collection York University, Packer Collection York University, Packer Collection York University, Packer Collection York University, Packer Collection York University, Packer Collection York University, Packer Collection York University, Packer Collection York University, Packer Collection York University, Packer Collection York University, Packer Collection York University, Packer Collection York University, Packer Collection York University, Packer Collection York University, Packer Collection York University, Packer Collection York University, Packer Collection York University, Packer Collection York University, Packer Collection York University, Packer Collection York University, Packer Collection York University, Packer Collection York University, Packer Collection York University, Packer Collection 
DIAL1413F12-TN DIAL1413F12 DIAL1413D05-SC DIAL1413D05 DIAL1413C09-TN DIAL1413C09 D0287G04-IL D0287G04 D0287G02-IL D0287G02 D0287G08-IL D0287G08 DIAL2385D05-NC DIAL2385D05-NC DIAL1413C02-TN DIAL1413C02 DIAL1413C03-TN DIAL1413C03 D0287G07-IL D0287G07 D0287G12-IL D0287G12 D0287H09-IL D0287H09 DIAL1413A03-IL DIAL1413A03 D0287C11-IL D0287C11 D0287C09-IL D0287C09 D0287D02-MI D0287D02 D0287D06-IL D0287D06 B03765B08-MD B03765B08-MD D-3253C08-NM D-3253C08 DIAL2374A05-UT DIAL2374A05 DIAL2387C12-TX DIAL2387C12 DIAL2374C06-UT DIAL2374C06 DIAL2374A06-UT DIAL2374A06 DIAL1849C11-CO DIAL1849C11 DIAL1409B10-TX DIAL1409B10 DIAL1409E06-TX DIAL1409E06 DIAL2387B03-NM DIAL2387B03 DIAL2386H10-UT DIAL2386H10 D-3253D03-NM D-3253D03 DIAL2374F08-UT DIAL2374F08 DIAL2388D11-NM DIAL2388D11-NM DIAL2386F01-NV DIAL2386F01 D00608D11-NM D00608D11 DIAL2374F09-UT DIAL2374F09 DIAL2386D09-UT DIAL2386D09 DIAL1849E03-AZ DIAL1849E03 DIAL2388E03-NM DIAL2388E03-NM DIAL2388B11-UT DIAL2388B11-UT B-3252H08-NV B-3252H08 DIAL2374A07-UT DIAL2374A07 D00608H06-NV D00608H06 DIAL1413F04-TN DIAL1413F04 DIAL1390H04-SC DIAL1390H04 DIAL1847H11-MD DIAL1847H11 D1414B02-NC D1414B02 DIAL1413G06-NC DIAL1413G06 DIAL1845B07-CA DIAL1845B07 DIAL1845A06-CA DIAL1845A06 DIAL1845B01-CA DIAL1845B01 DIAL1845C02-CA DIAL1845C02 D00608H09-OR D00608H09 D00607H02-CA D00607H02 D00607D09-CA D00607D09 D00607F10-CA D00607F10 D01559D12-BC D01559D12
York University, Packer Collection York University, Packer Collection York University, Packer Collection York University, Packer Collection York University, Packer Collection York University, Packer Collection York University, Packer Collection York University, Packer Collection York University, Packer Collection York University, Packer Collection York University, Packer Collection York University, Packer Collection York University, Packer Collection York University, Packer Collection York University, Packer Collection York University, Packer Collection York University, Packer Collection York University, Packer Collection York University, Packer Collection York University, Packer Collection York University, Packer Collection York University, Packer Collection York University, Packer Collection York University, Packer Collection York University, Packer Collection York University, Packer Collection York University, Packer Collection York University, Packer Collection York University, Packer Collection York University, Packer Collection York University, Packer Collection York University, Packer Collection York University, Packer Collection York University, Packer Collection York University, Packer Collection York University, Packer Collection York University, Packer Collection York University, Packer Collection York University, Packer Collection York University, Packer Collection York University, Packer Collection York University, Packer Collection York University, Packer Collection York University, Packer Collection York University, Packer Collection York University, Packer Collection York University, Packer Collection York University, Packer Collection York University, Packer Collection York University, Packer Collection York University, Packer Collection York University, Packer Collection York University, Packer Collection York University, Packer Collection York University, Packer Collection 
$\begin{array}{ll}\text { D-3253F09-KY } & \text { D-3253F09 } \\ \text { D0288F05-SC } & \text { D0288F05 } \\ \text { D0287D12-IL } & \text { D0287D12 } \\ \text { D0287F08-IL } & \text { D0287F08 }\end{array}$

DIAL1846G12-SC DIAL1846G12

D0287F05-IL D0287F05

DIAL1413H01-NC DIAL1413H01

D0287H02-IL D0287H02

DIAL1391D05-NC DIAL1391D05

DIAL1409B08-VA DIAL1409B08

DIAL1409B09-VA DIAL1409B09

DIAL1413C10-NC DIAL1413C10

DIAL1413B07-IL DIAL1413B07

DIAL1413D02-TN DIAL1413D02

DIAL1391E06-SC DIAL1391E06

D0288G07-NC D0288G07

DIAL1413G02-TN DIAL1413G02

D0287F03-IL D0287F03

DIAL1390G08-NC DIAL1390G08

DIAL1413G01-TN DIAL1413G01

D0287C06-IL D0287C06

DIAL1391F08-ON DIAL1391F08

DIAL1413H06-NC DIAL1413H06

DIAL1391F02-NC DIAL1391F02

DIAL1391B02-SC DIAL1391B02

DIAL1849G10-MA DIAL1849G10

D-3253A10-BC D-3253A10

D-3253A04-BC D-3253A04

DIAL2388B03-UT DIAL2388B03-UT

DIAL1847G08-OR DIAL1847G08

DIAL1409D08-OR DIAL1409D08

DIAL1847G09-OR DIAL1847G09

DIAL1847G11-OR DIAL1847G11

D00608A10-CA D00608A10

DIAL2386H12-UT DIAL2386H12

DIAL2386H02-WA DIAL2386H02

D00607D10-CA D00607D10

D00607D07-CA D00607D07

DIAL2386D03-UT DIAL2386D03

DIAL2386D05-UT DIAL2386D05

06717H07-MA CCDB-06717 H07

DIAL2385D09-WV DIAL2385D09-WV

D01560D09-IA D01560D09

B03751H11-MA B03751H11-MA

D01560E05-IA D01560E05

B03750B04-MA B03750B04-MA

B04743H04-MA B04743H04-MA

06735G03-MA CCDB-06735G03

D-3253B07-BC D-3253B07

D-3253A07-BC D-3253A07

D-3253B05-BC D-3253B05

D-3253C02-BC D-3253C02

DIAL1845C08-CA DIAL1845C08

D-3253B09-BC D-3253B09

DIAL1845C07-CA DIAL1845C07
York University, Packer Collection York University, Packer Collection York University, Packer Collection York University, Packer Collection York University, Packer Collection York University, Packer Collection York University, Packer Collection York University, Packer Collection York University, Packer Collection York University, Packer Collection York University, Packer Collection York University, Packer Collection York University, Packer Collection York University, Packer Collection York University, Packer Collection York University, Packer Collection York University, Packer Collection York University, Packer Collection York University, Packer Collection York University, Packer Collection York University, Packer Collection York University, Packer Collection York University, Packer Collection York University, Packer Collection York University, Packer Collection York University, Packer Collection York University, Packer Collection York University, Packer Collection York University, Packer Collection York University, Packer Collection York University, Packer Collection York University, Packer Collection York University, Packer Collection York University, Packer Collection York University, Packer Collection York University, Packer Collection York University, Packer Collection York University, Packer Collection York University, Packer Collection York University, Packer Collection York University, Packer Collection York University, Packer Collection York University, Packer Collection York University, Packer Collection York University, Packer Collection York University, Packer Collection York University, Packer Collection York University, Packer Collection York University, Packer Collection York University, Packer Collection York University, Packer Collection York University, Packer Collection York University, Packer Collection York University, Packer Collection York University, Packer Collection 


\begin{tabular}{|c|c|c|c|}
\hline D0295F03-CO & D0295F03 & & York University, Packer Collection \\
\hline D-3253A03-BC & D-3253A03 & BBSL705988 & York University, Packer Collection \\
\hline D00608D06-BC & D00608D06 & & York University, Packer Collection \\
\hline D00608E08-BC & D00608E08 & & York University, Packer Collection \\
\hline D00608D08-BC & D00608D08 & & York University, Packer Collection \\
\hline D00607C01-CO & D00607C01 & & York University, Packer Collection \\
\hline DIAL1845G08-BC & DIAL1845G08 & & York University, Packer Collection \\
\hline DIAL2374E06-WA & DIAL2374E06 & BBSL696000 & York University, Packer Collection \\
\hline DIAL1409C10-OR & DIAL1409C10 & & York University, Packer Collection \\
\hline DIAL1845F09-BC & DIAL1845F09 & & York University, Packer Collection \\
\hline DIAL1845D07-CA & DIAL1845D07 & & York University, Packer Collection \\
\hline DIAL1845D06-CA & DIAL1845D06 & & York University, Packer Collection \\
\hline DIAL1845D11-CA & DIAL1845D11 & & York University, Packer Collection \\
\hline DIAL1845E04-CA & DIAL1845E04 & & York University, Packer Collection \\
\hline DIAL1845F03-BC & DIAL1845F03 & & York University, Packer Collection \\
\hline DIAL1845G05-BC & DIAL1845G05 & & York University, Packer Collection \\
\hline 03767G11-BC & 03767G11-BC & & York University, Packer Collection \\
\hline DIAL2387E11-BC & DIAL2387E11 & & York University, Packer Collection \\
\hline DIAL2387F03-BC & DIAL2387F03 & & York University, Packer Collection \\
\hline DIAL2387A05-ID & DIAL2387A05 & & York University, Packer Collection \\
\hline DIAL2386A05-CA & DIAL2386A05 & BBSL563254 & York University, Packer Collection \\
\hline D00608D10-BC & D00608D10 & & York University, Packer Collection \\
\hline DIAL2386G12-ID & DIAL2386G12 & & York University, Packer Collection \\
\hline D00608C09-CO & D00608C09 & & York University, Packer Collection \\
\hline $\mathrm{D} 00608 \mathrm{C} 02-\mathrm{CO}$ & D00608C02 & & York University, Packer Collection \\
\hline D00608F04-BC & D00608F04 & & York University, Packer Collection \\
\hline D01562A04-MT & D01562A04 & & York University, Packer Collection \\
\hline D0295H06-CO & D0295H06 & & York University, Packer Collection \\
\hline B03765E10-BC & B03765E10-BC & & York University, Packer Collection \\
\hline D-3253C01-BC & D-3253C01 & BBSL705721 & York University, Packer Collection \\
\hline DIAL2385E08-MD & DIAL2385E08-MD & & York University, Packer Collection \\
\hline DIAL1848H12-PE & DIAL1848H12 & & York University, Packer Collection \\
\hline DIAL1845H05-BC & DIAL1845H05 & & York University, Packer Collection \\
\hline D-3253A02-BC & D-3253A02 & BBSL706086 & York University, Packer Collection \\
\hline D00609G06-NS & D00609G06 & & York University, Packer Collection \\
\hline DIAL0294F05-MD & \#035032 & & York University, Packer Collection \\
\hline DIAL0294H11-NB & DIAL0294H11 & & York University, Packer Collection \\
\hline D00607G08-BC & D00607G08 & & York University, Packer Collection \\
\hline DIAL1846A08-NC & DIAL1846A08 & & York University, Packer Collection \\
\hline DIAL1845H08-BC & DIAL1845H08 & & York University, Packer Collection \\
\hline DIAL1845F08-BC & DIAL1845F08 & & York University, Packer Collection \\
\hline DIAL1391B11-NC & DIAL1391B11 & & York University, Packer Collection \\
\hline D00609G04-NS & D00609G04 & & York University, Packer Collection \\
\hline DIAL1848D05-NS & DIAL1848D05 & & York University, Packer Collection \\
\hline DIAL1409H12-ON & JIAL1409H12 & & York University, Packer Collection \\
\hline DIAL1845H02-BC & DIAL1845H02 & & York University, Packer Collection \\
\hline DIAL1845G01-BC & DIAL1845G01 & & York University, Packer Collection \\
\hline DIAL1845G02-BC & DIAL1845G02 & & York University, Packer Collection \\
\hline DIAL1845F04-BC & DIAL1845F04 & & York University, Packer Collection \\
\hline DIAL1409B05-NC & DIAL1409B05 & & York University, Packer Collection \\
\hline DIAL1845F06-BC & DIAL1845F06 & & York University, Packer Collection \\
\hline DIAL1845F07-BC & DIAL1845F07 & & York University, Packer Collection \\
\hline DIAL1413E07-NC & DIAL1413E07 & & York University, Packer Collection \\
\hline DIAL1848F09-PE & DIAL1848F09 & & York University, Packer Collection \\
\hline DIAL1848A05-NB & DIAL1848A05 & & York University, Packer Collection \\
\hline
\end{tabular}


DIAL1848A06-NB DIAL1848A06 DIAL1848G06-PE DIAL1848G06 DIAL1849H06-ME DIAL1849H06 DIAL2388D05-CO DIAL2388D05-CO D01562D02-ON D01562D02 D00607A05-AB D00607A05 D00607C10-AB D00607C10 D00607C09-AB D00607C09 DIAL1391A07-NC DIAL1391A07 D0295B10-ON D0295B10 D0295C01-ON D0295C01 DIAL1391C02-NC DIAL1391C02 DIAL1391B03-OH DIAL1391B03 DIAL1391C01-NC DIAL1391C01 DIAL2388D04-CO DIAL2388D04-CO 06745E10-ON 06745E10-ON DIAL1413E02-FL DIAL1413E02 DIAL1413E04-FL DIAL1413E04 DIAL1413E06-FL DIAL1413E06 04485F04-MI 04485F04-MI 04485F08-MI 04485F08-MI 04485E08-MI 04485E08-MI DIAL1848C01-NB DIAL1848C01 04485F09-MI 04485F09-MI 04485F11-MI 04485F11-MI 04485H06-MI 04485H06-MI DIAL1846A04-ON DIAL1846A04 04485H04-MI 04485H04-MI DIAL1848B11-NB DIAL1848B11 D00609G02-NS D00609G02 D00609H09-NS D00609H09 DIAL1845E03-TX DIAL1845E03 DIAL1846D07-MS DIAL1846D07 DIAL1846F11-MS DIAL1846F11 DIAL1391A11-SC DIAL1391A11 DIAL2385E07-WV DIAL2385E07-WV BEE0289B05-MN BEE0289B05 DIAL0286H09-MB DIAL0286H09 DIAL0286H04-MB DIAL0286H04 D0295B08-ON D0295B08 DIAL1390C12-ON DIAL1390C12 DIAL0292H06-WV\#018478 DIAL1839G07-MN DIAL1839G07-MN 06732C04-WV 06732C04-WV D00607B07-KS D00607B07 DIAL1409B01-NC DIAL1409B01 DIAL1390C07-ON DIAL1390C07 DIAL1390C04-ON DIAL1390C04 DIAL0286H06-MB DIAL0286H06 DIAL1846B05-MD DIAL1846B05 DIAL1847H12-MD DIAL1847H12 DIAL2388E04-AL DIAL2388E04-AL DIAL1846H04-MS DIAL1846H04 DIAL1846E02-MS DIAL1846E02 DIAL1846E09-MS DIAL1846E09
York University, Packer Collection York University, Packer Collection York University, Packer Collection York University, Packer Collection York University, Packer Collection York University, Packer Collection York University, Packer Collection York University, Packer Collection York University, Packer Collection York University, Packer Collection York University, Packer Collection York University, Packer Collection York University, Packer Collection York University, Packer Collection York University, Packer Collection York University, Packer Collection York University, Packer Collection York University, Packer Collection York University, Packer Collection York University, Packer Collection York University, Packer Collection York University, Packer Collection York University, Packer Collection York University, Packer Collection York University, Packer Collection York University, Packer Collection York University, Packer Collection York University, Packer Collection York University, Packer Collection York University, Packer Collection York University, Packer Collection York University, Packer Collection York University, Packer Collection York University, Packer Collection York University, Packer Collection York University, Packer Collection York University, Packer Collection York University, Packer Collection York University, Packer Collection York University, Packer Collection York University, Packer Collection York University, Packer Collection York University, Packer Collection York University, Packer Collection York University, Packer Collection York University, Packer Collection York University, Packer Collection York University, Packer Collection York University, Packer Collection York University, Packer Collection York University, Packer Collection York University, Packer Collection York University, Packer Collection York University, Packer Collection York University, Packer Collection 
DIAL1846E06-MS DIAL1846E06 DIAL1846D05-MS DIAL1846D05 DIAL2387F06-TX DIAL2387F06 D0288F08-NC D0288F08 BEE0286B09-GA BEE0286B09 D0288F10-SC D0288F10 D01559E03-BC D01559E03 D01559F06-BC D01559F06 D00608B10-BC D00608B10 D00608D04-BC D00608D04 D00609H07-BC D00609H07 D00609H03-BC D00609H03 D00609H05-BC D00609H05 DIAL2388B05-WA DIAL2388B05-WA D01561E05-CA D01561E05 CCDB - 01565 H3 CCDB - 01565 H3 CCDB - 01565 H8 CCDB - 01565 H8 D1414C03-VA D1414C03 DIAL1846D08-VA DIAL1846D08 DIAL1848C05-MD DIAL1848C05 D1414D08-VA D1414D08 D1414C06-VA D1414C06 D1414C05-VA D1414C05 D1414C04-VA D1414C04 D01561E08-NY D01561E08 DIAL1849F05-DC DIAL1849F05 DIAL1409B02-NC DIAL1409B02 DIAL1409A01-ON DIAL1409A01 D-3253E12-FL D-3253E12 D-3253E02-FL D-3253E02 D01559A03-FL D01559A03 D01561H08-FL D01561H08 D01559D09-FL D01559D09 D01562D03-FL D01562D03 D-3253B01-BC D-3253B01 D-3253A05-BC D-3253A05 D-3253B10-BC D-3253B10 D-3253B11-BC D-3253B11 D-3253B08-BC D-3253B08 D-3262G02-UT D-3262G02-UT D-3253A08-BC D-3253A08 DIAL1845C12-CA DIAL1845C12 DIAL1845D10-CA DIAL1845D10 DIAL2387E12-BC DIAL2387E12 D00607F07-CA D00607F07 BEE1850F04-OR BEE1850F04 D00607G10-BC D00607G10 DIAL2386C03-CA DIAL2386C03 DIAL2374A11-UT DIAL2374A11 DIAL2386D08-UT DIAL2386D08 DIAL2386D06-UT DIAL2386D06 DIAL2386C04-CA DIAL2386C04 D01559E12-BC D01559E12 DIAL2374D09-UT DIAL2374D09 DIAL2374E11-WA DIAL2374E11
BBSL566129 BBSL370394 ZION19814 ZION121776 BBSL564884

BBSL437336 BBSL696899
York University, Packer Collection York University, Packer Collection York University, Packer Collection York University, Packer Collection York University, Packer Collection York University, Packer Collection York University, Packer Collection York University, Packer Collection York University, Packer Collection York University, Packer Collection York University, Packer Collection York University, Packer Collection York University, Packer Collection York University, Packer Collection York University, Packer Collection York University, Packer Collection York University, Packer Collection York University, Packer Collection York University, Packer Collection York University, Packer Collection York University, Packer Collection York University, Packer Collection York University, Packer Collection York University, Packer Collection York University, Packer Collection York University, Packer Collection York University, Packer Collection York University, Packer Collection York University, Packer Collection York University, Packer Collection York University, Packer Collection York University, Packer Collection York University, Packer Collection York University, Packer Collection

BBSL703865 York University, Packer Collection York University, Packer Collection York University, Packer Collection York University, Packer Collection York University, Packer Collection York University, Packer Collection York University, Packer Collection York University, Packer Collection York University, Packer Collection York University, Packer Collection York University, Packer Collection York University, Packer Collection York University, Packer Collection York University, Packer Collection York University, Packer Collection York University, Packer Collection York University, Packer Collection York University, Packer Collection York University, Packer Collection York University, Packer Collection York University, Packer Collection 


\begin{tabular}{|c|c|c|c|}
\hline 06732F01-WY & 06732F01-WY & & York University, Packer Collection \\
\hline DIAL2385C01-MD & DIAL2385C01-MD & & York University, Packer Collection \\
\hline DIAL1849G01-ME & DIAL1849G01 & & York University, Packer Collection \\
\hline 04485A08-MD & 04485A08-MD & & York University, Packer Collection \\
\hline D00609G08-NS & D00609G08 & & York University, Packer Collection \\
\hline DIAL1849F12-MA & DIAL1849F12 & & York University, Packer Collection \\
\hline DIAL1848F03-ON & DIAL1848F03 & DIAL1412F03 & York University, Packer Collection \\
\hline D01560B11-VT & D01560B11 & & York University, Packer Collection \\
\hline DIAL1848F05-PE & DIAL1848F05 & DIAL1412F05 & York University, Packer Collection \\
\hline D0288B10-NC & D0288B10 & & York University, Packer Collection \\
\hline D1414C10-VA & D1414C10 & & York University, Packer Collection \\
\hline D1414C09-VA & D1414C09 & & York University, Packer Collection \\
\hline D-3253F11-FL & D-3253F11 & & York University, Packer Collection \\
\hline D1414F02-NC & D1414F02 & & York University, Packer Collection \\
\hline D1414E12-NC & D1414E12 & & York University, Packer Collection \\
\hline D1414E11-NC & D1414E11 & & York University, Packer Collection \\
\hline D0288C03-SC & $\mathrm{D} 0288 \mathrm{C} 03$ & & York University, Packer Collection \\
\hline D1414C08-VA & D1414C08 & & York University, Packer Collection \\
\hline D0288B11-NC & D0288B11 & & York University, Packer Collection \\
\hline DIAL1846D11-MS & DIAL1846D11 & & York University, Packer Collection \\
\hline DIAL1849F03-NC & DIAL1849F03 & & York University, Packer Collection \\
\hline D1414D09-VA & D1414D09 & & York University, Packer Collection \\
\hline B03765B03-VA & B03765B03-VA & & York University, Packer Collection \\
\hline D0288C11-SC & D0288C11 & & York University, Packer Collection \\
\hline D1414F05-NC & D1414F05 & & York University, Packer Collection \\
\hline D1414C07-VA & D1414C07 & & York University, Packer Collection \\
\hline D0295D12-MD & D0295D12 & & York University, Packer Collection \\
\hline D1414B06-VA & D1414B06 & & York University, Packer Collection \\
\hline DIAL0293B05-MD & \#011917 & & York University, Packer Collection \\
\hline D1414B07-VA & D1414B07 & & York University, Packer Collection \\
\hline D00607D12-MA & D00607D12 & & York University, Packer Collection \\
\hline D00608F12-IN & D00608F12 & & York University, Packer Collection \\
\hline D1414B08-VA & D1414B08 & & York University, Packer Collection \\
\hline D1414E01-VA & D1414E01 & & York University, Packer Collection \\
\hline D1414B09-VA & D1414B09 & & York University, Packer Collection \\
\hline DIAL1849G02-MA & DIAL1849G02 & & York University, Packer Collection \\
\hline DIAL1413E12-TN & DIAL1413E12 & & York University, Packer Collection \\
\hline DIAL0294F11-VA & \#048070 & & York University, Packer Collection \\
\hline DIAL1847B03-WV & / DIAL1847B03 & & York University, Packer Collection \\
\hline DIAL1848C11-NY & DIAL1848C11 & DIAL1412C11 & York University, Packer Collection \\
\hline D0295E06-MD & D0295E06 & & York University, Packer Collection \\
\hline DIAL1846B08-WV & DIAL1846B08 & & York University, Packer Collection \\
\hline BEE0286D09-VA & BEE0286D09 & & York University, Packer Collection \\
\hline DIAL1391A04-NC & DIAL1391A04 & & York University, Packer Collection \\
\hline DIAL1390G11-NC & DIAL1390G11 & & York University, Packer Collection \\
\hline DIAL1849H08-VT & DIAL1849H08 & & York University, Packer Collection \\
\hline DIAL2385B03-MB & DIAL2385B03-MB & & York University, Packer Collection \\
\hline DIAL2387F09-NM & I DIAL2387F09 & & York University, Packer Collection \\
\hline DIAL2387H11-NM & DIAL2387H11 & & York University, Packer Collection \\
\hline D0287A11-CO & D0287A11 & & York University, Packer Collection \\
\hline DIAL2385A10-MB & DIAL2385A10-MB & & York University, Packer Collection \\
\hline D0295G05-CO & D0295G05 & & York University, Packer Collection \\
\hline DIAL2385A04-CO & DIAL2385A04-CO & & York University, Packer Collection \\
\hline D0287B11-CO & D0287B11 & & York University, Packer Collection \\
\hline D01561B01-MT & D01561B01 & & York University, Packer Collection \\
\hline
\end{tabular}


06732F09-WY 06732F09-WY D0295G10-CO D0295G10

DIAL1390C09-ON DIAL1390C09

DIAL1849G09-MA DIAL1849G09

DIAL1409A06-NC DIAL1409A06

DIAL1409A11-NC DIAL1409A11

DIAL1845G06-BC DIAL1845G06

DIAL1845H10-BC DIAL1845H10

DIAL1845G04-BC DIAL1845G04

DIAL1845F05-BC DIAL1845F05

DIAL1845H03-BC DIAL1845H03

DIAL1845H11-BC DIAL1845H11

D00608G06-BC D00608G06

DIAL1845H12-BC DIAL1845H12

DIAL1845G03-BC DIAL1845G03

DIAL1845G07-BC DIAL1845G07

DIAL1845G12-BC DIAL1845G12

DIAL1845F12-BC DIAL1845F12

DIAL1845F10-BC DIAL1845F10

DIAL1845F02-BC DIAL1845F02

DIAL1845G09-BC DIAL1845G09

DIAL1845G10-BC DIAL1845G10

DIAL1845G11-BC DIAL1845G11

DIAL2387H05-CO DIAL2387H05

B03765E05-BC B03765E05-BC

CCDB-00611 H09 CCDB-00611 H09

D01559D04-AB D01559D04

DIAL2385A08-SK DIAL2385A08-SK

D01559G01-AB D01559G01

DIAL2385B02-SK DIAL2385B02-SK

DIAL0293B11-WV \#032687

DIAL1413A02-IL DIAL1413A02

DIAL1409A08-NC DIAL1409A08

DIAL1390E09-ON DIAL1390E09

DIAL1413B05-IL DIAL1413B05

D00609C03-WI D00609C03

D01560C09-IA D01560C09

CCDB-01557 B01 CCDB-01557 B01

DIAL1390D01-NT DIAL1390D01

DIAL1390C10-NT DIAL1390C10

DIAL2384B09-YT DIAL2384B09

D0295F10-CO D0295F10

DIAL0286G05-MB DIAL0286G05

DIAL0286G06-MB DIAL0286G06

DIAL0293B09-IN perpunctatum \#12484

D00609H10-NS D00609H10

DIAL0286G07-MB DIAL0286G07

DIAL0286G03-MB DIAL0286G03

D01561B02-MT D01561B02

D0287A03-CO D0287A03

DIAL0286G10-MB DIAL0286G10

DIAL0286G09-MB DIAL0286G09

DIAL0286G04-MB DIAL0286G04

DIAL0286H03-MB DIAL0286H03

DIAL0293C07-MN pictum \#03
York University, Packer Collection

York University, Packer Collection

York University, Packer Collection

York University, Packer Collection

York University, Packer Collection

York University, Packer Collection

York University, Packer Collection

York University, Packer Collection

York University, Packer Collection

York University, Packer Collection

York University, Packer Collection

York University, Packer Collection

York University, Packer Collection

York University, Packer Collection

York University, Packer Collection

York University, Packer Collection

York University, Packer Collection

York University, Packer Collection

York University, Packer Collection

York University, Packer Collection

York University, Packer Collection

York University, Packer Collection

York University, Packer Collection

York University, Packer Collection

York University, Packer Collection

York University, Packer Collection

York University, Packer Collection

York University, Packer Collection

York University, Packer Collection

York University, Packer Collection

York University, Packer Collection

York University, Packer Collection

York University, Packer Collection

York University, Packer Collection

York University, Packer Collection

York University, Packer Collection

York University, Packer Collection

York University, Packer Collection

York University, Packer Collection

York University, Packer Collection

York University, Packer Collection

York University, Packer Collection

York University, Packer Collection

York University, Packer Collection

York University, Packer Collection

York University, Packer Collection

York University, Packer Collection

York University, Packer Collection

York University, Packer Collection

York University, Packer Collection

York University, Packer Collection

York University, Packer Collection

York University, Packer Collection

York University, Packer Collection

York University, Packer Collection 
DIAL0293C08-MN pictum \#04 DIAL0293C06-MN\#02 DIAL0294C01-IN \#15765 DIAL0293C05-MN\#01 DIAL0286F07-MB DIAL0286F07 DIAL0286F08-MB DIAL0286F08 DIAL0286F06-MB DIAL0286F06 DIAL2385B11-MI DIAL2385B11-MI D00609B09-WI D00609B09 D1414D12-VA D1414D12 DIAL1391D02-NC DIAL1391D02 DIAL0294F12-NY \#046092 DIAL1390B05-ON DIAL1390B05 04485G12-MI 04485G12-MI DIAL1846A06-ON DIAL1846A06 DIAL1847H06-MD DIAL1847H06 DIAL1391A01-VA DIAL1391A01 DIAL1413C07-TN DIAL1413C07 04485F01-MI 04485F01-MI DIAL1409A05-NC DIAL1409A05 DIAL1413A08-VA DIAL1413A08 DIAL1390H05-NC DIAL1390H05 DIAL1413F05-TN DIAL1413F05 DIAL1413D03-NC DIAL1413D03 DIAL1413B04-ON DIAL1413B04 BEE1850F06-MN BEE1850F06 DIAL1391C12-NC DIAL1391C12 D00607G06-BC D00607G06 DIAL0294H10-NB DIAL0294H10 DIAL1848A02-NB DIAL1848A02 DIAL1848G02-NB DIAL1848G02 DIAL1848A01-NB DIAL1848A01 DIAL2388E07-NB DIAL2388E07-NB DIAL1391F05-NT DIAL1391F05 CCDB-00611 F10. CCDB-00611 F10 D01560D11-DC D01560D11 DIAL2385C11-DC DIAL2385C11-DC DIAL1413D08-NC DIAL1413D08 DIAL0293D07-WV BBSL429788 DIAL0293D03-MD\#028159 DIAL1849H02-DC DIAL1849H02 DIAL1847H09-MD DIAL1847H09 DIAL1848E09-MD DIAL1848E09 D01560B02-DC D01560B02 D01560B05-DC D01560B05 DIAL1849F08-MD DIAL1849F08 D00609E03-MT D00609E03 D0295H01-CO D0295H01 D01559F01-AB D01559F01 DIAL2386G09-WA DIAL2386G09 D0295G03-CO D0295G03 DIAL2384C05-AB DIAL2384C05 DIAL1848F01-CO DIAL1848F01 D01559C10-AB D01559C10 DIAL2386H04-WA DIAL2386H04
York University, Packer Collection York University, Packer Collection York University, Packer Collection York University, Packer Collection York University, Packer Collection York University, Packer Collection York University, Packer Collection York University, Packer Collection York University, Packer Collection York University, Packer Collection York University, Packer Collection York University, Packer Collection York University, Packer Collection York University, Packer Collection York University, Packer Collection York University, Packer Collection York University, Packer Collection York University, Packer Collection York University, Packer Collection York University, Packer Collection York University, Packer Collection York University, Packer Collection York University, Packer Collection York University, Packer Collection York University, Packer Collection York University, Packer Collection York University, Packer Collection York University, Packer Collection York University, Packer Collection York University, Packer Collection York University, Packer Collection York University, Packer Collection York University, Packer Collection York University, Packer Collection York University, Packer Collection York University, Packer Collection York University, Packer Collection York University, Packer Collection York University, Packer Collection York University, Packer Collection York University, Packer Collection York University, Packer Collection York University, Packer Collection York University, Packer Collection York University, Packer Collection York University, Packer Collection York University, Packer Collection York University, Packer Collection York University, Packer Collection York University, Packer Collection York University, Packer Collection York University, Packer Collection York University, Packer Collection York University, Packer Collection York University, Packer Collection 


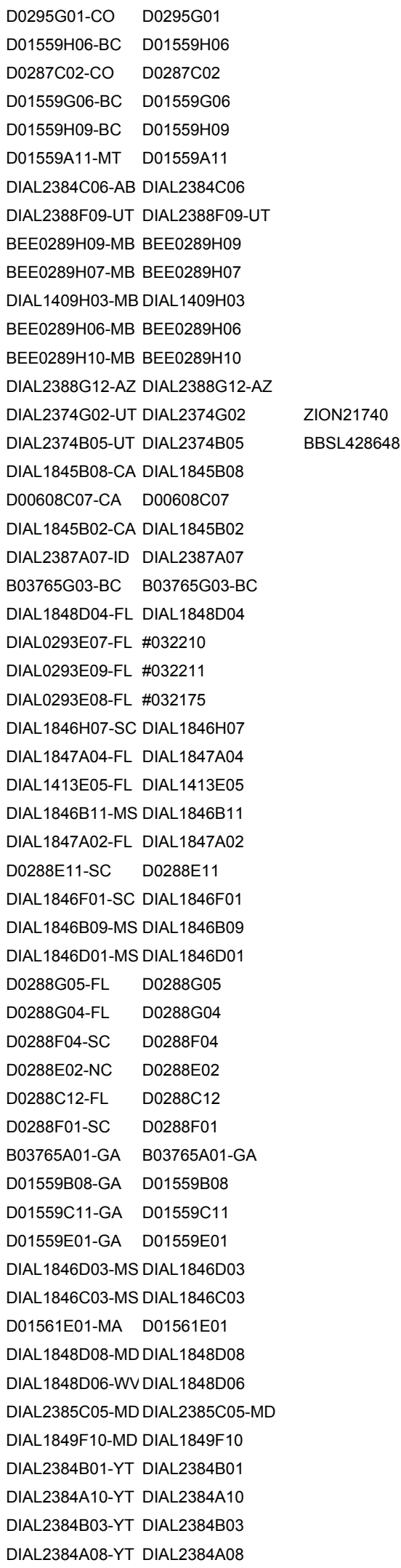

ZION21740

BBSL428648

York University, Packer Collection York University, Packer Collection York University, Packer Collection York University, Packer Collection York University, Packer Collection York University, Packer Collection York University, Packer Collection York University, Packer Collection York University, Packer Collection York University, Packer Collection York University, Packer Collection York University, Packer Collection York University, Packer Collection York University, Packer Collection York University, Packer Collection York University, Packer Collection York University, Packer Collection York University, Packer Collection York University, Packer Collection York University, Packer Collection York University, Packer Collection York University, Packer Collection York University, Packer Collection York University, Packer Collection York University, Packer Collection York University, Packer Collection York University, Packer Collection York University, Packer Collection York University, Packer Collection York University, Packer Collection York University, Packer Collection York University, Packer Collection York University, Packer Collection York University, Packer Collection York University, Packer Collection York University, Packer Collection York University, Packer Collection York University, Packer Collection York University, Packer Collection York University, Packer Collection York University, Packer Collection York University, Packer Collection York University, Packer Collection York University, Packer Collection York University, Packer Collection York University, Packer Collection York University, Packer Collection York University, Packer Collection York University, Packer Collection York University, Packer Collection York University, Packer Collection York University, Packer Collection York University, Packer Collection York University, Packer Collection York University, Packer Collection 


\begin{tabular}{|c|c|c|c|}
\hline \multirow{2}{*}{\multicolumn{3}{|c|}{$\begin{array}{l}\text { B01558C07-AB CCDB-01558C7 } \\
\text { DIAL2387C04-NM DIAL2387C04 }\end{array}$}} & York University, Packer Collection \\
\hline & & & York University, Packer Collection \\
\hline D00609B02-MT & \multicolumn{2}{|l|}{ D00609B02 } & York University, Packer Collection \\
\hline D01562A01-MT & \multicolumn{2}{|l|}{ D01562A01 } & York University, Packer Collection \\
\hline DIAL2374G03-NV & / DIAL2374G03 & \multirow[t]{10}{*}{ BBSL577864 } & York University, Packer Collection \\
\hline 03767G04-BC & 03767G04-BC & & York University, Packer Collection \\
\hline CCDB-00611 H02 & 2 CCDB-00611 H02 & & York University, Packer Collection \\
\hline D0295H07-CO & D0295H07 & & York University, Packer Collection \\
\hline CCDB-01557 A01 & 1 CCDB-01557 A01 & & York University, Packer Collection \\
\hline DIAL2384C03-AB & 3 DIAL2384C03 & & York University, Packer Collection \\
\hline D0295H03-CO & $\mathrm{D} 0295 \mathrm{H} 03$ & & York University, Packer Collection \\
\hline D01559G12-BC & D01559G12 & & York University, Packer Collection \\
\hline D00607H03-SK & $\mathrm{D} 00607 \mathrm{H} 03$ & & York University, Packer Collection \\
\hline DIAL2386G03-BC & DIAL2386G03 & & York University, Packer Collection \\
\hline DIAL2374B07-UT & DIAL2374B07 & \multirow[t]{12}{*}{ BBSL475705 } & York University, Packer Collection \\
\hline DIAL2388C07-NV & / DIAL2388C07-NV & & York University, Packer Collection \\
\hline D01561E07-SK & D01561E07 & & York University, Packer Collection \\
\hline D01561E02-SK & D01561E02 & & York University, Packer Collection \\
\hline D01562D12-BC & D01562D12 & & York University, Packer Collection \\
\hline D01559A08-AB & D01559A08 & & York University, Packer Collection \\
\hline 04486E05-WA & 04486E05-WA & & York University, Packer Collection \\
\hline CCDB-00611 H11 & 1 CCDB-00611 H11 & & York University, Packer Collection \\
\hline 03767G06-BC & 03767G06-BC & & York University, Packer Collection \\
\hline B03765E06-BC & B03765E06-BC & & York University, Packer Collection \\
\hline CCDB-01557 A04 & 4 CCDB-01557 A04 & & York University, Packer Collection \\
\hline 06732E12-WY & 06732E12-WY & & York University, Packer Collection \\
\hline DIAL2386A08-NV & DIAL2386A08 & \multirow[t]{29}{*}{ BBSL530045 } & York University, Packer Collection \\
\hline D01562D10-ON & D01562D10 & & York University, Packer Collection \\
\hline DIAL0286H10-MB & 3 DIAL0286H10 & & York University, Packer Collection \\
\hline DIAL0291H07-MB & 3 DIAL0291H07 & & York University, Packer Collection \\
\hline DIAL0291H05-MB & 3 DIAL0291H05 & & York University, Packer Collection \\
\hline DIAL0291H06-MB & 3 DIAL0291H06 & & York University, Packer Collection \\
\hline DIAL0286H11-MB & 3 DIAL0286H11 & & York University, Packer Collection \\
\hline DIAL0286H07-MB & 3 DIAL0286H07 & & York University, Packer Collection \\
\hline DIAL0286H08-MB & 3 DIAL0286H08 & & York University, Packer Collection \\
\hline B03765F12-BC & B03765F12-BC & & York University, Packer Collection \\
\hline DIAL1409H04-MB & 3 DIAL1409H04 & & York University, Packer Collection \\
\hline DIAL1409H02-MB & 3 DIAL1409H02 & & York University, Packer Collection \\
\hline CCDB-01557 A09 & CCDB-01557 A09 & & York University, Packer Collection \\
\hline D01562C04-MB & D01562C04 & & York University, Packer Collection \\
\hline D01560A10-WI & D01560A10 & & York University, Packer Collection \\
\hline DIAL0291H08-MB & 3 DIAL0291H08 & & York University, Packer Collection \\
\hline D01562E02-MB & D01562E02 & & York University, Packer Collection \\
\hline D01562C09-ON & D01562C09 & & York University, Packer Collection \\
\hline CCDB-01557 A07. & CCDB-01557 A07 & & York University, Packer Collection \\
\hline B03765F09-BC & B03765F09-BC & & York University, Packer Collection \\
\hline CCDB-01557 A10 & CCDB-01557 A10 & & York University, Packer Collection \\
\hline CCDB-01557 A11. & CCDB-01557 A11 & & York University, Packer Collection \\
\hline B03765F06-BC & B03765F06-BC & & York University, Packer Collection \\
\hline D00608F06-BC & D00608F06 & & York University, Packer Collection \\
\hline B03751H05-BC & B03751H05-BC & & York University, Packer Collection \\
\hline D01559E08-BC & D01559E08 & & York University, Packer Collection \\
\hline D00607E12-UT & D00607E12 & & York University, Packer Collection \\
\hline D00608E01-BC & D00608E01 & & York University, Packer Collection \\
\hline D01561F06-BC & D01561F06 & & York University, Packer Collection \\
\hline
\end{tabular}




\begin{tabular}{|c|c|c|c|c|}
\hline B03765E12-BC & B03765E12-BC & & & York University, Packer Collection \\
\hline D0295F01-CO & D0295F01 & & & York University, Packer Collection \\
\hline DIAL2374E05-WA & DIAL2374E05 & BBSL696917 & & York University, Packer Collection \\
\hline CCDB-00611 H04 & CCDB-00611 H04 & & & York University, Packer Collection \\
\hline D01559G09-BC & D01559G09 & & & York University, Packer Collection \\
\hline D0295G11-CO & D0295G11 & & & York University, Packer Collection \\
\hline DIAL2387C03-ID & DIAL2387C03 & & & York University, Packer Collection \\
\hline D0295F04-CO & D0295F04 & & & York University, Packer Collection \\
\hline DIAL2374E04-WA & DIAL2374E04 & BBSL696048 & & York University, Packer Collection \\
\hline D01559B11-BC & D01559B11 & & & York University, Packer Collection \\
\hline D01559C03-BC & D01559C03 & & & York University, Packer Collection \\
\hline D0295H11-CO & D0295H11 & & & York University, Packer Collection \\
\hline D0287C01-CO & $\mathrm{D} 0287 \mathrm{C} 01$ & & & York University, Packer Collection \\
\hline D0287B01-CO & D0287B01 & & & York University, Packer Collection \\
\hline D01562A10-MT & D01562A10 & & & York University, Packer Collection \\
\hline D0295G06-CO & D0295G06 & & & York University, Packer Collection \\
\hline CCDB-00611 H03 & 3 CCDB-00611 H03 & & & York University, Packer Collection \\
\hline DIAL2388D12-NM & I DIAL2388D12-NM & & & York University, Packer Collection \\
\hline DIAL0293H09-AZ & DIAL0293H09 & & & York University, Packer Collection \\
\hline D-3262F07-NM & D-3262F07-NM & & & York University, Packer Collection \\
\hline D0295G07-CO & D0295G07 & & & York University, Packer Collection \\
\hline DIAL1848C04-MN & NDIAL1848C04 & & DIAL1412C04 & York University, Packer Collection \\
\hline DIAL1848F02-MN & DIAL1848F02 & & & York University, Packer Collection \\
\hline DIAL1848B05-AZ & DIAL1848B05 & & DIAL1412B05 & York University, Packer Collection \\
\hline DIAL1849B05-AZ & DIAL1849B05 & & & York University, Packer Collection \\
\hline DIAL1847F03-TX & DIAL1847F03 & & & York University, Packer Collection \\
\hline DIAL1849E10-AZ & DIAL1849E10 & & & York University, Packer Collection \\
\hline D0287A06-CO & D0287A06 & & & York University, Packer Collection \\
\hline DIAL2374F12-UT & DIAL2374F12 & ZION21139 & & York University, Packer Collection \\
\hline D0295G02-CO & D0295G02 & & & York University, Packer Collection \\
\hline DIAL1849E02-CO & DIAL1849E02 & & & York University, Packer Collection \\
\hline DIAL1848B01-AZ & DIAL1848B01 & & DIAL1412B01 & York University, Packer Collection \\
\hline DIAL1848C03-MN & N DIAL1848C03 & & DIAL1412C03 & York University, Packer Collection \\
\hline D01561B03-CA & D01561B03 & & & York University, Packer Collection \\
\hline DIAL2386B02-NV & DIAL2386B02 & BBSL575216 & & York University, Packer Collection \\
\hline DIAL2374G01-UT & DIAL2374G01 & ZION22020 & & York University, Packer Collection \\
\hline DIAL2388E02-NM & I DIAL2388E02-NM & & & York University, Packer Collection \\
\hline DIAL1849C06-CO & DIAL1849C06 & & & York University, Packer Collection \\
\hline DIAL0286G11-MB & BIAL0286G11 & & & York University, Packer Collection \\
\hline D0287A04-CO & D0287A04 & & & York University, Packer Collection \\
\hline D0295H04-CO & $\mathrm{D} 0295 \mathrm{H} 04$ & & & York University, Packer Collection \\
\hline D0295G04-CO & D0295G04 & & & York University, Packer Collection \\
\hline DIAL0286G12-MB & 3 DIAL0286G12 & & & York University, Packer Collection \\
\hline DIAL0286G08-MB & 3 DIAL0286G08 & & & York University, Packer Collection \\
\hline 06732H03-CO & 06732H03-CO & & & York University, Packer Collection \\
\hline DIAL2385E11-NS & DIAL2385E11-NS & & & York University, Packer Collection \\
\hline DIAL2385A02-NS & DIAL2385A02-NS & & & York University, Packer Collection \\
\hline DIAL2385F01-NS & DIAL2385F01-NS & & & York University, Packer Collection \\
\hline DIAL2385FF4-NS & DIAL2385F04-NS & & & York University, Packer Collection \\
\hline DIAL1848D03-NS & DIAL1848D03 & & & York University, Packer Collection \\
\hline DIAL1848D02-NS & DIAL1848D02 & & & York University, Packer Collection \\
\hline D01560A05-WI & D01560A05 & & & York University, Packer Collection \\
\hline DIAL2384H08-MD & DIAL2384H08-MD & & & York University, Packer Collection \\
\hline DIAL1849H05-MD & DIAL1849H05 & & & York University, Packer Collection \\
\hline DIAL0294B06-IN & \#6716 & & & York University, Packer Collection \\
\hline
\end{tabular}


DIAL1848G09-AB DIAL1848G09 D01562E03-NS D01562E03 DIAL2385A01-SK DIAL2385A01-SK DIAL2385A05-SK DIAL2385A05-SK D01560C07-IA D01560C07 D-3253F03-MO D-3253F03 D0288E01-VA D0288E01 DIAL2385E09-NS DIAL2385E09-NS D0288C10-VA D0288C10 DIAL0294F07-MD \#045294 DIAL0293B04-MD obongum 020194 DIAL0294G03-MC \#012348 DIAL0293B03-MD oblongum 020202 DIAL0294G04-ML \#012349 DIAL0294C08-IN /lineat\#15367 DIAL0292A03-MD abanci 019384 DIAL0292A04-MD abanci 009068 B04743G07-MA B04743G07-MA B04743G01-MA B04743G01-MA DIAL1846F08-WV DIAL1846F08 DIAL1848C12-WV DIAL1848C12 B04743D05-NS B04743D05-NS D00608E10-IN D00608E10 D01562B05-ON D01562B05 B04743H06-MA B04743H06-MA DIAL1391E05-TN DIAL1391E05 D00607E07-CT D00607E07 D0288B02-MD D0288B02 D01559E06-ON D01559E06 D00607A10-CT D00607A10 DIAL1849G06-NH DIAL1849G06 D01560A01-WI D01560A01 B03750B02-MA B03750B02-MA CCDB-00611 G08 CCDB-00611 G08 CCDB-00611 F03. CCDB-00611 F03 B04743G03-MA B04743G03-MA B04743H02-MA B04743H02-MA CCDB-00611 G03 CCDB-00611 G03 CCDB-00611 G04 CCDB-00611 G04 B04743D03-NS B04743D03-NS D00608E12-IN D00608E12 D01562E07-NS D01562E07 D01560C12-IA D01560C12 DIAL0286F12-MB DIAL0286F12 DIAL0286F10-MB DIAL0286F10 DIAL1848H03-AB DIAL1848H03 DIAL1390B01-ON DIAL1390B01 DIAL0293E04-FL \#031875 D-3253D09-FL D-3253D09 D-3253E04-FL D-3253E04 D01561D10-FL D01561D10 D01559A04-FL D01559A04 D0295D02-DC D0295D02 D00608B05-ON D00608B05 D00608B03-ON D00608B03
York University, Packer Collection York University, Packer Collection York University, Packer Collection York University, Packer Collection York University, Packer Collection York University, Packer Collection York University, Packer Collection York University, Packer Collection York University, Packer Collection York University, Packer Collection York University, Packer Collection York University, Packer Collection York University, Packer Collection York University, Packer Collection York University, Packer Collection York University, Packer Collection York University, Packer Collection York University, Packer Collection York University, Packer Collection York University, Packer Collection York University, Packer Collection York University, Packer Collection York University, Packer Collection York University, Packer Collection York University, Packer Collection York University, Packer Collection York University, Packer Collection York University, Packer Collection York University, Packer Collection York University, Packer Collection York University, Packer Collection York University, Packer Collection York University, Packer Collection York University, Packer Collection York University, Packer Collection York University, Packer Collection York University, Packer Collection York University, Packer Collection York University, Packer Collection York University, Packer Collection York University, Packer Collection York University, Packer Collection York University, Packer Collection York University, Packer Collection York University, Packer Collection York University, Packer Collection York University, Packer Collection York University, Packer Collection York University, Packer Collection York University, Packer Collection York University, Packer Collection York University, Packer Collection York University, Packer Collection York University, Packer Collection York University, Packer Collection 
D1414C02-VA D1414C02

DIAL0294E01-VA \#032884

DIAL0294D12-NY \#046015

D1414B10-VA D1414B10

D1414C01-VA D1414C01

D-3253F06-MO D-3253F06

DIAL1846B06-MS DIAL1846B06

DIAL1847H10-MD DIAL1847H10

DIAL1846H10-MD DIAL1846H10

DIAL1846B04-MS DIAL1846B04

DIAL1413A09-VA DIAL1413A09

B01566D04-TX B01566D04

D0288E08-WV D0288E08

D1414B11-VA D1414B11

DIAL1391E02-SC DIAL1391E02

DIAL1391E01-SC DIAL1391E01

D0288G09-SC D0288G09

D0288E10-NC D0288E10

D0288E05-WV D0288E05

D0288D12-WV D0288D12

D0295E11-DC D0295E11

D0295E10-DC D0295E10

BEE0286E07-VA BEE0286E07

D00607C02-MO D00607C02

D00607A01-NE D00607A01

06732C02-DC 06732C02-DC

DIAL2387C06-YT DIAL2387C06

DIAL1845E09-NL DIAL1845E09

DIAL1845E06-NL DIAL1845E06

DIAL2384A11-YT DIAL2384A11

DIAL1391C03-NC DIAL1391C03

DIAL2384A03-YT DIAL2384A03

DIAL2384A02-YT DIAL2384A02

DIAL1845E12-NL DIAL1845E12

DIAL1845F01-NL DIAL1845F01

DIAL2387B10-YT DIAL2387B10

DIAL1391C04-NC DIAL1391C04

DIAL2387B04-YT DIAL2387B04

DIAL2384A06-YT DIAL2384A06

DIAL1848A10-NB DIAL1848A10

DIAL2384A09-YT DIAL2384A09

DIAL2384B08-YT DIAL2384B08

DIAL2384A01-YT DIAL2384A01

DIAL2384B02-YT DIAL2384B02

DIAL2384B10-YT DIAL2384B10

DIAL1390C01-ON DIAL1390C01

DIAL1390C03-ON DIAL1390C03

BEE1850G11-ME BEE1850G11

D1414H01-VA D1414H01

DIAL0292A11-WV admirandum 010937

DIAL0294E06-MD \#041908

DIAL2385F02-MD DIAL2385F02-MD

DIAL0294E12-MD \#023397

DIAL0294E07-IN \#021006

DIAL0293B10-WV perpunctatum 032749
York University, Packer Collection

York University, Packer Collection

York University, Packer Collection

York University, Packer Collection

York University, Packer Collection

York University, Packer Collection

York University, Packer Collection

York University, Packer Collection

York University, Packer Collection

York University, Packer Collection

York University, Packer Collection

York University, Packer Collection

York University, Packer Collection

York University, Packer Collection

York University, Packer Collection

York University, Packer Collection

York University, Packer Collection

York University, Packer Collection

York University, Packer Collection

York University, Packer Collection York University, Packer Collection York University, Packer Collection York University, Packer Collection York University, Packer Collection York University, Packer Collection York University, Packer Collection York University, Packer Collection York University, Packer Collection York University, Packer Collection York University, Packer Collection York University, Packer Collection York University, Packer Collection York University, Packer Collection York University, Packer Collection York University, Packer Collection York University, Packer Collection York University, Packer Collection York University, Packer Collection York University, Packer Collection York University, Packer Collection York University, Packer Collection York University, Packer Collection York University, Packer Collection York University, Packer Collection York University, Packer Collection York University, Packer Collection York University, Packer Collection York University, Packer Collection York University, Packer Collection York University, Packer Collection York University, Packer Collection York University, Packer Collection York University, Packer Collection York University, Packer Collection York University, Packer Collection 
DIAL0293F02-NC \#023965 D00607H11-KS D00607H11 B01558D07-NC CCDB-01558D7 D00608A07-KS D00608A07 D0288C05-DC D0288C05 DIAL1409B07-NC DIAL1409B07 DIAL1846D04-MS DIAL1846D04 DIAL1413H02-NC DIAL1413H02 D0288D08-NC D0288D08 DIAL2385C12-SC DIAL2385C12-SC DIAL1849C10-CO DIAL1849C10 D00607B03-KS D00607B03 D00607H09-KS D00607H09 06732E01-MD 06732E01-MD D1414A06-IL D1414A06 B03765B09-GA B03765B09-GA B01558D08-NC CCDB-01558D8 B01558D03-NC CCDB-01558D3 B01558C12-NC CCDB-01558C12 D0288B08-SC D0288B08 D0288B06-NC D0288B06 DIAL1391D08-NC DIAL1391D08 DIAL1391E10-NC DIAL1391E10 DIAL0293F04-TN versans 000503 DIAL0293F05-TN \#000504 DIAL1391C05-NC DIAL1391C05 DIAL1391A08-NC DIAL1391A08 DIAL1849H07-ME DIAL1849H07 DIAL1848F07-PE DIAL1848F07 DIAL1848E08-WV DIAL1848E08 D00607F12-NS D00607F12 DIAL2385C06-MD DIAL2385C06-MD D1414D03-VA D1414D03 DIAL1846C07-WV DIAL1846C07 D0287D08-IL D0287D08 DIAL0293B02-DC DIAL0293B02 DIAL1391H03-NC DIAL1391H03 DIAL1847H05-MD DIAL1847H05 DIAL0294G01-ML\#035111 DIAL0294C06-IL \#9074 DIAL0294C07-IN \#15 DIAL0294D11-NY \#046076 DIAL1848E02-MD DIAL1848E02 D0295B06-ON D0295B06 DIAL1391H02-NC DIAL1391H02 DIAL1391D01-NC DIAL1391D01 DIAL1391H08-NC DIAL1391H08 DIAL1847H04-MD DIAL1847H04 DIAL1391G12-NC DIAL1391G12 DIAL0292B09-MD big rohweri 016054 DIAL0292C01-WV big rohweri 011054 DIAL0292B11-MD big rohweri 013959 DIAL0292C02-MD big rohweri 016026 DIAL1413G10-NC DIAL1413G10 DIAL1391C10-NC DIAL1391C10
York University, Packer Collection York University, Packer Collection York University, Packer Collection York University, Packer Collection York University, Packer Collection York University, Packer Collection York University, Packer Collection York University, Packer Collection York University, Packer Collection York University, Packer Collection York University, Packer Collection York University, Packer Collection York University, Packer Collection York University, Packer Collection York University, Packer Collection York University, Packer Collection York University, Packer Collection York University, Packer Collection York University, Packer Collection York University, Packer Collection York University, Packer Collection York University, Packer Collection York University, Packer Collection York University, Packer Collection York University, Packer Collection York University, Packer Collection York University, Packer Collection York University, Packer Collection York University, Packer Collection York University, Packer Collection York University, Packer Collection York University, Packer Collection York University, Packer Collection York University, Packer Collection York University, Packer Collection York University, Packer Collection York University, Packer Collection York University, Packer Collection York University, Packer Collection York University, Packer Collection York University, Packer Collection York University, Packer Collection York University, Packer Collection York University, Packer Collection York University, Packer Collection York University, Packer Collection York University, Packer Collection York University, Packer Collection York University, Packer Collection York University, Packer Collection York University, Packer Collection York University, Packer Collection York University, Packer Collection York University, Packer Collection York University, Packer Collection 
DIAL1391B10-NC DIAL1391B10 06732E06-WV 06732E06-WV D0287H06-IL D0287H06 D0287F01-IL D0287F01 D0287H08-IL D0287H08 DIAL1413G09-NC DIAL1413G09 D0287H12-IL D0287H12 DIAL1409C01-PQ DIAL1409C01 DIAL1409B11-PQ DIAL1409B11 DIAL1846F03-MD DIAL1846F03 DIAL1847H01-MD DIAL1847H01 DIAL1847H02-MD DIAL1847H02 DIAL1846B03-MD DIAL1846B03 D00607H07-KS D00607H07 DIAL1391H05-NC DIAL1391H05 DIAL1391B07-NC DIAL1391B07 DIAL1391B05-NC DIAL1391B05 D0287E12-IL D0287E12 D0287E06-IL D0287E06 DIAL1413B03-IL DIAL1413B03 DIAL1390A02-NC DIAL1390A02 DIAL1390A01-NC DIAL1390A01 DIAL1390F02-NC DIAL1390F02 DIAL1413G08-NC DIAL1413G08 DIAL1391H12-NC DIAL1391H12 DIAL1413H07-NC DIAL1413H07 DIAL1413H04-NC DIAL1413H04 DIAL1391B09-NC DIAL1391B09 DIAL1391B08-NC DIAL1391B08 D0287H11-IL D0287H11 DIAL1391B04-NC DIAL1391B04 DIAL1391H07-NC DIAL1391H07 D0287E02-IL D0287E02 DIAL1391B06-NC DIAL1391B06 DIAL1848F04-MD DIAL1848F04 DIAL1413H09-NC DIAL1413H09 D0287E08-IL D0287E08 D0295B07-ON D0295B07 D0295B01-ON D0295B01 D0295B02-ON D0295B02 D0287H07-IL D0287H07 DIAL1391H11-NC DIAL1391H11 D0287G11-IL D0287G11 D0287H03-IL D0287H03 D0287H04-IL D0287H04 D0287H05-IL D0287H05 D0287G05-IL D0287G05 D0287E11-IL D0287E11 D0287E07-IL D0287E07 D0287D07-IL D0287D07 D0287C05-IL D0287C05 D1414E06-VA D1414E06 D0287C03-IL D0287C03 D0287C04-IL D0287C04 D0287E09-IL D0287E09
York University, Packer Collection York University, Packer Collection York University, Packer Collection York University, Packer Collection York University, Packer Collection York University, Packer Collection York University, Packer Collection York University, Packer Collection York University, Packer Collection York University, Packer Collection York University, Packer Collection York University, Packer Collection York University, Packer Collection York University, Packer Collection York University, Packer Collection York University, Packer Collection York University, Packer Collection York University, Packer Collection York University, Packer Collection York University, Packer Collection York University, Packer Collection York University, Packer Collection York University, Packer Collection York University, Packer Collection York University, Packer Collection York University, Packer Collection York University, Packer Collection York University, Packer Collection York University, Packer Collection York University, Packer Collection York University, Packer Collection York University, Packer Collection York University, Packer Collection York University, Packer Collection York University, Packer Collection York University, Packer Collection York University, Packer Collection York University, Packer Collection York University, Packer Collection York University, Packer Collection York University, Packer Collection York University, Packer Collection York University, Packer Collection York University, Packer Collection York University, Packer Collection York University, Packer Collection York University, Packer Collection York University, Packer Collection York University, Packer Collection York University, Packer Collection York University, Packer Collection York University, Packer Collection York University, Packer Collection York University, Packer Collection York University, Packer Collection 


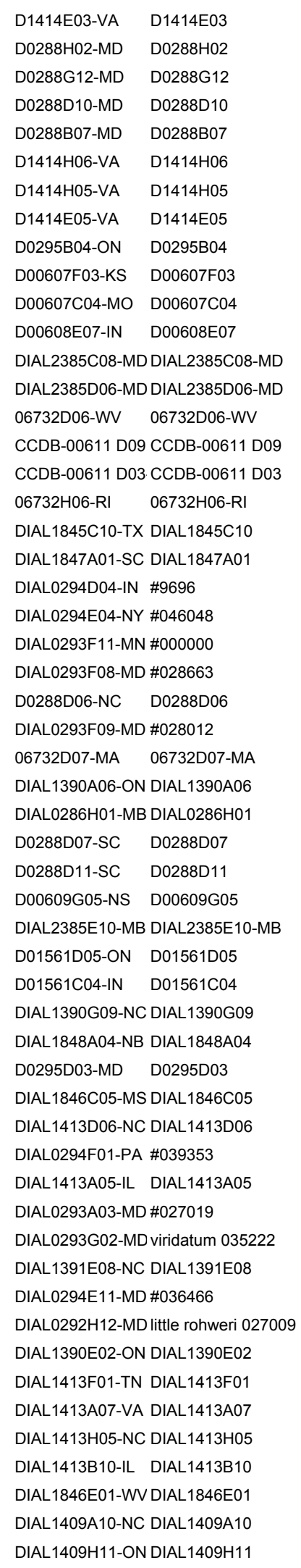

\begin{abstract}
York University, Packer Collection York University, Packer Collection York University, Packer Collection York University, Packer Collection York University, Packer Collection York University, Packer Collection York University, Packer Collection York University, Packer Collection York University, Packer Collection York University, Packer Collection York University, Packer Collection York University, Packer Collection York University, Packer Collection York University, Packer Collection York University, Packer Collection York University, Packer Collection York University, Packer Collection York University, Packer Collection York University, Packer Collection York University, Packer Collection York University, Packer Collection York University, Packer Collection York University, Packer Collection York University, Packer Collection York University, Packer Collection York University, Packer Collection York University, Packer Collection York University, Packer Collection York University, Packer Collection York University, Packer Collection York University, Packer Collection York University, Packer Collection York University, Packer Collection York University, Packer Collection York University, Packer Collection York University, Packer Collection York University, Packer Collection York University, Packer Collection York University, Packer Collection York University, Packer Collection York University, Packer Collection York University, Packer Collection York University, Packer Collection York University, Packer Collection York University, Packer Collection York University, Packer Collection York University, Packer Collection York University, Packer Collection York University, Packer Collection York University, Packer Collection York University, Packer Collection York University, Packer Collection York University, Packer Collection York University, Packer Collection York University, Packer Collection
\end{abstract}


DIAL1390H03-SC DIAL1390H03 DIAL1390C06-ON DIAL1390C06 DIAL1390H02-SC DIAL1390H02 DIAL1390G05-NC DIAL1390G05 DIAL1391A02-VA DIAL1391A02 DIAL1413D07-NC DIAL1413D07 DIAL1848F06-MD DIAL1848F06 D1414A01-YT D1414A01 DIAL2387F08-BC DIAL2387F08 DIAL1848C02-YT DIAL1848C02 BEE0286C11-ON BEE0286C11 D00608H10-OR D00608H10 04485A10-VA 04485A10-VA DIAL1848D10-MD DIAL1848D10 DIAL1390D09-ON DIAL1390D09 DIAL1848B06-WV DIAL1848B06 DIAL1848C08-WV DIAL1848C08
York University, Packer Collection York University, Packer Collection York University, Packer Collection York University, Packer Collection York University, Packer Collection York University, Packer Collection York University, Packer Collection York University, Packer Collection York University, Packer Collection York University, Packer Collection York University, Packer Collection York University, Packer Collection York University, Packer Collection York University, Packer Collection York University, Packer Collection York University, Packer Collection York University, Packer Collection 


\begin{tabular}{|c|c|c|c|c|c|}
\hline \multirow{2}{*}{$\begin{array}{l}\text { Project: } \\
\text { Created : } \\
\text { Sample ID }\end{array}$} & \multicolumn{2}{|c|}{ SEARCH: Process ids(1283 ids) } & & User: & Jason Gibbs \\
\hline & Phylum & Class & Order & Family & Subfamily \\
\hline D00608F07-IN & Arthropoda & Insecta & Hymenoptera & Halictidae & Halictinae \\
\hline DIAL2386F10-UT & Arthropoda & Insecta & Hymenoptera & Halictidae & Halictinae \\
\hline DIAL2386C10-UT & Arthropoda & Insecta & Hymenoptera & Halictidae & Halictinae \\
\hline DIAL2384C07-AB & Arthropoda & Insecta & Hymenoptera & Halictidae & Halictinae \\
\hline DIAL2386C08-UT & Arthropoda & Insecta & Hymenoptera & Halictidae & Halictinae \\
\hline DIAL2374H06-UT & Arthropoda & Insecta & Hymenoptera & Halictidae & Halictinae \\
\hline DIAL2385D04-ON & Arthropoda & Insecta & Hymenoptera & Halictidae & Halictinae \\
\hline DIAL2385E03-MD & Arthropoda & Insecta & Hymenoptera & Halictidae & Halictinae \\
\hline D01562A03-MI & Arthropoda & Insecta & Hymenoptera & Halictidae & Halictinae \\
\hline DIAL1839G06-MN & Arthropoda & Insecta & Hymenoptera & Halictidae & Halictinae \\
\hline DIAL1839G08-MN & Arthropoda & Insecta & Hymenoptera & Halictidae & Halictinae \\
\hline DIAL0292H11-MD & Arthropoda & Insecta & Hymenoptera & Halictidae & Halictinae \\
\hline DIAL1409C04-ON & Arthropoda & Insecta & Hymenoptera & Halictidae & Halictinae \\
\hline DIAL2387C05-ON & Arthropoda & Insecta & Hymenoptera & Halictidae & Halictinae \\
\hline D01561D09-ON & Arthropoda & Insecta & Hymenoptera & Halictidae & Halictinae \\
\hline 04791B03-FL & Arthropoda & Insecta & Hymenoptera & Halictidae & Halictinae \\
\hline DIAL0292B02-NJ & Arthropoda & Insecta & Hymenoptera & Halictidae & Halictinae \\
\hline DIAL0286F05-MB & Arthropoda & Insecta & Hymenoptera & Halictidae & Halictinae \\
\hline D00609G09-NS & Arthropoda & Insecta & Hymenoptera & Halictidae & Halictinae \\
\hline DIAL1839G12-MN & Arthropoda & Insecta & Hymenoptera & Halictidae & Halictinae \\
\hline DIAL1839G09-MN & Arthropoda & Insecta & Hymenoptera & Halictidae & Halictinae \\
\hline 06732D10-PA & Arthropoda & Insecta & Hymenoptera & Halictidae & Halictinae \\
\hline BEE1850G06-ME & Arthropoda & Insecta & Hymenoptera & Halictidae & Halictinae \\
\hline DIAL2386G02-BC & Arthropoda & Insecta & Hymenoptera & Halictidae & Halictinae \\
\hline DIAL1848A08-NB & Arthropoda & Insecta & Hymenoptera & Halictidae & Halictinae \\
\hline DIAL1849A09-CO & Arthropoda & Insecta & Hymenoptera & Halictidae & Halictinae \\
\hline DIAL0286F03-MB & Arthropoda & Insecta & Hymenoptera & Halictidae & Halictinae \\
\hline DIAL2384C02-AB & Arthropoda & Insecta & Hymenoptera & Halictidae & Halictinae \\
\hline D01559F12-BC & Arthropoda & Insecta & Hymenoptera & Halictidae & Halictinae \\
\hline DIAL2386G07-WA & Arthropoda & Insecta & Hymenoptera & Halictidae & Halictinae \\
\hline DIAL2387E09-AB & Arthropoda & Insecta & Hymenoptera & Halictidae & Halictinae \\
\hline D01559F10-BC & Arthropoda & Insecta & Hymenoptera & Halictidae & Halictinae \\
\hline D01559H03-BC & Arthropoda & Insecta & Hymenoptera & Halictidae & Halictinae \\
\hline D01559H10-BC & Arthropoda & Insecta & Hymenoptera & Halictidae & Halictinae \\
\hline DIAL2387B05-WA & Arthropoda & Insecta & Hymenoptera & Halictidae & Halictinae \\
\hline DIAL0294A03-IN & Arthropoda & Insecta & Hymenoptera & Halictidae & Halictinae \\
\hline DIAL0292B05-IN & Arthropoda & Insecta & Hymenoptera & Halictidae & Halictinae \\
\hline BEE0289C05-MN & Arthropoda & Insecta & Hymenoptera & Halictidae & Halictinae \\
\hline CCDB-00611 C12 & Arthropoda & Insecta & Hymenoptera & Halictidae & Halictinae \\
\hline D-3253D12-WI & Arthropoda & Insecta & Hymenoptera & Halictidae & Halictinae \\
\hline D0287D01-MI & Arthropoda & Insecta & Hymenoptera & Halictidae & Halictinae \\
\hline DIAL1390D06-ON & Arthropoda & Insecta & Hymenoptera & Halictidae & Halictinae \\
\hline DIAL1846F06-PA & Arthropoda & Insecta & Hymenoptera & Halictidae & Halictinae \\
\hline DIAL1390F04-NC & Arthropoda & Insecta & Hymenoptera & Halictidae & Halictinae \\
\hline DIAL0292E12-IN & Arthropoda & Insecta & Hymenoptera & Halictidae & Halictinae \\
\hline DIAL1849F01-WV & Arthropoda & Insecta & Hymenoptera & Halictidae & Halictinae \\
\hline DIAL0292B01-WV & Arthropoda & Insecta & Hymenoptera & Halictidae & Halictinae \\
\hline DIAL0292B07-WV & Arthropoda & Insecta & Hymenoptera & Halictidae & Halictinae \\
\hline DIAL0292B08-WV & Arthropoda & Insecta & Hymenoptera & Halictidae & Halictinae \\
\hline DIAL0292F02-WV & Arthropoda & Insecta & Hymenoptera & Halictidae & Halictinae \\
\hline DIAL1849E12-WV & Arthropoda & Insecta & Hymenoptera & Halictidae & Halictinae \\
\hline DIAL0292E11-WV & Arthropoda & Insecta & Hymenoptera & Halictidae & Halictinae \\
\hline
\end{tabular}




\begin{tabular}{|c|c|c|c|c|c|}
\hline DIAL1390F06-NC & Arthropoda & Insecta & Hymenoptera & Halictidae & Halictinae \\
\hline DIAL1390G04-NC & Arthropoda & Insecta & Hymenoptera & Halictidae & Halictinae \\
\hline DIAL1390G06-NC & Arthropoda & Insecta & Hymenoptera & Halictidae & Halictinae \\
\hline DIAL1390G02-WV & Arthropoda & Insecta & Hymenoptera & Halictidae & Halictinae \\
\hline DIAL1848D09-MD & Arthropoda & Insecta & Hymenoptera & Halictidae & Halictinae \\
\hline DIAL1846D12-MS & Arthropoda & Insecta & Hymenoptera & Halictidae & Halictinae \\
\hline D1414H02-VA & Arthropoda & Insecta & Hymenoptera & Halictidae & Halictinae \\
\hline DIAL2384H09-SC & Arthropoda & Insecta & Hymenoptera & Halictidae & Halictinae \\
\hline D01559C09-GA & Arthropoda & Insecta & Hymenoptera & Halictidae & Halictinae \\
\hline D1414E02-VA & Arthropoda & Insecta & Hymenoptera & Halictidae & Halictinae \\
\hline DIAL0293C03-NJ & Arthropoda & Insecta & Hymenoptera & Halictidae & Halictinae \\
\hline DIAL0293C04-NJ & Arthropoda & Insecta & Hymenoptera & Halictidae & Halictinae \\
\hline D01562B06-MD & Arthropoda & Insecta & Hymenoptera & Halictidae & Halictinae \\
\hline D01562B02-MD & Arthropoda & Insecta & Hymenoptera & Halictidae & Halictinae \\
\hline D01562C12-MD & Arthropoda & Insecta & Hymenoptera & Halictidae & Halictinae \\
\hline D01562B10-ON & Arthropoda & Insecta & Hymenoptera & Halictidae & Halictinae \\
\hline D01562C08-ON & Arthropoda & Insecta & Hymenoptera & Halictidae & Halictinae \\
\hline DIAL0294G02-MD & Arthropoda & Insecta & Hymenoptera & Halictidae & Halictinae \\
\hline DIAL1848B03-ON & Arthropoda & Insecta & Hymenoptera & Halictidae & Halictinae \\
\hline D00609B05-WI & Arthropoda & Insecta & Hymenoptera & Halictidae & Halictinae \\
\hline D00609G03-NS & Arthropoda & Insecta & Hymenoptera & Halictidae & Halictinae \\
\hline D0288F07-SC & Arthropoda & Insecta & Hymenoptera & Halictidae & Halictinae \\
\hline D0288F09-SC & Arthropoda & Insecta & Hymenoptera & Halictidae & Halictinae \\
\hline DIAL0294A05-in & Arthropoda & Insecta & Hymenoptera & Halictidae & Halictinae \\
\hline DIAL0292E08-MD & Arthropoda & Insecta & Hymenoptera & Halictidae & Halictinae \\
\hline 06732D09-VA & Arthropoda & Insecta & Hymenoptera & Halictidae & Halictinae \\
\hline D00609C07-KS & Arthropoda & Insecta & Hymenoptera & Halictidae & Halictinae \\
\hline DIAL1846B10-WV & Arthropoda & Insecta & Hymenoptera & Halictidae & Halictinae \\
\hline DIAL1390C08-ON & Arthropoda & Insecta & Hymenoptera & Halictidae & Halictinae \\
\hline DIAL1409A09-NC & Arthropoda & Insecta & Hymenoptera & Halictidae & Halictinae \\
\hline DIAL1846G04-VA & Arthropoda & Insecta & Hymenoptera & Halictidae & Halictinae \\
\hline DIAL1390B02-ON & Arthropoda & Insecta & Hymenoptera & Halictidae & Halictinae \\
\hline DIAL1848E12-MD & Arthropoda & Insecta & Hymenoptera & Halictidae & Halictinae \\
\hline DIAL1413C12-TN & Arthropoda & Insecta & Hymenoptera & Halictidae & Halictinae \\
\hline D01559G11-BC & Arthropoda & Insecta & Hymenoptera & Halictidae & Halictinae \\
\hline DIAL1845D09-CA & Arthropoda & Insecta & Hymenoptera & Halictidae & Halictinae \\
\hline DIAL2374G12-CA & Arthropoda & Insecta & Hymenoptera & Halictidae & Halictinae \\
\hline DIAL1845C05-CA & Arthropoda & Insecta & Hymenoptera & Halictidae & Halictinae \\
\hline DIAL1845D03-CA & Arthropoda & Insecta & Hymenoptera & Halictidae & Halictinae \\
\hline DIAL1845C11-CA & Arthropoda & Insecta & Hymenoptera & Halictidae & Halictinae \\
\hline DIAL2388C12-UT & Arthropoda & Insecta & Hymenoptera & Halictidae & Halictinae \\
\hline DIAL2374G11-CA & Arthropoda & Insecta & Hymenoptera & Halictidae & Halictinae \\
\hline D00607E04-CA & Arthropoda & Insecta & Hymenoptera & Halictidae & Halictinae \\
\hline D01559E10-BC & Arthropoda & Insecta & Hymenoptera & Halictidae & Halictinae \\
\hline D01559G04-BC & Arthropoda & Insecta & Hymenoptera & Halictidae & Halictinae \\
\hline DIAL2388B06-UT & Arthropoda & Insecta & Hymenoptera & Halictidae & Halictinae \\
\hline D00607D05-CA & Arthropoda & Insecta & Hymenoptera & Halictidae & Halictinae \\
\hline DIAL2374H09-UT & Arthropoda & Insecta & Hymenoptera & Halictidae & Halictinae \\
\hline DIAL2385C03-MD & Arthropoda & Insecta & Hymenoptera & Halictidae & Halictinae \\
\hline DIAL1413E11-SC & Arthropoda & Insecta & Hymenoptera & Halictidae & Halictinae \\
\hline DIAL0292D03-NC & Arthropoda & Insecta & Hymenoptera & Halictidae & Halictinae \\
\hline DIAL0294E03-VA & Arthropoda & Insecta & Hymenoptera & Halictidae & Halictinae \\
\hline DIAL0294E02-TN & Arthropoda & Insecta & Hymenoptera & Halictidae & Halictinae \\
\hline DIAL0293E11-NC & Arthropoda & Insecta & Hymenoptera & Halictidae & Halictinae \\
\hline DIAL0293E10-MD & Arthropoda & Insecta & Hymenoptera & Halictidae & Halictinae \\
\hline
\end{tabular}




\begin{tabular}{|c|c|c|c|c|c|}
\hline D0288C04-DC & Arthropoda & Insecta & Hymenoptera & Halictidae & Halictinae \\
\hline DIAL0293E12-DC & Arthropoda & Insecta & Hymenoptera & Halictidae & Halictinae \\
\hline D1414H07-VA & Arthropoda & Insecta & Hymenoptera & Halictidae & Halictinae \\
\hline BEE0286D06-VA & Arthropoda & Insecta & Hymenoptera & Halictidae & Halictinae \\
\hline DIAL1413D04-NC & Arthropoda & Insecta & Hymenoptera & Halictidae & Halictinae \\
\hline DIAL1391D10-NC & Arthropoda & Insecta & Hymenoptera & Halictidae & Halictinae \\
\hline D1414E08-VA & Arthropoda & Insecta & Hymenoptera & Halictidae & Halictinae \\
\hline DIAL1409B06-NC & Arthropoda & Insecta & Hymenoptera & Halictidae & Halictinae \\
\hline DIAL1413E08-NC & Arthropoda & Insecta & Hymenoptera & Halictidae & Halictinae \\
\hline D1414E04-VA & Arthropoda & Insecta & Hymenoptera & Halictidae & Halictinae \\
\hline DIAL1391A09-SC & Arthropoda & Insecta & Hymenoptera & Halictidae & Halictinae \\
\hline DIAL1413F07-TN & Arthropoda & Insecta & Hymenoptera & Halictidae & Halictinae \\
\hline DIAL1391D09-NC & Arthropoda & Insecta & Hymenoptera & Halictidae & Halictinae \\
\hline DIAL1391D07-NC & Arthropoda & Insecta & Hymenoptera & Halictidae & Halictinae \\
\hline DIAL1413D01-TN & Arthropoda & Insecta & Hymenoptera & Halictidae & Halictinae \\
\hline D1414E07-VA & Arthropoda & Insecta & Hymenoptera & Halictidae & Halictinae \\
\hline D0288C01-DC & Arthropoda & Insecta & Hymenoptera & Halictidae & Halictinae \\
\hline D0288C06-SC & Arthropoda & Insecta & Hymenoptera & Halictidae & Halictinae \\
\hline D0288D09-SC & Arthropoda & Insecta & Hymenoptera & Halictidae & Halictinae \\
\hline D00607E06-MO & Arthropoda & Insecta & Hymenoptera & Halictidae & Halictinae \\
\hline D00607A09-MO & Arthropoda & Insecta & Hymenoptera & Halictidae & Halictinae \\
\hline D0288G03-SC & Arthropoda & Insecta & Hymenoptera & Halictidae & Halictinae \\
\hline D0288F02-SC & Arthropoda & Insecta & Hymenoptera & Halictidae & Halictinae \\
\hline DIAL1391B01-SC & Arthropoda & Insecta & Hymenoptera & Halictidae & Halictinae \\
\hline DIAL1391E04-SC & Arthropoda & Insecta & Hymenoptera & Halictidae & Halictinae \\
\hline DIAL1391D11-SC & Arthropoda & Insecta & Hymenoptera & Halictidae & Halictinae \\
\hline DIAL1413B06-IL & Arthropoda & Insecta & Hymenoptera & Halictidae & Halictinae \\
\hline D1414A07-IL & Arthropoda & Insecta & Hymenoptera & Halictidae & Halictinae \\
\hline DIAL1413B11-IL & Arthropoda & Insecta & Hymenoptera & Halictidae & Halictinae \\
\hline 04485C03-MD & Arthropoda & Insecta & Hymenoptera & Halictidae & Halictinae \\
\hline D00607F06-MD & Arthropoda & Insecta & Hymenoptera & Halictidae & Halictinae \\
\hline D-3253D11-FL & Arthropoda & Insecta & Hymenoptera & Halictidae & Halictinae \\
\hline D00607B02-KS & Arthropoda & Insecta & Hymenoptera & Halictidae & Halictinae \\
\hline DIAL0292D09-MD & Arthropoda & Insecta & Hymenoptera & Halictidae & Halictinae \\
\hline DIAL0292D10-MD & Arthropoda & Insecta & Hymenoptera & Halictidae & Halictinae \\
\hline DIAL0292D08-NC & Arthropoda & Insecta & Hymenoptera & Halictidae & Halictinae \\
\hline DIAL0292D12-MD & Arthropoda & Insecta & Hymenoptera & Halictidae & Halictinae \\
\hline DIAL0292D11-MD & Arthropoda & Insecta & Hymenoptera & Halictidae & Halictinae \\
\hline DIAL1846H06-VA & Arthropoda & Insecta & Hymenoptera & Halictidae & Halictinae \\
\hline DIAL1846C01-MS & Arthropoda & Insecta & Hymenoptera & Halictidae & Halictinae \\
\hline DIAL1846E11-MS & Arthropoda & Insecta & Hymenoptera & Halictidae & Halictinae \\
\hline DIAL1409E03-TX & Arthropoda & Insecta & Hymenoptera & Halictidae & Halictinae \\
\hline DIAL1846G07-MS & Arthropoda & Insecta & Hymenoptera & Halictidae & Halictinae \\
\hline DIAL1849F11-DE & Arthropoda & Insecta & Hymenoptera & Halictidae & Halictinae \\
\hline DIAL1849G03-DE & Arthropoda & Insecta & Hymenoptera & Halictidae & Halictinae \\
\hline DIAL1391D04-NC & Arthropoda & Insecta & Hymenoptera & Halictidae & Halictinae \\
\hline 06732C08-MA & Arthropoda & Insecta & Hymenoptera & Halictidae & Halictinae \\
\hline 06732C10-VA & Arthropoda & Insecta & Hymenoptera & Halictidae & Halictinae \\
\hline D-3253D07-FL & Arthropoda & Insecta & Hymenoptera & Halictidae & Halictinae \\
\hline B01566H05-TX & Arthropoda & Insecta & Hymenoptera & Halictidae & Halictinae \\
\hline DIAL0292E02-MD & Arthropoda & Insecta & Hymenoptera & Halictidae & Halictinae \\
\hline DIAL0294F02-NC & Arthropoda & Insecta & Hymenoptera & Halictidae & Halictinae \\
\hline DIAL0294F03-NC & Arthropoda & Insecta & Hymenoptera & Halictidae & Halictinae \\
\hline DIAL0294E05-NC & Arthropoda & Insecta & Hymenoptera & Halictidae & Halictinae \\
\hline DIAL0292E04-MD & Arthropoda & Insecta & Hymenoptera & Halictidae & Halictinae \\
\hline
\end{tabular}




\begin{tabular}{|c|c|c|c|c|c|}
\hline DIAL1413D12-FL & Arthropoda & Insecta & Hymenoptera & Halictidae & Halictinae \\
\hline DIAL1846C06-MS & Arthropoda & Insecta & Hymenoptera & Halictidae & Halictinae \\
\hline DIAL1846H02-MS & Arthropoda & Insecta & Hymenoptera & Halictidae & Halictinae \\
\hline DIAL1846C11-MS & Arthropoda & Insecta & Hymenoptera & Halictidae & Halictinae \\
\hline CCDB-00611 E05 & Arthropoda & Insecta & Hymenoptera & Halictidae & Halictinae \\
\hline BEE0286C08-NC & Arthropoda & Insecta & Hymenoptera & Halictidae & Halictinae \\
\hline DIAL0294A11-IN & Arthropoda & Insecta & Hymenoptera & Halictidae & Halictinae \\
\hline DIAL0294A10-IN & Arthropoda & Insecta & Hymenoptera & Halictidae & Halictinae \\
\hline DIAL1390C02-ON & Arthropoda & Insecta & Hymenoptera & Halictidae & Halictinae \\
\hline BEE0286A05-GA & Arthropoda & Insecta & Hymenoptera & Halictidae & Halictinae \\
\hline DIAL0294A09-in & Arthropoda & Insecta & Hymenoptera & Halictidae & Halictinae \\
\hline DIAL0292E09-MD & Arthropoda & Insecta & Hymenoptera & Halictidae & Halictinae \\
\hline DIAL1845H09-BC & Arthropoda & Insecta & Hymenoptera & Halictidae & Halictinae \\
\hline DIAL1845H07-BC & Arthropoda & Insecta & Hymenoptera & Halictidae & Halictinae \\
\hline DIAL0292E07-ME & Arthropoda & Insecta & Hymenoptera & Halictidae & Halictinae \\
\hline DIAL1845F11-BC & Arthropoda & Insecta & Hymenoptera & Halictidae & Halictinae \\
\hline DIAL1391A03-NC & Arthropoda & Insecta & Hymenoptera & Halictidae & Halictinae \\
\hline BEE0289D05-MN & Arthropoda & Insecta & Hymenoptera & Halictidae & Halictinae \\
\hline BEE0289D07-MN & Arthropoda & Insecta & Hymenoptera & Halictidae & Halictinae \\
\hline DIAL1391A05-NC & Arthropoda & Insecta & Hymenoptera & Halictidae & Halictinae \\
\hline D00609G07-NS & Arthropoda & Insecta & Hymenoptera & Halictidae & Halictinae \\
\hline DIAL1848A03-NB & Arthropoda & Insecta & Hymenoptera & Halictidae & Halictinae \\
\hline BEE1850G05-ME & Arthropoda & Insecta & Hymenoptera & Halictidae & Halictinae \\
\hline BEE0289D06-MN & Arthropoda & Insecta & Hymenoptera & Halictidae & Halictinae \\
\hline D01561G01-MD & Arthropoda & Insecta & Hymenoptera & Halictidae & Halictinae \\
\hline DIAL2386H03-WA & Arthropoda & Insecta & Hymenoptera & Halictidae & Halictinae \\
\hline DIAL1391A06-NC & Arthropoda & Insecta & Hymenoptera & Halictidae & Halictinae \\
\hline D0287G06-IL & Arthropoda & Insecta & Hymenoptera & Halictidae & Halictinae \\
\hline D0288G10-ON & Arthropoda & Insecta & Hymenoptera & Halictidae & Halictinae \\
\hline DIAL2386G06-BC & Arthropoda & Insecta & Hymenoptera & Halictidae & Halictinae \\
\hline 03767G09-BC & Arthropoda & Insecta & Hymenoptera & Halictidae & Halictinae \\
\hline 06732B12-NY & Arthropoda & Insecta & Hymenoptera & Halictidae & Halictinae \\
\hline DIAL2386F07-WA & Arthropoda & Insecta & Hymenoptera & Halictidae & Halictinae \\
\hline DIAL2386H05-WA & Arthropoda & Insecta & Hymenoptera & Halictidae & Halictinae \\
\hline D-3253E10-OK & Arthropoda & Insecta & Hymenoptera & Halictidae & Halictinae \\
\hline D00607B09-KS & Arthropoda & Insecta & Hymenoptera & Halictidae & Halictinae \\
\hline DIAL1409D07-TX & Arthropoda & Insecta & Hymenoptera & Halictidae & Halictinae \\
\hline DIAL1409D04-TX & Arthropoda & Insecta & Hymenoptera & Halictidae & Halictinae \\
\hline DIAL1409E08-TX & Arthropoda & Insecta & Hymenoptera & Halictidae & Halictinae \\
\hline DIAL1409E10-TX & Arthropoda & Insecta & Hymenoptera & Halictidae & Halictinae \\
\hline DIAL1409F01-TX & Arthropoda & Insecta & Hymenoptera & Halictidae & Halictinae \\
\hline D00607B01-KS & Arthropoda & Insecta & Hymenoptera & Halictidae & Halictinae \\
\hline DIAL1848F12-NC & Arthropoda & Insecta & Hymenoptera & Halictidae & Halictinae \\
\hline D01561B12-ON & Arthropoda & Insecta & Hymenoptera & Halictidae & Halictinae \\
\hline DIAL1848F10-ON & Arthropoda & Insecta & Hymenoptera & Halictidae & Halictinae \\
\hline DIAL1848G01-ON & Arthropoda & Insecta & Hymenoptera & Halictidae & Halictinae \\
\hline D01561E03-ON & Arthropoda & Insecta & Hymenoptera & Halictidae & Halictinae \\
\hline DIAL2374G04-UT & Arthropoda & Insecta & Hymenoptera & Halictidae & Halictinae \\
\hline DIAL2388B04-UT & Arthropoda & Insecta & Hymenoptera & Halictidae & Halictinae \\
\hline DIAL2388C03-UT & Arthropoda & Insecta & Hymenoptera & Halictidae & Halictinae \\
\hline DIAL2386B05-NV & Arthropoda & Insecta & Hymenoptera & Halictidae & Halictinae \\
\hline DIAL2384C08-AB & Arthropoda & Insecta & Hymenoptera & Halictidae & Halictinae \\
\hline DIAL2374G05-UT & Arthropoda & Insecta & Hymenoptera & Halictidae & Halictinae \\
\hline DIAL0294D02-IN & Arthropoda & Insecta & Hymenoptera & Halictidae & Halictinae \\
\hline D01560D03-IA & Arthropoda & Insecta & Hymenoptera & Halictidae & Halictinae \\
\hline
\end{tabular}




\begin{tabular}{|c|c|c|c|c|c|}
\hline D01560A07-WI & Arthropoda & Insecta & Hymenoptera & Halictidae & Halictinae \\
\hline DIAL1409C09-ON & Arthropoda & Insecta & Hymenoptera & Halictidae & Halictinae \\
\hline DIAL1390A09-ON & Arthropoda & Insecta & Hymenoptera & Halictidae & Halictinae \\
\hline DIAL1839G05-MN & Arthropoda & Insecta & Hymenoptera & Halictidae & Halictinae \\
\hline D0288D03-IN & Arthropoda & Insecta & Hymenoptera & Halictidae & Halictinae \\
\hline DIAL1413G03-NC & Arthropoda & Insecta & Hymenoptera & Halictidae & Halictinae \\
\hline D0295C04-ON & Arthropoda & Insecta & Hymenoptera & Halictidae & Halictinae \\
\hline DIAL1391H04-NC & Arthropoda & Insecta & Hymenoptera & Halictidae & Halictinae \\
\hline DIAL1849G11-MA & Arthropoda & Insecta & Hymenoptera & Halictidae & Halictinae \\
\hline DIAL1839H01-MN & Arthropoda & Insecta & Hymenoptera & Halictidae & Halictinae \\
\hline D0295C05-ON & Arthropoda & Insecta & Hymenoptera & Halictidae & Halictinae \\
\hline D01562C02-MB & Arthropoda & Insecta & Hymenoptera & Halictidae & Halictinae \\
\hline D01562B07-MI & Arthropoda & Insecta & Hymenoptera & Halictidae & Halictinae \\
\hline B03765D08-IA & Arthropoda & Insecta & Hymenoptera & Halictidae & Halictinae \\
\hline 04485E09-MI & Arthropoda & Insecta & Hymenoptera & Halictidae & Halictinae \\
\hline B03765D06-IA & Arthropoda & Insecta & Hymenoptera & Halictidae & Halictinae \\
\hline 04485E02-MI & Arthropoda & Insecta & Hymenoptera & Halictidae & Halictinae \\
\hline B03765D11-IA & Arthropoda & Insecta & Hymenoptera & Halictidae & Halictinae \\
\hline B03765E01-IA & Arthropoda & Insecta & Hymenoptera & Halictidae & Halictinae \\
\hline D1414G06-DC & Arthropoda & Insecta & Hymenoptera & Halictidae & Halictinae \\
\hline DIAL1846H11-DC & Arthropoda & Insecta & Hymenoptera & Halictidae & Halictinae \\
\hline BEE0289A03-MN & Arthropoda & Insecta & Hymenoptera & Halictidae & Halictinae \\
\hline DIAL1846E03-PA & Arthropoda & Insecta & Hymenoptera & Halictidae & Halictinae \\
\hline D0295E03-VA & Arthropoda & Insecta & Hymenoptera & Halictidae & Halictinae \\
\hline BEE0289A07-MN & Arthropoda & Insecta & Hymenoptera & Halictidae & Halictinae \\
\hline DIAL0294H08-ON & Arthropoda & Insecta & Hymenoptera & Halictidae & Halictinae \\
\hline DIAL1390F12-NC & Arthropoda & Insecta & Hymenoptera & Halictidae & Halictinae \\
\hline D0295C07-ON & Arthropoda & Insecta & Hymenoptera & Halictidae & Halictinae \\
\hline DIAL2387C02-ON & Arthropoda & Insecta & Hymenoptera & Halictidae & Halictinae \\
\hline DIAL1849H03-DC & Arthropoda & Insecta & Hymenoptera & Halictidae & Halictinae \\
\hline DIAL1846A05-ON & Arthropoda & Insecta & Hymenoptera & Halictidae & Halictinae \\
\hline DIAL1846A07-ON & Arthropoda & Insecta & Hymenoptera & Halictidae & Halictinae \\
\hline DIAL1846A02-ON & Arthropoda & Insecta & Hymenoptera & Halictidae & Halictinae \\
\hline DIAL1390F08-WV & Arthropoda & Insecta & Hymenoptera & Halictidae & Halictinae \\
\hline DIAL1391F06-ON & Arthropoda & Insecta & Hymenoptera & Halictidae & Halictinae \\
\hline DIAL1391G07-ON & Arthropoda & Insecta & Hymenoptera & Halictidae & Halictinae \\
\hline DIAL1390F03-ON & Arthropoda & Insecta & Hymenoptera & Halictidae & Halictinae \\
\hline DIAL1846B02-ON & Arthropoda & Insecta & Hymenoptera & Halictidae & Halictinae \\
\hline DIAL1846A03-ON & Arthropoda & Insecta & Hymenoptera & Halictidae & Halictinae \\
\hline DIAL1409C02-ON & Arthropoda & Insecta & Hymenoptera & Halictidae & Halictinae \\
\hline DIAL1409C03-ON & Arthropoda & Insecta & Hymenoptera & Halictidae & Halictinae \\
\hline DIAL1409C07-ON & Arthropoda & Insecta & Hymenoptera & Halictidae & Halictinae \\
\hline DIAL1409C08-ON & Arthropoda & Insecta & Hymenoptera & Halictidae & Halictinae \\
\hline D01562C10-MB & Arthropoda & Insecta & Hymenoptera & Halictidae & Halictinae \\
\hline DIAL1390E01-ON & Arthropoda & Insecta & Hymenoptera & Halictidae & Halictinae \\
\hline DIAL1390F01-ON & Arthropoda & Insecta & Hymenoptera & Halictidae & Halictinae \\
\hline DIAL1390G01-NC & Arthropoda & Insecta & Hymenoptera & Halictidae & Halictinae \\
\hline DIAL1390F09-ON & Arthropoda & Insecta & Hymenoptera & Halictidae & Halictinae \\
\hline DIAL1390F05-ON & Arthropoda & Insecta & Hymenoptera & Halictidae & Halictinae \\
\hline DIAL1391G03-ON & Arthropoda & Insecta & Hymenoptera & Halictidae & Halictinae \\
\hline DIAL1390E03-ON & Arthropoda & Insecta & Hymenoptera & Halictidae & Halictinae \\
\hline DIAL1390E06-ON & Arthropoda & Insecta & Hymenoptera & Halictidae & Halictinae \\
\hline DIAL1390E07-ON & Arthropoda & Insecta & Hymenoptera & Halictidae & Halictinae \\
\hline DIAL1391G05-ON & Arthropoda & Insecta & Hymenoptera & Halictidae & Halictinae \\
\hline DIAL2387B11-ON & Arthropoda & Insecta & Hymenoptera & Halictidae & Halictinae \\
\hline
\end{tabular}




\begin{tabular}{|c|c|c|c|c|c|}
\hline BEE1850E08-MN & Arthropoda & Insecta & Hymenoptera & Halictidae & Halictinae \\
\hline D01561A03-IN & Arthropoda & Insecta & Hymenoptera & Halictidae & Halictinae \\
\hline DIAL1391G02-ON & Arthropoda & Insecta & Hymenoptera & Halictidae & Halictinae \\
\hline D01560A04-WI & Arthropoda & Insecta & Hymenoptera & Halictidae & Halictinae \\
\hline D01560B10-ON & Arthropoda & Insecta & Hymenoptera & Halictidae & Halictinae \\
\hline DIAL1849G08-WV & Arthropoda & Insecta & Hymenoptera & Halictidae & Halictinae \\
\hline DIAL2387B06-ON & Arthropoda & Insecta & Hymenoptera & Halictidae & Halictinae \\
\hline D1414G04-DC & Arthropoda & Insecta & Hymenoptera & Halictidae & Halictinae \\
\hline 04485F02-MI & Arthropoda & Insecta & Hymenoptera & Halictidae & Halictinae \\
\hline DIAL1390D04-ON & Arthropoda & Insecta & Hymenoptera & Halictidae & Halictinae \\
\hline DIAL1409A02-ON & Arthropoda & Insecta & Hymenoptera & Halictidae & Halictinae \\
\hline DIAL2387C07-ON & Arthropoda & Insecta & Hymenoptera & Halictidae & Halictinae \\
\hline DIAL1391H06-NC & Arthropoda & Insecta & Hymenoptera & Halictidae & Halictinae \\
\hline D-3253D06-FL & Arthropoda & Insecta & Hymenoptera & Halictidae & Halictinae \\
\hline BEE0286A11-GA & Arthropoda & Insecta & Hymenoptera & Halictidae & Halictinae \\
\hline DIAL1846F10-SC & Arthropoda & Insecta & Hymenoptera & Halictidae & Halictinae \\
\hline DIAL1846F12-SC & Arthropoda & Insecta & Hymenoptera & Halictidae & Halictinae \\
\hline BEE0286B08-GA & Arthropoda & Insecta & Hymenoptera & Halictidae & Halictinae \\
\hline DIAL0294C11-IN & Arthropoda & Insecta & Hymenoptera & Halictidae & Halictinae \\
\hline DIAL0294D01-IN & Arthropoda & Insecta & Hymenoptera & Halictidae & Halictinae \\
\hline DIAL2385E04-ON & Arthropoda & Insecta & Hymenoptera & Halictidae & Halictinae \\
\hline DIAL0294C12-IL & Arthropoda & Insecta & Hymenoptera & Halictidae & Halictinae \\
\hline DIAL0293E02-MD & Arthropoda & Insecta & Hymenoptera & Halictidae & Halictinae \\
\hline DIAL0293E01-IN & Arthropoda & Insecta & Hymenoptera & Halictidae & Halictinae \\
\hline D00608G05-IN & Arthropoda & Insecta & Hymenoptera & Halictidae & Halictinae \\
\hline D00608G01-IN & Arthropoda & Insecta & Hymenoptera & Halictidae & Halictinae \\
\hline D01561A09-MD & Arthropoda & Insecta & Hymenoptera & Halictidae & Halictinae \\
\hline D01561C07-MD & Arthropoda & Insecta & Hymenoptera & Halictidae & Halictinae \\
\hline DIAL2385C07-DE & Arthropoda & Insecta & Hymenoptera & Halictidae & Halictinae \\
\hline D00607F08-MA & Arthropoda & Insecta & Hymenoptera & Halictidae & Halictinae \\
\hline BEE1850F05-NY & Arthropoda & Insecta & Hymenoptera & Halictidae & Halictinae \\
\hline DIAL2385B04-NY & Arthropoda & Insecta & Hymenoptera & Halictidae & Halictinae \\
\hline D1414H03-VA & Arthropoda & Insecta & Hymenoptera & Halictidae & Halictinae \\
\hline DIAL2384H12-NY & Arthropoda & Insecta & Hymenoptera & Halictidae & Halictinae \\
\hline D01559D03-MA & Arthropoda & Insecta & Hymenoptera & Halictidae & Halictinae \\
\hline B04743G05-MA & Arthropoda & Insecta & Hymenoptera & Halictidae & Halictinae \\
\hline DIAL1846G03-VA & Arthropoda & Insecta & Hymenoptera & Halictidae & Halictinae \\
\hline D1414H12-VA & Arthropoda & Insecta & Hymenoptera & Halictidae & Halictinae \\
\hline D1414H10-VA & Arthropoda & Insecta & Hymenoptera & Halictidae & Halictinae \\
\hline DIAL0294D09-NC & Arthropoda & Insecta & Hymenoptera & Halictidae & Halictinae \\
\hline D1414C12-VA & Arthropoda & Insecta & Hymenoptera & Halictidae & Halictinae \\
\hline DIAL0294D08-NC & Arthropoda & Insecta & Hymenoptera & Halictidae & Halictinae \\
\hline DIAL1413D09-FL & Arthropoda & Insecta & Hymenoptera & Halictidae & Halictinae \\
\hline DIAL1846G01-VA & Arthropoda & Insecta & Hymenoptera & Halictidae & Halictinae \\
\hline DIAL1848E10-MD & Arthropoda & Insecta & Hymenoptera & Halictidae & Halictinae \\
\hline DIAL1413A10-VA & Arthropoda & Insecta & Hymenoptera & Halictidae & Halictinae \\
\hline DIAL1413A11-VA & Arthropoda & Insecta & Hymenoptera & Halictidae & Halictinae \\
\hline CCDB-00611 D02 & Arthropoda & Insecta & Hymenoptera & Halictidae & Halictinae \\
\hline 04485G02-MI & Arthropoda & Insecta & Hymenoptera & Halictidae & Halictinae \\
\hline DIAL1848D01-WI & Arthropoda & Insecta & Hymenoptera & Halictidae & Halictinae \\
\hline DIAL1846E08-WV & Arthropoda & Insecta & Hymenoptera & Halictidae & Halictinae \\
\hline DIAL1391D03-NC & Arthropoda & Insecta & Hymenoptera & Halictidae & Halictinae \\
\hline DIAL1391C11-NC & Arthropoda & Insecta & Hymenoptera & Halictidae & Halictinae \\
\hline D1414H08-VA & Arthropoda & Insecta & Hymenoptera & Halictidae & Halictinae \\
\hline DIAL1413H11-NC & Arthropoda & Insecta & Hymenoptera & Halictidae & Halictinae \\
\hline
\end{tabular}




\begin{tabular}{|c|c|c|c|c|c|}
\hline DIAL1391A10-SC & Arthropoda & Insecta & Hymenoptera & Halictidae & Halictinae \\
\hline D1414G12-VA & Arthropoda & Insecta & Hymenoptera & Halictidae & Halictinae \\
\hline DIAL1413E09-NC & Arthropoda & Insecta & Hymenoptera & Halictidae & Halictinae \\
\hline DIAL1413B01-VA & Arthropoda & Insecta & Hymenoptera & Halictidae & Halictinae \\
\hline DIAL1413G07-NC & Arthropoda & Insecta & Hymenoptera & Halictidae & Halictinae \\
\hline D00607H10-KS & Arthropoda & Insecta & Hymenoptera & Halictidae & Halictinae \\
\hline D0288A11-MD & Arthropoda & Insecta & Hymenoptera & Halictidae & Halictinae \\
\hline D0288A09-WV & Arthropoda & Insecta & Hymenoptera & Halictidae & Halictinae \\
\hline D0287D03-IL & Arthropoda & Insecta & Hymenoptera & Halictidae & Halictinae \\
\hline D0288C02-DC & Arthropoda & Insecta & Hymenoptera & Halictidae & Halictinae \\
\hline D0287E03-IL & Arthropoda & Insecta & Hymenoptera & Halictidae & Halictinae \\
\hline DIAL0294A01-IN & Arthropoda & Insecta & Hymenoptera & Halictidae & Halictinae \\
\hline D0287F07-IL & Arthropoda & Insecta & Hymenoptera & Halictidae & Halictinae \\
\hline D0287E04-IL & Arthropoda & Insecta & Hymenoptera & Halictidae & Halictinae \\
\hline DIAL1846F05-WV & Arthropoda & Insecta & Hymenoptera & Halictidae & Halictinae \\
\hline DIAL0294A02-il & Arthropoda & Insecta & Hymenoptera & Halictidae & Halictinae \\
\hline DIAL0294E08-MD & Arthropoda & Insecta & Hymenoptera & Halictidae & Halictinae \\
\hline DIAL0294F06-VA & Arthropoda & Insecta & Hymenoptera & Halictidae & Halictinae \\
\hline DIAL0294E10-VA & Arthropoda & Insecta & Hymenoptera & Halictidae & Halictinae \\
\hline DIAL0294E09-MD & Arthropoda & Insecta & Hymenoptera & Halictidae & Halictinae \\
\hline DIAL0294F08-MD & Arthropoda & Insecta & Hymenoptera & Halictidae & Halictinae \\
\hline DIAL0292A09-VA & Arthropoda & Insecta & Hymenoptera & Halictidae & Halictinae \\
\hline DIAL1413A01-IL & Arthropoda & Insecta & Hymenoptera & Halictidae & Halictinae \\
\hline DIAL1390G10-NC & Arthropoda & Insecta & Hymenoptera & Halictidae & Halictinae \\
\hline DIAL0294F04-VA & Arthropoda & Insecta & Hymenoptera & Halictidae & Halictinae \\
\hline DIAL1391D06-NC & Arthropoda & Insecta & Hymenoptera & Halictidae & Halictinae \\
\hline B03765B12-MD & Arthropoda & Insecta & Hymenoptera & Halictidae & Halictinae \\
\hline DIAL1847H08-MD & Arthropoda & Insecta & Hymenoptera & Halictidae & Halictinae \\
\hline DIAL1391G09-MI & Arthropoda & Insecta & Hymenoptera & Halictidae & Halictinae \\
\hline DIAL1413H08-NC & Arthropoda & Insecta & Hymenoptera & Halictidae & Halictinae \\
\hline DIAL0293C02-NC & Arthropoda & Insecta & Hymenoptera & Halictidae & Halictinae \\
\hline DIAL0292A08-VA & Arthropoda & Insecta & Hymenoptera & Halictidae & Halictinae \\
\hline DIAL1413A04-IL & Arthropoda & Insecta & Hymenoptera & Halictidae & Halictinae \\
\hline DIAL1413A06-IL & Arthropoda & Insecta & Hymenoptera & Halictidae & Halictinae \\
\hline DIAL1413G05-NC & Arthropoda & Insecta & Hymenoptera & Halictidae & Halictinae \\
\hline DIAL1391E03-SC & Arthropoda & Insecta & Hymenoptera & Halictidae & Halictinae \\
\hline DIAL1413B08-IL & Arthropoda & Insecta & Hymenoptera & Halictidae & Halictinae \\
\hline DIAL1846E12-SC & Arthropoda & Insecta & Hymenoptera & Halictidae & Halictinae \\
\hline DIAL1846B01-TN & Arthropoda & Insecta & Hymenoptera & Halictidae & Halictinae \\
\hline DIAL1846A11-NC & Arthropoda & Insecta & Hymenoptera & Halictidae & Halictinae \\
\hline DIAL1846E05-MS & Arthropoda & Insecta & Hymenoptera & Halictidae & Halictinae \\
\hline DIAL1413H03-NC & Arthropoda & Insecta & Hymenoptera & Halictidae & Halictinae \\
\hline DIAL1413C04-TN & Arthropoda & Insecta & Hymenoptera & Halictidae & Halictinae \\
\hline D0287F02-IL & Arthropoda & Insecta & Hymenoptera & Halictidae & Halictinae \\
\hline DIAL1413F02-TN & Arthropoda & Insecta & Hymenoptera & Halictidae & Halictinae \\
\hline D0288C08-DC & Arthropoda & Insecta & Hymenoptera & Halictidae & Halictinae \\
\hline DIAL1413C08-TN & Arthropoda & Insecta & Hymenoptera & Halictidae & Halictinae \\
\hline DIAL1413C05-TN & Arthropoda & Insecta & Hymenoptera & Halictidae & Halictinae \\
\hline DIAL1413B02-VA & Arthropoda & Insecta & Hymenoptera & Halictidae & Halictinae \\
\hline DIAL1413C06-TN & Arthropoda & Insecta & Hymenoptera & Halictidae & Halictinae \\
\hline DIAL1391G08-MI & Arthropoda & Insecta & Hymenoptera & Halictidae & Halictinae \\
\hline DIAL1413C11-NC & Arthropoda & Insecta & Hymenoptera & Halictidae & Halictinae \\
\hline D0287G01-IL & Arthropoda & Insecta & Hymenoptera & Halictidae & Halictinae \\
\hline DIAL1413B09-IL & Arthropoda & Insecta & Hymenoptera & Halictidae & Halictinae \\
\hline DIAL1413C01-IL & Arthropoda & Insecta & Hymenoptera & Halictidae & Halictinae \\
\hline
\end{tabular}




\begin{tabular}{|c|c|c|c|c|c|}
\hline DIAL1413F12-TN & Arthropoda & Insecta & Hymenoptera & Halictidae & Halictinae \\
\hline DIAL1413D05-SC & Arthropoda & Insecta & Hymenoptera & Halictidae & Halictinae \\
\hline DIAL1413C09-TN & Arthropoda & Insecta & Hymenoptera & Halictidae & Halictinae \\
\hline D0287G04-IL & Arthropoda & Insecta & Hymenoptera & Halictidae & Halictinae \\
\hline D0287G02-IL & Arthropoda & Insecta & Hymenoptera & Halictidae & Halictinae \\
\hline D0287G08-IL & Arthropoda & Insecta & Hymenoptera & Halictidae & Halictinae \\
\hline DIAL2385D05-NC & Arthropoda & Insecta & Hymenoptera & Halictidae & Halictinae \\
\hline DIAL1413C02-TN & Arthropoda & Insecta & Hymenoptera & Halictidae & Halictinae \\
\hline DIAL1413C03-TN & Arthropoda & Insecta & Hymenoptera & Halictidae & Halictinae \\
\hline D0287G07-IL & Arthropoda & Insecta & Hymenoptera & Halictidae & Halictinae \\
\hline D0287G12-IL & Arthropoda & Insecta & Hymenoptera & Halictidae & Halictinae \\
\hline D0287H09-IL & Arthropoda & Insecta & Hymenoptera & Halictidae & Halictinae \\
\hline DIAL1413A03-IL & Arthropoda & Insecta & Hymenoptera & Halictidae & Halictinae \\
\hline D0287C11-IL & Arthropoda & Insecta & Hymenoptera & Halictidae & Halictinae \\
\hline D0287C09-IL & Arthropoda & Insecta & Hymenoptera & Halictidae & Halictinae \\
\hline D0287D02-MI & Arthropoda & Insecta & Hymenoptera & Halictidae & Halictinae \\
\hline D0287D06-IL & Arthropoda & Insecta & Hymenoptera & Halictidae & Halictinae \\
\hline B03765B08-MD & Arthropoda & Insecta & Hymenoptera & Halictidae & Halictinae \\
\hline D-3253C08-NM & Arthropoda & Insecta & Hymenoptera & Halictidae & Halictinae \\
\hline DIAL2374A05-UT & Arthropoda & Insecta & Hymenoptera & Halictidae & Halictinae \\
\hline DIAL2387C12-TX & Arthropoda & Insecta & Hymenoptera & Halictidae & Halictinae \\
\hline DIAL2374C06-UT & Arthropoda & Insecta & Hymenoptera & Halictidae & Halictinae \\
\hline DIAL2374A06-UT & Arthropoda & Insecta & Hymenoptera & Halictidae & Halictinae \\
\hline DIAL1849C11-CO & Arthropoda & Insecta & Hymenoptera & Halictidae & Halictinae \\
\hline DIAL1409B10-TX & Arthropoda & Insecta & Hymenoptera & Halictidae & Halictinae \\
\hline DIAL1409E06-TX & Arthropoda & Insecta & Hymenoptera & Halictidae & Halictinae \\
\hline DIAL2387B03-NM & Arthropoda & Insecta & Hymenoptera & Halictidae & Halictinae \\
\hline DIAL2386H10-UT & Arthropoda & Insecta & Hymenoptera & Halictidae & Halictinae \\
\hline D-3253D03-NM & Arthropoda & Insecta & Hymenoptera & Halictidae & Halictinae \\
\hline DIAL2374F08-UT & Arthropoda & Insecta & Hymenoptera & Halictidae & Halictinae \\
\hline DIAL2388D11-NM & Arthropoda & Insecta & Hymenoptera & Halictidae & Halictinae \\
\hline DIAL2386F01-NV & Arthropoda & Insecta & Hymenoptera & Halictidae & Halictinae \\
\hline D00608D11-NM & Arthropoda & Insecta & Hymenoptera & Halictidae & Halictinae \\
\hline DIAL2374F09-UT & Arthropoda & Insecta & Hymenoptera & Halictidae & Halictinae \\
\hline DIAL2386D09-UT & Arthropoda & Insecta & Hymenoptera & Halictidae & Halictinae \\
\hline DIAL1849E03-AZ & Arthropoda & Insecta & Hymenoptera & Halictidae & Halictinae \\
\hline DIAL2388E03-NM & Arthropoda & Insecta & Hymenoptera & Halictidae & Halictinae \\
\hline DIAL2388B11-UT & Arthropoda & Insecta & Hymenoptera & Halictidae & Halictinae \\
\hline B-3252H08-NV & Arthropoda & Insecta & Hymenoptera & Halictidae & Halictinae \\
\hline DIAL2374A07-UT & Arthropoda & Insecta & Hymenoptera & Halictidae & Halictinae \\
\hline D00608H06-NV & Arthropoda & Insecta & Hymenoptera & Halictidae & Halictinae \\
\hline DIAL1413F04-TN & Arthropoda & Insecta & Hymenoptera & Halictidae & Halictinae \\
\hline DIAL1390H04-SC & Arthropoda & Insecta & Hymenoptera & Halictidae & Halictinae \\
\hline DIAL1847H11-MD & Arthropoda & Insecta & Hymenoptera & Halictidae & Halictinae \\
\hline D1414B02-NC & Arthropoda & Insecta & Hymenoptera & Halictidae & Halictinae \\
\hline DIAL1413G06-NC & Arthropoda & Insecta & Hymenoptera & Halictidae & Halictinae \\
\hline DIAL1845B07-CA & Arthropoda & Insecta & Hymenoptera & Halictidae & Halictinae \\
\hline DIAL1845A06-CA & Arthropoda & Insecta & Hymenoptera & Halictidae & Halictinae \\
\hline DIAL1845B01-CA & Arthropoda & Insecta & Hymenoptera & Halictidae & Halictinae \\
\hline DIAL1845C02-CA & Arthropoda & Insecta & Hymenoptera & Halictidae & Halictinae \\
\hline D00608H09-OR & Arthropoda & Insecta & Hymenoptera & Halictidae & Halictinae \\
\hline D00607H02-CA & Arthropoda & Insecta & Hymenoptera & Halictidae & Halictinae \\
\hline D00607D09-CA & Arthropoda & Insecta & Hymenoptera & Halictidae & Halictinae \\
\hline D00607F10-CA & Arthropoda & Insecta & Hymenoptera & Halictidae & Halictinae \\
\hline D01559D12-BC & Arthropoda & Insecta & Hymenoptera & Halictidae & Halictinae \\
\hline
\end{tabular}




\begin{tabular}{|c|c|c|c|c|c|}
\hline D-3253F09-KY & Arthropoda & Insecta & Hymenoptera & Halictidae & Halictinae \\
\hline D0288F05-SC & Arthropoda & Insecta & Hymenoptera & Halictidae & Halictinae \\
\hline D0287D12-IL & Arthropoda & Insecta & Hymenoptera & Halictidae & Halictinae \\
\hline D0287F08-IL & Arthropoda & Insecta & Hymenoptera & Halictidae & Halictinae \\
\hline DIAL1846G12-SC & Arthropoda & Insecta & Hymenoptera & Halictidae & Halictinae \\
\hline D0287F05-IL & Arthropoda & Insecta & Hymenoptera & Halictidae & Halictinae \\
\hline DIAL1413H01-NC & Arthropoda & Insecta & Hymenoptera & Halictidae & Halictinae \\
\hline D0287H02-IL & Arthropoda & Insecta & Hymenoptera & Halictidae & Halictinae \\
\hline DIAL1391D05-NC & Arthropoda & Insecta & Hymenoptera & Halictidae & Halictinae \\
\hline DIAL1409B08-VA & Arthropoda & Insecta & Hymenoptera & Halictidae & Halictinae \\
\hline DIAL1409B09-VA & Arthropoda & Insecta & Hymenoptera & Halictidae & Halictinae \\
\hline DIAL1413C10-NC & Arthropoda & Insecta & Hymenoptera & Halictidae & Halictinae \\
\hline DIAL1413B07-IL & Arthropoda & Insecta & Hymenoptera & Halictidae & Halictinae \\
\hline DIAL1413D02-TN & Arthropoda & Insecta & Hymenoptera & Halictidae & Halictinae \\
\hline DIAL1391E06-SC & Arthropoda & Insecta & Hymenoptera & Halictidae & Halictinae \\
\hline D0288G07-NC & Arthropoda & Insecta & Hymenoptera & Halictidae & Halictinae \\
\hline DIAL1413G02-TN & Arthropoda & Insecta & Hymenoptera & Halictidae & Halictinae \\
\hline D0287F03-IL & Arthropoda & Insecta & Hymenoptera & Halictidae & Halictinae \\
\hline DIAL1390G08-NC & Arthropoda & Insecta & Hymenoptera & Halictidae & Halictinae \\
\hline DIAL1413G01-TN & Arthropoda & Insecta & Hymenoptera & Halictidae & Halictinae \\
\hline D0287C06-IL & Arthropoda & Insecta & Hymenoptera & Halictidae & Halictinae \\
\hline DIAL1391F08-ON & Arthropoda & Insecta & Hymenoptera & Halictidae & Halictinae \\
\hline DIAL1413H06-NC & Arthropoda & Insecta & Hymenoptera & Halictidae & Halictinae \\
\hline DIAL1391F02-NC & Arthropoda & Insecta & Hymenoptera & Halictidae & Halictinae \\
\hline DIAL1391B02-SC & Arthropoda & Insecta & Hymenoptera & Halictidae & Halictinae \\
\hline DIAL1849G10-MA & Arthropoda & Insecta & Hymenoptera & Halictidae & Halictinae \\
\hline D-3253A10-BC & Arthropoda & Insecta & Hymenoptera & Halictidae & Halictinae \\
\hline D-3253A04-BC & Arthropoda & Insecta & Hymenoptera & Halictidae & Halictinae \\
\hline DIAL2388B03-UT & Arthropoda & Insecta & Hymenoptera & Halictidae & Halictinae \\
\hline DIAL1847G08-OR & Arthropoda & Insecta & Hymenoptera & Halictidae & Halictinae \\
\hline DIAL1409D08-OR & Arthropoda & Insecta & Hymenoptera & Halictidae & Halictinae \\
\hline DIAL1847G09-OR & Arthropoda & Insecta & Hymenoptera & Halictidae & Halictinae \\
\hline DIAL1847G11-OR & Arthropoda & Insecta & Hymenoptera & Halictidae & Halictinae \\
\hline D00608A10-CA & Arthropoda & Insecta & Hymenoptera & Halictidae & Halictinae \\
\hline DIAL2386H12-UT & Arthropoda & Insecta & Hymenoptera & Halictidae & Halictinae \\
\hline DIAL2386H02-WA & Arthropoda & Insecta & Hymenoptera & Halictidae & Halictinae \\
\hline D00607D10-CA & Arthropoda & Insecta & Hymenoptera & Halictidae & Halictinae \\
\hline D00607D07-CA & Arthropoda & Insecta & Hymenoptera & Halictidae & Halictinae \\
\hline DIAL2386D03-UT & Arthropoda & Insecta & Hymenoptera & Halictidae & Halictinae \\
\hline DIAL2386D05-UT & Arthropoda & Insecta & Hymenoptera & Halictidae & Halictinae \\
\hline 06717H07-MA & Arthropoda & Insecta & Hymenoptera & Halictidae & Halictinae \\
\hline DIAL2385D09-WV & Arthropoda & Insecta & Hymenoptera & Halictidae & Halictinae \\
\hline D01560D09-IA & Arthropoda & Insecta & Hymenoptera & Halictidae & Halictinae \\
\hline B03751H11-MA & Arthropoda & Insecta & Hymenoptera & Halictidae & Halictinae \\
\hline D01560E05-IA & Arthropoda & Insecta & Hymenoptera & Halictidae & Halictinae \\
\hline В03750B04-MA & Arthropoda & Insecta & Hymenoptera & Halictidae & Halictinae \\
\hline B04743H04-MA & Arthropoda & Insecta & Hymenoptera & Halictidae & Halictinae \\
\hline 06735G03-MA & Arthropoda & Insecta & Hymenoptera & Halictidae & Halictinae \\
\hline D-3253B07-BC & Arthropoda & Insecta & Hymenoptera & Halictidae & Halictinae \\
\hline D-3253A07-BC & Arthropoda & Insecta & Hymenoptera & Halictidae & Halictinae \\
\hline D-3253B05-BC & Arthropoda & Insecta & Hymenoptera & Halictidae & Halictinae \\
\hline D-3253C02-BC & Arthropoda & Insecta & Hymenoptera & Halictidae & Halictinae \\
\hline DIAL1845C08-CA & Arthropoda & Insecta & Hymenoptera & Halictidae & Halictinae \\
\hline D-3253B09-BC & Arthropoda & Insecta & Hymenoptera & Halictidae & Halictinae \\
\hline DIAL1845C07-CA & Arthropoda & Insecta & Hymenoptera & Halictidae & Halictinae \\
\hline
\end{tabular}




\begin{tabular}{|c|c|c|c|c|c|}
\hline D0295F03-CO & Arthropoda & Insecta & Hymenoptera & Halictidae & Halictinae \\
\hline D-3253A03-BC & Arthropoda & Insecta & Hymenoptera & Halictidae & Halictinae \\
\hline D00608D06-BC & Arthropoda & Insecta & Hymenoptera & Halictidae & Halictinae \\
\hline D00608E08-BC & Arthropoda & Insecta & Hymenoptera & Halictidae & Halictinae \\
\hline D00608D08-BC & Arthropoda & Insecta & Hymenoptera & Halictidae & Halictinae \\
\hline D00607C01-CO & Arthropoda & Insecta & Hymenoptera & Halictidae & Halictinae \\
\hline DIAL1845G08-BC & Arthropoda & Insecta & Hymenoptera & Halictidae & Halictinae \\
\hline DIAL2374E06-WA & Arthropoda & Insecta & Hymenoptera & Halictidae & Halictinae \\
\hline DIAL1409C10-OR & Arthropoda & Insecta & Hymenoptera & Halictidae & Halictinae \\
\hline DIAL1845F09-BC & Arthropoda & Insecta & Hymenoptera & Halictidae & Halictinae \\
\hline DIAL1845D07-CA & Arthropoda & Insecta & Hymenoptera & Halictidae & Halictinae \\
\hline DIAL1845D06-CA & Arthropoda & Insecta & Hymenoptera & Halictidae & Halictinae \\
\hline DIAL1845D11-CA & Arthropoda & Insecta & Hymenoptera & Halictidae & Halictinae \\
\hline DIAL1845E04-CA & Arthropoda & Insecta & Hymenoptera & Halictidae & Halictinae \\
\hline DIAL1845F03-BC & Arthropoda & Insecta & Hymenoptera & Halictidae & Halictinae \\
\hline DIAL1845G05-BC & Arthropoda & Insecta & Hymenoptera & Halictidae & Halictinae \\
\hline 03767G11-BC & Arthropoda & Insecta & Hymenoptera & Halictidae & Halictinae \\
\hline DIAL2387E11-BC & Arthropoda & Insecta & Hymenoptera & Halictidae & Halictinae \\
\hline DIAL2387F03-BC & Arthropoda & Insecta & Hymenoptera & Halictidae & Halictinae \\
\hline DIAL2387A05-ID & Arthropoda & Insecta & Hymenoptera & Halictidae & Halictinae \\
\hline DIAL2386A05-CA & Arthropoda & Insecta & Hymenoptera & Halictidae & Halictinae \\
\hline D00608D10-BC & Arthropoda & Insecta & Hymenoptera & Halictidae & Halictinae \\
\hline DIAL2386G12-ID & Arthropoda & Insecta & Hymenoptera & Halictidae & Halictinae \\
\hline D00608C09-CO & Arthropoda & Insecta & Hymenoptera & Halictidae & Halictinae \\
\hline D00608C02-CO & Arthropoda & Insecta & Hymenoptera & Halictidae & Halictinae \\
\hline D00608F04-BC & Arthropoda & Insecta & Hymenoptera & Halictidae & Halictinae \\
\hline D01562A04-MT & Arthropoda & Insecta & Hymenoptera & Halictidae & Halictinae \\
\hline D0295H06-CO & Arthropoda & Insecta & Hymenoptera & Halictidae & Halictinae \\
\hline B03765E10-BC & Arthropoda & Insecta & Hymenoptera & Halictidae & Halictinae \\
\hline D-3253C01-BC & Arthropoda & Insecta & Hymenoptera & Halictidae & Halictinae \\
\hline DIAL2385E08-MD & Arthropoda & Insecta & Hymenoptera & Halictidae & Halictinae \\
\hline DIAL1848H12-PE & Arthropoda & Insecta & Hymenoptera & Halictidae & Halictinae \\
\hline DIAL1845H05-BC & Arthropoda & Insecta & Hymenoptera & Halictidae & Halictinae \\
\hline D-3253A02-BC & Arthropoda & Insecta & Hymenoptera & Halictidae & Halictinae \\
\hline D00609G06-NS & Arthropoda & Insecta & Hymenoptera & Halictidae & Halictinae \\
\hline DIAL0294F05-MD & Arthropoda & Insecta & Hymenoptera & Halictidae & Halictinae \\
\hline DIAL0294H11-NB & Arthropoda & Insecta & Hymenoptera & Halictidae & Halictinae \\
\hline D00607G08-BC & Arthropoda & Insecta & Hymenoptera & Halictidae & Halictinae \\
\hline DIAL1846A08-NC & Arthropoda & Insecta & Hymenoptera & Halictidae & Halictinae \\
\hline DIAL1845H08-BC & Arthropoda & Insecta & Hymenoptera & Halictidae & Halictinae \\
\hline DIAL1845F08-BC & Arthropoda & Insecta & Hymenoptera & Halictidae & Halictinae \\
\hline DIAL1391B11-NC & Arthropoda & Insecta & Hymenoptera & Halictidae & Halictinae \\
\hline D00609G04-NS & Arthropoda & Insecta & Hymenoptera & Halictidae & Halictinae \\
\hline DIAL1848D05-NS & Arthropoda & Insecta & Hymenoptera & Halictidae & Halictinae \\
\hline DIAL1409H12-ON & Arthropoda & Insecta & Hymenoptera & Halictidae & Halictinae \\
\hline DIAL1845H02-BC & Arthropoda & Insecta & Hymenoptera & Halictidae & Halictinae \\
\hline DIAL1845G01-BC & Arthropoda & Insecta & Hymenoptera & Halictidae & Halictinae \\
\hline DIAL1845G02-BC & Arthropoda & Insecta & Hymenoptera & Halictidae & Halictinae \\
\hline DIAL1845F04-BC & Arthropoda & Insecta & Hymenoptera & Halictidae & Halictinae \\
\hline DIAL1409B05-NC & Arthropoda & Insecta & Hymenoptera & Halictidae & Halictinae \\
\hline DIAL1845F06-BC & Arthropoda & Insecta & Hymenoptera & Halictidae & Halictinae \\
\hline DIAL1845F07-BC & Arthropoda & Insecta & Hymenoptera & Halictidae & Halictinae \\
\hline DIAL1413E07-NC & Arthropoda & Insecta & Hymenoptera & Halictidae & Halictinae \\
\hline DIAL1848F09-PE & Arthropoda & Insecta & Hymenoptera & Halictidae & Halictinae \\
\hline DIAL1848A05-NB & Arthropoda & Insecta & Hymenoptera & Halictidae & Halictinae \\
\hline
\end{tabular}




\begin{tabular}{|c|c|c|c|c|c|}
\hline DIAL1848A06-NB & Arthropoda & Insecta & Hymenoptera & Halictidae & Halictinae \\
\hline DIAL1848G06-PE & Arthropoda & Insecta & Hymenoptera & Halictidae & Halictinae \\
\hline DIAL1849H06-ME & Arthropoda & Insecta & Hymenoptera & Halictidae & Halictinae \\
\hline DIAL2388D05-CO & Arthropoda & Insecta & Hymenoptera & Halictidae & Halictinae \\
\hline D01562D02-ON & Arthropoda & Insecta & Hymenoptera & Halictidae & Halictinae \\
\hline D00607A05-AB & Arthropoda & Insecta & Hymenoptera & Halictidae & Halictinae \\
\hline D00607C10-AB & Arthropoda & Insecta & Hymenoptera & Halictidae & Halictinae \\
\hline D00607C09-AB & Arthropoda & Insecta & Hymenoptera & Halictidae & Halictinae \\
\hline DIAL1391A07-NC & Arthropoda & Insecta & Hymenoptera & Halictidae & Halictinae \\
\hline D0295B10-ON & Arthropoda & Insecta & Hymenoptera & Halictidae & Halictinae \\
\hline D0295C01-ON & Arthropoda & Insecta & Hymenoptera & Halictidae & Halictinae \\
\hline DIAL1391C02-NC & Arthropoda & Insecta & Hymenoptera & Halictidae & Halictinae \\
\hline DIAL1391B03-OH & Arthropoda & Insecta & Hymenoptera & Halictidae & Halictinae \\
\hline DIAL1391C01-NC & Arthropoda & Insecta & Hymenoptera & Halictidae & Halictinae \\
\hline DIAL2388D04-CO & Arthropoda & Insecta & Hymenoptera & Halictidae & Halictinae \\
\hline 06745E10-ON & Arthropoda & Insecta & Hymenoptera & Halictidae & Halictinae \\
\hline DIAL1413E02-FL & Arthropoda & Insecta & Hymenoptera & Halictidae & Halictinae \\
\hline DIAL1413E04-FL & Arthropoda & Insecta & Hymenoptera & Halictidae & Halictinae \\
\hline DIAL1413E06-FL & Arthropoda & Insecta & Hymenoptera & Halictidae & Halictinae \\
\hline 04485F04-MI & Arthropoda & Insecta & Hymenoptera & Halictidae & Halictinae \\
\hline 04485F08-MI & Arthropoda & Insecta & Hymenoptera & Halictidae & Halictinae \\
\hline 04485E08-MI & Arthropoda & Insecta & Hymenoptera & Halictidae & Halictinae \\
\hline DIAL1848C01-NB & Arthropoda & Insecta & Hymenoptera & Halictidae & Halictinae \\
\hline 04485F09-MI & Arthropoda & Insecta & Hymenoptera & Halictidae & Halictinae \\
\hline 04485F11-MI & Arthropoda & Insecta & Hymenoptera & Halictidae & Halictinae \\
\hline 04485H06-MI & Arthropoda & Insecta & Hymenoptera & Halictidae & Halictinae \\
\hline DIAL1846A04-ON & Arthropoda & Insecta & Hymenoptera & Halictidae & Halictinae \\
\hline 04485H04-MI & Arthropoda & Insecta & Hymenoptera & Halictidae & Halictinae \\
\hline DIAL1848B11-NB & Arthropoda & Insecta & Hymenoptera & Halictidae & Halictinae \\
\hline D00609G02-NS & Arthropoda & Insecta & Hymenoptera & Halictidae & Halictinae \\
\hline D00609H09-NS & Arthropoda & Insecta & Hymenoptera & Halictidae & Halictinae \\
\hline DIAL1845E03-TX & Arthropoda & Insecta & Hymenoptera & Halictidae & Halictinae \\
\hline DIAL1846D07-MS & Arthropoda & Insecta & Hymenoptera & Halictidae & Halictinae \\
\hline DIAL1846F11-MS & Arthropoda & Insecta & Hymenoptera & Halictidae & Halictinae \\
\hline DIAL1391A11-SC & Arthropoda & Insecta & Hymenoptera & Halictidae & Halictinae \\
\hline DIAL2385E07-WV & Arthropoda & Insecta & Hymenoptera & Halictidae & Halictinae \\
\hline BEE0289B05-MN & Arthropoda & Insecta & Hymenoptera & Halictidae & Halictinae \\
\hline DIAL0286H09-MB & Arthropoda & Insecta & Hymenoptera & Halictidae & Halictinae \\
\hline DIAL0286H04-MB & Arthropoda & Insecta & Hymenoptera & Halictidae & Halictinae \\
\hline D0295B08-ON & Arthropoda & Insecta & Hymenoptera & Halictidae & Halictinae \\
\hline DIAL1390C12-ON & Arthropoda & Insecta & Hymenoptera & Halictidae & Halictinae \\
\hline DIAL0292H06-WV & Arthropoda & Insecta & Hymenoptera & Halictidae & Halictinae \\
\hline DIAL1839G07-MN & Arthropoda & Insecta & Hymenoptera & Halictidae & Halictinae \\
\hline 06732C04-WV & Arthropoda & Insecta & Hymenoptera & Halictidae & Halictinae \\
\hline D00607B07-KS & Arthropoda & Insecta & Hymenoptera & Halictidae & Halictinae \\
\hline DIAL1409B01-NC & Arthropoda & Insecta & Hymenoptera & Halictidae & Halictinae \\
\hline DIAL1390C07-ON & Arthropoda & Insecta & Hymenoptera & Halictidae & Halictinae \\
\hline DIAL1390C04-ON & Arthropoda & Insecta & Hymenoptera & Halictidae & Halictinae \\
\hline DIAL0286H06-MB & Arthropoda & Insecta & Hymenoptera & Halictidae & Halictinae \\
\hline DIAL1846B05-MD & Arthropoda & Insecta & Hymenoptera & Halictidae & Halictinae \\
\hline DIAL1847H12-MD & Arthropoda & Insecta & Hymenoptera & Halictidae & Halictinae \\
\hline DIAL2388E04-AL & Arthropoda & Insecta & Hymenoptera & Halictidae & Halictinae \\
\hline DIAL1846H04-MS & Arthropoda & Insecta & Hymenoptera & Halictidae & Halictinae \\
\hline DIAL1846E02-MS & Arthropoda & Insecta & Hymenoptera & Halictidae & Halictinae \\
\hline DIAL1846E09-MS & Arthropoda & Insecta & Hymenoptera & Halictidae & Halictinae \\
\hline
\end{tabular}




\begin{tabular}{|c|c|c|c|c|c|}
\hline DIAL1846E06-MS & Arthropoda & Insecta & Hymenoptera & Halictidae & Halictinae \\
\hline DIAL1846D05-MS & Arthropoda & Insecta & Hymenoptera & Halictidae & Halictinae \\
\hline DIAL2387F06-TX & Arthropoda & Insecta & Hymenoptera & Halictidae & Halictinae \\
\hline D0288F08-NC & Arthropoda & Insecta & Hymenoptera & Halictidae & Halictinae \\
\hline BEE0286B09-GA & Arthropoda & Insecta & Hymenoptera & Halictidae & Halictinae \\
\hline D0288F10-SC & Arthropoda & Insecta & Hymenoptera & Halictidae & Halictinae \\
\hline D01559E03-BC & Arthropoda & Insecta & Hymenoptera & Halictidae & Halictinae \\
\hline D01559F06-BC & Arthropoda & Insecta & Hymenoptera & Halictidae & Halictinae \\
\hline D00608B10-BC & Arthropoda & Insecta & Hymenoptera & Halictidae & Halictinae \\
\hline D00608D04-BC & Arthropoda & Insecta & Hymenoptera & Halictidae & Halictinae \\
\hline D00609H07-BC & Arthropoda & Insecta & Hymenoptera & Halictidae & Halictinae \\
\hline D00609H03-BC & Arthropoda & Insecta & Hymenoptera & Halictidae & Halictinae \\
\hline D00609H05-BC & Arthropoda & Insecta & Hymenoptera & Halictidae & Halictinae \\
\hline DIAL2388B05-WA & Arthropoda & Insecta & Hymenoptera & Halictidae & Halictinae \\
\hline D01561E05-CA & Arthropoda & Insecta & Hymenoptera & Halictidae & Halictinae \\
\hline CCDB - 01565 H3 & Arthropoda & Insecta & Hymenoptera & Halictidae & Halictinae \\
\hline CCDB - 01565 H8 & Arthropoda & Insecta & Hymenoptera & Halictidae & Halictinae \\
\hline D1414C03-VA & Arthropoda & Insecta & Hymenoptera & Halictidae & Halictinae \\
\hline DIAL1846D08-VA & Arthropoda & Insecta & Hymenoptera & Halictidae & Halictinae \\
\hline DIAL1848C05-MD & Arthropoda & Insecta & Hymenoptera & Halictidae & Halictinae \\
\hline D1414D08-VA & Arthropoda & Insecta & Hymenoptera & Halictidae & Halictinae \\
\hline D1414C06-VA & Arthropoda & Insecta & Hymenoptera & Halictidae & Halictinae \\
\hline D1414C05-VA & Arthropoda & Insecta & Hymenoptera & Halictidae & Halictinae \\
\hline D1414C04-VA & Arthropoda & Insecta & Hymenoptera & Halictidae & Halictinae \\
\hline D01561E08-NY & Arthropoda & Insecta & Hymenoptera & Halictidae & Halictinae \\
\hline DIAL1849F05-DC & Arthropoda & Insecta & Hymenoptera & Halictidae & Halictinae \\
\hline DIAL1409B02-NC & Arthropoda & Insecta & Hymenoptera & Halictidae & Halictinae \\
\hline DIAL1409A01-ON & Arthropoda & Insecta & Hymenoptera & Halictidae & Halictinae \\
\hline D-3253E12-FL & Arthropoda & Insecta & Hymenoptera & Halictidae & Halictinae \\
\hline D-3253E02-FL & Arthropoda & Insecta & Hymenoptera & Halictidae & Halictinae \\
\hline D01559A03-FL & Arthropoda & Insecta & Hymenoptera & Halictidae & Halictinae \\
\hline D01561H08-FL & Arthropoda & Insecta & Hymenoptera & Halictidae & Halictinae \\
\hline D01559D09-FL & Arthropoda & Insecta & Hymenoptera & Halictidae & Halictinae \\
\hline D01562D03-FL & Arthropoda & Insecta & Hymenoptera & Halictidae & Halictinae \\
\hline D-3253B01-BC & Arthropoda & Insecta & Hymenoptera & Halictidae & Halictinae \\
\hline D-3253A05-BC & Arthropoda & Insecta & Hymenoptera & Halictidae & Halictinae \\
\hline D-3253B10-BC & Arthropoda & Insecta & Hymenoptera & Halictidae & Halictinae \\
\hline D-3253B11-BC & Arthropoda & Insecta & Hymenoptera & Halictidae & Halictinae \\
\hline D-3253B08-BC & Arthropoda & Insecta & Hymenoptera & Halictidae & Halictinae \\
\hline D-3262G02-UT & Arthropoda & Insecta & Hymenoptera & Halictidae & Halictinae \\
\hline D-3253A08-BC & Arthropoda & Insecta & Hymenoptera & Halictidae & Halictinae \\
\hline DIAL1845C12-CA & Arthropoda & Insecta & Hymenoptera & Halictidae & Halictinae \\
\hline DIAL1845D10-CA & Arthropoda & Insecta & Hymenoptera & Halictidae & Halictinae \\
\hline DIAL2387E12-BC & Arthropoda & Insecta & Hymenoptera & Halictidae & Halictinae \\
\hline D00607F07-CA & Arthropoda & Insecta & Hymenoptera & Halictidae & Halictinae \\
\hline BEE1850F04-OR & Arthropoda & Insecta & Hymenoptera & Halictidae & Halictinae \\
\hline D00607G10-BC & Arthropoda & Insecta & Hymenoptera & Halictidae & Halictinae \\
\hline DIAL2386C03-CA & Arthropoda & Insecta & Hymenoptera & Halictidae & Halictinae \\
\hline DIAL2374A11-UT & Arthropoda & Insecta & Hymenoptera & Halictidae & Halictinae \\
\hline DIAL2386D08-UT & Arthropoda & Insecta & Hymenoptera & Halictidae & Halictinae \\
\hline DIAL2386D06-UT & Arthropoda & Insecta & Hymenoptera & Halictidae & Halictinae \\
\hline DIAL2386C04-CA & Arthropoda & Insecta & Hymenoptera & Halictidae & Halictinae \\
\hline D01559E12-BC & Arthropoda & Insecta & Hymenoptera & Halictidae & Halictinae \\
\hline DIAL2374D09-UT & Arthropoda & Insecta & Hymenoptera & Halictidae & Halictinae \\
\hline DIAL2374E11-WA & Arthropoda & Insecta & Hymenoptera & Halictidae & Halictinae \\
\hline
\end{tabular}




\begin{tabular}{|c|c|c|c|c|c|}
\hline 06732F01-WY & Arthropoda & Insecta & Hymenoptera & Halictidae & Halictinae \\
\hline DIAL2385C01-MD & Arthropoda & Insecta & Hymenoptera & Halictidae & Halictinae \\
\hline DIAL1849G01-ME & Arthropoda & Insecta & Hymenoptera & Halictidae & Halictinae \\
\hline 04485A08-MD & Arthropoda & Insecta & Hymenoptera & Halictidae & Halictinae \\
\hline D00609G08-NS & Arthropoda & Insecta & Hymenoptera & Halictidae & Halictinae \\
\hline DIAL1849F12-MA & Arthropoda & Insecta & Hymenoptera & Halictidae & Halictinae \\
\hline DIAL1848F03-ON & Arthropoda & Insecta & Hymenoptera & Halictidae & Halictinae \\
\hline D01560B11-VT & Arthropoda & Insecta & Hymenoptera & Halictidae & Halictinae \\
\hline DIAL1848F05-PE & Arthropoda & Insecta & Hymenoptera & Halictidae & Halictinae \\
\hline D0288B10-NC & Arthropoda & Insecta & Hymenoptera & Halictidae & Halictinae \\
\hline D1414C10-VA & Arthropoda & Insecta & Hymenoptera & Halictidae & Halictinae \\
\hline D1414C09-VA & Arthropoda & Insecta & Hymenoptera & Halictidae & Halictinae \\
\hline D-3253F11-FL & Arthropoda & Insecta & Hymenoptera & Halictidae & Halictinae \\
\hline D1414F02-NC & Arthropoda & Insecta & Hymenoptera & Halictidae & Halictinae \\
\hline D1414E12-NC & Arthropoda & Insecta & Hymenoptera & Halictidae & Halictinae \\
\hline D1414E11-NC & Arthropoda & Insecta & Hymenoptera & Halictidae & Halictinae \\
\hline D0288C03-SC & Arthropoda & Insecta & Hymenoptera & Halictidae & Halictinae \\
\hline D1414C08-VA & Arthropoda & Insecta & Hymenoptera & Halictidae & Halictinae \\
\hline D0288B11-NC & Arthropoda & Insecta & Hymenoptera & Halictidae & Halictinae \\
\hline DIAL1846D11-MS & Arthropoda & Insecta & Hymenoptera & Halictidae & Halictinae \\
\hline DIAL1849F03-NC & Arthropoda & Insecta & Hymenoptera & Halictidae & Halictinae \\
\hline D1414D09-VA & Arthropoda & Insecta & Hymenoptera & Halictidae & Halictinae \\
\hline B03765B03-VA & Arthropoda & Insecta & Hymenoptera & Halictidae & Halictinae \\
\hline D0288C11-SC & Arthropoda & Insecta & Hymenoptera & Halictidae & Halictinae \\
\hline D1414F05-NC & Arthropoda & Insecta & Hymenoptera & Halictidae & Halictinae \\
\hline D1414C07-VA & Arthropoda & Insecta & Hymenoptera & Halictidae & Halictinae \\
\hline D0295D12-MD & Arthropoda & Insecta & Hymenoptera & Halictidae & Halictinae \\
\hline D1414B06-VA & Arthropoda & Insecta & Hymenoptera & Halictidae & Halictinae \\
\hline DIAL0293B05-MD & Arthropoda & Insecta & Hymenoptera & Halictidae & Halictinae \\
\hline D1414B07-VA & Arthropoda & Insecta & Hymenoptera & Halictidae & Halictinae \\
\hline D00607D12-MA & Arthropoda & Insecta & Hymenoptera & Halictidae & Halictinae \\
\hline D00608F12-IN & Arthropoda & Insecta & Hymenoptera & Halictidae & Halictinae \\
\hline D1414B08-VA & Arthropoda & Insecta & Hymenoptera & Halictidae & Halictinae \\
\hline D1414E01-VA & Arthropoda & Insecta & Hymenoptera & Halictidae & Halictinae \\
\hline D1414B09-VA & Arthropoda & Insecta & Hymenoptera & Halictidae & Halictinae \\
\hline DIAL1849G02-MA & Arthropoda & Insecta & Hymenoptera & Halictidae & Halictinae \\
\hline DIAL1413E12-TN & Arthropoda & Insecta & Hymenoptera & Halictidae & Halictinae \\
\hline DIAL0294F11-VA & Arthropoda & Insecta & Hymenoptera & Halictidae & Halictinae \\
\hline DIAL1847B03-WV & Arthropoda & Insecta & Hymenoptera & Halictidae & Halictinae \\
\hline DIAL1848C11-NY & Arthropoda & Insecta & Hymenoptera & Halictidae & Halictinae \\
\hline D0295E06-MD & Arthropoda & Insecta & Hymenoptera & Halictidae & Halictinae \\
\hline DIAL1846B08-WV & Arthropoda & Insecta & Hymenoptera & Halictidae & Halictinae \\
\hline BEE0286D09-VA & Arthropoda & Insecta & Hymenoptera & Halictidae & Halictinae \\
\hline DIAL1391A04-NC & Arthropoda & Insecta & Hymenoptera & Halictidae & Halictinae \\
\hline DIAL1390G11-NC & Arthropoda & Insecta & Hymenoptera & Halictidae & Halictinae \\
\hline DIAL1849H08-VT & Arthropoda & Insecta & Hymenoptera & Halictidae & Halictinae \\
\hline DIAL2385B03-MB & Arthropoda & Insecta & Hymenoptera & Halictidae & Halictinae \\
\hline DIAL2387F09-NM & Arthropoda & Insecta & Hymenoptera & Halictidae & Halictinae \\
\hline DIAL2387H11-NM & Arthropoda & Insecta & Hymenoptera & Halictidae & Halictinae \\
\hline D0287A11-CO & Arthropoda & Insecta & Hymenoptera & Halictidae & Halictinae \\
\hline DIAL2385A10-MB & Arthropoda & Insecta & Hymenoptera & Halictidae & Halictinae \\
\hline D0295G05-CO & Arthropoda & Insecta & Hymenoptera & Halictidae & Halictinae \\
\hline DIAL2385A04-CO & Arthropoda & Insecta & Hymenoptera & Halictidae & Halictinae \\
\hline D0287B11-CO & Arthropoda & Insecta & Hymenoptera & Halictidae & Halictinae \\
\hline D01561B01-MT & Arthropoda & Insecta & Hymenoptera & Halictidae & Halictinae \\
\hline
\end{tabular}




\begin{tabular}{|c|c|c|c|c|c|}
\hline 06732F09-WY & Arthropoda & Insecta & Hymenoptera & Halictidae & Halictinae \\
\hline D0295G10-CO & Arthropoda & Insecta & Hymenoptera & Halictidae & Halictinae \\
\hline DIAL1390C09-ON & Arthropoda & Insecta & Hymenoptera & Halictidae & Halictinae \\
\hline DIAL1849G09-MA & Arthropoda & Insecta & Hymenoptera & Halictidae & Halictinae \\
\hline DIAL1409A06-NC & Arthropoda & Insecta & Hymenoptera & Halictidae & Halictinae \\
\hline DIAL1409A11-NC & Arthropoda & Insecta & Hymenoptera & Halictidae & Halictinae \\
\hline DIAL1845G06-BC & Arthropoda & Insecta & Hymenoptera & Halictidae & Halictinae \\
\hline DIAL1845H10-BC & Arthropoda & Insecta & Hymenoptera & Halictidae & Halictinae \\
\hline DIAL1845G04-BC & Arthropoda & Insecta & Hymenoptera & Halictidae & Halictinae \\
\hline DIAL1845F05-BC & Arthropoda & Insecta & Hymenoptera & Halictidae & Halictinae \\
\hline DIAL1845H03-BC & Arthropoda & Insecta & Hymenoptera & Halictidae & Halictinae \\
\hline DIAL1845H11-BC & Arthropoda & Insecta & Hymenoptera & Halictidae & Halictinae \\
\hline D00608G06-BC & Arthropoda & Insecta & Hymenoptera & Halictidae & Halictinae \\
\hline DIAL1845H12-BC & Arthropoda & Insecta & Hymenoptera & Halictidae & Halictinae \\
\hline DIAL1845G03-BC & Arthropoda & Insecta & Hymenoptera & Halictidae & Halictinae \\
\hline DIAL1845G07-BC & Arthropoda & Insecta & Hymenoptera & Halictidae & Halictinae \\
\hline DIAL1845G12-BC & Arthropoda & Insecta & Hymenoptera & Halictidae & Halictinae \\
\hline DIAL1845F12-BC & Arthropoda & Insecta & Hymenoptera & Halictidae & Halictinae \\
\hline DIAL1845F10-BC & Arthropoda & Insecta & Hymenoptera & Halictidae & Halictinae \\
\hline DIAL1845F02-BC & Arthropoda & Insecta & Hymenoptera & Halictidae & Halictinae \\
\hline DIAL1845G09-BC & Arthropoda & Insecta & Hymenoptera & Halictidae & Halictinae \\
\hline DIAL1845G10-BC & Arthropoda & Insecta & Hymenoptera & Halictidae & Halictinae \\
\hline DIAL1845G11-BC & Arthropoda & Insecta & Hymenoptera & Halictidae & Halictinae \\
\hline DIAL2387H05-CO & Arthropoda & Insecta & Hymenoptera & Halictidae & Halictinae \\
\hline B03765E05-BC & Arthropoda & Insecta & Hymenoptera & Halictidae & Halictinae \\
\hline CCDB-00611 H09-( & ( Arthropoda & Insecta & Hymenoptera & Halictidae & Halictinae \\
\hline D01559D04-AB & Arthropoda & Insecta & Hymenoptera & Halictidae & Halictinae \\
\hline DIAL2385A08-SK & Arthropoda & Insecta & Hymenoptera & Halictidae & Halictinae \\
\hline D01559G01-AB & Arthropoda & Insecta & Hymenoptera & Halictidae & Halictinae \\
\hline DIAL2385B02-SK & Arthropoda & Insecta & Hymenoptera & Halictidae & Halictinae \\
\hline DIAL0293B11-WV & Arthropoda & Insecta & Hymenoptera & Halictidae & Halictinae \\
\hline DIAL1413A02-IL & Arthropoda & Insecta & Hymenoptera & Halictidae & Halictinae \\
\hline DIAL1409A08-NC & Arthropoda & Insecta & Hymenoptera & Halictidae & Halictinae \\
\hline DIAL1390E09-ON & Arthropoda & Insecta & Hymenoptera & Halictidae & Halictinae \\
\hline DIAL1413B05-IL & Arthropoda & Insecta & Hymenoptera & Halictidae & Halictinae \\
\hline D00609C03-WI & Arthropoda & Insecta & Hymenoptera & Halictidae & Halictinae \\
\hline D01560C09-IA & Arthropoda & Insecta & Hymenoptera & Halictidae & Halictinae \\
\hline CCDB-01557 B01 & Arthropoda & Insecta & Hymenoptera & Halictidae & Halictinae \\
\hline DIAL1390D01-NT & Arthropoda & Insecta & Hymenoptera & Halictidae & Halictinae \\
\hline DIAL1390C10-NT & Arthropoda & Insecta & Hymenoptera & Halictidae & Halictinae \\
\hline DIAL2384B09-YT & Arthropoda & Insecta & Hymenoptera & Halictidae & Halictinae \\
\hline D0295F10-CO & Arthropoda & Insecta & Hymenoptera & Halictidae & Halictinae \\
\hline DIAL0286G05-MB & Arthropoda & Insecta & Hymenoptera & Halictidae & Halictinae \\
\hline DIAL0286G06-MB & Arthropoda & Insecta & Hymenoptera & Halictidae & Halictinae \\
\hline DIAL0293B09-IN & Arthropoda & Insecta & Hymenoptera & Halictidae & Halictinae \\
\hline D00609H10-NS & Arthropoda & Insecta & Hymenoptera & Halictidae & Halictinae \\
\hline DIAL0286G07-MB & Arthropoda & Insecta & Hymenoptera & Halictidae & Halictinae \\
\hline DIAL0286G03-MB & Arthropoda & Insecta & Hymenoptera & Halictidae & Halictinae \\
\hline D01561B02-MT & Arthropoda & Insecta & Hymenoptera & Halictidae & Halictinae \\
\hline D0287A03-CO & Arthropoda & Insecta & Hymenoptera & Halictidae & Halictinae \\
\hline DIAL0286G10-MB & Arthropoda & Insecta & Hymenoptera & Halictidae & Halictinae \\
\hline DIAL0286G09-MB & Arthropoda & Insecta & Hymenoptera & Halictidae & Halictinae \\
\hline DIAL0286G04-MB & Arthropoda & Insecta & Hymenoptera & Halictidae & Halictinae \\
\hline DIAL0286H03-MB & Arthropoda & Insecta & Hymenoptera & Halictidae & Halictinae \\
\hline DIAL0293C07-MN & Arthropoda & Insecta & Hymenoptera & Halictidae & Halictinae \\
\hline
\end{tabular}




\begin{tabular}{|c|c|c|c|c|c|}
\hline DIAL0293C08-MN & Arthropoda & Insecta & Hymenoptera & Halictidae & Halictinae \\
\hline DIAL0293C06-MN & Arthropoda & Insecta & Hymenoptera & Halictidae & Halictinae \\
\hline DIAL0294C01-IN & Arthropoda & Insecta & Hymenoptera & Halictidae & Halictinae \\
\hline DIAL0293C05-MN & Arthropoda & Insecta & Hymenoptera & Halictidae & Halictinae \\
\hline DIAL0286F07-MB & Arthropoda & Insecta & Hymenoptera & Halictidae & Halictinae \\
\hline DIAL0286F08-MB & Arthropoda & Insecta & Hymenoptera & Halictidae & Halictinae \\
\hline DIAL0286F06-MB & Arthropoda & Insecta & Hymenoptera & Halictidae & Halictinae \\
\hline DIAL2385B11-MI & Arthropoda & Insecta & Hymenoptera & Halictidae & Halictinae \\
\hline D00609B09-WI & Arthropoda & Insecta & Hymenoptera & Halictidae & Halictinae \\
\hline D1414D12-VA & Arthropoda & Insecta & Hymenoptera & Halictidae & Halictinae \\
\hline DIAL1391D02-NC & Arthropoda & Insecta & Hymenoptera & Halictidae & Halictinae \\
\hline DIAL0294F12-NY & Arthropoda & Insecta & Hymenoptera & Halictidae & Halictinae \\
\hline DIAL1390B05-ON & Arthropoda & Insecta & Hymenoptera & Halictidae & Halictinae \\
\hline 04485G12-MI & Arthropoda & Insecta & Hymenoptera & Halictidae & Halictinae \\
\hline DIAL1846A06-ON & Arthropoda & Insecta & Hymenoptera & Halictidae & Halictinae \\
\hline DIAL1847H06-MD & Arthropoda & Insecta & Hymenoptera & Halictidae & Halictinae \\
\hline DIAL1391A01-VA & Arthropoda & Insecta & Hymenoptera & Halictidae & Halictinae \\
\hline DIAL1413C07-TN & Arthropoda & Insecta & Hymenoptera & Halictidae & Halictinae \\
\hline 04485F01-MI & Arthropoda & Insecta & Hymenoptera & Halictidae & Halictinae \\
\hline DIAL1409A05-NC & Arthropoda & Insecta & Hymenoptera & Halictidae & Halictinae \\
\hline DIAL1413A08-VA & Arthropoda & Insecta & Hymenoptera & Halictidae & Halictinae \\
\hline DIAL1390H05-NC & Arthropoda & Insecta & Hymenoptera & Halictidae & Halictinae \\
\hline DIAL1413F05-TN & Arthropoda & Insecta & Hymenoptera & Halictidae & Halictinae \\
\hline DIAL1413D03-NC & Arthropoda & Insecta & Hymenoptera & Halictidae & Halictinae \\
\hline DIAL1413B04-ON & Arthropoda & Insecta & Hymenoptera & Halictidae & Halictinae \\
\hline BEE1850F06-MN & Arthropoda & Insecta & Hymenoptera & Halictidae & Halictinae \\
\hline DIAL1391C12-NC & Arthropoda & Insecta & Hymenoptera & Halictidae & Halictinae \\
\hline D00607G06-BC & Arthropoda & Insecta & Hymenoptera & Halictidae & Halictinae \\
\hline DIAL0294H10-NB & Arthropoda & Insecta & Hymenoptera & Halictidae & Halictinae \\
\hline DIAL1848A02-NB & Arthropoda & Insecta & Hymenoptera & Halictidae & Halictinae \\
\hline DIAL1848G02-NB & Arthropoda & Insecta & Hymenoptera & Halictidae & Halictinae \\
\hline DIAL1848A01-NB & Arthropoda & Insecta & Hymenoptera & Halictidae & Halictinae \\
\hline DIAL2388E07-NB & Arthropoda & Insecta & Hymenoptera & Halictidae & Halictinae \\
\hline DIAL1391F05-NT & Arthropoda & Insecta & Hymenoptera & Halictidae & Halictinae \\
\hline CCDB-00611 F10-C & (Arthropoda & Insecta & Hymenoptera & Halictidae & Halictinae \\
\hline D01560D11-DC & Arthropoda & Insecta & Hymenoptera & Halictidae & Halictinae \\
\hline DIAL2385C11-DC & Arthropoda & Insecta & Hymenoptera & Halictidae & Halictinae \\
\hline DIAL1413D08-NC & Arthropoda & Insecta & Hymenoptera & Halictidae & Halictinae \\
\hline DIAL0293D07-WV & Arthropoda & Insecta & Hymenoptera & Halictidae & Halictinae \\
\hline DIAL0293D03-MD & Arthropoda & Insecta & Hymenoptera & Halictidae & Halictinae \\
\hline DIAL1849H02-DC & Arthropoda & Insecta & Hymenoptera & Halictidae & Halictinae \\
\hline DIAL1847H09-MD & Arthropoda & Insecta & Hymenoptera & Halictidae & Halictinae \\
\hline DIAL1848E09-MD & Arthropoda & Insecta & Hymenoptera & Halictidae & Halictinae \\
\hline D01560B02-DC & Arthropoda & Insecta & Hymenoptera & Halictidae & Halictinae \\
\hline D01560B05-DC & Arthropoda & Insecta & Hymenoptera & Halictidae & Halictinae \\
\hline DIAL1849F08-MD & Arthropoda & Insecta & Hymenoptera & Halictidae & Halictinae \\
\hline D00609E03-MT & Arthropoda & Insecta & Hymenoptera & Halictidae & Halictinae \\
\hline D0295H01-CO & Arthropoda & Insecta & Hymenoptera & Halictidae & Halictinae \\
\hline D01559F01-AB & Arthropoda & Insecta & Hymenoptera & Halictidae & Halictinae \\
\hline DIAL2386G09-WA & Arthropoda & Insecta & Hymenoptera & Halictidae & Halictinae \\
\hline D0295G03-CO & Arthropoda & Insecta & Hymenoptera & Halictidae & Halictinae \\
\hline DIAL2384C05-AB & Arthropoda & Insecta & Hymenoptera & Halictidae & Halictinae \\
\hline DIAL1848F01-CO & Arthropoda & Insecta & Hymenoptera & Halictidae & Halictinae \\
\hline D01559C10-AB & Arthropoda & Insecta & Hymenoptera & Halictidae & Halictinae \\
\hline DIAL2386H04-WA & Arthropoda & Insecta & Hymenoptera & Halictidae & Halictinae \\
\hline
\end{tabular}




\begin{tabular}{|c|c|c|c|c|c|}
\hline D0295G01-CO & Arthropoda & Insecta & Hymenoptera & Halictidae & Halictinae \\
\hline D01559H06-BC & Arthropoda & Insecta & Hymenoptera & Halictidae & Halictinae \\
\hline D0287C02-CO & Arthropoda & Insecta & Hymenoptera & Halictidae & Halictinae \\
\hline D01559G06-BC & Arthropoda & Insecta & Hymenoptera & Halictidae & Halictinae \\
\hline D01559H09-BC & Arthropoda & Insecta & Hymenoptera & Halictidae & Halictinae \\
\hline D01559A11-MT & Arthropoda & Insecta & Hymenoptera & Halictidae & Halictinae \\
\hline DIAL2384C06-AB & Arthropoda & Insecta & Hymenoptera & Halictidae & Halictinae \\
\hline DIAL2388F09-UT & Arthropoda & Insecta & Hymenoptera & Halictidae & Halictinae \\
\hline BEE0289H09-MB & Arthropoda & Insecta & Hymenoptera & Halictidae & Halictinae \\
\hline BEE0289H07-MB & Arthropoda & Insecta & Hymenoptera & Halictidae & Halictinae \\
\hline DIAL1409H03-MB & Arthropoda & Insecta & Hymenoptera & Halictidae & Halictinae \\
\hline BEE0289H06-MB & Arthropoda & Insecta & Hymenoptera & Halictidae & Halictinae \\
\hline BEE0289H10-MB & Arthropoda & Insecta & Hymenoptera & Halictidae & Halictinae \\
\hline DIAL2388G12-AZ & Arthropoda & Insecta & Hymenoptera & Halictidae & Halictinae \\
\hline DIAL2374G02-UT & Arthropoda & Insecta & Hymenoptera & Halictidae & Halictinae \\
\hline DIAL2374B05-UT & Arthropoda & Insecta & Hymenoptera & Halictidae & Halictinae \\
\hline DIAL1845B08-CA & Arthropoda & Insecta & Hymenoptera & Halictidae & Halictinae \\
\hline D00608C07-CA & Arthropoda & Insecta & Hymenoptera & Halictidae & Halictinae \\
\hline DIAL1845B02-CA & Arthropoda & Insecta & Hymenoptera & Halictidae & Halictinae \\
\hline DIAL2387A07-ID & Arthropoda & Insecta & Hymenoptera & Halictidae & Halictinae \\
\hline B03765G03-BC & Arthropoda & Insecta & Hymenoptera & Halictidae & Halictinae \\
\hline DIAL1848D04-FL & Arthropoda & Insecta & Hymenoptera & Halictidae & Halictinae \\
\hline DIAL0293E07-FL & Arthropoda & Insecta & Hymenoptera & Halictidae & Halictinae \\
\hline DIAL0293E09-FL & Arthropoda & Insecta & Hymenoptera & Halictidae & Halictinae \\
\hline DIAL0293E08-FL & Arthropoda & Insecta & Hymenoptera & Halictidae & Halictinae \\
\hline DIAL1846H07-SC & Arthropoda & Insecta & Hymenoptera & Halictidae & Halictinae \\
\hline DIAL1847A04-FL & Arthropoda & Insecta & Hymenoptera & Halictidae & Halictinae \\
\hline DIAL1413E05-FL & Arthropoda & Insecta & Hymenoptera & Halictidae & Halictinae \\
\hline DIAL1846B11-MS & Arthropoda & Insecta & Hymenoptera & Halictidae & Halictinae \\
\hline DIAL1847A02-FL & Arthropoda & Insecta & Hymenoptera & Halictidae & Halictinae \\
\hline D0288E11-SC & Arthropoda & Insecta & Hymenoptera & Halictidae & Halictinae \\
\hline DIAL1846F01-SC & Arthropoda & Insecta & Hymenoptera & Halictidae & Halictinae \\
\hline DIAL1846B09-MS & Arthropoda & Insecta & Hymenoptera & Halictidae & Halictinae \\
\hline DIAL1846D01-MS & Arthropoda & Insecta & Hymenoptera & Halictidae & Halictinae \\
\hline D0288G05-FL & Arthropoda & Insecta & Hymenoptera & Halictidae & Halictinae \\
\hline D0288G04-FL & Arthropoda & Insecta & Hymenoptera & Halictidae & Halictinae \\
\hline D0288F04-SC & Arthropoda & Insecta & Hymenoptera & Halictidae & Halictinae \\
\hline D0288E02-NC & Arthropoda & Insecta & Hymenoptera & Halictidae & Halictinae \\
\hline D0288C12-FL & Arthropoda & Insecta & Hymenoptera & Halictidae & Halictinae \\
\hline D0288F01-SC & Arthropoda & Insecta & Hymenoptera & Halictidae & Halictinae \\
\hline B03765A01-GA & Arthropoda & Insecta & Hymenoptera & Halictidae & Halictinae \\
\hline D01559B08-GA & Arthropoda & Insecta & Hymenoptera & Halictidae & Halictinae \\
\hline D01559C11-GA & Arthropoda & Insecta & Hymenoptera & Halictidae & Halictinae \\
\hline D01559E01-GA & Arthropoda & Insecta & Hymenoptera & Halictidae & Halictinae \\
\hline DIAL1846D03-MS & Arthropoda & Insecta & Hymenoptera & Halictidae & Halictinae \\
\hline DIAL1846C03-MS & Arthropoda & Insecta & Hymenoptera & Halictidae & Halictinae \\
\hline D01561E01-MA & Arthropoda & Insecta & Hymenoptera & Halictidae & Halictinae \\
\hline DIAL1848D08-MD & Arthropoda & Insecta & Hymenoptera & Halictidae & Halictinae \\
\hline DIAL1848D06-WV & Arthropoda & Insecta & Hymenoptera & Halictidae & Halictinae \\
\hline DIAL2385C05-MD & Arthropoda & Insecta & Hymenoptera & Halictidae & Halictinae \\
\hline DIAL1849F10-MD & Arthropoda & Insecta & Hymenoptera & Halictidae & Halictinae \\
\hline DIAL2384B01-YT & Arthropoda & Insecta & Hymenoptera & Halictidae & Halictinae \\
\hline DIAL2384A10-YT & Arthropoda & Insecta & Hymenoptera & Halictidae & Halictinae \\
\hline DIAL2384B03-YT & Arthropoda & Insecta & Hymenoptera & Halictidae & Halictinae \\
\hline DIAL2384A08-YT & Arthropoda & Insecta & Hymenoptera & Halictidae & Halictinae \\
\hline
\end{tabular}




\begin{tabular}{|c|c|c|c|c|c|}
\hline B01558C07-AB & Arthropoda & Insecta & Hymenoptera & Halictidae & Halictinae \\
\hline DIAL2387C04-NM & Arthropoda & Insecta & Hymenoptera & Halictidae & Halictinae \\
\hline D00609B02-MT & Arthropoda & Insecta & Hymenoptera & Halictidae & Halictinae \\
\hline D01562A01-MT & Arthropoda & Insecta & Hymenoptera & Halictidae & Halictinae \\
\hline DIAL2374G03-NV & Arthropoda & Insecta & Hymenoptera & Halictidae & Halictinae \\
\hline 03767G04-BC & Arthropoda & Insecta & Hymenoptera & Halictidae & Halictinae \\
\hline CCDB-00611 H02 & Arthropoda & Insecta & Hymenoptera & Halictidae & Halictinae \\
\hline D0295H07-CO & Arthropoda & Insecta & Hymenoptera & Halictidae & Halictinae \\
\hline CCDB-01557 A01 & Arthropoda & Insecta & Hymenoptera & Halictidae & Halictinae \\
\hline DIAL2384C03-AB & Arthropoda & Insecta & Hymenoptera & Halictidae & Halictinae \\
\hline D0295H03-CO & Arthropoda & Insecta & Hymenoptera & Halictidae & Halictinae \\
\hline D01559G12-BC & Arthropoda & Insecta & Hymenoptera & Halictidae & Halictinae \\
\hline D00607H03-SK & Arthropoda & Insecta & Hymenoptera & Halictidae & Halictinae \\
\hline DIAL2386G03-BC & Arthropoda & Insecta & Hymenoptera & Halictidae & Halictinae \\
\hline DIAL2374B07-UT & Arthropoda & Insecta & Hymenoptera & Halictidae & Halictinae \\
\hline DIAL2388C07-NV & Arthropoda & Insecta & Hymenoptera & Halictidae & Halictinae \\
\hline D01561E07-SK & Arthropoda & Insecta & Hymenoptera & Halictidae & Halictinae \\
\hline D01561E02-SK & Arthropoda & Insecta & Hymenoptera & Halictidae & Halictinae \\
\hline D01562D12-BC & Arthropoda & Insecta & Hymenoptera & Halictidae & Halictinae \\
\hline D01559A08-AB & Arthropoda & Insecta & Hymenoptera & Halictidae & Halictinae \\
\hline 04486E05-WA & Arthropoda & Insecta & Hymenoptera & Halictidae & Halictinae \\
\hline CCDB-00611 H11 & Arthropoda & Insecta & Hymenoptera & Halictidae & Halictinae \\
\hline 03767G06-BC & Arthropoda & Insecta & Hymenoptera & Halictidae & Halictinae \\
\hline B03765E06-BC & Arthropoda & Insecta & Hymenoptera & Halictidae & Halictinae \\
\hline CCDB-01557 A04 & Arthropoda & Insecta & Hymenoptera & Halictidae & Halictinae \\
\hline 06732E12-WY & Arthropoda & Insecta & Hymenoptera & Halictidae & Halictinae \\
\hline DIAL2386A08-NV & Arthropoda & Insecta & Hymenoptera & Halictidae & Halictinae \\
\hline D01562D10-ON & Arthropoda & Insecta & Hymenoptera & Halictidae & Halictinae \\
\hline DIAL0286H10-MB & Arthropoda & Insecta & Hymenoptera & Halictidae & Halictinae \\
\hline DIAL0291H07-MB & Arthropoda & Insecta & Hymenoptera & Halictidae & Halictinae \\
\hline DIAL0291H05-MB & Arthropoda & Insecta & Hymenoptera & Halictidae & Halictinae \\
\hline DIAL0291H06-MB & Arthropoda & Insecta & Hymenoptera & Halictidae & Halictinae \\
\hline DIAL0286H11-MB & Arthropoda & Insecta & Hymenoptera & Halictidae & Halictinae \\
\hline DIAL0286H07-MB & Arthropoda & Insecta & Hymenoptera & Halictidae & Halictinae \\
\hline DIAL0286H08-MB & Arthropoda & Insecta & Hymenoptera & Halictidae & Halictinae \\
\hline B03765F12-BC & Arthropoda & Insecta & Hymenoptera & Halictidae & Halictinae \\
\hline DIAL1409H04-MB & Arthropoda & Insecta & Hymenoptera & Halictidae & Halictinae \\
\hline DIAL1409H02-MB & Arthropoda & Insecta & Hymenoptera & Halictidae & Halictinae \\
\hline CCDB-01557 A09-ז & Arthropoda & Insecta & Hymenoptera & Halictidae & Halictinae \\
\hline D01562C04-MB & Arthropoda & Insecta & Hymenoptera & Halictidae & Halictinae \\
\hline D01560A10-WI & Arthropoda & Insecta & Hymenoptera & Halictidae & Halictinae \\
\hline DIAL0291H08-MB & Arthropoda & Insecta & Hymenoptera & Halictidae & Halictinae \\
\hline D01562E02-MB & Arthropoda & Insecta & Hymenoptera & Halictidae & Halictinae \\
\hline D01562C09-ON & Arthropoda & Insecta & Hymenoptera & Halictidae & Halictinae \\
\hline CCDB-01557 A07-ז & Arthropoda & Insecta & Hymenoptera & Halictidae & Halictinae \\
\hline B03765F09-BC & Arthropoda & Insecta & Hymenoptera & Halictidae & Halictinae \\
\hline CCDB-01557 A10-I & Arthropoda & Insecta & Hymenoptera & Halictidae & Halictinae \\
\hline CCDB-01557 A11- & Arthropoda & Insecta & Hymenoptera & Halictidae & Halictinae \\
\hline B03765F06-BC & Arthropoda & Insecta & Hymenoptera & Halictidae & Halictinae \\
\hline D00608F06-BC & Arthropoda & Insecta & Hymenoptera & Halictidae & Halictinae \\
\hline В03751H05-BC & Arthropoda & Insecta & Hymenoptera & Halictidae & Halictinae \\
\hline D01559E08-BC & Arthropoda & Insecta & Hymenoptera & Halictidae & Halictinae \\
\hline D00607E12-UT & Arthropoda & Insecta & Hymenoptera & Halictidae & Halictinae \\
\hline D00608E01-BC & Arthropoda & Insecta & Hymenoptera & Halictidae & Halictinae \\
\hline D01561F06-BC & Arthropoda & Insecta & Hymenoptera & Halictidae & Halictinae \\
\hline
\end{tabular}




\begin{tabular}{|c|c|c|c|c|c|}
\hline B03765E12-BC & Arthropoda & Insecta & Hymenoptera & Halictidae & Halictinae \\
\hline D0295F01-CO & Arthropoda & Insecta & Hymenoptera & Halictidae & Halictinae \\
\hline DIAL2374E05-WA & Arthropoda & Insecta & Hymenoptera & Halictidae & Halictinae \\
\hline CCDB-00611 H04 & Arthropoda & Insecta & Hymenoptera & Halictidae & Halictinae \\
\hline D01559G09-BC & Arthropoda & Insecta & Hymenoptera & Halictidae & Halictinae \\
\hline D0295G11-CO & Arthropoda & Insecta & Hymenoptera & Halictidae & Halictinae \\
\hline DIAL2387C03-ID & Arthropoda & Insecta & Hymenoptera & Halictidae & Halictinae \\
\hline D0295F04-CO & Arthropoda & Insecta & Hymenoptera & Halictidae & Halictinae \\
\hline DIAL2374E04-WA & Arthropoda & Insecta & Hymenoptera & Halictidae & Halictinae \\
\hline D01559B11-BC & Arthropoda & Insecta & Hymenoptera & Halictidae & Halictinae \\
\hline D01559C03-BC & Arthropoda & Insecta & Hymenoptera & Halictidae & Halictinae \\
\hline D0295H11-CO & Arthropoda & Insecta & Hymenoptera & Halictidae & Halictinae \\
\hline D0287C01-CO & Arthropoda & Insecta & Hymenoptera & Halictidae & Halictinae \\
\hline D0287B01-CO & Arthropoda & Insecta & Hymenoptera & Halictidae & Halictinae \\
\hline D01562A10-MT & Arthropoda & Insecta & Hymenoptera & Halictidae & Halictinae \\
\hline D0295G06-CO & Arthropoda & Insecta & Hymenoptera & Halictidae & Halictinae \\
\hline CCDB-00611 H03 & Arthropoda & Insecta & Hymenoptera & Halictidae & Halictinae \\
\hline DIAL2388D12-NM & Arthropoda & Insecta & Hymenoptera & Halictidae & Halictinae \\
\hline DIAL0293H09-AZ & Arthropoda & Insecta & Hymenoptera & Halictidae & Halictinae \\
\hline D-3262F07-NM & Arthropoda & Insecta & Hymenoptera & Halictidae & Halictinae \\
\hline D0295G07-CO & Arthropoda & Insecta & Hymenoptera & Halictidae & Halictinae \\
\hline DIAL1848C04-MN & Arthropoda & Insecta & Hymenoptera & Halictidae & Halictinae \\
\hline DIAL1848F02-MN & Arthropoda & Insecta & Hymenoptera & Halictidae & Halictinae \\
\hline DIAL1848B05-AZ & Arthropoda & Insecta & Hymenoptera & Halictidae & Halictinae \\
\hline DIAL1849B05-AZ & Arthropoda & Insecta & Hymenoptera & Halictidae & Halictinae \\
\hline DIAL1847F03-TX & Arthropoda & Insecta & Hymenoptera & Halictidae & Halictinae \\
\hline DIAL1849E10-AZ & Arthropoda & Insecta & Hymenoptera & Halictidae & Halictinae \\
\hline D0287A06-CO & Arthropoda & Insecta & Hymenoptera & Halictidae & Halictinae \\
\hline DIAL2374F12-UT & Arthropoda & Insecta & Hymenoptera & Halictidae & Halictinae \\
\hline D0295G02-CO & Arthropoda & Insecta & Hymenoptera & Halictidae & Halictinae \\
\hline DIAL1849E02-CO & Arthropoda & Insecta & Hymenoptera & Halictidae & Halictinae \\
\hline DIAL1848B01-AZ & Arthropoda & Insecta & Hymenoptera & Halictidae & Halictinae \\
\hline DIAL1848C03-MN & Arthropoda & Insecta & Hymenoptera & Halictidae & Halictinae \\
\hline D01561B03-CA & Arthropoda & Insecta & Hymenoptera & Halictidae & Halictinae \\
\hline DIAL2386B02-NV & Arthropoda & Insecta & Hymenoptera & Halictidae & Halictinae \\
\hline DIAL2374G01-UT & Arthropoda & Insecta & Hymenoptera & Halictidae & Halictinae \\
\hline DIAL2388E02-NM & Arthropoda & Insecta & Hymenoptera & Halictidae & Halictinae \\
\hline DIAL1849C06-CO & Arthropoda & Insecta & Hymenoptera & Halictidae & Halictinae \\
\hline DIAL0286G11-MB & Arthropoda & Insecta & Hymenoptera & Halictidae & Halictinae \\
\hline D0287A04-CO & Arthropoda & Insecta & Hymenoptera & Halictidae & Halictinae \\
\hline D0295H04-CO & Arthropoda & Insecta & Hymenoptera & Halictidae & Halictinae \\
\hline D0295G04-CO & Arthropoda & Insecta & Hymenoptera & Halictidae & Halictinae \\
\hline DIAL0286G12-MB & Arthropoda & Insecta & Hymenoptera & Halictidae & Halictinae \\
\hline DIAL0286G08-MB & Arthropoda & Insecta & Hymenoptera & Halictidae & Halictinae \\
\hline 06732H03-CO & Arthropoda & Insecta & Hymenoptera & Halictidae & Halictinae \\
\hline DIAL2385E11-NS & Arthropoda & Insecta & Hymenoptera & Halictidae & Halictinae \\
\hline DIAL2385A02-NS & Arthropoda & Insecta & Hymenoptera & Halictidae & Halictinae \\
\hline DIAL2385F01-NS & Arthropoda & Insecta & Hymenoptera & Halictidae & Halictinae \\
\hline DIAL2385F04-NS & Arthropoda & Insecta & Hymenoptera & Halictidae & Halictinae \\
\hline DIAL1848D03-NS & Arthropoda & Insecta & Hymenoptera & Halictidae & Halictinae \\
\hline DIAL1848D02-NS & Arthropoda & Insecta & Hymenoptera & Halictidae & Halictinae \\
\hline D01560A05-WI & Arthropoda & Insecta & Hymenoptera & Halictidae & Halictinae \\
\hline DIAL2384H08-MD & Arthropoda & Insecta & Hymenoptera & Halictidae & Halictinae \\
\hline DIAL1849H05-MD & Arthropoda & Insecta & Hymenoptera & Halictidae & Halictinae \\
\hline DIAL0294B06-IN & Arthropoda & Insecta & Hymenoptera & Halictidae & Halictinae \\
\hline
\end{tabular}




\begin{tabular}{|c|c|c|c|c|c|}
\hline DIAL1848G09-AB & Arthropoda & Insecta & Hymenoptera & Halictidae & Halictinae \\
\hline D01562E03-NS & Arthropoda & Insecta & Hymenoptera & Halictidae & Halictinae \\
\hline DIAL2385A01-SK & Arthropoda & Insecta & Hymenoptera & Halictidae & Halictinae \\
\hline DIAL2385A05-SK & Arthropoda & Insecta & Hymenoptera & Halictidae & Halictinae \\
\hline D01560C07-IA & Arthropoda & Insecta & Hymenoptera & Halictidae & Halictinae \\
\hline D-3253F03-MO & Arthropoda & Insecta & Hymenoptera & Halictidae & Halictinae \\
\hline D0288E01-VA & Arthropoda & Insecta & Hymenoptera & Halictidae & Halictinae \\
\hline DIAL2385E09-NS & Arthropoda & Insecta & Hymenoptera & Halictidae & Halictinae \\
\hline D0288C10-VA & Arthropoda & Insecta & Hymenoptera & Halictidae & Halictinae \\
\hline DIAL0294F07-MD & Arthropoda & Insecta & Hymenoptera & Halictidae & Halictinae \\
\hline DIAL0293B04-MD & Arthropoda & Insecta & Hymenoptera & Halictidae & Halictinae \\
\hline DIAL0294G03-MD & Arthropoda & Insecta & Hymenoptera & Halictidae & Halictinae \\
\hline DIAL0293B03-MD & Arthropoda & Insecta & Hymenoptera & Halictidae & Halictinae \\
\hline DIAL0294G04-MD & Arthropoda & Insecta & Hymenoptera & Halictidae & Halictinae \\
\hline DIAL0294C08-IN & Arthropoda & Insecta & Hymenoptera & Halictidae & Halictinae \\
\hline DIAL0292A03-MD & Arthropoda & Insecta & Hymenoptera & Halictidae & Halictinae \\
\hline DIAL0292A04-MD & Arthropoda & Insecta & Hymenoptera & Halictidae & Halictinae \\
\hline B04743G07-MA & Arthropoda & Insecta & Hymenoptera & Halictidae & Halictinae \\
\hline B04743G01-MA & Arthropoda & Insecta & Hymenoptera & Halictidae & Halictinae \\
\hline DIAL1846F08-WV & Arthropoda & Insecta & Hymenoptera & Halictidae & Halictinae \\
\hline DIAL1848C12-WV & Arthropoda & Insecta & Hymenoptera & Halictidae & Halictinae \\
\hline B04743D05-NS & Arthropoda & Insecta & Hymenoptera & Halictidae & Halictinae \\
\hline D00608E10-IN & Arthropoda & Insecta & Hymenoptera & Halictidae & Halictinae \\
\hline D01562B05-ON & Arthropoda & Insecta & Hymenoptera & Halictidae & Halictinae \\
\hline B04743H06-MA & Arthropoda & Insecta & Hymenoptera & Halictidae & Halictinae \\
\hline DIAL1391E05-TN & Arthropoda & Insecta & Hymenoptera & Halictidae & Halictinae \\
\hline D00607E07-CT & Arthropoda & Insecta & Hymenoptera & Halictidae & Halictinae \\
\hline D0288B02-MD & Arthropoda & Insecta & Hymenoptera & Halictidae & Halictinae \\
\hline D01559E06-ON & Arthropoda & Insecta & Hymenoptera & Halictidae & Halictinae \\
\hline D00607A10-CT & Arthropoda & Insecta & Hymenoptera & Halictidae & Halictinae \\
\hline DIAL1849G06-NH & Arthropoda & Insecta & Hymenoptera & Halictidae & Halictinae \\
\hline D01560A01-WI & Arthropoda & Insecta & Hymenoptera & Halictidae & Halictinae \\
\hline В03750B02-MA & Arthropoda & Insecta & Hymenoptera & Halictidae & Halictinae \\
\hline \multicolumn{2}{|c|}{ CCDB-00611 G08-( Arthropoda } & Insecta & Hymenoptera & Halictidae & Halictinae \\
\hline \multicolumn{2}{|c|}{ CCDB-00611 F03-( Arthropoda } & Insecta & Hymenoptera & Halictidae & Halictinae \\
\hline B04743G03-MA & Arthropoda & Insecta & Hymenoptera & Halictidae & Halictinae \\
\hline B04743H02-MA & Arthropoda & Insecta & Hymenoptera & Halictidae & Halictinae \\
\hline \multicolumn{2}{|c|}{ CCDB-00611 G03-( Arthropoda } & Insecta & Hymenoptera & Halictidae & Halictinae \\
\hline \multicolumn{2}{|c|}{ CCDB-00611 G04-( Arthropoda } & Insecta & Hymenoptera & Halictidae & Halictinae \\
\hline B04743D03-NS & Arthropoda & Insecta & Hymenoptera & Halictidae & Halictinae \\
\hline D00608E12-IN & Arthropoda & Insecta & Hymenoptera & Halictidae & Halictinae \\
\hline D01562E07-NS & Arthropoda & Insecta & Hymenoptera & Halictidae & Halictinae \\
\hline D01560C12-IA & Arthropoda & Insecta & Hymenoptera & Halictidae & Halictinae \\
\hline DIAL0286F12-MB & Arthropoda & Insecta & Hymenoptera & Halictidae & Halictinae \\
\hline DIAL0286F10-MB & Arthropoda & Insecta & Hymenoptera & Halictidae & Halictinae \\
\hline DIAL1848H03-AB & Arthropoda & Insecta & Hymenoptera & Halictidae & Halictinae \\
\hline DIAL1390B01-ON & Arthropoda & Insecta & Hymenoptera & Halictidae & Halictinae \\
\hline DIAL0293E04-FL & Arthropoda & Insecta & Hymenoptera & Halictidae & Halictinae \\
\hline D-3253D09-FL & Arthropoda & Insecta & Hymenoptera & Halictidae & Halictinae \\
\hline D-3253E04-FL & Arthropoda & Insecta & Hymenoptera & Halictidae & Halictinae \\
\hline D01561D10-FL & Arthropoda & Insecta & Hymenoptera & Halictidae & Halictinae \\
\hline D01559A04-FL & Arthropoda & Insecta & Hymenoptera & Halictidae & Halictinae \\
\hline D0295D02-DC & Arthropoda & Insecta & Hymenoptera & Halictidae & Halictinae \\
\hline D00608B05-ON & Arthropoda & Insecta & Hymenoptera & Halictidae & Halictinae \\
\hline D00608B03-ON & Arthropoda & Insecta & Hymenoptera & Halictidae & Halictinae \\
\hline
\end{tabular}




\begin{tabular}{|c|c|c|c|c|c|}
\hline D1414C02-VA & Arthropoda & Insecta & Hymenoptera & Halictidae & Halictinae \\
\hline DIAL0294E01-VA & Arthropoda & Insecta & Hymenoptera & Halictidae & Halictinae \\
\hline DIAL0294D12-NY & Arthropoda & Insecta & Hymenoptera & Halictidae & Halictinae \\
\hline D1414B10-VA & Arthropoda & Insecta & Hymenoptera & Halictidae & Halictinae \\
\hline D1414C01-VA & Arthropoda & Insecta & Hymenoptera & Halictidae & Halictinae \\
\hline D-3253F06-MO & Arthropoda & Insecta & Hymenoptera & Halictidae & Halictinae \\
\hline DIAL1846B06-MS & Arthropoda & Insecta & Hymenoptera & Halictidae & Halictinae \\
\hline DIAL1847H10-MD & Arthropoda & Insecta & Hymenoptera & Halictidae & Halictinae \\
\hline DIAL1846H10-MD & Arthropoda & Insecta & Hymenoptera & Halictidae & Halictinae \\
\hline DIAL1846B04-MS & Arthropoda & Insecta & Hymenoptera & Halictidae & Halictinae \\
\hline DIAL1413A09-VA & Arthropoda & Insecta & Hymenoptera & Halictidae & Halictinae \\
\hline B01566D04-TX & Arthropoda & Insecta & Hymenoptera & Halictidae & Halictinae \\
\hline D0288E08-WV & Arthropoda & Insecta & Hymenoptera & Halictidae & Halictinae \\
\hline D1414B11-VA & Arthropoda & Insecta & Hymenoptera & Halictidae & Halictinae \\
\hline DIAL1391E02-SC & Arthropoda & Insecta & Hymenoptera & Halictidae & Halictinae \\
\hline DIAL1391E01-SC & Arthropoda & Insecta & Hymenoptera & Halictidae & Halictinae \\
\hline D0288G09-SC & Arthropoda & Insecta & Hymenoptera & Halictidae & Halictinae \\
\hline D0288E10-NC & Arthropoda & Insecta & Hymenoptera & Halictidae & Halictinae \\
\hline D0288E05-WV & Arthropoda & Insecta & Hymenoptera & Halictidae & Halictinae \\
\hline D0288D12-WV & Arthropoda & Insecta & Hymenoptera & Halictidae & Halictinae \\
\hline D0295E11-DC & Arthropoda & Insecta & Hymenoptera & Halictidae & Halictinae \\
\hline D0295E10-DC & Arthropoda & Insecta & Hymenoptera & Halictidae & Halictinae \\
\hline BEE0286E07-VA & Arthropoda & Insecta & Hymenoptera & Halictidae & Halictinae \\
\hline D00607C02-MO & Arthropoda & Insecta & Hymenoptera & Halictidae & Halictinae \\
\hline D00607A01-NE & Arthropoda & Insecta & Hymenoptera & Halictidae & Halictinae \\
\hline 06732C02-DC & Arthropoda & Insecta & Hymenoptera & Halictidae & Halictinae \\
\hline DIAL2387C06-YT & Arthropoda & Insecta & Hymenoptera & Halictidae & Halictinae \\
\hline DIAL1845E09-NL & Arthropoda & Insecta & Hymenoptera & Halictidae & Halictinae \\
\hline DIAL1845E06-NL & Arthropoda & Insecta & Hymenoptera & Halictidae & Halictinae \\
\hline DIAL2384A11-YT & Arthropoda & Insecta & Hymenoptera & Halictidae & Halictinae \\
\hline DIAL1391C03-NC & Arthropoda & Insecta & Hymenoptera & Halictidae & Halictinae \\
\hline DIAL2384A03-YT & Arthropoda & Insecta & Hymenoptera & Halictidae & Halictinae \\
\hline DIAL2384A02-YT & Arthropoda & Insecta & Hymenoptera & Halictidae & Halictinae \\
\hline DIAL1845E12-NL & Arthropoda & Insecta & Hymenoptera & Halictidae & Halictinae \\
\hline DIAL1845F01-NL & Arthropoda & Insecta & Hymenoptera & Halictidae & Halictinae \\
\hline DIAL2387B10-YT & Arthropoda & Insecta & Hymenoptera & Halictidae & Halictinae \\
\hline DIAL1391C04-NC & Arthropoda & Insecta & Hymenoptera & Halictidae & Halictinae \\
\hline DIAL2387B04-YT & Arthropoda & Insecta & Hymenoptera & Halictidae & Halictinae \\
\hline DIAL2384A06-YT & Arthropoda & Insecta & Hymenoptera & Halictidae & Halictinae \\
\hline DIAL1848A10-NB & Arthropoda & Insecta & Hymenoptera & Halictidae & Halictinae \\
\hline DIAL2384A09-YT & Arthropoda & Insecta & Hymenoptera & Halictidae & Halictinae \\
\hline DIAL2384B08-YT & Arthropoda & Insecta & Hymenoptera & Halictidae & Halictinae \\
\hline DIAL2384A01-YT & Arthropoda & Insecta & Hymenoptera & Halictidae & Halictinae \\
\hline DIAL2384B02-YT & Arthropoda & Insecta & Hymenoptera & Halictidae & Halictinae \\
\hline DIAL2384B10-YT & Arthropoda & Insecta & Hymenoptera & Halictidae & Halictinae \\
\hline DIAL1390C01-ON & Arthropoda & Insecta & Hymenoptera & Halictidae & Halictinae \\
\hline DIAL1390C03-ON & Arthropoda & Insecta & Hymenoptera & Halictidae & Halictinae \\
\hline BEE1850G11-ME & Arthropoda & Insecta & Hymenoptera & Halictidae & Halictinae \\
\hline D1414H01-VA & Arthropoda & Insecta & Hymenoptera & Halictidae & Halictinae \\
\hline DIAL0292A11-WV & Arthropoda & Insecta & Hymenoptera & Halictidae & Halictinae \\
\hline DIAL0294E06-MD & Arthropoda & Insecta & Hymenoptera & Halictidae & Halictinae \\
\hline DIAL2385F02-MD & Arthropoda & Insecta & Hymenoptera & Halictidae & Halictinae \\
\hline DIAL0294E12-MD & Arthropoda & Insecta & Hymenoptera & Halictidae & Halictinae \\
\hline DIAL0294E07-IN & Arthropoda & Insecta & Hymenoptera & Halictidae & Halictinae \\
\hline DIAL0293B10-WV & Arthropoda & Insecta & Hymenoptera & Halictidae & Halictinae \\
\hline
\end{tabular}




\begin{tabular}{|c|c|c|c|c|c|}
\hline DIAL0293F02-NC & Arthropoda & Insecta & Hymenoptera & Halictidae & Halictinae \\
\hline D00607H11-KS & Arthropoda & Insecta & Hymenoptera & Halictidae & Halictinae \\
\hline B01558D07-NC & Arthropoda & Insecta & Hymenoptera & Halictidae & Halictinae \\
\hline D00608A07-KS & Arthropoda & Insecta & Hymenoptera & Halictidae & Halictinae \\
\hline D0288C05-DC & Arthropoda & Insecta & Hymenoptera & Halictidae & Halictinae \\
\hline DIAL1409B07-NC & Arthropoda & Insecta & Hymenoptera & Halictidae & Halictinae \\
\hline DIAL1846D04-MS & Arthropoda & Insecta & Hymenoptera & Halictidae & Halictinae \\
\hline DIAL1413H02-NC & Arthropoda & Insecta & Hymenoptera & Halictidae & Halictinae \\
\hline D0288D08-NC & Arthropoda & Insecta & Hymenoptera & Halictidae & Halictinae \\
\hline DIAL2385C12-SC & Arthropoda & Insecta & Hymenoptera & Halictidae & Halictinae \\
\hline DIAL1849C10-CO & Arthropoda & Insecta & Hymenoptera & Halictidae & Halictinae \\
\hline D00607B03-KS & Arthropoda & Insecta & Hymenoptera & Halictidae & Halictinae \\
\hline D00607H09-KS & Arthropoda & Insecta & Hymenoptera & Halictidae & Halictinae \\
\hline 06732E01-MD & Arthropoda & Insecta & Hymenoptera & Halictidae & Halictinae \\
\hline D1414A06-IL & Arthropoda & Insecta & Hymenoptera & Halictidae & Halictinae \\
\hline B03765B09-GA & Arthropoda & Insecta & Hymenoptera & Halictidae & Halictinae \\
\hline B01558D08-NC & Arthropoda & Insecta & Hymenoptera & Halictidae & Halictinae \\
\hline B01558D03-NC & Arthropoda & Insecta & Hymenoptera & Halictidae & Halictinae \\
\hline B01558C12-NC & Arthropoda & Insecta & Hymenoptera & Halictidae & Halictinae \\
\hline D0288B08-SC & Arthropoda & Insecta & Hymenoptera & Halictidae & Halictinae \\
\hline D0288B06-NC & Arthropoda & Insecta & Hymenoptera & Halictidae & Halictinae \\
\hline DIAL1391D08-NC & Arthropoda & Insecta & Hymenoptera & Halictidae & Halictinae \\
\hline DIAL1391E10-NC & Arthropoda & Insecta & Hymenoptera & Halictidae & Halictinae \\
\hline DIAL0293F04-TN & Arthropoda & Insecta & Hymenoptera & Halictidae & Halictinae \\
\hline DIAL0293F05-TN & Arthropoda & Insecta & Hymenoptera & Halictidae & Halictinae \\
\hline DIAL1391C05-NC & Arthropoda & Insecta & Hymenoptera & Halictidae & Halictinae \\
\hline DIAL1391A08-NC & Arthropoda & Insecta & Hymenoptera & Halictidae & Halictinae \\
\hline DIAL1849H07-ME & Arthropoda & Insecta & Hymenoptera & Halictidae & Halictinae \\
\hline DIAL1848F07-PE & Arthropoda & Insecta & Hymenoptera & Halictidae & Halictinae \\
\hline DIAL1848E08-WV & Arthropoda & Insecta & Hymenoptera & Halictidae & Halictinae \\
\hline D00607F12-NS & Arthropoda & Insecta & Hymenoptera & Halictidae & Halictinae \\
\hline DIAL2385C06-MD & Arthropoda & Insecta & Hymenoptera & Halictidae & Halictinae \\
\hline D1414D03-VA & Arthropoda & Insecta & Hymenoptera & Halictidae & Halictinae \\
\hline DIAL1846C07-WV & Arthropoda & Insecta & Hymenoptera & Halictidae & Halictinae \\
\hline D0287D08-IL & Arthropoda & Insecta & Hymenoptera & Halictidae & Halictinae \\
\hline DIAL0293B02-DC & Arthropoda & Insecta & Hymenoptera & Halictidae & Halictinae \\
\hline DIAL1391H03-NC & Arthropoda & Insecta & Hymenoptera & Halictidae & Halictinae \\
\hline DIAL1847H05-MD & Arthropoda & Insecta & Hymenoptera & Halictidae & Halictinae \\
\hline DIAL0294G01-MD & Arthropoda & Insecta & Hymenoptera & Halictidae & Halictinae \\
\hline DIAL0294C06-IL & Arthropoda & Insecta & Hymenoptera & Halictidae & Halictinae \\
\hline DIAL0294C07-IN & Arthropoda & Insecta & Hymenoptera & Halictidae & Halictinae \\
\hline DIAL0294D11-NY & Arthropoda & Insecta & Hymenoptera & Halictidae & Halictinae \\
\hline DIAL1848E02-MD & Arthropoda & Insecta & Hymenoptera & Halictidae & Halictinae \\
\hline D0295B06-ON & Arthropoda & Insecta & Hymenoptera & Halictidae & Halictinae \\
\hline DIAL1391H02-NC & Arthropoda & Insecta & Hymenoptera & Halictidae & Halictinae \\
\hline DIAL1391D01-NC & Arthropoda & Insecta & Hymenoptera & Halictidae & Halictinae \\
\hline DIAL1391H08-NC & Arthropoda & Insecta & Hymenoptera & Halictidae & Halictinae \\
\hline DIAL1847H04-MD & Arthropoda & Insecta & Hymenoptera & Halictidae & Halictinae \\
\hline DIAL1391G12-NC & Arthropoda & Insecta & Hymenoptera & Halictidae & Halictinae \\
\hline DIAL0292B09-MD & Arthropoda & Insecta & Hymenoptera & Halictidae & Halictinae \\
\hline DIAL0292C01-WV & Arthropoda & Insecta & Hymenoptera & Halictidae & Halictinae \\
\hline DIAL0292B11-MD & Arthropoda & Insecta & Hymenoptera & Halictidae & Halictinae \\
\hline DIAL0292C02-MD & Arthropoda & Insecta & Hymenoptera & Halictidae & Halictinae \\
\hline DIAL1413G10-NC & Arthropoda & Insecta & Hymenoptera & Halictidae & Halictinae \\
\hline DIAL1391C10-NC & Arthropoda & Insecta & Hymenoptera & Halictidae & Halictinae \\
\hline
\end{tabular}




\begin{tabular}{|c|c|c|c|c|c|}
\hline DIAL1391B10-NC & Arthropoda & Insecta & Hymenoptera & Halictidae & Halictinae \\
\hline 06732E06-WV & Arthropoda & Insecta & Hymenoptera & Halictidae & Halictinae \\
\hline D0287H06-IL & Arthropoda & Insecta & Hymenoptera & Halictidae & Halictinae \\
\hline D0287F01-IL & Arthropoda & Insecta & Hymenoptera & Halictidae & Halictinae \\
\hline D0287H08-IL & Arthropoda & Insecta & Hymenoptera & Halictidae & Halictinae \\
\hline DIAL1413G09-NC & Arthropoda & Insecta & Hymenoptera & Halictidae & Halictinae \\
\hline D0287H12-IL & Arthropoda & Insecta & Hymenoptera & Halictidae & Halictinae \\
\hline DIAL1409C01-PQ & Arthropoda & Insecta & Hymenoptera & Halictidae & Halictinae \\
\hline DIAL1409B11-PQ & Arthropoda & Insecta & Hymenoptera & Halictidae & Halictinae \\
\hline DIAL1846F03-MD & Arthropoda & Insecta & Hymenoptera & Halictidae & Halictinae \\
\hline DIAL1847H01-MD & Arthropoda & Insecta & Hymenoptera & Halictidae & Halictinae \\
\hline DIAL1847H02-MD & Arthropoda & Insecta & Hymenoptera & Halictidae & Halictinae \\
\hline DIAL1846B03-MD & Arthropoda & Insecta & Hymenoptera & Halictidae & Halictinae \\
\hline D00607H07-KS & Arthropoda & Insecta & Hymenoptera & Halictidae & Halictinae \\
\hline DIAL1391H05-NC & Arthropoda & Insecta & Hymenoptera & Halictidae & Halictinae \\
\hline DIAL1391B07-NC & Arthropoda & Insecta & Hymenoptera & Halictidae & Halictinae \\
\hline DIAL1391B05-NC & Arthropoda & Insecta & Hymenoptera & Halictidae & Halictinae \\
\hline D0287E12-IL & Arthropoda & Insecta & Hymenoptera & Halictidae & Halictinae \\
\hline D0287E06-IL & Arthropoda & Insecta & Hymenoptera & Halictidae & Halictinae \\
\hline DIAL1413B03-IL & Arthropoda & Insecta & Hymenoptera & Halictidae & Halictinae \\
\hline DIAL1390A02-NC & Arthropoda & Insecta & Hymenoptera & Halictidae & Halictinae \\
\hline DIAL1390A01-NC & Arthropoda & Insecta & Hymenoptera & Halictidae & Halictinae \\
\hline DIAL1390F02-NC & Arthropoda & Insecta & Hymenoptera & Halictidae & Halictinae \\
\hline DIAL1413G08-NC & Arthropoda & Insecta & Hymenoptera & Halictidae & Halictinae \\
\hline DIAL1391H12-NC & Arthropoda & Insecta & Hymenoptera & Halictidae & Halictinae \\
\hline DIAL1413H07-NC & Arthropoda & Insecta & Hymenoptera & Halictidae & Halictinae \\
\hline DIAL1413H04-NC & Arthropoda & Insecta & Hymenoptera & Halictidae & Halictinae \\
\hline DIAL1391B09-NC & Arthropoda & Insecta & Hymenoptera & Halictidae & Halictinae \\
\hline DIAL1391B08-NC & Arthropoda & Insecta & Hymenoptera & Halictidae & Halictinae \\
\hline D0287H11-IL & Arthropoda & Insecta & Hymenoptera & Halictidae & Halictinae \\
\hline DIAL1391B04-NC & Arthropoda & Insecta & Hymenoptera & Halictidae & Halictinae \\
\hline DIAL1391H07-NC & Arthropoda & Insecta & Hymenoptera & Halictidae & Halictinae \\
\hline D0287E02-IL & Arthropoda & Insecta & Hymenoptera & Halictidae & Halictinae \\
\hline DIAL1391B06-NC & Arthropoda & Insecta & Hymenoptera & Halictidae & Halictinae \\
\hline DIAL1848F04-MD & Arthropoda & Insecta & Hymenoptera & Halictidae & Halictinae \\
\hline DIAL1413H09-NC & Arthropoda & Insecta & Hymenoptera & Halictidae & Halictinae \\
\hline D0287E08-IL & Arthropoda & Insecta & Hymenoptera & Halictidae & Halictinae \\
\hline D0295B07-ON & Arthropoda & Insecta & Hymenoptera & Halictidae & Halictinae \\
\hline D0295B01-ON & Arthropoda & Insecta & Hymenoptera & Halictidae & Halictinae \\
\hline D0295B02-ON & Arthropoda & Insecta & Hymenoptera & Halictidae & Halictinae \\
\hline D0287H07-IL & Arthropoda & Insecta & Hymenoptera & Halictidae & Halictinae \\
\hline DIAL1391H11-NC & Arthropoda & Insecta & Hymenoptera & Halictidae & Halictinae \\
\hline D0287G11-IL & Arthropoda & Insecta & Hymenoptera & Halictidae & Halictinae \\
\hline D0287H03-IL & Arthropoda & Insecta & Hymenoptera & Halictidae & Halictinae \\
\hline D0287H04-IL & Arthropoda & Insecta & Hymenoptera & Halictidae & Halictinae \\
\hline D0287H05-IL & Arthropoda & Insecta & Hymenoptera & Halictidae & Halictinae \\
\hline D0287G05-IL & Arthropoda & Insecta & Hymenoptera & Halictidae & Halictinae \\
\hline D0287E11-IL & Arthropoda & Insecta & Hymenoptera & Halictidae & Halictinae \\
\hline D0287E07-IL & Arthropoda & Insecta & Hymenoptera & Halictidae & Halictinae \\
\hline D0287D07-IL & Arthropoda & Insecta & Hymenoptera & Halictidae & Halictinae \\
\hline D0287C05-IL & Arthropoda & Insecta & Hymenoptera & Halictidae & Halictinae \\
\hline D1414E06-VA & Arthropoda & Insecta & Hymenoptera & Halictidae & Halictinae \\
\hline D0287C03-IL & Arthropoda & Insecta & Hymenoptera & Halictidae & Halictinae \\
\hline D0287C04-IL & Arthropoda & Insecta & Hymenoptera & Halictidae & Halictinae \\
\hline D0287E09-IL & Arthropoda & Insecta & Hymenoptera & Halictidae & Halictinae \\
\hline
\end{tabular}




\begin{tabular}{|c|c|c|c|c|c|}
\hline D1414E03-VA & Arthropoda & Insecta & Hymenoptera & Halictidae & Halictinae \\
\hline D0288H02-MD & Arthropoda & Insecta & Hymenoptera & Halictidae & Halictinae \\
\hline D0288G12-MD & Arthropoda & Insecta & Hymenoptera & Halictidae & Halictinae \\
\hline D0288D10-MD & Arthropoda & Insecta & Hymenoptera & Halictidae & Halictinae \\
\hline D0288B07-MD & Arthropoda & Insecta & Hymenoptera & Halictidae & Halictinae \\
\hline D1414H06-VA & Arthropoda & Insecta & Hymenoptera & Halictidae & Halictinae \\
\hline D1414H05-VA & Arthropoda & Insecta & Hymenoptera & Halictidae & Halictinae \\
\hline D1414E05-VA & Arthropoda & Insecta & Hymenoptera & Halictidae & Halictinae \\
\hline D0295B04-ON & Arthropoda & Insecta & Hymenoptera & Halictidae & Halictinae \\
\hline D00607F03-KS & Arthropoda & Insecta & Hymenoptera & Halictidae & Halictinae \\
\hline D00607C04-MO & Arthropoda & Insecta & Hymenoptera & Halictidae & Halictinae \\
\hline D00608E07-IN & Arthropoda & Insecta & Hymenoptera & Halictidae & Halictinae \\
\hline DIAL2385C08-MD & Arthropoda & Insecta & Hymenoptera & Halictidae & Halictinae \\
\hline DIAL2385D06-MD & Arthropoda & Insecta & Hymenoptera & Halictidae & Halictinae \\
\hline 06732D06-WV & Arthropoda & Insecta & Hymenoptera & Halictidae & Halictinae \\
\hline CCDB-00611 D09 & Arthropoda & Insecta & Hymenoptera & Halictidae & Halictinae \\
\hline CCDB-00611 D03-I & Arthropoda & Insecta & Hymenoptera & Halictidae & Halictinae \\
\hline 06732H06-RI & Arthropoda & Insecta & Hymenoptera & Halictidae & Halictinae \\
\hline DIAL1845C10-TX & Arthropoda & Insecta & Hymenoptera & Halictidae & Halictinae \\
\hline DIAL1847A01-SC & Arthropoda & Insecta & Hymenoptera & Halictidae & Halictinae \\
\hline DIAL0294D04-IN & Arthropoda & Insecta & Hymenoptera & Halictidae & Halictinae \\
\hline DIAL0294E04-NY & Arthropoda & Insecta & Hymenoptera & Halictidae & Halictinae \\
\hline DIAL0293F11-MN & Arthropoda & Insecta & Hymenoptera & Halictidae & Halictinae \\
\hline DIAL0293F08-MD & Arthropoda & Insecta & Hymenoptera & Halictidae & Halictinae \\
\hline D0288D06-NC & Arthropoda & Insecta & Hymenoptera & Halictidae & Halictinae \\
\hline DIAL0293F09-MD & Arthropoda & Insecta & Hymenoptera & Halictidae & Halictinae \\
\hline 06732D07-MA & Arthropoda & Insecta & Hymenoptera & Halictidae & Halictinae \\
\hline DIAL1390A06-ON & Arthropoda & Insecta & Hymenoptera & Halictidae & Halictinae \\
\hline DIAL0286H01-MB & Arthropoda & Insecta & Hymenoptera & Halictidae & Halictinae \\
\hline D0288D07-SC & Arthropoda & Insecta & Hymenoptera & Halictidae & Halictinae \\
\hline D0288D11-SC & Arthropoda & Insecta & Hymenoptera & Halictidae & Halictinae \\
\hline D00609G05-NS & Arthropoda & Insecta & Hymenoptera & Halictidae & Halictinae \\
\hline DIAL2385E10-MB & Arthropoda & Insecta & Hymenoptera & Halictidae & Halictinae \\
\hline D01561D05-ON & Arthropoda & Insecta & Hymenoptera & Halictidae & Halictinae \\
\hline D01561C04-IN & Arthropoda & Insecta & Hymenoptera & Halictidae & Halictinae \\
\hline DIAL1390G09-NC & Arthropoda & Insecta & Hymenoptera & Halictidae & Halictinae \\
\hline DIAL1848A04-NB & Arthropoda & Insecta & Hymenoptera & Halictidae & Halictinae \\
\hline D0295D03-MD & Arthropoda & Insecta & Hymenoptera & Halictidae & Halictinae \\
\hline DIAL1846C05-MS & Arthropoda & Insecta & Hymenoptera & Halictidae & Halictinae \\
\hline DIAL1413D06-NC & Arthropoda & Insecta & Hymenoptera & Halictidae & Halictinae \\
\hline DIAL0294F01-PA & Arthropoda & Insecta & Hymenoptera & Halictidae & Halictinae \\
\hline DIAL1413A05-IL & Arthropoda & Insecta & Hymenoptera & Halictidae & Halictinae \\
\hline DIAL0293A03-MD & Arthropoda & Insecta & Hymenoptera & Halictidae & Halictinae \\
\hline DIAL0293G02-MD & Arthropoda & Insecta & Hymenoptera & Halictidae & Halictinae \\
\hline DIAL1391E08-NC & Arthropoda & Insecta & Hymenoptera & Halictidae & Halictinae \\
\hline DIAL0294E11-MD & Arthropoda & Insecta & Hymenoptera & Halictidae & Halictinae \\
\hline DIAL0292H12-MD & Arthropoda & Insecta & Hymenoptera & Halictidae & Halictinae \\
\hline DIAL1390E02-ON & Arthropoda & Insecta & Hymenoptera & Halictidae & Halictinae \\
\hline DIAL1413F01-TN & Arthropoda & Insecta & Hymenoptera & Halictidae & Halictinae \\
\hline DIAL1413A07-VA & Arthropoda & Insecta & Hymenoptera & Halictidae & Halictinae \\
\hline DIAL1413H05-NC & Arthropoda & Insecta & Hymenoptera & Halictidae & Halictinae \\
\hline DIAL1413B10-IL & Arthropoda & Insecta & Hymenoptera & Halictidae & Halictinae \\
\hline DIAL1846E01-WV & Arthropoda & Insecta & Hymenoptera & Halictidae & Halictinae \\
\hline DIAL1409A10-NC & Arthropoda & Insecta & Hymenoptera & Halictidae & Halictinae \\
\hline DIAL1409H11-ON & Arthropoda & Insecta & Hymenoptera & Halictidae & Halictinae \\
\hline
\end{tabular}




$\begin{array}{llllll}\text { DIAL1390H03-SC } & \text { Arthropoda } & \text { Insecta } & \text { Hymenoptera } & \text { Halictidae } & \text { Halictinae } \\ \text { DIAL1390C06-ON } & \text { Arthropoda } & \text { Insecta } & \text { Hymenoptera } & \text { Halictidae } & \text { Halictinae } \\ \text { DIAL1390H02-SC } & \text { Arthropoda } & \text { Insecta } & \text { Hymenoptera } & \text { Halictidae } & \text { Halictinae } \\ \text { DIAL1390G05-NC } & \text { Arthropoda } & \text { Insecta } & \text { Hymenoptera } & \text { Halictidae } & \text { Halictinae } \\ \text { DIAL1391A02-VA } & \text { Arthropoda } & \text { Insecta } & \text { Hymenoptera } & \text { Halictidae } & \text { Halictinae } \\ \text { DIAL1413D07-NC } & \text { Arthropoda } & \text { Insecta } & \text { Hymenoptera } & \text { Halictidae } & \text { Halictinae } \\ \text { DIAL1848F06-MD } & \text { Arthropoda } & \text { Insecta } & \text { Hymenoptera } & \text { Halictidae } & \text { Halictinae } \\ \text { D1414A01-YT } & \text { Arthropoda } & \text { Insecta } & \text { Hymenoptera } & \text { Halictidae } & \text { Halictinae } \\ \text { DIAL2387F08-BC } & \text { Arthropoda } & \text { Insecta } & \text { Hymenoptera } & \text { Halictidae } & \text { Halictinae } \\ \text { DIAL1848C02-YT } & \text { Arthropoda } & \text { Insecta } & \text { Hymenoptera } & \text { Halictidae } & \text { Halictinae } \\ \text { BEE0286C11-ON } & \text { Arthropoda } & \text { Insecta } & \text { Hymenoptera } & \text { Halictidae } & \text { Halictinae } \\ \text { D00608H10-OR } & \text { Arthropoda } & \text { Insecta } & \text { Hymenoptera } & \text { Halictidae } & \text { Halictinae } \\ \text { 04485A10-VA } & \text { Arthropoda } & \text { Insecta } & \text { Hymenoptera } & \text { Halictidae } & \text { Halictinae } \\ \text { DIAL1848D10-MD } & \text { Arthropoda } & \text { Insecta } & \text { Hymenoptera } & \text { Halictidae } & \text { Halictinae } \\ \text { DIAL1390D09-ON } & \text { Arthropoda } & \text { Insecta } & \text { Hymenoptera } & \text { Halictidae } & \text { Halictinae } \\ \text { DIAL1848B06-WV } & \text { Arthropoda } & \text { Insecta } & \text { Hymenoptera } & \text { Halictidae } & \text { Halictinae } \\ \text { DIAL1848C08-WV } & \text { Arthropoda } & \text { Insecta } & \text { Hymenoptera } & \text { Halictidae } & \text { Halictinae }\end{array}$




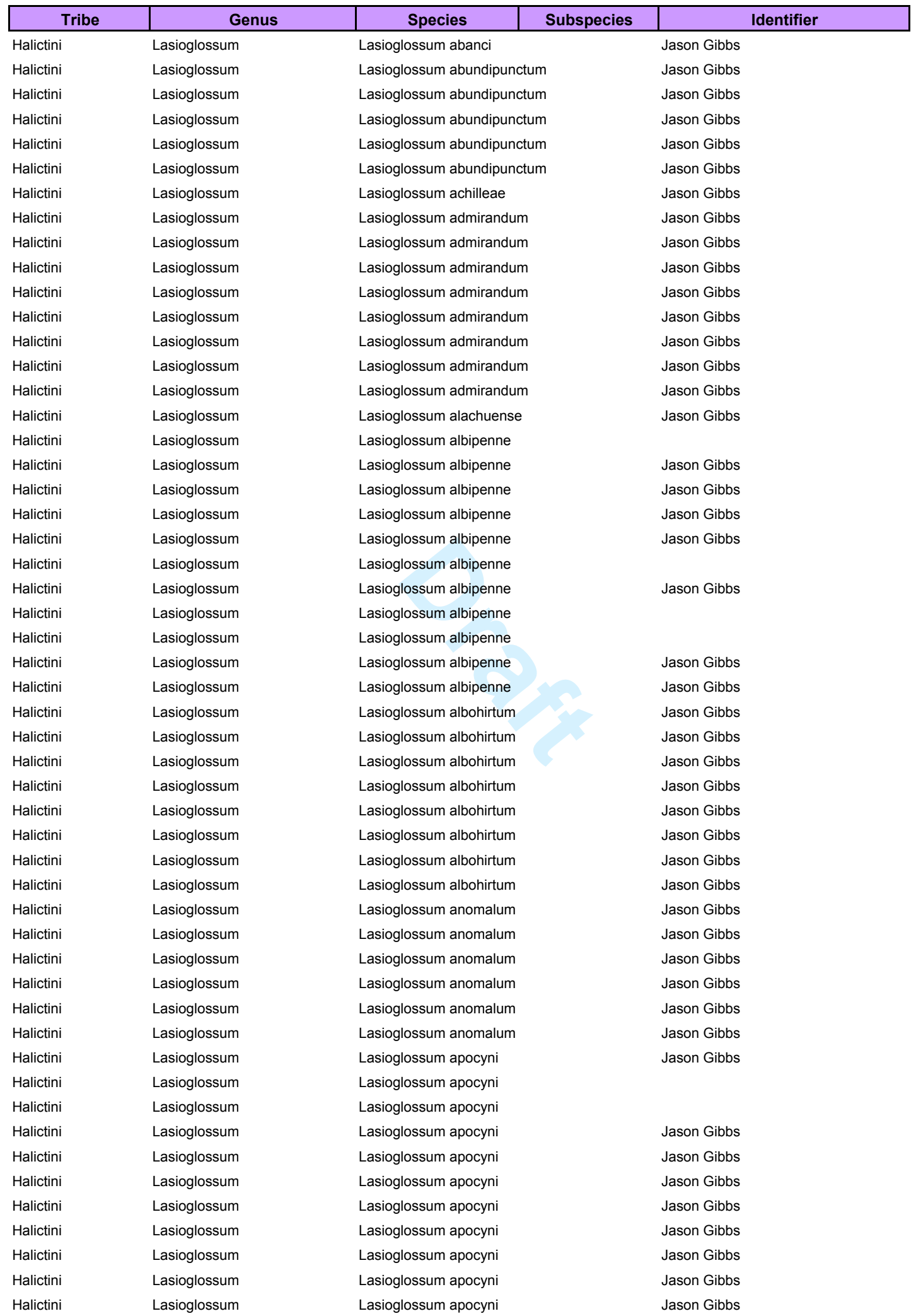




\begin{tabular}{|c|c|c|c|}
\hline Halictini & Lasioglossum & Lasioglossum apocyni & \\
\hline Halictini & Lasioglossum & Lasioglossum apocyni & Jason Gibbs \\
\hline Halictini & Lasioglossum & Lasioglossum apocyni & \\
\hline Halictini & Lasioglossum & Lasioglossum apocyni & Jason Gibbs \\
\hline Halictini & Lasioglossum & Lasioglossum apocyni & Jason Gibbs \\
\hline Halictini & Lasioglossum & Lasioglossum apopkense & \\
\hline Halictini & Lasioglossum & Lasioglossum apopkense & Jason Gibbs \\
\hline Halictini & Lasioglossum & Lasioglossum apopkense & Jason Gibbs \\
\hline Halictini & Lasioglossum & Lasioglossum apopkense & Jason Gibbs \\
\hline Halictini & Lasioglossum & Lasioglossum apopkense & \\
\hline Halictini & Lasioglossum & Lasioglossum arantium & Jason Gibbs \\
\hline Halictini & Lasioglossum & Lasioglossum arantium & Jason Gibbs \\
\hline Halictini & Lasioglossum & Lasioglossum arantium & Jason Gibbs \\
\hline Halictini & Lasioglossum & Lasioglossum arantium & Jason Gibbs \\
\hline Halictini & Lasioglossum & Lasioglossum arantium & Jason Gibbs \\
\hline Halictini & Lasioglossum & Lasioglossum atwoodi & Jason Gibbs \\
\hline Halictini & Lasioglossum & Lasioglossum atwoodi & Jason Gibbs \\
\hline Halictini & Lasioglossum & Lasioglossum atwoodi & Jason Gibbs \\
\hline Halictini & Lasioglossum & Lasioglossum atwoodi & Jason Gibbs \\
\hline Halictini & Lasioglossum & Lasioglossum atwoodi & Jason Gibbs \\
\hline Halictini & Lasioglossum & Lasioglossum atwoodi & Jason Gibbs \\
\hline Halictini & Lasioglossum & Lasioglossum batya & Jason Gibbs \\
\hline Halictini & Lasioglossum & Lasioglossum batya & Jason Gibbs \\
\hline Halictini & Lasioglossum & Lasioglossum bruneri & \\
\hline Halictini & Lasioglossum & Lasioglossum bruneri & Jason Gibbs \\
\hline Halictini & Lasioglossum & Lasioglossum bruneri & \\
\hline Halictini & Lasioglossum & Lasioglossum bruneri & Jason Gibbs \\
\hline Halictini & Lasioglossum & Lasioglossum bruneri & \\
\hline Halictini & Lasioglossum & Lasioglossum bruneri & \\
\hline Halictini & Lasioglossum & Lasioglossum bruneri & Jason Gibbs \\
\hline Halictini & Lasioglossum & Lasioglossum bruneri & Jason Gibbs \\
\hline Halictini & Lasioglossum & Lasioglossum bruneri & \\
\hline Halictini & Lasioglossum & Lasioglossum bruneri & Jason Gibbs \\
\hline Halictini & Lasioglossum & Lasioglossum bruneri & Jason Gibbs \\
\hline Halictini & Lasioglossum & Lasioglossum brunneiventre & Jason Gibbs \\
\hline Halictini & Lasioglossum & Lasioglossum brunneiventre & Jason Gibbs \\
\hline Halictini & Lasioglossum & Lasioglossum brunneiventre & Jason Gibbs \\
\hline Halictini & Lasioglossum & Lasioglossum brunneiventre & Jason Gibbs \\
\hline Halictini & Lasioglossum & Lasioglossum brunneiventre & Jason Gibbs \\
\hline Halictini & Lasioglossum & Lasioglossum brunneiventre & Jason Gibbs \\
\hline Halictini & Lasioglossum & Lasioglossum brunneiventre & Jason Gibbs \\
\hline Halictini & Lasioglossum & Lasioglossum brunneiventre & Jason Gibbs \\
\hline Halictini & Lasioglossum & Lasioglossum brunneiventre & Jason Gibbs \\
\hline Halictini & Lasioglossum & Lasioglossum brunneiventre & Jason Gibbs \\
\hline Halictini & Lasioglossum & Lasioglossum brunneiventre & Jason Gibbs \\
\hline Halictini & Lasioglossum & Lasioglossum brunneiventre & Jason Gibbs \\
\hline Halictini & Lasioglossum & Lasioglossum brunneiventre & Jason Gibbs \\
\hline Halictini & Lasioglossum & Lasioglossum brunneiventre & Jason Gibbs \\
\hline Halictini & Lasioglossum & Lasioglossum callidum & Jason Gibbs \\
\hline Halictini & Lasioglossum & Lasioglossum callidum & Jason Gibbs \\
\hline Halictini & Lasioglossum & Lasioglossum callidum & Jason Gibbs \\
\hline Halictini & Lasioglossum & Lasioglossum callidum & Jason Gibbs \\
\hline Halictini & Lasioglossum & Lasioglossum callidum & Jason Gibbs \\
\hline Halictini & Lasioglossum & Lasioglossum callidum & Jason Gibbs \\
\hline Halictini & Lasioglossum & Lasioglossum callidum & Jason Gibbs \\
\hline
\end{tabular}




\begin{tabular}{|c|c|c|c|}
\hline Halictini & Lasioglossum & Lasioglossum callidum & Jason Gibbs \\
\hline Halictini & Lasioglossum & Lasioglossum callidum & Jason Gibbs \\
\hline Halictini & Lasioglossum & Lasioglossum callidum & Jason Gibbs \\
\hline Halictini & Lasioglossum & Lasioglossum callidum & Jason Gibbs \\
\hline Halictini & Lasioglossum & Lasioglossum callidum & Jason Gibbs \\
\hline Halictini & Lasioglossum & Lasioglossum callidum & Jason Gibbs \\
\hline Halictini & Lasioglossum & Lasioglossum callidum & Jason Gibbs \\
\hline Halictini & Lasioglossum & Lasioglossum callidum & Jason Gibbs \\
\hline Halictini & Lasioglossum & Lasioglossum callidum & Jason Gibbs \\
\hline Halictini & Lasioglossum & Lasioglossum callidum & Jason Gibbs \\
\hline Halictini & Lasioglossum & Lasioglossum callidum & Jason Gibbs \\
\hline Halictini & Lasioglossum & Lasioglossum callidum & Jason Gibbs \\
\hline Halictini & Lasioglossum & Lasioglossum callidum & Jason Gibbs \\
\hline Halictini & Lasioglossum & Lasioglossum callidum & Jason Gibbs \\
\hline Halictini & Lasioglossum & Lasioglossum callidum & Jason Gibbs \\
\hline Halictini & Lasioglossum & Lasioglossum callidum & Jason Gibbs \\
\hline Halictini & Lasioglossum & Lasioglossum callidum & Jason Gibbs \\
\hline Halictini & Lasioglossum & Lasioglossum callidum & Jason Gibbs \\
\hline Halictini & Lasioglossum & Lasioglossum callidum & Jason Gibbs \\
\hline Halictini & Lasioglossum & Lasioglossum callidum & Jason Gibbs \\
\hline Halictini & Lasioglossum & Lasioglossum callidum & Jason Gibbs \\
\hline Halictini & Lasioglossum & Lasioglossum callidum & Jason Gibbs \\
\hline Halictini & Lasioglossum & Lasioglossum callidum & Jason Gibbs \\
\hline Halictini & Lasioglossum & Lasioglossum callidum & Jason Gibbs \\
\hline Halictini & Lasioglossum & Lasioglossum callidum & Jason Gibbs \\
\hline Halictini & Lasioglossum & Lasioglossum callidum & Jason Gibbs \\
\hline Halictini & Lasioglossum & Lasioglossum carlinvillense & Jason Gibbs \\
\hline Halictini & Lasioglossum & Lasioglossum carlinvillense & Jason Gibbs \\
\hline Halictini & Lasioglossum & Lasioglossum carlinvillense & Jason Gibbs \\
\hline Halictini & Lasioglossum & Lasioglossum ceanothi & \\
\hline Halictini & Lasioglossum & Lasioglossum ceanothi & Jason Gibbs \\
\hline Halictini & Lasioglossum & Lasioglossum cf. stuartense & Jason Gibbs \\
\hline Halictini & Lasioglossum & Lasioglossum coreopsis & Jason Gibbs \\
\hline Halictini & Lasioglossum & Lasioglossum coreopsis & Jason Gibbs \\
\hline Halictini & Lasioglossum & Lasioglossum coreopsis & Jason Gibbs \\
\hline Halictini & Lasioglossum & Lasioglossum coreopsis & Jason Gibbs \\
\hline Halictini & Lasioglossum & Lasioglossum coreopsis & Jason Gibbs \\
\hline Halictini & Lasioglossum & Lasioglossum coreopsis & Jason Gibbs \\
\hline Halictini & Lasioglossum & Lasioglossum coreopsis & \\
\hline Halictini & Lasioglossum & Lasioglossum coreopsis & \\
\hline Halictini & Lasioglossum & Lasioglossum coreopsis & \\
\hline Halictini & Lasioglossum & Lasioglossum coreopsis & \\
\hline Halictini & Lasioglossum & Lasioglossum coreopsis & \\
\hline Halictini & Lasioglossum & Lasioglossum coreopsis & Jason Gibbs \\
\hline Halictini & Lasioglossum & Lasioglossum coreopsis & Jason Gibbs \\
\hline Halictini & Lasioglossum & Lasioglossum coreopsis & \\
\hline Halictini & Lasioglossum & Lasioglossum coreopsis & \\
\hline Halictini & Lasioglossum & Lasioglossum coreopsis & \\
\hline Halictini & Lasioglossum & Lasioglossum creberrimum & Jason Gibbs \\
\hline Halictini & Lasioglossum & Lasioglossum creberrimum & John L. Neff \\
\hline Halictini & Lasioglossum & Lasioglossum creberrimum & Jason Gibbs \\
\hline Halictini & Lasioglossum & Lasioglossum creberrimum & \\
\hline Halictini & Lasioglossum & Lasioglossum creberrimum & \\
\hline Halictini & Lasioglossum & Lasioglossum creberrimum & \\
\hline Halictini & Lasioglossum & Lasioglossum creberrimum & Jason Gibbs \\
\hline
\end{tabular}




\begin{tabular}{|c|c|c|c|}
\hline Halictini & Lasioglossum & Lasioglossum creberrimum & Jason Gibbs \\
\hline Halictini & Lasioglossum & Lasioglossum creberrimum & \\
\hline Halictini & Lasioglossum & Lasioglossum creberrimum & \\
\hline Halictini & Lasioglossum & Lasioglossum creberrimum & \\
\hline Halictini & Lasioglossum & Lasioglossum creberrimum & Jason Gibbs \\
\hline Halictini & Lasioglossum & Lasioglossum cressonii & Jason Gibbs \\
\hline Halictini & Lasioglossum & Lasioglossum cressonii & \\
\hline Halictini & Lasioglossum & Lasioglossum cressonii & \\
\hline Halictini & Lasioglossum & Lasioglossum cressonii & \\
\hline Halictini & Lasioglossum & Lasioglossum cressonii & Jason Gibbs \\
\hline Halictini & Lasioglossum & Lasioglossum cressonii & \\
\hline Halictini & Lasioglossum & Lasioglossum cressonii & \\
\hline Halictini & Lasioglossum & Lasioglossum cressonii & \\
\hline Halictini & Lasioglossum & Lasioglossum cressonii & \\
\hline Halictini & Lasioglossum & Lasioglossum cressonii & \\
\hline Halictini & Lasioglossum & Lasioglossum cressonii & \\
\hline Halictini & Lasioglossum & Lasioglossum cressonii & \\
\hline Halictini & Lasioglossum & Lasioglossum cressonii & Jason Gibbs \\
\hline Halictini & Lasioglossum & Lasioglossum cressonii & Jason Gibbs \\
\hline Halictini & Lasioglossum & Lasioglossum cressonii & \\
\hline Halictini & Lasioglossum & Lasioglossum cressonii & Jason Gibbs \\
\hline Halictini & Lasioglossum & Lasioglossum cressonii & Jason Gibbs \\
\hline Halictini & Lasioglossum & Lasioglossum cressonii & Sam W. Droege \\
\hline Halictini & Lasioglossum & Lasioglossum cressonii & Jason Gibbs \\
\hline Halictini & Lasioglossum & Lasioglossum cressonii & Jason Gibbs \\
\hline Halictini & Lasioglossum & Lasioglossum cressonii & \\
\hline Halictini & Lasioglossum & Lasioglossum cressonii & \\
\hline Halictini & Lasioglossum & Lasioglossum cressonii & \\
\hline Halictini & Lasioglossum & Lasioglossum cressonii & \\
\hline Halictini & Lasioglossum & Lasioglossum cressonii & \\
\hline Halictini & Lasioglossum & Lasioglossum cressonii & Jason Gibbs \\
\hline Halictini & Lasioglossum & Lasioglossum cressonii & \\
\hline Halictini & Lasioglossum & Lasioglossum dashwoodi & Jason Gibbs \\
\hline Halictini & Lasioglossum & Lasioglossum dashwoodi & Jason Gibbs \\
\hline Halictini & Lasioglossum & Lasioglossum disparile & \\
\hline Halictini & Lasioglossum & Lasioglossum disparile & Jason Gibbs \\
\hline Halictini & Lasioglossum & Lasioglossum disparile & Jason Gibbs \\
\hline Halictini & Lasioglossum & Lasioglossum disparile & Jason Gibbs \\
\hline Halictini & Lasioglossum & Lasioglossum disparile & Jason Gibbs \\
\hline Halictini & Lasioglossum & Lasioglossum disparile & Jason Gibbs \\
\hline Halictini & Lasioglossum & Lasioglossum disparile & Jason Gibbs \\
\hline Halictini & Lasioglossum & Lasioglossum disparile & Jason Gibbs \\
\hline Halictini & Lasioglossum & Lasioglossum disparile & Jason Gibbs \\
\hline Halictini & Lasioglossum & Lasioglossum dreisbachi & Jason Gibbs \\
\hline Halictini & Lasioglossum & Lasioglossum dreisbachi & Jason Gibbs \\
\hline Halictini & Lasioglossum & Lasioglossum dreisbachi & Jason Gibbs \\
\hline Halictini & Lasioglossum & Lasioglossum dreisbachi & Jason Gibbs \\
\hline Halictini & Lasioglossum & Lasioglossum ebmerellum & Jason Gibbs \\
\hline Halictini & Lasioglossum & Lasioglossum ebmerellum & Jason Gibbs \\
\hline Halictini & Lasioglossum & Lasioglossum ebmerellum & Jason Gibbs \\
\hline Halictini & Lasioglossum & Lasioglossum ebmerellum & Jason Gibbs \\
\hline Halictini & Lasioglossum & Lasioglossum ebmerellum & Jason Gibbs \\
\hline Halictini & Lasioglossum & Lasioglossum ebmerellum & Jason Gibbs \\
\hline Halictini & Lasioglossum & Lasioglossum ellisiae & Jason Gibbs \\
\hline Halictini & Lasioglossum & Lasioglossum ellisiae & Jason Gibbs \\
\hline
\end{tabular}




\begin{tabular}{|c|c|c|c|}
\hline Halictini & Lasioglossum & Lasioglossum ellisiae & Jason Gibbs \\
\hline Halictini & Lasioglossum & Lasioglossum ellisiae & Jason Gibbs \\
\hline Halictini & Lasioglossum & Lasioglossum ellisiae & Jason Gibbs \\
\hline Halictini & Lasioglossum & Lasioglossum ellisiae & Jason Gibbs \\
\hline Halictini & Lasioglossum & Lasioglossum ellisiae & Jason Gibbs \\
\hline Halictini & Lasioglossum & Lasioglossum ellisiae & Jason Gibbs \\
\hline Halictini & Lasioglossum & Lasioglossum ellisiae & Jason Gibbs \\
\hline Halictini & Lasioglossum & Lasioglossum ellisiae & Jason Gibbs \\
\hline Halictini & Lasioglossum & Lasioglossum ellisiae & Jason Gibbs \\
\hline Halictini & Lasioglossum & Lasioglossum ellisiae & Jason Gibbs \\
\hline Halictini & Lasioglossum & Lasioglossum ellisiae & Jason Gibbs \\
\hline Halictini & Lasioglossum & Lasioglossum ellisiae & Jason Gibbs \\
\hline Halictini & Lasioglossum & Lasioglossum ellisiae & Jason Gibbs \\
\hline Halictini & Lasioglossum & Lasioglossum ellisiae & Jason Gibbs \\
\hline Halictini & Lasioglossum & Lasioglossum ellisiae & Jason Gibbs \\
\hline Halictini & Lasioglossum & Lasioglossum ellisiae & Jason Gibbs \\
\hline Halictini & Lasioglossum & Lasioglossum ellisiae & Jason Gibbs \\
\hline Halictini & Lasioglossum & Lasioglossum ellisiae & Jason Gibbs \\
\hline Halictini & Lasioglossum & Lasioglossum ellisiae & Jason Gibbs \\
\hline Halictini & Lasioglossum & Lasioglossum ephialtum & Jason Gibbs \\
\hline Halictini & Lasioglossum & Lasioglossum ephialtum & Jason Gibbs \\
\hline Halictini & Lasioglossum & Lasioglossum ephialtum & Jason Gibbs \\
\hline Halictini & Lasioglossum & Lasioglossum ephialtum & Jason Gibbs \\
\hline Halictini & Lasioglossum & Lasioglossum ephialtum & Jason Gibbs \\
\hline Halictini & Lasioglossum & Lasioglossum ephialtum & Jason Gibbs \\
\hline Halictini & Lasioglossum & Lasioglossum ephialtum & Jason Gibbs \\
\hline Halictini & Lasioglossum & Lasioglossum ephialtum & Jason Gibbs \\
\hline Halictini & Lasioglossum & Lasioglossum ephialtum & Jason Gibbs \\
\hline Halictini & Lasioglossum & Lasioglossum ephialtum & Jason Gibbs \\
\hline Halictini & Lasioglossum & Lasioglossum ephialtum & Jason Gibbs \\
\hline Halictini & Lasioglossum & Lasioglossum ephialtum & Jason Gibbs \\
\hline Halictini & Lasioglossum & Lasioglossum ephialtum & Jason Gibbs \\
\hline Halictini & Lasioglossum & Lasioglossum ephialtum & Jason Gibbs \\
\hline Halictini & Lasioglossum & Lasioglossum ephialtum & Jason Gibbs \\
\hline Halictini & Lasioglossum & Lasioglossum ephialtum & Jason Gibbs \\
\hline Halictini & Lasioglossum & Lasioglossum ephialtum & Jason Gibbs \\
\hline Halictini & Lasioglossum & Lasioglossum ephialtum & Jason Gibbs \\
\hline Halictini & Lasioglossum & Lasioglossum ephialtum & Jason Gibbs \\
\hline Halictini & Lasioglossum & Lasioglossum ephialtum & Jason Gibbs \\
\hline Halictini & Lasioglossum & Lasioglossum ephialtum & Jason Gibbs \\
\hline Halictini & Lasioglossum & Lasioglossum ephialtum & Jason Gibbs \\
\hline Halictini & Lasioglossum & Lasioglossum ephialtum & Jason Gibbs \\
\hline Halictini & Lasioglossum & Lasioglossum ephialtum & Jason Gibbs \\
\hline Halictini & Lasioglossum & Lasioglossum ephialtum & Jason Gibbs \\
\hline Halictini & Lasioglossum & Lasioglossum ephialtum & Jason Gibbs \\
\hline Halictini & Lasioglossum & Lasioglossum ephialtum & Jason Gibbs \\
\hline Halictini & Lasioglossum & Lasioglossum ephialtum & Jason Gibbs \\
\hline Halictini & Lasioglossum & Lasioglossum ephialtum & Jason Gibbs \\
\hline Halictini & Lasioglossum & Lasioglossum ephialtum & Jason Gibbs \\
\hline Halictini & Lasioglossum & Lasioglossum ephialtum & Jason Gibbs \\
\hline Halictini & Lasioglossum & Lasioglossum ephialtum & Jason Gibbs \\
\hline Halictini & Lasioglossum & Lasioglossum ephialtum & Jason Gibbs \\
\hline Halictini & Lasioglossum & Lasioglossum ephialtum & Jason Gibbs \\
\hline Halictini & Lasioglossum & Lasioglossum ephialtum & Jason Gibbs \\
\hline Halictini & Lasioglossum & Lasioglossum ephialtum & Jason Gibbs \\
\hline
\end{tabular}




\begin{tabular}{|c|c|c|c|}
\hline Halictini & Lasioglossum & Lasioglossum ephialtum & Jason Gibbs \\
\hline Halictini & Lasioglossum & Lasioglossum ephialtum & Jason Gibbs \\
\hline Halictini & Lasioglossum & Lasioglossum ephialtum & Jason Gibbs \\
\hline Halictini & Lasioglossum & Lasioglossum ephialtum & Jason Gibbs \\
\hline Halictini & Lasioglossum & Lasioglossum ephialtum & Jason Gibbs \\
\hline Halictini & Lasioglossum & Lasioglossum ephialtum & Jason Gibbs \\
\hline Halictini & Lasioglossum & Lasioglossum ephialtum & Jason Gibbs \\
\hline Halictini & Lasioglossum & Lasioglossum ephialtum & Jason Gibbs \\
\hline Halictini & Lasioglossum & Lasioglossum ephialtum & Jason Gibbs \\
\hline Halictini & Lasioglossum & Lasioglossum fattigi & Jason Gibbs \\
\hline Halictini & Lasioglossum & Lasioglossum fattigi & Jason Gibbs \\
\hline Halictini & Lasioglossum & Lasioglossum fattigi & Jason Gibbs \\
\hline Halictini & Lasioglossum & Lasioglossum fattigi & Jason Gibbs \\
\hline Halictini & Lasioglossum & Lasioglossum flaveriae & \\
\hline Halictini & Lasioglossum & Lasioglossum floridanum & Jason Gibbs \\
\hline Halictini & Lasioglossum & Lasioglossum floridanum & Jason Gibbs \\
\hline Halictini & Lasioglossum & Lasioglossum floridanum & Jason Gibbs \\
\hline Halictini & Lasioglossum & Lasioglossum floridanum & Jason Gibbs \\
\hline Halictini & Lasioglossum & Lasioglossum foveolatum & Jason Gibbs \\
\hline Halictini & Lasioglossum & Lasioglossum foveolatum & Jason Gibbs \\
\hline Halictini & Lasioglossum & Lasioglossum foveolatum & Jason Gibbs \\
\hline Halictini & Lasioglossum & Lasioglossum foveolatum & Jason Gibbs \\
\hline Halictini & Lasioglossum & Lasioglossum foveolatum & Jason Gibbs \\
\hline Halictini & Lasioglossum & Lasioglossum foveolatum & Jason Gibbs \\
\hline Halictini & Lasioglossum & Lasioglossum foveolatum & Jason Gibbs \\
\hline Halictini & Lasioglossum & Lasioglossum foveolatum & Jason Gibbs \\
\hline Halictini & Lasioglossum & Lasioglossum foveolatum & Jason Gibbs \\
\hline Halictini & Lasioglossum & Lasioglossum foveolatum & Jason Gibbs \\
\hline Halictini & Lasioglossum & Lasioglossum georgeickworti & Jason Gibbs \\
\hline Halictini & Lasioglossum & Lasioglossum georgeickworti & Jason Gibbs \\
\hline Halictini & Lasioglossum & Lasioglossum georgeickworti & Jason Gibbs \\
\hline Halictini & Lasioglossum & Lasioglossum georgeickworti & Jason Gibbs \\
\hline Halictini & Lasioglossum & Lasioglossum georgeickworti & Jason Gibbs \\
\hline Halictini & Lasioglossum & Lasioglossum georgeickworti & Jason Gibbs \\
\hline Halictini & Lasioglossum & Lasioglossum georgeickworti & Jason Gibbs \\
\hline Halictini & Lasioglossum & Lasioglossum georgeickworti & Jason Gibbs \\
\hline Halictini & Lasioglossum & Lasioglossum halophitum & Jason Gibbs \\
\hline Halictini & Lasioglossum & Lasioglossum halophitum & \\
\hline Halictini & Lasioglossum & Lasioglossum halophitum & \\
\hline Halictini & Lasioglossum & Lasioglossum halophitum & \\
\hline Halictini & Lasioglossum & Lasioglossum halophitum & \\
\hline Halictini & Lasioglossum & Lasioglossum halophitum & \\
\hline Halictini & Lasioglossum & Lasioglossum halophitum & Jason Gibbs \\
\hline Halictini & Lasioglossum & Lasioglossum halophitum & Jason Gibbs \\
\hline Halictini & Lasioglossum & Lasioglossum halophitum & \\
\hline Halictini & Lasioglossum & Lasioglossum halophitum & Jason Gibbs \\
\hline Halictini & Lasioglossum & Lasioglossum halophitum & Jason Gibbs \\
\hline Halictini & Lasioglossum & Lasioglossum hartii & Jason Gibbs \\
\hline Halictini & Lasioglossum & Lasioglossum hartii & Jason Gibbs \\
\hline Halictini & Lasioglossum & Lasioglossum hemimelas & \\
\hline Halictini & Lasioglossum & Lasioglossum heterognathum & \\
\hline Halictini & Lasioglossum & Lasioglossum heterognathum & \\
\hline Halictini & Lasioglossum & Lasioglossum heterognathum & \\
\hline Halictini & Lasioglossum & Lasioglossum hitchensi & Jason Gibbs \\
\hline Halictini & Lasioglossum & Lasioglossum hitchensi & Jason Gibbs \\
\hline
\end{tabular}




\begin{tabular}{|c|c|c|c|}
\hline Halictini & Lasioglossum & Lasioglossum hitchensi & Jason Gibbs \\
\hline Halictini & Lasioglossum & Lasioglossum hitchensi & Jason Gibbs \\
\hline Halictini & Lasioglossum & Lasioglossum hitchensi & Jason Gibbs \\
\hline Halictini & Lasioglossum & Lasioglossum hitchensi & Jason Gibbs \\
\hline Halictini & Lasioglossum & Lasioglossum hitchensi & Jason Gibbs \\
\hline Halictini & Lasioglossum & Lasioglossum hitchensi & Jason Gibbs \\
\hline Halictini & Lasioglossum & Lasioglossum hitchensi & Jason Gibbs \\
\hline Halictini & Lasioglossum & Lasioglossum hitchensi & Jason Gibbs \\
\hline Halictini & Lasioglossum & Lasioglossum hitchensi & Jason Gibbs \\
\hline Halictini & Lasioglossum & Lasioglossum hitchensi & Jason Gibbs \\
\hline Halictini & Lasioglossum & Lasioglossum hitchensi & Jason Gibbs \\
\hline Halictini & Lasioglossum & Lasioglossum hitchensi & Jason Gibbs \\
\hline Halictini & Lasioglossum & Lasioglossum hitchensi & Jason Gibbs \\
\hline Halictini & Lasioglossum & Lasioglossum hitchensi & Jason Gibbs \\
\hline Halictini & Lasioglossum & Lasioglossum hitchensi & Jason Gibbs \\
\hline Halictini & Lasioglossum & Lasioglossum hitchensi & Jason Gibbs \\
\hline Halictini & Lasioglossum & Lasioglossum hitchensi & Jason Gibbs \\
\hline Halictini & Lasioglossum & Lasioglossum hitchensi & Jason Gibbs \\
\hline Halictini & Lasioglossum & Lasioglossum hitchensi & Jason Gibbs \\
\hline Halictini & Lasioglossum & Lasioglossum hitchensi & Jason Gibbs \\
\hline Halictini & Lasioglossum & Lasioglossum hitchensi & Jason Gibbs \\
\hline Halictini & Lasioglossum & Lasioglossum hitchensi & Jason Gibbs \\
\hline Halictini & Lasioglossum & Lasioglossum hitchensi & Jason Gibbs \\
\hline Halictini & Lasioglossum & Lasioglossum hitchensi & Jason Gibbs \\
\hline Halictini & Lasioglossum & Lasioglossum hitchensi & Jason Gibbs \\
\hline Halictini & Lasioglossum & Lasioglossum hitchensi & Jason Gibbs \\
\hline Halictini & Lasioglossum & Lasioglossum hitchensi & Jason Gibbs \\
\hline Halictini & Lasioglossum & Lasioglossum hitchensi & Jason Gibbs \\
\hline Halictini & Lasioglossum & Lasioglossum hitchensi & Jason Gibbs \\
\hline Halictini & Lasioglossum & Lasioglossum hitchensi & Jason Gibbs \\
\hline Halictini & Lasioglossum & Lasioglossum hitchensi & Jason Gibbs \\
\hline Halictini & Lasioglossum & Lasioglossum hitchensi & Jason Gibbs \\
\hline Halictini & Lasioglossum & Lasioglossum hitchensi & Jason Gibbs \\
\hline Halictini & Lasioglossum & Lasioglossum hitchensi & Jason Gibbs \\
\hline Halictini & Lasioglossum & Lasioglossum hitchensi & Jason Gibbs \\
\hline Halictini & Lasioglossum & Lasioglossum hitchensi & Jason Gibbs \\
\hline Halictini & Lasioglossum & Lasioglossum hitchensi & Jason Gibbs \\
\hline Halictini & Lasioglossum & Lasioglossum hitchensi & Jason Gibbs \\
\hline Halictini & Lasioglossum & Lasioglossum hitchensi & Jason Gibbs \\
\hline Halictini & Lasioglossum & Lasioglossum hitchensi & Jason Gibbs \\
\hline Halictini & Lasioglossum & Lasioglossum hitchensi & Jason Gibbs \\
\hline Halictini & Lasioglossum & Lasioglossum hitchensi & Jason Gibbs \\
\hline Halictini & Lasioglossum & Lasioglossum hitchensi & Jason Gibbs \\
\hline Halictini & Lasioglossum & Lasioglossum hitchensi & Jason Gibbs \\
\hline Halictini & Lasioglossum & Lasioglossum hitchensi & Jason Gibbs \\
\hline Halictini & Lasioglossum & Lasioglossum hitchensi & Jason Gibbs \\
\hline Halictini & Lasioglossum & Lasioglossum hitchensi & Jason Gibbs \\
\hline Halictini & Lasioglossum & Lasioglossum hitchensi & Jason Gibbs \\
\hline Halictini & Lasioglossum & Lasioglossum hitchensi & Jason Gibbs \\
\hline Halictini & Lasioglossum & Lasioglossum hitchensi & Jason Gibbs \\
\hline Halictini & Lasioglossum & Lasioglossum hitchensi & Jason Gibbs \\
\hline Halictini & Lasioglossum & Lasioglossum hitchensi & Jason Gibbs \\
\hline Halictini & Lasioglossum & Lasioglossum hitchensi & Jason Gibbs \\
\hline Halictini & Lasioglossum & Lasioglossum hitchensi & Jason Gibbs \\
\hline Halictini & Lasioglossum & Lasioglossum hitchensi & Jason Gibbs \\
\hline
\end{tabular}




\begin{tabular}{|c|c|c|c|}
\hline Halictini & Lasioglossum & Lasioglossum hitchensi & Jason Gibbs \\
\hline Halictini & Lasioglossum & Lasioglossum hitchensi & Jason Gibbs \\
\hline Halictini & Lasioglossum & Lasioglossum hitchensi & Jason Gibbs \\
\hline Halictini & Lasioglossum & Lasioglossum hitchensi & Jason Gibbs \\
\hline Halictini & Lasioglossum & Lasioglossum hitchensi & Jason Gibbs \\
\hline Halictini & Lasioglossum & Lasioglossum hitchensi & Jason Gibbs \\
\hline Halictini & Lasioglossum & Lasioglossum hitchensi & Jason Gibbs \\
\hline Halictini & Lasioglossum & Lasioglossum hitchensi & Jason Gibbs \\
\hline Halictini & Lasioglossum & Lasioglossum hitchensi & Jason Gibbs \\
\hline Halictini & Lasioglossum & Lasioglossum hitchensi & Jason Gibbs \\
\hline Halictini & Lasioglossum & Lasioglossum hitchensi & Jason Gibbs \\
\hline Halictini & Lasioglossum & Lasioglossum hitchensi & Jason Gibbs \\
\hline Halictini & Lasioglossum & Lasioglossum hitchensi & Jason Gibbs \\
\hline Halictini & Lasioglossum & Lasioglossum hitchensi & Jason Gibbs \\
\hline Halictini & Lasioglossum & Lasioglossum hitchensi & Jason Gibbs \\
\hline Halictini & Lasioglossum & Lasioglossum hitchensi & Jason Gibbs \\
\hline Halictini & Lasioglossum & Lasioglossum hitchensi & Jason Gibbs \\
\hline Halictini & Lasioglossum & Lasioglossum hitchensi & Jason Gibbs \\
\hline Halictini & Lasioglossum & Lasioglossum hudsoniellum & Jason Gibbs \\
\hline Halictini & Lasioglossum & Lasioglossum hudsoniellum & \\
\hline Halictini & Lasioglossum & Lasioglossum hudsoniellum & \\
\hline Halictini & Lasioglossum & Lasioglossum hudsoniellum & \\
\hline Halictini & Lasioglossum & Lasioglossum hudsoniellum & \\
\hline Halictini & Lasioglossum & Lasioglossum hudsoniellum & Jason Gibbs \\
\hline Halictini & Lasioglossum & Lasioglossum hudsoniellum & Jason Gibbs \\
\hline Halictini & Lasioglossum & Lasioglossum hudsoniellum & \\
\hline Halictini & Lasioglossum & Lasioglossum hudsoniellum & Jason Gibbs \\
\hline Halictini & Lasioglossum & Lasioglossum hudsoniellum & \\
\hline Halictini & Lasioglossum & Lasioglossum hudsoniellum & Jason Gibbs \\
\hline Halictini & Lasioglossum & Lasioglossum hudsoniellum & Jason Gibbs \\
\hline Halictini & Lasioglossum & Lasioglossum hudsoniellum & Jason Gibbs \\
\hline Halictini & Lasioglossum & Lasioglossum hudsoniellum & \\
\hline Halictini & Lasioglossum & Lasioglossum hudsoniellum & Jason Gibbs \\
\hline Halictini & Lasioglossum & Lasioglossum hudsoniellum & \\
\hline Halictini & Lasioglossum & Lasioglossum hudsoniellum & Jason Gibbs \\
\hline Halictini & Lasioglossum & Lasioglossum hudsoniellum & \\
\hline Halictini & Lasioglossum & Lasioglossum hudsoniellum & Jason Gibbs \\
\hline Halictini & Lasioglossum & Lasioglossum hyalinum & Jason Gibbs \\
\hline Halictini & Lasioglossum & Lasioglossum hyalinum & Jason Gibbs \\
\hline Halictini & Lasioglossum & Lasioglossum hyalinum & Jason Gibbs \\
\hline Halictini & Lasioglossum & Lasioglossum hyalinum & Jason Gibbs \\
\hline Halictini & Lasioglossum & Lasioglossum illinoense & Jason Gibbs \\
\hline Halictini & Lasioglossum & Lasioglossum illinoense & \\
\hline Halictini & Lasioglossum & Lasioglossum illinoense & Jason Gibbs \\
\hline Halictini & Lasioglossum & Lasioglossum illinoense & \\
\hline Halictini & Lasioglossum & Lasioglossum illinoense & Jason Gibbs \\
\hline Halictini & Lasioglossum & Lasioglossum imbrex & Jason Gibbs \\
\hline Halictini & Lasioglossum & Lasioglossum imbrex & Jason Gibbs \\
\hline Halictini & Lasioglossum & Lasioglossum imbrex & Jason Gibbs \\
\hline Halictini & Lasioglossum & Lasioglossum imbrex & Jason Gibbs \\
\hline Halictini & Lasioglossum & Lasioglossum imbrex & Jason Gibbs \\
\hline Halictini & Lasioglossum & Lasioglossum imbrex & Jason Gibbs \\
\hline Halictini & Lasioglossum & Lasioglossum imbrex & Jason Gibbs \\
\hline Halictini & Lasioglossum & Lasioglossum imbrex & Jason Gibbs \\
\hline Halictini & Lasioglossum & Lasioglossum imbrex & Jason Gibbs \\
\hline
\end{tabular}




\begin{tabular}{|c|c|c|c|}
\hline Halictini & Lasioglossum & Lasioglossum imitatum & Jason Gibbs \\
\hline Halictini & Lasioglossum & Lasioglossum imitatum & \\
\hline Halictini & Lasioglossum & Lasioglossum imitatum & \\
\hline Halictini & Lasioglossum & Lasioglossum imitatum & \\
\hline Halictini & Lasioglossum & Lasioglossum imitatum & \\
\hline Halictini & Lasioglossum & Lasioglossum imitatum & \\
\hline Halictini & Lasioglossum & Lasioglossum imitatum & Jason Gibbs \\
\hline Halictini & Lasioglossum & Lasioglossum imitatum & \\
\hline Halictini & Lasioglossum & Lasioglossum imitatum & \\
\hline Halictini & Lasioglossum & Lasioglossum imitatum & Jason Gibbs \\
\hline Halictini & Lasioglossum & Lasioglossum imitatum & Jason Gibbs \\
\hline Halictini & Lasioglossum & Lasioglossum imitatum & Jason Gibbs \\
\hline Halictini & Lasioglossum & Lasioglossum imitatum & Jason Gibbs \\
\hline Halictini & Lasioglossum & Lasioglossum imitatum & Jason Gibbs \\
\hline Halictini & Lasioglossum & Lasioglossum imitatum & \\
\hline Halictini & Lasioglossum & Lasioglossum imitatum & \\
\hline Halictini & Lasioglossum & Lasioglossum imitatum & Jason Gibbs \\
\hline Halictini & Lasioglossum & Lasioglossum imitatum & \\
\hline Halictini & Lasioglossum & Lasioglossum imitatum & \\
\hline Halictini & Lasioglossum & Lasioglossum imitatum & Jason Gibbs \\
\hline Halictini & Lasioglossum & Lasioglossum imitatum & \\
\hline Halictini & Lasioglossum & Lasioglossum imitatum & \\
\hline Halictini & Lasioglossum & Lasioglossum imitatum & Jason Gibbs \\
\hline Halictini & Lasioglossum & Lasioglossum imitatum & \\
\hline Halictini & Lasioglossum & Lasioglossum imitatum & \\
\hline Halictini & Lasioglossum & Lasioglossum imitatum & \\
\hline Halictini & Lasioglossum & Lasioglossum incompletum & \\
\hline Halictini & Lasioglossum & Lasioglossum incompletum & \\
\hline Halictini & Lasioglossum & Lasioglossum incompletum & \\
\hline Halictini & Lasioglossum & Lasioglossum incompletum & \\
\hline Halictini & Lasioglossum & Lasioglossum incompletum & \\
\hline Halictini & Lasioglossum & Lasioglossum incompletum & \\
\hline Halictini & Lasioglossum & Lasioglossum incompletum & \\
\hline Halictini & Lasioglossum & Lasioglossum incompletum & Jason Gibbs \\
\hline Halictini & Lasioglossum & Lasioglossum incompletum & \\
\hline Halictini & Lasioglossum & Lasioglossum incompletum & Jason Gibbs \\
\hline Halictini & Lasioglossum & Lasioglossum incompletum & Jason Gibbs \\
\hline Halictini & Lasioglossum & Lasioglossum incompletum & Jason Gibbs \\
\hline Halictini & Lasioglossum & Lasioglossum incompletum & \\
\hline Halictini & Lasioglossum & Lasioglossum incompletum & \\
\hline Halictini & Lasioglossum & Lasioglossum izawsum & Jason Gibbs \\
\hline Halictini & Lasioglossum & Lasioglossum izawsum & Jason Gibbs \\
\hline Halictini & Lasioglossum & Lasioglossum katherineae & Jason Gibbs \\
\hline Halictini & Lasioglossum & Lasioglossum katherineae & Jason Gibbs \\
\hline Halictini & Lasioglossum & Lasioglossum katherineae & Jason Gibbs \\
\hline Halictini & Lasioglossum & Lasioglossum katherineae & Jason Gibbs \\
\hline Halictini & Lasioglossum & Lasioglossum katherineae & Jason Gibbs \\
\hline Halictini & Lasioglossum & Lasioglossum katherineae & Jason Gibbs \\
\hline Halictini & Lasioglossum & Lasioglossum knereri & Jason Gibbs \\
\hline Halictini & Lasioglossum & Lasioglossum knereri & Jason Gibbs \\
\hline Halictini & Lasioglossum & Lasioglossum knereri & Jason Gibbs \\
\hline Halictini & Lasioglossum & Lasioglossum knereri & Jason Gibbs \\
\hline Halictini & Lasioglossum & Lasioglossum knereri & Jason Gibbs \\
\hline Halictini & Lasioglossum & Lasioglossum knereri & Jason Gibbs \\
\hline Halictini & Lasioglossum & Lasioglossum knereri & Jason Gibbs \\
\hline
\end{tabular}




\begin{tabular}{|c|c|c|c|}
\hline Halictini & Lasioglossum & Lasioglossum knereri & Jason Gibbs \\
\hline Halictini & Lasioglossum & Lasioglossum knereri & Jason Gibbs \\
\hline Halictini & Lasioglossum & Lasioglossum knereri & Jason Gibbs \\
\hline Halictini & Lasioglossum & Lasioglossum knereri & Jason Gibbs \\
\hline Halictini & Lasioglossum & Lasioglossum knereri & Jason Gibbs \\
\hline Halictini & Lasioglossum & Lasioglossum knereri & Jason Gibbs \\
\hline Halictini & Lasioglossum & Lasioglossum knereri & Jason Gibbs \\
\hline Halictini & Lasioglossum & Lasioglossum knereri & Jason Gibbs \\
\hline Halictini & Lasioglossum & Lasioglossum knereri & Jason Gibbs \\
\hline Halictini & Lasioglossum & Lasioglossum knereri & Jason Gibbs \\
\hline Halictini & Lasioglossum & Lasioglossum knereri & Jason Gibbs \\
\hline Halictini & Lasioglossum & Lasioglossum knereri & Jason Gibbs \\
\hline Halictini & Lasioglossum & Lasioglossum knereri & Jason Gibbs \\
\hline Halictini & Lasioglossum & Lasioglossum knereri & Jason Gibbs \\
\hline Halictini & Lasioglossum & Lasioglossum knereri & Jason Gibbs \\
\hline Halictini & Lasioglossum & Lasioglossum knereri & Jason Gibbs \\
\hline Halictini & Lasioglossum & Lasioglossum knereri & Jason Gibbs \\
\hline Halictini & Lasioglossum & Lasioglossum knereri & Jason Gibbs \\
\hline Halictini & Lasioglossum & Lasioglossum knereri & Jason Gibbs \\
\hline Halictini & Lasioglossum & Lasioglossum knereri & Jason Gibbs \\
\hline Halictini & Lasioglossum & Lasioglossum knereri & Jason Gibbs \\
\hline Halictini & Lasioglossum & Lasioglossum knereri & Jason Gibbs \\
\hline Halictini & Lasioglossum & Lasioglossum knereri & Jason Gibbs \\
\hline Halictini & Lasioglossum & Lasioglossum knereri & Jason Gibbs \\
\hline Halictini & Lasioglossum & Lasioglossum knereri & Jason Gibbs \\
\hline Halictini & Lasioglossum & Lasioglossum knereri & Jason Gibbs \\
\hline Halictini & Lasioglossum & Lasioglossum knereri & Jason Gibbs \\
\hline Halictini & Lasioglossum & Lasioglossum knereri & Jason Gibbs \\
\hline Halictini & Lasioglossum & Lasioglossum knereri & Jason Gibbs \\
\hline Halictini & Lasioglossum & Lasioglossum laevissimum & \\
\hline Halictini & Lasioglossum & Lasioglossum laevissimum & \\
\hline Halictini & Lasioglossum & Lasioglossum laevissimum & \\
\hline Halictini & Lasioglossum & Lasioglossum laevissimum & Jason Gibbs \\
\hline Halictini & Lasioglossum & Lasioglossum laevissimum & \\
\hline Halictini & Lasioglossum & Lasioglossum laevissimum & Jason Gibbs \\
\hline Halictini & Lasioglossum & Lasioglossum laevissimum & \\
\hline Halictini & Lasioglossum & Lasioglossum laevissimum & \\
\hline Halictini & Lasioglossum & Lasioglossum laevissimum & Jason Gibbs \\
\hline Halictini & Lasioglossum & Lasioglossum laevissimum & Jason Gibbs \\
\hline Halictini & Lasioglossum & Lasioglossum laevissimum & Jason Gibbs \\
\hline Halictini & Lasioglossum & Lasioglossum laevissimum & Jason Gibbs \\
\hline Halictini & Lasioglossum & Lasioglossum laevissimum & Jason Gibbs \\
\hline Halictini & Lasioglossum & Lasioglossum laevissimum & Jason Gibbs \\
\hline Halictini & Lasioglossum & Lasioglossum laevissimum & Jason Gibbs \\
\hline Halictini & Lasioglossum & Lasioglossum laevissimum & Jason Gibbs \\
\hline Halictini & Lasioglossum & Lasioglossum laevissimum & Jason Gibbs \\
\hline Halictini & Lasioglossum & Lasioglossum laevissimum & Jason Gibbs \\
\hline Halictini & Lasioglossum & Lasioglossum laevissimum & Jason Gibbs \\
\hline Halictini & Lasioglossum & Lasioglossum laevissimum & Jason Gibbs \\
\hline Halictini & Lasioglossum & Lasioglossum laevissimum & Jason Gibbs \\
\hline Halictini & Lasioglossum & Lasioglossum laevissimum & Jason Gibbs \\
\hline Halictini & Lasioglossum & Lasioglossum laevissimum & Jason Gibbs \\
\hline Halictini & Lasioglossum & Lasioglossum laevissimum & Jason Gibbs \\
\hline Halictini & Lasioglossum & Lasioglossum laevissimum & Jason Gibbs \\
\hline Halictini & Lasioglossum & Lasioglossum laevissimum & \\
\hline
\end{tabular}




\begin{tabular}{|c|c|c|c|}
\hline Halictini & Lasioglossum & Lasioglossum laevissimum & Jason Gibbs \\
\hline Halictini & Lasioglossum & Lasioglossum laevissimum & \\
\hline Halictini & Lasioglossum & Lasioglossum laevissimum & \\
\hline Halictini & Lasioglossum & Lasioglossum laevissimum & Jason Gibbs \\
\hline Halictini & Lasioglossum & Lasioglossum laevissimum & Jason Gibbs \\
\hline Halictini & Lasioglossum & Lasioglossum laevissimum & Jason Gibbs \\
\hline Halictini & Lasioglossum & Lasioglossum laevissimum & Jason Gibbs \\
\hline Halictini & Lasioglossum & Lasioglossum laevissimum & Jason Gibbs \\
\hline Halictini & Lasioglossum & Lasioglossum laevissimum & \\
\hline Halictini & Lasioglossum & Lasioglossum laevissimum & \\
\hline Halictini & Lasioglossum & Lasioglossum laevissimum & \\
\hline Halictini & Lasioglossum & Lasioglossum laevissimum & \\
\hline Halictini & Lasioglossum & Lasioglossum laevissimum & \\
\hline Halictini & Lasioglossum & Lasioglossum laevissimum & Jason Gibbs \\
\hline Halictini & Lasioglossum & Lasioglossum laevissimum & Jason Gibbs \\
\hline Halictini & Lasioglossum & Lasioglossum laevissimum & \\
\hline Halictini & Lasioglossum & Lasioglossum lepidii & Jason Gibbs \\
\hline Halictini & Lasioglossum & Lasioglossum lepidii & Jason Gibbs \\
\hline Halictini & Lasioglossum & Lasioglossum lepidii & Jason Gibbs \\
\hline Halictini & Lasioglossum & Lasioglossum leucocomum & Jason Gibbs \\
\hline Halictini & Lasioglossum & Lasioglossum leucocomum & Jason Gibbs \\
\hline Halictini & Lasioglossum & Lasioglossum leucocomum & Jason Gibbs \\
\hline Halictini & Lasioglossum & Lasioglossum leucocomum & Jason Gibbs \\
\hline Halictini & Lasioglossum & Lasioglossum leucocomum & Jason Gibbs \\
\hline Halictini & Lasioglossum & Lasioglossum leucocomum & Jason Gibbs \\
\hline Halictini & Lasioglossum & Lasioglossum leucocomum & Jason Gibbs \\
\hline Halictini & Lasioglossum & Lasioglossum leucocomum & Jason Gibbs \\
\hline Halictini & Lasioglossum & Lasioglossum leucocomum & Jason Gibbs \\
\hline Halictini & Lasioglossum & Lasioglossum leucocomum & Jason Gibbs \\
\hline Halictini & Lasioglossum & Lasioglossum leucocomum & Jason Gibbs \\
\hline Halictini & Lasioglossum & Lasioglossum leucocomum & Jason Gibbs \\
\hline Halictini & Lasioglossum & Lasioglossum leviense & Jason Gibbs \\
\hline Halictini & Lasioglossum & Lasioglossum leviense & Jason Gibbs \\
\hline Halictini & Lasioglossum & Lasioglossum leviense & Jason Gibbs \\
\hline Halictini & Lasioglossum & Lasioglossum leviense & Jason Gibbs \\
\hline Halictini & Lasioglossum & Lasioglossum lineatulum & \\
\hline Halictini & Lasioglossum & Lasioglossum lineatulum & Jason Gibbs \\
\hline Halictini & Lasioglossum & Lasioglossum lineatulum & \\
\hline Halictini & Lasioglossum & Lasioglossum lineatulum & \\
\hline Halictini & Lasioglossum & Lasioglossum lineatulum & \\
\hline Halictini & Lasioglossum & Lasioglossum lineatulum & \\
\hline Halictini & Lasioglossum & Lasioglossum lineatulum & Jason Gibbs \\
\hline Halictini & Lasioglossum & Lasioglossum lineatulum & Jason Gibbs \\
\hline Halictini & Lasioglossum & Lasioglossum lineatulum & \\
\hline Halictini & Lasioglossum & Lasioglossum lineatulum & Jason Gibbs \\
\hline Halictini & Lasioglossum & Lasioglossum lineatulum & Jason Gibbs \\
\hline Halictini & Lasioglossum & Lasioglossum lineatulum & \\
\hline Halictini & Lasioglossum & Lasioglossum lineatulum & \\
\hline Halictini & Lasioglossum & Lasioglossum lineatulum & \\
\hline Halictini & Lasioglossum & Lasioglossum lionotum & Jason Gibbs \\
\hline Halictini & Lasioglossum & Lasioglossum lionotum & Jason Gibbs \\
\hline Halictini & Lasioglossum & Lasioglossum lionotum & Jason Gibbs \\
\hline Halictini & Lasioglossum & Lasioglossum longifrons & Jason Gibbs \\
\hline Halictini & Lasioglossum & Lasioglossum longifrons & Jason Gibbs \\
\hline Halictini & Lasioglossum & Lasioglossum longifrons & Jason Gibbs \\
\hline
\end{tabular}




\begin{tabular}{|c|c|c|c|}
\hline Halictini & Lasioglossum & Lasioglossum longifrons & Jason Gibbs \\
\hline Halictini & Lasioglossum & Lasioglossum longifrons & Jason Gibbs \\
\hline Halictini & Lasioglossum & Lasioglossum longifrons & Jason Gibbs \\
\hline Halictini & Lasioglossum & Lasioglossum longifrons & Jason Gibbs \\
\hline Halictini & Lasioglossum & Lasioglossum longifrons & Jason Gibbs \\
\hline Halictini & Lasioglossum & Lasioglossum longifrons & Jason Gibbs \\
\hline Halictini & Lasioglossum & Lasioglossum macroprosopum & Jason Gibbs \\
\hline Halictini & Lasioglossum & Lasioglossum macroprosopum & Jason Gibbs \\
\hline Halictini & Lasioglossum & Lasioglossum marinense & Jason Gibbs \\
\hline Halictini & Lasioglossum & Lasioglossum marinense & Jason Gibbs \\
\hline Halictini & Lasioglossum & Lasioglossum marinense & Jason Gibbs \\
\hline Halictini & Lasioglossum & Lasioglossum marinense & Jason Gibbs \\
\hline Halictini & Lasioglossum & Lasioglossum marinense & Jason Gibbs \\
\hline Halictini & Lasioglossum & Lasioglossum marinense & Jason Gibbs \\
\hline Halictini & Lasioglossum & Lasioglossum marinense & Jason Gibbs \\
\hline Halictini & Lasioglossum & Lasioglossum marinense & Jason Gibbs \\
\hline Halictini & Lasioglossum & Lasioglossum marinense & Jason Gibbs \\
\hline Halictini & Lasioglossum & Lasioglossum marinum & \\
\hline Halictini & Lasioglossum & Lasioglossum marinum & \\
\hline Halictini & Lasioglossum & Lasioglossum marinum & Jason Gibbs \\
\hline Halictini & Lasioglossum & Lasioglossum marinum & Jason Gibbs \\
\hline Halictini & Lasioglossum & Lasioglossum marinum & \\
\hline Halictini & Lasioglossum & Lasioglossum marinum & \\
\hline Halictini & Lasioglossum & Lasioglossum marinum & \\
\hline Halictini & Lasioglossum & Lasioglossum marinum & Jason Gibbs \\
\hline Halictini & Lasioglossum & Lasioglossum michiganense & Jason Gibbs \\
\hline Halictini & Lasioglossum & Lasioglossum michiganense & Jason Gibbs \\
\hline Halictini & Lasioglossum & Lasioglossum michiganense & Jason Gibbs \\
\hline Halictini & Lasioglossum & Lasioglossum miniatulum & \\
\hline Halictini & Lasioglossum & Lasioglossum miniatulum & \\
\hline Halictini & Lasioglossum & Lasioglossum miniatulum & Jason Gibbs \\
\hline Halictini & Lasioglossum & Lasioglossum miniatulum & Jason Gibbs \\
\hline Halictini & Lasioglossum & Lasioglossum miniatulum & Jason Gibbs \\
\hline Halictini & Lasioglossum & Lasioglossum miniatulum & Jason Gibbs \\
\hline Halictini & Lasioglossum & Lasioglossum nevadense & Jason Gibbs \\
\hline Halictini & Lasioglossum & Lasioglossum nevadense & \\
\hline Halictini & Lasioglossum & Lasioglossum nevadense & Jason Gibbs \\
\hline Halictini & Lasioglossum & Lasioglossum nevadense & Jason Gibbs \\
\hline Halictini & Lasioglossum & Lasioglossum nevadense & Jason Gibbs \\
\hline Halictini & Lasioglossum & Lasioglossum nevadense & Jason Gibbs \\
\hline Halictini & Lasioglossum & Lasioglossum nevadense & Jason Gibbs \\
\hline Halictini & Lasioglossum & Lasioglossum nevadense & \\
\hline Halictini & Lasioglossum & Lasioglossum nevadense & \\
\hline Halictini & Lasioglossum & Lasioglossum nevadense & Jason Gibbs \\
\hline Halictini & Lasioglossum & Lasioglossum nevadense & Jason Gibbs \\
\hline Halictini & Lasioglossum & Lasioglossum nevadense & \\
\hline Halictini & Lasioglossum & Lasioglossum nevadense & Jason Gibbs \\
\hline Halictini & Lasioglossum & Lasioglossum nevadense & \\
\hline Halictini & Lasioglossum & Lasioglossum nevadense & Jason Gibbs \\
\hline Halictini & Lasioglossum & Lasioglossum nevadense & \\
\hline Halictini & Lasioglossum & Lasioglossum nevadense & \\
\hline Halictini & Lasioglossum & Lasioglossum nevadense & \\
\hline Halictini & Lasioglossum & Lasioglossum nevadense & Jason Gibbs \\
\hline Halictini & Lasioglossum & Lasioglossum nevadense & \\
\hline Halictini & Lasioglossum & Lasioglossum nevadense & \\
\hline
\end{tabular}




\begin{tabular}{|c|c|c|c|}
\hline Halictini & Lasioglossum & Lasioglossum nevadense & Jason Gibbs \\
\hline Halictini & Lasioglossum & Lasioglossum nigroviride & Jason Gibbs \\
\hline Halictini & Lasioglossum & Lasioglossum nigroviride & Jason Gibbs \\
\hline Halictini & Lasioglossum & Lasioglossum nigroviride & Jason Gibbs \\
\hline Halictini & Lasioglossum & Lasioglossum nigroviride & Jason Gibbs \\
\hline Halictini & Lasioglossum & Lasioglossum nigroviride & Jason Gibbs \\
\hline Halictini & Lasioglossum & Lasioglossum nigroviride & Jason Gibbs \\
\hline Halictini & Lasioglossum & Lasioglossum nigroviride & Jason Gibbs \\
\hline Halictini & Lasioglossum & Lasioglossum novascotiae & Jason Gibbs \\
\hline Halictini & Lasioglossum & Lasioglossum nymphale & \\
\hline Halictini & Lasioglossum & Lasioglossum nymphale & \\
\hline Halictini & Lasioglossum & Lasioglossum nymphale & \\
\hline Halictini & Lasioglossum & Lasioglossum nymphale & \\
\hline Halictini & Lasioglossum & Lasioglossum nymphale & \\
\hline Halictini & Lasioglossum & Lasioglossum nymphale & \\
\hline Halictini & Lasioglossum & Lasioglossum nymphale & \\
\hline Halictini & Lasioglossum & Lasioglossum nymphale & \\
\hline Halictini & Lasioglossum & Lasioglossum nymphale & \\
\hline Halictini & Lasioglossum & Lasioglossum nymphale & \\
\hline Halictini & Lasioglossum & Lasioglossum nymphale & \\
\hline Halictini & Lasioglossum & Lasioglossum nymphale & Jason Gibbs \\
\hline Halictini & Lasioglossum & Lasioglossum nymphale & \\
\hline Halictini & Lasioglossum & Lasioglossum nymphale & Jason Gibbs \\
\hline Halictini & Lasioglossum & Lasioglossum nymphale & \\
\hline Halictini & Lasioglossum & Lasioglossum nymphale & \\
\hline Halictini & Lasioglossum & Lasioglossum nymphale & \\
\hline Halictini & Lasioglossum & Lasioglossum oblongum & \\
\hline Halictini & Lasioglossum & Lasioglossum oblongum & \\
\hline Halictini & Lasioglossum & Lasioglossum oblongum & Jason Gibbs \\
\hline Halictini & Lasioglossum & Lasioglossum oblongum & \\
\hline Halictini & Lasioglossum & Lasioglossum oblongum & Jason Gibbs \\
\hline Halictini & Lasioglossum & Lasioglossum oblongum & Jason Gibbs \\
\hline Halictini & Lasioglossum & Lasioglossum oblongum & \\
\hline Halictini & Lasioglossum & Lasioglossum oblongum & \\
\hline Halictini & Lasioglossum & Lasioglossum oblongum & \\
\hline Halictini & Lasioglossum & Lasioglossum oblongum & Jason Gibbs \\
\hline Halictini & Lasioglossum & Lasioglossum obscurum & Jason Gibbs \\
\hline Halictini & Lasioglossum & Lasioglossum obscurum & \\
\hline Halictini & Lasioglossum & Lasioglossum obscurum & \\
\hline Halictini & Lasioglossum & Lasioglossum obscurum & Jason Gibbs \\
\hline Halictini & Lasioglossum & Lasioglossum obscurum & \\
\hline Halictini & Lasioglossum & Lasioglossum obscurum & \\
\hline Halictini & Lasioglossum & Lasioglossum obscurum & Jason Gibbs \\
\hline Halictini & Lasioglossum & Lasioglossum obscurum & \\
\hline Halictini & Lasioglossum & Lasioglossum obscurum & \\
\hline Halictini & Lasioglossum & Lasioglossum obscurum & Jason Gibbs \\
\hline Halictini & Lasioglossum & Lasioglossum occidentale & Jason Gibbs \\
\hline Halictini & Lasioglossum & Lasioglossum occidentale & Jason Gibbs \\
\hline Halictini & Lasioglossum & Lasioglossum occidentale & Jason Gibbs \\
\hline Halictini & Lasioglossum & Lasioglossum occidentale & \\
\hline Halictini & Lasioglossum & Lasioglossum occidentale & Jason Gibbs \\
\hline Halictini & Lasioglossum & Lasioglossum occidentale & Jason Gibbs \\
\hline Halictini & Lasioglossum & Lasioglossum occidentale & Jason Gibbs \\
\hline Halictini & Lasioglossum & Lasioglossum occidentale & \\
\hline Halictini & Lasioglossum & Lasioglossum occidentale & Jason Gibbs \\
\hline
\end{tabular}




\begin{tabular}{|c|c|c|c|}
\hline Halictini & Lasioglossum & Lasioglossum occidentale & Jason Gibbs \\
\hline Halictini & Lasioglossum & Lasioglossum occidentale & Jason Gibbs \\
\hline Halictini & Lasioglossum & Lasioglossum oceanicum & Jason Gibbs \\
\hline Halictini & Lasioglossum & Lasioglossum oceanicum & Jason Gibbs \\
\hline Halictini & Lasioglossum & Lasioglossum oceanicum & Jason Gibbs \\
\hline Halictini & Lasioglossum & Lasioglossum oceanicum & Jason Gibbs \\
\hline Halictini & Lasioglossum & Lasioglossum pacatum & \\
\hline Halictini & Lasioglossum & Lasioglossum pacatum & Jason Gibbs \\
\hline Halictini & Lasioglossum & Lasioglossum pacatum & Jason Gibbs \\
\hline Halictini & Lasioglossum & Lasioglossum pacatum & \\
\hline Halictini & Lasioglossum & Lasioglossum pacatum & \\
\hline Halictini & Lasioglossum & Lasioglossum pacatum & Jason Gibbs \\
\hline Halictini & Lasioglossum & Lasioglossum pacatum & Jason Gibbs \\
\hline Halictini & Lasioglossum & Lasioglossum pacatum & \\
\hline Halictini & Lasioglossum & Lasioglossum pacatum & Jason Gibbs \\
\hline Halictini & Lasioglossum & Lasioglossum pacatum & Jason Gibbs \\
\hline Halictini & Lasioglossum & Lasioglossum pacatum & \\
\hline Halictini & Lasioglossum & Lasioglossum pacatum & Jason Gibbs \\
\hline Halictini & Lasioglossum & Lasioglossum pacatum & Jason Gibbs \\
\hline Halictini & Lasioglossum & Lasioglossum pacatum & \\
\hline Halictini & Lasioglossum & Lasioglossum pacatum & Jason Gibbs \\
\hline Halictini & Lasioglossum & Lasioglossum pacatum & \\
\hline Halictini & Lasioglossum & Lasioglossum pacatum & \\
\hline Halictini & Lasioglossum & Lasioglossum pacatum & Jason Gibbs \\
\hline Halictini & Lasioglossum & Lasioglossum pacatum & Jason Gibbs \\
\hline Halictini & Lasioglossum & Lasioglossum pacatum & Jason Gibbs \\
\hline Halictini & Lasioglossum & Lasioglossum packeri & Jason Gibbs \\
\hline Halictini & Lasioglossum & Lasioglossum packeri & Jason Gibbs \\
\hline Halictini & Lasioglossum & Lasioglossum packeri & Jason Gibbs \\
\hline Halictini & Lasioglossum & Lasioglossum packeri & Jason Gibbs \\
\hline Halictini & Lasioglossum & Lasioglossum paradmirandum & Jason Gibbs \\
\hline Halictini & Lasioglossum & Lasioglossum paradmirandum & Jason Gibbs \\
\hline Halictini & Lasioglossum & Lasioglossum paradmirandum & Jason Gibbs \\
\hline Halictini & Lasioglossum & Lasioglossum paradmirandum & Jason Gibbs \\
\hline Halictini & Lasioglossum & Lasioglossum paradmirandum & Jason Gibbs \\
\hline Halictini & Lasioglossum & Lasioglossum paradmirandum & Jason Gibbs \\
\hline Halictini & Lasioglossum & Lasioglossum paradmirandum & Jason Gibbs \\
\hline Halictini & Lasioglossum & Lasioglossum paradmirandum & Jason Gibbs \\
\hline Halictini & Lasioglossum & Lasioglossum pavoninum & \\
\hline Halictini & Lasioglossum & Lasioglossum pavoninum & Jason Gibbs \\
\hline Halictini & Lasioglossum & Lasioglossum pavoninum & Jason Gibbs \\
\hline Halictini & Lasioglossum & Lasioglossum perpunctatum & \\
\hline Halictini & Lasioglossum & Lasioglossum perpunctatum & Jason Gibbs \\
\hline Halictini & Lasioglossum & Lasioglossum perpunctatum & Jason Gibbs \\
\hline Halictini & Lasioglossum & Lasioglossum perpunctatum & \\
\hline Halictini & Lasioglossum & Lasioglossum perpunctatum & Jason Gibbs \\
\hline Halictini & Lasioglossum & Lasioglossum perpunctatum & Jason Gibbs \\
\hline Halictini & Lasioglossum & Lasioglossum perpunctatum & Jason Gibbs \\
\hline Halictini & Lasioglossum & Lasioglossum perpunctatum & Jason Gibbs \\
\hline Halictini & Lasioglossum & Lasioglossum perpunctatum & \\
\hline Halictini & Lasioglossum & Lasioglossum perpunctatum & Jason Gibbs \\
\hline Halictini & Lasioglossum & Lasioglossum perpunctatum & Jason Gibbs \\
\hline Halictini & Lasioglossum & Lasioglossum perpunctatum & Jason Gibbs \\
\hline Halictini & Lasioglossum & Lasioglossum perpunctatum & \\
\hline Halictini & Lasioglossum & Lasioglossum pictum & Jason Gibbs \\
\hline
\end{tabular}




\begin{tabular}{|c|c|c|c|}
\hline Halictini & Lasioglossum & Lasioglossum pictum & \\
\hline Halictini & Lasioglossum & Lasioglossum pictum & Jason Gibbs \\
\hline Halictini & Lasioglossum & Lasioglossum pictum & \\
\hline Halictini & Lasioglossum & Lasioglossum pictum & Jason Gibbs \\
\hline Halictini & Lasioglossum & Lasioglossum pictum & Jason Gibbs \\
\hline Halictini & Lasioglossum & Lasioglossum pictum & Jason Gibbs \\
\hline Halictini & Lasioglossum & Lasioglossum pictum & Jason Gibbs \\
\hline Halictini & Lasioglossum & Lasioglossum pictum & Jason Gibbs \\
\hline Halictini & Lasioglossum & Lasioglossum pilosum & Jason Gibbs \\
\hline Halictini & Lasioglossum & Lasioglossum pilosum & \\
\hline Halictini & Lasioglossum & Lasioglossum pilosum & Jason Gibbs \\
\hline Halictini & Lasioglossum & Lasioglossum pilosum & \\
\hline Halictini & Lasioglossum & Lasioglossum pilosum & \\
\hline Halictini & Lasioglossum & Lasioglossum pilosum & Jason Gibbs \\
\hline Halictini & Lasioglossum & Lasioglossum pilosum & Jason Gibbs \\
\hline Halictini & Lasioglossum & Lasioglossum pilosum & \\
\hline Halictini & Lasioglossum & Lasioglossum pilosum & Jason Gibbs \\
\hline Halictini & Lasioglossum & Lasioglossum pilosum & Jason Gibbs \\
\hline Halictini & Lasioglossum & Lasioglossum pilosum & Jason Gibbs \\
\hline Halictini & Lasioglossum & Lasioglossum pilosum & Jason Gibbs \\
\hline Halictini & Lasioglossum & Lasioglossum pilosum & Jason Gibbs \\
\hline Halictini & Lasioglossum & Lasioglossum pilosum & Jason Gibbs \\
\hline Halictini & Lasioglossum & Lasioglossum pilosum & Jason Gibbs \\
\hline Halictini & Lasioglossum & Lasioglossum pilosum & Jason Gibbs \\
\hline Halictini & Lasioglossum & Lasioglossum pilosum & Jason Gibbs \\
\hline Halictini & Lasioglossum & Lasioglossum pilosum & Jason Gibbs \\
\hline Halictini & Lasioglossum & Lasioglossum pilosum & Jason Gibbs \\
\hline Halictini & Lasioglossum & Lasioglossum planatum & Jason Gibbs \\
\hline Halictini & Lasioglossum & Lasioglossum planatum & Jason Gibbs \\
\hline Halictini & Lasioglossum & Lasioglossum planatum & Jason Gibbs \\
\hline Halictini & Lasioglossum & Lasioglossum planatum & Jason Gibbs \\
\hline Halictini & Lasioglossum & Lasioglossum planatum & Jason Gibbs \\
\hline Halictini & Lasioglossum & Lasioglossum planatum & Jason Gibbs \\
\hline Halictini & Lasioglossum & Lasioglossum planatum & Jason Gibbs \\
\hline Halictini & Lasioglossum & Lasioglossum planatum & Jason Gibbs \\
\hline Halictini & Lasioglossum & Lasioglossum platyparium & Jason Gibbs \\
\hline Halictini & Lasioglossum & Lasioglossum platyparium & Jason Gibbs \\
\hline Halictini & Lasioglossum & Lasioglossum platyparium & Jason Gibbs \\
\hline Halictini & Lasioglossum & Lasioglossum platyparium & Jason Gibbs \\
\hline Halictini & Lasioglossum & Lasioglossum platyparium & Jason Gibbs \\
\hline Halictini & Lasioglossum & Lasioglossum platyparium & \\
\hline Halictini & Lasioglossum & Lasioglossum platyparium & \\
\hline Halictini & Lasioglossum & Lasioglossum platyparium & Jason Gibbs \\
\hline Halictini & Lasioglossum & Lasioglossum platyparium & Jason Gibbs \\
\hline Halictini & Lasioglossum & Lasioglossum platyparium & Jason Gibbs \\
\hline Halictini & Lasioglossum & Lasioglossum platyparium & Jason Gibbs \\
\hline Halictini & Lasioglossum & Lasioglossum prasinogaster & Jason Gibbs \\
\hline Halictini & Lasioglossum & Lasioglossum prasinogaster & Jason Gibbs \\
\hline Halictini & Lasioglossum & Lasioglossum prasinogaster & Jason Gibbs \\
\hline Halictini & Lasioglossum & Lasioglossum prasinogaster & Jason Gibbs \\
\hline Halictini & Lasioglossum & Lasioglossum prasinogaster & Jason Gibbs \\
\hline Halictini & Lasioglossum & Lasioglossum prasinogaster & Jason Gibbs \\
\hline Halictini & Lasioglossum & Lasioglossum prasinogaster & Jason Gibbs \\
\hline Halictini & Lasioglossum & Lasioglossum prasinogaster & Jason Gibbs \\
\hline Halictini & Lasioglossum & Lasioglossum prasinogaster & Jason Gibbs \\
\hline
\end{tabular}




\begin{tabular}{|c|c|c|c|}
\hline Halictini & Lasioglossum & Lasioglossum prasinogaster & Jason Gibbs \\
\hline Halictini & Lasioglossum & Lasioglossum prasinogaster & Jason Gibbs \\
\hline Halictini & Lasioglossum & Lasioglossum prasinogaster & Jason Gibbs \\
\hline Halictini & Lasioglossum & Lasioglossum prasinogaster & Jason Gibbs \\
\hline Halictini & Lasioglossum & Lasioglossum prasinogaster & Jason Gibbs \\
\hline Halictini & Lasioglossum & Lasioglossum prasinogaster & Jason Gibbs \\
\hline Halictini & Lasioglossum & Lasioglossum prasinogaster & Jason Gibbs \\
\hline Halictini & Lasioglossum & Lasioglossum prasinogaster & Jason Gibbs \\
\hline Halictini & Lasioglossum & Lasioglossum pruinosum & Jason Gibbs \\
\hline Halictini & Lasioglossum & Lasioglossum pruinosum & Jason Gibbs \\
\hline Halictini & Lasioglossum & Lasioglossum pruinosum & Jason Gibbs \\
\hline Halictini & Lasioglossum & Lasioglossum pruinosum & Jason Gibbs \\
\hline Halictini & Lasioglossum & Lasioglossum pruinosum & \\
\hline Halictini & Lasioglossum & Lasioglossum pruinosum & Jason Gibbs \\
\hline Halictini & Lasioglossum & Lasioglossum pruinosum & Jason Gibbs \\
\hline Halictini & Lasioglossum & Lasioglossum pruinosum & \\
\hline Halictini & Lasioglossum & Lasioglossum punctatoventre & \\
\hline Halictini & Lasioglossum & Lasioglossum punctatoventre & Jason Gibbs \\
\hline Halictini & Lasioglossum & Lasioglossum punctatoventre & \\
\hline Halictini & Lasioglossum & Lasioglossum punctatoventre & \\
\hline Halictini & Lasioglossum & Lasioglossum punctatoventre & Jason Gibbs \\
\hline Halictini & Lasioglossum & Lasioglossum puteulanum & \\
\hline Halictini & Lasioglossum & Lasioglossum puteulanum & Jason Gibbs \\
\hline Halictini & Lasioglossum & Lasioglossum puteulanum & Jason Gibbs \\
\hline Halictini & Lasioglossum & Lasioglossum puteulanum & Jason Gibbs \\
\hline Halictini & Lasioglossum & Lasioglossum puteulanum & \\
\hline Halictini & Lasioglossum & Lasioglossum puteulanum & \\
\hline Halictini & Lasioglossum & Lasioglossum puteulanum & Jason Gibbs \\
\hline Halictini & Lasioglossum & Lasioglossum puteulanum & Jason Gibbs \\
\hline Halictini & Lasioglossum & Lasioglossum puteulanum & \\
\hline Halictini & Lasioglossum & Lasioglossum puteulanum & \\
\hline Halictini & Lasioglossum & Lasioglossum puteulanum & \\
\hline Halictini & Lasioglossum & Lasioglossum puteulanum & Jason Gibbs \\
\hline Halictini & Lasioglossum & Lasioglossum puteulanum & Jason Gibbs \\
\hline Halictini & Lasioglossum & Lasioglossum puteulanum & \\
\hline Halictini & Lasioglossum & Lasioglossum puteulanum & \\
\hline Halictini & Lasioglossum & Lasioglossum puteulanum & \\
\hline Halictini & Lasioglossum & Lasioglossum puteulanum & \\
\hline Halictini & Lasioglossum & Lasioglossum puteulanum & Jason Gibbs \\
\hline Halictini & Lasioglossum & Lasioglossum puteulanum & \\
\hline Halictini & Lasioglossum & Lasioglossum puteulanum & \\
\hline Halictini & Lasioglossum & Lasioglossum raleighense & Jason Gibbs \\
\hline Halictini & Lasioglossum & Lasioglossum raleighense & Jason Gibbs \\
\hline Halictini & Lasioglossum & Lasioglossum raleighense & Jason Gibbs \\
\hline Halictini & Lasioglossum & Lasioglossum reticulatum & \\
\hline Halictini & Lasioglossum & Lasioglossum rozeni & Jason Gibbs \\
\hline Halictini & Lasioglossum & Lasioglossum rozeni & Jason Gibbs \\
\hline Halictini & Lasioglossum & Lasioglossum rozeni & Jason Gibbs \\
\hline Halictini & Lasioglossum & Lasioglossum rozeni & Jason Gibbs \\
\hline Halictini & Lasioglossum & Lasioglossum rozeni & Jason Gibbs \\
\hline Halictini & Lasioglossum & Lasioglossum rozeni & Jason Gibbs \\
\hline Halictini & Lasioglossum & Lasioglossum rufulipes & Jason Gibbs \\
\hline Halictini & Lasioglossum & Lasioglossum rufulipes & Jason Gibbs \\
\hline Halictini & Lasioglossum & Lasioglossum rufulipes & Jason Gibbs \\
\hline Halictini & Lasioglossum & Lasioglossum rufulipes & Jason Gibbs \\
\hline
\end{tabular}




\begin{tabular}{|c|c|c|c|}
\hline Halictini & Lasioglossum & Lasioglossum ruidosense & Jason Gibbs \\
\hline Halictini & Lasioglossum & Lasioglossum ruidosense & Jason Gibbs \\
\hline Halictini & Lasioglossum & Lasioglossum ruidosense & Jason Gibbs \\
\hline Halictini & Lasioglossum & Lasioglossum ruidosense & Jason Gibbs \\
\hline Halictini & Lasioglossum & Lasioglossum ruidosense & \\
\hline Halictini & Lasioglossum & Lasioglossum ruidosense & Jason Gibbs \\
\hline Halictini & Lasioglossum & Lasioglossum ruidosense & Jason Gibbs \\
\hline Halictini & Lasioglossum & Lasioglossum ruidosense & \\
\hline Halictini & Lasioglossum & Lasioglossum ruidosense & Jason Gibbs \\
\hline Halictini & Lasioglossum & Lasioglossum ruidosense & Jason Gibbs \\
\hline Halictini & Lasioglossum & Lasioglossum ruidosense & \\
\hline Halictini & Lasioglossum & Lasioglossum ruidosense & Jason Gibbs \\
\hline Halictini & Lasioglossum & Lasioglossum ruidosense & Jason Gibbs \\
\hline Halictini & Lasioglossum & Lasioglossum ruidosense & \\
\hline Halictini & Lasioglossum & Lasioglossum ruidosense & \\
\hline Halictini & Lasioglossum & Lasioglossum ruidosense & Jason Gibbs \\
\hline Halictini & Lasioglossum & Lasioglossum ruidosense & Jason Gibbs \\
\hline Halictini & Lasioglossum & Lasioglossum ruidosense & Jason Gibbs \\
\hline Halictini & Lasioglossum & Lasioglossum ruidosense & Jason Gibbs \\
\hline Halictini & Lasioglossum & Lasioglossum ruidosense & Jason Gibbs \\
\hline Halictini & Lasioglossum & Lasioglossum ruidosense & Jason Gibbs \\
\hline Halictini & Lasioglossum & Lasioglossum ruidosense & Jason Gibbs \\
\hline Halictini & Lasioglossum & Lasioglossum ruidosense & Jason Gibbs \\
\hline Halictini & Lasioglossum & Lasioglossum ruidosense & Jason Gibbs \\
\hline Halictini & Lasioglossum & Lasioglossum ruidosense & Jason Gibbs \\
\hline Halictini & Lasioglossum & Lasioglossum ruidosense & \\
\hline Halictini & Lasioglossum & Lasioglossum ruidosense & Jason Gibbs \\
\hline Halictini & Lasioglossum & Lasioglossum sagax & Jason Gibbs \\
\hline Halictini & Lasioglossum & Lasioglossum sagax & \\
\hline Halictini & Lasioglossum & Lasioglossum sagax & Jason Gibbs \\
\hline Halictini & Lasioglossum & Lasioglossum sagax & Jason Gibbs \\
\hline Halictini & Lasioglossum & Lasioglossum sagax & Jason Gibbs \\
\hline Halictini & Lasioglossum & Lasioglossum sagax & Jason Gibbs \\
\hline Halictini & Lasioglossum & Lasioglossum sagax & \\
\hline Halictini & Lasioglossum & Lasioglossum sagax & \\
\hline Halictini & Lasioglossum & Lasioglossum sagax & Jason Gibbs \\
\hline Halictini & Lasioglossum & Lasioglossum sagax & Jason Gibbs \\
\hline Halictini & Lasioglossum & Lasioglossum sagax & Jason Gibbs \\
\hline Halictini & Lasioglossum & Lasioglossum sagax & Jason Gibbs \\
\hline Halictini & Lasioglossum & Lasioglossum sagax & Jason Gibbs \\
\hline Halictini & Lasioglossum & Lasioglossum sagax & Jason Gibbs \\
\hline Halictini & Lasioglossum & Lasioglossum sagax & Jason Gibbs \\
\hline Halictini & Lasioglossum & Lasioglossum sagax & Jason Gibbs \\
\hline Halictini & Lasioglossum & Lasioglossum sagax & Jason Gibbs \\
\hline Halictini & Lasioglossum & Lasioglossum sagax & Jason Gibbs \\
\hline Halictini & Lasioglossum & Lasioglossum sagax & Jason Gibbs \\
\hline Halictini & Lasioglossum & Lasioglossum sagax & Jason Gibbs \\
\hline Halictini & Lasioglossum & Lasioglossum sagax & Jason Gibbs \\
\hline Halictini & Lasioglossum & Lasioglossum sagax & Jason Gibbs \\
\hline Halictini & Lasioglossum & Lasioglossum sandhousiellum & Jason Gibbs \\
\hline Halictini & Lasioglossum & Lasioglossum sandhousiellum & Jason Gibbs \\
\hline Halictini & Lasioglossum & Lasioglossum sandhousiellum & Jason Gibbs \\
\hline Halictini & Lasioglossum & Lasioglossum sandhousiellum & Jason Gibbs \\
\hline Halictini & Lasioglossum & Lasioglossum sandhousiellum & Jason Gibbs \\
\hline Halictini & Lasioglossum & Lasioglossum sandhousiellum & Jason Gibbs \\
\hline
\end{tabular}




\begin{tabular}{|c|c|c|c|}
\hline Halictini & Lasioglossum & Lasioglossum sandhousiellum & Jason Gibbs \\
\hline Halictini & Lasioglossum & Lasioglossum sedi & \\
\hline Halictini & Lasioglossum & Lasioglossum sedi & \\
\hline Halictini & Lasioglossum & Lasioglossum sedi & Jason Gibbs \\
\hline Halictini & Lasioglossum & Lasioglossum sedi & Jason Gibbs \\
\hline Halictini & Lasioglossum & Lasioglossum sedi & \\
\hline Halictini & Lasioglossum & Lasioglossum sedi & Jason Gibbs \\
\hline Halictini & Lasioglossum & Lasioglossum sedi & \\
\hline Halictini & Lasioglossum & Lasioglossum sedi & Jason Gibbs \\
\hline Halictini & Lasioglossum & Lasioglossum sedi & Jason Gibbs \\
\hline Halictini & Lasioglossum & Lasioglossum sedi & Jason Gibbs \\
\hline Halictini & Lasioglossum & Lasioglossum sedi & \\
\hline Halictini & Lasioglossum & Lasioglossum sedi & \\
\hline Halictini & Lasioglossum & Lasioglossum sedi & \\
\hline Halictini & Lasioglossum & Lasioglossum sedi & Jason Gibbs \\
\hline Halictini & Lasioglossum & Lasioglossum sedi & \\
\hline Halictini & Lasioglossum & Lasioglossum sedi & Jason Gibbs \\
\hline Halictini & Lasioglossum & Lasioglossum semicaeruleum & Jason Gibbs \\
\hline Halictini & Lasioglossum & Lasioglossum semicaeruleum & Jason Gibbs \\
\hline Halictini & Lasioglossum & Lasioglossum semicaeruleum & Jason Gibbs \\
\hline Halictini & Lasioglossum & Lasioglossum semicaeruleum & Jason Gibbs \\
\hline Halictini & Lasioglossum & Lasioglossum semicaeruleum & Jason Gibbs \\
\hline Halictini & Lasioglossum & Lasioglossum semicaeruleum & Jason Gibbs \\
\hline Halictini & Lasioglossum & Lasioglossum semicaeruleum & Jason Gibbs \\
\hline Halictini & Lasioglossum & Lasioglossum semicaeruleum & Jason Gibbs \\
\hline Halictini & Lasioglossum & Lasioglossum semicaeruleum & Jason Gibbs \\
\hline Halictini & Lasioglossum & Lasioglossum semicaeruleum & Jason Gibbs \\
\hline Halictini & Lasioglossum & Lasioglossum semicaeruleum & Jason Gibbs \\
\hline Halictini & Lasioglossum & Lasioglossum semicaeruleum & Jason Gibbs \\
\hline Halictini & Lasioglossum & Lasioglossum semicaeruleum & Jason Gibbs \\
\hline Halictini & Lasioglossum & Lasioglossum semicaeruleum & Jason Gibbs \\
\hline Halictini & Lasioglossum & Lasioglossum semicaeruleum & Jason Gibbs \\
\hline Halictini & Lasioglossum & Lasioglossum semicaeruleum & Jason Gibbs \\
\hline Halictini & Lasioglossum & Lasioglossum semicaeruleum & Jason Gibbs \\
\hline Halictini & Lasioglossum & Lasioglossum semicaeruleum & Jason Gibbs \\
\hline Halictini & Lasioglossum & Lasioglossum semicaeruleum & Jason Gibbs \\
\hline Halictini & Lasioglossum & Lasioglossum semicaeruleum & Jason Gibbs \\
\hline Halictini & Lasioglossum & Lasioglossum semicaeruleum & Jason Gibbs \\
\hline Halictini & Lasioglossum & Lasioglossum semicaeruleum & Jason Gibbs \\
\hline Halictini & Lasioglossum & Lasioglossum semicaeruleum & Jason Gibbs \\
\hline Halictini & Lasioglossum & Lasioglossum semicaeruleum & Jason Gibbs \\
\hline Halictini & Lasioglossum & Lasioglossum semicaeruleum & Jason Gibbs \\
\hline Halictini & Lasioglossum & Lasioglossum semicaeruleum & Jason Gibbs \\
\hline Halictini & Lasioglossum & Lasioglossum semicaeruleum & Jason Gibbs \\
\hline Halictini & Lasioglossum & Lasioglossum semicaeruleum & \\
\hline Halictini & Lasioglossum & Lasioglossum sheffieldi & Jason Gibbs \\
\hline Halictini & Lasioglossum & Lasioglossum sheffieldi & Jason Gibbs \\
\hline Halictini & Lasioglossum & Lasioglossum sheffieldi & Jason Gibbs \\
\hline Halictini & Lasioglossum & Lasioglossum sheffieldi & Jason Gibbs \\
\hline Halictini & Lasioglossum & Lasioglossum sheffieldi & Jason Gibbs \\
\hline Halictini & Lasioglossum & Lasioglossum sheffieldi & Jason Gibbs \\
\hline Halictini & Lasioglossum & Lasioglossum sheffieldi & Jason Gibbs \\
\hline Halictini & Lasioglossum & Lasioglossum simplex & Jason Gibbs \\
\hline Halictini & Lasioglossum & Lasioglossum simplex & Jason Gibbs \\
\hline Halictini & Lasioglossum & Lasioglossum smilacinae & Jason Gibbs \\
\hline
\end{tabular}




\begin{tabular}{|c|c|c|c|}
\hline Halictini & Lasioglossum & Lasioglossum sp. nov A & Jason Gibbs \\
\hline Halictini & Lasioglossum & Lasioglossum subversans & Jason Gibbs \\
\hline Halictini & Lasioglossum & Lasioglossum subversans & Jason Gibbs \\
\hline Halictini & Lasioglossum & Lasioglossum subversans & Jason Gibbs \\
\hline Halictini & Lasioglossum & Lasioglossum subviridatum & Jason Gibbs \\
\hline Halictini & Lasioglossum & Lasioglossum subviridatum & Jason Gibbs \\
\hline Halictini & Lasioglossum & Lasioglossum subviridatum & \\
\hline Halictini & Lasioglossum & Lasioglossum subviridatum & Jason Gibbs \\
\hline Halictini & Lasioglossum & Lasioglossum subviridatum & Jason Gibbs \\
\hline Halictini & Lasioglossum & Lasioglossum subviridatum & Jason Gibbs \\
\hline Halictini & Lasioglossum & Lasioglossum subviridatum & \\
\hline Halictini & Lasioglossum & Lasioglossum subviridatum & \\
\hline Halictini & Lasioglossum & Lasioglossum subviridatum & Jason Gibbs \\
\hline Halictini & Lasioglossum & Lasioglossum subviridatum & \\
\hline Halictini & Lasioglossum & Lasioglossum subviridatum & Jason Gibbs \\
\hline Halictini & Lasioglossum & Lasioglossum subviridatum & \\
\hline Halictini & Lasioglossum & Lasioglossum subviridatum & \\
\hline Halictini & Lasioglossum & Lasioglossum subviridatum & Jason Gibbs \\
\hline Halictini & Lasioglossum & Lasioglossum subviridatum & Jason Gibbs \\
\hline Halictini & Lasioglossum & Lasioglossum subviridatum & \\
\hline Halictini & Lasioglossum & Lasioglossum subviridatum & Jason Gibbs \\
\hline Halictini & Lasioglossum & Lasioglossum subviridatum & Jason Gibbs \\
\hline Halictini & Lasioglossum & Lasioglossum subviridatum & Jason Gibbs \\
\hline Halictini & Lasioglossum & Lasioglossum subviridatum & Jason Gibbs \\
\hline Halictini & Lasioglossum & Lasioglossum subviridatum & Jason Gibbs \\
\hline Halictini & Lasioglossum & Lasioglossum subviridatum & Jason Gibbs \\
\hline Halictini & Lasioglossum & Lasioglossum subviridatum & Jason Gibbs \\
\hline Halictini & Lasioglossum & Lasioglossum subviridatum & \\
\hline Halictini & Lasioglossum & Lasioglossum subviridatum & Jason Gibbs \\
\hline Halictini & Lasioglossum & Lasioglossum subviridatum & Jason Gibbs \\
\hline Halictini & Lasioglossum & Lasioglossum subviridatum & Jason Gibbs \\
\hline Halictini & Lasioglossum & Lasioglossum subviridatum & Jason Gibbs \\
\hline Halictini & Lasioglossum & Lasioglossum subviridatum & Jason Gibbs \\
\hline Halictini & Lasioglossum & Lasioglossum subviridatum & Jason Gibbs \\
\hline Halictini & Lasioglossum & Lasioglossum subviridatum & Jason Gibbs \\
\hline Halictini & Lasioglossum & Lasioglossum subviridatum & Jason Gibbs \\
\hline Halictini & Lasioglossum & Lasioglossum subviridatum & Jason Gibbs \\
\hline Halictini & Lasioglossum & Lasioglossum subviridatum & Jason Gibbs \\
\hline Halictini & Lasioglossum & Lasioglossum subviridatum & Jason Gibbs \\
\hline Halictini & Lasioglossum & Lasioglossum subviridatum & Jason Gibbs \\
\hline Halictini & Lasioglossum & Lasioglossum subviridatum & Jason Gibbs \\
\hline Halictini & Lasioglossum & Lasioglossum subviridatum & Jason Gibbs \\
\hline Halictini & Lasioglossum & Lasioglossum succinipenne & Jason Gibbs \\
\hline Halictini & Lasioglossum & Lasioglossum succinipenne & Jason Gibbs \\
\hline Halictini & Lasioglossum & Lasioglossum succinipenne & Jason Gibbs \\
\hline Halictini & Lasioglossum & Lasioglossum succinipenne & \\
\hline Halictini & Lasioglossum & Lasioglossum succinipenne & Jason Gibbs \\
\hline Halictini & Lasioglossum & Lasioglossum tamiamense & Jason Gibbs \\
\hline Halictini & Lasioglossum & Lasioglossum tarponense & \\
\hline Halictini & Lasioglossum & Lasioglossum tarponense & \\
\hline Halictini & Lasioglossum & Lasioglossum tarponense & Jason Gibbs \\
\hline Halictini & Lasioglossum & Lasioglossum tarponense & Jason Gibbs \\
\hline Halictini & Lasioglossum & Lasioglossum taylorae & Jason Gibbs \\
\hline Halictini & Lasioglossum & Lasioglossum taylorae & Jason Gibbs \\
\hline Halictini & Lasioglossum & Lasioglossum taylorae & Jason Gibbs \\
\hline
\end{tabular}




\begin{tabular}{|c|c|c|c|}
\hline Halictini & Lasioglossum & Lasioglossum tegulare & \\
\hline Halictini & Lasioglossum & Lasioglossum tegulare & \\
\hline Halictini & Lasioglossum & Lasioglossum tegulare & \\
\hline Halictini & Lasioglossum & Lasioglossum tegulare & \\
\hline Halictini & Lasioglossum & Lasioglossum tegulare & \\
\hline Halictini & Lasioglossum & Lasioglossum tegulare & \\
\hline Halictini & Lasioglossum & Lasioglossum tegulare & \\
\hline Halictini & Lasioglossum & Lasioglossum tegulare & \\
\hline Halictini & Lasioglossum & Lasioglossum tegulare & \\
\hline Halictini & Lasioglossum & Lasioglossum tegulare & \\
\hline Halictini & Lasioglossum & Lasioglossum tegulare & Jason Gibbs \\
\hline Halictini & Lasioglossum & Lasioglossum tegulare & John L. Neff \\
\hline Halictini & Lasioglossum & Lasioglossum tegulare & \\
\hline Halictini & Lasioglossum & Lasioglossum tegulare & Jason Gibbs \\
\hline Halictini & Lasioglossum & Lasioglossum tegulare & Jason Gibbs \\
\hline Halictini & Lasioglossum & Lasioglossum tegulare & \\
\hline Halictini & Lasioglossum & Lasioglossum tegulare & \\
\hline Halictini & Lasioglossum & Lasioglossum tegulare & \\
\hline Halictini & Lasioglossum & Lasioglossum tegulare & \\
\hline Halictini & Lasioglossum & Lasioglossum tegulare & \\
\hline Halictini & Lasioglossum & Lasioglossum tegulare & \\
\hline Halictini & Lasioglossum & Lasioglossum tegulare & \\
\hline Halictini & Lasioglossum & Lasioglossum tegulare & Jason Gibbs \\
\hline Halictini & Lasioglossum & Lasioglossum tegulare & Jason Gibbs \\
\hline Halictini & Lasioglossum & Lasioglossum tegulare & Jason Gibbs \\
\hline Halictini & Lasioglossum & Lasioglossum tegulare & \\
\hline Halictini & Lasioglossum & Lasioglossum tenax & Jason Gibbs \\
\hline Halictini & Lasioglossum & Lasioglossum tenax & Jason Gibbs \\
\hline Halictini & Lasioglossum & Lasioglossum tenax & Jason Gibbs \\
\hline Halictini & Lasioglossum & Lasioglossum tenax & Jason Gibbs \\
\hline Halictini & Lasioglossum & Lasioglossum tenax & Jason Gibbs \\
\hline Halictini & Lasioglossum & Lasioglossum tenax & Jason Gibbs \\
\hline Halictini & Lasioglossum & Lasioglossum tenax & Jason Gibbs \\
\hline Halictini & Lasioglossum & Lasioglossum tenax & Jason Gibbs \\
\hline Halictini & Lasioglossum & Lasioglossum tenax & Jason Gibbs \\
\hline Halictini & Lasioglossum & Lasioglossum tenax & Jason Gibbs \\
\hline Halictini & Lasioglossum & Lasioglossum tenax & Jason Gibbs \\
\hline Halictini & Lasioglossum & Lasioglossum tenax & Jason Gibbs \\
\hline Halictini & Lasioglossum & Lasioglossum tenax & \\
\hline Halictini & Lasioglossum & Lasioglossum tenax & Jason Gibbs \\
\hline Halictini & Lasioglossum & Lasioglossum tenax & Jason Gibbs \\
\hline Halictini & Lasioglossum & Lasioglossum tenax & Jason Gibbs \\
\hline Halictini & Lasioglossum & Lasioglossum tenax & Jason Gibbs \\
\hline Halictini & Lasioglossum & Lasioglossum tenax & Jason Gibbs \\
\hline Halictini & Lasioglossum & Lasioglossum tenax & Jason Gibbs \\
\hline Halictini & Lasioglossum & Lasioglossum timothyi & Jason Gibbs \\
\hline Halictini & Lasioglossum & Lasioglossum timothyi & Jason Gibbs \\
\hline Halictini & Lasioglossum & Lasioglossum timothyi & Jason Gibbs \\
\hline Halictini & Lasioglossum & Lasioglossum trigeminum & Jason Gibbs \\
\hline Halictini & Lasioglossum & Lasioglossum trigeminum & Jason Gibbs \\
\hline Halictini & Lasioglossum & Lasioglossum trigeminum & Jason Gibbs \\
\hline Halictini & Lasioglossum & Lasioglossum trigeminum & Jason Gibbs \\
\hline Halictini & Lasioglossum & Lasioglossum trigeminum & Jason Gibbs \\
\hline Halictini & Lasioglossum & Lasioglossum trigeminum & Jason Gibbs \\
\hline Halictini & Lasioglossum & Lasioglossum trigeminum & Jason Gibbs \\
\hline
\end{tabular}




\begin{tabular}{|c|c|c|c|}
\hline Halictini & Lasioglossum & Lasioglossum trigeminum & Jason Gibbs \\
\hline Halictini & Lasioglossum & Lasioglossum trigeminum & Jason Gibbs \\
\hline Halictini & Lasioglossum & Lasioglossum trigeminum & Jason Gibbs \\
\hline Halictini & Lasioglossum & Lasioglossum trigeminum & Jason Gibbs \\
\hline Halictini & Lasioglossum & Lasioglossum trigeminum & Jason Gibbs \\
\hline Halictini & Lasioglossum & Lasioglossum trigeminum & Jason Gibbs \\
\hline Halictini & Lasioglossum & Lasioglossum trigeminum & Jason Gibbs \\
\hline Halictini & Lasioglossum & Lasioglossum trigeminum & Jason Gibbs \\
\hline Halictini & Lasioglossum & Lasioglossum trigeminum & Jason Gibbs \\
\hline Halictini & Lasioglossum & Lasioglossum trigeminum & Jason Gibbs \\
\hline Halictini & Lasioglossum & Lasioglossum trigeminum & Jason Gibbs \\
\hline Halictini & Lasioglossum & Lasioglossum trigeminum & Jason Gibbs \\
\hline Halictini & Lasioglossum & Lasioglossum trigeminum & Jason Gibbs \\
\hline Halictini & Lasioglossum & Lasioglossum trigeminum & Jason Gibbs \\
\hline Halictini & Lasioglossum & Lasioglossum trigeminum & Jason Gibbs \\
\hline Halictini & Lasioglossum & Lasioglossum trigeminum & Jason Gibbs \\
\hline Halictini & Lasioglossum & Lasioglossum trigeminum & Jason Gibbs \\
\hline Halictini & Lasioglossum & Lasioglossum trigeminum & Jason Gibbs \\
\hline Halictini & Lasioglossum & Lasioglossum trigeminum & Jason Gibbs \\
\hline Halictini & Lasioglossum & Lasioglossum trigeminum & Jason Gibbs \\
\hline Halictini & Lasioglossum & Lasioglossum trigeminum & Jason Gibbs \\
\hline Halictini & Lasioglossum & Lasioglossum trigeminum & Jason Gibbs \\
\hline Halictini & Lasioglossum & Lasioglossum versans & \\
\hline Halictini & Lasioglossum & Lasioglossum versans & \\
\hline Halictini & Lasioglossum & Lasioglossum versans & Jason Gibbs \\
\hline Halictini & Lasioglossum & Lasioglossum versans & \\
\hline Halictini & Lasioglossum & Lasioglossum versans & \\
\hline Halictini & Lasioglossum & Lasioglossum versans & Jason Gibbs \\
\hline Halictini & Lasioglossum & Lasioglossum versans & Jason Gibbs \\
\hline Halictini & Lasioglossum & Lasioglossum versans & Jason Gibbs \\
\hline Halictini & Lasioglossum & Lasioglossum versans & Jason Gibbs \\
\hline Halictini & Lasioglossum & Lasioglossum versatum & Jason Gibbs \\
\hline Halictini & Lasioglossum & Lasioglossum versatum & Jason Gibbs \\
\hline Halictini & Lasioglossum & Lasioglossum versatum & Jason Gibbs \\
\hline Halictini & Lasioglossum & Lasioglossum versatum & Jason Gibbs \\
\hline Halictini & Lasioglossum & Lasioglossum versatum & Jason Gibbs \\
\hline Halictini & Lasioglossum & Lasioglossum versatum & Jason Gibbs \\
\hline Halictini & Lasioglossum & Lasioglossum versatum & Jason Gibbs \\
\hline Halictini & Lasioglossum & Lasioglossum versatum & Jason Gibbs \\
\hline Halictini & Lasioglossum & Lasioglossum versatum & Jason Gibbs \\
\hline Halictini & Lasioglossum & Lasioglossum versatum & Jason Gibbs \\
\hline Halictini & Lasioglossum & Lasioglossum versatum & Jason Gibbs \\
\hline Halictini & Lasioglossum & Lasioglossum versatum & Jason Gibbs \\
\hline Halictini & Lasioglossum & Lasioglossum versatum & Jason Gibbs \\
\hline Halictini & Lasioglossum & Lasioglossum versatum & Jason Gibbs \\
\hline Halictini & Lasioglossum & Lasioglossum versatum & Jason Gibbs \\
\hline Halictini & Lasioglossum & Lasioglossum versatum & Jason Gibbs \\
\hline Halictini & Lasioglossum & Lasioglossum versatum & Jason Gibbs \\
\hline Halictini & Lasioglossum & Lasioglossum versatum & Jason Gibbs \\
\hline Halictini & Lasioglossum & Lasioglossum versatum & Jason Gibbs \\
\hline Halictini & Lasioglossum & Lasioglossum versatum & Jason Gibbs \\
\hline Halictini & Lasioglossum & Lasioglossum versatum & Jason Gibbs \\
\hline Halictini & Lasioglossum & Lasioglossum versatum & Jason Gibbs \\
\hline Halictini & Lasioglossum & Lasioglossum versatum & Jason Gibbs \\
\hline Halictini & Lasioglossum & Lasioglossum versatum & Jason Gibbs \\
\hline
\end{tabular}




\begin{tabular}{|c|c|c|c|}
\hline Halictini & Lasioglossum & Lasioglossum versatum & Jason Gibbs \\
\hline Halictini & Lasioglossum & Lasioglossum versatum & \\
\hline Halictini & Lasioglossum & Lasioglossum versatum & Jason Gibbs \\
\hline Halictini & Lasioglossum & Lasioglossum versatum & Jason Gibbs \\
\hline Halictini & Lasioglossum & Lasioglossum versatum & Jason Gibbs \\
\hline Halictini & Lasioglossum & Lasioglossum versatum & Jason Gibbs \\
\hline Halictini & Lasioglossum & Lasioglossum versatum & Jason Gibbs \\
\hline Halictini & Lasioglossum & Lasioglossum versatum & Jason Gibbs \\
\hline Halictini & Lasioglossum & Lasioglossum versatum & Jason Gibbs \\
\hline Halictini & Lasioglossum & Lasioglossum versatum & Jason Gibbs \\
\hline Halictini & Lasioglossum & Lasioglossum versatum & Jason Gibbs \\
\hline Halictini & Lasioglossum & Lasioglossum versatum & Jason Gibbs \\
\hline Halictini & Lasioglossum & Lasioglossum versatum & Jason Gibbs \\
\hline Halictini & Lasioglossum & Lasioglossum versatum & Jason Gibbs \\
\hline Halictini & Lasioglossum & Lasioglossum versatum & Jason Gibbs \\
\hline Halictini & Lasioglossum & Lasioglossum versatum & Jason Gibbs \\
\hline Halictini & Lasioglossum & Lasioglossum versatum & Jason Gibbs \\
\hline Halictini & Lasioglossum & Lasioglossum versatum & Jason Gibbs \\
\hline Halictini & Lasioglossum & Lasioglossum versatum & Jason Gibbs \\
\hline Halictini & Lasioglossum & Lasioglossum versatum & Jason Gibbs \\
\hline Halictini & Lasioglossum & Lasioglossum versatum & Jason Gibbs \\
\hline Halictini & Lasioglossum & Lasioglossum versatum & Jason Gibbs \\
\hline Halictini & Lasioglossum & Lasioglossum versatum & Jason Gibbs \\
\hline Halictini & Lasioglossum & Lasioglossum versatum & Jason Gibbs \\
\hline Halictini & Lasioglossum & Lasioglossum versatum & Jason Gibbs \\
\hline Halictini & Lasioglossum & Lasioglossum versatum & Jason Gibbs \\
\hline Halictini & Lasioglossum & Lasioglossum versatum & Jason Gibbs \\
\hline Halictini & Lasioglossum & Lasioglossum versatum & Jason Gibbs \\
\hline Halictini & Lasioglossum & Lasioglossum versatum & Jason Gibbs \\
\hline Halictini & Lasioglossum & Lasioglossum versatum & Jason Gibbs \\
\hline Halictini & Lasioglossum & Lasioglossum versatum & Jason Gibbs \\
\hline Halictini & Lasioglossum & Lasioglossum versatum & Jason Gibbs \\
\hline Halictini & Lasioglossum & Lasioglossum versatum & Jason Gibbs \\
\hline Halictini & Lasioglossum & Lasioglossum versatum & Jason Gibbs \\
\hline Halictini & Lasioglossum & Lasioglossum versatum & Jason Gibbs \\
\hline Halictini & Lasioglossum & Lasioglossum versatum & Jason Gibbs \\
\hline Halictini & Lasioglossum & Lasioglossum versatum & Jason Gibbs \\
\hline Halictini & Lasioglossum & Lasioglossum versatum & Jason Gibbs \\
\hline Halictini & Lasioglossum & Lasioglossum versatum & Jason Gibbs \\
\hline Halictini & Lasioglossum & Lasioglossum versatum & Jason Gibbs \\
\hline Halictini & Lasioglossum & Lasioglossum versatum & Jason Gibbs \\
\hline Halictini & Lasioglossum & Lasioglossum versatum & Jason Gibbs \\
\hline Halictini & Lasioglossum & Lasioglossum versatum & Jason Gibbs \\
\hline Halictini & Lasioglossum & Lasioglossum versatum & Jason Gibbs \\
\hline Halictini & Lasioglossum & Lasioglossum versatum & Jason Gibbs \\
\hline Halictini & Lasioglossum & Lasioglossum versatum & Jason Gibbs \\
\hline Halictini & Lasioglossum & Lasioglossum versatum & Jason Gibbs \\
\hline Halictini & Lasioglossum & Lasioglossum versatum & Jason Gibbs \\
\hline Halictini & Lasioglossum & Lasioglossum versatum & Jason Gibbs \\
\hline Halictini & Lasioglossum & Lasioglossum versatum & Jason Gibbs \\
\hline Halictini & Lasioglossum & Lasioglossum versatum & Jason Gibbs \\
\hline Halictini & Lasioglossum & Lasioglossum versatum & Jason Gibbs \\
\hline Halictini & Lasioglossum & Lasioglossum versatum & Jason Gibbs \\
\hline Halictini & Lasioglossum & Lasioglossum versatum & Jason Gibbs \\
\hline Halictini & Lasioglossum & Lasioglossum versatum & Jason Gibbs \\
\hline
\end{tabular}




\begin{tabular}{|c|c|c|c|}
\hline Halictini & Lasioglossum & Lasioglossum versatum & Jason Gibbs \\
\hline Halictini & Lasioglossum & Lasioglossum versatum & Jason Gibbs \\
\hline Halictini & Lasioglossum & Lasioglossum versatum & Jason Gibbs \\
\hline Halictini & Lasioglossum & Lasioglossum versatum & Jason Gibbs \\
\hline Halictini & Lasioglossum & Lasioglossum versatum & Jason Gibbs \\
\hline Halictini & Lasioglossum & Lasioglossum versatum & Jason Gibbs \\
\hline Halictini & Lasioglossum & Lasioglossum versatum & Jason Gibbs \\
\hline Halictini & Lasioglossum & Lasioglossum versatum & Jason Gibbs \\
\hline Halictini & Lasioglossum & Lasioglossum versatum & Jason Gibbs \\
\hline Halictini & Lasioglossum & Lasioglossum versatum & Jason Gibbs \\
\hline Halictini & Lasioglossum & Lasioglossum versatum & Jason Gibbs \\
\hline Halictini & Lasioglossum & Lasioglossum versatum & Jason Gibbs \\
\hline Halictini & Lasioglossum & Lasioglossum versatum & Jason Gibbs \\
\hline Halictini & Lasioglossum & Lasioglossum versatum & Jason Gibbs \\
\hline Halictini & Lasioglossum & Lasioglossum versatum & \\
\hline Halictini & Lasioglossum & Lasioglossum versatum & Jason Gibbs \\
\hline Halictini & Lasioglossum & Lasioglossum versatum & Jason Gibbs \\
\hline Halictini & Lasioglossum & Lasioglossum versatum & Jason Gibbs \\
\hline Halictini & Lasioglossum & Lasioglossum vierecki & \\
\hline Halictini & Lasioglossum & Lasioglossum vierecki & Jason Gibbs \\
\hline Halictini & Lasioglossum & Lasioglossum vierecki & \\
\hline Halictini & Lasioglossum & Lasioglossum vierecki & \\
\hline Halictini & Lasioglossum & Lasioglossum vierecki & Jason Gibbs \\
\hline Halictini & Lasioglossum & Lasioglossum vierecki & Jason Gibbs \\
\hline Halictini & Lasioglossum & Lasioglossum vierecki & \\
\hline Halictini & Lasioglossum & Lasioglossum vierecki & Jason Gibbs \\
\hline Halictini & Lasioglossum & Lasioglossum vierecki & \\
\hline Halictini & Lasioglossum & Lasioglossum vierecki & \\
\hline Halictini & Lasioglossum & Lasioglossum vierecki & Jason Gibbs \\
\hline Halictini & Lasioglossum & Lasioglossum vierecki & \\
\hline Halictini & Lasioglossum & Lasioglossum vierecki & \\
\hline Halictini & Lasioglossum & Lasioglossum viridatum & Jason Gibbs \\
\hline Halictini & Lasioglossum & Lasioglossum viridatum & \\
\hline Halictini & Lasioglossum & Lasioglossum viridatum & Jason Gibbs \\
\hline Halictini & Lasioglossum & Lasioglossum viridatum & Jason Gibbs \\
\hline Halictini & Lasioglossum & Lasioglossum viridatum & \\
\hline Halictini & Lasioglossum & Lasioglossum viridatum & Jason Gibbs \\
\hline Halictini & Lasioglossum & Lasioglossum weemsi & Jason Gibbs \\
\hline Halictini & Lasioglossum & Lasioglossum weemsi & Jason Gibbs \\
\hline Halictini & Lasioglossum & Lasioglossum weemsi & Jason Gibbs \\
\hline Halictini & Lasioglossum & Lasioglossum weemsi & Jason Gibbs \\
\hline Halictini & Lasioglossum & Lasioglossum weemsi & Jason Gibbs \\
\hline Halictini & Lasioglossum & Lasioglossum weemsi & Jason Gibbs \\
\hline Halictini & Lasioglossum & Lasioglossum weemsi & Jason Gibbs \\
\hline Halictini & Lasioglossum & Lasioglossum weemsi & Jason Gibbs \\
\hline Halictini & Lasioglossum & Lasioglossum weemsi & Jason Gibbs \\
\hline Halictini & Lasioglossum & Lasioglossum weemsi & Jason Gibbs \\
\hline Halictini & Lasioglossum & Lasioglossum weemsi & Jason Gibbs \\
\hline Halictini & Lasioglossum & Lasioglossum weemsi & Jason Gibbs \\
\hline Halictini & Lasioglossum & Lasioglossum weemsi & Jason Gibbs \\
\hline Halictini & Lasioglossum & Lasioglossum weemsi & Jason Gibbs \\
\hline Halictini & Lasioglossum & Lasioglossum weemsi & Jason Gibbs \\
\hline Halictini & Lasioglossum & Lasioglossum weemsi & Jason Gibbs \\
\hline Halictini & Lasioglossum & Lasioglossum weemsi & Jason Gibbs \\
\hline Halictini & Lasioglossum & Lasioglossum weemsi & Jason Gibbs \\
\hline
\end{tabular}




$\begin{array}{llll}\text { Halictini } & \text { Lasioglossum } & \text { Lasioglossum weemsi } & \text { Jason Gibbs } \\ \text { Halictini } & \text { Lasioglossum } & \text { Lasioglossum weemsi } & \text { Jason Gibbs } \\ \text { Halictini } & \text { Lasioglossum } & \text { Lasioglossum weemsi } & \text { Jason Gibbs } \\ \text { Halictini } & \text { Lasioglossum } & \text { Lasioglossum weemsi } & \text { Jason Gibbs } \\ \text { Halictini } & \text { Lasioglossum } & \text { Lasioglossum weemsi } & \text { Jason Gibbs } \\ \text { Halictini } & \text { Lasioglossum } & \text { Lasioglossum weemsi } & \text { Jason Gibbs } \\ \text { Halictini } & \text { Lasioglossum } & \text { Lasioglossum weemsi } & \text { Jason Gibbs } \\ \text { Halictini } & \text { Lasioglossum } & \text { Lasioglossum yukonae } & \text { Jason Gibbs } \\ \text { Halictini } & \text { Lasioglossum } & \text { Lasioglossum yukonae } & \text { Jason Gibbs } \\ \text { Halictini } & \text { Lasioglossum } & \text { Lasioglossum yukonae } & \text { Jason Gibbs } \\ \text { Halictini } & \text { Lasioglossum } & \text { Lasioglossum zephyrum } & \text { Jason Gibbs } \\ \text { Halictini } & \text { Lasioglossum } & \text { Lasioglossum zephyrum } & \text { Jason Gibbs } \\ \text { Halictini } & \text { Lasioglossum } & \text { Lasioglossum zephyrum } & \text { Jason Gibbs } \\ \text { Halictini } & \text { Lasioglossum } & \text { Lasioglossum zephyrum } & \text { Jason Gibbs } \\ \text { Halictini } & \text { Lasioglossum } & \text { Lasioglossum zephyrum } & \\ \text { Halictini } & \text { Lasioglossum } & \text { Lasioglossum zephyrum } & \text { Jason Gibbs } \\ \text { Halictini } & \text { Lasioglossum } & \text { Lasioglossum zephyrum } & \end{array}$




\begin{abstract}
jason.gibbs@umanitoba.ca jason.gibbs@umanitoba.ca jason.gibbs@umanitoba.ca jason.gibbs@umanitoba.ca jason.gibbs@umanitoba.ca jason.gibbs@umanitoba.ca jason.gibbs@umanitoba.ca jason.gibbs@umanitoba.ca jason.gibbs@umanitoba.ca jason.gibbs@umanitoba.ca jason.gibbs@umanitoba.ca jason.gibbs@umanitoba.ca jason.gibbs@umanitoba.ca jason.gibbs@umanitoba.ca jason.gibbs@umanitoba.ca jason.gibbs@umanitoba.ca jason.gibbs@umanitoba.ca jason.gibbs@umanitoba.ca jason.gibbs@umanitoba.ca jason.gibbs@umanitoba.ca jason.gibbs@umanitoba.ca jason.gibbs@umanitoba.ca jason.gibbs@umanitoba.ca jason.gibbs@umanitoba.ca jason.gibbs@umanitoba.ca jason.gibbs@umanitoba.ca jason.gibbs@umanitoba.ca jason.gibbs@umanitoba.ca jason.gibbs@umanitoba.ca

jason.gibbs@umanitoba.ca jason.gibbs@umanitoba.ca jason.gibbs@umanitoba.ca jason.gibbs@umanitoba.ca jason.gibbs@umanitoba.ca jason.gibbs@umanitoba.ca jason.gibbs@umanitoba.ca jason.gibbs@umanitoba.ca
\end{abstract}

jason.gibbs@umanitoba.ca jason.gibbs@umanitoba.ca

jason.gibbs@umanitoba.ca jInatctmi@yahoo.com

jason.gibbs@umanitoba.ca

jason.gibbs@umanitoba.ca 
jason.gibbs@umanitoba.ca

jason.gibbs@umanitoba.ca jason.gibbs@umanitoba.ca

jason.gibbs@umanitoba.ca

jason.gibbs@umanitoba.ca jason.gibbs@umanitoba.ca jason.gibbs@umanitoba.ca jason.gibbs@umanitoba.ca sdroege@usgs.gov jason.gibbs@umanitoba.ca jason.gibbs@umanitoba.ca

jason.gibbs@umanitoba.ca jason.gibbs@umanitoba.ca jason.gibbs@umanitoba.ca jason.gibbs@umanitoba.ca jason.gibbs@umanitoba.ca jason.gibbs@umanitoba.ca jason.gibbs@umanitoba.ca jason.gibbs@umanitoba.ca jason.gibbs@umanitoba.ca jason.gibbs@umanitoba.ca jason.gibbs@umanitoba.ca jason.gibbs@umanitoba.ca jason.gibbs@umanitoba.ca jason.gibbs@umanitoba.ca jason.gibbs@umanitoba.ca jason.gibbs@umanitoba.ca jason.gibbs@umanitoba.ca jason.gibbs@umanitoba.ca jason.gibbs@umanitoba.ca jason.gibbs@umanitoba.ca jason.gibbs@umanitoba.ca jason.gibbs@umanitoba.ca jason.gibbs@umanitoba.ca 
jason.gibbs@umanitoba.ca jason.gibbs@umanitoba.ca jason.gibbs@umanitoba.ca jason.gibbs@umanitoba.ca jason.gibbs@umanitoba.ca jason.gibbs@umanitoba.ca jason.gibbs@umanitoba.ca jason.gibbs@umanitoba.ca jason.gibbs@umanitoba.ca jason.gibbs@umanitoba.ca jason.gibbs@umanitoba.ca jason.gibbs@umanitoba.ca jason.gibbs@umanitoba.ca jason.gibbs@umanitoba.ca jason.gibbs@umanitoba.ca jason.gibbs@umanitoba.ca jason.gibbs@umanitoba.ca jason.gibbs@umanitoba.ca jason.gibbs@umanitoba.ca jason.gibbs@umanitoba.ca jason.gibbs@umanitoba.ca jason.gibbs@umanitoba.ca jason.gibbs@umanitoba.ca jason.gibbs@umanitoba.ca jason.gibbs@umanitoba.ca jason.gibbs@umanitoba.ca jason.gibbs@umanitoba.ca jason.gibbs@umanitoba.ca jason.gibbs@umanitoba.ca jason.gibbs@umanitoba.ca jason.gibbs@umanitoba.ca jason.gibbs@umanitoba.ca jason.gibbs@umanitoba.ca jason.gibbs@umanitoba.ca jason.gibbs@umanitoba.ca jason.gibbs@umanitoba.ca jason.gibbs@umanitoba.ca jason.gibbs@umanitoba.ca jason.gibbs@umanitoba.ca jason.gibbs@umanitoba.ca jason.gibbs@umanitoba.ca jason.gibbs@umanitoba.ca jason.gibbs@umanitoba.ca jason.gibbs@umanitoba.ca jason.gibbs@umanitoba.ca jason.gibbs@umanitoba.ca jason.gibbs@umanitoba.ca jason.gibbs@umanitoba.ca jason.gibbs@umanitoba.ca jason.gibbs@umanitoba.ca jason.gibbs@umanitoba.ca jason.gibbs@umanitoba.ca jason.gibbs@umanitoba.ca jason.gibbs@umanitoba.ca jason.gibbs@umanitoba.ca 
jason.gibbs@umanitoba.ca jason.gibbs@umanitoba.ca jason.gibbs@umanitoba.ca jason.gibbs@umanitoba.ca jason.gibbs@umanitoba.ca jason.gibbs@umanitoba.ca jason.gibbs@umanitoba.ca jason.gibbs@umanitoba.ca jason.gibbs@umanitoba.ca jason.gibbs@umanitoba.ca jason.gibbs@umanitoba.ca jason.gibbs@umanitoba.ca jason.gibbs@umanitoba.ca

jason.gibbs@umanitoba.ca jason.gibbs@umanitoba.ca jason.gibbs@umanitoba.ca jason.gibbs@umanitoba.ca jason.gibbs@umanitoba.ca jason.gibbs@umanitoba.ca jason.gibbs@umanitoba.ca jason.gibbs@umanitoba.ca jason.gibbs@umanitoba.ca jason.gibbs@umanitoba.ca jason.gibbs@umanitoba.ca jason.gibbs@umanitoba.ca jason.gibbs@umanitoba.ca jason.gibbs@umanitoba.ca jason.gibbs@umanitoba.ca jason.gibbs@umanitoba.ca jason.gibbs@umanitoba.ca jason.gibbs@umanitoba.ca jason.gibbs@umanitoba.ca jason.gibbs@umanitoba.ca jason.gibbs@umanitoba.ca jason.gibbs@umanitoba.ca jason.gibbs@umanitoba.ca

jason.gibbs@umanitoba.ca jason.gibbs@umanitoba.ca

jason.gibbs@umanitoba.ca jason.gibbs@umanitoba.ca jason.gibbs@umanitoba.ca jason.gibbs@umanitoba.ca 
jason.gibbs@umanitoba.ca jason.gibbs@umanitoba.ca jason.gibbs@umanitoba.ca jason.gibbs@umanitoba.ca jason.gibbs@umanitoba.ca jason.gibbs@umanitoba.ca jason.gibbs@umanitoba.ca jason.gibbs@umanitoba.ca jason.gibbs@umanitoba.ca jason.gibbs@umanitoba.ca jason.gibbs@umanitoba.ca barcode jason.gibbs@umanitoba.ca jason.gibbs@umanitoba.ca jason.gibbs@umanitoba.ca jason.gibbs@umanitoba.ca jason.gibbs@umanitoba.ca jason.gibbs@umanitoba.ca jason.gibbs@umanitoba.ca jason.gibbs@umanitoba.ca jason.gibbs@umanitoba.ca jason.gibbs@umanitoba.ca jason.gibbs@umanitoba.ca jason.gibbs@umanitoba.ca jason.gibbs@umanitoba.ca jason.gibbs@umanitoba.ca jason.gibbs@umanitoba.ca jason.gibbs@umanitoba.ca jason.gibbs@umanitoba.ca jason.gibbs@umanitoba.ca jason.gibbs@umanitoba.ca jason.gibbs@umanitoba.ca jason.gibbs@umanitoba.ca jason.gibbs@umanitoba.ca jason.gibbs@umanitoba.ca jason.gibbs@umanitoba.ca jason.gibbs@umanitoba.ca jason.gibbs@umanitoba.ca jason.gibbs@umanitoba.ca jason.gibbs@umanitoba.ca jason.gibbs@umanitoba.ca jason.gibbs@umanitoba.ca jason.gibbs@umanitoba.ca jason.gibbs@umanitoba.ca jason.gibbs@umanitoba.ca jason.gibbs@umanitoba.ca jason.gibbs@umanitoba.ca jason.gibbs@umanitoba.ca jason.gibbs@umanitoba.ca jason.gibbs@umanitoba.ca jason.gibbs@umanitoba.ca jason.gibbs@umanitoba.ca jason.gibbs@umanitoba.ca jason.gibbs@umanitoba.ca jason.gibbs@umanitoba.ca jason.gibbs@umanitoba.ca

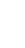


jason.gibbs@umanitoba.ca

jason.gibbs@umanitoba.ca

jason.gibbs@umanitoba.ca jason.gibbs@umanitoba.ca jason.gibbs@umanitoba.ca jason.gibbs@umanitoba.ca jason.gibbs@umanitoba.ca

jason.gibbs@umanitoba.ca

jason.gibbs@umanitoba.ca

jason.gibbs@umanitoba.ca

jason.gibbs@umanitoba.ca

jason.gibbs@umanitoba.ca jason.gibbs@umanitoba.ca jason.gibbs@umanitoba.ca

jason.gibbs@umanitoba.ca jason.gibbs@umanitoba.ca jason.gibbs@umanitoba.ca jason.gibbs@umanitoba.ca jason.gibbs@umanitoba.ca jason.gibbs@umanitoba.ca jason.gibbs@umanitoba.ca jason.gibbs@umanitoba.ca jason.gibbs@umanitoba.ca jason.gibbs@umanitoba.ca jason.gibbs@umanitoba.ca jason.gibbs@umanitoba.ca jason.gibbs@umanitoba.ca jason.gibbs@umanitoba.ca jason.gibbs@umanitoba.ca 


\begin{abstract}
jason.gibbs@umanitoba.ca jason.gibbs@umanitoba.ca jason.gibbs@umanitoba.ca jason.gibbs@umanitoba.ca jason.gibbs@umanitoba.ca jason.gibbs@umanitoba.ca jason.gibbs@umanitoba.ca jason.gibbs@umanitoba.ca jason.gibbs@umanitoba.ca jason.gibbs@umanitoba.ca jason.gibbs@umanitoba.ca jason.gibbs@umanitoba.ca jason.gibbs@umanitoba.ca jason.gibbs@umanitoba.ca jason.gibbs@umanitoba.ca jason.gibbs@umanitoba.ca jason.gibbs@umanitoba.ca jason.gibbs@umanitoba.ca jason.gibbs@umanitoba.ca jason.gibbs@umanitoba.ca jason.gibbs@umanitoba.ca jason.gibbs@umanitoba.ca jason.gibbs@umanitoba.ca jason.gibbs@umanitoba.ca jason.gibbs@umanitoba.ca jason.gibbs@umanitoba.ca jason.gibbs@umanitoba.ca jason.gibbs@umanitoba.ca jason.gibbs@umanitoba.ca
\end{abstract}

jason.gibbs@umanitoba.ca

jason.gibbs@umanitoba.ca

jason.gibbs@umanitoba.ca jason.gibbs@umanitoba.ca jason.gibbs@umanitoba.ca jason.gibbs@umanitoba.ca jason.gibbs@umanitoba.ca jason.gibbs@umanitoba.ca jason.gibbs@umanitoba.ca jason.gibbs@umanitoba.ca jason.gibbs@umanitoba.ca jason.gibbs@umanitoba.ca jason.gibbs@umanitoba.ca jason.gibbs@umanitoba.ca jason.gibbs@umanitoba.ca jason.gibbs@umanitoba.ca jason.gibbs@umanitoba.ca jason.gibbs@umanitoba.ca jason.gibbs@umanitoba.ca 
jason.gibbs@umanitoba.ca

jason.gibbs@umanitoba.ca jason.gibbs@umanitoba.ca jason.gibbs@umanitoba.ca jason.gibbs@umanitoba.ca jason.gibbs@umanitoba.ca

jason.gibbs@umanitoba.ca jason.gibbs@umanitoba.ca

jason.gibbs@umanitoba.ca jason.gibbs@umanitoba.ca jason.gibbs@umanitoba.ca jason.gibbs@umanitoba.ca jason.gibbs@umanitoba.ca jason.gibbs@umanitoba.ca jason.gibbs@umanitoba.ca jason.gibbs@umanitoba.ca jason.gibbs@umanitoba.ca jason.gibbs@umanitoba.ca jason.gibbs@umanitoba.ca jason.gibbs@umanitoba.ca jason.gibbs@umanitoba.ca jason.gibbs@umanitoba.ca jason.gibbs@umanitoba.ca jason.gibbs@umanitoba.ca jason.gibbs@umanitoba.ca jason.gibbs@umanitoba.ca jason.gibbs@umanitoba.ca

jason.gibbs@umanitoba.ca

jason.gibbs@umanitoba.ca jason.gibbs@umanitoba.ca

jason.gibbs@umanitoba.ca jason.gibbs@umanitoba.ca

jason.gibbs@umanitoba.ca jason.gibbs@umanitoba.ca jason.gibbs@umanitoba.ca jason.gibbs@umanitoba.ca jason.gibbs@umanitoba.ca jason.gibbs@umanitoba.ca 


\begin{abstract}
jason.gibbs@umanitoba.ca jason.gibbs@umanitoba.ca jason.gibbs@umanitoba.ca jason.gibbs@umanitoba.ca jason.gibbs@umanitoba.ca jason.gibbs@umanitoba.ca jason.gibbs@umanitoba.ca jason.gibbs@umanitoba.ca jason.gibbs@umanitoba.ca jason.gibbs@umanitoba.ca jason.gibbs@umanitoba.ca jason.gibbs@umanitoba.ca jason.gibbs@umanitoba.ca jason.gibbs@umanitoba.ca jason.gibbs@umanitoba.ca jason.gibbs@umanitoba.ca jason.gibbs@umanitoba.ca
\end{abstract}

jason.gibbs@umanitoba.ca jason.gibbs@umanitoba.ca

jason.gibbs@umanitoba.ca jason.gibbs@umanitoba.ca jason.gibbs@umanitoba.ca jason.gibbs@umanitoba.ca

jason.gibbs@umanitoba.ca jason.gibbs@umanitoba.ca jason.gibbs@umanitoba.ca jason.gibbs@umanitoba.ca jason.gibbs@umanitoba.ca

jason.gibbs@umanitoba.ca jason.gibbs@umanitoba.ca jason.gibbs@umanitoba.ca jason.gibbs@umanitoba.ca jason.gibbs@umanitoba.ca

jason.gibbs@umanitoba.ca jason.gibbs@umanitoba.ca jason.gibbs@umanitoba.ca jason.gibbs@umanitoba.ca

jason.gibbs@umanitoba.ca 
jason.gibbs@umanitoba.ca jason.gibbs@umanitoba.ca jason.gibbs@umanitoba.ca jason.gibbs@umanitoba.ca jason.gibbs@umanitoba.ca jason.gibbs@umanitoba.ca jason.gibbs@umanitoba.ca jason.gibbs@umanitoba.ca jason.gibbs@umanitoba.ca

\begin{abstract}
jason.gibbs@umanitoba.ca jason.gibbs@umanitoba.ca

jason.gibbs@umanitoba.ca jason.gibbs@umanitoba.ca jason.gibbs@umanitoba.ca
\end{abstract}

jason.gibbs@umanitoba.ca jason.gibbs@umanitoba.ca

jason.gibbs@umanitoba.ca

jason.gibbs@umanitoba.ca

jason.gibbs@umanitoba.ca jason.gibbs@umanitoba.ca jason.gibbs@umanitoba.ca jason.gibbs@umanitoba.ca

jason.gibbs@umanitoba.ca jason.gibbs@umanitoba.ca jason.gibbs@umanitoba.ca jason.gibbs@umanitoba.ca 


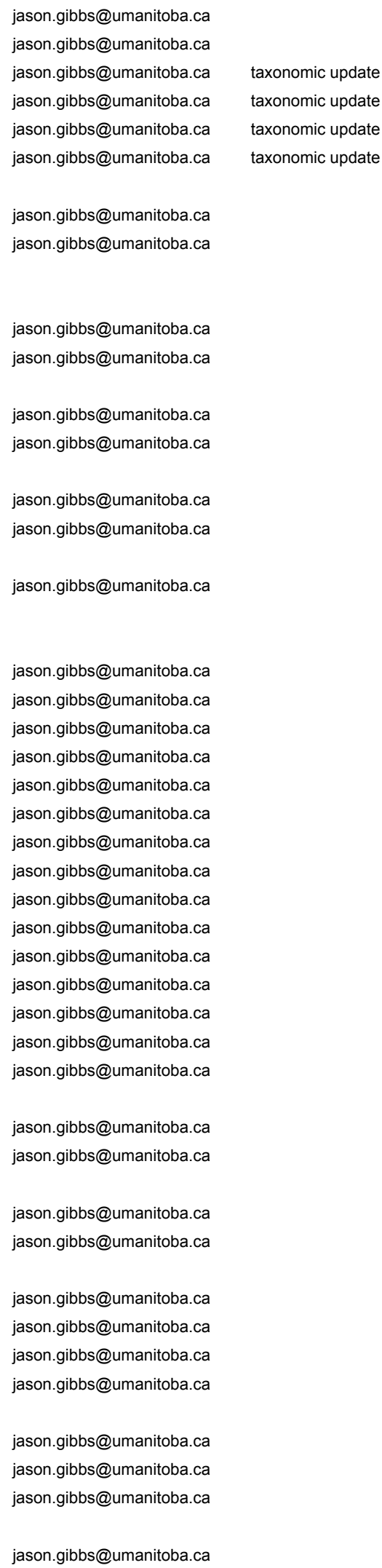

taxonomic update taxonomic update taxonomic update taxonomic update 


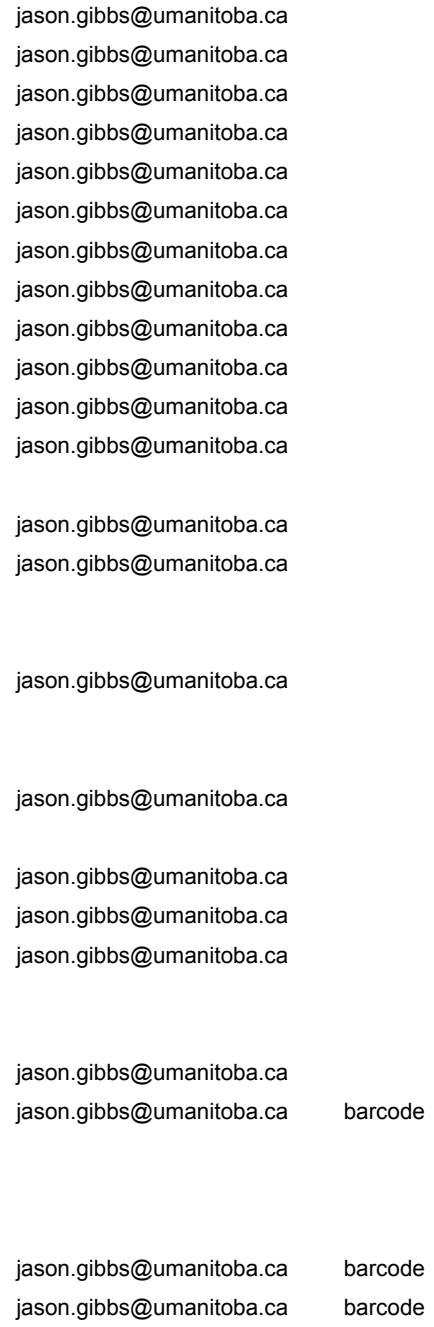

jason.gibbs@umanitoba.ca

jason.gibbs@umanitoba.ca jason.gibbs@umanitoba.ca jason.gibbs@umanitoba.ca

jason.gibbs@umanitoba.ca jason.gibbs@umanitoba.ca jason.gibbs@umanitoba.ca jason.gibbs@umanitoba.ca jason.gibbs@umanitoba.ca jason.gibbs@umanitoba.ca jason.gibbs@umanitoba.ca jason.gibbs@umanitoba.ca jason.gibbs@umanitoba.ca jason.gibbs@umanitoba.ca 
jason.gibbs@umanitoba.ca
jason.gibbs@umanitoba.ca
jason.gibbs@umanitoba.ca
jason.gibbs@umanitoba.ca
jason.gibbs@umanitoba.ca
jason.gibbs@umanitoba.ca
jason.gibbs@umanitoba.ca
jason.gibbs@umanitoba.ca
jason.gibbs@umanitoba.ca
jason.gibbs@umanitoba.ca

jason.gibbs@umanitoba.ca jason.gibbs@umanitoba.ca jason.gibbs@umanitoba.ca jason.gibbs@umanitoba.ca jason.gibbs@umanitoba.ca jason.gibbs@umanitoba.ca jason.gibbs@umanitoba.ca jason.gibbs@umanitoba.ca jason.gibbs@umanitoba.ca jason.gibbs@umanitoba.ca

jason.gibbs@umanitoba.ca jason.gibbs@umanitoba.ca

jason.gibbs@umanitoba.ca jason.gibbs@umanitoba.ca jason.gibbs@umanitoba.ca jason.gibbs@umanitoba.ca

jason.gibbs@umanitoba.ca jason.gibbs@umanitoba.ca jason.gibbs@umanitoba.ca jason.gibbs@umanitoba.ca jason.gibbs@umanitoba.ca jason.gibbs@umanitoba.ca jason.gibbs@umanitoba.ca jason.gibbs@umanitoba.ca jason.gibbs@umanitoba.ca jason.gibbs@umanitoba.ca jason.gibbs@umanitoba.ca jason.gibbs@umanitoba.ca jason.gibbs@umanitoba.ca jason.gibbs@umanitoba.ca jason.gibbs@umanitoba.ca jason.gibbs@umanitoba.ca jason.gibbs@umanitoba.ca jason.gibbs@umanitoba.ca jason.gibbs@umanitoba.ca jason.gibbs@umanitoba.ca 
jason.gibbs@umanitoba.ca

jason.gibbs@umanitoba.ca jason.gibbs@umanitoba.ca

jason.gibbs@umanitoba.ca

jason.gibbs@umanitoba.ca jason.gibbs@umanitoba.ca jason.gibbs@umanitoba.ca

jason.gibbs@umanitoba.ca

jason.gibbs@umanitoba.ca jason.gibbs@umanitoba.ca jason.gibbs@umanitoba.ca jason.gibbs@umanitoba.ca jason.gibbs@umanitoba.ca jason.gibbs@umanitoba.ca jason.gibbs@umanitoba.ca jason.gibbs@umanitoba.ca jason.gibbs@umanitoba.ca jason.gibbs@umanitoba.ca jason.gibbs@umanitoba.ca jason.gibbs@umanitoba.ca jason.gibbs@umanitoba.ca jason.gibbs@umanitoba.ca jason.gibbs@umanitoba.ca jason.gibbs@umanitoba.ca jason.gibbs@umanitoba.ca jason.gibbs@umanitoba.ca jason.gibbs@umanitoba.ca jason.gibbs@umanitoba.ca jason.gibbs@umanitoba.ca jason.gibbs@umanitoba.ca jason.gibbs@umanitoba.ca jason.gibbs@umanitoba.ca jason.gibbs@umanitoba.ca jason.gibbs@umanitoba.ca jason.gibbs@umanitoba.ca jason.gibbs@umanitoba.ca

jason.gibbs@umanitoba.ca jason.gibbs@umanitoba.ca jason.gibbs@umanitoba.ca jason.gibbs@umanitoba.ca jason.gibbs@umanitoba.ca jason.gibbs@umanitoba.ca jason.gibbs@umanitoba.ca jason.gibbs@umanitoba.ca jason.gibbs@umanitoba.ca jason.gibbs@umanitoba.ca 


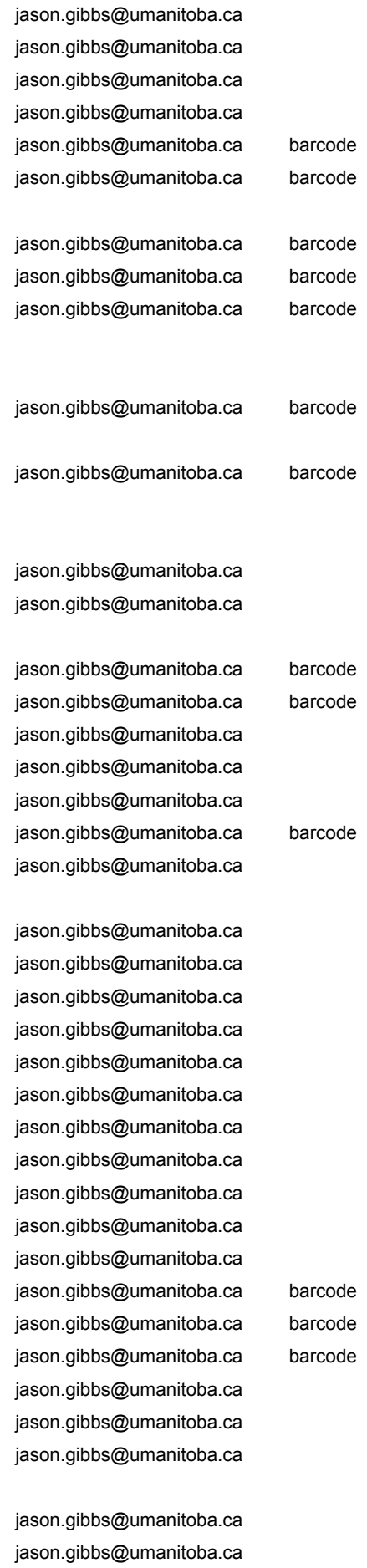


jason.gibbs@umanitoba.ca jInatctmi@yahoo.com

jason.gibbs@umanitoba.ca jason.gibbs@umanitoba.ca

jason.gibbs@umanitoba.ca jason.gibbs@umanitoba.ca jason.gibbs@umanitoba.ca

jason.gibbs@umanitoba.ca jason.gibbs@umanitoba.ca jason.gibbs@umanitoba.ca jason.gibbs@umanitoba.ca jason.gibbs@umanitoba.ca jason.gibbs@umanitoba.ca jason.gibbs@umanitoba.ca jason.gibbs@umanitoba.ca jason.gibbs@umanitoba.ca jason.gibbs@umanitoba.ca jason.gibbs@umanitoba.ca jason.gibbs@umanitoba.ca

jason.gibbs@umanitoba.ca jason.gibbs@umanitoba.ca jason.gibbs@umanitoba.ca jason.gibbs@umanitoba.ca jason.gibbs@umanitoba.ca jason.gibbs@umanitoba.ca jason.gibbs@umanitoba.ca jason.gibbs@umanitoba.ca jason.gibbs@umanitoba.ca jason.gibbs@umanitoba.ca jason.gibbs@umanitoba.ca jason.gibbs@umanitoba.ca jason.gibbs@umanitoba.ca jason.gibbs@umanitoba.ca jason.gibbs@umanitoba.ca jason.gibbs@umanitoba.ca 
jason.gibbs@umanitoba.ca jason.gibbs@umanitoba.ca jason.gibbs@umanitoba.ca jason.gibbs@umanitoba.ca jason.gibbs@umanitoba.ca jason.gibbs@umanitoba.ca jason.gibbs@umanitoba.ca jason.gibbs@umanitoba.ca jason.gibbs@umanitoba.ca jason.gibbs@umanitoba.ca jason.gibbs@umanitoba.ca jason.gibbs@umanitoba.ca jason.gibbs@umanitoba.ca jason.gibbs@umanitoba.ca jason.gibbs@umanitoba.ca jason.gibbs@umanitoba.ca jason.gibbs@umanitoba.ca jason.gibbs@umanitoba.ca jason.gibbs@umanitoba.ca jason.gibbs@umanitoba.ca jason.gibbs@umanitoba.ca jason.gibbs@umanitoba.ca jason.gibbs@umanitoba.ca jason.gibbs@umanitoba.ca jason.gibbs@umanitoba.ca jason.gibbs@umanitoba.ca jason.gibbs@umanitoba.ca jason.gibbs@umanitoba.ca jason.gibbs@umanitoba.ca jason.gibbs@umanitoba.ca jason.gibbs@umanitoba.ca jason.gibbs@umanitoba.ca jason.gibbs@umanitoba.ca jason.gibbs@umanitoba.ca jason.gibbs@umanitoba.ca jason.gibbs@umanitoba.ca jason.gibbs@umanitoba.ca jason.gibbs@umanitoba.ca jason.gibbs@umanitoba.ca jason.gibbs@umanitoba.ca jason.gibbs@umanitoba.ca jason.gibbs@umanitoba.ca jason.gibbs@umanitoba.ca jason.gibbs@umanitoba.ca jason.gibbs@umanitoba.ca jason.gibbs@umanitoba.ca jason.gibbs@umanitoba.ca jason.gibbs@umanitoba.ca jason.gibbs@umanitoba.ca jason.gibbs@umanitoba.ca jason.gibbs@umanitoba.ca jason.gibbs@umanitoba.ca jason.gibbs@umanitoba.ca 


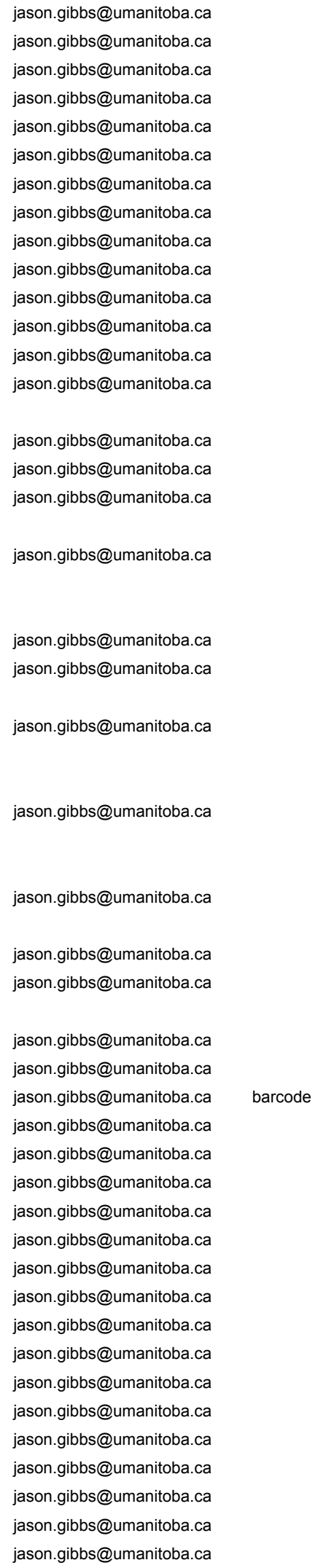


jason.gibbs@umanitoba.ca jason.gibbs@umanitoba.ca jason.gibbs@umanitoba.ca jason.gibbs@umanitoba.ca jason.gibbs@umanitoba.ca jason.gibbs@umanitoba.ca jason.gibbs@umanitoba.ca jason.gibbs@umanitoba.ca jason.gibbs@umanitoba.ca jason.gibbs@umanitoba.ca jason.gibbs@umanitoba.ca jason.gibbs@umanitoba.ca jason.gibbs@umanitoba.ca jason.gibbs@umanitoba.ca

jason.gibbs@umanitoba.ca 


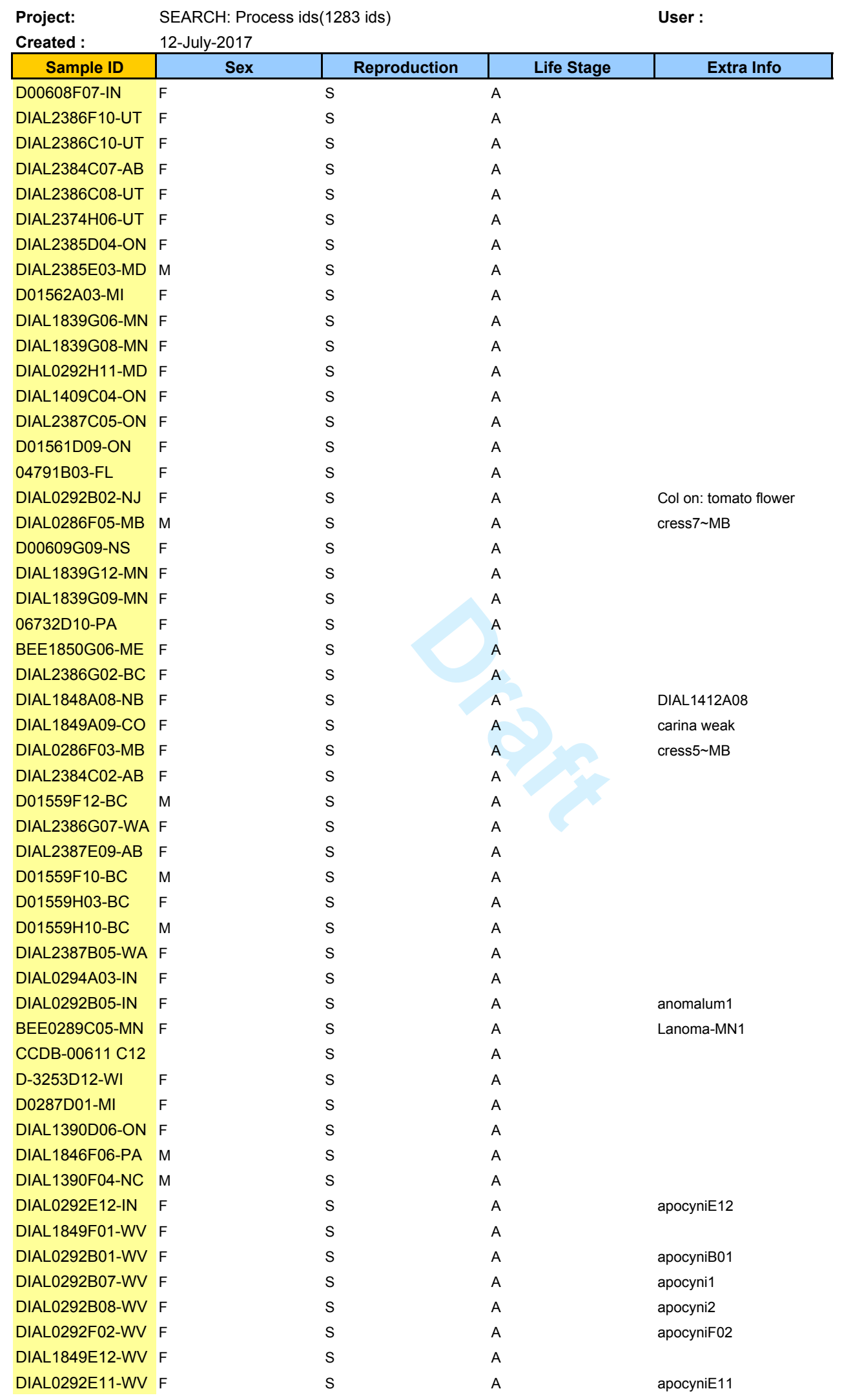




\begin{tabular}{|c|c|c|c|c|}
\hline DIAL1390F06-NC & $\mathrm{F}$ & $\mathrm{s}$ & A & \\
\hline DIAL1390G04-NC & $\mathrm{F}$ & S & A & \\
\hline DIAL1390G06-NC & $\mathrm{F}$ & S & A & \\
\hline DIAL1390G02-WV & M & $\mathrm{s}$ & A & \\
\hline DIAL1848D09-MD & $\mathrm{F}$ & S & A & $293 \mathrm{G} 09$ \\
\hline DIAL1846D12-MS & $\mathrm{F}$ & S & A & \\
\hline D1414H02-VA & $\mathrm{F}$ & S & A & \\
\hline DIAL2384H09-SC & $\mathrm{F}$ & S & A & \\
\hline D01559C09-GA & $\mathrm{F}$ & S & A & \\
\hline D1414E02-VA & $\mathrm{F}$ & S & A & \\
\hline DIAL0293C03-NJ & $\mathrm{F}$ & S & A & pictum1 \\
\hline DIAL0293C04-NJ & $\mathrm{F}$ & S & A & pictum2 \\
\hline D01562B06-MD & $\mathrm{F}$ & S & A & \\
\hline D01562B02-MD & $\mathrm{F}$ & S & A & \\
\hline D01562C12-MD & $\mathrm{F}$ & S & A & \\
\hline D01562B10-ON & $\mathrm{F}$ & S & A & \\
\hline D01562C08-ON & $\mathrm{F}$ & S & A & \\
\hline DIAL0294G02-MD & $\mathrm{F}$ & S & A & DIAL6MD \\
\hline DIAL1848B03-ON & $\mathrm{F}$ & S & A & \\
\hline D00609B05-WI & $\mathrm{F}$ & S & A & \\
\hline D00609G03-NS & $\mathrm{F}$ & $S$ & A & \\
\hline D0288F07-SC & $\mathrm{F}$ & S & A & \\
\hline D0288F09-SC & $\mathrm{F}$ & S & A & \\
\hline DIAL0294A05-in & $\mathrm{F}$ & S & A & brune $\sim$ IN2 \\
\hline DIAL0292E08-MD & $\mathrm{F}$ & S & A & bruneriE08 \\
\hline 06732D09-VA & $\mathrm{F}$ & $\mathrm{s}$ & A & \\
\hline D00609C07-KS & $\mathrm{F}$ & S & A & \\
\hline DIAL1846B10-WV & $\mathrm{F}$ & s & A & \\
\hline DIAL1390C08-ON & $M$ & S & A & \\
\hline DIAL1409A09-NC & M & $\mathrm{s}$ & A & \\
\hline DIAL1846G04-VA & $M$ & $S$ & A & \\
\hline DIAL1390B02-ON & $\mathrm{F}$ & $S$ & A & \\
\hline DIAL1848E12-MD & M & $S$ & A & DIAL1412E12 \\
\hline DIAL1413C12-TN & $\mathrm{F}$ & $S$ & A & \\
\hline D01559G11-BC & $\mathrm{F}$ & S & A & \\
\hline DIAL1845D09-CA & $\mathrm{F}$ & $S$ & A & \\
\hline DIAL2374G12-CA & $\mathrm{F}$ & $S$ & A & long face \\
\hline DIAL1845C05-CA & $\mathrm{F}$ & $S$ & A & \\
\hline DIAL1845D03-CA & $\mathrm{F}$ & $S$ & A & \\
\hline DIAL1845C11-CA & $\mathrm{F}$ & $S$ & A & \\
\hline DIAL2388C12-UT & $\mathrm{F}$ & $S$ & A & \\
\hline DIAL2374G11-CA & $\mathrm{F}$ & $S$ & A & long face \\
\hline D00607E04-CA & $\mathrm{F}$ & $S$ & A & \\
\hline D01559E10-BC & M & $S$ & A & \\
\hline D01559G04-BC & $\mathrm{F}$ & $s$ & A & \\
\hline DIAL2388B06-UT & $\mathrm{F}$ & $S$ & A & \\
\hline D00607D05-CA & $\mathrm{F}$ & $S$ & A & \\
\hline DIAL2374H09-UT & $\mathrm{F}$ & $S$ & A & \\
\hline DIAL2385C03-MD & M & $S$ & A & \\
\hline DIAL1413E11-SC & M & $S$ & A & \\
\hline DIAL0292D03-NC & $\mathrm{F}$ & $S$ & A & \\
\hline DIAL0294E03-VA & $\mathrm{F}$ & $S$ & A & versa2 VA \\
\hline DIAL0294E02-TN & $\mathrm{F}$ & S & A & versa1 TN \\
\hline DIAL0293E11-NC & $\mathrm{F}$ & $S$ & A & \\
\hline DIAL0293E10-MD & $\mathrm{F}$ & $\mathrm{s}$ & A & versatum 1 \\
\hline
\end{tabular}




\begin{tabular}{|c|c|c|c|c|}
\hline D0288C04-DC & $\mathrm{F}$ & $\mathrm{s}$ & A & \\
\hline DIAL0293E12-DC & $\mathrm{F}$ & $s$ & A & \\
\hline D1414H07-VA & $\mathrm{F}$ & S & A & \\
\hline BEE0286D06-VA & $\mathrm{F}$ & $s$ & A & versa1 VA \\
\hline DIAL1413D04-NC & $\mathrm{F}$ & S & A & \\
\hline DIAL1391D10-NC & $\mathrm{F}$ & $s$ & A & \\
\hline D1414E08-VA & $\mathrm{F}$ & S & A & \\
\hline DIAL1409B06-NC & $\mathrm{F}$ & s & A & \\
\hline DIAL1413E08-NC & $\mathrm{F}$ & s & A & \\
\hline D1414E04-VA & $\mathrm{F}$ & s & A & \\
\hline DIAL1391A09-SC & $\mathrm{F}$ & s & A & \\
\hline DIAL1413F07-TN & $\mathrm{F}$ & s & A & \\
\hline DIAL1391D09-NC & $\mathrm{F}$ & $S$ & A & \\
\hline DIAL1391D07-NC & $\mathrm{F}$ & S & A & \\
\hline DIAL1413D01-TN & $\mathrm{F}$ & $S$ & A & \\
\hline D1414E07-VA & $\mathrm{F}$ & s & A & \\
\hline D0288C01-DC & $\mathrm{F}$ & $S$ & A & \\
\hline D0288C06-SC & $\mathrm{F}$ & S & A & \\
\hline D0288D09-SC & $\mathrm{F}$ & s & A & \\
\hline D00607E06-MO & $\mathrm{F}$ & s & A & \\
\hline D00607A09-MO & $\mathrm{F}$ & s & A & \\
\hline D0288G03-SC & $\mathrm{F}$ & S & A & \\
\hline D0288F02-SC & $\mathrm{F}$ & S & A & \\
\hline DIAL1391B01-SC & $\mathrm{F}$ & s & A & \\
\hline DIAL1391E04-SC & $\mathrm{F}$ & s & A & \\
\hline DIAL1391D11-SC & $\mathrm{F}$ & s & A & \\
\hline DIAL1413B06-IL & $\mathrm{F}$ & s & A & CARLINVILLENSE \\
\hline D1414A07-IL & $\mathrm{F}$ & s & A & CARLINVILLENSE \\
\hline DIAL1413B11-IL & $\mathrm{F}$ & s & A & CARLINVILLENSE \\
\hline 04485C03-MD & $\mathrm{F}$ & s & A & \\
\hline D00607F06-MD & $\mathrm{F}$ & s & A & \\
\hline D-3253D11-FL & $\mathrm{F}$ & S & A & \\
\hline D00607B02-KS & M & S & A & \\
\hline DIAL0292D09-MD & $\mathrm{F}$ & s & A & coreopsis2 \\
\hline DIAL0292D10-MD & $\mathrm{F}$ & s & A & coreopsis3 \\
\hline DIAL0292D08-NC & $\mathrm{F}$ & S & A & coreopsis1 \\
\hline DIAL0292D12-MD & $\mathrm{F}$ & S & A & coreopsis5 \\
\hline DIAL0292D11-MD & $\mathrm{F}$ & S & A & coreopsis4 \\
\hline DIAL1846H06-VA & $\mathrm{F}$ & S & A & \\
\hline DIAL1846C01-MS & $\mathrm{F}$ & S & A & \\
\hline DIAL1846E11-MS & $\mathrm{F}$ & $S$ & A & \\
\hline DIAL1409E03-TX & $\mathrm{F}$ & S & A & \\
\hline DIAL1846G07-MS & $\mathrm{F}$ & S & A & \\
\hline DIAL1849F11-DE & $\mathrm{F}$ & $S$ & A & \\
\hline DIAL1849G03-DE & $\mathrm{F}$ & $S$ & A & \\
\hline DIAL1391D04-NC & $\mathrm{F}$ & S & A & \\
\hline 06732C08-MA & $\mathrm{F}$ & S & A & \\
\hline 06732C10-VA & $\mathrm{F}$ & S & A & \\
\hline D-3253D07-FL & $\mathrm{F}$ & $S$ & A & \\
\hline B01566H05-TX & $\mathrm{F}$ & $s$ & A & \\
\hline DIAL0292E02-MD & $\mathrm{F}$ & $S$ & A & crebberimum2 \\
\hline DIAL0294F02-NC & $\mathrm{F}$ & $S$ & A & crebe2 NC \\
\hline DIAL0294F03-NC & $\mathrm{F}$ & $\mathrm{s}$ & A & crebe3 NC \\
\hline DIAL0294E05-NC & $\mathrm{F}$ & $S$ & A & crebe1 NC \\
\hline DIAL0292E04-MD & $\mathrm{F}$ & $\mathrm{s}$ & A & crebberimum4 \\
\hline
\end{tabular}




\begin{tabular}{|c|c|c|c|c|}
\hline DIAL1413D12-FL & $\mathrm{F}$ & $\mathrm{s}$ & A & \\
\hline DIAL1846C06-MS & $\mathrm{F}$ & S & A & \\
\hline DIAL1846H02-MS & $\mathrm{F}$ & s & A & \\
\hline DIAL1846C11-MS & $\mathrm{F}$ & s & A & \\
\hline CCDB-00611 E05 & $\mathrm{F}$ & s & A & \\
\hline BEE0286C08-NC & $\mathrm{F}$ & $\mathrm{s}$ & A & cress2 NC \\
\hline DIAL0294A11-IN & $\mathrm{F}$ & s & A & cress $\sim \mathrm{IN} 2$ \\
\hline DIAL0294A10-IN & $\mathrm{F}$ & S & A & cress $\sim \mathrm{IN} 1$ \\
\hline DIAL1390C02-ON & $\mathrm{F}$ & S & A & \\
\hline BEE0286A05-GA & $\mathrm{F}$ & s & A & cress1 GA \\
\hline DIAL0294A09-in & $\mathrm{F}$ & s & A & cress IL1 \\
\hline DIAL0292E09-MD & $\mathrm{F}$ & S & A & \\
\hline DIAL1845H09-BC & $\mathrm{F}$ & $\mathrm{s}$ & A & \\
\hline DIAL1845H07-BC & $\mathrm{F}$ & s & A & \\
\hline DIAL0292E07-ME & $\mathrm{F}$ & s & A & \\
\hline DIAL1845F11-BC & $\mathrm{F}$ & S & A & \\
\hline DIAL1391A03-NC & M & S & A & \\
\hline BEE0289D05-MN & $\mathrm{F}$ & s & A & Lcress-MN1 \\
\hline BEE0289D07-MN & $\mathrm{F}$ & s & A & Lcress-MN3 \\
\hline DIAL1391A05-NC & M & s & A & \\
\hline D00609G07-NS & $\mathrm{F}$ & s & A & \\
\hline DIAL1848A03-NB & $\mathrm{F}$ & s & A & DIAL1412A03 \\
\hline BEE1850G05-ME & $\mathrm{F}$ & s & A & \\
\hline BEE0289D06-MN & $\mathrm{F}$ & s & A & Lcress-MN2 \\
\hline D01561G01-MD & M & s & A & \\
\hline DIAL2386H03-WA & $\mathrm{F}$ & s & A & \\
\hline DIAL1391A06-NC & $\mathrm{F}$ & s & A & \\
\hline D0287G06-IL & $\mathrm{F}$ & s & A & \\
\hline D0288G10-ON & $\mathrm{F}$ & s & A & \\
\hline DIAL2386G06-BC & $\mathrm{F}$ & s & A & \\
\hline 03767G09-BC & $\mathrm{F}$ & s & A & \\
\hline 06732B12-NY & $\mathrm{F}$ & s & A & \\
\hline DIAL2386F07-WA & M & s & A & \\
\hline DIAL2386H05-WA & $\mathrm{F}$ & s & A & WA-3 \\
\hline D-3253E10-OK & M & s & A & \\
\hline D00607B09-KS & $\mathrm{F}$ & s & A & \\
\hline DIAL1409D07-TX & $\mathrm{F}$ & s & A & \\
\hline DIAL1409D04-TX & $\mathrm{F}$ & S & A & \\
\hline DIAL1409E08-TX & $\mathrm{F}$ & s & A & \\
\hline DIAL1409E10-TX & $\mathrm{F}$ & s & A & \\
\hline DIAL1409F01-TX & $\mathrm{F}$ & s & A & \\
\hline D00607B01-KS & $\mathrm{F}$ & s & A & \\
\hline DIAL1848F12-NC & M & s & A & \\
\hline D01561B12-ON & $\mathrm{F}$ & S & A & \\
\hline DIAL1848F10-ON & $\mathrm{F}$ & S & A & \\
\hline DIAL1848G01-ON & $\mathrm{F}$ & s & A & \\
\hline D01561E03-ON & $\mathrm{F}$ & s & A & \\
\hline DIAL2374G04-UT & $\mathrm{F}$ & S & A & \\
\hline DIAL2388B04-UT & $\mathrm{F}$ & S & A & \\
\hline DIAL2388C03-UT & $\mathrm{F}$ & S & A & \\
\hline DIAL2386B05-NV & M & S & A & \\
\hline DIAL2384C08-AB & $\mathrm{F}$ & S & A & \\
\hline DIAL2374G05-UT & $\mathrm{F}$ & S & A & \\
\hline DIAL0294D02-IN & $\mathrm{F}$ & S & A & tegul IN1 \\
\hline D01560D03-IA & $\mathrm{F}$ & $\mathrm{s}$ & A & \\
\hline
\end{tabular}




\begin{tabular}{|c|c|c|c|c|}
\hline D01560A07-WI & M & S & A & \\
\hline DIAL1409C09-ON & $\mathrm{F}$ & $s$ & A & \\
\hline DIAL1390A09-ON & $\mathrm{F}$ & S & A & \\
\hline DIAL1839G05-MN & $\mathrm{F}$ & $s$ & A & \\
\hline D0288D03-IN & $\mathrm{F}$ & S & A & \\
\hline DIAL1413G03-NC & $\mathrm{F}$ & $s$ & A & \\
\hline D0295C04-ON & $\mathrm{F}$ & S & A & \\
\hline DIAL1391H04-NC & M & s & A & \\
\hline DIAL1849G11-MA & M & s & A & \\
\hline DIAL1839H01-MN & $\mathrm{F}$ & s & A & \\
\hline D0295C05-ON & $\mathrm{F}$ & S & A & \\
\hline D01562C02-MB & $\mathrm{F}$ & S & A & \\
\hline D01562B07-MI & $\mathrm{F}$ & S & A & \\
\hline B03765D08-IA & $\mathrm{F}$ & S & A & \\
\hline 04485E09-MI & $\mathrm{F}$ & S & A & \\
\hline B03765D06-IA & $\mathrm{F}$ & s & A & \\
\hline 04485E02-MI & $\mathrm{F}$ & $S$ & A & \\
\hline B03765D11-IA & $\mathrm{F}$ & S & A & \\
\hline B03765E01-IA & $\mathrm{F}$ & s & A & \\
\hline D1414G06-DC & $\mathrm{F}$ & s & A & \\
\hline DIAL1846H11-DC & M & $S$ & A & \\
\hline BEE0289A03-MN & $\mathrm{F}$ & S & A & LASIO-MN1 \\
\hline DIAL1846E03-PA & $\mathrm{F}$ & $\mathrm{s}$ & A & \\
\hline D0295E03-VA & $\mathrm{F}$ & s & A & \\
\hline BEE0289A07-MN & $\mathrm{F}$ & s & A & LASIO-MN3 \\
\hline DIAL0294H08-ON & $\mathrm{F}$ & s & A & rohwe1 ON \\
\hline DIAL1390F12-NC & $\mathrm{F}$ & s & A & \\
\hline D0295C07-ON & $\mathrm{F}$ & s & A & \\
\hline DIAL2387C02-ON & $\mathrm{F}$ & s & A & \\
\hline DIAL1849H03-DC & M & s & A & \\
\hline DIAL1846A05-ON & $\mathrm{F}$ & s & A & \\
\hline DIAL1846A07-ON & $\mathrm{F}$ & S & A & \\
\hline DIAL1846A02-ON & $\mathrm{F}$ & S & A & \\
\hline DIAL1390F08-WV & $\mathrm{F}$ & s & A & \\
\hline DIAL1391F06-ON & $\mathrm{F}$ & s & A & \\
\hline DIAL1391G07-ON & $\mathrm{F}$ & S & A & \\
\hline DIAL1390F03-ON & $\mathrm{F}$ & S & A & \\
\hline DIAL1846B02-ON & $\mathrm{F}$ & S & A & \\
\hline DIAL1846A03-ON & $\mathrm{F}$ & S & A & \\
\hline DIAL1409C02-ON & $\mathrm{F}$ & S & A & \\
\hline DIAL1409C03-ON & M & $S$ & A & \\
\hline DIAL1409C07-ON & $\mathrm{F}$ & S & A & \\
\hline DIAL1409C08-ON & $\mathrm{F}$ & S & A & \\
\hline D01562C10-MB & $\mathrm{F}$ & $S$ & A & \\
\hline DIAL1390E01-ON & $\mathrm{F}$ & $S$ & A & \\
\hline DIAL1390F01-ON & $\mathrm{F}$ & S & A & \\
\hline DIAL1390G01-NC & $\mathrm{F}$ & S & A & \\
\hline DIAL1390F09-ON & $\mathrm{F}$ & S & A & \\
\hline DIAL1390F05-ON & $\mathrm{F}$ & $S$ & A & \\
\hline DIAL1391G03-ON & M & $S$ & A & \\
\hline DIAL1390E03-ON & $\mathrm{F}$ & $S$ & A & \\
\hline DIAL1390E06-ON & $\mathrm{F}$ & $S$ & A & \\
\hline DIAL1390E07-ON & $\mathrm{F}$ & $S$ & A & \\
\hline DIAL1391G05-ON & $\mathrm{F}$ & $S$ & A & \\
\hline DIAL2387B11-ON & $\mathrm{F}$ & $\mathrm{s}$ & A & \\
\hline
\end{tabular}




\begin{tabular}{|c|c|c|c|c|}
\hline BEE1850E08-MN & $\mathrm{F}$ & $\mathrm{s}$ & A & \\
\hline D01561A03-IN & $\mathrm{F}$ & S & A & \\
\hline DIAL1391G02-ON & M & S & A & \\
\hline D01560A04-WI & M & S & A & \\
\hline D01560B10-ON & $\mathrm{F}$ & S & A & \\
\hline DIAL1849G08-WV & $\mathrm{F}$ & S & A & \\
\hline DIAL2387B06-ON & $\mathrm{F}$ & S & A & \\
\hline D1414G04-DC & $\mathrm{F}$ & S & A & \\
\hline 04485F02-MI & $\mathrm{F}$ & S & A & \\
\hline DIAL1390D04-ON & $\mathrm{F}$ & S & A & \\
\hline DIAL1409A02-ON & $\mathrm{F}$ & S & A & \\
\hline DIAL2387C07-ON & $\mathrm{F}$ & S & A & \\
\hline DIAL1391H06-NC & $\mathrm{F}$ & S & A & \\
\hline D-3253D06-FL & $\mathrm{F}$ & S & A & \\
\hline BEE0286A11-GA & M & s & A & Lpilos1 GA \\
\hline DIAL1846F10-SC & $\mathrm{F}$ & S & A & \\
\hline DIAL1846F12-SC & $\mathrm{F}$ & S & A & \\
\hline BEE0286B08-GA & $\mathrm{F}$ & S & A & Lpilos3 $\sim \mathrm{GA}$ \\
\hline DIAL0294C11-IN & $\mathrm{F}$ & S & A & supra IN1 \\
\hline DIAL0294D01-IN & $F$ & s & A & supra IN2 \\
\hline DIAL2385E04-ON & M & s & A & \\
\hline DIAL0294C12-IL & $\mathrm{F}$ & S & A & supra IL1 \\
\hline DIAL0293E02-MD & $\mathrm{F}$ & S & A & supraclypeatum3 \\
\hline DIAL0293E01-IN & $\mathrm{F}$ & S & A & supraclypeatum2 \\
\hline D00608G05-IN & M & s & A & \\
\hline D00608G01-IN & M & s & A & \\
\hline D01561A09-MD & $\mathrm{F}$ & S & A & \\
\hline D01561C07-MD & $\mathrm{F}$ & s & A & \\
\hline DIAL2385C07-DE & M & s & A & \\
\hline D00607F08-MA & M & s & A & \\
\hline BEE1850F05-NY & $\mathrm{F}$ & s & A & \\
\hline DIAL2385B04-NY & $\mathrm{F}$ & $s$ & A & \\
\hline D1414H03-VA & $\mathrm{F}$ & $S$ & A & \\
\hline DIAL2384H12-NY & $\mathrm{F}$ & S & A & \\
\hline D01559D03-MA & $\mathrm{F}$ & s & A & \\
\hline B04743G05-MA & $\mathrm{F}$ & $\mathrm{s}$ & A & \\
\hline DIAL1846G03-VA & M & $S$ & A & \\
\hline D1414H12-VA & $\mathrm{F}$ & $S$ & A & \\
\hline D1414H10-VA & $\mathrm{F}$ & $S$ & A & \\
\hline DIAL0294D09-NC & $\mathrm{F}$ & $s$ & A & halop2 NC \\
\hline D1414C12-VA & $\mathrm{F}$ & $S$ & A & \\
\hline DIAL0294D08-NC & $\mathrm{F}$ & $S$ & A & halop1 NC \\
\hline DIAL1413D09-FL & $\mathrm{F}$ & $S$ & A & \\
\hline DIAL1846G01-VA & M & $S$ & A & \\
\hline DIAL1848E10-MD & $\mathrm{F}$ & $s$ & A & \\
\hline DIAL1413A10-VA & $\mathrm{F}$ & $S$ & A & \\
\hline DIAL1413A11-VA & $\mathrm{F}$ & $S$ & A & \\
\hline CCDB-00611 D02 & & S & A & \\
\hline 04485G02-MI & $\mathrm{F}$ & s & A & \\
\hline DIAL1848D01-WI & $\mathrm{F}$ & S & A & DIAL1412D01 \\
\hline DIAL1846E08-WV & $\mathrm{F}$ & $s$ & A & \\
\hline DIAL1391D03-NC & $\mathrm{F}$ & $S$ & A & \\
\hline DIAL1391C11-NC & $\mathrm{F}$ & S & A & \\
\hline D1414H08-VA & $\mathrm{F}$ & $S$ & A & \\
\hline DIAL1413H11-NC & $\mathrm{F}$ & $\mathrm{s}$ & A & \\
\hline
\end{tabular}




\begin{tabular}{|c|c|c|c|c|}
\hline DIAL1391A10-SC & $\mathrm{F}$ & s & A & \\
\hline D1414G12-VA & $\mathrm{F}$ & S & A & \\
\hline DIAL1413E09-NC & $\mathrm{F}$ & $S$ & A & \\
\hline DIAL1413B01-VA & $\mathrm{F}$ & s & A & \\
\hline DIAL1413G07-NC & M & S & A & \\
\hline D00607H10-KS & $\mathrm{F}$ & $\mathrm{s}$ & A & \\
\hline D0288A11-MD & $\mathrm{F}$ & S & A & \\
\hline D0288A09-WV & $\mathrm{F}$ & S & A & \\
\hline D0287D03-IL & $\mathrm{F}$ & $s$ & A & \\
\hline D0288C02-DC & $\mathrm{F}$ & s & A & \\
\hline D0287E03-IL & $\mathrm{F}$ & S & A & \\
\hline DIAL0294A01-IN & $\mathrm{F}$ & s & A & admirlN1 \\
\hline D0287F07-IL & $\mathrm{F}$ & S & A & \\
\hline D0287E04-IL & $\mathrm{F}$ & S & A & \\
\hline DIAL1846F05-WV & M & S & A & \\
\hline DIAL0294A02-il & $\mathrm{F}$ & s & A & admir IL1 \\
\hline DIAL0294E08-MD & $\mathrm{F}$ & S & A & DIAL1MD \\
\hline DIAL0294F06-VA & $\mathrm{F}$ & S & A & laevi3 VA \\
\hline DIAL0294E10-VA & $\mathrm{F}$ & S & A & DIAL1VA \\
\hline DIAL0294E09-MD & $\mathrm{F}$ & s & A & DIAL2MD \\
\hline DIAL0294F08-MD & $\mathrm{F}$ & s & A & oblon MD2 \\
\hline DIAL0292A09-VA & $\mathrm{F}$ & S & A & admirandum4 \\
\hline DIAL1413A01-IL & $\mathrm{F}$ & $\mathrm{s}$ & A & \\
\hline DIAL1390G10-NC & $\mathrm{F}$ & s & A & \\
\hline DIAL0294F04-VA & $\mathrm{F}$ & s & A & laevi1 VA \\
\hline DIAL1391D06-NC & M & S & A & \\
\hline B03765B12-MD & M & S & A & \\
\hline DIAL1847H08-MD & M & s & A & \\
\hline DIAL1391G09-MI & $\mathrm{F}$ & s & A & \\
\hline DIAL1413H08-NC & $\mathrm{F}$ & s & A & \\
\hline DIAL0293C02-NC & $F$ & S & A & \\
\hline DIAL0292A08-VA & $\mathrm{F}$ & s & A & \\
\hline DIAL1413A04-IL & $\mathrm{F}$ & S & A & \\
\hline DIAL1413A06-IL & $\mathrm{F}$ & S & A & \\
\hline DIAL1413G05-NC & $\mathrm{F}$ & s & A & \\
\hline DIAL1391E03-SC & $\mathrm{F}$ & s & A & \\
\hline DIAL1413B08-IL & $\mathrm{F}$ & S & A & \\
\hline DIAL1846E12-SC & $\mathrm{F}$ & S & A & \\
\hline DIAL1846B01-TN & $\mathrm{F}$ & S & A & \\
\hline DIAL1846A11-NC & $\mathrm{F}$ & s & A & \\
\hline DIAL1846E05-MS & $\mathrm{F}$ & S & A & \\
\hline DIAL1413H03-NC & M & $S$ & A & \\
\hline DIAL1413C04-TN & $\mathrm{F}$ & S & A & \\
\hline D0287F02-IL & $\mathrm{F}$ & s & A & \\
\hline DIAL1413F02-TN & $\mathrm{F}$ & S & A & \\
\hline D0288C08-DC & $\mathrm{F}$ & S & A & \\
\hline DIAL1413C08-TN & $\mathrm{F}$ & S & A & \\
\hline DIAL1413C05-TN & M & s & A & \\
\hline DIAL1413B02-VA & $\mathrm{F}$ & s & A & \\
\hline DIAL1413C06-TN & M & S & A & \\
\hline DIAL1391G08-MI & $\mathrm{F}$ & s & A & \\
\hline DIAL1413C11-NC & $\mathrm{F}$ & S & A & \\
\hline D0287G01-IL & $\mathrm{F}$ & S & A & \\
\hline DIAL1413B09-IL & $\mathrm{F}$ & S & A & \\
\hline DIAL1413C01-IL & $\mathrm{F}$ & $\mathrm{s}$ & A & \\
\hline
\end{tabular}




\begin{tabular}{|c|c|c|c|c|}
\hline DIAL1413F12-TN & $\mathrm{F}$ & $S$ & A & \\
\hline DIAL1413D05-SC & $\mathrm{F}$ & S & A & \\
\hline DIAL1413C09-TN & $\mathrm{F}$ & S & A & \\
\hline D0287G04-IL & $\mathrm{F}$ & S & A & \\
\hline D0287G02-IL & $\mathrm{F}$ & $S$ & A & \\
\hline D0287G08-IL & $\mathrm{F}$ & S & A & \\
\hline DIAL2385D05-NC & $\mathrm{F}$ & S & A & \\
\hline DIAL1413C02-TN & $\mathrm{F}$ & S & A & \\
\hline DIAL1413C03-TN & $\mathrm{F}$ & S & A & \\
\hline D0287G07-IL & $\mathrm{F}$ & S & A & \\
\hline D0287G12-IL & $\mathrm{F}$ & S & A & \\
\hline D0287H09-IL & $\mathrm{F}$ & $S$ & A & \\
\hline DIAL1413A03-IL & $\mathrm{F}$ & S & A & \\
\hline D0287C11-IL & $\mathrm{F}$ & S & A & \\
\hline D0287C09-IL & $\mathrm{F}$ & S & A & \\
\hline D0287D02-MI & $\mathrm{F}$ & S & A & mes transverse striat \\
\hline D0287D06-IL & $\mathrm{F}$ & S & A & \\
\hline B03765B08-MD & M & S & A & \\
\hline D-3253C08-NM & $\mathrm{F}$ & s & A & \\
\hline DIAL2374A05-UT & $\mathrm{F}$ & S & A & \\
\hline DIAL2387C12-TX & $\mathrm{F}$ & $S$ & A & \\
\hline DIAL2374C06-UT & $\mathrm{F}$ & S & A & sp E5 \\
\hline DIAL2374A06-UT & $\mathrm{F}$ & S & A & \\
\hline DIAL1849C11-CO & $\mathrm{F}$ & S & A & \\
\hline DIAL1409B10-TX & $\mathrm{F}$ & $S$ & A & \\
\hline DIAL1409E06-TX & $\mathrm{F}$ & $S$ & A & \\
\hline DIAL2387B03-NM & $\mathrm{F}$ & S & A & \\
\hline DIAL2386H10-UT & $\mathrm{F}$ & s & A & \\
\hline D-3253D03-NM & $\mathrm{F}$ & $S$ & A & \\
\hline DIAL2374F08-UT & $\mathrm{F}$ & S & A & \\
\hline DIAL2388D11-NM & M & S & A & \\
\hline DIAL2386F01-NV & $\mathrm{F}$ & S & A & M10 \\
\hline D00608D11-NM & $\mathrm{F}$ & S & A & brown metasoma \\
\hline DIAL2374F09-UT & $\mathrm{F}$ & $S$ & A & \\
\hline DIAL2386D09-UT & $\mathrm{F}$ & S & A & $Z 16$ or 27 \\
\hline DIAL1849E03-AZ & $\mathrm{F}$ & S & A & \\
\hline DIAL2388E03-NM & M & S & A & \\
\hline DIAL2388B11-UT & $\mathrm{F}$ & S & A & \\
\hline B-3252H08-NV & $\mathrm{F}$ & S & A & \\
\hline DIAL2374A07-UT & $\mathrm{F}$ & S & A & \\
\hline D00608H06-NV & $\mathrm{F}$ & S & A & \\
\hline DIAL1413F04-TN & $\mathrm{F}$ & S & A & \\
\hline DIAL1390H04-SC & $\mathrm{F}$ & S & A & \\
\hline DIAL1847H11-MD & M & S & A & \\
\hline D1414B02-NC & $\mathrm{F}$ & S & A & \\
\hline DIAL1413G06-NC & $\mathrm{F}$ & S & A & \\
\hline DIAL1845B07-CA & $\mathrm{F}$ & s & A & \\
\hline DIAL1845A06-CA & $\mathrm{F}$ & $S$ & A & \\
\hline DIAL1845B01-CA & $\mathrm{F}$ & s & A & \\
\hline DIAL1845C02-CA & $\mathrm{F}$ & s & A & \\
\hline D00608H09-OR & $\mathrm{F}$ & s & A & \\
\hline D00607H02-CA & $\mathrm{F}$ & s & A & \\
\hline D00607D09-CA & $\mathrm{F}$ & s & A & \\
\hline D00607F10-CA & $\mathrm{F}$ & s & A & \\
\hline D01559D12-BC & $\mathrm{F}$ & s & A & \\
\hline
\end{tabular}




\begin{tabular}{|c|c|c|c|}
\hline D-3253F09-KY & M & $\mathrm{s}$ & A \\
\hline D0288F05-SC & $\mathrm{F}$ & $S$ & A \\
\hline D0287D12-IL & $\mathrm{F}$ & S & A \\
\hline D0287F08-IL & $\mathrm{F}$ & $s$ & A \\
\hline DIAL1846G12-SC & $\mathrm{F}$ & S & A \\
\hline D0287F05-IL & $\mathrm{F}$ & S & A \\
\hline DIAL1413H01-NC & M & S & A \\
\hline D0287H02-IL & $\mathrm{F}$ & s & A \\
\hline DIAL1391D05-NC & $\mathrm{F}$ & S & A \\
\hline DIAL1409B08-VA & M & S & A \\
\hline DIAL1409B09-VA & M & S & A \\
\hline DIAL1413C10-NC & $\mathrm{F}$ & s & A \\
\hline DIAL1413B07-IL & $\mathrm{F}$ & $\mathrm{s}$ & A \\
\hline DIAL1413D02-TN & $\mathrm{F}$ & S & A \\
\hline DIAL1391E06-SC & $\mathrm{F}$ & S & A \\
\hline D0288G07-NC & $\mathrm{F}$ & s & A \\
\hline DIAL1413G02-TN & $\mathrm{F}$ & $\mathrm{s}$ & A \\
\hline D0287F03-IL & $\mathrm{F}$ & S & A \\
\hline DIAL1390G08-NC & $\mathrm{F}$ & $\mathrm{s}$ & A \\
\hline DIAL1413G01-TN & $\mathrm{F}$ & s & A \\
\hline D0287C06-IL & $\mathrm{F}$ & s & A \\
\hline DIAL1391F08-ON & $\mathrm{F}$ & S & A \\
\hline DIAL1413H06-NC & $\mathrm{F}$ & $\mathrm{s}$ & A \\
\hline DIAL1391F02-NC & $\mathrm{F}$ & s & A \\
\hline DIAL1391B02-SC & $\mathrm{F}$ & s & A \\
\hline DIAL1849G10-MA & M & s & A \\
\hline D-3253A10-BC & $\mathrm{F}$ & s & A \\
\hline D-3253A04-BC & $\mathrm{F}$ & s & A \\
\hline DIAL2388B03-UT & $\mathrm{F}$ & s & A \\
\hline DIAL1847G08-OR & $\mathrm{F}$ & s & A \\
\hline DIAL1409D08-OR & $\mathrm{F}$ & s & A \\
\hline DIAL1847G09-OR & $\mathrm{F}$ & S & A \\
\hline DIAL1847G11-OR & $\mathrm{F}$ & S & A \\
\hline D00608A10-CA & $\mathrm{F}$ & s & A \\
\hline DIAL2386H12-UT & $\mathrm{F}$ & s & A \\
\hline DIAL2386H02-WA & $\mathrm{F}$ & S & A \\
\hline D00607D10-CA & $\mathrm{F}$ & S & A \\
\hline D00607D07-CA & M & $s$ & A \\
\hline DIAL2386D03-UT & $\mathrm{F}$ & S & A \\
\hline DIAL2386D05-UT & $\mathrm{F}$ & S & A \\
\hline 06717H07-MA & $\mathrm{F}$ & $S$ & A \\
\hline DIAL2385D09-WV & M & S & A \\
\hline D01560D09-IA & $\mathrm{F}$ & S & A \\
\hline B03751H11-MA & $\mathrm{F}$ & $\mathrm{s}$ & A \\
\hline D01560E05-IA & $\mathrm{F}$ & $S$ & A \\
\hline В03750B04-MA & $\mathrm{F}$ & S & A \\
\hline B04743H04-MA & $\mathrm{F}$ & S & A \\
\hline 06735G03-MA & M & S & A \\
\hline D-3253B07-BC & $\mathrm{F}$ & $S$ & A \\
\hline D-3253A07-BC & $\mathrm{F}$ & $\mathrm{s}$ & A \\
\hline D-3253B05-BC & $\mathrm{F}$ & $S$ & A \\
\hline D-3253C02-BC & $\mathrm{F}$ & $S$ & A \\
\hline DIAL1845C08-CA & $\mathrm{F}$ & $S$ & A \\
\hline D-3253B09-BC & $\mathrm{F}$ & $\mathrm{s}$ & A \\
\hline DIAL1845C07-CA & $\mathrm{F}$ & $\mathrm{s}$ & A \\
\hline
\end{tabular}




\begin{tabular}{|c|c|c|c|c|}
\hline D0295F03-CO & $\mathrm{F}$ & $\mathrm{s}$ & A & \\
\hline D-3253A03-BC & $\mathrm{F}$ & S & A & \\
\hline D00608D06-BC & $\mathrm{F}$ & S & A & \\
\hline D00608E08-BC & $\mathrm{F}$ & $\mathrm{s}$ & A & \\
\hline D00608D08-BC & $\mathrm{F}$ & S & A & \\
\hline D00607C01-CO & $\mathrm{F}$ & S & A & \\
\hline DIAL1845G08-BC & $\mathrm{F}$ & S & A & \\
\hline DIAL2374E06-WA & $\mathrm{F}$ & S & A & \\
\hline DIAL1409C10-OR & $\mathrm{F}$ & S & A & \\
\hline DIAL1845F09-BC & $\mathrm{F}$ & S & A & \\
\hline DIAL1845D07-CA & $\mathrm{F}$ & S & A & \\
\hline DIAL1845D06-CA & $\mathrm{F}$ & S & A & \\
\hline DIAL1845D11-CA & $\mathrm{F}$ & S & A & \\
\hline DIAL1845E04-CA & $\mathrm{F}$ & S & A & \\
\hline DIAL1845F03-BC & $\mathrm{F}$ & S & A & \\
\hline DIAL1845G05-BC & $\mathrm{F}$ & S & A & \\
\hline 03767G11-BC & $\mathrm{F}$ & S & A & \\
\hline DIAL2387E11-BC & $\mathrm{F}$ & S & A & \\
\hline DIAL2387F03-BC & $\mathrm{F}$ & S & A & \\
\hline DIAL2387A05-ID & $\mathrm{F}$ & S & A & \\
\hline DIAL2386A05-CA & M & $S$ & A & \\
\hline D00608D10-BC & $\mathrm{F}$ & S & A & \\
\hline DIAL2386G12-ID & $\mathrm{F}$ & S & A & \\
\hline D00608C09-CO & $\mathrm{F}$ & $S$ & A & \\
\hline D00608C02-CO & $\mathrm{F}$ & $S$ & A & \\
\hline D00608F04-BC & $\mathrm{F}$ & $S$ & A & \\
\hline D01562A04-MT & $\mathrm{F}$ & $S$ & A & \\
\hline D0295H06-CO & $\mathrm{F}$ & s & A & \\
\hline B03765E10-BC & $\mathrm{F}$ & $S$ & A & \\
\hline D-3253C01-BC & $\mathrm{F}$ & $S$ & A & \\
\hline DIAL2385E08-MD & M & $S$ & A & \\
\hline DIAL1848H12-PE & $\mathrm{F}$ & $s$ & A & DIAL1412H12 \\
\hline DIAL1845H05-BC & $\mathrm{F}$ & $S$ & A & \\
\hline D-3253A02-BC & $\mathrm{F}$ & $S$ & A & \\
\hline D00609G06-NS & $\mathrm{F}$ & $s$ & A & \\
\hline DIAL0294F05-MD & $\mathrm{F}$ & $S$ & A & laev2 MD \\
\hline DIAL0294H11-NB & $\mathrm{F}$ & $s$ & A & DIAL2NB \\
\hline D00607G08-BC & M & $S$ & A & \\
\hline DIAL1846A08-NC & $\mathrm{F}$ & $s$ & A & \\
\hline DIAL1845H08-BC & $\mathrm{F}$ & $\mathrm{s}$ & A & \\
\hline DIAL1845F08-BC & $\mathrm{F}$ & $s$ & A & \\
\hline DIAL1391B11-NC & $\mathrm{F}$ & $s$ & A & \\
\hline D00609G04-NS & $\mathrm{F}$ & s & A & \\
\hline DIAL1848D05-NS & $\mathrm{F}$ & s & A & DIAL1412D05 \\
\hline DIAL1409H12-ON & $\mathrm{F}$ & $\mathrm{s}$ & A & \\
\hline DIAL1845H02-BC & $\mathrm{F}$ & $s$ & A & \\
\hline DIAL1845G01-BC & $\mathrm{F}$ & $s$ & A & \\
\hline DIAL1845G02-BC & $\mathrm{F}$ & s & A & \\
\hline DIAL1845F04-BC & $\mathrm{F}$ & s & A & \\
\hline DIAL1409B05-NC & $\mathrm{F}$ & s & A & \\
\hline DIAL1845F06-BC & $\mathrm{F}$ & $s$ & A & \\
\hline DIAL1845F07-BC & $\mathrm{F}$ & $S$ & A & \\
\hline DIAL1413E07-NC & $\mathrm{F}$ & s & A & \\
\hline DIAL1848F09-PE & $\mathrm{F}$ & $S$ & A & DIAL1412F09 \\
\hline DIAL1848A05-NB & $\mathrm{F}$ & $\mathrm{s}$ & A & DIAL1412A05 \\
\hline
\end{tabular}




\begin{tabular}{|c|c|c|c|c|}
\hline DIAL1848A06-NB & $\mathrm{F}$ & s & A & DIAL1412A06 \\
\hline DIAL1848G06-PE & $\mathrm{F}$ & $\mathrm{s}$ & A & DIAL1412G06 \\
\hline DIAL1849H06-ME & M & $S$ & A & \\
\hline DIAL2388D05-CO & $\mathrm{F}$ & S & A & \\
\hline D01562D02-ON & M & S & A & \\
\hline D00607A05-AB & M & $\mathrm{s}$ & A & \\
\hline D00607C10-AB & M & S & A & \\
\hline D00607C09-AB & M & S & A & \\
\hline DIAL1391A07-NC & $\mathrm{F}$ & $s$ & A & \\
\hline D0295B10-ON & $\mathrm{F}$ & s & A & \\
\hline D0295C01-ON & $\mathrm{F}$ & S & A & \\
\hline DIAL1391C02-NC & $\mathrm{F}$ & S & A & \\
\hline DIAL1391B03-OH & $\mathrm{F}$ & $\mathrm{s}$ & A & \\
\hline DIAL1391C01-NC & $\mathrm{F}$ & S & A & \\
\hline DIAL2388D04-CO & $\mathrm{F}$ & S & A & \\
\hline 06745E10-ON & $\mathrm{F}$ & S & A & \\
\hline DIAL1413E02-FL & M & s & A & \\
\hline DIAL1413E04-FL & $\mathrm{F}$ & S & A & \\
\hline DIAL1413E06-FL & M & $\mathrm{s}$ & A & \\
\hline 04485F04-MI & M & s & A & \\
\hline 04485F08-MI & $\mathrm{F}$ & s & A & \\
\hline 04485E08-MI & M & S & A & \\
\hline DIAL1848C01-NB & $\mathrm{F}$ & $\mathrm{s}$ & A & DIAL1412C01 \\
\hline 04485F09-MI & $\mathrm{F}$ & s & A & \\
\hline 04485F11-MI & M & s & A & \\
\hline 04485H06-MI & $\mathrm{F}$ & S & A & \\
\hline DIAL1846A04-ON & $F$ & S & A & \\
\hline 04485H04-MI & M & s & A & \\
\hline DIAL1848B11-NB & $\mathrm{F}$ & s & A & DIAL1412B11 \\
\hline D00609G02-NS & $\mathrm{F}$ & s & A & \\
\hline D00609H09-NS & $\mathrm{F}$ & s & A & \\
\hline DIAL1845E03-TX & $\mathrm{F}$ & s & A & yellow legs \\
\hline DIAL1846D07-MS & $\mathrm{F}$ & S & A & yellowish legs \\
\hline DIAL1846F11-MS & $\mathrm{F}$ & S & A & \\
\hline DIAL1391A11-SC & $\mathrm{F}$ & s & A & \\
\hline DIAL2385E07-WV & M & s & A & \\
\hline BEE0289B05-MN & $\mathrm{F}$ & S & A & Ladmir-MN1 \\
\hline DIAL0286H09-MB & $\mathrm{F}$ & S & A & DIAL8-MB \\
\hline DIAL0286H04-MB & $\mathrm{F}$ & S & A & DIAL3-MB \\
\hline D0295B08-ON & $\mathrm{F}$ & s & A & \\
\hline DIAL1390C12-ON & $\mathrm{F}$ & S & A & \\
\hline DIAL0292H06-WV & $\mathrm{F}$ & $S$ & A & lineatulum1 \\
\hline DIAL1839G07-MN & $\mathrm{F}$ & S & A & \\
\hline 06732C04-WV & $\mathrm{F}$ & s & A & \\
\hline D00607B07-KS & $\mathrm{F}$ & S & A & \\
\hline DIAL1409B01-NC & M & S & A & \\
\hline DIAL1390C07-ON & $\mathrm{F}$ & S & A & \\
\hline DIAL1390C04-ON & $\mathrm{F}$ & s & A & \\
\hline DIAL0286H06-MB & $\mathrm{F}$ & S & A & DIAL5-MB \\
\hline DIAL1846B05-MD & M & S & A & \\
\hline DIAL1847H12-MD & M & s & A & \\
\hline DIAL2388E04-AL & $\mathrm{F}$ & S & A & \\
\hline DIAL1846H04-MS & $\mathrm{F}$ & $\mathrm{s}$ & A & \\
\hline DIAL1846E02-MS & $\mathrm{F}$ & S & A & \\
\hline DIAL1846E09-MS & $\mathrm{F}$ & $\mathrm{s}$ & A & \\
\hline
\end{tabular}




\begin{tabular}{|c|c|c|c|}
\hline DIAL1846E06-MS & $\mathrm{F}$ & $\mathrm{s}$ & A \\
\hline DIAL1846D05-MS & M & s & A \\
\hline DIAL2387F06-TX & M & s & A \\
\hline D0288F08-NC & $\mathrm{F}$ & s & A \\
\hline BEE0286B09-GA & $\mathrm{F}$ & s & A \\
\hline D0288F10-SC & $\mathrm{F}$ & s & A \\
\hline D01559E03-BC & $\mathrm{F}$ & s & A \\
\hline D01559F06-BC & $\mathrm{F}$ & s & A \\
\hline D00608B10-BC & $\mathrm{F}$ & s & A \\
\hline D00608D04-BC & $\mathrm{F}$ & s & A \\
\hline D00609H07-BC & $\mathrm{F}$ & s & A \\
\hline D00609H03-BC & $\mathrm{F}$ & S & A \\
\hline D00609H05-BC & $\mathrm{F}$ & s & A \\
\hline DIAL2388B05-WA & $\mathrm{F}$ & S & A \\
\hline D01561E05-CA & $\mathrm{F}$ & s & A \\
\hline CCDB - 01565 H3 & & s & A \\
\hline CCDB - 01565 H8 & & s & A \\
\hline D1414C03-VA & $\mathrm{F}$ & S & A \\
\hline DIAL1846D08-VA & $\mathrm{F}$ & s & A \\
\hline DIAL1848C05-MD & $\mathrm{F}$ & s & A \\
\hline D1414D08-VA & $\mathrm{F}$ & s & A \\
\hline D1414C06-VA & $\mathrm{F}$ & s & A \\
\hline D1414C05-VA & $\mathrm{F}$ & s & A \\
\hline D1414C04-VA & $\mathrm{F}$ & S & A \\
\hline D01561E08-NY & M & s & A \\
\hline DIAL1849F05-DC & $\mathrm{F}$ & S & A \\
\hline DIAL1409B02-NC & M & s & A \\
\hline DIAL1409A01-ON & $\mathrm{F}$ & S & A \\
\hline D-3253E12-FL & $\mathrm{F}$ & s & A \\
\hline D-3253E02-FL & $\mathrm{F}$ & S & A \\
\hline D01559A03-FL & $\mathrm{F}$ & s & A \\
\hline D01561H08-FL & $\mathrm{F}$ & S & A \\
\hline D01559D09-FL & $\mathrm{F}$ & s & A \\
\hline D01562D03-FL & $\mathrm{F}$ & S & A \\
\hline D-3253B01-BC & $\mathrm{F}$ & s & A \\
\hline D-3253A05-BC & $\mathrm{F}$ & S & A \\
\hline D-3253B10-BC & $\mathrm{F}$ & s & A \\
\hline D-3253B11-BC & $\mathrm{F}$ & s & A \\
\hline D-3253B08-BC & $\mathrm{F}$ & s & A \\
\hline D-3262G02-UT & $\mathrm{F}$ & S & A \\
\hline D-3253A08-BC & $\mathrm{F}$ & s & A \\
\hline DIAL1845C12-CA & $\mathrm{F}$ & S & A \\
\hline DIAL1845D10-CA & $\mathrm{F}$ & S & A \\
\hline DIAL2387E12-BC & $\mathrm{F}$ & $s$ & A \\
\hline D00607F07-CA & M & $S$ & A \\
\hline BEE1850F04-OR & $\mathrm{F}$ & s & A \\
\hline D00607G10-BC & $\mathrm{F}$ & s & A \\
\hline DIAL2386C03-CA & M & $s$ & A \\
\hline DIAL2374A11-UT & $\mathrm{F}$ & $S$ & A \\
\hline DIAL2386D08-UT & $\mathrm{F}$ & $S$ & A \\
\hline DIAL2386D06-UT & $\mathrm{F}$ & S & A \\
\hline DIAL2386C04-CA & M & $S$ & A \\
\hline D01559E12-BC & M & $S$ & A \\
\hline DIAL2374D09-UT & $\mathrm{F}$ & s & A \\
\hline DIAL2374E11-WA & $\mathrm{F}$ & $\mathrm{s}$ & A \\
\hline
\end{tabular}

Lcoreo1 GA 


\begin{tabular}{|c|c|c|c|c|}
\hline 06732F01-WY & $\mathrm{F}$ & s & A & \\
\hline DIAL2385C01-MD & M & S & A & \\
\hline DIAL1849G01-ME & $\mathrm{F}$ & $S$ & A & \\
\hline 04485A08-MD & M & s & A & \\
\hline D00609G08-NS & $\mathrm{F}$ & S & A & \\
\hline DIAL1849F12-MA & $\mathrm{F}$ & s & A & \\
\hline DIAL1848F03-ON & $\mathrm{F}$ & S & A & \\
\hline D01560B11-VT & M & S & A & \\
\hline DIAL1848F05-PE & $\mathrm{F}$ & $s$ & A & \\
\hline D0288B10-NC & $\mathrm{F}$ & s & A & \\
\hline D1414C10-VA & $\mathrm{F}$ & S & A & \\
\hline D1414C09-VA & $\mathrm{F}$ & S & A & \\
\hline D-3253F11-FL & $\mathrm{F}$ & S & A & \\
\hline D1414F02-NC & $\mathrm{F}$ & S & A & \\
\hline D1414E12-NC & $\mathrm{F}$ & S & A & \\
\hline D1414E11-NC & $\mathrm{F}$ & s & A & \\
\hline D0288C03-SC & $\mathrm{F}$ & $\mathrm{s}$ & A & \\
\hline D1414C08-VA & $\mathrm{F}$ & S & A & \\
\hline D0288B11-NC & $\mathrm{F}$ & S & A & \\
\hline DIAL1846D11-MS & $\mathrm{F}$ & s & A & \\
\hline DIAL1849F03-NC & $\mathrm{F}$ & s & A & \\
\hline D1414D09-VA & $\mathrm{F}$ & S & A & \\
\hline B03765B03-VA & $\mathrm{F}$ & S & A & \\
\hline D0288C11-SC & $\mathrm{F}$ & s & A & \\
\hline D1414F05-NC & $\mathrm{F}$ & s & A & \\
\hline D1414C07-VA & $\mathrm{F}$ & S & A & \\
\hline D0295D12-MD & $\mathrm{F}$ & S & A & \\
\hline D1414B06-VA & $\mathrm{F}$ & s & A & \\
\hline DIAL0293B05-MD & $\mathrm{F}$ & s & A & oblongum6 \\
\hline D1414B07-VA & $\mathrm{F}$ & s & A & \\
\hline D00607D12-MA & M & s & A & \\
\hline D00608F12-IN & M & s & A & \\
\hline D1414B08-VA & $\mathrm{F}$ & S & A & \\
\hline D1414E01-VA & $\mathrm{F}$ & S & A & \\
\hline D1414B09-VA & $\mathrm{F}$ & s & A & \\
\hline DIAL1849G02-MA & $\mathrm{F}$ & s & A & \\
\hline DIAL1413E12-TN & $\mathrm{F}$ & S & A & \\
\hline DIAL0294F11-VA & $\mathrm{F}$ & S & A & catte1 VA \\
\hline DIAL1847B03-WV & $\mathrm{F}$ & S & A & \\
\hline DIAL1848C11-NY & $\mathrm{F}$ & s & A & \\
\hline D0295E06-MD & $\mathrm{F}$ & S & A & \\
\hline DIAL1846B08-WV & $\mathrm{F}$ & $S$ & A & \\
\hline BEE0286D09-VA & $\mathrm{F}$ & S & A & catte1 VA \\
\hline DIAL1391A04-NC & $\mathrm{F}$ & s & A & \\
\hline DIAL1390G11-NC & $\mathrm{F}$ & S & A & \\
\hline DIAL1849H08-VT & M & S & A & \\
\hline DIAL2385B03-MB & $\mathrm{F}$ & S & A & \\
\hline DIAL2387F09-NM & $\mathrm{F}$ & s & A & \\
\hline DIAL2387H11-NM & M & s & A & \\
\hline D0287A11-CO & $\mathrm{F}$ & S & A & \\
\hline DIAL2385A10-MB & $\mathrm{F}$ & s & A & \\
\hline D0295G05-CO & $\mathrm{F}$ & S & A & \\
\hline DIAL2385A04-CO & $\mathrm{F}$ & S & A & \\
\hline D0287B11-CO & $\mathrm{F}$ & S & A & \\
\hline D01561B01-MT & $\mathrm{F}$ & $\mathrm{s}$ & A & \\
\hline
\end{tabular}




\begin{tabular}{|c|c|c|c|c|}
\hline 06732F09-WY & $\mathrm{F}$ & S & A & \\
\hline D0295G10-CO & $\mathrm{F}$ & S & A & \\
\hline DIAL1390C09-ON & $\mathrm{F}$ & $S$ & A & \\
\hline DIAL1849G09-MA & M & S & A & \\
\hline DIAL1409A06-NC & M & S & A & \\
\hline DIAL1409A11-NC & $\mathrm{F}$ & s & A & \\
\hline DIAL1845G06-BC & $\mathrm{F}$ & $S$ & A & \\
\hline DIAL1845H10-BC & $\mathrm{F}$ & S & A & \\
\hline DIAL1845G04-BC & $\mathrm{F}$ & s & A & \\
\hline DIAL1845F05-BC & $\mathrm{F}$ & s & A & \\
\hline DIAL1845H03-BC & $\mathrm{F}$ & $S$ & A & \\
\hline DIAL1845H11-BC & $\mathrm{F}$ & S & A & \\
\hline D00608G06-BC & $\mathrm{F}$ & S & A & \\
\hline DIAL1845H12-BC & $\mathrm{F}$ & S & A & \\
\hline DIAL1845G03-BC & $\mathrm{F}$ & $S$ & A & \\
\hline DIAL1845G07-BC & $\mathrm{F}$ & s & A & \\
\hline DIAL1845G12-BC & $\mathrm{F}$ & s & A & \\
\hline DIAL1845F12-BC & $\mathrm{F}$ & S & A & \\
\hline DIAL1845F10-BC & $\mathrm{F}$ & $S$ & A & \\
\hline DIAL1845F02-BC & $\mathrm{F}$ & $\mathrm{s}$ & A & \\
\hline DIAL1845G09-BC & $\mathrm{F}$ & S & A & \\
\hline DIAL1845G10-BC & $\mathrm{F}$ & S & A & \\
\hline DIAL1845G11-BC & $\mathrm{F}$ & $S$ & A & \\
\hline DIAL2387H05-CO & $\mathrm{F}$ & $\mathrm{s}$ & A & \\
\hline B03765E05-BC & $\mathrm{F}$ & $\mathrm{s}$ & A & \\
\hline CCDB-00611 H09-( & & S & A & \\
\hline D01559D04-AB & $\mathrm{F}$ & S & A & \\
\hline DIAL2385A08-SK & $\mathrm{F}$ & S & A & \\
\hline D01559G01-AB & $\mathrm{F}$ & S & A & \\
\hline DIAL2385B02-SK & $\mathrm{F}$ & $\mathrm{s}$ & A & \\
\hline DIAL0293B11-WV & $\mathrm{F}$ & S & A & laevissimumB11 \\
\hline DIAL1413A02-IL & $\mathrm{F}$ & S & A & \\
\hline DIAL1409A08-NC & $\mathrm{F}$ & S & A & \\
\hline DIAL1390E09-ON & $\mathrm{F}$ & $\mathrm{s}$ & A & \\
\hline DIAL1413B05-IL & $\mathrm{F}$ & s & A & \\
\hline D00609C03-WI & $\mathrm{F}$ & S & A & \\
\hline D01560C09-IA & $\mathrm{F}$ & S & A & \\
\hline CCDB-01557 B01 & & $S$ & A & \\
\hline DIAL1390D01-NT & $\mathrm{F}$ & s & A & \\
\hline DIAL1390C10-NT & $\mathrm{F}$ & $\mathrm{s}$ & A & \\
\hline DIAL2384B09-YT & M & S & A & \\
\hline D0295F10-CO & $\mathrm{F}$ & $S$ & A & \\
\hline DIAL0286G05-MB & $\mathrm{F}$ & S & A & perpu3 MB \\
\hline DIAL0286G06-MB & $\mathrm{F}$ & S & A & perpu4 MB \\
\hline DIAL0293B09-IN & $\mathrm{F}$ & S & A & \\
\hline D00609H10-NS & $\mathrm{F}$ & S & A & \\
\hline DIAL0286G07-MB & $\mathrm{F}$ & S & A & perpu5 MB \\
\hline DIAL0286G03-MB & $\mathrm{F}$ & S & A & perpu1 MB \\
\hline D01561B02-MT & $\mathrm{F}$ & S & A & \\
\hline D0287A03-CO & $\mathrm{F}$ & S & A & \\
\hline DIAL0286G10-MB & M & S & A & pruin3 MB \\
\hline DIAL0286G09-MB & M & S & A & pruin2 MB \\
\hline DIAL0286G04-MB & $\mathrm{F}$ & S & A & perpu2 MB \\
\hline DIAL0286H03-MB & $\mathrm{F}$ & S & A & DIAL2-MB \\
\hline DIAL0293C07-MN & $\mathrm{F}$ & s & A & \\
\hline
\end{tabular}




\begin{tabular}{|c|c|c|c|c|}
\hline DIAL0293C08-MN & $\mathrm{F}$ & s & A & \\
\hline DIAL0293C06-MN & $\mathrm{F}$ & $S$ & A & pictum4 \\
\hline DIAL0294C01-IN & $\mathrm{F}$ & $S$ & A & pictu IN1 \\
\hline DIAL0293C05-MN & $\mathrm{F}$ & S & A & pictum3 \\
\hline DIAL0286F07-MB & $\mathrm{F}$ & S & A & pictu2 MB \\
\hline DIAL0286F08-MB & $\mathrm{F}$ & S & A & pictu3 MB \\
\hline DIAL0286F06-MB & $\mathrm{F}$ & $S$ & A & pictu1 MB \\
\hline DIAL2385B11-MI & M & s & A & \\
\hline D00609B09-WI & $\mathrm{F}$ & $\mathrm{s}$ & A & \\
\hline D1414D12-VA & $\mathrm{F}$ & s & A & \\
\hline DIAL1391D02-NC & $\mathrm{F}$ & $S$ & A & \\
\hline DIAL0294F12-NY & $\mathrm{F}$ & s & A & pilos1 NY \\
\hline DIAL1390B05-ON & $\mathrm{F}$ & s & A & \\
\hline 04485G12-MI & $\mathrm{F}$ & S & A & \\
\hline DIAL1846A06-ON & $\mathrm{F}$ & s & A & \\
\hline DIAL1847H06-MD & M & s & A & \\
\hline DIAL1391A01-VA & $\mathrm{F}$ & $\mathrm{s}$ & A & \\
\hline DIAL1413C07-TN & $\mathrm{F}$ & s & A & \\
\hline 04485F01-MI & $\mathrm{F}$ & s & A & \\
\hline DIAL1409A05-NC & $\mathrm{F}$ & $s$ & A & \\
\hline DIAL1413A08-VA & $\mathrm{F}$ & $s$ & A & \\
\hline DIAL1390H05-NC & $\mathrm{F}$ & s & A & \\
\hline DIAL1413F05-TN & $\mathrm{F}$ & $\mathrm{s}$ & A & \\
\hline DIAL1413D03-NC & $\mathrm{F}$ & $s$ & A & \\
\hline DIAL1413B04-ON & $\mathrm{F}$ & $s$ & A & \\
\hline BEE1850F06-MN & $\mathrm{F}$ & $s$ & A & \\
\hline DIAL1391C12-NC & $\mathrm{F}$ & $s$ & A & \\
\hline D00607G06-BC & $\mathrm{F}$ & $s$ & A & \\
\hline DIAL0294H10-NB & $\mathrm{F}$ & $s$ & A & DIAL1NB \\
\hline DIAL1848A02-NB & $\mathrm{F}$ & s & A & DIAL1412A02 \\
\hline DIAL1848G02-NB & $\mathrm{F}$ & $s$ & A & $293 \mathrm{H} 06$ \\
\hline DIAL1848A01-NB & $\mathrm{F}$ & s & A & DIAL1412A01 \\
\hline DIAL2388E07-NB & $\mathrm{F}$ & $S$ & A & \\
\hline DIAL1391F05-NT & $\mathrm{F}$ & $s$ & A & \\
\hline CCDB-00611 F10-C & & $s$ & A & \\
\hline D01560D11-DC & $\mathrm{F}$ & s & A & \\
\hline DIAL2385C11-DC & M & $S$ & A & \\
\hline DIAL1413D08-NC & $\mathrm{F}$ & S & A & \\
\hline DIAL0293D07-WV & $\mathrm{F}$ & $S$ & A & P. platyparium5 \\
\hline DIAL0293D03-MD & $\mathrm{F}$ & s & A & P. platyparium1 \\
\hline DIAL1849H02-DC & M & $S$ & A & \\
\hline DIAL1847H09-MD & M & $S$ & A & \\
\hline DIAL1848E09-MD & $\mathrm{F}$ & $S$ & A & \\
\hline D01560B02-DC & $\mathrm{F}$ & $S$ & A & \\
\hline D01560B05-DC & $\mathrm{F}$ & $S$ & A & \\
\hline DIAL1849F08-MD & $\mathrm{F}$ & $S$ & A & \\
\hline D00609E03-MT & $\mathrm{F}$ & $S$ & A & \\
\hline D0295H01-CO & $\mathrm{F}$ & s & A & \\
\hline D01559F01-AB & M & $S$ & A & \\
\hline DIAL2386G09-WA & $\mathrm{F}$ & $S$ & A & WA-4 \\
\hline D0295G03-CO & $\mathrm{F}$ & $S$ & A & \\
\hline DIAL2384C05-AB & $\mathrm{F}$ & $S$ & A & \\
\hline DIAL1848F01-CO & $\mathrm{F}$ & $\mathrm{s}$ & A & DIAL1412F01 \\
\hline D01559C10-AB & $\mathrm{F}$ & $S$ & A & \\
\hline DIAL2386H04-WA & $\mathrm{F}$ & $\mathrm{s}$ & A & WA-4 \\
\hline
\end{tabular}




\begin{tabular}{|c|c|c|c|c|}
\hline D0295G01-CO & $\mathrm{F}$ & $\mathrm{s}$ & A & \\
\hline D01559H06-BC & $\mathrm{F}$ & s & A & \\
\hline D0287C02-CO & $\mathrm{F}$ & s & A & \\
\hline D01559G06-BC & M & s & A & \\
\hline D01559H09-BC & $M$ & s & A & \\
\hline D01559A11-MT & $\mathrm{F}$ & s & A & \\
\hline DIAL2384C06-AB & $M$ & s & A & \\
\hline DIAL2388F09-UT & M & s & A & \\
\hline BEE0289H09-MB & $\mathrm{F}$ & s & A & pruin-MB4 \\
\hline BEE0289H07-MB & $\mathrm{F}$ & s & A & pruin-MB2 \\
\hline DIAL1409H03-MB & $M$ & s & A & \\
\hline BEE0289H06-MB & $\mathrm{F}$ & s & A & pruin-MB1 \\
\hline BEE0289H10-MB & $\mathrm{F}$ & S & A & LASIO-MB1 \\
\hline DIAL2388G12-AZ & $\mathrm{F}$ & S & A & \\
\hline DIAL2374G02-UT & $\mathrm{F}$ & S & A & $\mathrm{Z} 5$ \\
\hline DIAL2374B05-UT & $\mathrm{F}$ & s & A & \\
\hline DIAL1845B08-CA & $\mathrm{F}$ & s & A & \\
\hline D00608C07-CA & $\mathrm{F}$ & S & A & \\
\hline DIAL1845B02-CA & $\mathrm{F}$ & s & A & \\
\hline DIAL2387A07-ID & $\mathrm{F}$ & S & A & \\
\hline B03765G03-BC & $\mathrm{F}$ & s & A & \\
\hline DIAL1848D04-FL & M & s & A & \\
\hline DIAL0293E07-FL & $\mathrm{F}$ & S & A & tegulare2 \\
\hline DIAL0293E09-FL & $\mathrm{F}$ & s & A & tegulare4 \\
\hline DIAL0293E08-FL & $\mathrm{F}$ & s & A & tegulare3 \\
\hline DIAL1846H07-SC & $\mathrm{F}$ & s & A & \\
\hline DIAL1847A04-FL & M & s & A & \\
\hline DIAL1413E05-FL & $\mathrm{F}$ & s & A & \\
\hline DIAL1846B11-MS & $\mathrm{F}$ & s & A & \\
\hline DIAL1847A02-FL & M & s & A & \\
\hline D0288E11-SC & $\mathrm{F}$ & s & A & \\
\hline DIAL1846F01-SC & M & s & A & \\
\hline DIAL1846B09-MS & $\mathrm{F}$ & S & A & \\
\hline DIAL1846D01-MS & $\mathrm{F}$ & s & A & \\
\hline D0288G05-FL & $\mathrm{F}$ & s & A & \\
\hline D0288G04-FL & $\mathrm{F}$ & s & A & \\
\hline D0288F04-SC & $\mathrm{F}$ & S & A & \\
\hline D0288E02-NC & $\mathrm{F}$ & s & A & \\
\hline D0288C12-FL & $\mathrm{F}$ & s & A & \\
\hline D0288F01-SC & $\mathrm{F}$ & s & A & \\
\hline B03765A01-GA & $\mathrm{F}$ & s & A & \\
\hline D01559B08-GA & $\mathrm{F}$ & S & A & \\
\hline D01559C11-GA & $\mathrm{F}$ & S & A & \\
\hline D01559E01-GA & $\mathrm{F}$ & s & A & \\
\hline DIAL1846D03-MS & $\mathrm{F}$ & s & A & \\
\hline DIAL1846C03-MS & $\mathrm{F}$ & s & A & \\
\hline D01561E01-MA & M & S & A & \\
\hline DIAL1848D08-MD & $\mathrm{F}$ & s & A & \\
\hline DIAL1848D06-WV & $\mathrm{F}$ & s & A & \\
\hline DIAL2385C05-MD & M & s & A & \\
\hline DIAL1849F10-MD & $\mathrm{F}$ & s & A & \\
\hline DIAL2384B01-YT & M & s & A & \\
\hline DIAL2384A10-YT & M & s & A & \\
\hline DIAL2384B03-YT & M & s & A & \\
\hline DIAL2384A08-YT & M & $\mathrm{s}$ & A & \\
\hline
\end{tabular}




\begin{tabular}{|c|c|c|c|c|}
\hline B01558C07-AB & $\mathrm{F}$ & s & A & \\
\hline DIAL2387C04-NM & $\mathrm{F}$ & $S$ & A & \\
\hline D00609B02-MT & $\mathrm{F}$ & $S$ & A & \\
\hline D01562A01-MT & $\mathrm{F}$ & $s$ & A & \\
\hline DIAL2374G03-NV & M & S & A & \\
\hline 03767G04-BC & $\mathrm{F}$ & s & A & \\
\hline CCDB-00611 H02 & & $S$ & A & \\
\hline D0295H07-CO & $\mathrm{F}$ & s & A & \\
\hline CCDB-01557 A01 & & s & A & \\
\hline DIAL2384C03-AB & $\mathrm{F}$ & s & A & \\
\hline D0295H03-CO & $\mathrm{F}$ & s & A & \\
\hline D01559G12-BC & $\mathrm{F}$ & s & A & \\
\hline D00607H03-SK & $\mathrm{F}$ & $\mathrm{s}$ & A & \\
\hline DIAL2386G03-BC & $\mathrm{F}$ & S & A & BC-12 kkk \\
\hline DIAL2374B07-UT & $\mathrm{F}$ & s & A & \\
\hline DIAL2388C07-NV & $\mathrm{F}$ & s & A & \\
\hline D01561E07-SK & $\mathrm{F}$ & $S$ & A & \\
\hline D01561E02-SK & $\mathrm{F}$ & s & A & \\
\hline D01562D12-BC & $\mathrm{F}$ & s & A & \\
\hline D01559A08-AB & $\mathrm{F}$ & $s$ & A & \\
\hline 04486E05-WA & $\mathrm{F}$ & $s$ & A & \\
\hline CCDB-00611 H11 & & s & A & \\
\hline 03767G06-BC & $\mathrm{F}$ & $\mathrm{s}$ & A & \\
\hline B03765E06-BC & $\mathrm{F}$ & $s$ & A & \\
\hline CCDB-01557 A04 & & $s$ & A & \\
\hline 06732E12-WY & $\mathrm{F}$ & $s$ & A & \\
\hline DIAL2386A08-NV & M & $s$ & A & \\
\hline D01562D10-ON & $\mathrm{F}$ & $s$ & A & \\
\hline DIAL0286H10-MB & $\mathrm{F}$ & $s$ & A & DIAL9-MB \\
\hline DIAL0291H07-MB & $\mathrm{F}$ & s & A & LASIO-ON2 \\
\hline DIAL0291H05-MB & $\mathrm{F}$ & $s$ & A & laevi-MB1 \\
\hline DIAL0291H06-MB & $\mathrm{F}$ & s & A & LASIO-ON1 \\
\hline DIAL0286H11-MB & $\mathrm{F}$ & $S$ & A & DIAL10-MB \\
\hline DIAL0286H07-MB & $\mathrm{F}$ & $s$ & A & DIAL6-MB \\
\hline DIAL0286H08-MB & $\mathrm{F}$ & $s$ & A & DIAL7-MB \\
\hline B03765F12-BC & $\mathrm{F}$ & s & A & \\
\hline DIAL1409H04-MB & $\mathrm{F}$ & $S$ & A & \\
\hline DIAL1409H02-MB & $\mathrm{F}$ & S & A & \\
\hline CCDB-01557 A09-^ & & $S$ & A & \\
\hline D01562C04-MB & $\mathrm{F}$ & s & A & \\
\hline D01560A10-WI & M & $S$ & A & \\
\hline DIAL0291H08-MB & M & $S$ & A & LASIO-ON3 \\
\hline D01562E02-MB & $\mathrm{F}$ & $S$ & A & \\
\hline D01562C09-ON & $\mathrm{F}$ & $S$ & A & \\
\hline CCDB-01557 A07-I & & $S$ & A & \\
\hline B03765F09-BC & $\mathrm{F}$ & $S$ & A & \\
\hline CCDB-01557 A10- & & $S$ & A & \\
\hline CCDB-01557 A11- & & s & A & \\
\hline B03765F06-BC & $\mathrm{F}$ & $S$ & A & \\
\hline D00608F06-BC & $\mathrm{F}$ & $S$ & A & \\
\hline В03751Н05-BC & $\mathrm{F}$ & $S$ & A & \\
\hline D01559E08-BC & M & $S$ & A & \\
\hline D00607E12-UT & $\mathrm{F}$ & $S$ & A & \\
\hline D00608E01-BC & $\mathrm{F}$ & S & A & \\
\hline D01561F06-BC & $\mathrm{F}$ & $\mathrm{s}$ & A & \\
\hline
\end{tabular}




\begin{tabular}{|c|c|c|c|c|}
\hline B03765E12-BC & $\mathrm{F}$ & $s$ & A & \\
\hline D0295F01-CO & $\mathrm{F}$ & S & A & \\
\hline DIAL2374E05-WA & $\mathrm{F}$ & S & A & ok 1 \\
\hline CCDB-00611 H04 & & S & A & \\
\hline D01559G09-BC & M & S & A & \\
\hline D0295G11-CO & $\mathrm{F}$ & S & A & \\
\hline DIAL2387C03-ID & $\mathrm{F}$ & S & A & \\
\hline D0295F04-CO & $\mathrm{F}$ & S & A & \\
\hline DIAL2374E04-WA & $\mathrm{F}$ & S & A & \\
\hline D01559B11-BC & $\mathrm{F}$ & S & A & \\
\hline D01559C03-BC & $\mathrm{F}$ & S & A & \\
\hline D0295H11-CO & $\mathrm{F}$ & S & A & \\
\hline D0287C01-CO & $\mathrm{F}$ & S & A & \\
\hline D0287B01-CO & $\mathrm{F}$ & S & A & \\
\hline D01562A10-MT & $\mathrm{F}$ & S & A & \\
\hline D0295G06-CO & $\mathrm{F}$ & s & A & \\
\hline CCDB-00611 H03 & M & S & A & \\
\hline DIAL2388D12-NM & $\mathrm{F}$ & S & A & \\
\hline DIAL0293H09-AZ & $\mathrm{F}$ & S & A & \\
\hline D-3262F07-NM & $\mathrm{F}$ & s & A & \\
\hline D0295G07-CO & $\mathrm{F}$ & s & A & \\
\hline DIAL1848C04-MN & $\mathrm{F}$ & S & A & \\
\hline DIAL1848F02-MN & $\mathrm{F}$ & S & A & DIAL1412F02 \\
\hline DIAL1848B05-AZ & $\mathrm{F}$ & S & A & \\
\hline DIAL1849B05-AZ & M & s & A & \\
\hline DIAL1847F03-TX & $\mathrm{F}$ & s & A & \\
\hline DIAL1849E10-AZ & $\mathrm{F}$ & S & A & \\
\hline D0287A06-CO & $\mathrm{F}$ & s & A & \\
\hline DIAL2374F12-UT & $\mathrm{F}$ & s & A & \\
\hline D0295G02-CO & $\mathrm{F}$ & s & A & \\
\hline DIAL1849E02-CO & $\mathrm{F}$ & S & A & \\
\hline DIAL1848B01-AZ & $\mathrm{F}$ & S & A & \\
\hline DIAL1848C03-MN & $\mathrm{F}$ & S & A & \\
\hline D01561B03-CA & M & S & A & \\
\hline DIAL2386B02-NV & M & S & A & \\
\hline DIAL2374G01-UT & $\mathrm{F}$ & S & A & \\
\hline DIAL2388E02-NM & M & S & A & \\
\hline DIAL1849C06-CO & $\mathrm{F}$ & S & A & \\
\hline DIAL0286G11-MB & $\mathrm{F}$ & S & A & pruin4 MB \\
\hline D0287A04-CO & $\mathrm{F}$ & S & A & \\
\hline D0295H04-CO & $\mathrm{F}$ & S & A & \\
\hline D0295G04-CO & $\mathrm{F}$ & S & A & \\
\hline DIAL0286G12-MB & $\mathrm{F}$ & S & A & pruin5 MB \\
\hline DIAL0286G08-MB & $\mathrm{F}$ & s & A & pruin1 MB \\
\hline 06732H03-CO & $\mathrm{F}$ & s & A & \\
\hline DIAL2385E11-NS & $\mathrm{F}$ & S & A & \\
\hline DIAL2385A02-NS & $F$ & S & A & \\
\hline DIAL2385F01-NS & M & S & A & \\
\hline DIAL2385F04-NS & M & s & A & \\
\hline DIAL1848D03-NS & $\mathrm{F}$ & s & A & DIAL1412D03 \\
\hline DIAL1848D02-NS & $\mathrm{F}$ & s & A & DIAL1412D02 \\
\hline D01560A05-WI & $\mathrm{F}$ & S & A & \\
\hline DIAL2384H08-MD & $\mathrm{F}$ & s & A & \\
\hline DIAL1849H05-MD & M & s & A & \\
\hline DIAL0294B06-IN & $\mathrm{F}$ & S & A & laevi IN1 \\
\hline
\end{tabular}




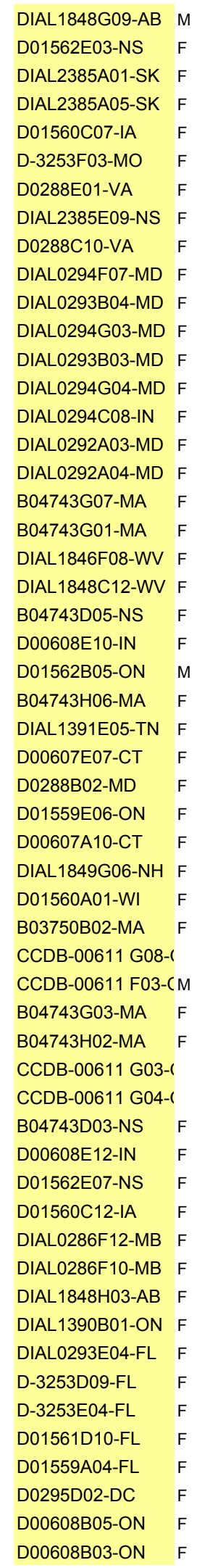

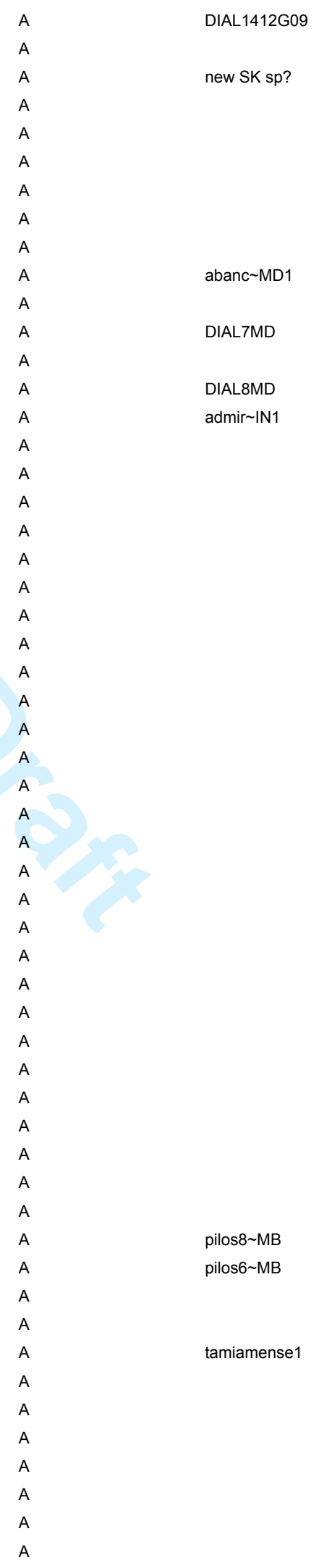




\begin{tabular}{|c|c|c|c|c|}
\hline D1414C02-VA & $\mathrm{F}$ & $s$ & A & \\
\hline DIAL0294E01-VA & $\mathrm{F}$ & S & A & tegul VA1 \\
\hline DIAL0294D12-NY & $\mathrm{F}$ & S & A & tegul NY1 \\
\hline D1414B10-VA & $\mathrm{F}$ & S & A & \\
\hline D1414C01-VA & $\mathrm{F}$ & S & A & \\
\hline D-3253F06-MO & $\mathrm{F}$ & S & A & \\
\hline DIAL1846B06-MS & $\mathrm{F}$ & S & A & \\
\hline DIAL1847H10-MD & $M$ & S & A & \\
\hline DIAL1846H10-MD & $\mathrm{F}$ & s & A & \\
\hline DIAL1846B04-MS & $\mathrm{F}$ & S & A & \\
\hline DIAL1413A09-VA & $\mathrm{F}$ & S & A & \\
\hline B01566D04-TX & $\mathrm{F}$ & S & A & \\
\hline D0288E08-WV & $\mathrm{F}$ & S & A & \\
\hline D1414B11-VA & $\mathrm{F}$ & S & A & \\
\hline DIAL1391E02-SC & M & s & A & \\
\hline DIAL1391E01-SC & $\mathrm{F}$ & S & A & \\
\hline D0288G09-SC & $\mathrm{F}$ & s & A & \\
\hline D0288E10-NC & $\mathrm{F}$ & S & A & \\
\hline D0288E05-WV & $\mathrm{F}$ & s & A & \\
\hline D0288D12-WV & $\mathrm{F}$ & $S$ & A & \\
\hline D0295E11-DC & $\mathrm{F}$ & $S$ & A & \\
\hline D0295E10-DC & $\mathrm{F}$ & S & A & \\
\hline BEE0286E07-VA & $\mathrm{F}$ & s & A & tegul1 VA \\
\hline D00607C02-MO & $\mathrm{F}$ & $S$ & A & \\
\hline D00607A01-NE & $\mathrm{F}$ & $S$ & A & \\
\hline 06732C02-DC & $\mathrm{F}$ & $S$ & A & \\
\hline DIAL2387C06-YT & $\mathrm{F}$ & S & A & \\
\hline DIAL1845E09-NL & $\mathrm{F}$ & $S$ & A & \\
\hline DIAL1845E06-NL & $\mathrm{F}$ & $S$ & A & \\
\hline DIAL2384A11-YT & M & S & A & \\
\hline DIAL1391C03-NC & $M$ & S & A & \\
\hline DIAL2384A03-YT & $M$ & S & A & \\
\hline DIAL2384A02-YT & $M$ & S & A & \\
\hline DIAL1845E12-NL & $\mathrm{F}$ & $s$ & A & \\
\hline DIAL1845F01-NL & $\mathrm{F}$ & S & A & \\
\hline DIAL2387B10-YT & $\mathrm{F}$ & S & A & \\
\hline DIAL1391C04-NC & $\mathrm{F}$ & S & A & \\
\hline DIAL2387B04-YT & $\mathrm{F}$ & s & A & \\
\hline DIAL2384A06-YT & $\mathrm{F}$ & $s$ & A & \\
\hline DIAL1848A10-NB & $\mathrm{F}$ & S & A & DIAL1412A10 \\
\hline DIAL2384A09-YT & $M$ & S & A & \\
\hline DIAL2384B08-YT & $\mathrm{F}$ & s & A & \\
\hline DIAL2384A01-YT & M & s & A & \\
\hline DIAL2384B02-YT & $M$ & $s$ & A & \\
\hline DIAL2384B10-YT & M & s & A & \\
\hline DIAL1390C01-ON & $\mathrm{F}$ & S & A & \\
\hline DIAL1390C03-ON & $\mathrm{F}$ & s & A & \\
\hline BEE1850G11-ME & $\mathrm{F}$ & s & A & \\
\hline D1414H01-VA & $\mathrm{F}$ & s & A & \\
\hline DIAL0292A11-WV & $\mathrm{F}$ & s & A & \\
\hline DIAL0294E06-MD & $\mathrm{F}$ & s & A & admir1 MD \\
\hline DIAL2385F02-MD & M & s & A & \\
\hline DIAL0294E12-MD & $\mathrm{F}$ & s & A & DIAL4MD \\
\hline DIAL0294E07-IN & $\mathrm{F}$ & S & A & admir2 IN \\
\hline DIAL0293B10-WV & $\mathrm{F}$ & s & A & \\
\hline
\end{tabular}




\begin{tabular}{|c|c|c|c|c|}
\hline DIAL0293F02-NC & $\mathrm{F}$ & S & A & versatum5 \\
\hline D00607H11-KS & M & $s$ & A & \\
\hline B01558D07-NC & $\mathrm{F}$ & S & A & \\
\hline D00608A07-KS & $\mathrm{F}$ & S & A & \\
\hline D0288C05-DC & $\mathrm{F}$ & S & A & \\
\hline DIAL1409B07-NC & $\mathrm{F}$ & S & A & \\
\hline DIAL1846D04-MS & $\mathrm{F}$ & S & A & \\
\hline DIAL1413H02-NC & $\mathrm{F}$ & s & A & \\
\hline D0288D08-NC & $\mathrm{F}$ & S & A & \\
\hline DIAL2385C12-SC & M & s & A & \\
\hline DIAL1849C10-CO & $\mathrm{F}$ & S & A & \\
\hline D00607B03-KS & $\mathrm{F}$ & S & A & \\
\hline D00607H09-KS & M & $\mathrm{s}$ & A & \\
\hline 06732E01-MD & $\mathrm{F}$ & S & A & \\
\hline D1414A06-IL & $\mathrm{F}$ & $S$ & A & \\
\hline B03765B09-GA & M & s & A & \\
\hline B01558D08-NC & $\mathrm{F}$ & $S$ & A & \\
\hline B01558D03-NC & M & S & A & \\
\hline B01558C12-NC & $\mathrm{F}$ & s & A & \\
\hline D0288B08-SC & $\mathrm{F}$ & s & A & \\
\hline D0288B06-NC & $\mathrm{F}$ & $S$ & A & \\
\hline DIAL1391D08-NC & $\mathrm{F}$ & S & A & \\
\hline DIAL1391E10-NC & $\mathrm{F}$ & S & A & \\
\hline DIAL0293F04-TN & $\mathrm{F}$ & s & A & \\
\hline DIAL0293F05-TN & $\mathrm{F}$ & s & A & versans3 \\
\hline DIAL1391C05-NC & $\mathrm{F}$ & s & A & \\
\hline DIAL1391A08-NC & $\mathrm{F}$ & s & A & \\
\hline DIAL1849H07-ME & M & s & A & \\
\hline DIAL1848F07-PE & $\mathrm{F}$ & s & A & \\
\hline DIAL1848E08-WV & $M$ & s & A & DIAL1412E08 \\
\hline D00607F12-NS & $\mathrm{F}$ & s & A & \\
\hline DIAL2385C06-MD & M & S & A & \\
\hline D1414D03-VA & $\mathrm{F}$ & S & A & \\
\hline DIAL1846C07-WV & $\mathrm{F}$ & s & A & \\
\hline D0287D08-IL & $\mathrm{F}$ & s & A & \\
\hline DIAL0293B02-DC & $\mathrm{F}$ & S & A & \\
\hline DIAL1391H03-NC & M & S & A & \\
\hline DIAL1847H05-MD & M & S & A & \\
\hline DIAL0294G01-MD & $\mathrm{F}$ & S & A & DIAL5MD \\
\hline DIAL0294C06-IL & $\mathrm{F}$ & S & A & rohwe IL1 \\
\hline DIAL0294C07-IN & $\mathrm{F}$ & $S$ & A & rohwe IN2 \\
\hline DIAL0294D11-NY & $\mathrm{F}$ & S & A & rohwe NY1 \\
\hline DIAL1848E02-MD & $\mathrm{F}$ & S & A & DIAL1412E02 \\
\hline D0295B06-ON & $\mathrm{F}$ & $S$ & A & \\
\hline DIAL1391H02-NC & M & $S$ & A & \\
\hline DIAL1391D01-NC & M & S & A & \\
\hline DIAL1391H08-NC & M & S & A & \\
\hline DIAL1847H04-MD & M & S & A & \\
\hline DIAL1391G12-NC & $\mathrm{F}$ & $S$ & A & gynandromorph \\
\hline DIAL0292B09-MD & $\mathrm{F}$ & $S$ & A & \\
\hline DIAL0292C01-WV & $\mathrm{F}$ & $S$ & A & \\
\hline DIAL0292B11-MD & $\mathrm{F}$ & $S$ & A & \\
\hline DIAL0292C02-MD & $\mathrm{F}$ & $S$ & A & \\
\hline DIAL1413G10-NC & M & $S$ & A & \\
\hline DIAL1391C10-NC & M & $\mathrm{s}$ & A & \\
\hline
\end{tabular}




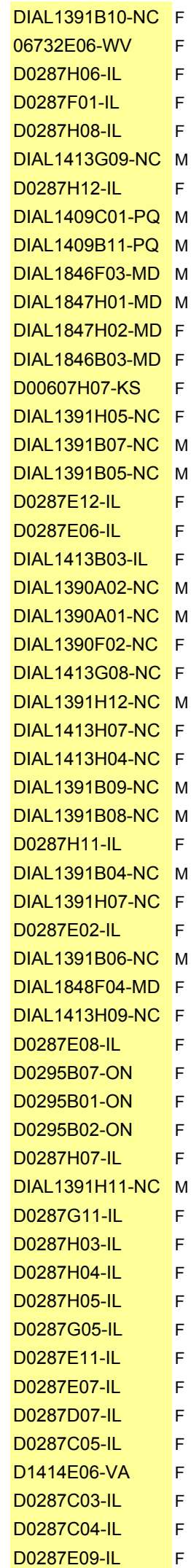

$293 \mathrm{H} 02$ 


\begin{tabular}{|c|c|c|c|c|}
\hline D1414E03-VA & $\mathrm{F}$ & $\mathrm{s}$ & A & \\
\hline D0288H02-MD & $\mathrm{F}$ & s & A & \\
\hline D0288G12-MD & $\mathrm{F}$ & $\mathrm{s}$ & A & \\
\hline D0288D10-MD & $\mathrm{F}$ & S & A & \\
\hline D0288B07-MD & $\mathrm{F}$ & $\mathrm{s}$ & A & \\
\hline D1414H06-VA & $\mathrm{F}$ & S & A & \\
\hline D1414H05-VA & $\mathrm{F}$ & S & A & \\
\hline D1414E05-VA & $\mathrm{F}$ & $\mathrm{s}$ & A & \\
\hline D0295B04-ON & $\mathrm{F}$ & S & A & \\
\hline D00607F03-KS & $\mathrm{F}$ & S & A & \\
\hline D00607C04-MO & $\mathrm{F}$ & S & A & \\
\hline D00608E07-IN & M & $\mathrm{s}$ & A & \\
\hline DIAL2385C08-MD & M & S & A & \\
\hline DIAL2385D06-MD & M & S & A & \\
\hline 06732D06-WV & $\mathrm{F}$ & S & A & \\
\hline CCDB-00611 D09 & & $\mathrm{s}$ & A & \\
\hline CCDB-00611 D03-I & & $\mathrm{s}$ & A & \\
\hline 06732H06-RI & M & $\mathrm{s}$ & A & \\
\hline DIAL1845C10-TX & $\mathrm{F}$ & S & A & \\
\hline DIAL1847A01-SC & M & S & A & \\
\hline DIAL0294D04-IN & $\mathrm{F}$ & S & A & viere IN1 \\
\hline DIAL0294E04-NY & $\mathrm{F}$ & $\mathrm{s}$ & A & viere1 NY \\
\hline DIAL0293F11-MN & $\mathrm{F}$ & S & A & vierecki4 \\
\hline DIAL0293F08-MD & $\mathrm{F}$ & S & A & vierecki1 \\
\hline D0288D06-NC & $\mathrm{F}$ & S & A & \\
\hline DIAL0293F09-MD & $\mathrm{F}$ & $s$ & A & vierecki2 \\
\hline 06732D07-MA & $\mathrm{F}$ & S & A & \\
\hline DIAL1390A06-ON & $\mathrm{F}$ & S & A & \\
\hline DIAL0286H01-MB & M & S & A & viere1 MB \\
\hline D0288D07-SC & $\mathrm{F}$ & S & A & \\
\hline D0288D11-SC & $\mathrm{F}$ & $s$ & A & \\
\hline D00609G05-NS & $\mathrm{F}$ & S & A & \\
\hline DIAL2385E10-MB & $\mathrm{F}$ & S & A & \\
\hline D01561D05-ON & $\mathrm{F}$ & S & A & \\
\hline D01561C04-IN & $\mathrm{F}$ & S & A & \\
\hline DIAL1390G09-NC & $\mathrm{F}$ & S & A & \\
\hline DIAL1848A04-NB & $\mathrm{F}$ & S & A & DIAL1412A04 \\
\hline D0295D03-MD & $\mathrm{F}$ & $s$ & A & \\
\hline DIAL1846C05-MS & $\mathrm{F}$ & $S$ & A & yellow legs \\
\hline DIAL1413D06-NC & $\mathrm{F}$ & $s$ & A & \\
\hline DIAL0294F01-PA & $\mathrm{F}$ & S & A & admir1 PA \\
\hline DIAL1413A05-IL & $\mathrm{F}$ & $s$ & A & \\
\hline DIAL0293A03-MD & $\mathrm{F}$ & $\mathrm{s}$ & A & atlanticumA03 \\
\hline DIAL0293G02-MD & $\mathrm{F}$ & $s$ & A & \\
\hline DIAL1391E08-NC & $\mathrm{F}$ & $s$ & A & \\
\hline DIAL0294E11-MD & $\mathrm{F}$ & $s$ & A & DIAL3MD \\
\hline DIAL0292H12-MD & $\mathrm{F}$ & $S$ & A & \\
\hline DIAL1390E02-ON & $\mathrm{F}$ & $s$ & A & \\
\hline DIAL1413F01-TN & $\mathrm{F}$ & $S$ & A & \\
\hline DIAL1413A07-VA & $\mathrm{F}$ & $s$ & A & \\
\hline DIAL1413H05-NC & $\mathrm{F}$ & $S$ & A & \\
\hline DIAL1413B10-IL & $\mathrm{F}$ & $s$ & A & \\
\hline DIAL1846E01-WV & $\mathrm{F}$ & $S$ & A & \\
\hline DIAL1409A10-NC & $\mathrm{F}$ & $S$ & A & \\
\hline DIAL1409H11-ON & $\mathrm{F}$ & $\mathrm{s}$ & A & \\
\hline
\end{tabular}




\begin{abstract}
DIAL1390H03-SC F DIAL1390C06-ON F DIAL1390H02-SC F DIAL1390G05-NC F DIAL1391A02-VA F DIAL1413D07-NC F DIAL1848F06-MD F D1414A01-YT $F$ DIAL2387F08-BC F DIAL1848C02-YT F BEE0286C11-ON F D00608H10-OR $F$ 04485A10-VA M DIAL1848D10-MD F DIAL1390D09-ON F DIAL1848B06-WV M DIAL1848C08-WV M
\end{abstract}

A

A

A

A

A

A

A A

A

A

A

A

A

A

A

A

A
293H03

zephy2 ON

odd specimen

1390G03 
Jason Gibbs

\begin{tabular}{|l|l|l|l|}
\hline Notes & Voucher Status & Tissue Descriptor & External URLs \\
\hline
\end{tabular}

sp. C

White pan trap

Col on: Monarda fistulosa

original ID by S.D. as fattigi

20-Sep-year? Original ID by S.D. as admiran

20-Sep-year?

20-Sep-year?

original ID by S.D. as fattigi

original ID by S.D. as fattigi 
White pan trap

original ID by S.D. as cressonii 
mix up?

Sep or Oct-03 
Col on: Claytonia virginica

Blue pan trap

Blue pan trap

Blue pan trap 
apical half of $\mathrm{T} 2$ very sparsely punctate 
Col on: Chamaecrista fasciculata

Col on: Tradescantia ohienis

Col on: surnac

white pan trap

nr halophitum with clypeus red 
Col on: Ranunculus abortivus

Yellow pan trap

near admirandum with relatively dense punct

close to flaveriae 
dorsal surface of propodeum truncate with cc 
ID-11 
original ID by S. D. as perpunctatum 
bowl survey

Yellow pan trap

bowl survey

Paralictus; pan trap

Paralictus 
near pruinosum

HOLOTYPE 
near laevissimum

near laevissimum

near laevissimum 
Col on: Selix humilis 
Yellow pan trap

close to viridatum

close to viridatum; Collection Date: 15-Jul-?

size of specimen unusually small 
Collection Date: 20-Sep-? 
Sep or Oct-03

Yellow pan trap

Col on: Barbarea vulgaris

Specimen is asymmetric. Half male/Half femi

Collection Date: 21-Sep-? 
Whilte pan trap

bowl survey

original ID by S. D. as little rohweri

acarinium incomplete, tegula pale, dorsal sur 
obscure punctures visible on pleura

sculpturing unusual 293610

https://mc06.manuscriptcentral.com/genome-pubs 
\begin{tabular}{l|l} 
Associated Taxa & Associated Specimens \\
\hline
\end{tabular} 


\begin{tabular}{|c|c|c|c|c|c|}
\hline $\begin{array}{l}\text { Project: } \\
\text { Created : }\end{array}$ & $\begin{array}{l}\text { SEARCH: Proce } \\
\text { 12-July-2017 }\end{array}$ & ess ids(1283 ids) & & User: & Jason Gibbs \\
\hline Sample ID & Collectors & Collection Date & Country/Ocean & State/Province & Region \\
\hline D00608F07-IN & R. Grundel & 05-Aug-2004 & United States & Indiana & Porter Co. \\
\hline DIAL2386F10-UT & & 16-Jun-2005 & United States & Utah & \\
\hline DIAL2386C10-UT & & & United States & Utah & \\
\hline DIAL2384C07-AB & Gibbs \& Sheffield & 15-Jun-2007 & Canada & Alberta & \\
\hline DIAL2386C08-UT & & & United States & Utah & \\
\hline DIAL2374H06-UT & & 28-May-1998 & United States & Utah & \\
\hline DIAL2385D04-ON & & 03-Aug-2001 & Canada & Ontario & \\
\hline DIAL2385E03-MD & N Allen & 22-Sep-2004 & United States & Maryland & \\
\hline D01562A03-MI & & & United States & Michigan & \\
\hline DIAL1839G06-MN & RL Andres & 21-Jul-2006 & United States & Minnesota & Clay Co. \\
\hline DIAL1839G08-MN & RL Andres & 21-Jul-2006 & United States & Minnesota & Clay Co. \\
\hline DIAL0292H11-MD & SW Droege & 22-Jul-2004 & United States & Maryland & Pr. George's Co. \\
\hline DIAL1409C04-ON & J Gibbs & 07-Oct-2006 & Canada & Ontario & Toronto \\
\hline DIAL2387C05-ON & E Willis & & Canada & Ontario & \\
\hline D01561D09-ON & & 26-Sep-2003 & Canada & Ontario & Escarpment \\
\hline 04791B03-FL & A. Vanderby & 04-Jun-2005 & United States & Florida & \\
\hline DIAL0292B02-NJ & H Gaines & 21-Jul-2004 & United States & New Jersey & Hunterdon Co. \\
\hline DIAL0286F05-MB & A. M. Patenaude & 23-Aug-2005 & Canada & Manitoba & YellowQuill Mxd. \\
\hline D00609G09-NS & S. Rigby & 07-Jun-1999 & Canada & Nova Scotia & \\
\hline DIAL1839G12-MN & RL Andres & 02-Jun-2006 & United States & Minnesota & Clay Co. \\
\hline DIAL1839G09-MN & RL Andres & 21-Jul-2006 & United States & Minnesota & Clay Co. \\
\hline 06732D10-PA & A. Smith, N. Kline & 20-Aug-2008 & United States & Pennsylvania & \\
\hline BEE1850G06-ME & HW Ikerd & & United States & Maine & Hancock Co. \\
\hline DIAL2386G02-BC & J. Gibbs & & Canada & British Columbia & \\
\hline DIAL1848A08-NB & M Gravel & 31-Jul-2005 & Canada & New Brunswick & Kent Co. \\
\hline DIAL1849A09-CO & Kearns \& Oliveras & 02-Jun-2005 & United States & Colorado & Boulder Co. \\
\hline DIAL0286F03-MB & A. M. Patenaude & 23-Aug-2005 & Canada & Manitoba & YellowQuill Mxd. \\
\hline DIAL2384C02-AB & Gibbs \& Sheffield & 20-Jun-2007 & Canada & Alberta & \\
\hline D01559F12-BC & LR Best & 12-Jul-2008 & Canada & British Columbia & Okanagan-Smilkameen \\
\hline DIAL2386G07-WA & & & United States & Washington & \\
\hline DIAL2387E09-AB & Gibbs \& Sheffield & 20-Jun-2007 & Canada & Alberta & \\
\hline D01559F10-BC & LR Best & 27-Jul-2008 & Canada & British Columbia & Okanagan-Smilkameen \\
\hline D01559H03-BC & LR Best & 27-Jul-2008 & Canada & British Columbia & Okanagan-Smilkameen \\
\hline D01559H10-BC & LR Best & 18-Jul-2008 & Canada & British Columbia & Okanagan-Smilkameen \\
\hline DIAL2387B05-WA & Gibbs \& Sheffield & 29-May-2007 & United States & Washington & Columbia Co. \\
\hline DIAL0294A03-IN & RP Jean & 01-Jul-2003 & United States & Indiana & Jasper Co. \\
\hline DIAL0292B05-IN & RP Jean & 16-Jul-2003 & United States & Indiana & Jasper Co. \\
\hline BEE0289C05-MN & L Packer & 01-Jun-2005 & United States & Minnesota & Minnetonka \\
\hline CCDB-00611 C12 & J.K Tuell & & United States & Michigan & Tuscola \\
\hline D-3253D12-WI & & 05-Sep-2007 & United States & Wisconsin & \\
\hline D0287D01-MI & J Gibbs \& C Sheffi & fi 27-Jun-2006 & United States & Michigan & Shiawassee \\
\hline DIAL1390D06-ON & E.Willis & 20-Jun-2005 & Canada & Ontario & Toronto \\
\hline DIAL1846F06-PA & SW Droege & 23-Aug-2005 & United States & Pennsylvania & Philadelphia Co. \\
\hline DIAL1390F04-NC & & 15-Aug-2006 & & & near Charleston \\
\hline DIAL0292E12-IN & RP Jean & 11-May-1999 & United States & Indiana & Vigo Co. \\
\hline DIAL1849F01-WV & SW Droege & 11-Jul-2005 & United States & West Virginia & Hampshire Co. \\
\hline DIAL0292B01-WV & SW Droege & & United States & West Virginia & Hampshire Co. \\
\hline DIAL0292B07-WV & SW Droege & & United States & West Virginia & Hampshire Co. \\
\hline DIAL0292B08-WV & SW Droege & & United States & West Virginia & Hampshire Co. \\
\hline DIAL0292F02-WV & SW Droege & 30-May-2004 & United States & West Virginia & Hampshire Co. \\
\hline DIAL1849E12-WV & SW Droege & 11-Jul-2005 & United States & West Virginia & Hampshire Co. \\
\hline DIAL0292E11-WV & SW Droege & 30-May-2004 & United States & West Virginia & Hampshire Co. \\
\hline
\end{tabular}




\begin{tabular}{|c|c|c|c|c|c|}
\hline DIAL1390F06-NC & & 15-Aug-2006 & & & near Charleston \\
\hline DIAL1390G04-NC & & 15-Aug-2006 & United States & North Carolina & near Charleston \\
\hline DIAL1390G06-NC & & 15-Aug-2006 & & & near Charleston \\
\hline DIAL1390G02-WV & & 15-Aug-2006 & United States & West Virginia & near Charleston \\
\hline DIAL1848D09-MD & SW Droege & & United States & Maryland & Garrett Co. \\
\hline DIAL1846D12-MS & SW Droege & 04-Jun-2006 & United States & Mississippi & Jackson Co. \\
\hline D1414H02-VA & SW Droege & 01-Jul-2006 & United States & Virginia & Assateague Island \\
\hline DIAL2384H09-SC & & 11-Jul-2007 & United States & South Carolina & \\
\hline D01559C09-GA & J Hanula \& S Horr & r 18-Jul-2008 & United States & Georgia & Jasper Co. \\
\hline D1414E02-VA & SW Droege & & United States & Virginia & Assateague Island \\
\hline DIAL0293C03-NJ & B. Ahlstrom & 17-Jun-2003 & United States & New Jersey & Atlantic Co. \\
\hline DIAL0293C04-NJ & R Winfree & 06-Jun-2003 & United States & New Jersey & Atlantic Co. \\
\hline D01562B06-MD & & 22-Jul-2008 & United States & Maryland & Worcester Co. \\
\hline D01562B02-MD & & 22-Jul-2008 & United States & Maryland & Worcester Co. \\
\hline D01562C12-MD & & 22-Jul-2008 & United States & Maryland & Worcester Co. \\
\hline D01562B10-ON & E. Proctor & 19-Jun-2007 & Canada & Ontario & \\
\hline D01562C08-ON & E. Proctor & 19-Jun-2007 & Canada & Ontario & \\
\hline DIAL0294G02-MD & & & United States & Maryland & \\
\hline DIAL1848B03-ON & E.Willis & & Canada & Ontario & \\
\hline D00609B05-WI & A. Wolf & 25-May-2005 & United States & Wisconsin & \\
\hline D00609G03-NS & Sheffield, Rigby \& & 10-Jul-2003 & Canada & Nova Scotia & \\
\hline D0288F07-SC & SW Droege & & United States & South Carolina & Chesterfield Co \\
\hline D0288F09-SC & SW Droege & 18-May-2006 & United States & South Carolina & Chesterfield Co \\
\hline DIAL0294A05-in & RP Jean & 01-Jul-2003 & United States & Indiana & Jasper Co. \\
\hline DIAL0292E08-MD & A. Lohr & 27-Jul-2004 & United States & Maryland & Anne Arundel Co. \\
\hline 06732D09-VA & M. Mackinnon, R. & 14-Aug-2008 & United States & Virginia & \\
\hline D00609C07-KS & J. Hopwood & 09-Aug-2004 & United States & Kansas & \\
\hline DIAL1846B10-WV & SW Droege & 30-May-2005 & United States & West Virginia & Hampshire Co. \\
\hline DIAL1390C08-ON & PJ Carson & & Canada & Ontario & Norfolk CO \\
\hline DIAL1409A09-NC & J Gibbs & 13-Aug-2006 & United States & North Carolina & near Jefferson \\
\hline DIAL1846G04-VA & SW Droege & 30-Jun-2006 & United States & Virginia & Assateague $\mathrm{I}$. \\
\hline DIAL1390B02-ON & PJ Carson & & Canada & Ontario & Norfolk CO \\
\hline DIAL1848E12-MD & Z Riegel & 21-Aug-2005 & United States & Maryland & Pr. George's Co. \\
\hline DIAL1413C12-TN & J Gibbs & 05-Aug-2006 & United States & Tennessee & Great Smoky Mountain \\
\hline D01559G11-BC & LR Best & 29-Jul-2008 & Canada & British Columbia & Okanagan-Smilkameen \\
\hline DIAL1845D09-CA & Fuerst, Briggs & & United States & California & Mariposa Co. \\
\hline DIAL2374G12-CA & & & United States & California & \\
\hline DIAL1845C05-CA & Briggs, Stephens & & United States & California & Mariposa Co. \\
\hline DIAL1845D03-CA & Fuerst, Briggs & & United States & California & Mariposa Co. \\
\hline DIAL1845C11-CA & Fuerst, Briggs & & United States & California & Mariposa Co. \\
\hline DIAL2388C12-UT & & 26-May-2006 & United States & Utah & Washington Co. \\
\hline DIAL2374G11-CA & & & United States & California & \\
\hline D00607E04-CA & S.W. Droege & 30-May-2008 & United States & California & Los Angeles Co. \\
\hline D01559E10-BC & LR Best & 15-Aug-2008 & Canada & British Columbia & Okanagan-Smilkameen \\
\hline D01559G04-BC & LR Best & 28-Jul-2008 & Canada & British Columbia & Okanagan-Smilkameen \\
\hline DIAL2388B06-UT & & 05-Jul-2006 & United States & Utah & Washington Co. \\
\hline D00607D05-CA & S.W. Droege & 30-May-2008 & United States & California & Los Angeles Co. \\
\hline DIAL2374H09-UT & T Toler & 11-May-1998 & United States & Utah & Tooele Co. \\
\hline DIAL2385C03-MD & & 30-Sep-2003 & United States & Maryland & \\
\hline DIAL1413E11-SC & J Gibbs & 08-Aug-2006 & United States & South Carolina & S of Anderson \\
\hline DIAL0292D03-NC & R Jackowski & 01-Oct-2003 & United States & North Carolina & Union Co. \\
\hline DIAL0294E03-VA & SW Droege & 08-Jun-2005 & United States & Virginia & Suffolk City Co. \\
\hline DIAL0294E02-TN & SW Droege & 08-Mar-2006 & United States & Tennessee & Sevier Co. \\
\hline DIAL0293E11-NC & R Jackowski & 01-Oct-2003 & United States & North Carolina & Union Co. \\
\hline DIAL0293E10-MD & SW Droege & 03-Sep-2003 & United States & Maryland & \\
\hline
\end{tabular}




\begin{tabular}{|c|c|c|c|c|c|}
\hline D0288C04-DC & S Rudy & & United States & District of Columbia & \\
\hline DIAL0293E12-DC & C. Osborn & 30-Jul-2004 & United States & District of Columbia & \\
\hline D1414H07-VA & SW Droege & & United States & Virginia & Assateague Island \\
\hline BEE0286D06-VA & Amro Zayed & 06-Jun-2005 & United States & Virginia & $\mathrm{KOA}$ \\
\hline DIAL1413D04-NC & J Gibbs & 07-Aug-2006 & United States & North Carolina & Junaluska Meadow \\
\hline DIAL1391D10-NC & C. Sheffield & 10-Aug-2006 & United States & North Carolina & E of Charlotte \\
\hline D1414E08-VA & SW Droege & & United States & Virginia & Assateague Island \\
\hline DIAL1409B06-NC & J Gibbs & 10-Aug-2006 & United States & North Carolina & E of Charlotte \\
\hline DIAL1413E08-NC & J Gibbs & 08-Aug-2006 & United States & North Carolina & Bryson City \\
\hline D1414E04-VA & SW Droege & & United States & Virginia & Assateague Island \\
\hline DIAL1391A09-SC & J Gibbs & 09-Aug-2006 & United States & South Carolina & Okanee Co. \\
\hline DIAL1413F07-TN & J Gibbs & 08-Aug-2006 & United States & Tennessee & near Gatlinburg \\
\hline DIAL1391D09-NC & C. Sheffield & 10-Aug-2006 & United States & North Carolina & E of Charlotte \\
\hline DIAL1391D07-NC & C. Sheffield & 10-Aug-2006 & United States & North Carolina & E of Charlotte \\
\hline DIAL1413D01-TN & J Gibbs & 05-Aug-2006 & United States & Tennessee & Great Smoky Mountain \\
\hline D1414E07-VA & SW Droege & & United States & Virginia & Assateague Island \\
\hline D0288C01-DC & S Rudy & & United States & District of Columbia & \\
\hline D0288C06-SC & SW Droege & & United States & South Carolina & Chesterfield Co \\
\hline D0288D09-SC & SW Droege & & United States & South Carolina & Chesterfield Co \\
\hline D00607E06-MO & M.S. Arduser & 20-Jun-2005 & United States & Missouri & Newton Co. \\
\hline D00607A09-MO & M.S. Arduser & 02-Jun-2005 & United States & Missouri & Newton Co. \\
\hline D0288G03-SC & SW Droege & & United States & South Carolina & Chesterfield Co \\
\hline D0288F02-SC & SW Droege & & United States & South Carolina & Chesterfield Co \\
\hline DIAL1391B01-SC & J Gibbs & 09-Aug-2006 & United States & South Carolina & Okanee Co. \\
\hline DIAL1391E04-SC & J Gibbs & 09-Aug-2006 & United States & South Carolina & Okanee Co. \\
\hline DIAL1391D11-SC & J Gibbs & 09-Aug-2006 & United States & South Carolina & Okanee Co. \\
\hline DIAL1413B06-IL & C. Sheffield & 25-Jun-2006 & United States & Illinois & Litchfield \\
\hline D1414A07-IL & J Gibbs & 24-Jun-2006 & United States & Illinois & Macoupin Co. \\
\hline DIAL1413B11-IL & Gibbs \& Sheffield & 25-Jun-2006 & United States & Illinois & Macoupin Co. \\
\hline 04485C03-MD & & 09-Jul-2007 & United States & Maryland & \\
\hline D00607F06-MD & B. Hollister & 13-May-2005 & United States & Maryland & Anne Arundel Co. \\
\hline D-3253D11-FL & & & United States & Florida & \\
\hline D00607B02-KS & J. Hopwood & 26-Aug-2004 & United States & Kansas & Douglas Co. \\
\hline DIAL0292D09-MD & Haramis, Arch & 13-Aug-2003 & United States & Maryland & Pr. George's Co. \\
\hline DIAL0292D10-MD & LE Hilden & 17-Jul-2002 & United States & Maryland & Pr. George's Co. \\
\hline DIAL0292D08-NC & R Jackowski & & United States & North Carolina & Union Co. \\
\hline DIAL0292D12-MD & LE Hilden & 17-Jul-2002 & United States & Maryland & Pr. George's Co. \\
\hline DIAL0292D11-MD & R Andrus & 25-Sep-2004 & United States & Maryland & Pr. George's Co. \\
\hline DIAL1846H06-VA & SW Droege & 01-Jul-2006 & United States & Virginia & Assateague I. \\
\hline DIAL1846C01-MS & SW Droege & 04-Jun-2006 & United States & Mississippi & Jackson Co. \\
\hline DIAL1846E11-MS & SW Droege & 04-Jun-2006 & United States & Mississippi & Jackson Co. \\
\hline DIAL1409E03-TX & HW Ikerd & 27-Apr-2002 & United States & Texas & Denton Co. \\
\hline DIAL1846G07-MS & SW Droege & 04-Jun-2006 & United States & Mississippi & Jackson Co. \\
\hline DIAL1849F11-DE & SW Droege & 26-Aug-2006 & United States & Delaware & Fenwick Island \\
\hline DIAL1849G03-DE & SW Droege & 26-Aug-2005 & United States & Delaware & Fenwick Island \\
\hline DIAL1391D04-NC & C. Sheffield & 10-Aug-2006 & United States & North Carolina & E of Charlotte \\
\hline 06732C08-MA & DB & 06-Aug-2008 & United States & Massachusetts & \\
\hline 06732C10-VA & B. Staff & 20-Aug-2008 & United States & Virginia & \\
\hline D-3253D07-FL & & 03-Jun-2007 & United States & Florida & Martin Co. \\
\hline B01566H05-TX & J.L. Neff & 19-Apr-2008 & United States & Texas & Tyler Co. \\
\hline DIAL0292E02-MD & SW Droege & 21-Aug-2003 & United States & Maryland & Dorchester Co. \\
\hline DIAL0294F02-NC & & & United States & North Carolina & \\
\hline DIAL0294F03-NC & & & United States & North Carolina & \\
\hline DIAL0294E05-NC & SW Droege & 07-Jun-2005 & United States & North Carolina & Hyde Co. \\
\hline DIAL0292E04-MD & SW Droege & 21-Aug-2003 & United States & Maryland & Dorchester Co. \\
\hline
\end{tabular}




\begin{tabular}{|c|c|c|c|c|c|}
\hline DIAL1413D12-FL & JA Genaro & 30-Aug-2006 & United States & Florida & Miami \\
\hline DIAL1846C06-MS & SW Droege & 04-Jun-2006 & United States & Mississippi & Jackson Co. \\
\hline DIAL1846H02-MS & SW Droege & 04-Jun-2006 & United States & Mississippi & Jackson Co. \\
\hline DIAL1846C11-MS & SW Droege & 04-Jun-2006 & United States & Mississippi & Jackson Co. \\
\hline CCDB-00611 E05 & Ronquist lab & 16-May-2005 & United States & Florida & Wakulla co. \\
\hline BEE0286C08-NC & Amro Zayed & 04-Jun-2005 & United States & North Carolina & Blude Ridge Pkwy \\
\hline DIAL0294A11-IN & RP Jean & 13-Apr-2001 & United States & Indiana & Vigo Co. \\
\hline DIAL0294A10-IN & RP Jean & 01-Jul-2003 & United States & Indiana & Jasper Co. \\
\hline DIAL1390C02-ON & Alana Taylor & & Canada & Ontario & Norfolk CO \\
\hline BEE0286A05-GA & Amro Zayed & 01-Jun-2005 & United States & Georgia & Hwy 60 \\
\hline DIAL0294A09-in & RP Jean & 02-Jul-2003 & United States & Illinois & Kankakee Co. \\
\hline DIAL0292E09-MD & SW Droege & 01-Jan-2004 & United States & Maryland & Garrett Co. \\
\hline DIAL1845H09-BC & C. Ratti & 19-Jun-2003 & Canada & British Columbia & Banns \\
\hline DIAL1845H07-BC & C. Ratti & 01-Jun-2003 & Canada & British Columbia & Fisher \\
\hline DIAL0292E07-ME & HW Ikerd & 20-Jun-2002 & United States & Maine & Hancock Co. \\
\hline DIAL1845F11-BC & C. Ratti & 14-May-2003 & Canada & British Columbia & Fisher \\
\hline DIAL1391A03-NC & C. Sheffield & 13-Aug-2006 & United States & North Carolina & Grandfather Mtn. \\
\hline BEE0289D05-MN & L Packer & 01-Jun-2005 & United States & Minnesota & Minnetonka \\
\hline BEE0289D07-MN & L Packer & 01-Jun-2005 & United States & Minnesota & Minnetonka \\
\hline DIAL1391A05-NC & C. Sheffield & 13-Aug-2006 & United States & North Carolina & Grandfather Mtn. \\
\hline D00609G07-NS & Sheffield, Rigby \& & 24-Jun-2003 & Canada & Nova Scotia & \\
\hline DIAL1848A03-NB & M Gravel & 31-Jul-2005 & Canada & New Brunswick & Kent Co. \\
\hline BEE1850G05-ME & HW Ikerd & & United States & Maine & Hancock Co. \\
\hline BEE0289D06-MN & L Packer & 01-Jun-2005 & United States & Minnesota & Minnetonka \\
\hline D01561G01-MD & S.W. Droege & 03-Sep-2003 & United States & Maryland & \\
\hline DIAL2386H03-WA & & & United States & Washington & \\
\hline DIAL1391A06-NC & C. Sheffield & 13-Aug-2006 & United States & North Carolina & Grandfather Mtn. \\
\hline D0287G06-IL & J Gibbs \& C Sheffi & & United States & Illinois & Macoupin Co. \\
\hline D0288G10-ON & J Gibbs & 17-Jun-2006 & Canada & Ontario & \\
\hline DIAL2386G06-BC & & & Canada & British Columbia & \\
\hline 03767G09-BC & L.McKinnon & 15-May-2009 & Canada & British Columbia & Cowichan Garry Oak Ri \\
\hline 06732B12-NY & L. Shapiro & 18-Aug-2008 & United States & New York & Suffolk Co. \\
\hline DIAL2386F07-WA & & 09-Aug-2004 & United States & Washington & \\
\hline DIAL2386H05-WA & & & United States & Washington & \\
\hline D-3253E10-OK & & & United States & Oklahoma & \\
\hline D00607B09-KS & J. Hopwood & 02-Aug-2004 & United States & Kansas & Douglas Co. \\
\hline DIAL1409D07-TX & HW Ikerd & 27-Apr-2002 & United States & Texas & Denton Co. \\
\hline DIAL1409D04-TX & HW Ikerd & 27-Apr-2002 & United States & Texas & Denton Co. \\
\hline DIAL1409E08-TX & HW Ikerd & 27-Apr-2002 & United States & Texas & Denton Co. \\
\hline DIAL1409E10-TX & HW Ikerd & 27-Apr-2002 & United States & Texas & Denton Co. \\
\hline DIAL1409F01-TX & HW Ikerd & 27-Apr-2002 & United States & Texas & Denton Co. \\
\hline D00607B01-KS & J. Hopwood & 09-Aug-2004 & United States & Kansas & Douglas Co. \\
\hline DIAL1848F12-NC & R Jackowski & & United States & North Carolina & Union Co. \\
\hline D01561B12-ON & & 09-Sep-2003 & Canada & Ontario & Brock W \\
\hline DIAL1848F10-ON & V MacPhail & 07-Jun-2006 & Canada & Ontario & Wentworth Co. \\
\hline DIAL1848G01-ON & V MacPhail & 07-Jun-2006 & Canada & Ontario & Wentworth Co. \\
\hline D01561E03-ON & V. MacPhail & 07-Jun-2006 & Canada & Ontario & Wentworth Co. \\
\hline DIAL2374G04-UT & & & United States & Utah & \\
\hline DIAL2388B04-UT & & 02-Jun-2006 & United States & Utah & Washington Co. \\
\hline DIAL2388C03-UT & & 24-May-2006 & United States & Utah & Washington Co. \\
\hline DIAL2386B05-NV & & & United States & Nevada & \\
\hline DIAL2384C08-AB & Gibbs \& Sheffield & 13-Jun-2007 & Canada & Alberta & \\
\hline DIAL2374G05-UT & & & United States & Utah & \\
\hline DIAL0294D02-IN & RP Jean & 16-Jul-2003 & United States & Indiana & Jasper Co. \\
\hline D01560D03-IA & J. Krippel & 14-Aug-2005 & United States & lowa & Allamakee Co. \\
\hline
\end{tabular}




\begin{tabular}{|c|c|c|c|c|c|}
\hline D01560A07-WI & A. Wolf & 23-Jul-2006 & United States & Wisconsin & Avoca Co. \\
\hline DIAL1409C09-ON & J Gibbs & 01-Oct-2006 & Canada & Ontario & Toronto \\
\hline DIAL1390A09-ON & PJ Carson & & Canada & Ontario & Norfolk CO \\
\hline DIAL1839G05-MN & RL Andres & 21-Jul-2006 & United States & Minnesota & Clay Co. \\
\hline D0288D03-IN & SW Droege & 26-Jul-2003 & United States & Indiana & Jackson Co, \\
\hline DIAL1413G03-NC & J Gibbs & 08-Aug-2006 & United States & North Carolina & S. of Bryson City \\
\hline D0295C04-ON & J Gibbs & 24-May-2006 & Canada & Ontario & \\
\hline DIAL1391H04-NC & J Gibbs & 06-Aug-2006 & United States & North Carolina & Great Smoky Mtn NP \\
\hline DIAL1849G11-MA & MF Veit & 19-Jul-2006 & United States & Massachusetts & Franklin Co. \\
\hline DIAL1839H01-MN & RL Andres & 21-Jul-2006 & United States & Minnesota & Clay Co. \\
\hline D0295C05-ON & J Gibbs & 24-May-2006 & Canada & Ontario & \\
\hline D01562C02-MB & A.M. Patenaude & 05-Aug-2007 & Canada & Manitoba & \\
\hline D01562B07-MI & & & United States & Michigan & \\
\hline B03765D08-IA & J. Hopwood & 19-Jun-2007 & United States & lowa & Story Co. \\
\hline 04485E09-MI & & 30-Jul-2009 & United States & Michigan & \\
\hline B03765D06-IA & J. Hopwood & 01-Aug-2007 & United States & lowa & Story Co. \\
\hline 04485E02-MI & & 23-Sep-2009 & United States & Michigan & \\
\hline B03765D11-IA & J. Hopwood & 31-Jul-2007 & United States & lowa & Story Co. \\
\hline B03765E01-IA & J. Hopwood & 20-Jun-2007 & United States & lowa & Story Co. \\
\hline D1414G06-DC & Pascarella & & United States & District of Columbia & Fairfax Co. \\
\hline DIAL1846H11-DC & & 08-Jul-2005 & United States & District of Columbia & \\
\hline BEE0289A03-MN & L Packer & 01-Jun-2005 & United States & Minnesota & Kingsview Ln, North \\
\hline DIAL1846E03-PA & SW Droege & 23-Aug-2005 & United States & Pennsylvania & Philadelphia Co. \\
\hline D0295E03-VA & H Katki & & United States & Virginia & Fairfax Co. \\
\hline BEE0289A07-MN & L Packer & 01-Jun-2005 & United States & Minnesota & Kingsview Ln, North \\
\hline DIAL0294H08-ON & & & Canada & Ontario & Toronto \\
\hline DIAL1390F12-NC & & 15-Aug-2006 & & & near Charleston \\
\hline D0295C07-ON & J Gibbs & & Canada & Ontario & \\
\hline DIAL2387C02-ON & E Willis & & Canada & Ontario & \\
\hline DIAL1849H03-DC & C Osborn & 16-Jul-2004 & United States & District of Columbia & Washington DC \\
\hline DIAL1846A05-ON & L Packer & 17-Jul-2006 & Canada & Ontario & \\
\hline DIAL1846A07-ON & L Packer & 17-Jul-2006 & Canada & Ontario & \\
\hline DIAL1846A02-ON & L Packer & 17-Jul-2006 & Canada & Ontario & \\
\hline DIAL1390F08-WV & & 15-Aug-2006 & & & near Charleston \\
\hline DIAL1391F06-ON & J Gibbs & 23-Sep-2006 & Canada & Ontario & Toronto \\
\hline DIAL1391G07-ON & J Gibbs & 18-Aug-2006 & Canada & Ontario & Toronto \\
\hline DIAL1390F03-ON & Jason Gibbs & & Canada & Ontario & Queen's University Biol \\
\hline DIAL1846B02-ON & L Packer & 17-Jul-2006 & Canada & Ontario & \\
\hline DIAL1846A03-ON & L Packer & 17-Jul-2006 & Canada & Ontario & \\
\hline DIAL1409C02-ON & J Gibbs & 06-Oct-2006 & Canada & Ontario & Toronto \\
\hline DIAL1409C03-ON & J Gibbs & 06-Oct-2006 & Canada & Ontario & Toronto \\
\hline DIAL1409C07-ON & J Gibbs & & Canada & Ontario & Toronto \\
\hline DIAL1409C08-ON & J Gibbs & & Canada & Ontario & Toronto \\
\hline D01562C10-MB & A.M. Patenaude & 09-Jun-2007 & Canada & Manitoba & \\
\hline DIAL1390E01-ON & E.Willis & & Canada & Ontario & Toronto \\
\hline DIAL1390F01-ON & Jason Gibbs & & Canada & Ontario & Queen's University Biol \\
\hline DIAL1390G01-NC & & 15-Aug-2006 & & & near Charleston \\
\hline DIAL1390F09-ON & & & Canada & Ontario & Queen's University Biol \\
\hline DIAL1390F05-ON & Jason Gibbs & & Canada & Ontario & \\
\hline DIAL1391G03-ON & J Gibbs & 23-Sep-2006 & Canada & Ontario & Toronto \\
\hline DIAL1390E03-ON & E.Willis & & Canada & Ontario & Toronto \\
\hline DIAL1390E06-ON & E.Willis & & Canada & Ontario & Toronto \\
\hline DIAL1390E07-ON & E.Willis & & Canada & Ontario & Toronto \\
\hline DIAL1391G05-ON & J Gibbs & 23-Sep-2006 & Canada & Ontario & Toronto \\
\hline DIAL2387B11-ON & E Willis & & Canada & Ontario & \\
\hline
\end{tabular}




\begin{tabular}{|c|c|c|c|c|c|}
\hline BEE1850E08-MN & Pimm \& Wageniu & =17-Aug-2004 & United States & Minnesota & Douglas Co. \\
\hline D01561A03-IN & R. Grundel & 04-May-2004 & United States & Indiana & Porter Co. \\
\hline DIAL1391G02-ON & J Gibbs & 23-Sep-2006 & Canada & Ontario & Toronto \\
\hline D01560A04-WI & A. Wolf & 23-Jul-2006 & United States & Wisconsin & Avoca Co. \\
\hline D01560B10-ON & E. Willlis & 04-Aug-2006 & Canada & Ontario & York Co. \\
\hline DIAL1849G08-WV & SW Droege & 11-Jul-2005 & United States & West Virginia & Hampshire Co. \\
\hline DIAL2387B06-ON & E Willis & 17-Aug-2006 & Canada & Ontario & \\
\hline D1414G04-DC & Pascarella & & United States & District of Columbia & Fairfax Co. \\
\hline 04485F02-MI & & 12-Aug-2009 & United States & Michigan & \\
\hline DIAL1390D04-ON & E.Willis & 20-Jun-2005 & Canada & Ontario & Toronto \\
\hline DIAL1409A02-ON & H Ngo & 03-Oct-2006 & Canada & Ontario & Toronto \\
\hline DIAL2387C07-ON & E Willis & 17-Aug-2006 & Canada & Ontario & York University \\
\hline DIAL1391H06-NC & J Gibbs & 06-Aug-2006 & United States & North Carolina & Great Smoky Mtn NP \\
\hline D-3253D06-FL & & & United States & Florida & \\
\hline BEE0286A11-GA & Amro Zayed & 02-Jun-2005 & United States & Georgia & PAVO \\
\hline DIAL1846F10-SC & SW Droege & 07-Sep-2006 & United States & South Carolina & Chesterfield Co. \\
\hline DIAL1846F12-SC & SW Droege & 07-Sep-2006 & United States & South Carolina & Chesterfield Co. \\
\hline BEE0286B08-GA & Amro Zayed & 02-Jun-2005 & United States & Georgia & PAVO \\
\hline DIAL0294C11-IN & RP Jean & 01-Aug-2001 & United States & Indiana & Newton Co. \\
\hline DIAL0294D01-IN & RP Jean & 22-Jun-2003 & United States & Indiana & Jasper Co. \\
\hline DIAL2385E04-ON & PJ Carson & & Canada & Ontario & \\
\hline DIAL0294C12-IL & RP Jean & 02-Jul-2003 & United States & Illinois & Kankakee Co. \\
\hline DIAL0293E02-MD & Russell, Droege & 06-Jun-2001 & United States & Maryland & PG Co. \\
\hline DIAL0293E01-IN & RP Jean & 22-May-2003 & United States & Indiana & Jasper Co. \\
\hline D00608G05-IN & R. Grundel & 09-Aug-2004 & United States & Indiana & Porter Co. \\
\hline D00608G01-IN & R. Grundel & & United States & Indiana & Lake Co. \\
\hline D01561A09-MD & M. Gates & & United States & Maryland & Calvert Co. \\
\hline D01561C07-MD & M. Gates & & United States & Maryland & Calvert Co. \\
\hline DIAL2385C07-DE & SW Droege & & United States & Delaware & \\
\hline D00607F08-MA & M.F. Veit & 14-Jul-2006 & United States & Massachusetts & Plymouth Co. \\
\hline BEE1850F05-NY & SW Droege & 08-Sep-2005 & United States & New York & Suffolk Co. \\
\hline DIAL2385B04-NY & SW Droege & 07-Sep-2005 & United States & New York & \\
\hline D1414H03-VA & SW Droege & 30-Jun-2006 & United States & Virginia & Assateague Island \\
\hline DIAL2384H12-NY & SW. Droege & 06-Sep-2005 & United States & New York & Suffolk Co. \\
\hline D01559D03-MA & & 21-Aug-2008 & United States & Massachusetts & Parker River NWR \\
\hline B04743G05-MA & & 30-Jul-2008 & United States & Massachusetts & FRANKLIN CO. \\
\hline DIAL1846G03-VA & SW Droege & 30-Jun-2006 & United States & Virginia & Assateague I. \\
\hline D1414H12-VA & SW Droege & & United States & Virginia & Assateague Island \\
\hline D1414H10-VA & SW Droege & & United States & Virginia & Accomack Co. \\
\hline DIAL0294D09-NC & SW Droege & 07-Jun-2005 & United States & North Carolina & Hyde Co. \\
\hline D1414C12-VA & SW Droege & & United States & Virginia & Assateague Island \\
\hline DIAL0294D08-NC & SW Droege & 07-Jun-2005 & United States & North Carolina & Hyde Co. \\
\hline DIAL1413D09-FL & JA Genaro & 30-Aug-2006 & United States & Florida & Miami \\
\hline DIAL1846G01-VA & SW Droege & 01-Jul-2006 & United States & Virginia & Assateague I. \\
\hline DIAL1848E10-MD & SW Droege & 21-Aug-2003 & United States & Maryland & Dorchester Co. \\
\hline DIAL1413A10-VA & SW Droege & 01-Jul-2006 & United States & Virginia & Accomac Co. \\
\hline DIAL1413A11-VA & SW Droege & 01-Jul-2006 & United States & Virginia & Assateague $\mathrm{I}$. \\
\hline CCDB-00611 D02 & J.K Tuell & & United States & Michigan & Barry \\
\hline 04485G02-MI & & 25-Jun-2009 & United States & Michigan & \\
\hline DIAL1848D01-WI & A Wolf & 24-May-2005 & United States & Wisconsin & Oconto Co. \\
\hline DIAL1846E08-WV & SW Droege & 30-Mar-2006 & United States & West Virginia & Hampshire Co. \\
\hline DIAL1391D03-NC & C. Sheffield & 06-Aug-2006 & United States & North Carolina & Great Smoky Mountain \\
\hline DIAL1391C11-NC & C. Sheffield & 06-Aug-2006 & United States & North Carolina & Great Smoky Mountain \\
\hline D1414H08-VA & SW Droege & & United States & Virginia & Assateague Island \\
\hline DIAL1413H11-NC & J Gibbs & 08-Aug-2006 & United States & North Carolina & S. of Bryson City \\
\hline
\end{tabular}




\begin{tabular}{|c|c|c|c|c|c|}
\hline DIAL1391A10-SC & J Gibbs & 09-Aug-2006 & United States & South Carolina & Okanee Co. \\
\hline D1414G12-VA & SW Droege & & United States & Virginia & Assateague Island \\
\hline DIAL1413E09-NC & J Gibbs & 08-Aug-2006 & United States & North Carolina & Bryson City \\
\hline DIAL1413B01-VA & SW Droege & 01-Jul-2006 & United States & Virginia & Assateague I. \\
\hline DIAL1413G07-NC & J Gibbs & 08-Aug-2006 & United States & North Carolina & S. of Bryson City \\
\hline D00607H10-KS & J. Hopwood & 26-Jul-2004 & United States & Kansas & Douglas Co. \\
\hline D0288A11-MD & WE Steiner \& JM ؛ & 05-Sep-2005 & United States & Maryland & Talbot Co. \\
\hline D0288A09-WV & SW Droege & & United States & West Virginia & Berkley Co. \\
\hline D0287D03-IL & J Gibbs \& C Sheffi & & United States & Illinois & Macoupin Co. \\
\hline D0288C02-DC & SW Droege & & United States & District of Columbia & \\
\hline D0287E03-IL & J Gibbs \& C Sheffi & & United States & Illinois & Macoupin Co. \\
\hline DIAL0294A01-IN & RP Jean & 24-Apr-2002 & United States & Indiana & Vigo Co. \\
\hline D0287F07-IL & J Gibbs \& C Sheffi & & United States & Illinois & Macoupin Co. \\
\hline D0287E04-IL & J Gibbs \& C Sheffi & & United States & Illinois & Macoupin Co. \\
\hline DIAL1846F05-WV & SW Droege & 17-Sep-2005 & United States & West Virginia & Hampshire Co. \\
\hline DIAL0294A02-il & RP Jean & 11-Apr-2003 & United States & Illinois & Kankakee Co. \\
\hline DIAL0294E08-MD & N Allen & 21-Sep-2004 & United States & Maryland & Pr. George's Co. \\
\hline DIAL0294F06-VA & & & United States & Virginia & \\
\hline DIAL0294E10-VA & SW Droege & 08-Jun-2005 & United States & Virginia & Suffolk City Co. \\
\hline DIAL0294E09-MD & SW Droege & 13-Apr-2005 & United States & Maryland & Pr. George's Co. \\
\hline DIAL0294F08-MD & & & United States & Maryland & \\
\hline DIAL0292A09-VA & T Olivier & 14-Apr-2003 & United States & Virginia & Albemarle Co. \\
\hline DIAL1413A01-IL & J Gibbs & 25-Jun-2006 & United States & Illinois & Montgomery Co. \\
\hline DIAL1390G10-NC & & 06-Aug-2006 & United States & North Carolina & Cataloochee \\
\hline DIAL0294F04-VA & & & United States & Virginia & \\
\hline DIAL1391D06-NC & C. Sheffield & 10-Aug-2006 & United States & North Carolina & E of Charlotte \\
\hline B03765B12-MD & S.W. Droege & 05-Oct-2009 & United States & Maryland & Dorchester Co. \\
\hline DIAL1847H08-MD & M Ibrahim & 30-Sep-2003 & United States & Maryland & Prince George's Co, \\
\hline DIAL1391G09-MI & Colla \& Willis & 21-Sep-2006 & United States & Michigan & St. Clair \\
\hline DIAL1413H08-NC & J Gibbs & 08-Aug-2006 & United States & North Carolina & S. of Bryson City \\
\hline DIAL0293C02-NC & R Jackowski & 01-Oct-2003 & United States & North Carolina & Union Co. \\
\hline DIAL0292A08-VA & D Smith & 24-Apr-2003 & United States & Virginia & Albemarle Co. \\
\hline DIAL1413A04-IL & J Gibbs & 25-Jun-2006 & United States & Illinois & Montgomery Co. \\
\hline DIAL1413A06-IL & J Gibbs & 25-Jun-2006 & United States & Illinois & Montgomery Co. \\
\hline DIAL1413G05-NC & J Gibbs & 08-Aug-2006 & United States & North Carolina & S. of Bryson City \\
\hline DIAL1391E03-SC & J Gibbs & 09-Aug-2006 & United States & South Carolina & Okanee Co. \\
\hline DIAL1413B08-IL & C. Sheffield & 25-Jun-2006 & United States & Illinois & Litchfield \\
\hline DIAL1846E12-SC & SW Droege & 07-Sep-2006 & United States & South Carolina & C. Sandhills NWR \\
\hline DIAL1846B01-TN & C Sheffield & 05-Aug-2006 & United States & Tennessee & Great Smoky Mountain \\
\hline DIAL1846A11-NC & C Sheffield & 07-Aug-2006 & United States & North Carolina & \\
\hline DIAL1846E05-MS & SW Droege & 04-Jun-2006 & United States & Mississippi & Jackson Co. \\
\hline DIAL1413H03-NC & J Gibbs & 08-Aug-2006 & United States & North Carolina & S. of Bryson City \\
\hline DIAL1413C04-TN & J Gibbs & 05-Aug-2006 & United States & Tennessee & Great Smoky Mountain \\
\hline D0287F02-IL & J Gibbs \& C Sheffi & & United States & Illinois & Macoupin Co. \\
\hline DIAL1413F02-TN & J Gibbs & 08-Aug-2006 & United States & Tennessee & near Gatlinburg \\
\hline D0288C08-DC & S Rudy & & United States & District of Columbia & \\
\hline DIAL1413C08-TN & J Gibbs & 05-Aug-2006 & United States & Tennessee & Great Smoky Mountain \\
\hline DIAL1413C05-TN & J Gibbs & 05-Aug-2006 & United States & Tennessee & Great Smoky Mountain \\
\hline DIAL1413B02-VA & SW Droege & 01-Jul-2006 & United States & Virginia & Assateague $\mathrm{I}$. \\
\hline DIAL1413C06-TN & J Gibbs & 05-Aug-2006 & United States & Tennessee & Great Smoky Mountain \\
\hline DIAL1391G08-MI & Colla \& Willis & 21-Sep-2006 & United States & Michigan & St. Clair \\
\hline DIAL1413C11-NC & J Gibbs & 10-Aug-2006 & United States & North Carolina & \\
\hline D0287G01-IL & J Gibbs \& C Sheffi & & United States & Illinois & Macoupin Co. \\
\hline DIAL1413B09-IL & C. Sheffield & 25-Jun-2006 & United States & Illinois & Litchfield \\
\hline DIAL1413C01-IL & Gibbs \& Sheffield & 25-Jun-2006 & United States & Illinois & Macoupin Co. \\
\hline
\end{tabular}




\begin{tabular}{|c|c|c|c|c|c|}
\hline DIAL1413F12-TN & J Gibbs & 08-Aug-2006 & United States & Tennessee & near Gatlinburg \\
\hline DIAL1413D05-SC & C. Sheffield & 09-Aug-2006 & United States & South Carolina & Okanee Co. \\
\hline DIAL1413C09-TN & J Gibbs & 05-Aug-2006 & United States & Tennessee & Great Smoky Mountain \\
\hline D0287G04-IL & \multicolumn{2}{|c|}{ J Gibbs \& C Sheffi } & United States & Illinois & Macoupin Co. \\
\hline D0287G02-IL & \multicolumn{2}{|l|}{ J Gibbs \& C Sheffi } & United States & Illinois & Macoupin Co. \\
\hline D0287G08-IL & \multicolumn{2}{|l|}{ J Gibbs \& C Sheffi } & United States & Illinois & Macoupin Co. \\
\hline DIAL2385D05-NC & MF Veit & 08-Jul-2006 & United States & North Carolina & \\
\hline DIAL1413C02-TN & J Gibbs & 05-Aug-2006 & United States & Tennessee & Great Smoky Mountain \\
\hline DIAL1413C03-TN & J Gibbs & 05-Aug-2006 & United States & Tennessee & Great Smoky Mountain \\
\hline D0287G07-IL & \multicolumn{2}{|c|}{ J Gibbs \& C Sheffi } & United States & Illinois & Macoupin Co. \\
\hline D0287G12-IL & \multicolumn{2}{|l|}{ J Gibbs \& C Sheffi } & United States & Illinois & Macoupin Co. \\
\hline D0287H09-IL & \multicolumn{2}{|l|}{ J Gibbs \& C Sheffi } & United States & Illinois & Macoupin Co. \\
\hline DIAL1413A03-IL & J Gibbs & 25-Jun-2006 & United States & Illinois & Montgomery Co. \\
\hline D0287C11-IL & \multicolumn{2}{|c|}{ J Gibbs \& C Sheffi } & United States & Illinois & Macoupin Co. \\
\hline D0287C09-IL & \multicolumn{2}{|l|}{ J Gibbs \& C Sheffi } & United States & Illinois & Macoupin Co. \\
\hline D0287D02-MI & \multicolumn{2}{|c|}{ J Gibbs \& C Sheffi 27-Jun-2006 } & United States & Michigan & Eaton \\
\hline D0287D06-IL & \multicolumn{2}{|l|}{ J Gibbs \& C Sheffi } & United States & Illinois & Macoupin Co. \\
\hline B03765B08-MD & S.W. Droege & 05-Oct-2009 & United States & Maryland & Dorchester Co. \\
\hline D-3253C08-NM & \multirow{2}{*}{\multicolumn{2}{|c|}{ K. Wetherill }} & United States & New Mexico & \\
\hline DIAL2374A05-UT & & & United States & Utah & \\
\hline DIAL2387C12-TX & \multirow{3}{*}{ HW Ikerd } & \multirow[t]{3}{*}{ 27-Apr-2002 } & United States & Texas & Denton Co. \\
\hline DIAL2374C06-UT & & & United States & Utah & \\
\hline DIAL2374A06-UT & & & United States & Utah & \\
\hline DIAL1849C11-CO & \multicolumn{2}{|c|}{ Kearns \& Oliveras 07-Jul-2004 } & United States & Colorado & Boulder Co. \\
\hline DIAL1409B10-TX & L Packer & 15-May-2003 & United States & Texas & Lasalle Co. \\
\hline DIAL1409E06-TX & HW Ikerd & 27-Apr-2002 & United States & Texas & Denton Co. \\
\hline DIAL2387B03-NM & \multirow[t]{2}{*}{ Gibbs \& Sheffield } & \multirow[t]{2}{*}{ 10-Aug-2007 } & United States & New Mexico & Chaves Co. \\
\hline DIAL2386H10-UT & & & United States & Utah & \\
\hline D-3253D03-NM & \multicolumn{2}{|l|}{ K. Wetherill } & United States & New Mexico & \\
\hline DIAL2374F08-UT & B Hays & 02-May-2006 & United States & Utah & Washington Co. \\
\hline DIAL2388D11-NM & \multirow[t]{2}{*}{ J. Gibbs } & \multirow[t]{2}{*}{ 23-Aug-2007 } & United States & New Mexico & Hidalgo Co. \\
\hline DIAL2386F01-NV & & & United States & Nevada & \\
\hline D00608D11-NM & K. Wetherill & 19-Oct-2004 & United States & New Mexico & Socorro Co. \\
\hline DIAL2374F09-UT & & & United States & Utah & \\
\hline DIAL2386D09-UT & & & United States & Utah & \\
\hline DIAL1849E03-AZ & RL Minckley & 05-Jul-2001 & United States & Arizona & Cochise Co. \\
\hline DIAL2388E03-NM & J. Gibbs & 23-Aug-2007 & United States & New Mexico & Hidalgo Co. \\
\hline DIAL2388B11-UT & J Wilson & 06-May-2002 & United States & Utah & Garfield Co. \\
\hline B-3252H08-NV & & 05-Feb-2004 & United States & Nevada & Clark Co. \\
\hline DIAL2374A07-UT & O. Messinger & 17-May-2002 & United States & Utah & Kane Co. \\
\hline D00608H06-NV & E. Ahlstrom & 24-Aug-2004 & United States & Nevada & \\
\hline DIAL1413F04-TN & J Gibbs & 08-Aug-2006 & United States & Tennessee & near Gatlinburg \\
\hline DIAL1390H04-SC & & 09-Aug-2006 & United States & South Carolina & Okanee Co. \\
\hline DIAL1847H11-MD & S Aclander & 09-Sep-2005 & United States & Maryland & Prince George's Co, \\
\hline D1414B02-NC & J Gibbs & & United States & North Carolina & \\
\hline DIAL1413G06-NC & J Gibbs & 08-Aug-2006 & United States & North Carolina & S. of Bryson City \\
\hline DIAL1845B07-CA & L. Fuerst & & United States & California & Tuolomne Co. \\
\hline DIAL1845A06-CA & Briggs, Stephens & & United States & California & Mariposa Co. \\
\hline DIAL1845B01-CA & Fuerst, Gibbs, Ste & & United States & California & Mariposa Co. \\
\hline DIAL1845C02-CA & E. Stephens & & United States & California & Mariposa Co. \\
\hline D00608H09-OR & Gibbs \& Sheffield & 06-Jul-2007 & United States & Oregon & Baker Co. \\
\hline $\mathrm{D} 00607 \mathrm{H} 02-\mathrm{CA}$ & S.W. Droege & 01-Jun-2008 & United States & California & Santa Monica Mtns. \\
\hline D00607D09-CA & S.W. Droege & 31-May-2008 & United States & California & Santa Monica Mtns. \\
\hline D00607F10-CA & S.W. Droege & 30-May-2008 & United States & California & Los Angeles Co. \\
\hline D01559D12-BC & LR Best & 16-Jul-2008 & Canada & British Columbia & Okanagan-Smilkameen \\
\hline
\end{tabular}




\begin{tabular}{|c|c|c|c|c|c|}
\hline D-3253F09-KY & & 26-Jul-2007 & United States & Kentucky & \\
\hline D0288F05-SC & \multicolumn{2}{|l|}{ SW Droege } & United States & South Carolina & Chesterfield Co \\
\hline D0287D12-IL & \multicolumn{2}{|l|}{ J Gibbs \& C Sheffi } & United States & Illinois & Macoupin Co. \\
\hline D0287F08-IL & \multicolumn{2}{|l|}{ J Gibbs \& C Sheffi } & United States & Illinois & Macoupin Co. \\
\hline DIAL1846G12-SC & SW Droege & 06-Sep-2006 & United States & South Carolina & C. Sandhills NWR \\
\hline D0287F05-IL & \multicolumn{2}{|c|}{ J Gibbs \& C Sheffi } & United States & Illinois & Macoupin Co. \\
\hline DIAL1413H01-NC & J Gibbs & 08-Aug-2006 & United States & North Carolina & S. of Bryson City \\
\hline D0287H02-IL & \multicolumn{2}{|c|}{ J Gibbs \& C Sheffi } & United States & Illinois & Montgomery Co. \\
\hline DIAL1391D05-NC & C. Sheffield & 10-Aug-2006 & United States & North Carolina & E of Charlotte \\
\hline DIAL1409B08-VA & J Gibbs & 14-Aug-2006 & United States & Virginia & Mount Rogers \\
\hline DIAL1409B09-VA & J Gibbs & 14-Aug-2006 & United States & Virginia & Mount Rogers \\
\hline DIAL1413C10-NC & J Gibbs & 10-Aug-2006 & United States & North Carolina & \\
\hline DIAL1413B07-IL & C. Sheffield & 25-Jun-2006 & United States & Illinois & Litchfield \\
\hline DIAL1413D02-TN & J Gibbs & 05-Aug-2006 & United States & Tennessee & Great Smoky Mountair \\
\hline DIAL1391E06-SC & $J$ Gibbs & 09-Aug-2006 & United States & South Carolina & S of Anderson \\
\hline D0288G07-NC & SW Droege & & United States & North Carolina & Richmond Co. \\
\hline DIAL1413G02-TN & J Gibbs & 08-Aug-2006 & United States & Tennessee & near Gatlinburg \\
\hline D0287F03-IL & \multicolumn{2}{|c|}{ J Gibbs \& C Sheffi } & United States & Illinois & Macoupin Co. \\
\hline DIAL1390G08-NC & & 06-Aug-2006 & United States & North Carolina & Cataloochee \\
\hline DIAL1413G01-TN & J Gibbs & 08-Aug-2006 & United States & Tennessee & near Gatlinburg \\
\hline D0287C06-IL & \multicolumn{2}{|c|}{ J Gibbs \& C Sheffi } & United States & Illinois & Macoupin Co. \\
\hline DIAL1391F08-ON & J Gibbs & 23-Sep-2006 & Canada & Ontario & Toronto \\
\hline DIAL1413H06-NC & J Gibbs & 08-Aug-2006 & United States & North Carolina & S. of Bryson City \\
\hline DIAL1391F02-NC & C. Sheffield & 10-Aug-2006 & United States & North Carolina & E of Charlotte \\
\hline DIAL1391B02-SC & J Gibbs & 09-Aug-2006 & United States & South Carolina & Okanee Co. \\
\hline DIAL1849G10-MA & MF Veit & 19-Jul-2006 & United States & Massachusetts & Berkshire Co. \\
\hline D-3253A10-BC & E. Elle & & Canada & British Columbia & \\
\hline D-3253A04-BC & E. Elle & & Canada & British Columbia & \\
\hline DIAL2388B03-UT & & 14-May-1997 & United States & Utah & Tooele Co. \\
\hline DIAL1847G08-OR & E Soderholm & 13-Aug-2005 & United States & Oregon & Douglas Co. \\
\hline DIAL1409D08-OR & E Solderholm & 13-Aug-2005 & United States & Oregon & Douglas Co. \\
\hline DIAL1847G09-OR & E Soderholm & 13-Aug-2005 & United States & Oregon & Douglas Co. \\
\hline DIAL1847G11-OR & E Soderholm & 13-Aug-2005 & United States & Oregon & Douglas Co. \\
\hline D00608A10-CA & S.W. Droege & 31-May-2008 & United States & California & Santa Monica Mtns. \\
\hline DIAL2386H12-UT & & & United States & Utah & \\
\hline DIAL2386H02-WA & Gibbs \& Sheffield & 29-May-2007 & United States & Washington & Garfield Co. \\
\hline D00607D10-CA & S.W. Droege & 31-May-2008 & United States & California & Santa Monica Mtns. \\
\hline D00607D07-CA & S.W. Droege & 30-May-2008 & United States & California & Los Angeles Co. \\
\hline DIAL2386D03-UT & & & United States & Utah & \\
\hline DIAL2386D05-UT & & & United States & Utah & \\
\hline 06717H07-MA & J. Milam & 08-Oct-2008 & United States & Massachusetts & Franklin Co. \\
\hline DIAL2385D09-WV & sW Droege & 11-Jul-2002 & United States & West Virginia & \\
\hline D01560D09-IA & C. Owens & 18-Jul-2005 & United States & lowa & Allamakee Co. \\
\hline B03751H11-MA & J. Milam & 08-Oct-2008 & United States & Massachusetts & Franklin Co. \\
\hline D01560E05-IA & C. Hemsley & 20-May-2004 & United States & lowa & Emmet Co \\
\hline B03750B04-MA & J. Milam & 02-May-2008 & United States & Massachusetts & Franklin Co. \\
\hline B04743H04-MA & & 08-Oct-2008 & United States & Massachusetts & FRANKLIN CO. \\
\hline 06735G03-MA & J. Milam & 20-Oct-2009 & United States & Massachusetts & Franklin Co. \\
\hline D-3253B07-BC & E. Elle & & Canada & British Columbia & \\
\hline D-3253A07-BC & E. Elle & & Canada & British Columbia & \\
\hline D-3253B05-BC & E. Elle & & Canada & British Columbia & \\
\hline D-3253C02-BC & E. Elle & & Canada & British Columbia & \\
\hline DIAL1845C08-CA & Briggs, Stephens & & United States & California & Mariposa Co. \\
\hline D-3253B09-BC & E. Elle & 01-May-2005 & Canada & British Columbia & \\
\hline DIAL1845C07-CA & H. Briggs & 16-Jun-2005 & United States & California & Mariposa Co. \\
\hline
\end{tabular}




\begin{tabular}{|c|c|c|c|c|c|}
\hline D0295F03-CO & SW Droege & & United States & Colorado & Larimer Co. \\
\hline D-3253A03-BC & E. Elle & & Canada & British Columbia & \\
\hline D00608D06-BC & E. Elle & 01-May-2005 & Canada & British Columbia & District of Saanich \\
\hline D00608E08-BC & E. Elle & 28-Jun-2005 & Canada & British Columbia & \\
\hline D00608D08-BC & E. Elle & 30-May-2005 & Canada & British Columbia & Sooke Potholes Prov. F \\
\hline D00607C01-CO & S.W. Droege & 11-May-2004 & United States & Colorado & Jackson Co. \\
\hline DIAL1845G08-BC & C. Ratti & 26-May-2003 & Canada & British Columbia & Surrey \\
\hline DIAL2374E06-WA & & & United States & Washington & \\
\hline DIAL1409C10-OR & E Solderholm & 13-Aug-2005 & United States & Oregon & Douglas Co. \\
\hline DIAL1845F09-BC & C. Ratti & 14-May-2003 & Canada & British Columbia & Fisher \\
\hline DIAL1845D07-CA & Stephens, Ikerd & & United States & California & Tuolomne Co. \\
\hline DIAL1845D06-CA & Fuerst, Briggs & & United States & California & Tuolomne Co. \\
\hline DIAL1845D11-CA & H. Briggs \& L. Fue & & United States & California & Tuolomne Co. \\
\hline DIAL1845E04-CA & Fuerst, Briggs & & United States & California & Tuolomne Co. \\
\hline DIAL1845F03-BC & C. Ratti & 14-May-2003 & Canada & British Columbia & Surrey \\
\hline DIAL1845G05-BC & C. Ratti & 14-May-2003 & Canada & British Columbia & Fisher \\
\hline 03767G11-BC & E.Elle & 14-May-2009 & Canada & British Columbia & Cowichan Garry Oak R \\
\hline DIAL2387E11-BC & C Ratti & 23-Apr-2004 & Canada & British Columbia & \\
\hline DIAL2387F03-BC & C Ratti & 07-May-2004 & Canada & British Columbia & \\
\hline DIAL2387A05-ID & Gibbs \& Sheffield & 23-Jun-2007 & United States & Idaho & \\
\hline DIAL2386A05-CA & & 13-Aug-2004 & United States & California & \\
\hline D00608D10-BC & E. Elle & 20-Jun-2005 & Canada & British Columbia & Sooke Potholes Prov. F \\
\hline DIAL2386G12-ID & Gibbs \& Sheffield & 28-May-2007 & United States & Idaho & Valley County \\
\hline D00608C09-CO & S.W. Droege & 12-May-2004 & United States & Colorado & Jackson CO. \\
\hline D00608C02-CO & S.W. Droege & 11-May-2004 & United States & Colorado & Larimer Co. \\
\hline D00608F04-BC & E. Elle & 27-Apr-2005 & Canada & British Columbia & \\
\hline D01562A04-MT & S.W. Droege & 22-Jun-2008 & United States & Montana & Park Co. \\
\hline D0295H06-CO & SW Droege & & United States & Colorado & Jackson Co. \\
\hline B03765E10-BC & L.R. Best & 02-Aug-2009 & Canada & British Columbia & Kootenay Boundary \\
\hline D-3253C01-BC & E. Elle & & Canada & British Columbia & \\
\hline DIAL2385E08-MD & SW Droege & & United States & Maryland & \\
\hline DIAL1848H12-PE & V MacPhail & 07-Jul-2006 & Canada & Prince Edward Island & Queens Co. \\
\hline DIAL1845H05-BC & C. Ratti & 04-Jul-2003 & Canada & British Columbia & Edwards \\
\hline D-3253A02-BC & E. Elle & & Canada & British Columbia & \\
\hline D00609G06-NS & Sheffield, Rigby \& & 08-Jul-2003 & Canada & Nova Scotia & \\
\hline DIAL0294F05-MD & & & United States & Maryland & \\
\hline DIAL0294H11-NB & & & Canada & New Brunswick & \\
\hline D00607G08-BC & & 07-Aug-2001 & Canada & British Columbia & \\
\hline DIAL1846A08-NC & C Sheffield & 07-Aug-2006 & United States & North Carolina & \\
\hline DIAL1845H08-BC & C. Ratti & 03-Jul-2003 & Canada & British Columbia & Bauck \\
\hline DIAL1845F08-BC & C. Ratti & 13-May-2003 & Canada & British Columbia & Randowa \\
\hline DIAL1391B11-NC & C. Sheffield & 06-Aug-2006 & United States & North Carolina & Great Smoky Mountain \\
\hline D00609G04-NS & C. Sheffield & 29-May-2002 & Canada & Nova Scotia & \\
\hline DIAL1848D05-NS & C Sheffield & 29-May-2002 & Canada & Nova Scotia & Kings Co. \\
\hline DIAL1409H12-ON & E Willis & 06-Jun-2006 & Canada & Ontario & Toronto \\
\hline DIAL1845H02-BC & C. Ratti & 23-Apr-2004 & Canada & British Columbia & Bissett \\
\hline DIAL1845G01-BC & C. Ratti & 12-Jul-2003 & Canada & British Columbia & Edwards \\
\hline DIAL1845G02-BC & C. Ratti & 13-May-2003 & Canada & British Columbia & Randowa \\
\hline DIAL1845F04-BC & C. Ratti & 31-May-2003 & Canada & British Columbia & Beckmann \\
\hline DIAL1409B05-NC & J Gibbs & 13-Aug-2006 & United States & North Carolina & Grandfather Mtn. \\
\hline DIAL1845F06-BC & C. Ratti & 13-May-2003 & Canada & British Columbia & Randowa \\
\hline DIAL1845F07-BC & C. Ratti & 08-Jul-2003 & Canada & British Columbia & Edwards \\
\hline DIAL1413E07-NC & J Gibbs & 07-Aug-2006 & United States & North Carolina & Highlands \\
\hline DIAL1848F09-PE & V MacPhail & 06-Jul-2006 & Canada & Prince Edward Island & Queens Co. \\
\hline DIAL1848A05-NB & M Gravel & 31-Jul-2005 & Canada & New Brunswick & Kent Co. \\
\hline
\end{tabular}




\begin{tabular}{|c|c|c|c|c|c|}
\hline DIAL1848A06-NB & M Gravel & 31-Jul-2005 & Canada & New Brunswick & Kent Co. \\
\hline DIAL1848G06-PE & V MacPhail & 08-Jul-2006 & Canada & Prince Edward Island & Queens Co. \\
\hline DIAL1849H06-ME & MF Veit & 16-Aug-2006 & United States & Maine & Aroostock Co. \\
\hline DIAL2388D05-CO & J. Gibbs & & United States & Colorado & \\
\hline D01562D02-ON & E. Proctor & 11-Jul-2007 & Canada & Ontario & \\
\hline D00607A05-AB & Gibbs \& Sheffield & 02-Sep-2007 & Canada & Alberta & \\
\hline D00607C10-AB & Gibbs \& Sheffield & 02-Sep-2007 & Canada & Alberta & Hwy 21 \\
\hline D00607C09-AB & Gibbs \& Sheffield & 02-Sep-2007 & Canada & Alberta & Hwy 21 \\
\hline DIAL1391A07-NC & C. Sheffield & 13-Aug-2006 & United States & North Carolina & Grandfather Mtn. \\
\hline D0295B10-ON & J Gibbs & & Canada & Ontario & \\
\hline D0295C01-ON & J Gibbs & & Canada & Ontario & \\
\hline DIAL1391C02-NC & J Gibbs & 04-Aug-2006 & United States & North Carolina & Great Smoky Mountain \\
\hline DIAL1391B03-OH & J Gibbs & 16-Aug-2006 & United States & Ohio & near Cuyahoga Valley $\uparrow$ \\
\hline DIAL1391C01-NC & J Gibbs & 04-Aug-2006 & United States & North Carolina & Great Smoky Mountain \\
\hline DIAL2388D04-CO & J. Gibbs & & United States & Colorado & \\
\hline 06745E10-ON & L. Packer & 30-May-2010 & Canada & Ontario & York University, Keele ( \\
\hline DIAL1413E02-FL & JA Genaro & 31-Aug-2006 & United States & Florida & Miami \\
\hline DIAL1413E04-FL & JA Genaro & 31-Aug-2006 & United States & Florida & Miami \\
\hline DIAL1413E06-FL & JA Genaro & 31-Aug-2006 & United States & Florida & Miami \\
\hline 04485F04-MI & H. Gordon & 09-Jul-2009 & United States & Michigan & St. Joseph Co. \\
\hline 04485F08-MI & H. Gordon & 06-Jun-2009 & United States & Michigan & St. Joseph Co. \\
\hline 04485E08-MI & & 22-Jul-2009 & United States & Michigan & \\
\hline DIAL1848C01-NB & M Gravel & 31-Jul-2005 & Canada & New Brunswick & Kent Co. \\
\hline 04485F09-MI & H. Gordon & 27-Jun-2009 & United States & Michigan & St. Joseph Co. \\
\hline 04485F11-MI & & 28-Jul-2009 & United States & Michigan & \\
\hline 04485H06-MI & & 24-Jun-2009 & United States & Michigan & \\
\hline DIAL1846A04-ON & L Packer & 17-Jul-2006 & Canada & Ontario & \\
\hline 04485H04-MI & & 19-Aug-2009 & United States & Michigan & \\
\hline DIAL1848B11-NB & M Gravel & 31-Jul-2005 & Canada & New Brunswick & Kent Co. \\
\hline D00609G02-NS & Sheffield \& Rigby & 21-Jul-2003 & Canada & Nova Scotia & \\
\hline D00609H09-NS & C. Sheffield & 17-May-2003 & Canada & Nova Scotia & \\
\hline DIAL1845E03-TX & Abbott, Caesar & 12-Мay-2000 & United States & Texas & Bastrop Co. \\
\hline DIAL1846D07-MS & SW Droege & 04-Jun-2006 & United States & Mississippi & Jackson Co. \\
\hline DIAL1846F11-MS & SW Droege & 04-Jun-2006 & United States & Mississippi & Jackson Co. \\
\hline DIAL1391A11-SC & J Gibbs & 09-Aug-2006 & United States & South Carolina & Okanee Co. \\
\hline DIAL2385E07-WV & SW Droege & 14-Jul-2003 & United States & West Virginia & \\
\hline BEE0289B05-MN & L Packer & & United States & Minnesota & St. Paul \\
\hline DIAL0286H09-MB & A. M. Patenaude & 06-Jun-2005 & Canada & Manitoba & YellowQuill Mxd. \\
\hline DIAL0286H04-MB & A. M. Patenaude & 25-Jul-2005 & Canada & Manitoba & YellowQuill Mxd. \\
\hline D0295B08-ON & J Gibbs & & Canada & Ontario & \\
\hline DIAL1390C12-ON & Alana Taylor & & Canada & Ontario & Huron ON \\
\hline DIAL0292H06-WV & SW Droege & 24-May-2003 & United States & West Virginia & Hampshire Co. \\
\hline DIAL1839G07-MN & & 10-Jun-2005 & United States & Minnesota & Clay Co. \\
\hline 06732C04-WV & J. Whitaker & 11-May-2009 & United States & West Virginia & Pendleton Co. \\
\hline D00607B07-KS & J. Hopwood & 14-Jun-2005 & United States & Kansas & Franklin Co. \\
\hline DIAL1409B01-NC & J Gibbs & 13-Aug-2006 & United States & North Carolina & Grandfather Mtn. \\
\hline DIAL1390C07-ON & PJ Carson & & Canada & Ontario & Norfolk CO \\
\hline DIAL1390C04-ON & PJ Carson & & Canada & Ontario & Norfolk CO \\
\hline DIAL0286H06-MB & A. M. Patenaude & 06-Jun-2005 & Canada & Manitoba & YellowQuill Mxd. \\
\hline DIAL1846B05-MD & K Hutson & 22-Sep-2006 & United States & Maryland & Prince Georges Co. \\
\hline DIAL1847H12-MD & R Walker & 10-Sep-2005 & United States & Maryland & Prince George's Co, \\
\hline DIAL2388E04-AL & & 31-May-2007 & United States & Alabama & Clay Co. \\
\hline DIAL1846H04-MS & SW Droege & 04-Jun-2006 & United States & Mississippi & Jackson Co. \\
\hline DIAL1846E02-MS & SW Droege & 04-Jun-2006 & United States & Mississippi & Jackson Co. \\
\hline DIAL1846E09-MS & SW Droege & 04-Jun-2006 & United States & Mississippi & Jackson Co. \\
\hline
\end{tabular}




\begin{tabular}{|c|c|c|c|c|c|}
\hline DIAL1846E06-MS & SW Droege & 04-Jun-2006 & United States & Mississippi & Jackson Co. \\
\hline DIAL1846D05-MS & SW Droege & 04-Jun-2006 & United States & Mississippi & Jackson Co. \\
\hline DIAL2387F06-TX & & & United States & Texas & \\
\hline D0288F08-NC & SW Droege & 19-Мay-2006 & United States & North Carolina & Richmond Co. \\
\hline BEE0286B09-GA & Amro Zayed & 02-Jun-2005 & United States & Georgia & PAVO \\
\hline D0288F10-SC & SW Droege & & United States & South Carolina & Chesterfield Co \\
\hline D01559E03-BC & LR Best & 25-Jul-2008 & Canada & British Columbia & Okanagan-Smilkameen \\
\hline D01559F06-BC & LR Best & 27-Jul-2008 & Canada & British Columbia & Okanagan-Smilkameen \\
\hline D00608B10-BC & E. Elle & 28-Jun-2005 & Canada & British Columbia & \\
\hline D00608D04-BC & E. Elle & 26-Jul-2005 & Canada & British Columbia & Elk Falls Prov. Pk. \\
\hline D00609H07-BC & E. Ellle & 12-Jun-2005 & Canada & British Columbia & \\
\hline D00609H03-BC & E. Ellle & 25-Jun-2005 & Canada & British Columbia & \\
\hline D00609H05-BC & E. Ellle & 13-Jul-2005 & Canada & British Columbia & \\
\hline DIAL2388B05-WA & J. WIIson & 09-Aug-2004 & United States & Washington & Okanagan \\
\hline D01561E05-CA & H.W. Ikerd & 03-Aug-2005 & United States & California & Mariposa Co. \\
\hline CCDB - 01565 H3 & LR Best & 16-Jul-2008 & Canada & British Columbia & Okanagan - Similkame \\
\hline CCDB - 01565 H8 & LR Best & 13-Jul-2008 & Canada & British Columbia & Okanagan - Similkame \\
\hline D1414C03-VA & SW Droege & 01-Jul-2006 & United States & Virginia & Assateague Island \\
\hline DIAL1846D08-VA & SW Droege & 01-Jul-2006 & United States & Virginia & Assateague $\mathrm{I}$. \\
\hline DIAL1848C05-MD & & 18-Jul-2006 & United States & Maryland & Worchestor Co. \\
\hline D1414D08-VA & SW Droege & 01-Jul-2006 & United States & Virginia & Assateague Island \\
\hline D1414C06-VA & SW Droege & 01-Jul-2006 & United States & Virginia & Assateague Island \\
\hline D1414C05-VA & SW Droege & 01-Jul-2006 & United States & Virginia & Assateague Island \\
\hline D1414C04-VA & SW Droege & 01-Jul-2006 & United States & Virginia & Assateague Island \\
\hline D01561E08-NY & S.W. Droege & 08-Sep-2005 & United States & New York & Suffolk Co. \\
\hline DIAL1849F05-DC & E Keto & 18-Oct-2004 & United States & District of Columbia & \\
\hline DIAL1409B02-NC & J Gibbs & 13-Aug-2006 & United States & North Carolina & Grandfather Mtn. \\
\hline DIAL1409A01-ON & H Ngo & & Canada & Ontario & Toronto \\
\hline D-3253E12-FL & & & United States & Florida & \\
\hline D-3253E02-FL & & & United States & Florida & \\
\hline D01559A03-FL & SW Droege & 04-Jun-2007 & United States & Florida & Martin Co. \\
\hline D01561H08-FL & S.W. Droege & 04-Jun-2007 & United States & Florida & \\
\hline D01559D09-FL & SW Droege & 04-Jun-2007 & United States & Florida & Martin Co. \\
\hline D01562D03-FL & S.W. Droege & 04-Jun-2007 & United States & Florida & Martin Co. \\
\hline D-3253B01-BC & E. Elle & & Canada & British Columbia & \\
\hline D-3253A05-BC & E. Elle & & Canada & British Columbia & \\
\hline D-3253B10-BC & E. Elle & & Canada & British Columbia & \\
\hline D-3253B11-BC & E. Elle & & Canada & British Columbia & \\
\hline D-3253B08-BC & E. Elle & & Canada & British Columbia & \\
\hline D-3262G02-UT & L. Packer & 23-Jun-1999 & United States & Utah & kane co. \\
\hline D-3253A08-BC & E. Elle & & Canada & British Columbia & \\
\hline DIAL1845C12-CA & Ikerd, Stephens & & United States & California & Mariposa Co. \\
\hline DIAL1845D10-CA & Stephens \& Briggs & & United States & California & Mariposa Co. \\
\hline DIAL2387E12-BC & C Ratti & 05-Jul-2003 & Canada & British Columbia & \\
\hline D00607F07-CA & S.W. Droege & 31-May-2008 & United States & California & Los Angeles Co. \\
\hline BEE1850F04-OR & L Packer & 27-Jul-2001 & United States & Oregon & Lane Co. \\
\hline D00607G10-BC & & 22-Мay-2001 & Canada & British Columbia & \\
\hline DIAL2386C03-CA & & & United States & California & \\
\hline DIAL2374A11-UT & & 07-Sep-2000 & United States & Utah & Kane Co. \\
\hline DIAL2386D08-UT & & & United States & Utah & \\
\hline DIAL2386D06-UT & & & United States & Utah & \\
\hline DIAL2386C04-CA & & & United States & California & \\
\hline D01559E12-BC & LR Best & 16-Aug-2008 & Canada & British Columbia & Thompson-Nicola \\
\hline DIAL2374D09-UT & & & United States & Utah & \\
\hline DIAL2374E11-WA & & & United States & Washington & \\
\hline
\end{tabular}




\begin{tabular}{|c|c|c|c|c|c|}
\hline 06732F01-WY & J. Rykken & 28-Aug-2009 & United States & Wyoming & Park Co. \\
\hline DIAL2385C01-MD & SW Droege & & United States & Maryland & \\
\hline DIAL1849G01-ME & MF Veit & 18-Aug-2006 & United States & Maine & Aroostock Co. \\
\hline 04485A08-MD & & 09-Jul-2007 & United States & Maryland & Ann Arundel Co. \\
\hline D00609G08-NS & Jansen, Sheffield & :25-Jun-2003 & Canada & Nova Scotia & Kings Co. \\
\hline DIAL1849F12-MA & MF Veit & 28-May-2006 & United States & Massachusetts & Middlesex Co. \\
\hline DIAL1848F03-ON & V MacPhail & 07-Jun-2006 & Canada & Ontario & Wentworth Co. \\
\hline D01560B11-VT & M.F. Veit & 28-Jul-2006 & United States & Vermont & Lamoille Co. \\
\hline DIAL1848F05-PE & V MacPhail & 07-Jul-2006 & Canada & Prince Edward Island & Queens Co. \\
\hline D0288B10-NC & SW Droege & & United States & North Carolina & Moore Co. \\
\hline D1414C10-VA & SW Droege & & United States & Virginia & Assateague Island \\
\hline D1414C09-VA & SW Droege & & United States & Virginia & Assateague Island \\
\hline D-3253F11-FL & & & United States & Florida & \\
\hline D1414F02-NC & & & United States & North Carolina & Kill Devil Hills \\
\hline D1414E12-NC & & & United States & North Carolina & Kill Devil Hills \\
\hline D1414E11-NC & & & United States & North Carolina & Kill Devil Hills \\
\hline D0288C03-SC & SW Droege & & United States & South Carolina & Chesterfield Co \\
\hline D1414C08-VA & SW Droege & & United States & Virginia & Assateague Island \\
\hline D0288B11-NC & SW Droege & & United States & North Carolina & Moore Co. \\
\hline DIAL1846D11-MS & SW Droege & 04-Jun-2006 & United States & Mississippi & Jackson Co. \\
\hline DIAL1849F03-NC & E Soderholm & 13-Jul-2006 & United States & North Carolina & Dare Co. \\
\hline D1414D09-VA & SW Droege & & United States & Virginia & Assateague Island \\
\hline B03765B03-VA & W. Schmitz & 09-Aug-2008 & United States & Virginia & Accomack Co. \\
\hline D0288C11-SC & SW Droege & & United States & South Carolina & Chesterfield Co \\
\hline D1414F05-NC & & & United States & North Carolina & Kill Devil Hills \\
\hline D1414C07-VA & SW Droege & & United States & Virginia & Accomack Co. \\
\hline D0295D12-MD & SW Droege & & United States & Maryland & Montgomery Co. \\
\hline D1414B06-VA & SW Droege & & United States & Virginia & Assateague Island \\
\hline DIAL0293B05-MD & HW Ikerd & 31-Jul-2002 & United States & Maryland & Pr. George's Co. \\
\hline D1414B07-VA & SW Droege & & United States & Virginia & Assateague Island \\
\hline D00607D12-MA & M.F. Veit & 21-Jul-2006 & United States & Massachusetts & Worcester Co. \\
\hline D00608F12-IN & R. Grundel & 31-Aug-2004 & United States & Indiana & Porter Co. \\
\hline D1414B08-VA & SW Droege & & United States & Virginia & Assateague Island \\
\hline D1414E01-VA & SW Droege & & United States & Virginia & Assateague Island \\
\hline D1414B09-VA & SW Droege & & United States & Virginia & Assateague Island \\
\hline DIAL1849G02-MA & MF Veit & 05-Aug-2006 & United States & Massachusetts & Middlesex Co. \\
\hline DIAL1413E12-TN & J Gibbs & 08-Aug-2006 & United States & Tennessee & near Gatlinburg \\
\hline DIAL0294F11-VA & & & United States & Virginia & \\
\hline DIAL1847B03-WV & & 30-Jul-2005 & United States & West Virginia & Hampshire Co, \\
\hline DIAL1848C11-NY & Fetridge & 21-Jul-2005 & United States & New York & Westchester co. \\
\hline D0295E06-MD & SW Droege & & United States & Maryland & Montgomery Co. \\
\hline DIAL1846B08-WV & & 07-Aug-2006 & United States & West Virginia & Hampshire Co. \\
\hline BEE0286D09-VA & Amro Zayed & 06-Jun-2005 & United States & Virginia & KOA \\
\hline DIAL1391A04-NC & C. Sheffield & 13-Aug-2006 & United States & North Carolina & Grandfather Mtn. \\
\hline DIAL1390G11-NC & & 06-Aug-2006 & United States & North Carolina & Cataloochee \\
\hline DIAL1849H08-VT & MF Veit & 28-Jul-2006 & United States & Vermont & Lamoille Co. \\
\hline DIAL2385B03-MB & & 12-Jun-2007 & Canada & Manitoba & \\
\hline DIAL2387F09-NM & Gibbs \& Sheffield & & United States & New Mexico & \\
\hline DIAL2387H11-NM & Gibbs \& Sheffield & 06-Aug-2007 & United States & New Mexico & Colfax Co. \\
\hline D0287A11-CO & SW Droege & & United States & Colorado & Larimer Co. \\
\hline DIAL2385A10-MB & & 12-Jun-2007 & Canada & Manitoba & \\
\hline D0295G05-CO & SW Droege & & United States & Colorado & Larimer Co. \\
\hline DIAL2385A04-CO & SW Droege & 11-May-2004 & United States & Colorado & \\
\hline D0287B11-CO & SW Droege & & United States & Colorado & Larimer Co. \\
\hline D01561B01-MT & Gibbs \& Sheffield & & United States & Montana & \\
\hline
\end{tabular}




\begin{tabular}{|c|c|c|c|c|c|}
\hline 06732F09-WY & J. Rykken & 28-Aug-2009 & United States & Wyoming & Park Co. \\
\hline D0295G10-CO & SW Droege & 12-May-2004 & United States & Colorado & Larimer Co. \\
\hline DIAL1390C09-ON & PJ Carson & & Canada & Ontario & Norfolk CO \\
\hline DIAL1849G09-MA & MF Veit & 05-Aug-2006 & United States & Massachusetts & Middlesex Co. \\
\hline DIAL1409A06-NC & J Gibbs & 13-Aug-2006 & United States & North Carolina & near Jefferson \\
\hline DIAL1409A11-NC & J Gibbs & 13-Aug-2006 & United States & North Carolina & near Jefferson \\
\hline DIAL1845G06-BC & C. Ratti & 04-Jun-2003 & Canada & British Columbia & Surrey \\
\hline DIAL1845H10-BC & C. Ratti & 18-Jun-2004 & Canada & British Columbia & Bauck \\
\hline DIAL1845G04-BC & C. Ratti & 13-May-2003 & Canada & British Columbia & Randowa \\
\hline DIAL1845F05-BC & C. Ratti & 14-May-2003 & Canada & British Columbia & Surrey \\
\hline DIAL1845H03-BC & C. Ratti & 12-May-2003 & Canada & British Columbia & Bissett \\
\hline DIAL1845H11-BC & C. Ratti & 01-Jul-2003 & Canada & British Columbia & Hopcott \\
\hline D00608G06-BC & E. Elle & 11-May-2005 & Canada & British Columbia & Cowichan River Prov. F \\
\hline DIAL1845H12-BC & C. Ratti & 09-Apr-2004 & Canada & British Columbia & Fisher \\
\hline DIAL1845G03-BC & C. Ratti & 16-May-2003 & Canada & British Columbia & Randowa \\
\hline DIAL1845G07-BC & C. Ratti & 22-May-2003 & Canada & British Columbia & Beckmann \\
\hline DIAL1845G12-BC & C. Ratti & 25-May-2003 & Canada & British Columbia & Onnink \\
\hline DIAL1845F12-BC & C. Ratti & 19-May-2003 & Canada & British Columbia & Beckmann \\
\hline DIAL1845F10-BC & C. Ratti & 14-May-2003 & Canada & British Columbia & Surrey \\
\hline DIAL1845F02-BC & C. Ratti & 20-May-2003 & Canada & British Columbia & Surrey \\
\hline DIAL1845G09-BC & C. Ratti & 22-May-2003 & Canada & British Columbia & Beckmann \\
\hline DIAL1845G10-BC & C. Ratti & 31-May-2003 & Canada & British Columbia & Randowa \\
\hline DIAL1845G11-BC & C. Ratti & 14-May-2003 & Canada & British Columbia & Fisher \\
\hline DIAL2387H05-CO & Gibbs \& Sheffield & 15-Aug-2007 & United States & Colorado & Gilpin Co. \\
\hline B03765E05-BC & L.R. Best & 03-May-2009 & Canada & British Columbia & Okanagan-Similkameer \\
\hline CCDB-00611 H09-( & ( Gibbs and Sheffiel & | 01-Jul-2007 & United States & Oregon & Lane co. \\
\hline D01559D04-AB & Gibbs \& Sheffield & 13-Jun-2007 & Canada & Alberta & Cypress Co. \\
\hline DIAL2385A08-SK & & 04-Jun-2007 & Canada & Saskatchewan & \\
\hline D01559G01-AB & Gibbs \& Sheffield & 13-Jun-2007 & Canada & Alberta & Cypress Co. \\
\hline DIAL2385B02-SK & & 04-Jun-2007 & Canada & Saskatchewan & \\
\hline DIAL0293B11-WV & SW Droege & 30-May-2004 & United States & West Virginia & Hampshire Co. \\
\hline DIAL1413A02-IL & J Gibbs & 25-Jun-2006 & United States & Illinois & Montgomery Co. \\
\hline DIAL1409A08-NC & J Gibbs & 13-Aug-2006 & United States & North Carolina & near Jefferson \\
\hline DIAL1390E09-ON & E.Willis & 28-Jun-2005 & Canada & Ontario & Toronto \\
\hline DIAL1413B05-IL & C. Sheffield & 25-Jun-2006 & United States & Illinois & Dwight \\
\hline D00609C03-WI & A. Wolf & 23-Jul-2006 & United States & Wisconsin & \\
\hline D01560C09-IA & D. Christensen & 18-Jun-2005 & United States & lowa & Allamakee Co. \\
\hline CCDB-01557 B01 & Green & 20-Jul-2007 & United States & Tennessee & Rutherford \\
\hline DIAL1390D01-NT & A. Gunn & & Canada & Northwest Territories & Yellowknife \\
\hline DIAL1390C10-NT & A. Gunn & 30-Jun-2005 & Canada & Northwest Territories & Yellowknife \\
\hline DIAL2384B09-YT & J. Taylor & 22-Jul-1992 & Canada & Yukon Territory & \\
\hline D0295F10-CO & sW Droege & & United States & Colorado & Larimer Co. \\
\hline DIAL0286G05-MB & A. M. Patenaude & 06-Jun-2005 & Canada & Manitoba & YellowQuill Mxd. \\
\hline DIAL0286G06-MB & A. M. Patenaude & 01-Jun-2005 & Canada & Manitoba & YellowQuill Mxd. \\
\hline DIAL0293B09-IN & RP Jean & 01-Jul-2003 & United States & Indiana & Jasper Co. \\
\hline D00609H10-NS & Sheffield \& Rigby & 03-Jul-2002 & Canada & Nova Scotia & \\
\hline DIAL0286G07-MB & A. M. Patenaude & 15-Jun-2005 & Canada & Manitoba & YellowQuill Mxd. \\
\hline DIAL0286G03-MB & A. M. Patenaude & 20-Jun-2005 & Canada & Manitoba & YellowQuill Mxd. \\
\hline D01561B02-MT & Gibbs \& Sheffield & & United States & Montana & \\
\hline D0287A03-CO & SW Droege & & United States & Colorado & Larimer Co. \\
\hline DIAL0286G10-MB & A. M. Patenaude & 20-Jul-2005 & Canada & Manitoba & YellowQuill Mxd. \\
\hline DIAL0286G09-MB & A. M. Patenaude & 22-Aug-2005 & Canada & Manitoba & YellowQuill Mxd. \\
\hline DIAL0286G04-MB & A. M. Patenaude & 20-Jul-2005 & Canada & Manitoba & YellowQuill Mxd. \\
\hline DIAL0286H03-MB & A. M. Patenaude & 11-Sep-2005 & Canada & Manitoba & YellowQuill Mxd. \\
\hline DIAL0293C07-MN & SW Droege & 10-Jul-2001 & United States & Minnesota & Sher Co. \\
\hline
\end{tabular}




\begin{tabular}{|c|c|c|c|c|c|}
\hline DIAL0293C08-MN & SW Droege & 10-Jul-2001 & United States & Minnesota & Sher Co. \\
\hline DIAL0293C06-MN & SW Droege & 10-Jul-2001 & United States & Minnesota & Sher Co. \\
\hline DIAL0294C01-IN & RP Jean & 30-Jul-2003 & United States & Indiana & Newton Co. \\
\hline DIAL0293C05-MN & SW Droege & 10-Jul-2001 & United States & Minnesota & Sher Co. \\
\hline DIAL0286F07-MB & A. M. Patenaude & 16-Aug-2005 & Canada & Manitoba & YellowQuill Mxd. \\
\hline DIAL0286F08-MB & A. M. Patenaude & 15-Jun-2005 & Canada & Manitoba & YellowQuill Mxd. \\
\hline DIAL0286F06-MB & A. M. Patenaude & 20-Jun-2005 & Canada & Manitoba & YellowQuill Mxd. \\
\hline DIAL2385B11-MI & SW Droege & 18-Aug-2003 & United States & Michigan & \\
\hline D00609B09-WI & A. Wolf & 23-Jul-2006 & United States & Wisconsin & \\
\hline D1414D12-VA & SW Droege & & United States & Virginia & Assateague Island \\
\hline DIAL1391D02-NC & C. Sheffield & 06-Aug-2006 & United States & North Carolina & Great Smoky Mountain \\
\hline DIAL0294F12-NY & & & United States & New York & \\
\hline DIAL1390B05-ON & PJ Carson & & Canada & Ontario & Norfolk CO \\
\hline 04485G12-MI & & 30-Jul-2009 & United States & Michigan & \\
\hline DIAL1846A06-ON & L Packer & 17-Jul-2006 & Canada & Ontario & \\
\hline DIAL1847H06-MD & S Kolski & 20-Aug-2004 & United States & Maryland & Prince George's Co, \\
\hline DIAL1391A01-VA & C. Sheffield & 14-Aug-2006 & United States & Virginia & 5 miles S of Elk Creek \\
\hline DIAL1413C07-TN & J Gibbs & 05-Aug-2006 & United States & Tennessee & Great Smoky Mountain \\
\hline 04485F01-MI & & 22-Jul-2009 & United States & Michigan & \\
\hline DIAL1409A05-NC & J Gibbs & 14-Aug-2006 & United States & North Carolina & $\mathrm{N}$ of Doughton Park \\
\hline DIAL1413A08-VA & SW Droege & 01-Jul-2006 & United States & Virginia & Assateague $\mathrm{I}$. \\
\hline DIAL1390H05-NC & & 14-Aug-2006 & United States & North Carolina & $\mathrm{N}$ of Doughton Park \\
\hline DIAL1413F05-TN & J Gibbs & 08-Aug-2006 & United States & Tennessee & near Gatlinburg \\
\hline DIAL1413D03-NC & J Gibbs & 07-Aug-2006 & United States & North Carolina & Junaluska Meadow \\
\hline DIAL1413B04-ON & S Paiero & 14-Jul-2001 & Canada & Ontario & Haldimand-Norfolk \\
\hline BEE1850F06-MN & Pimm \& Wagenius & § 16-Jul-2004 & United States & Minnesota & Douglas Co. \\
\hline DIAL1391C12-NC & C. Sheffield & 06-Aug-2006 & United States & North Carolina & Great Smoky Mountain \\
\hline D00607G06-BC & & 11-Jul-2002 & Canada & British Columbia & \\
\hline DIAL0294H10-NB & & & Canada & New Brunswick & \\
\hline DIAL1848A02-NB & M Gravel & 31-Jul-2005 & Canada & New Brunswick & Kent Co. \\
\hline DIAL1848G02-NB & M Gravel & 31-Jul-2005 & Canada & New Brunswick & Kent Co. \\
\hline DIAL1848A01-NB & M Gravel & 31-Jul-2005 & Canada & New Brunswick & Kent Co. \\
\hline DIAL2388E07-NB & M. Gravel & 31-Jul-2005 & Canada & New Brunswick & Kent Co. \\
\hline DIAL1391F05-NT & Sheffield \& A Gun & 21-Jun-2005 & Canada & Northwest Territories & Fort Providence \\
\hline CCDB-00611 F10-C & A. Taylor & 23-Jun-2007 & Canada & Ontario & Lambton \\
\hline D01560D11-DC & E. Keto & 07-Oct-2004 & United States & District of Columbia & Washington DC \\
\hline DIAL2385C11-DC & & 18-Oct-2004 & United States & District of Columbia & \\
\hline DIAL1413D08-NC & J Gibbs & 07-Aug-2006 & United States & North Carolina & Junaluska Meadow \\
\hline DIAL0293D07-WV & S Droege & 07-Apr-1999 & United States & West Virginia & Jackson Co. \\
\hline DIAL0293D03-MD & N Allen & 22-Aug-2004 & United States & Maryland & Pr. George's Co. \\
\hline DIAL1849H02-DC & E Keto & 18-Oct-2004 & United States & District of Columbia & Washington DC \\
\hline DIAL1847H09-MD & sW Droege & 03-Sep-2003 & United States & Maryland & \\
\hline DIAL1848E09-MD & SW Droege & 02-Apr-2002 & United States & Maryland & Anne Arundel Co. \\
\hline D01560B02-DC & E. Keto & 07-Oct-2004 & United States & District of Columbia & Washington DC \\
\hline D01560B05-DC & E. Keto & 07-Oct-2004 & United States & District of Columbia & Washington DC \\
\hline DIAL1849F08-MD & Riegel \& Walk & 19-Apr-2006 & United States & Maryland & Pr. George's Co. \\
\hline D00609E03-MT & Gibbs \& Sheffield & 21-Jun-2007 & United States & Montana & \\
\hline D0295H01-CO & SW Droege & & United States & Colorado & Jackson Co. \\
\hline D01559F01-AB & LR Best & 07-Aug-2008 & Canada & British Columbia & Okanagan-Smilkameen \\
\hline DIAL2386G09-WA & & & United States & Washington & \\
\hline D0295G03-CO & SW Droege & & United States & Colorado & Jackson Co. \\
\hline DIAL2384C05-AB & Gibbs \& Sheffield & 11-Jun-2007 & Canada & Alberta & \\
\hline DIAL1848F01-CO & SW Droege & 12-May-2005 & United States & Colorado & Jackson Co. \\
\hline D01559C10-AB & Gibbs \& Sheffield & 13-Jun-2007 & Canada & Alberta & Cypress Co. \\
\hline DIAL2386H04-WA & & & United States & Washington & \\
\hline
\end{tabular}




\begin{tabular}{|c|c|c|c|c|c|}
\hline D0295G01-CO & SW Droege & & United States & Colorado & Jackson Co. \\
\hline D01559H06-BC & LR Best & 18-Jul-2008 & Canada & British Columbia & Okanagan-Smilkameen \\
\hline D0287C02-CO & SW Droege & & United States & Colorado & Jackson Co. \\
\hline D01559G06-BC & LR Best & 11-Jul-2008 & Canada & British Columbia & Okanagan-Smilkameen \\
\hline D01559H09-BC & LR Best & 18-Jul-2008 & Canada & British Columbia & Okanagan-Smilkameen \\
\hline D01559A11-MT & Gibbs \& Sheffield & 21-Jun-2007 & United States & Montana & Pondera Co. \\
\hline DIAL2384C06-AB & Gibbs \& Sheffield & & Canada & Alberta & \\
\hline DIAL2388F09-UT & & 12-Jul-2002 & United States & Utah & Garfield Co. \\
\hline BEE0289H09-MB & A. M. Patenaude & 20-Sep-2005 & Canada & Manitoba & YellowQuill Mxd. \\
\hline BEE0289H07-MB & A. M. Patenaude & 05-Sep-2005 & Canada & Manitoba & YellowQuill Mxd. \\
\hline DIAL1409H03-MB & A. M. Patenaude & 30-Aug-2005 & Canada & Manitoba & YellowQuill Mxd \\
\hline BEE0289H06-MB & A. M. Patenaude & 20-Sep-2005 & Canada & Manitoba & YellowQuill Mxd. \\
\hline BEE0289H10-MB & A. M. Patenaude & 16-Aug-2005 & Canada & Manitoba & YellowQuill Mxd. \\
\hline DIAL2388G12-AZ & L. Packer & 14-Jun-1999 & United States & Arizona & Apache Co. \\
\hline DIAL2374G02-UT & B. Hays & 05-Jul-2006 & United States & Utah & Washington Co. \\
\hline DIAL2374B05-UT & & & United States & Utah & \\
\hline DIAL1845B08-CA & Ikerd, Stephens & & United States & California & Mariposa Co. \\
\hline D00608C07-CA & Gibbs \& Sheffield & 03-Jul-2007 & United States & California & Siskiyou Co. \\
\hline DIAL1845B02-CA & Ikerd, Stephens & & United States & California & Mariposa Co. \\
\hline DIAL2387A07-ID & Gibbs \& Sheffield & 24-Jun-2007 & United States & Idaho & \\
\hline B03765G03-BC & L.R. Best & 04-May-2009 & Canada & British Columbia & Okanagan-Similkameer \\
\hline DIAL1848D04-FL & SW Droege & 25-Jan-2005 & United States & Florida & Broward Co. \\
\hline DIAL0293E07-FL & SW Droege & 27-Jan-2005 & United States & Florida & Broward Co. \\
\hline DIAL0293E09-FL & SW Droege & 27-Jan-2005 & United States & Florida & Broward Co. \\
\hline DIAL0293E08-FL & SW Droege & 27-Jan-2005 & United States & Florida & Broward Co. \\
\hline DIAL1846H07-SC & SW Droege & 07-Sep-2005 & United States & South Carolina & C. Sandhills NWR \\
\hline DIAL1847A04-FL & SW Droege & 27-Jan-2005 & United States & Florida & Broward Co. \\
\hline DIAL1413E05-FL & JA Genaro & 31-Aug-2006 & United States & Florida & Miami \\
\hline DIAL1846B11-MS & SW Droege & 04-Jun-2006 & United States & Mississippi & Jackson Co. \\
\hline DIAL1847A02-FL & SW Droege & 27-Jan-2005 & United States & Florida & Broward Co. \\
\hline D0288E11-SC & SW Droege & 19-May-2006 & United States & South Carolina & Chesterfield Co \\
\hline DIAL1846F01-SC & SW Droege & 06-Sep-2006 & United States & South Carolina & C. Sandhills NWR \\
\hline DIAL1846B09-MS & SW Droege & 04-Jun-2006 & United States & Mississippi & Jackson Co. \\
\hline DIAL1846D01-MS & SW Droege & 04-Jun-2006 & United States & Mississippi & Jackson Co. \\
\hline D0288G05-FL & SW Droege & 27-Jan-2005 & United States & Florida & Hendry Co. \\
\hline D0288G04-FL & SW Droege & 25-Jan-2005 & United States & Florida & Palm Beach Co. \\
\hline D0288F04-SC & SW Droege & 19-May-2006 & United States & South Carolina & Chesterfield Co \\
\hline D0288E02-NC & SW Droege & 19-May-2006 & United States & North Carolina & Moore Co. \\
\hline D0288C12-FL & SW Droege & 25-Jan-2005 & United States & Florida & Palm Beach Co. \\
\hline D0288F01-SC & SW Droege & 19-May-2006 & United States & South Carolina & Chesterfield Co \\
\hline B03765A01-GA & W. Calver & 23-Oct-2009 & United States & Georgia & Chatham Co. \\
\hline D01559B08-GA & J Hanula \& S Horr & r 18-Jul-2008 & United States & Georgia & \\
\hline D01559C11-GA & J Hanula \& S Horr & r 18-Jul-2008 & United States & Georgia & Jasper Co. \\
\hline D01559E01-GA & J Hanula \& S Horr & 18-Jul-2008 & United States & Georgia & Jasper Co. \\
\hline DIAL1846D03-MS & SW Droege & 04-Jun-2006 & United States & Mississippi & Jackson Co. \\
\hline DIAL1846C03-MS & W Steiner & 08-May-2005 & United States & Maryland & Talbot Co. \\
\hline D01561E01-MA & M.F. Veit & 01-Sep-2006 & United States & Massachusetts & Worchester Co. \\
\hline DIAL1848D08-MD & J Jackson & 02-Oct-2002 & United States & Maryland & Pr. George's Co. \\
\hline DIAL1848D06-WV & SW Droege & 20-Jul-2001 & United States & West Virginia & Hampshire Co. \\
\hline DIAL2385C05-MD & & 21-Jul-2004 & United States & Maryland & \\
\hline DIAL1849F10-MD & W Steiner & 08-Mar-2005 & United States & Maryland & Talbot Co. \\
\hline DIAL2384B01-YT & & 04-Aug-1992 & Canada & Yukon Territory & \\
\hline DIAL2384A10-YT & J. Taylor & 04-Aug-1992 & Canada & Yukon Territory & \\
\hline DIAL2384B03-YT & & 04-Aug-1992 & Canada & Yukon Territory & \\
\hline DIAL2384A08-YT & & 04-Aug-1992 & Canada & Yukon Territory & \\
\hline
\end{tabular}




\begin{tabular}{|c|c|c|c|c|c|}
\hline B01558C07-AB & Gibbs \& Sheffield & 12-Jun-2007 & Canada & Alberta & Division No. 2 \\
\hline DIAL2387C04-NM & Gibbs \& Sheffield & & United States & New Mexico & \\
\hline D00609B02-MT & Gibbs \& Sheffield & 23-Jun-2007 & United States & Montana & \\
\hline D01562A01-MT & S.W. Droege & 24-Jun-2008 & United States & Montana & Gallatin Co. \\
\hline DIAL2374G03-NV & & & United States & Nevada & \\
\hline 03767G04-BC & L.Neame & 09-May-2007 & Canada & British Columbia & Thetis Lake Regional $\mathrm{P}$ \\
\hline CCDB-00611 H02 & Gibbs and Sheffie & 27-Jun-2007 & United States & Oregon & Baker co. \\
\hline D0295H07-CO & SW Droege & & United States & Colorado & Jackson Co. \\
\hline CCDB-01557 A01 & Gibbs \& Sheffiled & 15-Jun-2007 & Canada & Alberta & Three Hills \\
\hline DIAL2384C03-AB & Gibbs \& Sheffield & 12-Jun-2007 & Canada & Alberta & \\
\hline D0295H03-CO & SW Droege & & United States & Colorado & Larimer Co. \\
\hline D01559G12-BC & LR Best & 29-Jul-2008 & Canada & British Columbia & Okanagan-Smilkameen \\
\hline D00607H03-SK & Goulet, Boudreaul & 04-Jun-2007 וג & Canada & Saskatchewan & Grassland Nat. Park \\
\hline DIAL2386G03-BC & & & Canada & British Columbia & \\
\hline DIAL2374B07-UT & & & United States & Utah & \\
\hline DIAL2388C07-NV & & 15-Jul-2005 & United States & Nevada & Clark Co. \\
\hline D01561E07-SK & Goulet, Boudreaul & 104-Jun-2007 וג & Canada & Saskatchewan & Grassland NP \\
\hline D01561E02-SK & Goulet, Boudreaul & 1) 04-Jun-2007 & Canada & Saskatchewan & Grassland NP \\
\hline D01562D12-BC & L.R. Best & 23-Aug-2008 & Canada & British Columbia & Okanagan-Simiilkamee \\
\hline D01559A08-AB & Gibbs \& Sheffield & 12-Jun-2007 & Canada & Alberta & Division 2 \\
\hline 04486E05-WA & C. Fimbel & 23-Jun-2009 & United States & Washington & Thurston County \\
\hline CCDB-00611 H11 & Gibbs and Sheffie & | 30-Jul-2007 & United States & Colorado & Park co. \\
\hline 03767G06-BC & L.Neame & 22-May-2007 & Canada & British Columbia & Mount Douglas Park, Vi \\
\hline B03765E06-BC & L.R. Best & 02-May-2009 & Canada & British Columbia & Okanagan-Similkameer \\
\hline CCDB-01557 A04 & Goulet, Boudreaul & 104-Jun-2007 & Canada & Saskatchewan & Grassland National Par \\
\hline 06732E12-WY & J. Rykken & 28-Aug-2009 & United States & Wyoming & Park Co. \\
\hline DIAL2386A08-NV & & 27-Aug-2004 & United States & Nevada & Clark County \\
\hline D01562D10-ON & E. Proctor & 27-May-2007 & Canada & Ontario & \\
\hline DIAL0286H10-MB & A. M. Patenaude & 16-May-2005 & Canada & Manitoba & YellowQuill Mxd. \\
\hline DIAL0291H07-MB & & & Canada & Manitoba & YellowQuill Mxd. \\
\hline DIAL0291H05-MB & A. M. Patenaude & & Canada & Manitoba & YellowQuill Mxd. \\
\hline DIAL0291H06-MB & & & Canada & Manitoba & YellowQuill Mxd. \\
\hline DIAL0286H11-MB & A. M. Patenaude & 16-May-2005 & Canada & Manitoba & YellowQuill Mxd. \\
\hline DIAL0286H07-MB & A. M. Patenaude & 06-Jun-2005 & Canada & Manitoba & YellowQuill Mxd. \\
\hline DIAL0286H08-MB & A. M. Patenaude & 15-Jun-2005 & Canada & Manitoba & YellowQuill Mxd. \\
\hline B03765F12-BC & L.R. Best & 22-Apr-2009 & Canada & British Columbia & Okanagan-Similkameer \\
\hline DIAL1409H04-MB & A. M. Patenaude & 24-May-2005 & Canada & Manitoba & YellowQuill Mxd \\
\hline DIAL1409H02-MB & A. M. Patenaude & 16-May-2005 & Canada & Manitoba & YellowQuill Mxd \\
\hline CCDB-01557 A09-I & r Goulet \& Boudrea & al 16-Jun-2007 & Canada & Manitoba & Camperville \\
\hline D01562C04-MB & A.M. Patenaude & 09-Jun-2007 & Canada & Manitoba & \\
\hline D01560A10-WI & A. Wolf & 30-Jul-2005 & United States & Wisconsin & Manitowoc Co. \\
\hline DIAL0291H08-MB & & 08-Aug-2005 & Canada & Manitoba & YellowQuill Mxd. \\
\hline D01562E02-MB & A.M. Patenaude & 03-Jun-2007 & Canada & Manitoba & \\
\hline D01562C09-ON & E. Proctor & 27-May-2007 & Canada & Ontario & \\
\hline CCDB-01557 A07-I & I Goulet \& Boudrea & al 16-Jun-2007 & Canada & Manitoba & Camperville \\
\hline B03765F09-BC & L.R. Best & 22-Apr-2009 & Canada & British Columbia & Okanagan-Similkameer \\
\hline ICCDB-01557 A10- & I Goulet \& Boudrea & al 11-Jun-2007 & Canada & Manitoba & Tailgrass Prairie Preser \\
\hline CCDB-01557 A11-I & I Goulet \& Boudrea & al 16-Jun-2007 & Canada & Manitoba & Winnipegosis \\
\hline B03765F06-BC & L.R. Best & 22-Apr-2009 & Canada & British Columbia & Okanagan-Similkameer \\
\hline D00608F06-BC & E. Elle & 12-May-2005 & Canada & British Columbia & Elk Falls Prov. Pk. \\
\hline В03751H05-BC & L.R. Best & 03-May-2009 & Canada & British Columbia & Okanagan-Similkamed \\
\hline D01559E08-BC & LR Best & 26-Jul-2008 & Canada & British Columbia & Okanagan-Smilkameen \\
\hline D00607E12-UT & L. Packer & 21-Jun-1999 & United States & Utah & Iron Co. \\
\hline D00608E01-BC & E. Elle & 25-Jun-2005 & Canada & British Columbia & Elk Falls Prov. Pk. \\
\hline D01561F06-BC & E. Elle & 26-May-2005 & Canada & British Columbia & Elk Falls Prov. Park \\
\hline
\end{tabular}




\begin{tabular}{|c|c|c|c|c|c|}
\hline B03765E12-BC & L.R. Best & 03-May-2009 & Canada & British Columbia & Okanagan-Similkameer \\
\hline D0295F01-CO & SW Droege & & United States & Colorado & Larimer Co. \\
\hline DIAL2374E05-WA & & & United States & Washington & \\
\hline CCDB-00611 H04 & Gibbs and Sheffiel & 27-Jun-2007 & United States & Oregon & Baker co. \\
\hline D01559G09-BC & LR Best & 18-Jul-2008 & Canada & British Columbia & Okanagan-Smilkameen \\
\hline D0295G11-CO & SW Droege & & United States & Colorado & Larimer Co. \\
\hline DIAL2387C03-ID & Gibbs \& Sheffield & 23-Jun-2007 & United States & Idaho & \\
\hline D0295F04-CO & SW Droege & & United States & Colorado & Larimer Co. \\
\hline DIAL2374E04-WA & & & United States & Washington & \\
\hline D01559B11-BC & LR Best & 13-Jul-2008 & Canada & British Columbia & Okanagan-Smilkameen \\
\hline D01559C03-BC & LR Best & 13-Jul-2008 & Canada & British Columbia & Okanagan-Smilkameen \\
\hline D0295H11-CO & SW Droege & & United States & Colorado & Jackson Co. \\
\hline D0287C01-CO & SW Droege & & United States & Colorado & Larimer Co. \\
\hline D0287B01-CO & SW Droege & & United States & Colorado & Jackson Co. \\
\hline D01562A10-MT & S.W. Droege & 22-Jun-2008 & United States & Montana & Park Co. \\
\hline D0295G06-CO & SW Droege & & United States & Colorado & Larimer Co. \\
\hline CCDB-00611 H03 & Gibbs and Sheffiel & 27-Jun-2007 & United States & Oregon & Baker co. \\
\hline DIAL2388D12-NM & J. Gibbs & 23-Aug-2007 & United States & New Mexico & Hidalgo Co. \\
\hline DIAL0293H09-AZ & Laurence Packer & & United States & Arizona & Cochise Co. \\
\hline D-3262F07-NM & K. Wetherill & & United States & New Mexico & Socorro Co. \\
\hline D0295G07-CO & SW Droege & & United States & Colorado & Larimer Co. \\
\hline DIAL1848C04-MN & Pimm \& Wagenius & \& 13-Jul-2004 & United States & Minnesota & Douglas Co. \\
\hline DIAL1848F02-MN & Pimm \& Wagenius & £21-Jul-2004 & United States & Minnesota & Douglas Co. \\
\hline DIAL1848B05-AZ & L Packer & 01-Aug-2005 & United States & Arizona & Willcox \\
\hline DIAL1849B05-AZ & RL Minckley & 03-Mar-2001 & United States & Arizona & Cochise Co. \\
\hline DIAL1847F03-TX & HW Ikerd & 27-Apr-2007 & United States & Texas & Denton Co. \\
\hline DIAL1849E10-AZ & L Packer & 01-Sep-2004 & United States & Arizona & Cochise Co. \\
\hline D0287A06-CO & SW Droege & 11-May-2004 & United States & Colorado & Larimer Co. \\
\hline DIAL2374F12-UT & B. Hays & 29-May-2006 & United States & Utah & Washington Co. \\
\hline D0295G02-CO & SW Droege & & United States & Colorado & Larimer Co. \\
\hline DIAL1849E02-CO & Kearns \& Oliveras & 08-Jun-2004 & United States & Colorado & Boulder Co. \\
\hline DIAL1848B01-AZ & L Packer & 01-Aug-2005 & United States & Arizona & Willcox \\
\hline DIAL1848C03-MN & Pimm \& Wagenius & s 26-Jul-2004 & United States & Minnesota & Douglas Co. \\
\hline D01561B03-CA & Gibbs \& Sheffield & & United States & California & \\
\hline DIAL2386B02-NV & & & United States & Nevada & \\
\hline DIAL2374G01-UT & & & United States & Utah & \\
\hline DIAL2388E02-NM & J. Gibbs & 23-Aug-2007 & United States & New Mexico & Hidalgo Co. \\
\hline DIAL1849C06-CO & Kearns \& Oliveras & 01-Jun-2005 & United States & Colorado & Boulder Co. \\
\hline DIAL0286G11-MB & A. M. Patenaude & 20-Sep-2005 & Canada & Manitoba & YellowQuill Mxd. \\
\hline D0287A04-CO & SW Droege & & United States & Colorado & Larimer Co. \\
\hline D0295H04-CO & SW Droege & & United States & Colorado & Larimer Co. \\
\hline D0295G04-CO & SW Droege & & United States & Colorado & Larimer Co. \\
\hline DIAL0286G12-MB & A. M. Patenaude & 28-Jun-2005 & Canada & Manitoba & YellowQuill Mxd. \\
\hline DIAL0286G08-MB & A. M. Patenaude & 05-Sep-2005 & Canada & Manitoba & YellowQuill Mxd. \\
\hline 06732H03-CO & Gibbs \& Sheffield & 31-Aug-2007 & United States & Colorado & Gilpin Co. \\
\hline DIAL2385E11-NS & C Sheffield & 01-Aug-2001 & Canada & Nova Scotia & \\
\hline DIAL2385A02-NS & C Sheffield & 18-Jul-2002 & Canada & Nova Scotia & \\
\hline DIAL2385F01-NS & C Sheffield & 20-Sep-2001 & Canada & Nova Scotia & \\
\hline DIAL2385F04-NS & C Sheffield & 09-Oct-2002 & Canada & Nova Scotia & \\
\hline DIAL1848D03-NS & C Sheffield & 29-May-2002 & Canada & Nova Scotia & Kings Co. \\
\hline DIAL1848D02-NS & C Sheffield & 29-May-2002 & Canada & Nova Scotia & Kings Co. \\
\hline D01560A05-WI & A. Wolf & 30-Jul-2005 & United States & Wisconsin & Manitowoc Co. \\
\hline DIAL2384H08-MD & SW. Droege & 13-Apr-2005 & United States & Maryland & \\
\hline DIAL1849H05-MD & SW Droege & 20-Sep-2006 & United States & Maryland & Worcester Co. \\
\hline DIAL0294B06-IN & RP Jean & 03-Apr-2003 & United States & Indiana & Starke Co. \\
\hline
\end{tabular}




\begin{tabular}{|c|c|c|c|c|c|}
\hline DIAL1848G09-AB & Finnamore \& Pollc & c 28-Jul-1994 & Canada & Alberta & CFB Suffield \\
\hline D01562E03-NS & C. Sheffield & 31-Jul-2002 & Canada & Nova Scotia & Kings Co. \\
\hline DIAL2385A01-SK & & 04-Jun-2007 & Canada & Saskatchewan & \\
\hline DIAL2385A05-SK & & 04-Jun-2007 & Canada & Saskatchewan & \\
\hline D01560C07-IA & C. Hemsley & 20-May-2004 & United States & lowa & Dickinson Co. \\
\hline D-3253F03-MO & & 29-Mar-1998 & United States & Missouri & \\
\hline D0288E01-VA & B. Steury & 11-Apr-2005 & United States & Virginia & Fairfax Co. \\
\hline DIAL2385E09-NS & C Sheffield & 20-Jun-2002 & Canada & Nova Scotia & \\
\hline D0288C10-VA & B. Steury & & United States & Virginia & Fairfax Co. \\
\hline DIAL0294F07-MD & & & United States & Maryland & \\
\hline DIAL0293B04-MD & SW Droege & 07-Jul-2001 & United States & Maryland & Pr. George's Co. \\
\hline DIAL0294G03-MD & & 16-Jul-2003 & United States & Maryland & \\
\hline DIAL0293B03-MD & SW Droege & 07-Jul-2001 & United States & Maryland & Pr. George's Co. \\
\hline DIAL0294G04-MD & & 15-Jul-2003 & United States & Maryland & \\
\hline DIAL0294C08-IN & RP Jean & 17-Jul-2003 & United States & Indiana & Newton Co. \\
\hline DIAL0292A03-MD & SW Droege & 13-Apr-2003 & United States & Maryland & Anne Arundel Co. \\
\hline DIAL0292A04-MD & & & United States & Maryland & Pr. George's Co. \\
\hline B04743G07-MA & & 15-May-2008 & United States & Massachusetts & FRANKLIN CO. \\
\hline B04743G01-MA & & 08-Oct-2008 & United States & Massachusetts & FRANKLIN CO. \\
\hline DIAL1846F08-WV & & 07-Aug-2006 & United States & West Virginia & Hampshire Co. \\
\hline DIAL1848C12-WV & SW Droege & 30-May-2004 & United States & West Virginia & Hampshire Co. \\
\hline B04743D05-NS & & 20-Jun-2002 & Canada & Nova Scotia & KINGS CO. \\
\hline D00608E10-IN & R. Grundel & 28-Apr-2004 & United States & Indiana & Jasper Co. \\
\hline D01562B05-ON & E. Proctor & 11-Jul-2007 & Canada & Ontario & \\
\hline B04743H06-MA & & 08-Aug-2009 & United States & Massachusetts & \\
\hline DIAL1391E05-TN & C. Sheffield & 05-Aug-2006 & United States & Tennessee & Great Smoky Mountain \\
\hline D00607E07-CT & Bricker \& Watson & 01-Jun-2006 & United States & Connecticut & New London Co. \\
\hline D0288B02-MD & HW Ikerd & & United States & Maryland & \\
\hline D01559E06-ON & A Taylor & 15-Jul-2008 & Canada & Ontario & Lambton Co. \\
\hline D00607A10-CT & Bricker \& Watson & 19-Jul-2006 & United States & Connecticut & New London Co. \\
\hline DIAL1849G06-NH & MF Veit & 23-Jun-2006 & United States & New Hampshire & Coos Co. \\
\hline D01560A01-WI & A. Wolf & 01-Jul-2005 & United States & Wisconsin & Grant Co. \\
\hline B03750B02-MA & J. Milam & 08-Oct-2008 & United States & Massachusetts & Franklin Co. \\
\hline CCDB-00611 G08-1 & (A. Taylor & 14-Jul-2008 & Canada & Ontario & Lambdon \\
\hline CCDB-00611 F03-C & (A. Taylor & 07-Jul-2007 & Canada & Ontario & Lambton \\
\hline B04743G03-MA & & 15-May-2008 & United States & Massachusetts & FRANKLIN CO. \\
\hline B04743H02-MA & & 03-Jul-2008 & United States & Massachusetts & FRANKLIN CO. \\
\hline CCDB-00611 G03-1 & (A. Taylor & 14-Jul-2008 & Canada & Ontario & Lambdon \\
\hline CCDB-00611 G04-I & (A. Taylor & 12-Aug-2008 & Canada & Ontario & Lambdon \\
\hline B04743D03-NS & & 06-Jun-2002 & Canada & Nova Scotia & KINGS CO. \\
\hline D00608E12-IN & R. Grundel & 25-Jun-2003 & United States & Indiana & Jasper Co. \\
\hline D01562E07-NS & C. Sheffield & 20-Jun-2002 & Canada & Nova Scotia & Kings Co. \\
\hline D01560C12-IA & K. Kwaiser & 17-Aug-2004 & United States & lowa & Dickinson Co. \\
\hline DIAL0286F12-MB & A. M. Patenaude & 01-Jun-2005 & Canada & Manitoba & YellowQuill Mxd. \\
\hline DIAL0286F10-MB & A. M. Patenaude & 29-Aug-2005 & Canada & Manitoba & YellowQuill Mxd. \\
\hline DIAL1848H03-AB & Finnamore \& Pollc & c 29-Jun-1994 & Canada & Alberta & CFB Suffield \\
\hline DIAL1390B01-ON & PJ Carson & & Canada & Ontario & Norfolk CO \\
\hline DIAL0293E04-FL & SW Droege & 27-Jan-2005 & United States & Florida & Hendry Co. \\
\hline D-3253D09-FL & & & United States & Florida & \\
\hline D-3253E04-FL & & & United States & Florida & \\
\hline D01561D10-FL & S.W. Droege & 04-Jun-2007 & United States & Florida & Martin Co. \\
\hline D01559A04-FL & SW Droege & 04-Jun-2007 & United States & Florida & Martin Co. \\
\hline D0295D02-DC & Pascarella & 24-Jun-2006 & United States & District of Columbia & \\
\hline D00608B05-ON & A. Taylor & 07-Jul-2007 & Canada & Ontario & Lambton \\
\hline D00608B03-ON & A. Taylor & 22-Jun-2007 & Canada & Ontario & Lambton \\
\hline
\end{tabular}




\begin{tabular}{|c|c|c|c|c|c|}
\hline D1414C02-VA & SW Droege & 01-Jul-2006 & United States & Virginia & Assateague Island \\
\hline DIAL0294E01-VA & SW Droege & 02-Oct-2004 & United States & Virginia & Fluvanna Co. \\
\hline DIAL0294D12-NY & SW Droege & 06-Sep-2005 & United States & New York & Suffolk Co. \\
\hline D1414B10-VA & SW Droege & 01-Jul-2006 & United States & Virginia & Assateague Island \\
\hline D1414C01-VA & SW Droege & & United States & Virginia & Accomack Co. \\
\hline D-3253F06-MO & & & United States & Missouri & \\
\hline DIAL1846B06-MS & SW Droege & 04-Jun-2006 & United States & Mississippi & Jackson Co. \\
\hline DIAL1847H10-MD & Haramis \& Arch & 12-Aug-2003 & United States & Maryland & Prince George's Co, \\
\hline DIAL1846H10-MD & M Gates & 07-Jul-2006 & United States & Maryland & Calvert Co. \\
\hline DIAL1846B04-MS & SW Droege & 04-Jun-2006 & United States & Mississippi & Jackson Co. \\
\hline DIAL1413A09-VA & SW Droege & 01-Jul-2006 & United States & Virginia & Assateague I. \\
\hline B01566D04-TX & L.Packer & 22-Мay-2003 & United States & Texas & Ceass Co. \\
\hline D0288E08-WV & SW Droege & 30-May-2004 & United States & West Virginia & Hampshire Co. \\
\hline D1414B11-VA & SW Droege & 01-Jul-2006 & United States & Virginia & Assateague Island \\
\hline DIAL1391E02-SC & J Gibbs & 09-Aug-2006 & United States & South Carolina & Okanee Co. \\
\hline DIAL1391E01-SC & J Gibbs & 09-Aug-2006 & United States & South Carolina & Okanee Co. \\
\hline D0288G09-SC & SW Droege & 19-May-2006 & United States & South Carolina & Chesterfield Co \\
\hline D0288E10-NC & R Jackowski & 01-Oct-2003 & United States & North Carolina & Union Co. \\
\hline D0288E05-WV & SW Droege & 30-May-2004 & United States & West Virginia & Hampshire Co. \\
\hline D0288D12-WV & SW Droege & 30-May-2004 & United States & West Virginia & Hampshire Co. \\
\hline D0295E11-DC & Pascarella & 24-Jun-2006 & United States & District of Columbia & \\
\hline D0295E10-DC & Pascarella & 24-Jun-2006 & United States & District of Columbia & \\
\hline BEE0286E07-VA & Amro Zayed & 07-Jun-2005 & United States & Virginia & Hwy 340 \\
\hline D00607C02-MO & R.P. Jean & 16-Jul-2006 & United States & Missouri & Ozark Co. \\
\hline D00607A01-NE & S.W. Droege & 10-May-2007 & United States & Nebraska & Douglas Co. \\
\hline 06732C02-DC & J. Whitaker & 08-Mar-2009 & United States & Washington & \\
\hline DIAL2387C06-YT & Goulet \& Boudrea। & | 08-Jul-2008 & Canada & Yukon Territory & Champagne \\
\hline DIAL1845E09-NL & M. Baird & 05-Jul-2006 & Canada & Newfoundland and Labra & Colliers \\
\hline DIAL1845E06-NL & M. Baird & 21-Jul-2006 & Canada & Newfoundland and Labra & Colliers \\
\hline DIAL2384A11-YT & & 04-Aug-1992 & Canada & Yukon Territory & \\
\hline DIAL1391C03-NC & J Gibbs & 04-Aug-2006 & United States & North Carolina & Great Smoky Mountain \\
\hline DIAL2384A03-YT & & 04-Aug-1992 & Canada & Yukon Territory & \\
\hline DIAL2384A02-YT & & 04-Aug-1992 & Canada & Yukon Territory & \\
\hline DIAL1845E12-NL & M. Baird & 07-Jul-2006 & Canada & Newfoundland and Labra & Colliers \\
\hline DIAL1845F01-NL & M. Baird & 05-Jul-2006 & Canada & Newfoundland and Labra & Colliers \\
\hline DIAL2387B10-YT & Goulet \& Boudreal & 108-Jul-2008 & Canada & Yukon Territory & Champagne \\
\hline DIAL1391C04-NC & J Gibbs & 04-Aug-2006 & United States & North Carolina & Great Smoky Mountain \\
\hline DIAL2387B04-YT & Goulet \& Boudreaı & | 08-Jul-2008 & Canada & Yukon Territory & Champagne \\
\hline DIAL2384A06-YT & & 04-Aug-1992 & Canada & Yukon Territory & \\
\hline DIAL1848A10-NB & M. Gravel & 31-Jul-2005 & Canada & New Brunswick & Kent Co. \\
\hline DIAL2384A09-YT & & 04-Aug-1992 & Canada & Yukon Territory & \\
\hline DIAL2384B08-YT & & 22-Jul-1992 & Canada & Yukon Territory & \\
\hline DIAL2384A01-YT & & 04-Aug-1992 & Canada & Yukon Territory & \\
\hline DIAL2384B02-YT & & 04-Aug-1992 & Canada & Yukon Territory & \\
\hline DIAL2384B10-YT & & 22-Jul-1992 & Canada & Yukon Territory & \\
\hline DIAL1390C01-ON & Alana Taylor & & Canada & Ontario & Norfolk CO \\
\hline DIAL1390C03-ON & Alana Taylor & & Canada & Ontario & Norfolk CO \\
\hline BEE1850G11-ME & HW Ikerd & 17-Jun-2002 & United States & Maine & Hancock Co. \\
\hline D1414H01-VA & SW Droege & & United States & Virginia & Assateague Island \\
\hline DIAL0292A11-WV & SW Droege & & United States & West Virginia & Hampshire Co. \\
\hline DIAL0294E06-MD & Z Riegel & 08-Sep-2005 & United States & Maryland & Pr. George's Co. \\
\hline DIAL2385F02-MD & K Hutson & 17-Sep-2006 & United States & Maryland & \\
\hline DIAL0294E12-MD & P Osenton & 14-Jun-2005 & United States & Maryland & Anne Arundel Co. \\
\hline DIAL0294E07-IN & SW Droege & 26-Jul-2003 & United States & Indiana & Jackson Co. \\
\hline DIAL0293B10-WV & SW Droege & 30-May-2004 & United States & West Virginia & Hampshire Co. \\
\hline
\end{tabular}




\begin{tabular}{|c|c|c|c|c|c|}
\hline DIAL0293F02-NC & R Jackowski & & United States & North Carolina & Union Co. \\
\hline D00607H11-KS & J. Hopwood & 09-Aug-2004 & United States & Kansas & Douglas Co. \\
\hline B01558D07-NC & E.J. Soderholm & 17-Jul-2008 & United States & North Carolina & Buncombe Co. \\
\hline D00608A07-KS & J. Hopwood & 06-Aug-2004 & United States & Kansas & Douglas Co. \\
\hline D0288C05-DC & S Rudy & & United States & District of Columbia & \\
\hline DIAL1409B07-NC & J Gibbs & 10-Aug-2006 & United States & North Carolina & E of Charlotte \\
\hline DIAL1846D04-MS & SW Droege & 04-Jun-2006 & United States & Mississippi & Jackson Co. \\
\hline DIAL1413H02-NC & J Gibbs & 08-Aug-2006 & United States & North Carolina & S. of Bryson City \\
\hline D0288D08-NC & SW Droege & 19-May-2006 & United States & North Carolina & Richmond Co. \\
\hline DIAL2385C12-SC & & 25-Oct-2006 & United States & South Carolina & \\
\hline DIAL1849C10-CO & Kearns \& Oliveras & 23-Jun-2004 & United States & Colorado & Boulder Co. \\
\hline D00607B03-KS & J. Hopwood & 02-Aug-2004 & United States & Kansas & Douglas Co. \\
\hline D00607H09-KS & J. Hopwood & 06-Aug-2004 & United States & Kansas & Douglas Co. \\
\hline 06732E01-MD & S.W.Droege & 01-Aug-2009 & United States & Maryland & Anne Arundel Co. \\
\hline D1414A06-IL & J Gibbs & & United States & Illinois & Macoupin Co. \\
\hline B03765B09-GA & W. Calver & 30-Oct-2009 & United States & Georgia & Chatham Co. \\
\hline B01558D08-NC & E.J. Soderholm & 17-Jul-2008 & United States & North Carolina & Buncombe Co. \\
\hline B01558D03-NC & E.J. Soderholm & 17-Jul-2008 & United States & North Carolina & Buncombe Co. \\
\hline B01558C12-NC & E.J. Soderholm & 17-Jul-2008 & United States & North Carolina & Buncombe Co. \\
\hline D0288B08-SC & SW Droege & & United States & South Carolina & Chesterfield Co \\
\hline D0288B06-NC & SW Droege & & United States & North Carolina & Richmond Co. \\
\hline DIAL1391D08-NC & C. Sheffield & 10-Aug-2006 & United States & North Carolina & E of Charlotte \\
\hline DIAL1391E10-NC & J Gibbs & 14-Aug-2006 & United States & North Carolina & $\mathrm{N}$ of Doughton Park \\
\hline DIAL0293F04-TN & HW Ikerd & 02-Apr-2002 & United States & Tennessee & Blount Co. \\
\hline DIAL0293F05-TN & HW Ikerd & 02-Apr-2002 & United States & Tennessee & Blount Co. \\
\hline DIAL1391C05-NC & J Gibbs & 04-Aug-2006 & United States & North Carolina & Great Smoky Mountain \\
\hline DIAL1391A08-NC & C. Sheffield & 13-Aug-2006 & United States & North Carolina & Grandfather Mtn. \\
\hline DIAL1849H07-ME & MF Veit & 17-Aug-2006 & United States & Maine & Aroostock Co. \\
\hline DIAL1848F07-PE & V MacPhail & 07-Jul-2006 & Canada & Prince Edward Island & Queens Co. \\
\hline DIAL1848E08-WV & SW Droege & 15-Aug-2004 & United States & West Virginia & Hampshire Co. \\
\hline D00607F12-NS & H. Goulet, A. Badi: & 14-Jun-2004 & Canada & Nova Scotia & \\
\hline DIAL2385C06-MD & HW Ikerd & & United States & Maryland & \\
\hline D1414D03-VA & SW Droege & & United States & Virginia & Assateague Island \\
\hline DIAL1846C07-WV & & 07-Aug-2006 & United States & West Virginia & Hampshire Co. \\
\hline D0287D08-IL & J Gibbs \& C Sheffi & & United States & Illinois & Macoupin Co. \\
\hline DIAL0293B02-DC & & 29-Jul-2004 & United States & District of Columbia & \\
\hline DIAL1391H03-NC & J Gibbs & 06-Aug-2006 & United States & North Carolina & Great Smoky Mtn NP \\
\hline DIAL1847H05-MD & S Kolski & 19-Aug-2004 & United States & Maryland & Prince George's Co, \\
\hline DIAL0294G01-MD & & & United States & Maryland & \\
\hline DIAL0294C06-IL & RP Jean & 26-Apr-2003 & United States & Illinois & Kankakee Co. \\
\hline DIAL0294C07-IN & RP Jean & 14-Apr-2000 & United States & Indiana & Vigo Co. \\
\hline DIAL0294D11-NY & SW Droege & 06-Sep-2005 & United States & New York & Suffolk Co. \\
\hline DIAL1848E02-MD & SW Droege & 04-Aug-2004 & United States & Maryland & Anne Arundel Co. \\
\hline D0295B06-ON & J Gibbs & & Canada & Ontario & \\
\hline DIAL1391H02-NC & J Gibbs & 06-Aug-2006 & United States & North Carolina & Great Smoky Mtn NP \\
\hline DIAL1391D01-NC & C. Sheffield & 06-Aug-2006 & United States & North Carolina & Great Smoky Mountain \\
\hline DIAL1391H08-NC & J Gibbs & 06-Aug-2006 & United States & North Carolina & Great Smoky Mtn NP \\
\hline DIAL1847H04-MD & S Kolski & 21-Jul-2004 & United States & Maryland & Prince George's Co, \\
\hline DIAL1391G12-NC & J Gibbs & 06-Aug-2006 & United States & North Carolina & Great Smoky Mtn NP \\
\hline DIAL0292B09-MD & SW Droege & 03-Apr-2003 & United States & Maryland & Pr. George's Co. \\
\hline DIAL0292C01-WV & SW Droege & & United States & West Virginia & Hampshire Co. \\
\hline DIAL0292B11-MD & SW Droege & 03-Apr-2003 & United States & Maryland & Pr. George's Co. \\
\hline DIAL0292C02-MD & SW Droege & 03-Apr-2003 & United States & Maryland & Pr. George's Co. \\
\hline DIAL1413G10-NC & J Gibbs & 08-Aug-2006 & United States & North Carolina & S. of Bryson City \\
\hline DIAL1391C10-NC & C. Sheffield & 10-Aug-2006 & United States & North Carolina & E of Charlotte \\
\hline
\end{tabular}




\begin{tabular}{|c|c|c|c|c|c|}
\hline DIAL1391B10-NC & J Gibbs & 06-Aug-2006 & United States & North Carolina & Great Smoky Mountain \\
\hline 06732E06-WV & J. Whitaker & 20-Jul-2008 & United States & West Virginia & Doddridge Co. \\
\hline D0287H06-IL & \multicolumn{2}{|c|}{ J Gibbs \& C Sheffi } & United States & Illinois & Macoupin Co. \\
\hline D0287F01-IL & \multicolumn{2}{|c|}{ J Gibbs \& C Sheffi } & United States & Illinois & Macoupin Co. \\
\hline D0287H08-IL & \multicolumn{2}{|c|}{ J Gibbs \& C Sheffi } & United States & Illinois & Macoupin Co. \\
\hline DIAL1413G09-NC & J Gibbs & 08-Aug-2006 & United States & North Carolina & S. of Bryson City \\
\hline D0287H12-IL & \multicolumn{2}{|c|}{ J Gibbs \& C Sheffi } & United States & Illinois & Macoupin Co. \\
\hline DIAL1409C01-PQ & J Gibbs & 07-Oct-2006 & Canada & Quebec & W of Hudson \\
\hline DIAL1409B11-PQ & J Gibbs & 07-Oct-2006 & Canada & Quebec & W of Hudson \\
\hline DIAL1846F03-MD & K Hutson & 21-Sep-2006 & United States & Maryland & Prince Georges Co. \\
\hline DIAL1847H01-MD & HW Ikerd & 06-Jul-2002 & United States & Maryland & Prince George's Co, \\
\hline DIAL1847H02-MD & HW Ikerd & 06-Jul-2002 & United States & Maryland & Prince George's Co, \\
\hline DIAL1846B03-MD & M Fell & 15-Apr-2005 & United States & Maryland & Howard Co. \\
\hline D00607H07-KS & J. Hopwood & 27-Jul-2004 & United States & Kansas & Douglas Co. \\
\hline DIAL1391H05-NC & J Gibbs & 06-Aug-2006 & United States & North Carolina & Great Smoky Mtn NP \\
\hline DIAL1391B07-NC & J Gibbs & 06-Aug-2006 & United States & North Carolina & Great Smoky Mountain \\
\hline DIAL1391B05-NC & J Gibbs & 06-Aug-2006 & United States & North Carolina & Great Smoky Mountain \\
\hline D0287E12-IL & \multicolumn{2}{|c|}{ J Gibbs \& C Sheffi } & United States & Illinois & Macoupin Co. \\
\hline D0287E06-IL & \multicolumn{2}{|c|}{ J Gibbs \& C Sheffi } & United States & Illinois & Macoupin Co. \\
\hline DIAL1413B03-IL & C. Sheffield & 24-Jun-2006 & United States & Illinois & Macoupin Co. \\
\hline DIAL1390A02-NC & Jason Gibbs & 06-Aug-2006 & United States & North Carolina & Great Smoky Mountain \\
\hline DIAL1390A01-NC & \multirow[t]{2}{*}{ Jason Gibbs } & 06-Aug-2006 & \multirow[t]{2}{*}{ United States } & \multirow[t]{2}{*}{ North Carolina } & Great Smoky Mountain \\
\hline DIAL1390F02-NC & & 15-Aug-2006 & & & near Charleston \\
\hline DIAL1413G08-NC & J Gibbs & 08-Aug-2006 & United States & North Carolina & S. of Bryson City \\
\hline DIAL1391H12-NC & J Gibbs & 06-Aug-2006 & United States & North Carolina & Great Smoky Mtn NP \\
\hline DIAL1413H07-NC & J Gibbs & 08-Aug-2006 & United States & North Carolina & S. of Bryson City \\
\hline DIAL1413H04-NC & J Gibbs & 08-Aug-2006 & United States & North Carolina & S. of Bryson City \\
\hline DIAL1391B09-NC & J Gibbs & 06-Aug-2006 & United States & North Carolina & Great Smoky Mountain \\
\hline DIAL1391B08-NC & J Gibbs & 06-Aug-2006 & United States & North Carolina & Great Smoky Mountain \\
\hline D0287H11-IL & \multicolumn{2}{|c|}{ J Gibbs \& C Sheffi } & United States & Illinois & Macoupin Co. \\
\hline DIAL1391B04-NC & J Gibbs & 06-Aug-2006 & United States & North Carolina & Great Smoky Mountain \\
\hline DIAL1391H07-NC & J Gibbs & 06-Aug-2006 & United States & North Carolina & Great Smoky Mtn NP \\
\hline D0287E02-IL & \multicolumn{2}{|c|}{ J Gibbs \& C Sheffi } & United States & Illinois & Macoupin Co. \\
\hline DIAL1391B06-NC & J Gibbs & 06-Aug-2006 & United States & North Carolina & Great Smoky Mountain \\
\hline DIAL1848F04-MD & R Andrus & 24-Sep-2004 & United States & Maryland & Anne Arundel Co. \\
\hline DIAL1413H09-NC & J Gibbs & 08-Aug-2006 & United States & North Carolina & S. of Bryson City \\
\hline D0287E08-IL & \multicolumn{2}{|c|}{ J Gibbs \& C Sheffi } & United States & Illinois & Macoupin Co. \\
\hline D0295B07-ON & \multicolumn{2}{|l|}{ J Gibbs } & Canada & Ontario & \\
\hline D0295B01-ON & \multicolumn{2}{|l|}{ J Gibbs } & Canada & Ontario & \\
\hline D0295B02-ON & \multicolumn{2}{|l|}{ J Gibbs } & Canada & Ontario & \\
\hline D0287H07-IL & \multicolumn{2}{|c|}{ J Gibbs \& C Sheffi } & United States & Illinois & Macoupin Co. \\
\hline DIAL1391H11-NC & J Gibbs & 06-Aug-2006 & United States & North Carolina & Great Smoky Mtn NP \\
\hline D0287G11-IL & \multicolumn{2}{|c|}{ J Gibbs \& C Sheffi } & United States & Illinois & Macoupin Co. \\
\hline D0287H03-IL & \multicolumn{2}{|c|}{ J Gibbs \& C Sheffi } & United States & Illinois & Macoupin Co. \\
\hline D0287H04-IL & \multicolumn{2}{|c|}{ J Gibbs \& C Sheffi } & United States & Illinois & Macoupin Co. \\
\hline D0287H05-IL & J Gibbs \& C & & United States & Illinois & Macoupin Co. \\
\hline D0287G05-IL & J Gibbs \& C & & United States & Illinois & Macoupin Co. \\
\hline D0287E11-IL & J Gibbs \& C & & United States & Illinois & Macoupin Co. \\
\hline D0287E07-IL & J Gibbs \& C & & United States & Illinois & Macoupin Co. \\
\hline D0287D07-IL & J Gibbs \& C & & United States & Illinois & Macoupin Co. \\
\hline D0287C05-IL & J Gibbs \& C & & United States & Illinois & Macoupin Co. \\
\hline D1414E06-VA & SW Droege & & United States & Virginia & Assateague Island \\
\hline D0287C03-IL & J Gibbs \& C & & United States & Illinois & Macoupin Co. \\
\hline D0287C04-IL & J Gibbs \& C & & United States & Illinois & Macoupin Co. \\
\hline D0287E09-IL & J Gibbs \& C & & United States & Illinois & Macoupin Co. \\
\hline
\end{tabular}




\begin{tabular}{|c|c|c|c|c|c|}
\hline D1414E03-VA & SW Droege & & United States & Virginia & Assateague Island \\
\hline D0288H02-MD & J Gibbs & & United States & Maryland & Frederick Co. \\
\hline D0288G12-MD & J Gibbs & & United States & Maryland & Frederick Co. \\
\hline D0288D10-MD & SW Droege & & United States & Maryland & Pr. George's Co. \\
\hline D0288B07-MD & SW Droege & & United States & Maryland & Pr. George's Co. \\
\hline D1414H06-VA & SW Droege & & United States & Virginia & Assateague Island \\
\hline D1414H05-VA & SW Droege & & United States & Virginia & Assateague Island \\
\hline D1414E05-VA & SW Droege & & United States & Virginia & Assateague Island \\
\hline D0295B04-ON & J Gibbs & & Canada & Ontario & \\
\hline D00607F03-KS & J. Hopwood & 01-Aug-2004 & United States & Kansas & Douglas Co. \\
\hline D00607C04-MO & M.S. Arduser & 24-Jun-2001 & United States & Missouri & Dent Co. \\
\hline D00608E07-IN & R. Grundel & 23-Jun-2004 & United States & Indiana & Porter Co. \\
\hline DIAL2385C08-MD & & 21-Jul-2004 & United States & Maryland & \\
\hline DIAL2385D06-MD & & 01-Aug-2005 & United States & Maryland & \\
\hline 06732D06-WV & J. Whitaker & 20-Jul-2008 & United States & West Virginia & Doddridge Co. \\
\hline CCDB-00611 D09 & J.K Tuell & & United States & Michigan & Cass \\
\hline CCDB-00611 D03-I & I J.K Tuell & & United States & Michigan & Calhoun \\
\hline 06732H06-RI & E. King, S. Paton & 28-Aug-2008 & United States & Rhode Island & \\
\hline DIAL1845C10-TX & Abbott, Caesar & 12-May-2000 & United States & Texas & Bastrop Co. \\
\hline DIAL1847A01-SC & SW Droege & 18-May-2006 & United States & South Carolina & Chesterfield Co. \\
\hline DIAL0294D04-IN & RP Jean & 27-Apr-2003 & United States & Indiana & Jasper Co. \\
\hline DIAL0294E04-NY & SW Droege & 06-Sep-2005 & United States & New York & Suffolk Co. \\
\hline DIAL0293F11-MN & SW Droege & 10-Jul-2001 & United States & Minnesota & Sher Co. \\
\hline DIAL0293F08-MD & A. Lohr & 27-Jul-2004 & United States & Maryland & Anne Arundel Co. \\
\hline D0288D06-NC & SW Droege & 19-May-2006 & United States & North Carolina & Moore Co. \\
\hline DIAL0293F09-MD & S Kolski & 20-Aug-2004 & United States & Maryland & Pr. George's Co. \\
\hline 06732D07-MA & $\mathrm{DB}$ & 22-Aug-2008 & United States & Massachusetts & \\
\hline DIAL1390A06-ON & PJ Carson & & Canada & Ontario & Norfolk CO \\
\hline DIAL0286H01-MB & A. M. Patenaude & 22-Aug-2005 & Canada & Manitoba & YellowQuill Mxd. \\
\hline D0288D07-SC & SW Droege & 19-May-2006 & United States & South Carolina & Chesterfield Co \\
\hline D0288D11-SC & SW Droege & 19-May-2006 & United States & South Carolina & Chesterfield Co \\
\hline D00609G05-NS & Sheffield, Rigby \& & 10-Jul-2003 & Canada & Nova Scotia & \\
\hline DIAL2385E10-MB & Boudreault \& Gou & | 11-Jun-2007 & Canada & Manitoba & \\
\hline D01561D05-ON & E. Willis & 30-Jul-2005 & Canada & Ontario & York Co. \\
\hline D01561C04-IN & R. Grundel & 05-Jun-2003 & United States & Indiana & Lake Co. \\
\hline DIAL1390G09-NC & J Gibbs & 06-Aug-2006 & United States & North Carolina & Cataloochee \\
\hline DIAL1848A04-NB & M Gravel & 31-Jul-2005 & Canada & New Brunswick & Kent Co. \\
\hline D0295D03-MD & SW Droege & 24-Jun-2006 & United States & Maryland & Montgomery Co. \\
\hline DIAL1846C05-MS & SW Droege & 04-Jun-2006 & United States & Mississippi & Jackson Co. \\
\hline DIAL1413D06-NC & J Gibbs & 07-Aug-2006 & United States & North Carolina & Junaluska Meadow \\
\hline DIAL0294F01-PA & & & United States & Pennsylvania & \\
\hline DIAL1413A05-IL & J Gibbs & 25-Jun-2006 & United States & Illinois & Montgomery Co. \\
\hline DIAL0293A03-MD & SW Droege & 19-Jul-2004 & United States & Maryland & Pr. George's Co. \\
\hline DIAL0293G02-MD & SW Droege & 01-Jan-2004 & United States & Maryland & Garrett Co. \\
\hline DIAL1391E08-NC & J Gibbs & 14-Aug-2006 & United States & North Carolina & $\mathrm{N}$ of Doughton Park \\
\hline DIAL0294E11-MD & P Osenton & 10-May-2005 & United States & Maryland & Pr. George's Co. \\
\hline DIAL0292H12-MD & SW Droege & 19-Jul-2004 & United States & Maryland & Pr. George's Co. \\
\hline DIAL1390E02-ON & E.Willis & & Canada & Ontario & Toronto \\
\hline DIAL1413F01-TN & J Gibbs & 08-Aug-2006 & United States & Tennessee & near Gatlinburg \\
\hline DIAL1413A07-VA & SW Droege & 01-Jul-2006 & United States & Virginia & Accomac Co. \\
\hline DIAL1413H05-NC & J Gibbs & 08-Aug-2006 & United States & North Carolina & S. of Bryson City \\
\hline DIAL1413B10-IL & Gibbs \& Sheffield & 25-Jun-2006 & United States & Illinois & Macoupin Co. \\
\hline DIAL1846E01-WV & & 08-Aug-2006 & United States & West Virginia & Hampshire Co. \\
\hline DIAL1409A10-NC & J Gibbs & 13-Aug-2006 & United States & North Carolina & near Jefferson \\
\hline DIAL1409H11-ON & E Willis & 06-Jun-2006 & Canada & Ontario & Toronto \\
\hline
\end{tabular}




\begin{tabular}{|c|c|c|c|c|c|}
\hline DIAL1390H03-SC & & 09-Aug-2006 & United States & South Carolina & Okanee Co. \\
\hline DIAL1390C06-ON & PJ Carson & & Canada & Ontario & Norfolk CO \\
\hline DIAL1390H02-SC & & 09-Aug-2006 & United States & South Carolina & Okanee Co. \\
\hline DIAL1390G05-NC & & 15-Aug-2006 & United States & North Carolina & near Charleston \\
\hline DIAL1391A02-VA & C. Sheffield & 14-Aug-2006 & United States & Virginia & 5miles S of Elk Creek \\
\hline DIAL1413D07-NC & J Gibbs & 07-Aug-2006 & United States & North Carolina & Junaluska Meadow \\
\hline DIAL1848F06-MD & SW Droege & 03-Sep-2003 & United States & Maryland & \\
\hline D1414A01-YT & L Packer & & Canada & Yukon Territory & \\
\hline DIAL2387F08-BC & C Ratti & 25-Jun-2004 & Canada & British Columbia & \\
\hline DIAL1848C02-YT & Laurence Packer & & Canada & Yukon Territory & Carcross Dunes \\
\hline BEE0286C11-ON & JA Genaro & 30-Apr-2006 & Canada & Ontario & North York \\
\hline D00608H10-OR & Gibbs \& Sheffield & 06-Jul-2007 & United States & Oregon & Baker Co. \\
\hline 04485A10-VA & & 18-Jul-2009 & United States & West Virginia & \\
\hline DIAL1848D10-MD & S Kolski & 20-Aug-2004 & United States & Maryland & Pr. Georgelll's Co. \\
\hline DIAL1390D09-ON & E.Willis & & Canada & Ontario & Toronto \\
\hline DIAL1848B06-WV & J Gibbs & 15-Aug-2006 & United States & West Virginia & near Charleston \\
\hline DIAL1848C08-WV & J Gibbs & 15-Aug-2006 & United States & West Virginia & near Charleston \\
\hline
\end{tabular}




\begin{tabular}{|c|c|c|c|c|c|c|}
\hline Sector & Exact Site & Lat & Lon & Elev & Depth & Elevation Precision \\
\hline \multirow[t]{9}{*}{ Indiana Dunes NL } & & 41.652 & -87.071 & & & \\
\hline & & 37.2982 & -111.157 & & & \\
\hline & & 51.495 & -113.232 & & & \\
\hline & & 40.3681 & -113.054 & & & \\
\hline & & 42.714 & -80.453 & & & \\
\hline & & 39.024 & -76.92 & & & \\
\hline & & 47.066 & -96.449 & & & \\
\hline & & 47.046 & -96.419 & & & \\
\hline & & 39.028 & -76.923 & & & \\
\hline Gibbs Road & & 45.433 & -74.508 & & & \\
\hline \multirow[t]{2}{*}{ Apalachicola N.F. } & Post Office Bay & 30.059 & -84.984 & & & \\
\hline & & 40.56 & -74.96 & & & \\
\hline \multirow{4}{*}{\multicolumn{2}{|c|}{ Grass Prairie Preserv near Treesbank }} & 49.681 & -99.554 & & & \\
\hline & & 45.0694 & -64.484 & & & \\
\hline & & 46.856 & -96.472 & & & \\
\hline & & 47.066 & -96.45 & & & \\
\hline & Erie NWR & 41.629 & -79.964 & & & \\
\hline & & 44.361 & -68.23 & & & \\
\hline \multirow{2}{*}{\multicolumn{2}{|c|}{ St. Ignace }} & 46.707 & -65.054 & & & \\
\hline & & 39.964 & -105.265 & & & \\
\hline \multirow{3}{*}{\multicolumn{2}{|c|}{ Grass Prairie Preserv near Treesbank }} & 49.681 & -99.554 & & & \\
\hline & & 49.093 & -111.621 & & & \\
\hline & & 49.243 & -119.633 & & & \\
\hline \multirow{4}{*}{\multicolumn{2}{|c|}{ nr Painting on Stone }} & 49.0925 & -111.621 & 929 & & \\
\hline & & 49.089 & -119.536 & & & \\
\hline & & 49.09 & -119.538 & & & \\
\hline & & 49.688 & -119.776 & & & \\
\hline $10 \mathrm{~km}$ E Starbuck & Hwy 261 & 46.5048 & -118.037 & 461 & & \\
\hline \multicolumn{7}{|l|}{ Nipsco } \\
\hline \multicolumn{7}{|l|}{ Prairie Border } \\
\hline \multirow[t]{7}{*}{ University of Minneso } & Arboretum & 44.86 & -93.61 & & & \\
\hline & & 43.621 & -83.317 & & & \\
\hline & & 43.338 & -89.949 & & & \\
\hline & & 42.838 & -84.221 & & & \\
\hline & York University Ke & 43.774 & -79.505 & & & \\
\hline & & 39.963 & -75.177 & & & \\
\hline & & 38.412 & -81.648 & & & \\
\hline \multicolumn{7}{|l|}{ MTJ } \\
\hline & & 39.279 & -78.68 & & & \\
\hline & & 39.335 & -78.458 & & & \\
\hline & & 39.335 & -78.458 & & & \\
\hline & & 39.335 & -78.458 & & & \\
\hline & & 39.241 & -78.464 & & & \\
\hline & & 39.235 & -78.744 & & & \\
\hline & & 39.241 & -78.464 & & & \\
\hline
\end{tabular}




\begin{tabular}{|c|c|c|c|c|}
\hline & & 38.412 & -81.648 & \\
\hline & & 38.412 & -81.648 & \\
\hline & & 38.412 & -81.648 & \\
\hline & & 38.412 & -81.648 & \\
\hline & & 39.551 & -79.299 & \\
\hline & & 30.53 & -88.694 & \\
\hline & & 37.918 & -75.327 & \\
\hline & & 33.71 & -79.442 & \\
\hline & & 37.909 & -75.356 & \\
\hline & & 39.617 & -74.783 & \\
\hline & & 39.683 & -74.767 & \\
\hline & & 38.195 & -75.476 & \\
\hline & & 38.186 & -75.495 & \\
\hline & & 38.198 & -75.479 & \\
\hline Algonquin P.P. & Madawaska Lk. & 45.3285 & -78.3066 & \\
\hline Algonquin P.P. & Madawaska Lk. & 45.3285 & -78.3066 & \\
\hline & & 38.899 & -76.678 & \\
\hline & York University Keele & 43.775 & -79.496 & \\
\hline & & 44.5441 & -87.907 & \\
\hline & & 45.0845 & -63.73 & \\
\hline & & 34.579 & -80.233 & \\
\hline & & 34.579 & -80.233 & \\
\hline Prairie Border & & & & \\
\hline & & 38.928 & -76.668 & \\
\hline & Rappahnnck NWR & 38.014 & -76.888 & \\
\hline & & 38.4917 & -95.218 & \\
\hline & & 39.232 & -78.517 & \\
\hline & Pterophylla Plant Nurs & 42.384 & -80.344 & \\
\hline Blue Mtn Parkway & & 36.342 & -81.375 & \\
\hline & & 37.914 & -75.338 & \\
\hline & Pterophylla Plant Nurs & 42.384 & -80.344 & \\
\hline & & 38.949 & -76.732 & \\
\hline Cades Cove & Mill Creek & 35.583 & -83.837 & \\
\hline & & 49.03 & -119.492 & \\
\hline El Portal & Eagle Pk & 37.673 & -119.792 & 586 \\
\hline Yosemite NP & Yosem Vly & 37.73 & -119.61 & 1204 \\
\hline El Portal & Eagle Pk & 37.673 & -119.792 & 586 \\
\hline El Portal & Eagle Pk & 37.673 & -119.792 & 586 \\
\hline & & 37.268 & -112.91 & \\
\hline & & 34.112 & -118.777 & \\
\hline & & 49.029 & -119.492 & \\
\hline & & 49.03 & -119.493 & 415 \\
\hline & & 37.349 & -113.091 & \\
\hline & & 34.112 & -118.777 & \\
\hline & & 40.2014 & -112.92 & \\
\hline & & 38.681 & -77.096 & \\
\hline Hwy 28 near 1VA(?) & & 34.349 & -82.567 & \\
\hline & & 34.984 & -80.449 & \\
\hline & & 36.818 & -76.552 & \\
\hline & & 35.685 & -83.536 & \\
\hline & & 34.984 & -80.449 & \\
\hline
\end{tabular}




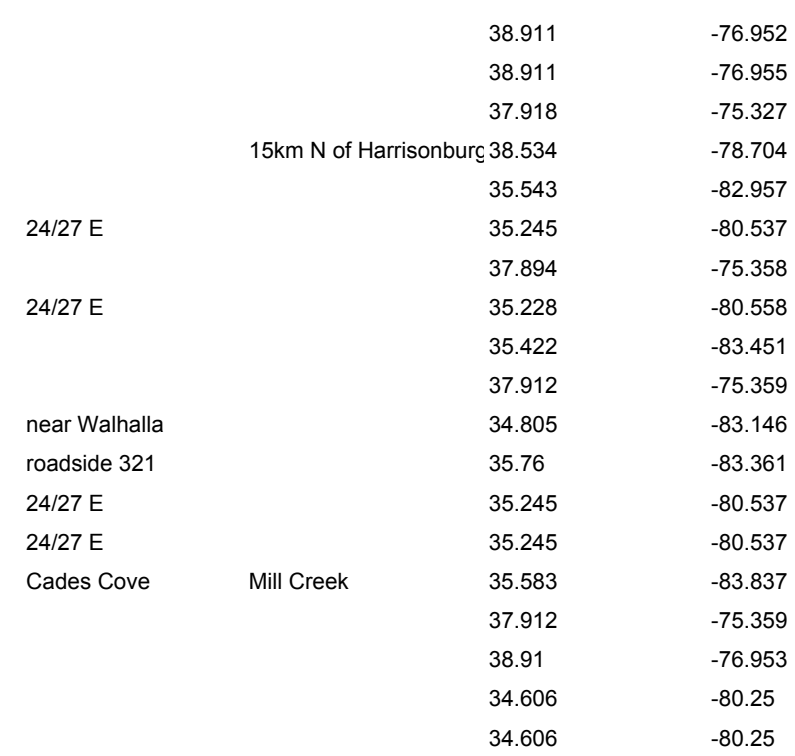

Diamond Grove Prairi

\begin{tabular}{|c|c|c|c|}
\hline & & 34.606 & -80.25 \\
\hline & & 34.606 & -80.25 \\
\hline near Walhalla & & 34.805 & -83.146 \\
\hline near Walhalla & & 34.813 & -83.137 \\
\hline near Walhalla & & 34.813 & -83.137 \\
\hline & & 39.148 & -89.667 \\
\hline Carlinville & & 39.278 & -89.89 \\
\hline E of Carlinville & & 39.279 & -89.796 \\
\hline & & 38.807 & -76.702 \\
\hline & & 38.8 & -76.698 \\
\hline & & 27.45 & -81.374 \\
\hline & & 39.061 & -95.204 \\
\hline & & 38.912 & -76.755 \\
\hline & & 39.04 & -78.77 \\
\hline & & 34.984 & -80.449 \\
\hline & & 39.04 & -78.77 \\
\hline & & 39.05 & -76.777 \\
\hline & & 37.94 & -75.315 \\
\hline & & 30.53 & -88.694 \\
\hline & & 30.53 & -88.694 \\
\hline & & 33.204 & -97.081 \\
\hline & & 30.53 & -88.694 \\
\hline & & 38.681 & -75.073 \\
\hline & & 38.681 & -75.073 \\
\hline $24 / 27 \mathrm{E}$ & & 35.245 & -80.537 \\
\hline & Park River NWR & 42.746 & -70.804 \\
\hline & Dismal Swmp NWR & 36.745 & -76.456 \\
\hline & & 27.092 & -80.129 \\
\hline & & 30.548 & -94.409 \\
\hline & & 38.46 & -76.139 \\
\hline & & 35.806 & -75.767 \\
\hline & & 35.806 & -75.767 \\
\hline & & 35.416 & -76.162 \\
\hline & & 38.432 & -76.159 \\
\hline
\end{tabular}


Homestead

$\begin{array}{rr}30.399 & -88.766 \\ 30.396 & -88.755 \\ 30.418 & -88.715 \\ 30.329 & -84.505 \\ 10 \mathrm{~km} \mathrm{~N} \text { of ASHEVILLE } 35.663 & -82.479\end{array}$

Landsbaum

Prairie Border

Turkey Point Provincial 42.422

$10 \mathrm{~km} \mathrm{~N}$ of DAHLONEG 34.675

$-80.199$

$-83.994$

Hooper Br.

$39.566 \quad-79.428$

44.361

$-68.23$

36.089

$-81.837$

University of Minneso Arboretum

University of Minneso Arboretum

36.089

$-81.837$

45.2046

St. Ignace

46.707

$-64.379$

$-65.054$

44.361

$-68.23$

University of Minneso Arboretum

36.089

$-81.837$

39.137

$-89.942$

Joker's Hill

48.809

41.051

48.6036

$-123.631$

$-72.331$

$-119.765$

38.906

33.204

33.204

33.204

33.204

33.204

38.819

34.984

$-95.156$

$-97.081$

$-97.081$

$-97.081$

$-97.081$

$-97.081$

$-95.363$

$-80.449$

$\begin{array}{ll}37.294 & -112.909 \\ 37.259 & -112.918 \\ & \\ 49.939 & -110.35\end{array}$

Nipsco

43.454

$-91.304$ 


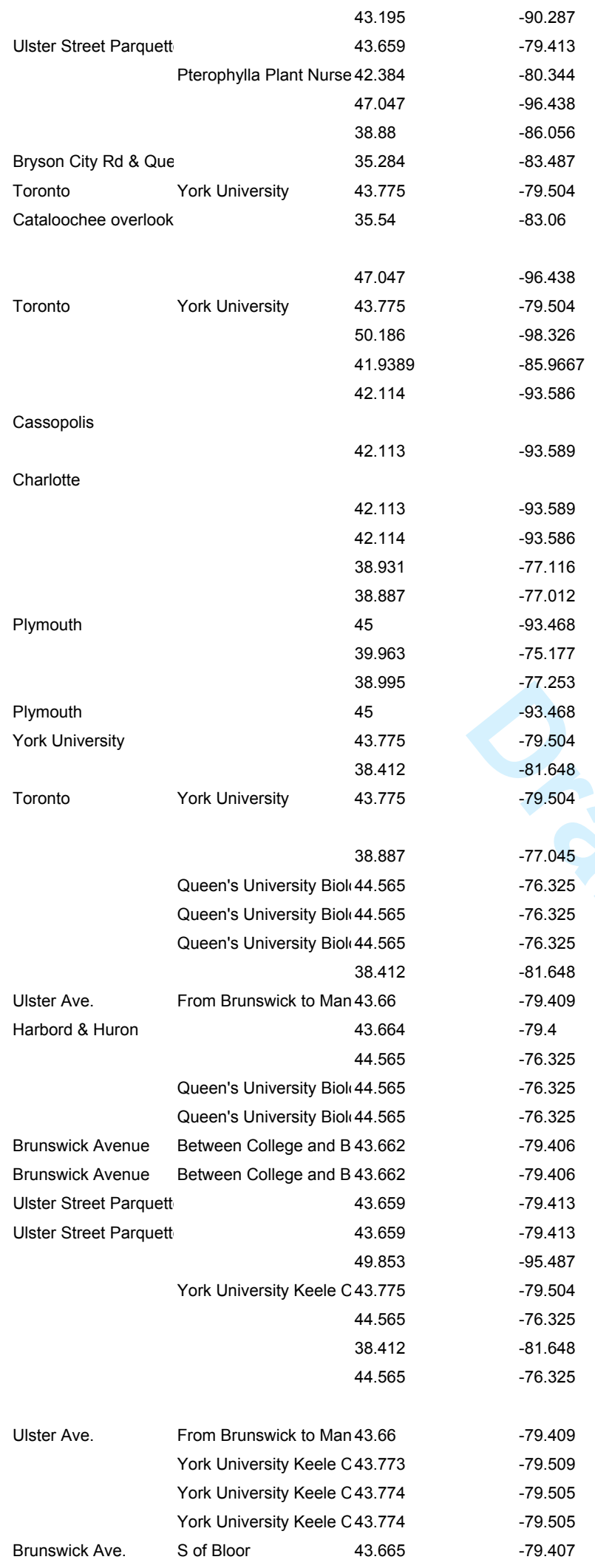




\begin{tabular}{|c|c|c|c|}
\hline & & 45.798 & -95.732 \\
\hline Indiana Dune NL & & 41.601 & -87.203 \\
\hline \multirow[t]{5}{*}{ Ulster Ave. } & \multicolumn{2}{|c|}{ From Brunswick to Man 43.66} & -79.409 \\
\hline & & 43.195 & -90.287 \\
\hline & & 43.7739 & -79.5053 \\
\hline & & 39.216 & -78.748 \\
\hline & & 38.931 & -77.116 \\
\hline \multicolumn{4}{|l|}{ Grant Blanc } \\
\hline & \multicolumn{2}{|c|}{ York University Keele C 43.774} & -79.505 \\
\hline \multirow[t]{2}{*}{ York University } & & 43.772 & -79.496 \\
\hline & & 43.7728 & -79.5087 \\
\hline \multirow[t]{6}{*}{ Cataloochee overlook } & & 35.54 & -83.06 \\
\hline & & 26.448 & -82.041 \\
\hline & & 30.941 & -83.708 \\
\hline & & 34.53 & -80.222 \\
\hline & & 34.53 & -80.222 \\
\hline & & 30.941 & -83.708 \\
\hline \multicolumn{4}{|l|}{ Conrad } \\
\hline \multicolumn{4}{|l|}{ Prairie Border } \\
\hline & & 42.717 & -80.433 \\
\hline \multicolumn{4}{|l|}{ Hooper Br. } \\
\hline Laurel & \multicolumn{3}{|l|}{ PWRC } \\
\hline \multicolumn{4}{|l|}{ Nipsco } \\
\hline Indiana Dunes NL & Ogden dunes & 41.615 & -87.203 \\
\hline \multirow[t]{2}{*}{ Indiana Dunes NL } & & 41.611 & -87.274 \\
\hline & & 39.544 & -75.576 \\
\hline \multirow[t]{12}{*}{ Plymouth } & \multicolumn{3}{|l|}{ Myles Standish St. Fore } \\
\hline & & 41.128 & -72.272 \\
\hline & & 40.997 & -72.074 \\
\hline & & 37.981 & -75.281 \\
\hline & & 42.772 & -70.806 \\
\hline & & 42.57 & -72.536 \\
\hline & & 37.981 & -75.281 \\
\hline & & 37.958 & -75.315 \\
\hline & & 37.97 & -75.304 \\
\hline & & 35.416 & -76.162 \\
\hline & & 37.912 & -75.359 \\
\hline & & 35.416 & -76.162 \\
\hline \multicolumn{4}{|l|}{ Homestead } \\
\hline & & 37.98 & -75.293 \\
\hline & & 38.381 & -76.068 \\
\hline & & 37.97 & -75.304 \\
\hline & & 37.98 & -75.293 \\
\hline & & 42.687 & -85.209 \\
\hline & & 43.343 & -84.108 \\
\hline \multirow[t]{2}{*}{ Sunrise Lake Rd. } & & 45.224 & -88.459 \\
\hline & & 39.248 & -78.527 \\
\hline Cataloochee & Cataloochee overlook & 35.64 & -83.06 \\
\hline \multirow[t]{2}{*}{ Cataloochee } & Cataloochee overlook & 35.64 & -83.06 \\
\hline & & 37.914 & -75.338 \\
\hline Bryson City Rd \& Que & & 35.284 & -83.487 \\
\hline
\end{tabular}




\begin{tabular}{|c|c|c|}
\hline \multirow[t]{4}{*}{ near Walhalla } & 34.805 & -83.146 \\
\hline & 37.914 & -75.338 \\
\hline & 35.422 & -83.451 \\
\hline & 37.98 & -75.293 \\
\hline \multirow[t]{2}{*}{ Bryson City Rd \& Qu€ } & 35.284 & -83.487 \\
\hline & 38.819 & -95.363 \\
\hline \multirow[t]{5}{*}{ Wittman } & 38.8 & -76.283 \\
\hline & 39.519 & -77.841 \\
\hline & 39.303 & -89.882 \\
\hline & 38.868 & -77.001 \\
\hline & 39.302 & -89.789 \\
\hline \multirow[t]{4}{*}{ Sandford } & SW Ag. Field, Regan R & \\
\hline & 39.278 & -89.89 \\
\hline & 39.302 & -89.789 \\
\hline & 39.335 & -78.456 \\
\hline \multicolumn{3}{|l|}{ Hooper Br. } \\
\hline & 39.029 & -76.927 \\
\hline & 36.818 & -76.552 \\
\hline & 36.818 & -76.552 \\
\hline & 39.015 & -76.864 \\
\hline & 38.941 & -76.741 \\
\hline & 37.848 & -78.668 \\
\hline \multirow[t]{3}{*}{ E of Carlinville } & 39.26 & -89.549 \\
\hline & Great Smoky Mountain 35.628 & -83.1 \\
\hline & 36.818 & -76.552 \\
\hline \multirow[t]{4}{*}{$24 / 27 \mathrm{E}$} & 35.245 & -80.537 \\
\hline & 38.349 & -75.937 \\
\hline & 38.692 & -77.07 \\
\hline & 42.815 & -82.749 \\
\hline \multirow[t]{3}{*}{ Bryson City Rd \& Que } & 35.284 & -83.487 \\
\hline & 34.984 & -80.449 \\
\hline & 38.091 & -78.493 \\
\hline E of Carlinville & 39.26 & -89.549 \\
\hline E of Carlinville & 39.26 & -89.549 \\
\hline Bryson City Rd \& Qu€ & 35.284 & -83.487 \\
\hline \multirow[t]{3}{*}{ near Walhalla } & 34.813 & -83.137 \\
\hline & 39.148 & -89.667 \\
\hline & 34.547 & -80.177 \\
\hline \multirow[t]{3}{*}{ Cades Cove } & Hiate lane (Abhrams Cr 35.592 & -83.824 \\
\hline & Junaluska Meadow & -82.957 \\
\hline & 30.425 & -88.838 \\
\hline Bryson City Rd \& Qu€ & 35.284 & -83.487 \\
\hline \multirow[t]{2}{*}{ Cades Cove } & Hiate lane (Abhrams Cr 35.592 & -83.824 \\
\hline & 39.302 & -89.789 \\
\hline \multirow[t]{2}{*}{ roadside 321} & 35.76 & -83.361 \\
\hline & 38.868 & -77 \\
\hline Cades Cove & Hiate lane (Abhrams Cr 35.592 & -83.824 \\
\hline \multirow[t]{2}{*}{ Cades Cove } & Hiate lane (Abhrams Cr 35.592 & -83.824 \\
\hline & 37.914 & -75.338 \\
\hline \multirow[t]{5}{*}{ Cades Cove } & Hiate lane (Abhrams Cr 35.592 & -83.824 \\
\hline & 42.815 & -82.749 \\
\hline & 35.139 & -80.139 \\
\hline & 39.137 & -89.942 \\
\hline & 39.148 & -89.667 \\
\hline E of Carlinville & 39.279 & -89.796 \\
\hline
\end{tabular}




\begin{tabular}{|c|c|c|c|c|}
\hline roadside 321 & & 35.76 & -83.361 & \\
\hline near Walhalla & & 34.813 & -83.137 & \\
\hline \multirow[t]{5}{*}{ Cades Cove } & \multicolumn{2}{|c|}{ Hiate lane (Abhrams Cr 35.592} & \multicolumn{2}{|l|}{-83.824} \\
\hline & & 39.137 & -89.942 & \\
\hline & & 39.137 & -89.942 & \\
\hline & & 39.137 & -89.942 & \\
\hline & & 35.416 & -80.856 & \\
\hline Cades Cove & \multicolumn{2}{|c|}{ Barns at 1st farm parkir 35.588} & -83.821 & \\
\hline \multirow[t]{4}{*}{ Cades Cove } & \multicolumn{2}{|c|}{ Hiate lane (Abhrams Cr 35.592} & -83.824 & \\
\hline & & 39.137 & -89.942 & \\
\hline & & 39.137 & -89.942 & \\
\hline & & 39.302 & -89.789 & \\
\hline \multirow[t]{7}{*}{ E of Carlinville } & & 39.26 & -89.549 & \\
\hline & & 39.279 & -89.796 & \\
\hline & & 39.279 & -89.796 & \\
\hline & \multirow[t]{4}{*}{ Olivet } & 42.4128 & -84.9386 & \\
\hline & & 39.302 & -89.789 & \\
\hline & & 38.349 & -75.937 & \\
\hline & & 33.204 & -97.082 & \\
\hline \multicolumn{5}{|l|}{ Chaparrel Wildlife Ma } \\
\hline & & 33.204 & -97.081 & \\
\hline \multirow[t]{5}{*}{ Hwy 385} & \multirow[t]{2}{*}{$5 \mathrm{~km} \mathrm{~S}$ of Roswell } & 33.3618 & -104.512 & 1108 \\
\hline & & 37.1769 & -113.036 & \\
\hline & \multirow[t]{3}{*}{ 24.5mi S of Animas } & 31.603 & -108.868 & \\
\hline & & 36.42 & -114.551 & \\
\hline & & 37.268 & -112.91 & \\
\hline \multirow{6}{*}{\multicolumn{2}{|c|}{ San Bernardino NWR }} & 31.336 & -109.268 & \\
\hline & & 31.619 & -108.867 & \\
\hline & & 37.847 & -111.354 & \\
\hline & & 36.445 & -115.375 & \\
\hline & & 37.14 & -111.835 & \\
\hline & & 36.436 & -115.353 & \\
\hline roadside 321 & & 35.76 & -83.361 & \\
\hline \multirow[t]{2}{*}{ near Walhalla } & & 34.805 & -83.146 & \\
\hline & & 39.013 & -76.824 & \\
\hline \multicolumn{2}{|l|}{ Bryson City Rd \& Qu€ } & 35.284 & -83.487 & \\
\hline Yosemite NP & Poopenaut Vly & 37.934 & -119.8 & 1060 \\
\hline Yosemite NP & Tueeulala Falls & 37.96 & -119.779 & 1225 \\
\hline Yosemite NP & Foresta Rd & 37.673 & -119.79 & 576 \\
\hline \multirow[t]{6}{*}{ Yosemite NP } & Tueeulala Falls & 37.96 & -119.779 & 1225 \\
\hline & \multirow[t]{5}{*}{ Farewell Bend S.P. } & 44.3096 & -117.221 & 659 \\
\hline & & 34.046 & -118.898 & \\
\hline & & 34.142 & -118.777 & \\
\hline & & 34.112 & -118.777 & \\
\hline & & 49.042 & -119.511 & \\
\hline
\end{tabular}




\begin{tabular}{|c|c|c|c|c|}
\hline & & 36.929 & -84.883 & \\
\hline & & 34.519 & -80.221 & \\
\hline & & 39.302 & -89.789 & \\
\hline & & 39.278 & -89.89 & \\
\hline & & 34.547 & -80.177 & \\
\hline & & 39.278 & -89.89 & \\
\hline Bryson City Rd \& Que & & 35.284 & -83.487 & \\
\hline & & 39.267 & -89.623 & \\
\hline $24 / 27 \mathrm{E}$ & & 35.245 & -80.537 & \\
\hline Jeff Nat Forest & & 36.888 & -81.138 & \\
\hline Jeff Nat Forest & & 36.888 & -81.138 & \\
\hline & & 35.139 & -80.139 & \\
\hline & & 39.148 & -89.667 & \\
\hline Cades Cove & Mill Creek & 35.583 & -83.837 & \\
\hline Hwy 28 near 1VA(?) & & 34.349 & -82.567 & \\
\hline & & 34.863 & -79.836 & \\
\hline roadside 321 & & 35.76 & -83.361 & \\
\hline & & 39.278 & -89.89 & \\
\hline & Great Smoky Mountain & 35.628 & -83.1 & \\
\hline roadside 321 & & 35.76 & -83.361 & \\
\hline & & 39.246 & -89.918 & \\
\hline Ulster Ave. & From Brunswick to Man & 43.66 & -79.409 & \\
\hline Bryson City Rd \& Que & & 35.284 & -83.487 & \\
\hline $24 / 27 E$ & & 35.245 & -80.537 & \\
\hline near Walhalla & & 34.805 & -83.146 & \\
\hline & & 43.214 & -123.377 & \\
\hline & & 43.214 & -123.377 & \\
\hline & & 43.214 & -123.377 & \\
\hline & & 43.214 & -123.377 & \\
\hline & & 34.142 & -118.777 & \\
\hline & $10 \mathrm{~km} \mathrm{~S}$ of Dodge & 46.477 & -117.731 & 461 \\
\hline & & 34.142 & -118.777 & \\
\hline & & 34.113 & -118.778 & \\
\hline Montague WMA & & 42.568 & -72.519 & \\
\hline & & 39.301 & -78.436 & \\
\hline & & 43.454 & -91.304 & \\
\hline & & 42.567 & -72.533 & \\
\hline & & 43.442 & -94.873 & \\
\hline & & 42.57 & -72.436 & \\
\hline & & 42.572 & -72.523 & \\
\hline Montague WMA & & 42.565 & -72.536 & \\
\hline Yosemite NP & Merced Lk Tr & 37.744 & -119.432 & 2141 \\
\hline Yosemite NP & Elephant Rock & 37.703 & -119.743 & 1331 \\
\hline
\end{tabular}




\begin{tabular}{|c|c|c|c|c|}
\hline & & 40.683 & -105.398 & \\
\hline \multicolumn{2}{|l|}{ Konukson Park } & 48.79 & -123.257 & \\
\hline \multirow[t]{6}{*}{ Meachie Lake } & & 48.819 & -124.135 & \\
\hline & & 48.429 & -123.711 & \\
\hline & & 40.602 & -106.073 & \\
\hline & & 49.0983 & -122.775 & \\
\hline & & 43.214 & -123.377 & \\
\hline & & 49.1447 & -123.06 & \\
\hline Yosemite NP & Andrews Peak & 38.041 & -119.717 & 2394 \\
\hline Yosemite NP & Hodgon Meadow & & & \\
\hline Yosemite NP & Hodgon Meadow & 37.796 & -119.861 & 1414 \\
\hline \multirow[t]{4}{*}{ Yosemite NP } & Hodgon Meadow & 37.796 & -119.861 & 1414 \\
\hline & & 49.0983 & -122.775 & \\
\hline & & 49.1447 & -123.06 & \\
\hline & & 48.809 & -123.631 & \\
\hline Westham Island & & 49.088 & -123.136 & \\
\hline \multirow[t]{6}{*}{ Surrey } & & 49.098 & -122.774 & \\
\hline & & 44.3933 & -111.383 & 2032 \\
\hline & & 48.429 & -123.711 & \\
\hline & & 44.2978 & -116.089 & \\
\hline & & 40.602 & -106.073 & \\
\hline & & 40.683 & -105.398 & \\
\hline \multirow[t]{7}{*}{ Meachie Lake } & & 48.819 & -124.135 & \\
\hline & & 45.067 & -110.638 & \\
\hline & & 40.602 & -106.073 & \\
\hline & & 49.111 & -119.178 & 1295 \\
\hline & & 39.31 & -79.458 & \\
\hline & & 45.113 & -65.585 & \\
\hline & & 39.299 & -79.42 & \\
\hline Highlands & Nature Centre & 35.053 & -83.188 & \\
\hline \multirow[t]{2}{*}{ Cataloochee } & & 35.628 & -83.1 & \\
\hline & & 45.1198 & -64.273 & \\
\hline Avonport & & 45.12 & -64.273 & \\
\hline \multirow[t]{5}{*}{ York University } & Lawn Near CSEB & 43.774 & -79.505 & \\
\hline & & 49.148 & -123.065 & \\
\hline & & 36.089 & -81.837 & \\
\hline & & 35.053 & -83.188 & \\
\hline & & 46.3642 & -63.1661 & \\
\hline St. Ignace & & 46.707 & -65.054 & \\
\hline
\end{tabular}




\begin{tabular}{|c|c|c|c|}
\hline \multirow[t]{3}{*}{ St. Ignace } & & 46.707 & -65.054 \\
\hline & & 46.3638 & -63.1661 \\
\hline & & 46.796 & -68.4825 \\
\hline \multirow[t]{2}{*}{ Algonquin P.P. } & Sitting Duck Lk. & & \\
\hline & & 51.495 & -113.232 \\
\hline $15 \mathrm{~km} \mathrm{~S} \mathrm{Three} \mathrm{Hills}$ & & 51.495 & -113.232 \\
\hline \multirow[t]{2}{*}{$15 \mathrm{~km} \mathrm{~S} \mathrm{Three} \mathrm{Hills}$} & & 51.495 & -113.232 \\
\hline & & 36.089 & -81.837 \\
\hline Toronto & York University & 43.775 & -79.504 \\
\hline Toronto & York University & 43.775 & -79.504 \\
\hline \multirow[t]{2}{*}{ Swain Co. } & Andrew's Bald & 35.54 & -83.494 \\
\hline & & 41.241 & -81.545 \\
\hline \multirow[t]{3}{*}{ Swain Co. } & Andrew's Bald & 35.54 & -83.494 \\
\hline & & 39.9929 & -105.363 \\
\hline & & 43.775 & -79.5046 \\
\hline \multicolumn{4}{|l|}{ Westchester } \\
\hline \multicolumn{4}{|l|}{ Westchester } \\
\hline Westchester & & & \\
\hline
\end{tabular}

$41.852-85.609$

$\begin{array}{lll}\text { St. Ignace } & 46.707 & -65.054\end{array}$

East Leroy

Queen's University Biol $44.565 \quad-76.325$

$42.539-86.111$

St. Ignace $\quad 46.707 \quad-65.054$

$45.0628 \quad-64.488$

$45.135-64.489$

Stengl Biological Stn $6 \mathrm{ml} \mathrm{N}$ of Smithville

$\begin{array}{lll}\text { near Walhalla } & 34.805 & -83.146\end{array}$

$39.335 \quad-78.457$

394+Penn

Grass Prairie Preserv near Treesbank $\quad 49.691 \quad-99.575$

Grass Prairie Preserv near Treesbank $\quad 49.681 \quad-99.554$

$\begin{array}{llll}\text { Toronto } \quad \text { York University } & 43.775 & -79.504\end{array}$

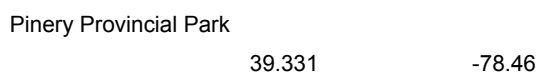

4mi E, 1mi S Glyndon $\quad 46.85 \quad-96.433$

$38.864 \quad-79.455$

$38.572 \quad-94.715$

$36.089-81.837$

Pterophylla Plant Nurse $42.384 \quad-80.344$

Pterophylla Plant Nurse $42.384 \quad-80.344$

Grass Prairie Preserv near Treesbank $\quad 49.691 \quad-99.575$

$38.962 \quad-76.746$

$38.942 \quad-76.892$

$33.323-86.293$

$30.425 \quad-88.838$

$30.396 \quad-88.755$

$30.366-88.732$ 


\begin{tabular}{|c|c|c|c|}
\hline & 30.396 & -88.755 & \\
\hline & 30.396 & -88.755 & \\
\hline \multicolumn{4}{|l|}{ Galveston } \\
\hline & 34.894 & -79.81 & \\
\hline & 30.941 & -83.708 & \\
\hline & 34.579 & -80.233 & \\
\hline & 49.089 & -119.534 & \\
\hline & 49.09 & -119.537 & \\
\hline \multirow[t]{10}{*}{ Meachie Lake } & 48.819 & -124.135 & \\
\hline & 50.043 & -125.326 & \\
\hline & 48.4639 & -123.563 & \\
\hline & 48.4639 & -123.563 & \\
\hline & 48.4639 & -123.563 & \\
\hline & 48.602 & -119.765 & \\
\hline & 49.045 & -119.531 & 780 \\
\hline & 49.244 & -119.632 & 925 \\
\hline & 37.981 & -75.281 & \\
\hline & 37.882 & -75.346 & \\
\hline
\end{tabular}

Assateague Island $\mathrm{N} \varepsilon$

$37.981-75.281$

$37.981-75.281$

$37.981-75.281$

$37.981-75.281$

$41.128 \quad-72.272$

$38.891-77.024$

$36.089-81.837$

$43.772 \quad-79.496$

$27.007-80.102$

$27.007-80.102$

$27.012-80.103$

$27.012-80.103$

$27.007-80.102$

$27.012-80.103$

$\begin{array}{lllll}\text { N of Kanab } & \text { Kanab creek } & & & \\ & & & & \\ \text { Yosemite NP } & \text { Ostrander Rocks } & 37.677 & -119.573 & 2114 \\ \text { Yosemite NP } & \text { El Capitan } & 37.723 & -119.637 & 1202 \\ \text { Pitt Meadows } & & 49.25 & -122.716 & \\ & & 34.097 & -118.813 & \end{array}$

nr McCreadie Hot Spr Hwy 58 E of Oak Ridge

$37.3019 \quad-111.144$

$50.743-120.886$ 


\begin{tabular}{|c|c|c|}
\hline & 44.948 & -110.701 \\
\hline & 39.346 & -79.484 \\
\hline & 38.809 & -76.702 \\
\hline ston & & \\
\hline
\end{tabular}

Field, 4.5 mi NE Hyde F

$\begin{array}{ll}35.284 & -79.314 \\ 37.94 & -75.315 \\ 37.98 & -75.293 \\ 27.741 & -81.578\end{array}$

$34.6 \quad-80.214$

$37.949 \quad-75.316$

$35.088 \quad-79.483$

$30.368 \quad-88.719$

$36.031 \quad-75.676$

$37.94 \quad-75.315$

$37.95 \quad-75.305$

$34.504-80.225$

\begin{tabular}{ll}
37.938 & -75.318 \\
\hline
\end{tabular}

$38.97 \quad-77.156$

$37.914 \quad-75.338$

$39.048 \quad-76.795$

$37.98 \quad-75.293$

Winchendon

Indiana Dunes NL

Lk. Denison Rec. Area 42.64

$-72.09$

$-87.071$

$-75.338$

$-75.34$

$-75.338$

roadside 321

$35.76 \quad-83.361$

39.193

$-78.42$

39.249

$-78.526$

Ossining

Clemmons Garden

$-77.156$

$-78.527$

$-78.704$

$-81.837$

$-83.1$

Great Smoky Mountain 35.628

$-98.25$

Hwy 64

49.196

$-104.678$

36.6369

$-105.398$

40.683

$-98.25$

49.196

$-105.189$

40.664

$-105.189$

40.664
40.683

$-105.398$

45.8305

$-111.896$ 


\begin{tabular}{|c|c|c|c|}
\hline & 44.969 & -110.7 & \\
\hline & 40.683 & -105.398 & \\
\hline \multicolumn{2}{|c|}{ Pterophylla Plant Nurse 42.384} & -80.344 & \\
\hline Blue Mtn Parkway & 36.342 & -81.375 & \\
\hline \multirow[t]{19}{*}{ Blue Mtn Parkway } & 36.342 & -81.375 & \\
\hline & 49.0983 & -122.792 & \\
\hline & 49.1281 & -122.418 & \\
\hline & 49.0983 & -122.792 & \\
\hline & 49.0883 & -123.164 & \\
\hline & 48.778 & -123.942 & \\
\hline & 49.1447 & -123.073 & \\
\hline & 49.1281 & -122.418 & \\
\hline & 49.21 & -122.246 & \\
\hline & 49.0783 & -122.388 & \\
\hline & 49.21 & -122.246 & \\
\hline & 49.0983 & -122.792 & \\
\hline & 49.0983 & -122.792 & \\
\hline & 49.21 & -122.246 & \\
\hline & 49.1281 & -122.418 & \\
\hline & 49.1447 & -123.073 & \\
\hline & 39.8701 & -105.466 & \\
\hline & 49.114 & -119.341 & 1109 \\
\hline & 44.27 & -124.108 & 7 \\
\hline \multirow[t]{2}{*}{$10 \mathrm{~km}$ S of Manyberri Hwy 501} & 49.2613 & -110.591 & 948 \\
\hline & 49.071 & -106.524 & \\
\hline \multirow[t]{3}{*}{$10 \mathrm{~km}$ S of Manyberri Hwy 501} & 49.2613 & -110.591 & 948 \\
\hline & 49.071 & -106.524 & \\
\hline & 39.341 & -78.464 & \\
\hline E of Carlinville & 39.26 & -89.549 & \\
\hline \multirow[t]{5}{*}{ Blue Mtn Parkway } & 36.342 & -81.375 & \\
\hline & C 43.7732 & -79.509 & \\
\hline & 43.1978 & -90.059 & \\
\hline & 43.454 & -91.304 & \\
\hline & 35.828 & -86.291 & \\
\hline Ingram Trail & 62.375 & -113.214 & \\
\hline \multirow[t]{3}{*}{ Ingram Trail } & 62.375 & -113.214 & \\
\hline & 64.097 & -138.527 & \\
\hline & 40.683 & -105.398 & \\
\hline Grass Prairie Preserv near Treesbank & 49.691 & -99.575 & \\
\hline Grass Prairie Preserv near Treesbank & 49.691 & -99.575 & \\
\hline \multicolumn{4}{|l|}{ Nipsco } \\
\hline & 45.1189 & -64.263 & \\
\hline Grass Prairie Preserv near Treesbank & 49.681 & -99.554 & \\
\hline \multirow[t]{3}{*}{ Grass Prairie Preserv near Treesbank } & 49.681 & -99.554 & \\
\hline & 45.8305 & -111.896 & \\
\hline & 40.683 & -105.398 & \\
\hline Grass Prairie Preserv near Treesbank & 49.691 & -99.575 & \\
\hline Grass Prairie Preserv near Treesbank & 49.681 & -99.554 & \\
\hline Grass Prairie Preserv near Treesbank & 49.691 & -99.575 & \\
\hline Grass Prairie Preserv near Treesbank & 49.691 & -99.575 & \\
\hline Sherburne NWR & & & \\
\hline
\end{tabular}




\begin{tabular}{|c|c|c|c|}
\hline Zimmerman & \multicolumn{3}{|l|}{ Sherburne NWR } \\
\hline Zimmerman & \multicolumn{3}{|l|}{ Sherburne NWR } \\
\hline \multicolumn{4}{|l|}{ Conrad } \\
\hline Zimmerman & \multicolumn{3}{|l|}{ Sherburne NWR } \\
\hline \multicolumn{2}{|c|}{ Grass Prairie Preserv near Treesbank } & 49.691 & -99.575 \\
\hline \multicolumn{2}{|c|}{ Grass Prairie Preserv near Treesbank } & 49.691 & -99.575 \\
\hline \multirow{4}{*}{\multicolumn{2}{|c|}{ Grass Prairie Preserv near Treesbank }} & 49.691 & -99.575 \\
\hline & & 42.554 & -86.044 \\
\hline & & 43.1978 & -90.059 \\
\hline & & 37.891 & -75.346 \\
\hline \multirow[t]{5}{*}{ Cataloochee } & Cataloochee overlook & 35.64 & -83.06 \\
\hline & \multicolumn{2}{|c|}{ Pterophylla Plant Nurse 42.384} & -80.344 \\
\hline & & 43.693 & -83.207 \\
\hline & \multicolumn{2}{|c|}{ Queen's University Biol 44.565} & -76.325 \\
\hline & & 38.958 & -76.744 \\
\hline \multicolumn{2}{|l|}{ Hwy 21} & 36.689 & -81.178 \\
\hline \multirow[t]{4}{*}{ Cades Cove } & \multicolumn{2}{|c|}{ Hiate lane (Abhrams Cr 35.592} & -83.824 \\
\hline & & 42.539 & -86.111 \\
\hline & & 36.448 & -81.029 \\
\hline & & 37.98 & -75.293 \\
\hline Blue Ridge Pkv & & 36.448 & -81.029 \\
\hline \multirow[t]{2}{*}{ roadside 321} & & 35.76 & -83.361 \\
\hline & & 35.543 & -82.957 \\
\hline \multicolumn{4}{|c|}{ Reg. Delhi-Simcoe Ré } \\
\hline & & 45.854 & -95.758 \\
\hline Cataloochee & \multirow[t]{10}{*}{ Cataloochee overlook } & 35.64 & -83.06 \\
\hline St. Ignace & & 46.707 & -65.054 \\
\hline St. Ignace & & 46.707 & -65.054 \\
\hline St. Ignace & & 46.7066 & -65.054 \\
\hline St. Ignace & & 46.707 & -65.054 \\
\hline \multirow[t]{5}{*}{ Roadside } & & 61.32 & -117.605 \\
\hline & & 43.272 & -81.82 \\
\hline & & 38.895 & -77.046 \\
\hline & & 38.886 & -77.037 \\
\hline & & 35.543 & -82.957 \\
\hline \multirow[t]{14}{*}{ Sheperdstown } & \multicolumn{2}{|l|}{ National Conservation 7} & \\
\hline & & 39.029 & -76.927 \\
\hline & & 38.886 & -77.034 \\
\hline & & 38.858 & -76.687 \\
\hline & & 38.888 & -77.045 \\
\hline & & 38.888 & -77.045 \\
\hline & & 39.037 & -76.872 \\
\hline & & 48.1014 & -112.053 \\
\hline & & 40.602 & -106.073 \\
\hline & & 49.03 & -119.492 \\
\hline & & 40.602 & -106.073 \\
\hline & & 49.463 & -112.456 \\
\hline & & 40.602 & -106.073 \\
\hline & ii Hwy 501 & 49.2613 & -110.591 \\
\hline
\end{tabular}


Oil Field Rd.

Trass Prairie Preserv near Treesbank

Grass Prairie Preserv near Treesbank

grass prairie preserve

Grass Prairie Preserv near Treesbank

Grass Prairie Preserv near Treesbank
$40.602-106.073$

$49.688-119.776$

$40.602-106.073$

$49.243-119.634$

$49.688 \quad-119.776$

48.1014

50.001

38.076

49.681

49.681

49.681

49.681

49.681

37.3459

Yosemite NP

Ostrander Rocks

37.672

41.256

37.672

Yosemite NP

Ostrander Rocks

Sawtooth Botanic Gard 43.6272

49.243

26.303

25.107

25.107

25.107

34.547

26.107

Westchester

$-114.355 \quad 1726$

26.107

34.623

34.56

30.366

30.53

26.312

26.349

34.504

35.284

26.349

34.623

32.139

30.425

38.8

42.61

39.057

Capon Bridge

Buffalo Gap camp

39.035

38.8

60.902

60.902

60.902

60.902
$-112.0531135$

$-110.644$

$-110.843$

$-99.554$

$-99.554$

$-98.446$

$-99.554$

$-99.554$

$-113.092$

$-119.569 \quad 2104$

$-122.04 \quad 125$

-119.569 2104

$-119.633935$

$-80.202$

$-80.264$

$-80.264$

$-80.264$

$-80.177$

$-80.264$

$-88.694$

$-80.264$

$-80.19$

$-80.256$

$-88.732$

$-88.694$

$-81.235$

$-80.276$

$-80.225$

$-79.314$

$-80.276$

$-80.19$

$-81.14$

$-88.838$

$-76.283$

$-72.1$

$-76.814$

$-76.874$

$-76.283$

$-135.432$

$-135.432$

$-135.432$

$-135.432$ 


\begin{tabular}{|c|c|c|c|}
\hline \multirow[t]{3}{*}{ Stafford Lake Park } & 49.728 & -112.458 & 855 \\
\hline & 44.8038 & -111.175 & \\
\hline & 45.825 & -110.91 & \\
\hline \multirow[t]{12}{*}{$6 \mathrm{~km}$ W Victoria CRD } & 48.467 & -123.466 & \\
\hline & 44.669 & -118.377 & \\
\hline & 40.602 & -106.073 & \\
\hline & 51.495 & -113.232 & \\
\hline & 49.745 & -112.533 & \\
\hline & 40.664 & -105.189 & \\
\hline & 49.207 & -119.614 & \\
\hline & 49.071 & -106.529 & \\
\hline & 36.288 & -115.653 & \\
\hline & 49.071 & -106.539 & \\
\hline & 49.071 & -106.529 & \\
\hline & 49.303 & -119.626 & \\
\hline \multirow[t]{3}{*}{ Stafford Lake Park } & 49.7276 & -112.458 & 855 \\
\hline & 46.903 & -122.739 & 125 \\
\hline & 38.991 & -105.43 & 2671 \\
\hline \multirow[t]{5}{*}{ District of Saanich } & 48.492 & -123.345 & \\
\hline & 49.207 & -119.613 & 745 \\
\hline & 49.071 & -106.529 & 830 \\
\hline & 44.949 & -110.705 & \\
\hline & 36.324 & -115.762 & \\
\hline \multicolumn{4}{|l|}{ Algonquin P.P. $\quad$ Crossbar Lk. } \\
\hline Grass Prairie Preserv near Treesbank & 49.681 & -99.554 & \\
\hline Grass Prairie Preserv near Treesbank & 49.691 & -99.575 & \\
\hline Grass Prairie Preserv near Treesbank & 49.681 & -99.554 & \\
\hline Grass Prairie Preserv near Treesbank & 49.691 & -99.575 & \\
\hline Grass Prairie Preserv near Treesbank & 49.681 & -99.554 & \\
\hline Grass Prairie Preserv near Treesbank & 49.681 & -99.554 & \\
\hline \multirow[t]{2}{*}{ Grass Prairie Preserv near Treesbank } & 49.681 & -99.554 & \\
\hline & 49.09 & -119.536 & 280 \\
\hline grass prairie preserve & 49.681 & -98.446 & \\
\hline \multirow[t]{4}{*}{ grass prairie preserve } & 49.691 & -98.425 & \\
\hline & 51.795 & -100.104 & 260 \\
\hline & 49.853 & -95.487 & \\
\hline & 43.962 & -87.701 & \\
\hline \multirow[t]{2}{*}{ Grass Prairie Preserv near Treesbank } & 49.681 & -99.554 & \\
\hline & 50.476 & -96.002 & \\
\hline \multirow[t]{9}{*}{ Algonquin P.P. } & & & \\
\hline & 51.795 & -100.104 & 260 \\
\hline & 49.09 & -119.536 & 280 \\
\hline & 49.074 & -96.766 & 292 \\
\hline & 51.651 & -99.945 & 251 \\
\hline & 49.09 & -119.536 & 280 \\
\hline & 50.043 & -125.326 & \\
\hline & 49.029 & -119.493 & \\
\hline & 49.114 & -119.342 & \\
\hline \multicolumn{4}{|l|}{ 1-4mi S of Cedar Brei } \\
\hline & 50.043 & -125.326 & \\
\hline & 50.049 & -125.326 & \\
\hline
\end{tabular}




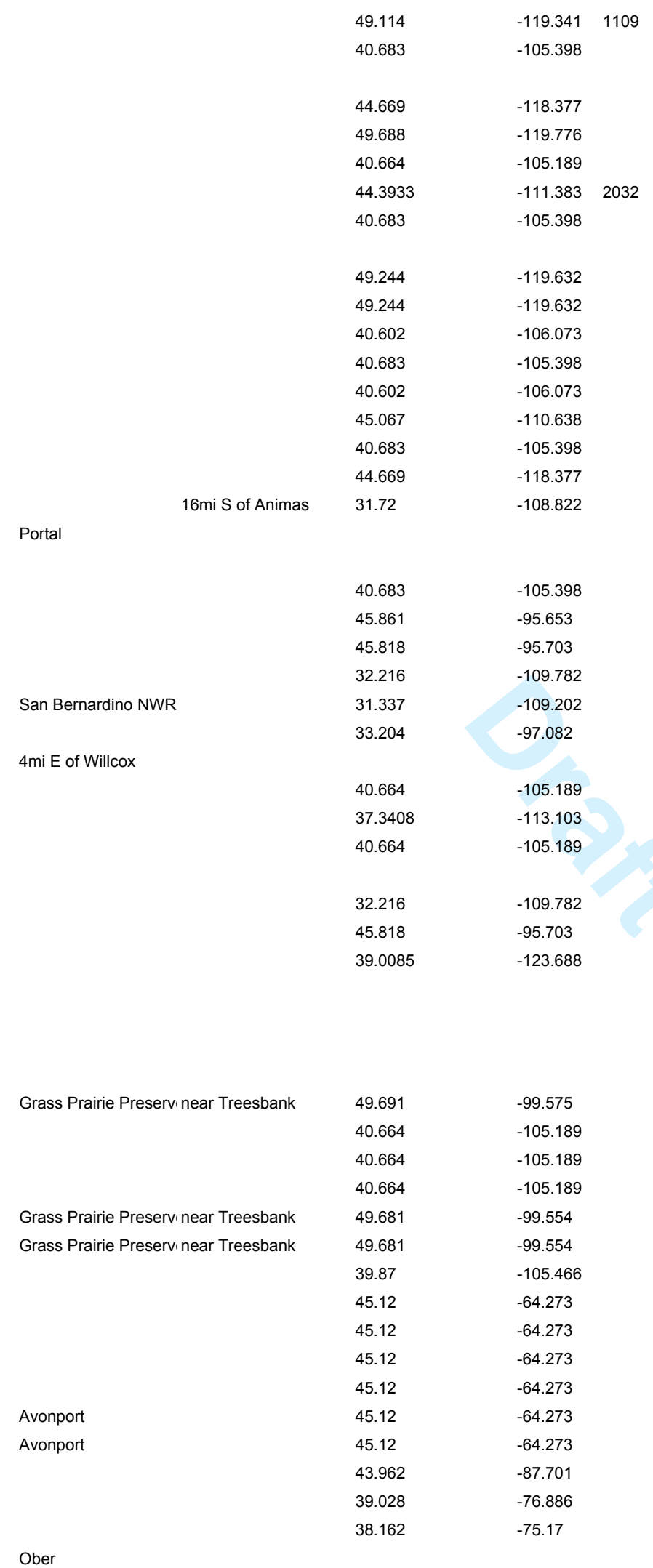




\begin{tabular}{|c|c|c|c|}
\hline \multirow[t]{14}{*}{ Amiens } & \multicolumn{3}{|l|}{ Mounted Rifles Rd. } \\
\hline & & 45.087 & -64.668 \\
\hline & & 49.071 & -106.524 \\
\hline & & 49.071 & -106.524 \\
\hline & & 43.439 & -95.182 \\
\hline & & 37.0921 & -91.0541 \\
\hline & & 38.967 & -77.169 \\
\hline & & 45.12 & -64.273 \\
\hline & & 38.961 & -77.145 \\
\hline & & 38.3 & -75.117 \\
\hline & & 38.899 & -76.678 \\
\hline & & 38.989 & -76.904 \\
\hline & & 38.899 & -76.678 \\
\hline & & 38.989 & -76.904 \\
\hline \multicolumn{4}{|l|}{ Conrad } \\
\hline & & 38.891 & -76.53 \\
\hline & & 38.91 & -76.684 \\
\hline & & 42.57 & -72.536 \\
\hline & & 42.564 & -72.534 \\
\hline & & 39.248 & -78.527 \\
\hline & & 39.346 & -78.403 \\
\hline & & 45.12 & -64.273 \\
\hline & & 41.157 & -86.978 \\
\hline Algonquin P.P. & \multicolumn{3}{|l|}{ Florence Lk. } \\
\hline \multicolumn{2}{|c|}{ SKYFIELDS ARBORE } & 42.622 & -72.22 \\
\hline Cades Cove & Mill Creek & 35.583 & -83.837 \\
\hline \multirow[t]{3}{*}{ Montville } & & 41.511 & -72.18 \\
\hline & Patuxent Wildlife & 39 & -76.7 \\
\hline & & 43.281 & -81.808 \\
\hline \multirow[t]{11}{*}{ Montville } & & 41.511 & -72.18 \\
\hline & & 43.003 & -91.026 \\
\hline & & 42.568 & -72.519 \\
\hline & & 43.263 & -81.838 \\
\hline & & 43.276 & -81.814 \\
\hline & & 42.564 & -72.539 \\
\hline & & 42.564 & -72.534 \\
\hline & & 43.26 & -81.839 \\
\hline & & 43.272 & -81.82 \\
\hline & & 45.12 & -64.273 \\
\hline & & 41.176 & -86.95 \\
\hline \multirow{2}{*}{\multicolumn{2}{|c|}{ Avonport }} & 45.12 & -64.273 \\
\hline & & 43.427 & -95.244 \\
\hline \multicolumn{2}{|c|}{ Grass Prairie Preserv near Treesbank } & 49.681 & -99.554 \\
\hline \multicolumn{2}{|c|}{ Grass Prairie Preserv near Treesbank } & 49.681 & -99.554 \\
\hline \multirow[t]{10}{*}{ Amiens } & & 50.628 & -110.306 \\
\hline & \multicolumn{2}{|c|}{ Pterophylla Plant Nurse 42.384} & -80.344 \\
\hline & & 26.311 & -81.267 \\
\hline & & 27.43 & -81.391 \\
\hline & & 27.43 & -81.391 \\
\hline & & 27.02 & -80.107 \\
\hline & & 27.012 & -80.103 \\
\hline & & 38.931 & -77.116 \\
\hline & & 43.233 & -81.865 \\
\hline & & 43.234 & -81.859 \\
\hline
\end{tabular}




\begin{tabular}{|c|c|c|c|c|}
\hline & & 37.962 & -75.311 & \\
\hline & & 37.753 & -78.162 & \\
\hline & & 37.958 & -75.315 & \\
\hline & & 37.938 & -75.318 & \\
\hline & & 30.366 & -88.732 & \\
\hline & & 38.912 & -76.755 & \\
\hline & & 38.536 & -76.518 & \\
\hline & & 30.456 & -88.784 & \\
\hline & & 37.962 & -75.108 & \\
\hline & & 39.346 & -78.403 & \\
\hline & & 37.962 & -75.311 & \\
\hline near Walhalla & & 34.813 & -83.137 & \\
\hline near Walhalla & & 34.813 & -83.137 & \\
\hline & & 34.637 & -80.176 & \\
\hline & & 34.984 & -80.449 & \\
\hline & & 39.346 & -78.403 & \\
\hline & & 39.351 & -78.509 & \\
\hline & & 38.931 & -77.116 & \\
\hline & & 38.931 & -77.116 & \\
\hline & $10 \mathrm{~km} \mathrm{~N}$ of SHENANDO & 38.564 & -78.606 & \\
\hline & & 41.28 & -95.907 & \\
\hline & District of Columbia & 38.948 & -77.08 & \\
\hline & & 60.787 & -136.436 & 733 \\
\hline Blueberry farm $\mathrm{C}$ & & & & \\
\hline Blueberry farm $\mathrm{c}$ & & & & \\
\hline & & 60.902 & -135.432 & \\
\hline Swain Co. & Andrew's Bald & 35.54 & -83.494 & \\
\hline & & 60.902 & -135.432 & \\
\hline & & 60.902 & -135.432 & \\
\hline Blueberry farm $\mathrm{C}$ & & & & \\
\hline Blueberry farm $\mathrm{c}$ & & & & \\
\hline & & 60.787 & -136.436 & 733 \\
\hline Swain Co. & Andrew's Bald & 35.54 & -83.494 & \\
\hline & & 60.787 & -136.436 & 733 \\
\hline & & 60.902 & -135.432 & \\
\hline & St. Ignace & 46.707 & -71.054 & \\
\hline & & 60.902 & -135.432 & \\
\hline & & 64.097 & -138.527 & \\
\hline & & 60.902 & -135.432 & \\
\hline & & 60.902 & -135.432 & \\
\hline & & 64.097 & -138.527 & \\
\hline & Turkey Point Provincial & 42.422 & -80.199 & \\
\hline & Turkey Point Provincial & 42.422 & -80.199 & \\
\hline & & 44.368 & -68.23 & \\
\hline & & 37.914 & -75.338 & \\
\hline & & 39.335 & -78.458 & \\
\hline & & 38.978 & -76.764 & \\
\hline & & 38.962 & -76.75 & \\
\hline & & 39.082 & -76.788 & \\
\hline & & 38.88 & -86.056 & \\
\hline & & 39.303 & -78.434 & \\
\hline
\end{tabular}




\begin{tabular}{|c|c|c|}
\hline & 34.984 & -80.449 \\
\hline & 38.819 & -95.363 \\
\hline & 35.606 & -82.455 \\
\hline & 38.905 & -76.955 \\
\hline $24 / 27 \mathrm{E}$ & 35.228 & -80.558 \\
\hline & 30.53 & -88.694 \\
\hline Bryson City Rd \& Que & 35.284 & -83.487 \\
\hline & 34.863 & -79.835 \\
\hline & 34.601 & -80.258 \\
\hline & 38.906 & -95.156 \\
\hline & 38.819 & -95.363 \\
\hline & 38.879 & -76.673 \\
\hline Carlinville & 39.278 & -89.89 \\
\hline & 32.132 & -81.134 \\
\hline & 35.606 & -82.455 \\
\hline & 35.613 & -82.449 \\
\hline & 35.611 & -82.445 \\
\hline & 34.54 & -80.232 \\
\hline & 34.894 & -79.81 \\
\hline $24 / 27 \mathrm{E}$ & 35.245 & -80.537 \\
\hline & 36.448 & -81.029 \\
\hline & 35.774 & -83.213 \\
\hline & 35.774 & -83.213 \\
\hline Swain Co. & Andrew's Bald & -83.494 \\
\hline & 36.089 & -81.837 \\
\hline & 39.322 & -78.43 \\
\hline Sydney & 46.145 & -70.962 \\
\hline & 39.05 & -76.816 \\
\hline & 37.912 & -75.359 \\
\hline & 39.248 & -78.527 \\
\hline & 39.302 & -89.789 \\
\hline & 38.9 & -76.958 \\
\hline Cataloochee overlook & 35.54 & -83.06 \\
\hline & 39.035 & -76.874 \\
\hline & 39.346 & -79.484 \\
\hline Hooper Br. & & \\
\hline MTJ & & \\
\hline & 38.894 & -76.614 \\
\hline Gibbs Road & West Side from Petite C 45.435 & -74.508 \\
\hline Cataloochee overlook & 35.54 & -83.06 \\
\hline Cataloochee & Cataloochee overlook & -83.06 \\
\hline Cataloochee overlook & 35.54 & -83.06 \\
\hline & 39.035 & -76.874 \\
\hline Cataloochee overlook & 35.54 & -83.06 \\
\hline & 38.699 & -77.048 \\
\hline & 39.335 & -78.458 \\
\hline & 38.689 & -77.024 \\
\hline & 38.699 & -77.048 \\
\hline Bryson City Rd \& Qu€ & 35.284 & -83.487 \\
\hline $24 / 27 \mathrm{E}$ & 35.245 & -80.537 \\
\hline
\end{tabular}




\begin{tabular}{|c|c|c|}
\hline \multirow[t]{5}{*}{ Cataloochee } & 35.625 & -83.114 \\
\hline & 39.226 & -80.843 \\
\hline & 39.302 & -89.789 \\
\hline & 39.302 & -89.789 \\
\hline & 39.302 & -89.789 \\
\hline \multirow[t]{2}{*}{ Bryson City Rd \& Que } & 35.284 & -83.487 \\
\hline & 39.302 & -89.789 \\
\hline Finnegan's Market & 45.473 & -74.186 \\
\hline \multirow[t]{6}{*}{ Finnegan's Market } & 45.473 & -74.186 \\
\hline & 38.963 & -76.748 \\
\hline & 39.049 & -76.809 \\
\hline & 39.049 & -76.809 \\
\hline & 39.212 & -76.878 \\
\hline & 39.045 & -95.204 \\
\hline Cataloochee overlook & 35.54 & -83.06 \\
\hline Cataloochee & 35.625 & -83.114 \\
\hline \multirow[t]{3}{*}{ Cataloochee } & 35.625 & -83.114 \\
\hline & 39.302 & -89.789 \\
\hline & 39.302 & -89.789 \\
\hline Carlinville & 39.246 & -89.918 \\
\hline Cataloochee & 35.625 & -83.114 \\
\hline \multirow[t]{2}{*}{ Cataloochee } & 35.625 & -83.114 \\
\hline & 38.412 & -81.648 \\
\hline Bryson City Rd \& Que & 35.284 & -83.487 \\
\hline Cataloochee overlook & 35.54 & -83.06 \\
\hline Bryson City Rd \& Qu€ & 35.284 & -83.487 \\
\hline Bryson City Rd \& Qu€ & 35.284 & -83.487 \\
\hline Cataloochee & 35.625 & -83.114 \\
\hline \multirow[t]{2}{*}{ Cataloochee } & 35.625 & -83.114 \\
\hline & 39.302 & -89.789 \\
\hline Cataloochee & 35.625 & -83.114 \\
\hline \multirow[t]{2}{*}{ Cataloochee overlook } & 35.54 & -83.06 \\
\hline & 39.302 & -89.789 \\
\hline \multirow[t]{2}{*}{ Cataloochee } & 35.625 & -83.114 \\
\hline & 38.964 & -76.685 \\
\hline \multirow[t]{2}{*}{ Bryson City Rd \& Que } & 35.284 & -83.487 \\
\hline & 39.302 & -89.789 \\
\hline Gibbs Road & West Side from Petite C 45.435 & -74.508 \\
\hline Gibbs Road & West Side from Petite C 45.435 & -74.508 \\
\hline \multirow[t]{2}{*}{ Gibbs Road } & West Side from Petite C 45.435 & -74.508 \\
\hline & 39.302 & -89.789 \\
\hline \multirow[t]{14}{*}{ Cataloochee overlook } & 35.54 & -83.06 \\
\hline & 39.137 & -89.942 \\
\hline & 39.267 & -89.623 \\
\hline & 39.302 & -89.789 \\
\hline & 39.302 & -89.789 \\
\hline & 39.137 & -89.942 \\
\hline & 39.302 & -89.789 \\
\hline & 39.302 & -89.789 \\
\hline & 39.302 & -89.789 \\
\hline & 39.246 & -89.918 \\
\hline & 37.912 & -75.359 \\
\hline & 39.246 & -89.918 \\
\hline & 39.246 & -89.918 \\
\hline & 39.302 & -89.789 \\
\hline
\end{tabular}




\begin{tabular}{|c|c|c|c|}
\hline & & 37.891 & -75.346 \\
\hline 550 at Monocacy Rive & $\epsilon$ by Creagerstown Park & 39.562 & -77.351 \\
\hline 550 at Monocacy Rive & є by Creagerstown Park & 39.562 & -77.351 \\
\hline & & 39.035 & -76.874 \\
\hline & & 39.035 & -76.874 \\
\hline & & 37.914 & -75.338 \\
\hline & & 37.98 & -75.293 \\
\hline & & 37.912 & -75.359 \\
\hline Gibbs Road & West Side from Petite C & 45.435 & -74.508 \\
\hline & & 38.906 & -95.156 \\
\hline Indiana Dunes NL & & 41.618 & -87.102 \\
\hline & & 39.035 & -76.874 \\
\hline & & 39.034 & -76.874 \\
\hline & & 39.226 & -80.843 \\
\hline & & 42.954 & -85.849 \\
\hline & & 42.181 & -85.132 \\
\hline & RI NWR - Trustom & 41.381 & -71.567 \\
\hline Stengl Biological Stn & $6 \mathrm{ml} \mathrm{N}$ of Smithville & & \\
\hline & & 34.6 & -80.214 \\
\hline Nipsco & & & \\
\hline Zimmerman & Sherburne NWR & & \\
\hline & & 39.928 & -76.668 \\
\hline & & 35.284 & -79.314 \\
\hline & & 38.976 & -76.749 \\
\hline & Park River NWR & 42.738 & -70.792 \\
\hline & Pterophylla Plant Nurse & 42.384 & -80.344 \\
\hline Grass Prairie Preserv & near Treesbank & 49.691 & -99.575 \\
\hline & & 34.504 & -80.225 \\
\hline & & 34.6 & -80.214 \\
\hline & & 45.0845 & -63.73 \\
\hline & & 49.147 & -96.68 \\
\hline & & 43.774 & -79.507 \\
\hline & & 41.607 & -87.286 \\
\hline & Great Smoky Mountain & 35.628 & -83.1 \\
\hline St. Ignace & & 46.707 & -71.054 \\
\hline & & 38.97 & -77.156 \\
\hline & & 30.386 & -88.753 \\
\hline & & 35.543 & -82.957 \\
\hline & & 39.963 & -75.177 \\
\hline E of Carlinville & & 39.26 & -89.549 \\
\hline & & 38.99 & -76.752 \\
\hline & & 39.551 & -79.299 \\
\hline & & 36.448 & -81.029 \\
\hline & & 39.056 & -76.82 \\
\hline & & 38.99 & -76.752 \\
\hline & York University Keele C & 43.775 & -79.504 \\
\hline roadside 321 & & 35.76 & -83.361 \\
\hline & & 37.938 & -75.318 \\
\hline Bryson City Rd \& Que & & 35.284 & -83.487 \\
\hline E of Carlinville & & 39.279 & -89.796 \\
\hline & & 39.248 & -78.527 \\
\hline Blue Mtn Parkway & & 36.342 & -81.375 \\
\hline York University & CSEB & 43.774 & -79.505 \\
\hline
\end{tabular}




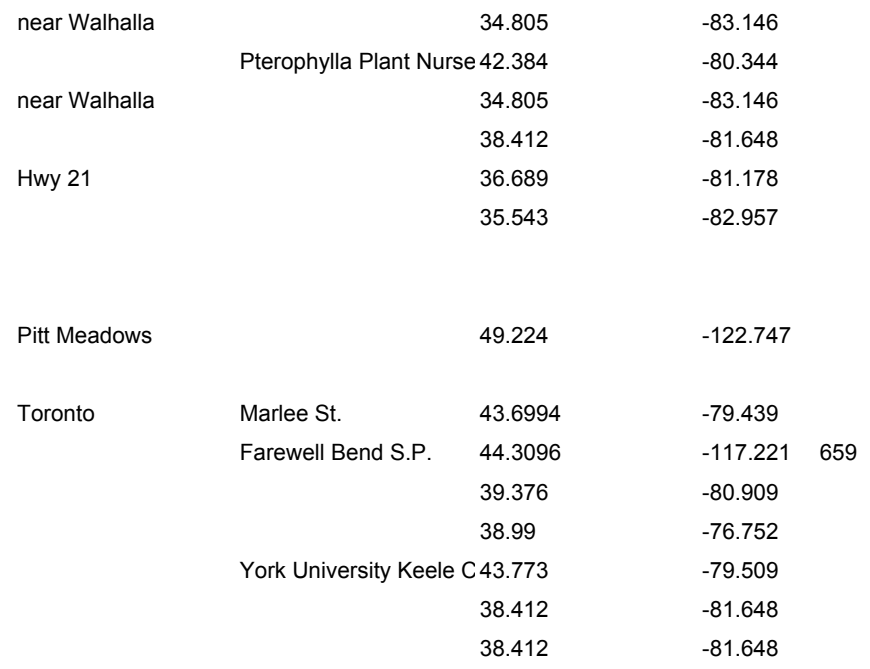


\begin{tabular}{l|l} 
Site Code & Collection Event ID \\
\hline
\end{tabular} 


\section{BOLD TaxonID Tree}

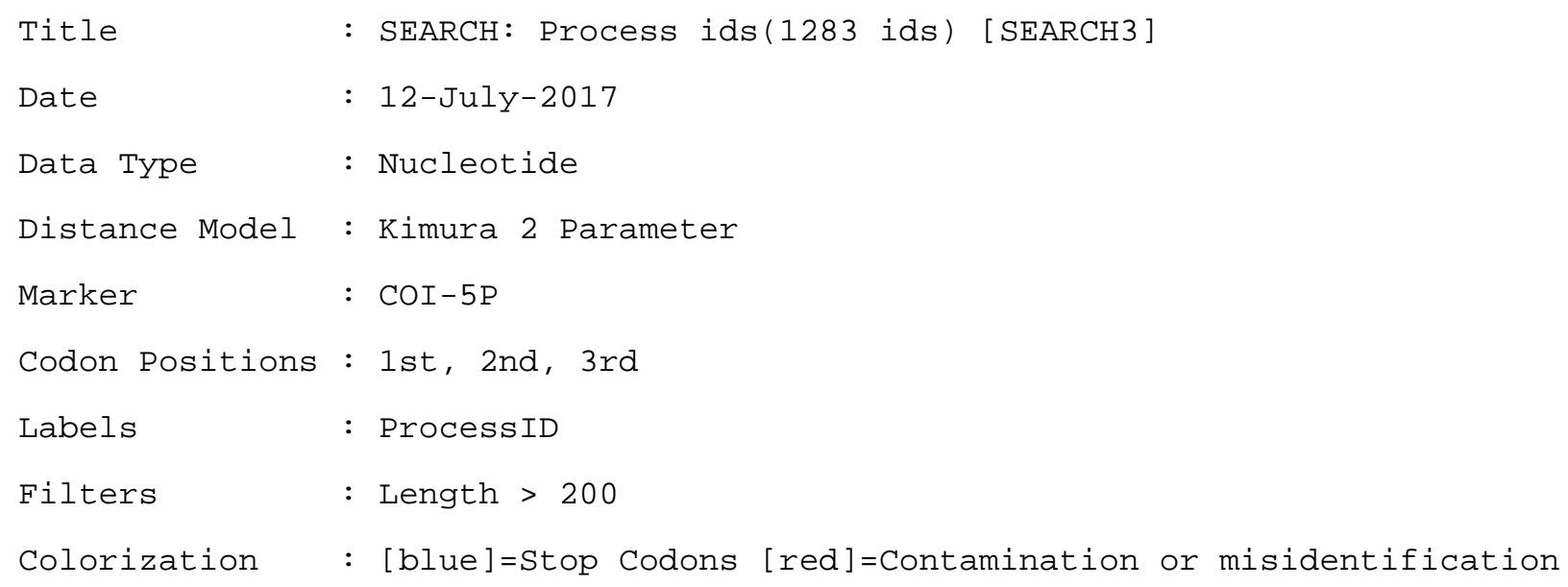


Genome

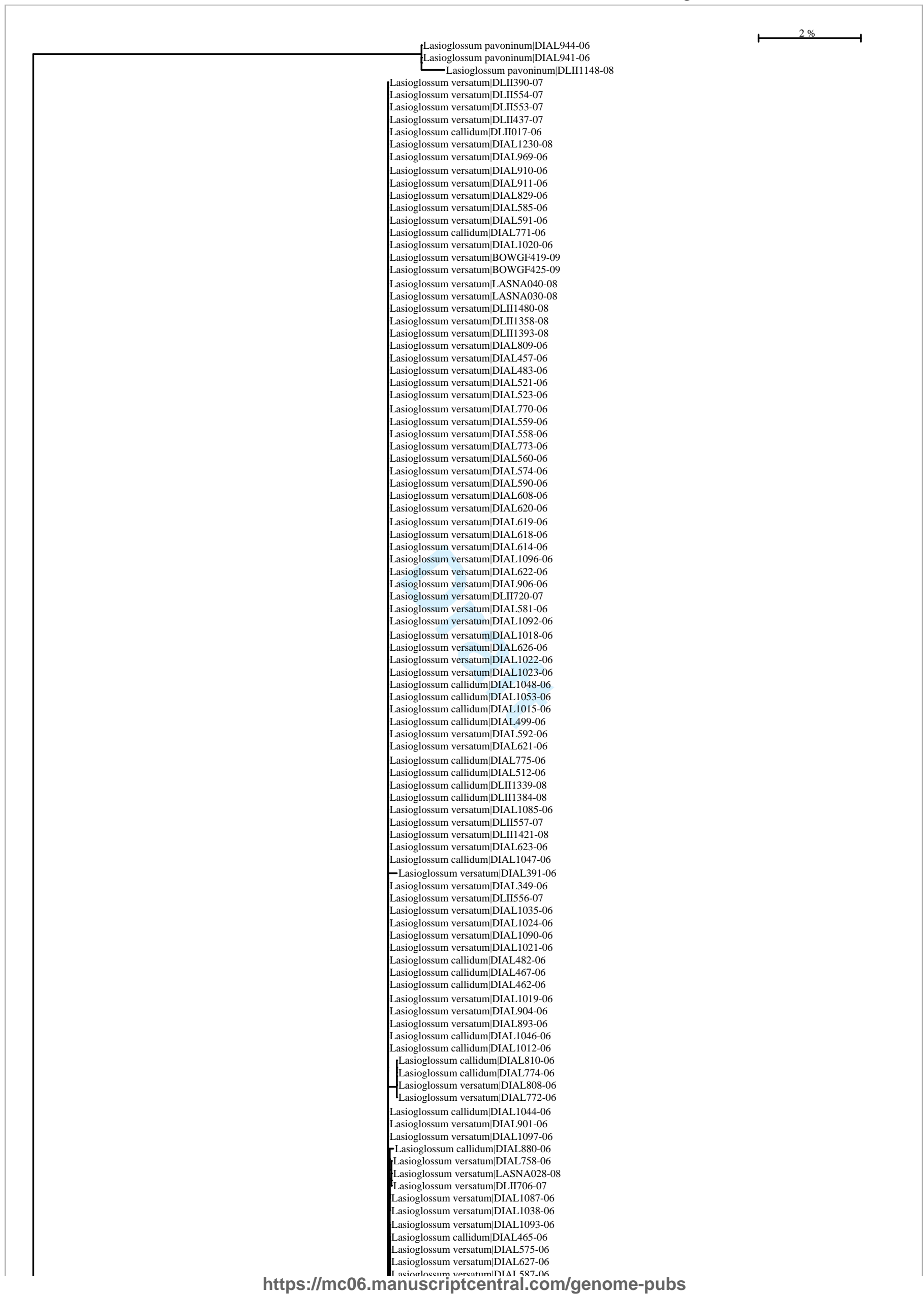




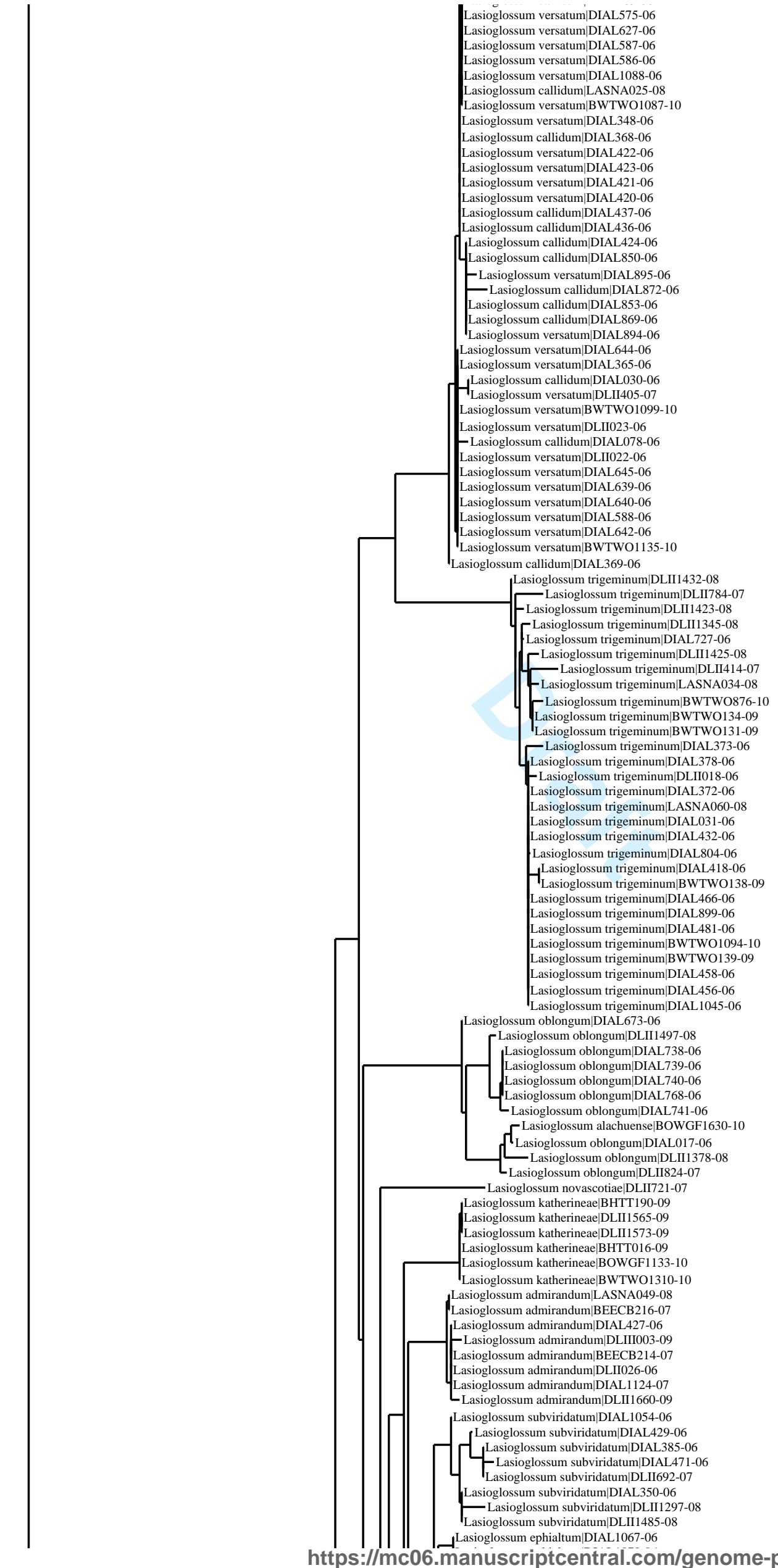

Lasioglossum versatum|DIAL575-06 Lasioglossum versatum|BWTW01087-10 callidum|DIAL369-06

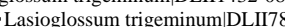

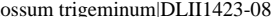
Lasioglossum trigeminum|DLIII345-08 作 - Lasioglossum trigeminum|BWTWO876-10 Lasioglossum trigeminum|BWTWO134-09 Laioglossm trigem - Lasioglossum trigeminum|DLII018-06 Lasioglossum trigeminum|DIAL372-06 Lasioglossum trigeminum|LASNA060-08 Lasioglossum trigeminum|DIAL432-06 Lasioglossum trigeminum|DIAL804-06 Lasioglossum trigeminum|DIAL418-06 ioglossum trigeminum|BWTWO138-09 Lasioglossum trigeminum|DIAL466-06 Lasioglossum trigeminum|DIAL481-06 Lasioglossum trigeminum|BWTWO1094-10 Lasioglossum trigeminum|BWTWO139-09 Lasioglossum trigeminum|DIAL458-06 Lasioglossum trigeminum|DIAL456-06 um oblongum|DIAL673-06 asioglossum oblongum|DLII1497-08 Lasioglossum oblongum|DIAL 738-06 Lasioglossum oblongum|DIAL 740-06 Lasioglossum admirandum|BEECB214-07 Lasioglossum subviridatum|DLII692-07

Lasioglossum subviridatum|DLI11297-08 


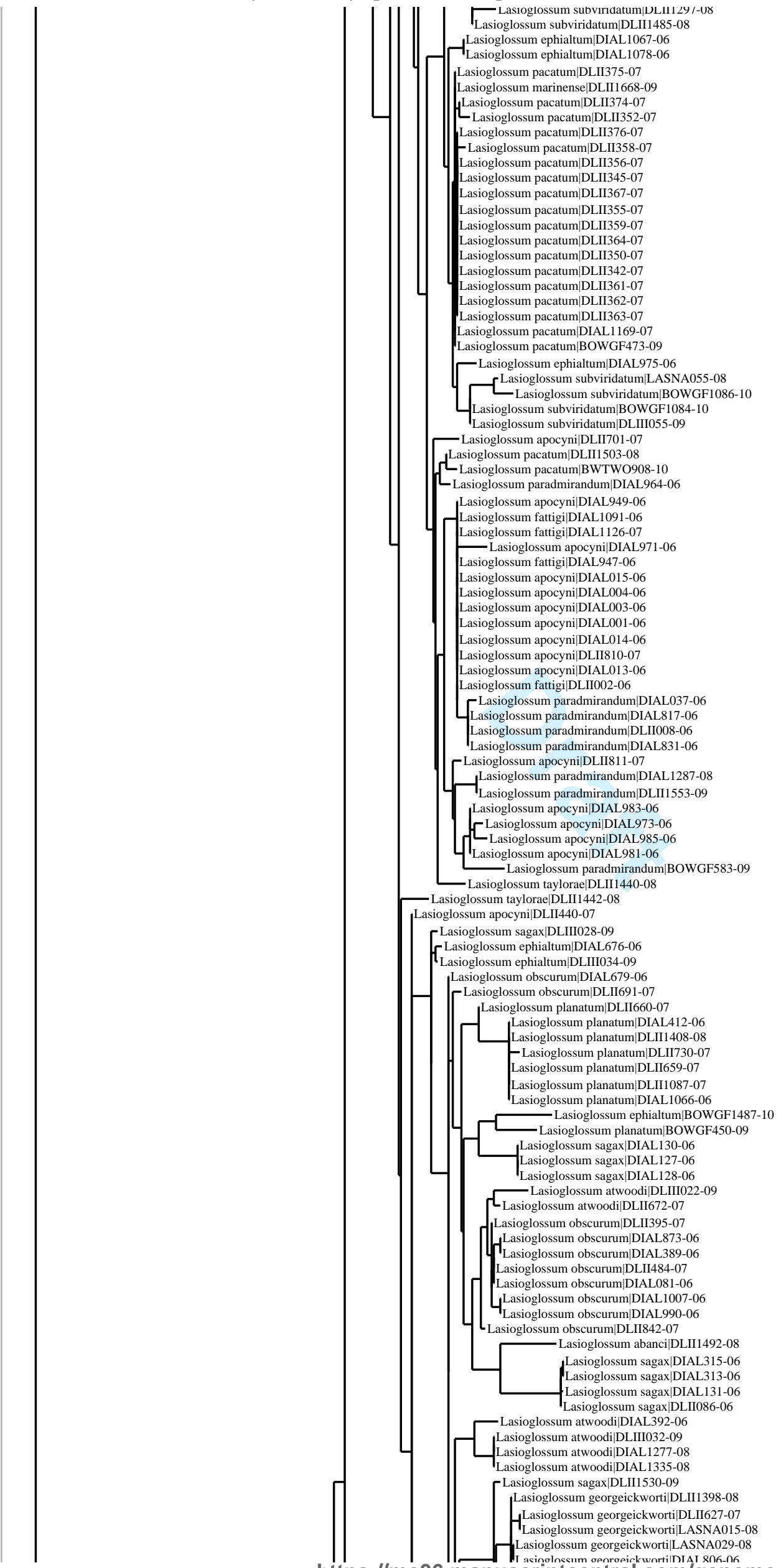




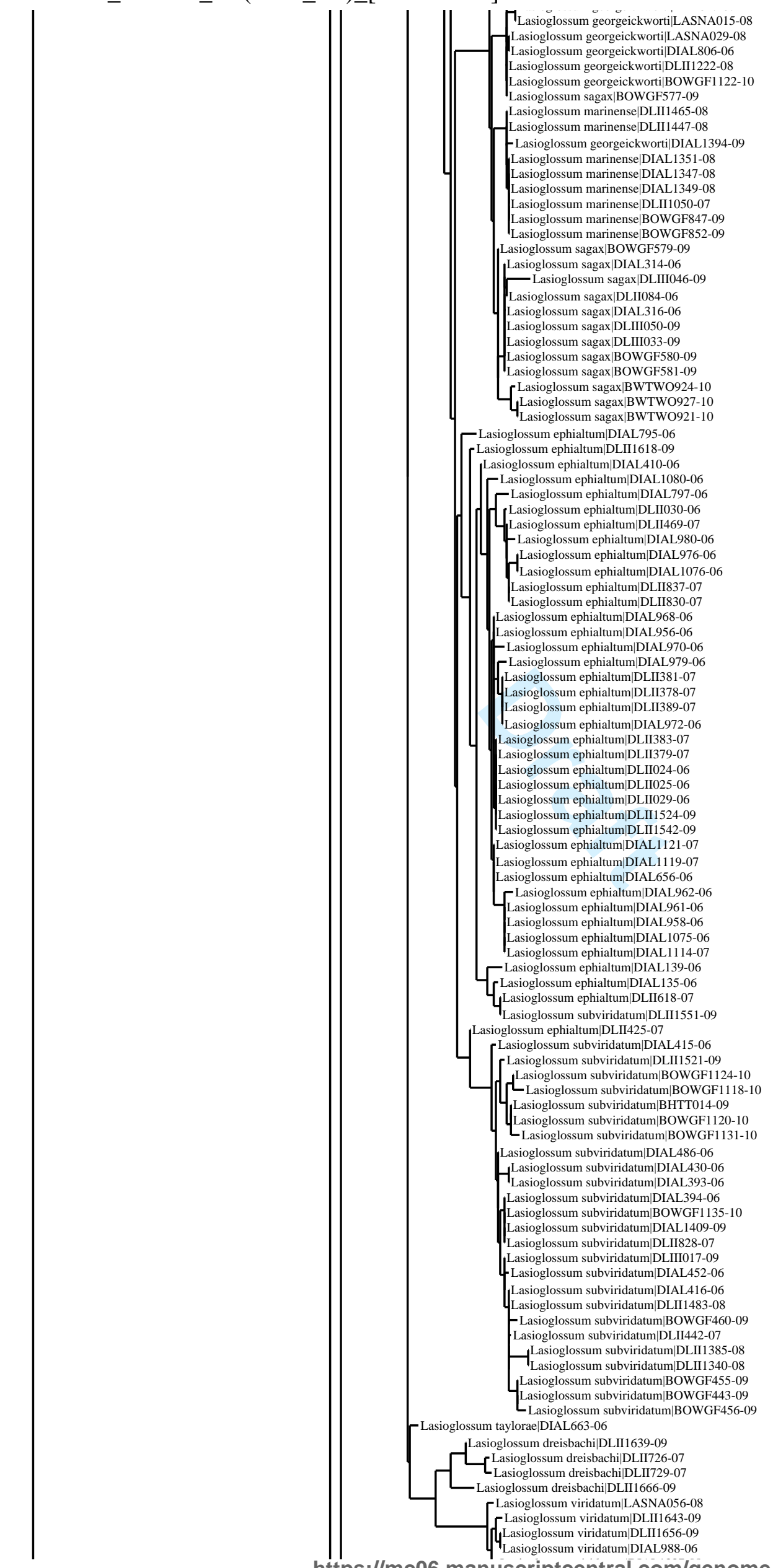




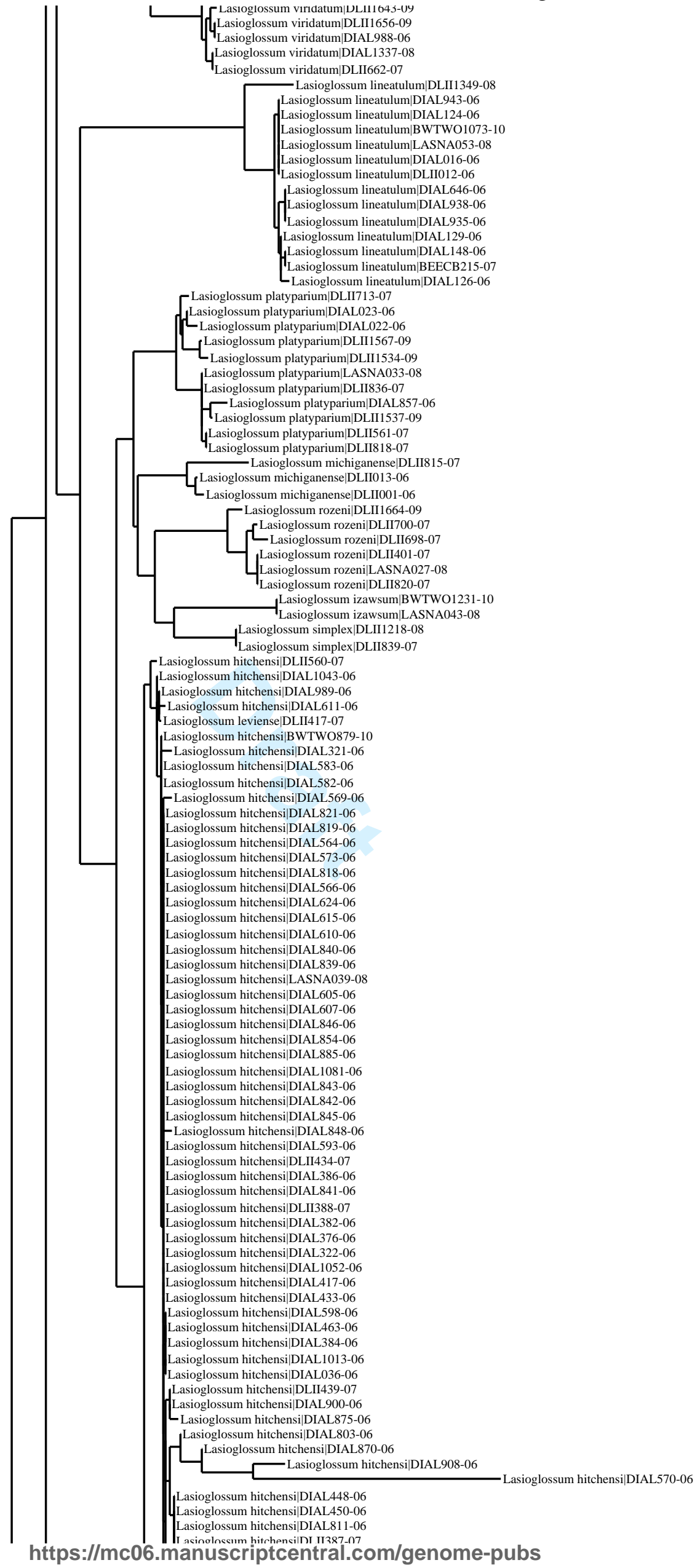




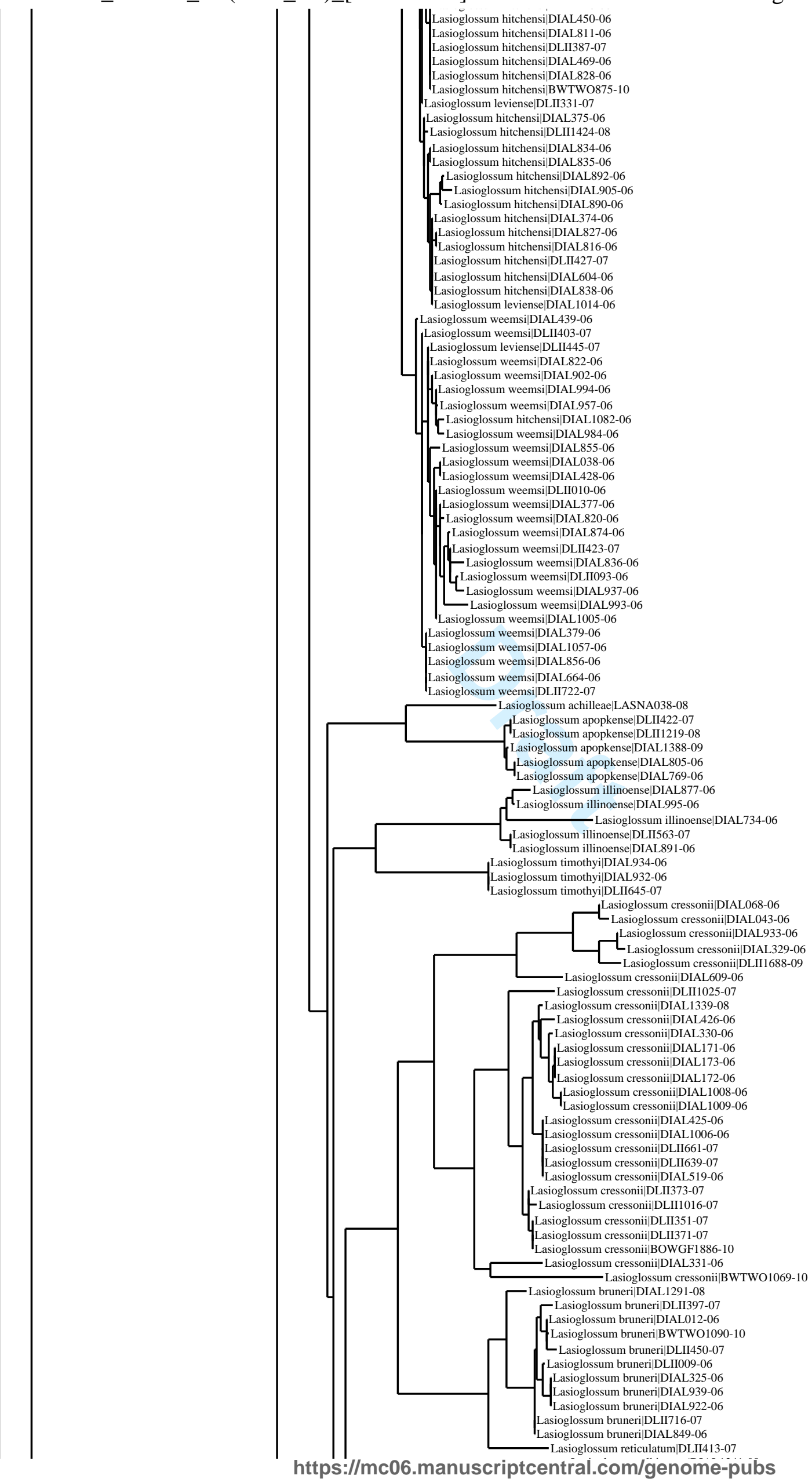


SEARCH:_Process_ids(1283_ids)_[SEARCH3] Wedome

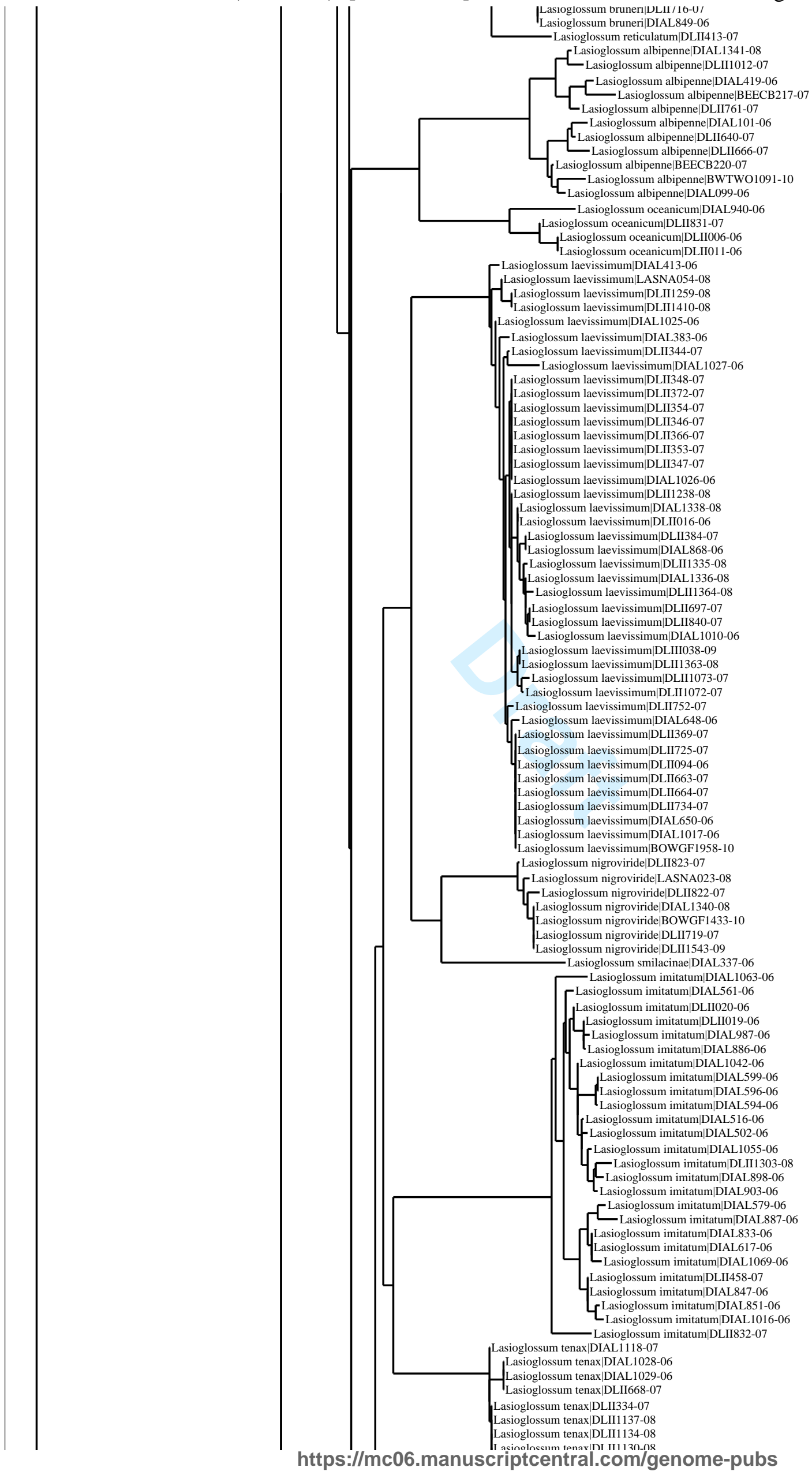

Page 446 of 452 


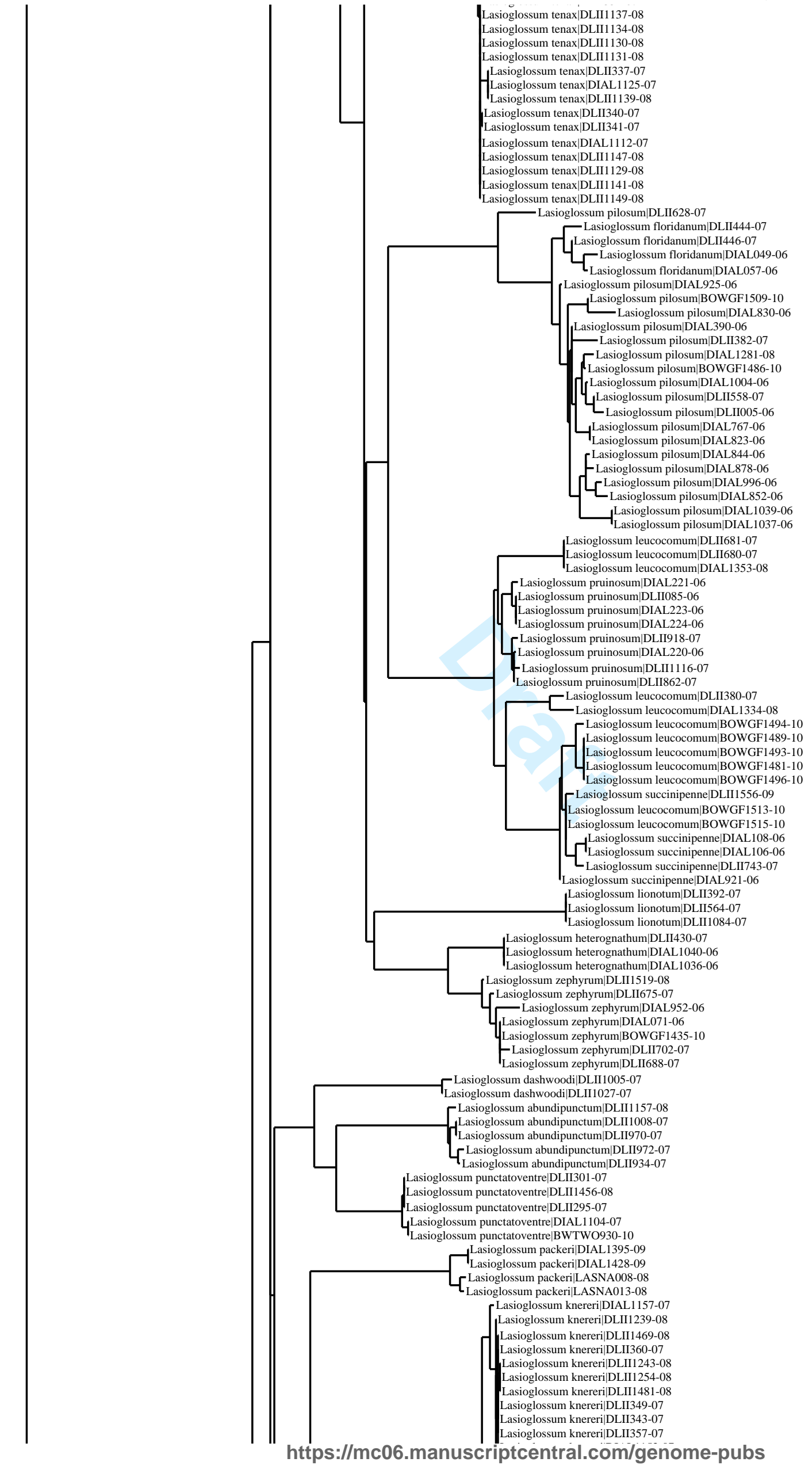




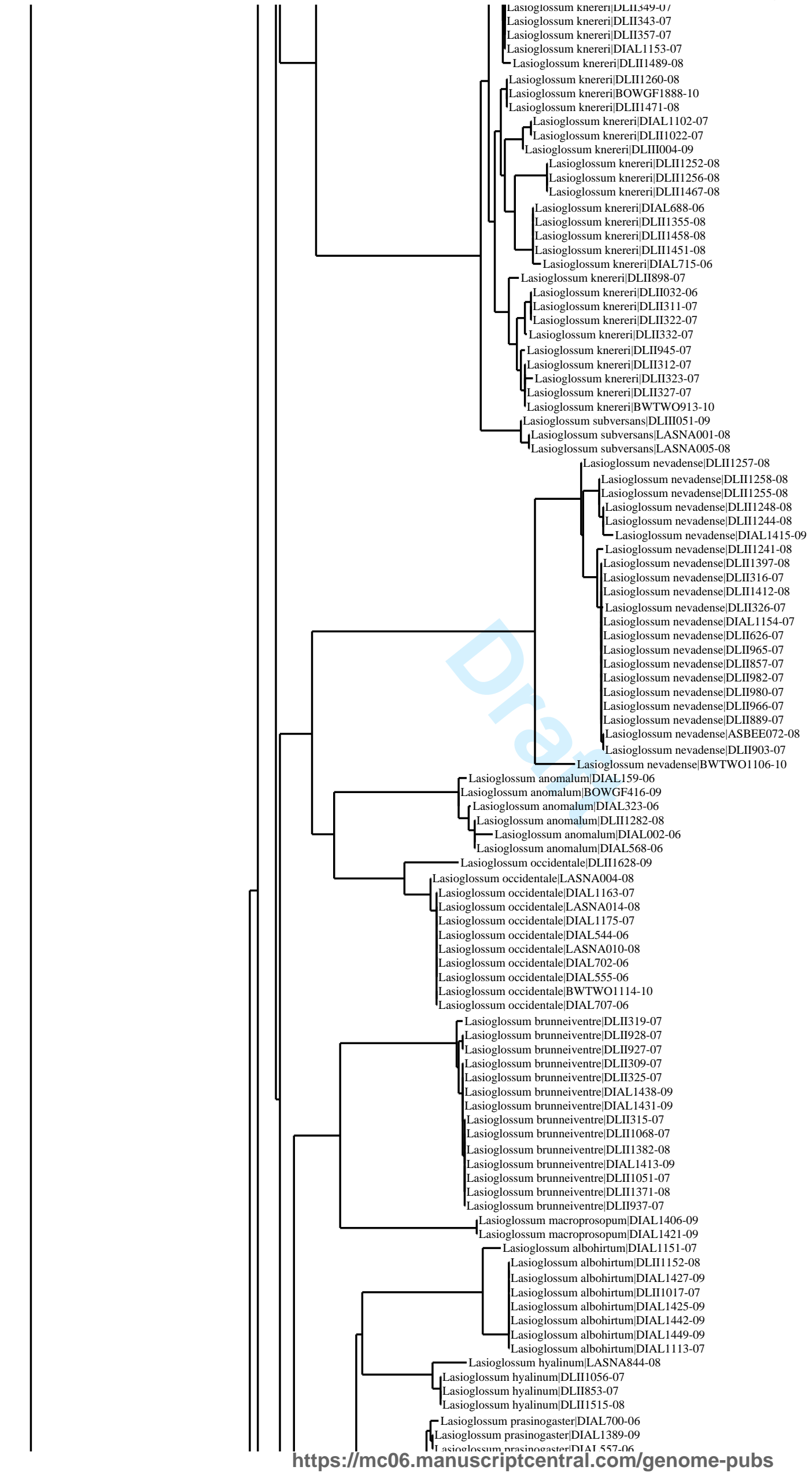




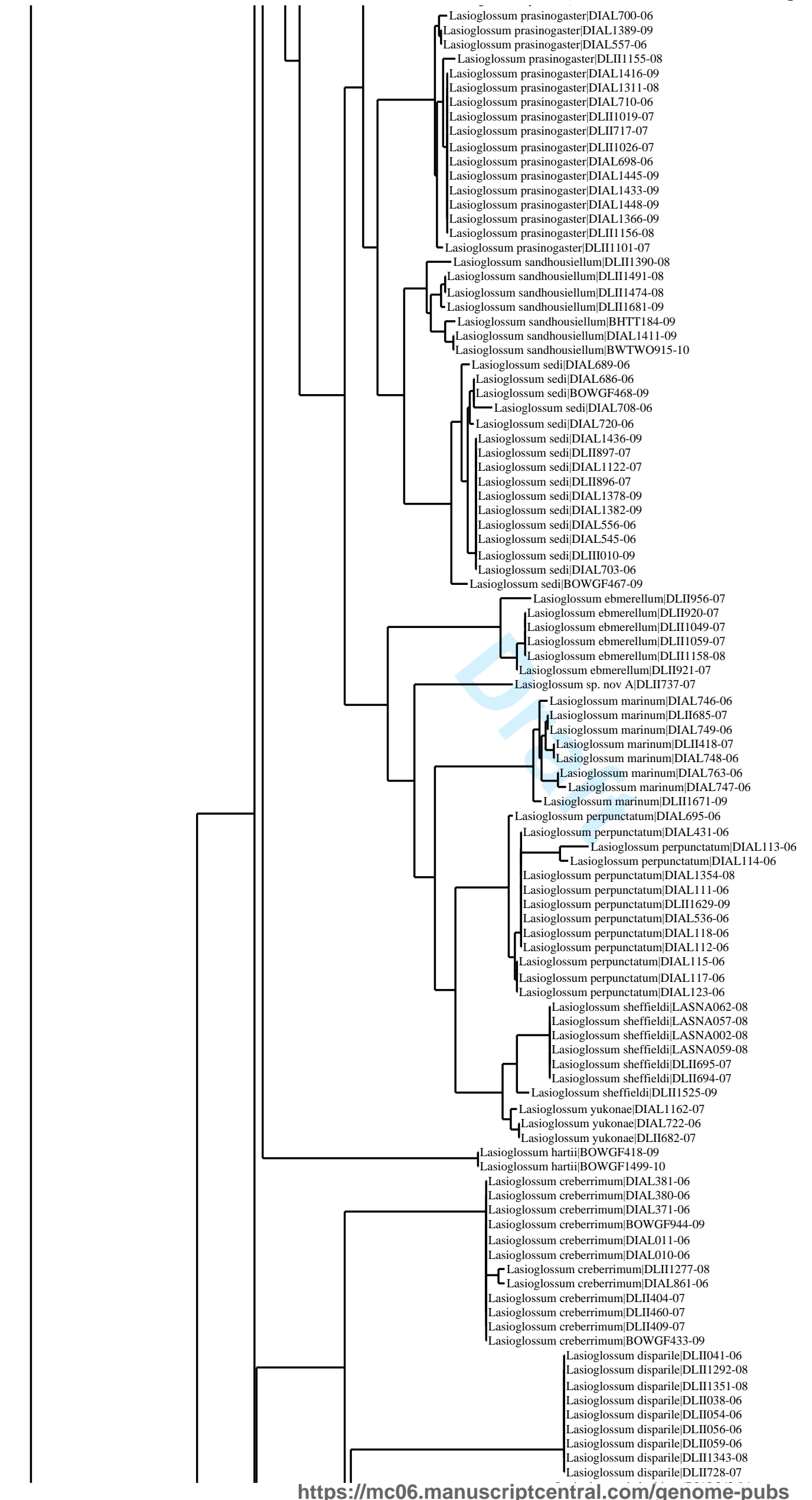




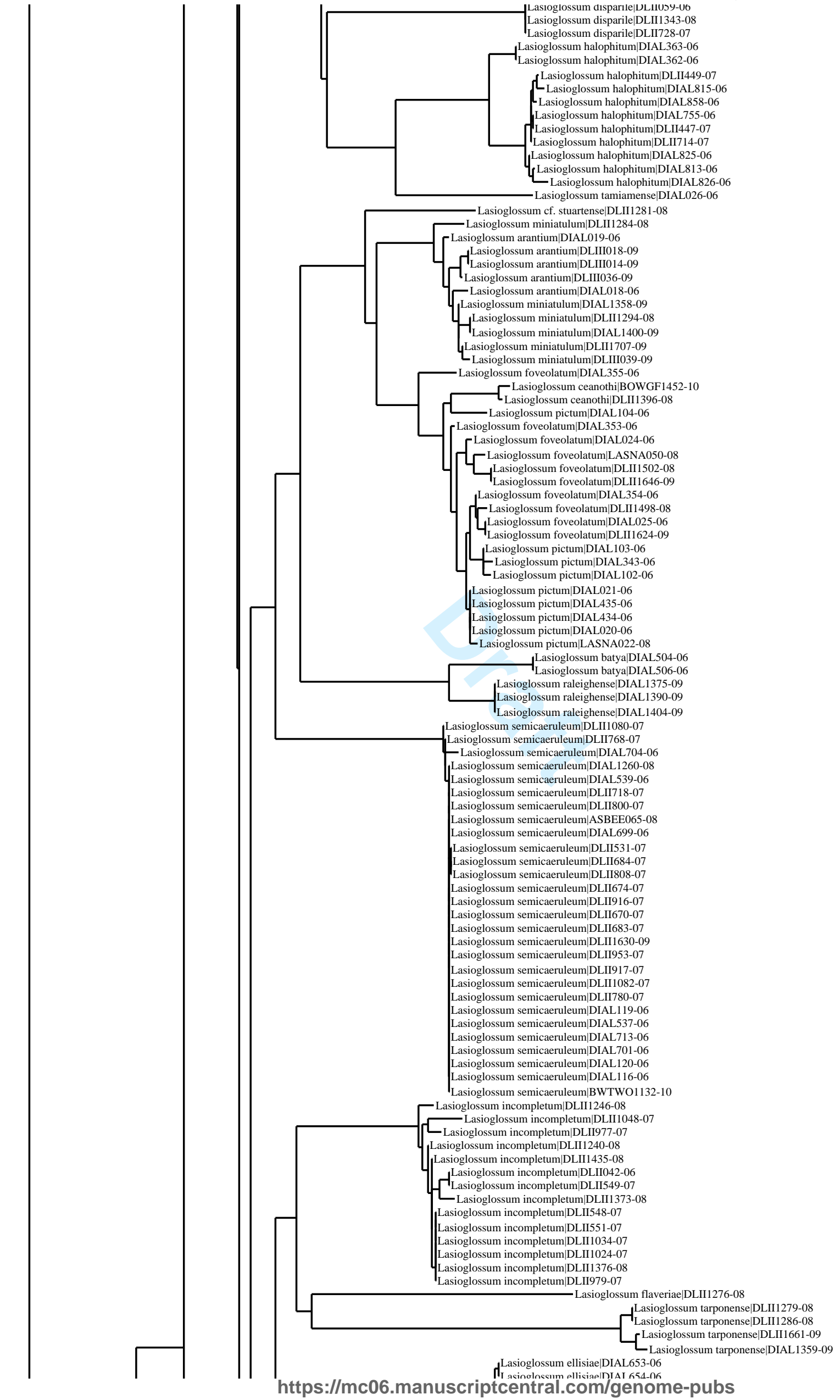




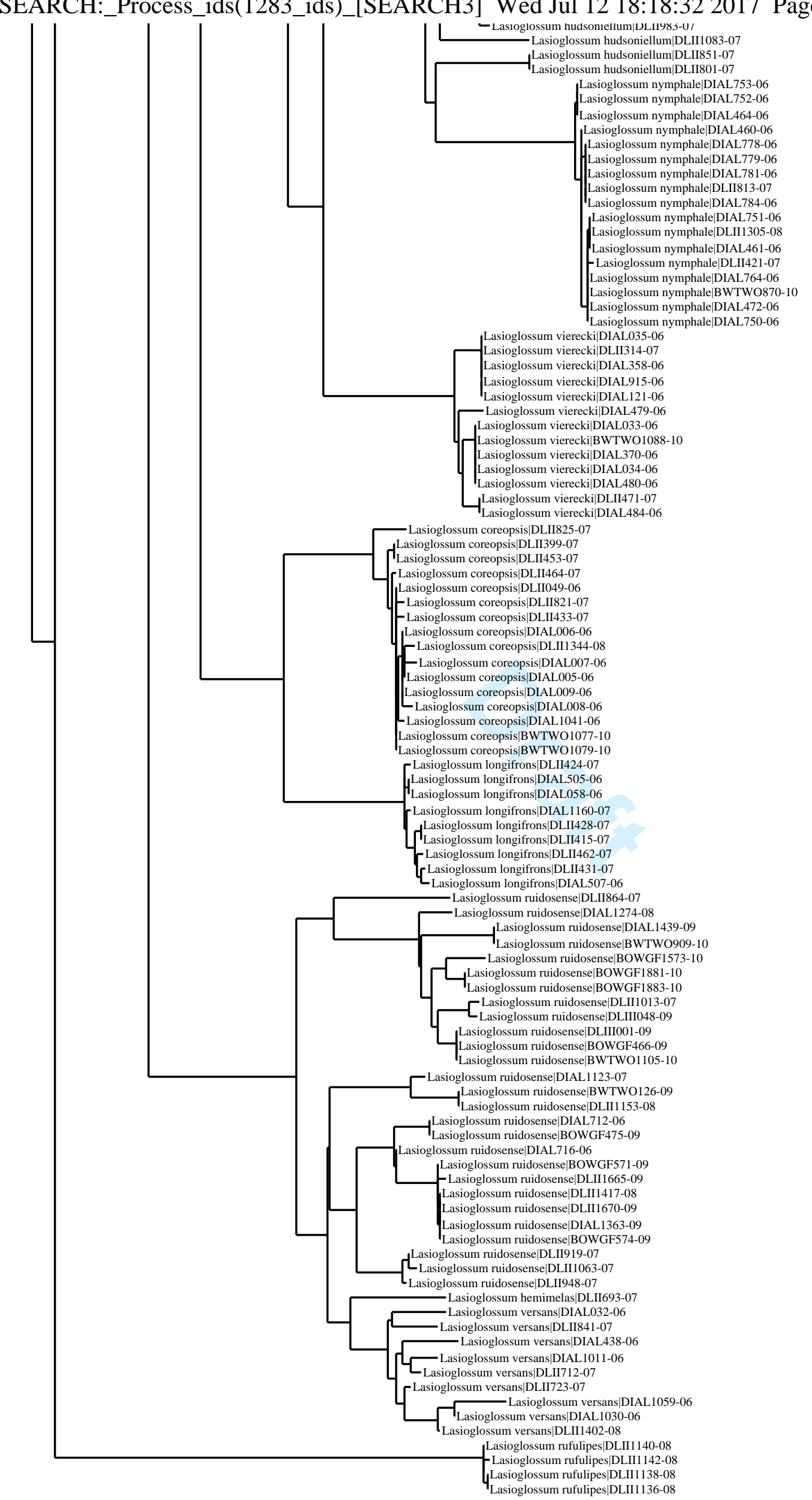

